Birte Arendt / Andreas Bieberstedt /

Klaas-Hinrich Ehlers (Hrsg.)

\title{
Niederdeutsch und regionale Umgangssprache in Mecklenburg-Vorpommern
}

Strukturelle, soziolinguistische und didaktische Aspekte

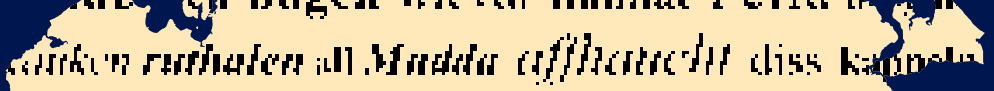

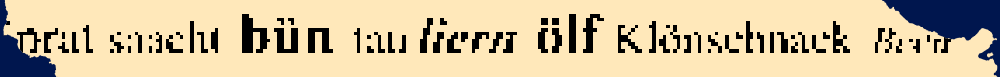

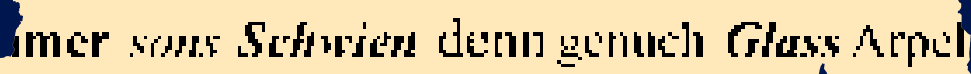

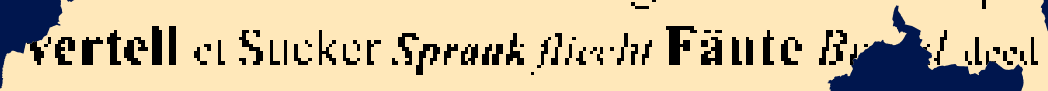

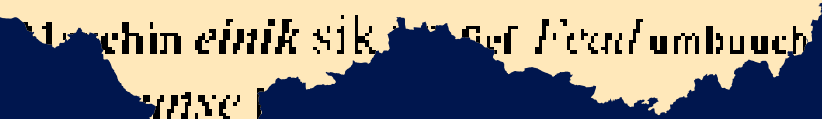


REGIONALSPRACHE UND REGIONALE KULTUR, MECKLENBURG-VORPOMMERN IM

OSTNIEDERDEUTSCHEN KONTEXT 1

\section{Birte Arendt / Andreas Bieberstedt / Klaas-Hinrich Ehlers (Hrsg.)}

\section{Niederdeutsch und regionale Umgangssprache in Mecklenburg-Vorpommern}

Im Mittelpunkt des Bandes steht der Wandel des Niederdeutschen und der regionalen Umgangssprache in MecklenburgVorpommern. Er vereint linguistische Beiträge, die Aspekte der Entwicklung und Struktur sowie des Gebrauchs regionaler Sprachformen in diesem Bundesland in den Blick nehmen. Der Fokus der Darstellungen liegt auf jüngeren und aktuellen Entwicklungen. Zugleich beziehen die Beiträge die Vorgeschichte der gegenwärtigen Sprachverhältnisse seit dem 19. Jahrhundert ein. Ein eigener Themenblock ist der institutionellen Vermittlung und Förderung des Niederdeutschen gewidmet. Damit trägt der Band der Tatsache Rechnung, dass sich die Vermittlung des Niederdeutschen heute zunehmend auf Bildungsinstitutionen verlagert und zum Gegenstand amtlicher Sprachpolitik geworden ist.

\section{Die Herausgeber}

Birte Arendt ist Wissenschaftliche Mitarbeiterin am Institut für Deutsche Philologie an der Universität Greifswald und Leiterin des Kompetenzzentrums Didaktik des Niederdeutschen. Ihre Forschungsfelder sind Spracheinstellungen, Spracherwerb und Regionalsprachvermittlung im Elementarbereich und in der Schule.

Andreas Bieberstedt ist Professor für niederdeutsche Sprache und Literatur an der Universität Rostock. Seine Schwerpunkte sind die Regionalsprachen- und Sprachbiographieforschung sowie die Sprachgeschichte des Niederdeutschen. Er beschäftigt sich unter anderem mit dem rezenten Wandel des Hamburgischen.

Klaas-Hinrich Ehlers ist Privatdozent für germanistische Linguistik an der Freien Universität Berlin. Er ist Mitarbeiter am Norddeutschen Sprachatlas und leitet ein von der Deutschen Forschungsgemeinschaft gefördertes Projekt zur Geschichte der mecklenburgischen Regionalsprache seit dem Zweiten Weltkrieg. 


\section{Niederdeutsch und regionale Umgangssprache}

in Mecklenburg-Vorpommern 


\section{REGIONALSPRACHE UND REGIONALE KULTUR MECKLENBURG-VORPOMMERN IM OSTNIEDERDEUTSCHEN KONTEXT}

Herausgegeben von Birte Arendt, Andreas Bieberstedt,

Klaas-Hinrich Ehlers, Christoph Schmitt

\section{BAND 1}

\section{PETER LANG}


Birte Arendt / Andreas Bieberstedt / Klaas-Hinrich Ehlers (Hrsg.)

\section{Niederdeutsch und regionale Umgangssprache in Mecklenburg-Vorpommern}

Strukturelle, soziolinguistische und didaktische Aspekte

\section{PETER LANG}




\section{Bibliografische Information der Deutschen Nationalbibliothek}

Die Deutsche Nationalbibliothek verzeichnet diese Publikation in der Deutschen Nationalbibliografie; detaillierte bibliografische Daten sind im Internet über http://dnb.d-nb.de abrufbar.

Die Umschlagabbildung wurde erstellt mit www.regionalsprache.de, Klaas-Hinrich Ehlers"

Der Druck dieses Bandes wurde ermöglicht durch eine finanzielle Förderung durch den Heimatverband Mecklenburg-Vorpommern e. V.

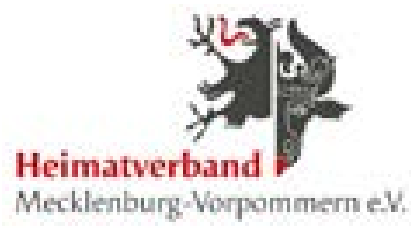

ISSN 2511-1213

ISBN 978-3-631-71824-7 (Print)

E-ISBN 978-3-631-71892-6 (E-PDF)

E-ISBN 978-3-631-71893-3 (EPUB)

E-ISBN 978-3-631-71894-0 (MOBI)

DOI 10.3726/b10975
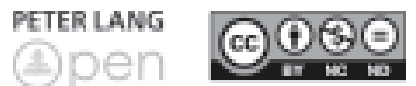

Open Access: Dieses Werk ist lizensiert unter der Creative Commons Lizenz Namensnennung - Nicht kommerziell - Keine Bearbeitungen 4.0 International (CC BY-NC-ND 4.0). Den vollständigen Lizenztext finden Sie unter: https://creativecommons.org/licenses/by-nc-nd/4.0/deed.de

(C) Birte Arendt / Andreas Bieberstedt / Klaas-Hinrich Ehlers, 2017

Peter Lang GmbH

Internationaler Verlag der Wissenschaften

Berlin

Peter Lang - Berlin · Bern · Bruxelles .

New York · Oxford $\cdot$ Warszawa $\cdot$ Wien

Diese Publikation wurde begutachtet.

www.peterlang.com 


\section{Inhaltsverzeichnis}

Birte Arendt, Andreas Bieberstedt, Klaas-Hinrich Ehlers

Niederdeutsch und regionale Umgangssprache in

Mecklenburg-Vorpommern: Einführende Bemerkungen 7

\section{Sprachraum, Sprachstrukturen und Sprachgebrauch in der Entwicklung}

Peter Rosenberg

Regionalsprache in Mecklenburg-Vorpommern

Matthias Vollmer

Anmerkungen zu den sprachlichen Verhältnissen Vorpommerns

in der ersten Hälfte des 19. Jahrhunderts

André Köhncke

Regionale Unterschiede und Veränderungen im Wortschatz

des Mecklenburgischen

Klaas-Hinrich Ehlers

Meihen, meiden, meigen ,mähen' - Zur Hiattilgung im

mecklenburgischen Niederdeutsch 99

Martin Hansen

Zum Wandel des Niederdeutschen auf der Insel Rügen zwischen

dem 19. und 21. Jahrhundert - Ein diachronischer Vergleich anhand ausgewählter Sprachmerkmale

Lars Vorberger

Hochdeutsch auf Rügen - Eine Untersuchung zum Regiolekt in Bergen

auf Rügen

Mona Biemann

„Wir haben's zwar gehört, konnten's verstehen und... aber selbst so richtig benutzt haben wir's auch nicht." Eine Fallstudie zum Wandel des Dialektgebrauchs in der Generationsfolge in einer mecklenburgischen Gemeinde 


\section{Förderung und Vermittlung des Niederdeutschen in Bildungsinstitutionen}

\section{Julia Mittelstädt}

Das Projekt „Niederdeutsch in der Kita“ - Bedingungen, Formen und Perspektiven der Niederdeutschvermittlung in Kindergärten Mecklenburg-Vorpommerns

Johanna Biedowicz

Frühkindlicher Niederdeutscherwerb. Eine exemplarische Studie zu einem Greifswalder Kindergarten

\section{Anne Fink}

Die Umsetzung der Verwaltungsvorschrift „Niederdeutsch in der Schule" - eine exemplarische Studie zum Niederdeutschangebot in den Schulen Greifswalds

Birte Arendt

Niederdeutsch als Studienfach - Formen, Entwicklungen und studentische Motivationen am Beispiel der Universität Greifswald

Hannah Reuter

Niederdeutschunterricht an der Volkshochschule - Kaffeeklatschersatz oder Spracherwerbskonzept mit Zukunft?

Regionalsprache in Mecklenburg-Vorpommern seit dem 18. Jahrhundert. Eine Bibliographie der sprachwissenschaftlichen Forschungsliteratur. Begründet von Ingrid Schröder, fortgesetzt von Andreas Bieberstedt und Klaas-Hinrich Ehlers 


\section{Birte Arendt, Andreas Bieberstedt, Klaas-Hinrich Ehlers}

\section{Niederdeutsch und regionale Umgangssprache in Mecklenburg-Vorpommern: Einführende Bemerkungen}

In den vergangenen Jahrzehnten nach 1945 und insbesondere seit den 1960er Jahren durchlief die Regionalsprache in Mecklenburg-Vorpommern, wie insgesamt im norddeutschen Raum, einen grundlegenden Wandelprozess. ${ }^{1}$ Dieser betrifft das gesamte in diesem Bundesland vorfindliche regionalsprachliche Spektrum, von den niederdeutschen (mecklenburgischen und pommerschen) Dialekten bis hin zu den hochdeutschen regionalen Umgangssprachen und Regionalakzenten. In Bezug auf die niederdeutschen Dialektvarietäten in Mecklenburg-Vorpommern lassen sich Veränderungen sowohl hinsichtlich deren Struktur und Wortschatz als auch in Hinsicht auf ihren Erwerb und Gebrauch sowie auf ihre Bewertung konstatieren. Der seit Jahrhunderten bestehende und in der Gegenwart zunehmend intensive Sprachkontakt zwischen Niederdeutsch und Hochdeutsch hat auf der strukturellen Ebene zu niederdeutsch-hochdeutschen Interferenzen geführt,

1 Unter Regionalsprache wird im Folgenden mit Lenz 2008, S. 2: „der regional markierte sprechsprachliche Gesamtbereich unterhalb der normierten und kodifizierten Standardsprache verstanden. Den tiefsten Pol einer Regionalsprache stellen die Lokaldialekte (Basisdialekte) dar, die mitunter von einem Ortspunkt zum nächsten variieren können. Sie weisen eine größere areale Vielfalt auf als die regionalen Umgangssprachen (Regiolekte), die oberhalb der Dialekte, aber unterhalb der Standardsprache anzusiedeln sind." Schmidt/Herrgen 2011, S. 66 zufolge handelt es sich bei einer Regionalsprache um ein ,vernetztes Gesamt an Varietäten und Sprechlagen, das horizontal durch die Strukturgrenzen der Dialektverbände/-regionen und vertikal durch Differenzen zu den nationalen Oralisierungsnormen der Standardvarietät begrenzt ist. " Ein Modell zur vertikalen Struktur der Regionalsprache im Norden der DDR (im Wesentlichen deckungsgleich mit dem heutigen Bundesland Mecklenburg-Vorpommern) schlägt Dahl 1974, S. 343-345 vor (ähnlich Gernentz 1975a). Dahl unterscheidet diesbezüglich niederdeutsche Mundart, Niederdeutsch mit hochdeutscher Beimischung, Hochdeutsch mit niederdeutschen Beimischungen (mundartnahe Umgangssprache), hochsprachennahes Hochdeutsch (umgangssprachliches Hochdeutsch), hochsprachliches Hochdeutsch (hochsprachliche Norm). Für die gegenwärtige Sprachsituation in Mecklenburg-Vorpommern bedarf Dahls Modell einer Überprüfung bzw. gegebenenfalls Modifizierung auf Basis aktuellen Datenmaterials, vgl. hierfür z. B. den Beitrag von Vorberger in diesem Band. 
die sich als Transfer hochdeutscher Elemente ins Niederdeutsche im lautlichen, morphologischen und lexikalischen Bereich, zum Teil auch in strukturellen Angleichungen auf syntaktischer Ebene äußern.

Solche Ausgleichserscheinungen sind für die regionalen Dialekte in Mecklenburg-Vorpommern bereits seit den 1970er Jahren wissenschaftlich beschrieben worden. ${ }^{2}$ Sie bewirken einen sukzessiven Abbau dialektaler Elemente und deren Ersetzung durch hochdeutsche oder hochdeutsch-nähere Formen und damit insgesamt eine Advergenz des Basisdialekts an das Standardhochdeutsche. ${ }^{3}$ Dieser für den gesamten norddeutschen Sprachraum zu beobachtende dialektale Abbauprozess zieht seinerseits Veränderungen in der dialektalen Raumstruktur nach sich. Dialektgrenzen verschieben sich bzw. lösen sich zum Teil auf, ${ }^{4}$ insgesamt werden kleinräumige Dialekte und Ortsmundarten zunehmend durch dialektale und regiolektale Sprachformen mit größerer kommunikativer Reichweite ersetzt. ${ }^{5}$ Zugleich ergeben sich Veränderungen im Bereich der regional geprägten hochdeutschen Umgangssprache, die sich zu einer standardnahen Varietät mit Restmerkmalen eines niederdeutschen Substrats und vereinzelten Markern der norddeutschen Regiolekte entwickelt hat. Insgesamt scheint sich auf diese Weise das Spektrum regionaler Sprechlagen kontinuierlich in Richtung hochdeutscher Standardsprache zu verschieben. Die Analyse und Beschreibung dieses sich wandelnden Gefüges an regionalen Sprachformen bildet eine der vordringlichsten Aufgaben der aktuellen Regionalsprachenforschung. Zudem steht nicht zu erwarten, dass die regionale Markierung des Sprechens ihre kommunikativpragmatischen Funktionen gänzlich verlieren wird und es zu einer vollständigen Aufgabe regionalsprachlicher Formen kommt. Vielmehr zeichnet es sich ab, dass diese auch neue, unter anderem sozialsymbolische Funktionen übernehmen. Die weitere Entwicklung der Regionalsprache in Mecklenburg-Vorpommern wird folglich ein spannendes Forschungsfeld der regional bezogenen Sprachwissenschaft bleiben, das eng mit den gesellschaftlichen und kulturellen Entwicklungen in der Region verschränkt ist.

Besonders gravierende Veränderungen zeigen sich beim Gebrauch des Niederdeutschen. Auf der kommunikativen Ebene führt der niederdeutschhochdeutsche Sprachkontakt bei Sprechern mit niederdeutscher Dialektkompetenz zu einer Sprachwahl zwischen Niederdeutsch und Hochdeutsch, die

2 Dahl 1974; Gernentz 1974.

3 Zum Begriff der Advergenz vgl. Vorberger/Schröder 2011, S. 137.

4 Vgl. Köhncke 2010, S. 534.

5 Vgl. Herrmann-Winter 1974, S. 173.

Birte Arendt, Andreas Bieberstedt and Klaas-Hinrich Ehlers - 978-3-631-71893-3 
situativ, adressatenabhängig und funktional gesteuert ist, sowie zu Kommunikationsstrategien, die durch Formen von Codeswitching, Codemixing und Codeshifting gekennzeichnet sind. ${ }^{6}$ Vor allem jedoch ist auf die rapid abnehmende Zahl aktiver Dialektsprecher in Mecklenburg-Vorpommern zu verweisen. Im Jahre 2007 führte das Bremer Institut für niederdeutsche Sprache eine repräsentative Umfrage in den norddeutschen Bundesländern zur Kenntnis, Verwendung und Bewertung des Niederdeutschen durch. ${ }^{7}$ Im Vergleich der norddeutschen Bundesländer zählt Mecklenburg-Vorpommern diesem Sprachzensus zufolge zu den Regionen mit relativ hoher Dialektstärke (nach dem dialektstarken Bundesland Schleswig-Holstein, aber vor dialektschwachen Bundesländern wie Brandenburg und Sachsen-Anhalt sowie den norddeutschen Stadtstaaten Hamburg und Bremen). Dabei ist zu bedenken, dass Norddeutschland insgesamt eine signifikant niedrigere Dialektstärke, d. h. eine prozentual geringere Zahl an Dialektsprechern sowie eine geringere Präsenz des Dialektes in der öffentlichen Kommunikation, als insbesondere die oberdeutschen Dialektregionen aufweist. Laut dem Sprachzensus des Instituts für niederdeutsche Sprache können aktuell 46 \% der norddeutschen Bevölkerung das Niederdeutsche gut oder sogar sehr gut verstehen, ${ }^{8}$ lediglich $14 \%$ der Befragten jedoch geben von sich an, es gut oder sogar sehr gut zu sprechen. ${ }^{9}$ Zum Vergleich: In einer ähnlich angelegten statistischen Erhebung aus dem Jahre 1984, ${ }^{10}$ in die allerdings lediglich die alten Bundesländer einbezogen waren, gaben immerhin noch $35 \%$ der Befragten an, Niederdeutsch gut oder sehr gut sprechen zu können. ${ }^{11}$ Dies bedeutet einen signifikanten Rückgang der Sprecherzahl um mehr als die Hälfte innerhalb von nur einer Generation. Unterstrichen wird die Brisanz dieses Prozesses noch dadurch, dass die Dialektkompetenz heute primär altersabhängig ist. Die Kenntnis des Niederdeutschen und die Fähigkeit zu seiner Verwendung sind in hohem Maße eine Frage der

6 Vgl. schon die frühe Untersuchung von Dahl 1974.

7 Möller 2008; Möller 2010.

8 Möller 2010, S. 152. Ein etwas anderes Bild zeigt eine repräsentative Nachfolgeerhebung, die das Institut für niederdeutsche Sprache zusammen mit dem Institut für Deutsche Sprache im Jahre 2016 durchführte. Dieser Erhebung zufolge liegt das Bundesland Mecklenburg-Vorpommern hinsichtlich der passiven Dialektkompetenz („Verstehen“) an der Spitze aller norddeutschen Bundesländer. 70,1 \% aller Befragten aus Mecklenburg-Vorpommern gaben hierbei von sich an, das Niederdeutsche gut oder sehr gut zu verstehen. Vgl. Institut für niederdeutsche Sprache 2016.

9 Möller 2010, S. 153.

10 Vgl. Stellmacher 1987.

11 Möller 2010, S. 154. 
Generationenzugehörigkeit und nunmehr weitgehend auf die ältere und älteste Sprechergeneration beschränkt. ${ }^{12}$

Die auch in Mecklenburg-Vorpommern stark gesunkene Dialektkompetenz innerhalb der jüngeren Bevölkerungsschichten hängt eng mit einem grundlegenden Wandel dialektaler Spracherwerbsmechanismen zusammen. „Klassische“ Spracherwerbsformen, die bis in die zweite Hälfte des 20. Jahrhunderts dominierten und bei denen der Dialekt als Erstsprache im Elternhaus, das Hochdeutsche hingegen parallel als zweite Erstsprache oder zu einem späteren Zeitpunkt als Zweitsprache in der Schule vermittelt wurde, sind durch neuere Formen abgelöst worden, bei denen die hochdeutsche Standardsprache den wesentlichen Fixpunkt für die Sprachsozialisation bildet. ${ }^{13}$ Der Erwerb des Hochdeutschen als Erstsprache stellt in der Gegenwart den absoluten Regelfall dar. Zugleich eignen sich Dialektsprecher in Mecklenburg-Vorpommern das Niederdeutsche häufig nicht mehr parallel zum Hochdeutschen an, sondern sukzessiv in neuen Erwerbszusammenhängen. Auf diese Weise verlieren die herkömmlichen dialektalen Sprachvermittler innerhalb der Familie stark an Bedeutung und es treten neue in Erscheinung. Hingewiesen sei hier vor allem auf die wachsende Relevanz vorschulischer und schulischer, aber auch universitärer Institutionen für die Vermittlung dialektaler Kompetenzen und Kenntnisse oder auch nur für eine Sensibilisierung für regionale Sprachformen. Gestützt zum Teil durch staatliche Sprachpolitik bemühen sich gegenwärtig verstärkt Bildungsinstitutionen darum, den Ausfall der familiären Sprachvermittler bei der primären intergenerationellen Vermittlung der Niederdeutschkompetenz durch einen gesteuerten sekundären Spracherwerb zu kompensieren. ${ }^{14}$

Als Folge der hier skizzierten Prozesse zieht sich das Niederdeutsche in Mecklenburg-Vorpommern seit Längerem fortschreitend aus der Öffentlichkeit zurück und hat seine Funktion als ein Mittel der Alltagskommunikation bereits weitgehend eingebüßt. ${ }^{15}$ Rückzugsorte des Dialektes sind der private Bereich, d.h. Familie und Verwandtschaft, Nachbarschaft und Freundeskreis. Auch in den Medien und im Bereich öffentlicher Kultur ist das Niederdeutsche weiterhin

12 Möller 2010, S. 155.

13 Vgl. hierzu Gernentz 1974, S. 240-241 sowie mit Bezug auf das hamburgische Niederdeutsch Bieberstedt 2015.

14 Möller 2010, S. 154-155. Zu Mecklenburg-Vorpommern vgl. Arendt/Gąszczyk 2015.

15 Wissenschaftliche Beobachtungen über einen Rückzug des Dialektes aus der Alltagskommunikation in der Region Mecklenburg-Vorpommern reichen bis in das Ende der 1960er Jahre zurück, vgl. Gernentz 1969, daneben auch Gernentz 1974; Gernentz 1975b; Herrmann-Winter 1979. 
präsent. ${ }^{16}$ Im selben Maße, in dem das Niederdeutsche jedoch an Relevanz als öffentliche Alltagssprache verliert, gewinnt es seit einigen Jahren an Bedeutung als eine Identifikationssprache. ${ }^{17}$ Die mehrhundertjährige Stigmatisierung des niederdeutschen Dialektes weicht seit längerer Zeit zunehmend seiner sozialsymbolischen Aufwertung als Ausdrucksmittel regionaler Identität und Kultur. ${ }^{18}$ Die vielfältigen Aktivitäten von Kulturvereinen und Bildungseinrichtungen zur Pflege und Weitervermittlung des regionalen Niederdeutsch legen davon ebenso Zeugnis ab wie das wachsende Interesse vieler Menschen an der heimatlichen Mundart sowie an entsprechenden Bildungsangeboten. Auf Seiten der Politik zeugen die Verankerung des Sprachenschutzes und der Niederdeutschpflege in die Landesverfassung von Mecklenburg-Vorpommern ${ }^{19}$ sowie die Aufnahme des Niederdeutschen als Regionalsprache in die EU-Charta der Regional- oder Minderheitensprachen im Jahre $1999^{20}$ von der gestiegenen Wertschätzung gegenüber regionalen Sprachformen. ${ }^{21}$ Der Erwerb und Gebrauch des Niederdeutschen stellt in der Gegenwart keine durch die elterliche Spracherziehung und die kindliche Umgebung bedingte Zwangsläufigkeit mehr dar, sondern zeigt sich vielmehr immer öfter als Ausdruck einer bewussten Entscheidung einzelner Personen für diese Sprachvarietät, die mit deren regionalen Identifikationspotential zusammenhängt. Damit zeichnen individuelle biographische Faktoren und persönliche Interessen im weitaus stärkeren Maße als früher für das Erlernen, die Nutzung und die Weitervermittlung des Niederdeutschen verantwortlich.

Der hier skizzierte Wandel der Regionalsprache ist zwar vor allem in Hinblick auf die Entwicklung des Niederdeutschen in seinen Grundzügen bekannt, aber von der Forschung bislang noch nicht hinreichend untersucht worden. Die Forschungslage zu den regionalen Varietäten in Mecklenburg-Vorpommern ist, trotz einer vergleichsweise weit zurückreichenden Forschungstradition sowie aufschlussreicher Einzelstudien und Forschungsprojekte in den vergangenen Jahren, immer noch als unzureichend zu bezeichnen. ${ }^{22}$ Ältere Arbeiten des 20. Jahr-

16 Vgl. dazu Arendt 2010, S. 217-239.

17 Vgl. dazu die Untersuchung zum Niederdeutschwandel in Hamburg von Jürgens 2015.

$18 \mathrm{Zu}$ dieser Entwicklung Schröder/Niebaum 2008, S. 7. Ebenso Möller 2010, S. 142-143.

19 So heißt es bereits in der Landesverfassung von 1993 in Artikel 16, Absatz 2: „Das Land schützt und fördert die Pflege der niederdeutschen Sprache."

20 Vgl. Europarat 1998.

21 Zum Niederdeutschdiskurs in der Politik vgl. Arendt 2010, S. 241-273.

22 Vgl. Scharioth 2015, S. 3. Nachfolgend werden lediglich einzelne relevante Studien zu den jeweiligen Forschungsbereichen aufgelistet, eine umfassende Beschreibung der Forschungssituation kann an dieser Stelle nicht geleistet werden. Vgl. dazu die Forschungsbibliographie in diesem Band. 
hunderts fokussieren die neuniederdeutschen Dialektvarietäten in Mecklenburg und Vorpommern unter dialektgeographischen ${ }^{23}$ sowie unter strukturellen, vor allem lautlichen Gesichtspunkten. ${ }^{24}$ Soziolinguistische Arbeiten zur Verwendung und Bewertung des Niederdeutschen und der regionalen Umgangssprachen im Norden der DDR ${ }^{25}$ sowie Untersuchungen zu dialektal-hochdeutschen Sprachkontakten und Sprachmischungen ${ }^{26}$ liegen seit den 1970er Jahren vor. Auch die Dialektlexikographie kann mit dem Mecklenburgischen und dem Pommerschen Wörterbuch, deren Vorarbeiten bis in das beginnende 20. Jahrhundert zurückreichen, auf eine lange Forschungstradition verweisen. Ähnliches gilt für ethnographische Studien in der Tradition und Nachfolge Richard Wossidlos. ${ }^{27}$

Aktuelle Studien zur rezenten Entwicklung sowie zum Wandel des regionalsprachlichen Spektrums in Mecklenburg-Vorpommern liegen dagegen nur in vergleichsweise bescheidener Zahl vor. An neueren dialektgeographischen Studien zur Struktur und Verteilung der neuniederdeutschen Mundarten in MecklenburgVorpommern sind die breit angelegten Untersuchungen von Köhncke zum Mecklenburgischen, der Sprachatlas von Herrmann-Winter zum Pommerschen sowie die diachrone Studie von Hansen zum Niederdeutschen auf der Insel Rügen hervorzuheben. ${ }^{28}$ Die rezente regionale Umgangssprache Vorpommerns am Beispiel von Stralsund fokussiert Kehrein..$^{29}$ Dialektlexikographisch angelegt sind die Arbeiten von Vollmer, ebenfalls zu den pommerschen Mundarten..$^{30}$ Über den Stand des Mecklenburgisch-Vorpommerschen in Bezug auf Dialektkompetenz und -gebrauch informiert der bereits angesprochene Sprachzensus aus dem Jahre 2007. Das gesamte regionalsprachliche Spektrum in Norddeutschland von den Basisdialekten bis zu den standardnahen Sprechlagen nimmt das von der Deutschen Forschungsgemeinschaft geförderte, universitätsübergreifende Projekt „Sprachvariation in Norddeutschland“ in den Blick. ${ }^{31}$ In diesem seit 2008 laufenden Forschungsprojekt, das sowohl die areale Differenzierung der Regionalsprache in Norddeutschland als auch Aspekte der Sprachvariation, des

23 Vgl. z. B. Brose 1955; Jacobs 1925/1926/1927; Teuchert 1927; Teuchert 1933.

24 Vgl. Prowatke 1973; Prowatke 1975.

25 Vgl. etwa Dittmar 1962; Gernentz 1974; Gernentz 1989; Herrmann-Winter 1974; Herrmann-Winter 1979; Herrmann-Winter 1987; Schönfeld 1981.

26 Vgl. Dahl 1974; Dahl 1981; Herrmann-Winter 1979; Schönfeld 1989.

27 Vgl. Bentzien/Neumann 1988; Rassow 1958; Rehfeld 1960.

28 Herrmann-Winter 2013; Hansen 2009; Köhncke 2010.

29 Kehrein 2012.

30 Vollmer 2008; Vollmer 2012.

31 Vgl. Schröder/Elmentaler 2009; Elmentaler/Rosenberg 2015. 
Sprachwissens und der Spracheinstellungen untersucht und damit strukturelle, variationslinguistische und perzeptionslinguistische Fragestellungen kombiniert, bilden die Dialektregionen Mecklenburg-Vorpommern und Mittelpommern (Uckermark) zwei der insgesamt 18 Erhebungsareale. Aus dem Zusammenhang dieses Forschungsprojekt ist vor kurzem der erste Band des Norddeutschen Sprachatlasses (NOSA) veröffentlicht worden, der die areale Verteilung und situative Varianz der kennzeichnenden Merkmale des Regiolekts auch für MecklenburgVorpommern und Mittelpommern kartographisch veranschaulicht. ${ }^{32}$

Fragen der Spracheinstellung gegenüber dem Niederdeutschen und ihrem Wandel in politischen, medialen und laienlinguistischen Diskursen thematisieren die neueren Studien von Arendt. ${ }^{33}$ Die Dissertation von Scharioth beleuchtet die regionalen Unterschiede in diesen Spracheinstellungen in einem Vergleich von Gewährsfrauen aus den Regionen Holstein, Mecklenburg, Vorpommern und Mittelpommern. ${ }^{34}$ Mit der Entwicklung der mecklenburgischen Regionalsprache nach 1945 setzt sich auch das von der Deutschen Forschungsgemeinschaft geförderte Projekt von Ehlers zur Geschichte der Regionalsprache in Mecklenburg seit 1945 auseinander, zu dem bereits erste Detailstudien vorliegen. Diese thematisieren insbesondere die sprachlichen Folgen der massiven Zuwanderung von Flüchtlingen und Vertriebenen in die Region. ${ }^{35}$ Zunehmend in den Vordergrund rücken zudem Aspekte der Sprachbildung und Sprachdidaktik mit Bezug auf das Niederdeutsche. ${ }^{36}$

Eine Schnittstelle zu regionalsprachlichen Problemfeldern bilden ebenso die ethnographischen Forschungsaktivitäten des Wossidlo-Archivs der Universität Rostock, einer ehemaligen Arbeitsstelle der Berliner Akademie der Wissenschaften..$^{37}$ Mit der 2014 erfolgten Onlinestellung der Sammlungen des mecklenburgischen Volkskundlers Richard Wossidlo ${ }^{38}$ wurde ein frei zugängliches Feldforschungsdaten-Repositorium („WossiDiA“) geschaffen, das über klassischvolkskundliche Quellen hinaus auch semantische und soziolinguistische Gruppierungen von Sprachmaterial, Hinweise über Sprachverwendungssituationen sowie die Zettelkästen für das Mecklenburgische Wörterbuch präsentiert. ${ }^{39}$

32 Elmentaler/Rosenberg 2015. Ein zweiter Atlas-Band zu den niederdeutschen Sprachlagen ist in Vorbereitung.

33 Arendt 2010; Arendt 2012.

34 Scharioth 2015.

35 Vgl. Ehlers 2011; Ehlers 2012; Ehlers 2013; Ehlers 2016.

36 Vgl. etwa Arendt/Gąszczyk 2015.

37 Vgl. Neumann 1994; Schmitt 2015.

38 Meyer/Schmitt/Schering 2014.

39 Schmitt 2014. 
Fragen des rezenten Wandels der Regionalsprache in MecklenburgVorpommern stehen auch im Mittelpunkt des vorliegenden Sammelbandes. Er vereint Beiträge von Linguistinnen und Linguisten, die Aspekte der Entwicklung, des Gebrauchs sowie der Bewertung und Vermittlung bzw. Förderung dialektaler und regiolektaler Sprachformen in diesem Bundesland in den Blick nehmen. Der Fokus der Darstellungen liegt auf jüngeren und aktuellen Entwicklungen. Zugleich beziehen die Beiträge die Vorgeschichte der gegenwärtigen Sprachverhältnisse seit dem 19. Jahrhundert ein. Gleichzeitig bildet der Sammelband den Auftakt der neu gegründeten wissenschaftlichen Publikationsreihe „Regionalsprache und regionale Kultur. Mecklenburg-Vorpommern im ostniederdeutschen Kontext“. Ziel dieser Publikationsreihe ist es, einerseits die bislang disparate Forschung zur Sprache und Kommunikation in dieser norddeutschen Region erstmals zusammenzuführen und auf einem gemeinsamen Forum fortlaufend zu präsentieren. Generell stehen hierbei das Gesamt und die Vielfalt an geschriebenen und gesprochenen Ausprägungen regionaler Sprache und Kommunikation im Mittelpunkt des Interesses. In diesem Sinne steht die Reihe nicht allein dialektologischen und regiolektalen Studien im engeren Sinne als Publikationsplattform offen, sondern ebenso linguistischen Untersuchungen zu fachsprachlichen, sondersprachlichen sowie sozio- und ethnolektalen Varietäten, die für das regionale kommunikative Gefüge Relevanz besitzen.

Die Reihe will andererseits die Zusammenhänge zwischen Entwicklungen im regionalsprachlichen Bereich und den Entwicklungen in anderen Bereichen der regionalen Kultur Mecklenburg-Vorpommerns herausstellen und dabei ein Untersuchungsfeld von historischen Formen der mecklenburgisch-vorpommerschen Volkskultur bis hin zu gegenwärtigen Trends der regionalen Alltagskultur und Populärkultur oder auch zu Entwicklungen in der regionalen Literatur aufspannen. Zu beobachten wäre etwa, ob sich parallel zum oben angesprochenen Wandel des Niederdeutschen von einer Kommunikationssprache zu einer Identifikationssprache auch in anderen Bereichen der Regionalkultur ein ähnlicher Funktionswandel abzeichnet. Korrespondiert den historischen Verschiebungen im komplexen Gefüge der mecklenburgisch-vorpommerschen Regionalsprache ein Strukturwandel auch in anderen Feldern der regionalen Kultur? Generell soll der fokussierte Blick auf Mecklenburg-Vorpommern, von dem unsere Reihe ihren Ausgang nimmt, die Frage nach der sich verändernden Rolle von Regionalität in den modernisierten Gesellschaften exemplarisch und empirisch zentriert ,auf den arealen Punkt bringen.

Der regionale Horizont der Reihe liegt zunächst zwar auf MecklenburgVorpommern, wird aber den umfassenden Kontext der ostniederdeutschen 
Sprachregionen ebenso wie die weitgespannten kulturellen Beziehungen des Landes zum Ostseeraum berücksichtigen und in vergleichender Perspektive einbeziehen. Mecklenburg-Vorpommern fungiert hierbei als Bezugsraum unter dreifacher Perspektive: erstens sprachlich, zweitens historisch-politisch und drittens kulturell. Als Erscheinungsform soll die Reihe sowohl einschlägige Monographien als auch thematisch fokussierte Sammelbände in teilweise wechselnder Gastherausgeberschaft umfassen.

Der vorliegende Sammelband ist als Einführung in aktuelle Probleme und Ergebnisse der Dialekt- und Regionalsprachenforschung zum mecklenburgischvorpommerschen Raum und zugleich als Hinführung zu den sprachwissenschaftlichen Themenbereichen der nachfolgenden Reihentitel angelegt. Im Fokus dieses Eröffnungsbandes stehen vor allem die traditionellen mecklenburgischen und pommerschen Dialekte, die sowohl aus struktureller und verwendungsbezogener als auch aus didaktischer Perspektive betrachtet werden. Damit greift der Eröffnungsband zwei Themenbereiche auf, die im Zentrum der aktuellen Forschungsdiskussion stehen. Die Beiträge präsentieren Detailstudien sowie ausgewählte Ergebnisse umfangreicherer Forschungsvorhaben und -projekte. Sie verdeutlichen mit ihrer thematischen Vielfalt die große Bandbreite der wissenschaftlichen Auseinandersetzung mit dialektalen und regiolektalen Sprachformen in Mecklenburg-Vorpommern sowie die Diversität der Fragestellungen und Methoden, mit denen sich linguistische Teildisziplinen wie etwa Dialektgeographie, Variationslinguistik, Soziolinguistik, aber auch Sprachkontaktforschung, Diskurslinguistik und Sprachdidaktik dem Phänomen der Entwicklung und des Gebrauchs der Regionalsprache in der Gegenwart und jüngeren Geschichte Mecklenburgs und Vorpommerns nähern. Angesichts der zunehmenden Verlagerung der Sprachvermittlung auf Bildungsinstitutionen widmet unser Band der Frage des gesteuerten Niederdeutscherwerbs einen eigenen Themenblock. Hier werden unlängst abgeschlossene oder laufende Initiativen und Projekte der Niederdeutschvermittlung in Mecklenburg-Vorpommern vorgestellt, auf ihre institutionellen und motivationalen Rahmenbedingungen befragt und in ihrer Rolle für den Spracherhalt des Niederdeutschen untersucht.

Thematisch gliedern sich die Beiträge des Eröffnungsbandes damit in die zwei Bereiche „Sprachraum, Sprachstrukturen und Sprachgebrauch in der Entwicklung“ und „Förderung und Vermittlung des Niederdeutschen in Bildungsinstitutionen“. Eingeleitet wird der Sammelband von einer Überblicksdarstellung Peter Rosenbergs zur Regionalsprache in Mecklenburg-Vorpommern, der auf Basis des aktuellen Norddeutschen Sprachatlasses die rezente Entwicklung der regionalen hochdeutschen Sprachlagen in diesem Bundesland nachzeichnet. 
Der Beitrag bietet zugleich eine kompakte Skizze der bisherigen Forschung zur mecklenburgisch-vorpommerschen Regionalsprache. In einem historischen Rückblick auf das Niederdeutsch im vorpommerschen Raum in der ersten Hälfte des 19. Jahrhunderts analysiert der Beitrag von Matthias Vollmer sowohl zeitgenössische metasprachliche Äußerungen zum Gebrauch des Niederdeutschen als auch objektsprachliche Daten und gewinnt auf diese Weise Rückschlüsse auf sprachstrukturelle Phänomene wie auch auf das damalige Varietätenspektrum in Vorpommern und auf die Entwicklung einer städtisch geprägten niederdeutschen Prestigevarietät. Die diachronische Entwicklung von Sprachräumen und Sprachstrukturen fokussieren sodann die nachfolgenden Beiträge von Köhncke, Ehlers, Hansen und Vorberger. Dialektgeographisch angelegt ist der Beitrag von André Köhncke zu Entwicklungstendenzen im Wortschatz des Mecklenburgischen, der anhand ausgewählter Beispiele lexikalische Unterschiede innerhalb der mecklenburgischen Dialekte aufzeigt und deren zunehmende Vereinheitlichung unter dem Einfluss des Hochdeutschen sichtbar macht. Klaas-Hinrich Ehlers untersucht in seinem Beitrag „Meihen, meiden, meigen ,mähen“" das phonetische Phänomen der Hiattilgung, deren Entwicklung seit dem ausgehenden 19. Jahrhundert beispielhaft für die strukturelle Annäherung des jüngeren mecklenburgischen Niederdeutsch an den hochdeutschen Standard und für den Ausgleich regionaler sprachlicher Besonderheiten in der Dialektlandschaft MecklenburgVorpommerns steht. Dem strukturellen Wandel des Pommerschen auf der Insel Rügen zwischen dem 19. und 21. Jahrhundert geht der Beitrag von Martin Hansen nach, der anhand von Datenmaterial (Wenkersätzen) aus dem 19. Jahrhundert und von Sprachaufzeichungen aus den Jahren 1962 und 1963 sowie aus den Jahren 2006 und 2007 die diachrone Annäherung des regionalen Niederdeutsch an das System der hochdeutschen Standardsprache auf verschiedenen Sprachebenen nachweist. Ebenfalls mit den Sprachverhältnissen auf der Insel Rügen beschäftigt sich der Beitrag „Hochdeutsch auf Rügen“ von Lars Vorberger, der am Beispiel der Stadt Bergen den hochdeutschen Teil des regionalsprachlichen Spektrums untersucht und hierfür Aufnahmen von Sprechern aus drei Generationen in unterschiedlichen Gesprächssituationen auf ihre regiolektalen Merkmale analysiert. Einen sprachbiographischen Ansatz verfolgt die Fallstudie von Mona Biemann zum Wandel von Dialektgebrauch und Dialektvermittlung über mehrere Generationsfolgen von Einwohnern der mecklenburgischen Gemeinde Satow.

Breiten Raum nehmen mit den Beiträgen von Mittelstädt, Biedowicz, Fink, Arendt und Reuter Studien zum Thema „Förderung und Vermittlung des Niederdeutschen in Bildungsinstitutionen" ein. Sie verdeutlichen die steigende Relevanz 
institutioneller Spracherwerbsmöglichkeiten für das Niederdeutsche angesichts sinkender Sprecherzahlen und einer weitgehend ausfallenden Dialektvermittlung durch elterliche Spracherzieher. In den Blick genommen wird die Vermittlung des Niederdeutschen in institutionellen Kontexten in Mecklenburg-Vorpommern, die von der vorschulischen Sprachbildung in regionalen Kindertagesstätten, über die schulische und universitäre Niederdeutschvermittlung bis hin zu den Angeboten zum Niederdeutscherwerb an den hiesigen Volkshochschulen reicht. Ergebnisse eines Pilotprojektes zur vorschulischen Niederdeutschvermittlung in ausgewählten Kindergärten Mecklenburg-Vorpommerns aus dem Jahre 2010/11 referiert der Beitrag von Julia Mittelstädt anhand der Auswertung von Fragebögen, in denen die am Projekt beteiligten Erzieherinnen Auskunft über die jeweiligen sprachlichen Voraussetzungen, Ziele, Methoden und Ergebnisse der dialektalen Spracherziehung in ihren Einrichtungen geben. Mit Aspekten des frühkindlichen dialektalen Zweitspracherwerbs setzt sich auch die Untersuchung von Johanna Biedowicz auseinander, die Verlauf und Ergebnisse einer Studie zur vorschulischen Niederdeutschvermittlung in einem Greifswalder Kindergarten referiert. Aspekte der Niederdeutschvermittlung im schulischen Kontext thematisiert der Beitrag von Anne Fink, die anhand einer exemplarischen Studie am Beispiel der Stadt Greifswald die Umsetzung der Verwaltungsvorschrift „Niederdeutsch in der Schule“ des Landes Mecklenburg-Vorpommern aus dem Jahre 2003 untersucht und dafür Fragebogenerhebungen, narrative Interviews mit Lehrerinnen und Lehrern sowie teilnehmende Beobachtungen auswertet. Niederdeutsch als universitäres Studienfach steht im Mittelpunkt des Beitrages von Birte Arendt, die Formen und Entwicklungen der Niederdeutschlehre an der Universität Greifswald beschreibt und studentische Motivationen für den Erwerb dieser Sprache diskutiert. Abgeschlossen wird der Themenbereich „Sprachförderung und Sprachbildung" durch den Artikel von Hannah Reuter, die der Frage nachgeht, welchen Beitrag die Volkshochschulen des Landes Mecklenburg-Vorpommern zur Ausbildung potenzieller Multiplikatoren des Niederdeutschen und damit zu dessen Bewahrung leisten können.

Abgerundet wird der Sammelband schließlich von einer Bibliographie der wissenschaftlichen Forschungsliteratur zur Regionalsprache in MecklenburgVorpommern ab 1700, die von Ingrid Schröder begründet wurde und die erstmalig einen Überblick über die bisherige Forschungslage erlaubt, an die mit diesem Eröffnungsband unserer Reihe und ihren Folgetiteln angeknüpft werden soll. Sie verzeichnet Überblicksdarstellungen, Einzelstudien und wissenschaftliche Beiträge, die sich unter verschiedensten Perspektiven mit der Entwicklung und dem aktuellen Stand der neuniederdeutschen Dialektvarianten und der hochdeut- 
schen regionalen Umgangssprache auf dem Gebiet des heutigen Bundeslandes Mecklenburg-Vorpommern (und zum Teil darüber hinaus) auseinandersetzen.

Die Drucklegung dieser Publikation wurde ermöglicht durch eine finanzielle Förderung durch den Heimatverband Mecklenburg-Vorpommern e. V., dem an dieser Stelle herzlich dafür gedankt sei. Eingebunden in die Realisierung des Publikationsprojektes war ebenso die Gesellschaft zur Förderung des Wossidlo-Archivs. Unser spezieller Dank gilt zudem Dr. Christoph Schmitt, Leiter des Instituts für Volkskunde an der Universität Rostock, für seine engagierte Unterstützung dieser Publikation.

\section{Literatur}

Arendt, Birte: Niederdeutschdiskurse. Spracheinstellungen im Kontext von Laien, Printmedien und Politik (Philologische Studien und Quellen; 224). Berlin 2010.

Arendt, Birte: „deswegen hab ich mich auch nich getr!AU!t zu sprechen“. Spracheinstellungsmuster und Sprachgebrauch Jugendlicher bezüglich des Niederdeutschen im sozialen Netzwerk »Plattdüütschkring «. In: Muttersprache. Vierteljahresschrift der Gesellschaft für deutsche Sprache (2012), S. 1-25.

Arendt, Birte/Gąszczyk, Alicja: Second language immersion programmes at kindergarten - as illustrated by Polish and Low German. In: Minsterium für Bildung und Kultur des Saarlandes (Hrsg.): Multilingualism creates opportunities. Saarbrücken 2015, S. 54-68.

Bentzien, Ulrich/Neumann, Siegfried (Hrsg.): Mecklenburgische Volkskunde. Rostock 1988.

Bieberstedt, Andreas: „In meinem Elternhaus wurde nur Plattdeutsch gesprochen." Sprachbiographische Konzeptionen Hamburger Dialektsprecher zum frühen Spracherwerb. In: Langhanke, Robert (Hrsg.): Sprache, Literatur, Raum. Festgabe für Willy Diercks. Bielefeld 2015, S. 205-237.

Brose, Gerhard: Zur Dialektgeographie der pommersch-neumärkischen Grenzzone. In: Zeitschrift für Mundartforschung 23 (1955), S. 22-37.

Dahl, Eva-Sophie: Interferenz und Alternanz - zwei Typen der Sprachschichtenmischung im Norden der Deutschen Demokratischen Republik. In: Ising, Gerhard (Hrsg.): Aktuelle Probleme der sprachlichen Kommunikation. Berlin 1974, S. 339-388.

Dahl, Eva-Sophie: Niederdeutsche Verbalkomposita als Interferenzmerkmale im gesprochenen Hochdeutsch der Nordbezirke. Grundlagen und Grenzen ihres Auftretens. In: Zentralinstitut für Sprachwissenschaft der Akademie der Wissenschaften der DDR (Hrsg.): Das Niederdeutsche in Geschichte und Gegenwart. 1. Bd. Berlin 1981, S. 105-111. 
Dittmar, Lisa: Die gegenwärtige Sprachsituation auf dem Fischland und dem Darß. In: Wissenschaftliche Zeitschrift der Universität Rostock. Gesellschaftsund sprachwissenschaftliche Reihe 11 (1962), S. 313-318.

Elmentaler, Michael/Rosenberg, Peter: Norddeutscher Sprachatlas (NOSA). Bd. 1: Regiolektale Sprachlagen (Deutsche Dialektgeographie; 133.1). Hildesheim/ Zürich/New York 2015.

Ehlers, Klaas-Hinrich: Schlesische und sudetendeutsche Plattschnacker. Eine Fallstudie zur sprachlichen Integration der Vertriebenen in MecklenburgVorpommern. In: Bohemia. Zeitschrift für Geschichte und Kultur der böhmischen Länder 51, H. 2 (2011), S. 345-357.

Ehlers, Klaas-Hinrich: Forschungsprojekt: Kontaktlinguistische Untersuchungen zur sprachlichen Akkulturation Heimatvertriebener in Mecklenburg. In: brücken. Germanistisches Jahrbuch Tschechien-Slowakei NF 19, H. 1-2 (2012), S. 358-374.

Ehlers, Klaas-Hinrich: Führte die Immigration der Heimatvertriebenen nach 1945 zu Dialektverlust und Nivellierung regionalsprachlicher Differenzen? Beobachtungen aus einer Untersuchungsregion in Mecklenburg. In: Niederdeutsches Jahrbuch 136 (2013), S. 97-116.

Ehlers, Klaas-Hinrich: „Uns’re Leut“ - Akkulturation und Abgrenzung einer karpatendeutschen Vertriebenengruppe in Mecklenburg. In: Zückert, Martin/ Schvarc, Michal/Meier, Jörg (Hrsg.): Migration - Zentrum und Peripherie kulturelle Vielfalt. Neue Zugänge zur Geschichte der Deutschen in der Slowakei. Leipzig 2016, S. 161-197.

Europarat: Europäische Charta der Regional- oder Minderheitensprachen. 1998. [Online-Ressource: https://www.coe.int/de/web/conventions/full-list/-/ conventions/treaty/148 (Stand: 24.11.2016)]

Gernentz, Hans Joachim: Über den Auflösungsprozeß der Mundart im Norden der Deutschen Demokratischen Republik. In: Actes du Xe Congres International des Linguistes. 1. Bd. Bukarest 1969, S. 677-684.

Gernentz, Hans Joachim: Die kommunikative Funktion der niederdeutschen Mundart und hochdeutschen Umgangssprache im Norden der Deutschen Demokratischen Republik, unter besonderer Berücksichtigung der Interferenz und der Alternanz zwischen diesen beiden sprachlichen Existenzformen. In: Studia Germanica Gandensia 15 (1974), S. 209-244.

Gernentz, Hans Joachim: System und Verwendung der Existenzformen des Deutschen im Norden der Deutschen Demokratischen Republik. In: Wissenschaftliche Zeitschrift der Universität Rostock. Gesellschafts- und sprachwissenschaftliche Reihe 24 (1975a), S. 385-393. 
Gernentz, Hans Joachim: Wann und wie wird heute niederdeutsch gesprochen? In: Theil, Hans-Joachim (Red.): Niederdeutsch heute. Materialien einer Arbeitstagung des Freundeskreises Niederdeutsche Sprache und Literatur im Kulturbund der DDR, Kreisleitung Rostock 16.-17. November 1974. Rostock 1975b, S. 12-19.

Gernentz, Hans Joachim: Zur Entwicklung und Bewertung des Niederdeutschen in Mecklenburg und Vorpommern. In: Greifswalder Germanistische Forschungen 11 (1989), S. 48-58.

Hansen, Martin: Zum Wandel des Niederdeutschen auf der Insel Rügen zwischen dem 19. und 21. Jahrhundert. Ein diachronischer Vergleich anhand ausgewählter Sprachmerkmale. Masterarbeit Greifswald 2009.

Herrmann-Winter, Renate: Auswirkungen der sozialistischen Produktionsweise in der Landwirtschaft auf die sprachliche Kommunikation in den Nordbezirken der Deutschen Demokratischen Republik. In: Ising, Gerhard (Hrsg.): Aktuelle Probleme der sprachlichen Kommunikation. Soziolinguistische Studien zur sprachlichen Situation in der Deutschen Demokratischen Republik. Berlin 1974, S. 135-190.

Herrmann-Winter, Renate: Studien zur gesprochenen Sprache im Norden der DDR. Soziolinguistische Untersuchungen im Kreis Greifswald (Akademie der Wissenschaften der DDR. Zentralinstitut für Sprachwissenschaft, Reihe Sprache und Gesellschaft; 14). Berlin 1979.

Herrmann-Winter, Renate: Zum Zusammenhang von Verwendungsweisen und Bewertungen des Niederdeutschen im Norden der DDR. In: Zeitschrift für Phonetik, Sprachwissenschaft und Kommunikationsforschung 40, H. 6 (1987), S. 822-829.

Herrmann-Winter, Renate: Sprachatlas für Rügen und die vorpommersche Küste. Kartographie Martin Hansen. Rostock 2013.

Institut für niederdeutsche Sprache: Umfrage zum Niederdeutschen. Erste Ergebnisse. 2016. Institut für niederdeutsche Sprache 2016 [Online-Ressource: http://www.ins-bremen.de/de/sprache/umfrage/erste-ergebnisse.html (Stand: 24.11.2016)]

Jacobs, Hugo: Dialektgeographie Südmecklenburgs zwischen Lübz und Hagenow. In: Teuthonista 2 (1925/26), S. 46-55 und 107-133; 3 (1926/27), S. 119-152 und 241-262.

Jürgens, Carolin: Niederdeutsch im Wandel. Sprachgebrauchswandel und Sprachwahrnehmung in Hamburg (Deutsche Dialektgeographie; 119). Hildesheim/ Zürich/New York 2015.

Kehrein, Roland: Regionalsprachliche Spektren im Raum. Zur linguistischen Struktur der Vertikale (ZDL Beihefte; 152). Stuttgart 2012. 
Köhncke, André: Mecklenburgisch heute - Bestandsaufnahme eines niederdeutschen Dialektes. Rostock 2010. [Online-Ressource: urn:nbn:de:gbv:28diss2011-0137-3 (Stand: 24.11.2016)]

Lenz, Alexandra: Vom Dialekt zur regionalen Umgangssprache - Zur Vielfalt regionaler Sprechweisen. In: Munske, Horst Haider (Hrsg.): Sterben die Dialekte aus? Vorträge am Interdisziplinären Zentrum für Dialektforschung an der Friedrich-Alexander-Universität Erlangen-Nürnberg, 22.10.-10.12.2007. 2008. [Online-Ressource: http://www.dialektforschung.phil.uni-erlangen.de/ sterbendialekte (Stand: 29.10.2016)]

Mecklenburgisches Wörterbuch. Hrsg. von der Sächsischen Akademie der Wissenschaften zu Leipzig. Aus den Sammlungen Richard Wossidlos und aus den Ergänzungen und nach der Anlage Hermann Teucherts. 7 Bde. Berlin 1942-1992. Unveränd., verkleinerter Nachdruck der Erstauflage von 1937-1992. Neumünster 1996. Rothe, Christian: Nachtrag und Index. Neumünster 1998.

Meyer, Holger/Schmitt, Christoph/Schering, Alf-Christian: WossiDiA - The Digital Wossidlo Archive. In: Meyer, Holger/Schmitt, Christoph/Janssen, Stefanie/ Schering, Alf-Christian (Hrsg.): Corpora ethnographica online. Strategien der Digitalisierung kultureller Archive und ihrer Präsentation im Internet. Münster/New York 2014, S. 61-84.

Möller, Frerk: Plattdeutsch im 21. Jahrhundert. Bestandsaufnahme und Perspektiven. Mit einem Aufsatz von Michael Windzio (Schriften des Instituts für niederdeutsche Sprache; 34). Leer 2008.

Möller, Frerk: Niederdeutsch im 21. Jahrhundert. Bestandsaufnahme und Perspektiven. In: Niederdeutsches Jahrbuch 133 (2010), S. 141-163.

Neumann, Siegfried: Richard Wossidlo und das Wossidlo-Archiv in Rostock. Von der volkskundlichen Sammlung des Privatgelehrten zum Institut für Volkskunde in Mecklenburg-Vorpommern. Rostock 1994.

Pommersches Wörterbuch. Begründet von Wolfgang Stammler. Fortgesetzt von Hans-Friedrich Rosenfeld. Hrsg. von Renate Herrmann-Winter. Sächsische Akademie der Wissenschaften-Leipzig. Kommission für die Mundartwörterbücher. Bd. 1 ff. Berlin 1997 ff.

Prowatke, Christa: Zum gegenwärtigen Lautstand der niederdeutschen Mundart in der DDR. Untersuchungen von Wortmaterial aus den drei Nordbezirken unter Berücksichtigung phonologischer Erkenntnisse. Diss. Rostock, 1973.

Prowatke, Christa: Untersuchungen von niederdeutschem Wortmaterial aus den drei Nordbezirken der DDR unter Berücksichtigung phonologischer Erkenntnisse. In: Wissenschaftliche Zeitschrift der Universität Rostock. Gesellschaftsund sprachwissenschaftliche Reihe 24 (1975), S. 395-401. 
Rassow, Margarethe: Fischersprache und Brauchtum im Lande zwischen dem Darß und der unteren Oder (Veröffentlichungen des Instituts für Deutsche Sprache und Literatur der Deutsche Akademie der Wissenschaften zu Berlin; 12). Berlin 1958.

Rehfeld, Irmgard: Sprache und volkskundliche Geltung des Wasser- und Windmüllers im ehemaligen Pommern. 2. Bde. Diss. Greifswald 1960.

Scharioth, Claudia: Regionales Sprechen und Identität. Eine Studie zum Sprachgebrauch, zu Spracheinstellungen und zu Identitätskonstruktionen von Frauen in Schleswig-Holstein und Mecklenburg-Vorpommern (Deutsche Dialektgeographie; 120). Hildesheim/Zürich/New York 2015.

Schmidt, Jürgen Erich/Herrgen, Joachim: Sprachdynamik. Eine Einführung in die moderne Regionalsprachenforschung (Grundlagen der Germanistik; 49). Berlin 2011.

Schmitt, Christoph: Szenarien semantischer Vernetzung zwischen regionalethnographischen und dialektlexikographischen Korpora im OnlineProjekt „WossiDiA“. In: Bühler, Rudolf/Bürkle, Rebekka/Leonhardt, Nina Kim (Hrsg.): Sprachkultur - Regionalkultur. Neue Felder kulturwissenschaftlicher Dialektforschung. Tübingen 2014, S. 255-286.

Schmitt, Christoph: Folklore research at the University of Rostock: the Wossidlo Archive, its development and present situation. In: FF network 47 (2015), S. 22-27.

Schönfeld, Helmut: Gruppenspezifische Unterschiede bei der Verwendung und Bewertung des Niederdeutschen in der DDR. In: Das Niederdeutsche in Geschichte und Gegenwart (Zentralinstitut für Sprachwissenschaft. Reihe A. Arbeitsberichte; 75.2). Hrsg. vom Zentralinstitut für Sprachwissenschaft der Akademie der Wissenschaften der DDR, Berlin 1981, S. 112-120.

Schönfeld, Helmut: Sprache und Sprachvariation in der Stadt. Zu sprachlichen Entwicklungen und zur Sprachvariation in Berlin und anderen Städten im Nordteil der DDR. Berlin 1989.

Schröder, Ingrid/Niebaum, Hermann: Vorwort. In: Möller, Frerk: Plattdeutsch im 21. Jahrhundert. Bestandsaufnahmen und Perspektiven (Schriften des Instituts für niederdeutsche Sprache; 34). Leer 2008, S. 7-8.

Schröder, Ingrid/Elmentaler, Michael: Sprachvariation in Norddeutschland (SiN). In: Niederdeutsches Jahrbuch 132 (2009), S. 41-68.

Stellmacher, Dieter: Wer spricht Platt? Zur Lage des Niederdeutschen heute. Eine kurze Bestandsaufnahme (Schriften des Instituts für niederdeutsche Sprache. Reihe: Dokumentation; 14). Leer 1987.

Teuchert, Hermann: Deutsche Mundarten. Mecklenburgisch (Lautbibliothek. Phonetische Platten und Umschriften; 21). Berlin 1927. 
Teuchert, Hermann/Schmitt, Alfred: Deutsche Mundarten. Mecklenburgisch II und Pommersch (Lautbibliothek. Phonetische Platten und Umschriften; 35). Berlin 1933.

Vollmer, Matthias: Zur Dialektlexikographie in Pommern: Kosegartens Wörterbuch der Niederdeutschen Sprache älterer und neuerer Zeit. In: Niederdeutsches Jahrbuch 131 (2008), S. 113-132.

Vollmer, Matthias: Die ostpommerschen Dialekte. 2 Bde. Habilitationsschrift Greifswald 2012.

Vorberger, Lars/Schröder, Ingrid: Standardadvergenz im nördlichen Niedersachsen? - Eine exemplarische Analyse von Sprecherinnen aus drei Generationen. In: Niederdeutsches Jahrbuch 134 (2011), S. 137-147. 
Birte Arendt, Andreas Bieberstedt and Klaas-Hinrich Ehlers - 978-3-631-71893-3

Downloaded from PubFactory at 01/11/2019 10:11:06AM

via free access 


\section{Sprachraum, Sprachstrukturen und Sprachgebrauch in der Entwicklung}


Birte Arendt, Andreas Bieberstedt and Klaas-Hinrich Ehlers - 978-3-631-71893-3

Downloaded from PubFactory at 01/11/2019 10:11:06AM

via free access 


\title{
Peter Rosenberg \\ Regionalsprache in Mecklenburg-Vorpommern
}

\begin{abstract}
The regional language in the state of Mecklenburg-Western Pomerania in the North East of Germany contains the regional colloquial language (regiolect) and the Low German varieties which are described in both their historical and present state. A comprehensive overview of former and recent research reveals an ongoing spread of regiolectal varieties and a decline of Low German. The process is described in terms of linguistic features and explained on the basis of the social, political, and cultural history of the region.
\end{abstract}

\section{Einleitung}

Mit dem Norddeutschen Sprachatlas, der aus den Ergebnissen des Projekts „Sprachvariation in Norddeutschland (SiN)“ entstanden ist, liegt eine umfassende Beschreibung der Sprachvariation in Norddeutschland in ihren regiolektalen Sprachlagen vor. ${ }^{1}$ Im Atlas kennzeichnen zahlreiche Karten und Abbildungen auch den aktuellen Stand der Sprachentwicklung in Mecklenburg-Vorpommern. In seiner Einleitung wird diese Entwicklung bezogen auf die „hochdeutschen“ Sprachlagen nachgezeichnet. Im Folgenden soll auf dieser Grundlage eine Zusammenschau der Entwicklung des Regiolekts und des Niederdeutschen vorgenommen werden, deren Gebrauch sich in einem komplexen - komplementären, konkurrierenden oder synergetischen - Abhängigkeitsverhältnis befindet. Die folgenden Ausführungen sind selbstverständlich nicht mehr als eine Skizze der ausschlaggebenden Entwicklungslinien.

\section{Historische Entwicklung und areale Gliederung der mecklenburgisch-vorpommerschen Regionalsprache}

Der mecklenburgisch-vorpommersche Raum zeigt unterhalb des Standards eine "großlandschaftliche Sprachschicht“" , die - trotz der erheblichen Distanz der Basisdialekte vom Standard - nur in geringem Maße vom Standarddeutschen abweicht. ${ }^{3}$ Hierin unterscheidet sich der mecklenburgisch-vorpommersche Re-

1 Elmentaler/Rosenberg 2015.

2 Mihm 2000, S. 2116. Vgl. Schönfeld 1990, S. 117.

3 Vgl. Schönfeld 1990, S. 117; Kehrein 2012, S. 312. 
giolekt gravierend vom südlich angrenzenden berlinisch-brandenburgischen, der deutlichere regionale Eigenheiten bei weitgehend aufgegebenen Dialekten besitzt. ${ }^{4}$

Der mecklenburgisch-vorpommersche Regiolekt weist eine Nord-SüdGliederung und eine gewisse Ost-West-Differenzierung auf. Beide arealen Differenzierungen sind alt und prägten bereits die „koloniale(n) Misch- und Ausgleichsmundarten, deren spezifisches Gepräge weitgehend durch Herkunft und Zahl der jeweiligen Neusiedler bestimmt wurde“. ${ }^{5}$ Die deutschsprachige Besiedlung des Raums ist das Ergebnis „zweier Siedlerströme, eines nd. an der

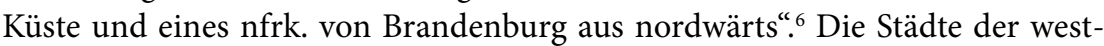
pommerschen Küste hatten folglich Lübecker Stadtrecht (Stralsund ab 1234, Greifswald 1250, Wolgast 1257), die Städte des Oderkeils Magdeburger Stadtrecht (Stettin 1243, Garz/Oder 1249, Penkun 1284). ${ }^{7}$

Die Ost-West-Differenzierung des mecklenburgischen Niederdeutsch gegenüber dem Mittelpommerschen ist markant mit in den westlichen Landesteilen älteren Formen, die sie mit Holstein und Nordniedersachsen verbinden (z.B. westliche Diphthongierung ([kaokn] ,Kuchen' gegenüber mittelpommerschem Monophthong [ko:kn]). ${ }^{8}$ Alt ist auch die Sonderentwicklung von Mecklenburg-Strelitz, dem alten Land Stargard, das aus der Mark Brandenburg besiedelt worden ist. ${ }^{9}$ Eine gewisse areale Gliederung besteht also innerhalb des Mecklenburgisch-Vorpommerschen bereits in den Basisdialekten, etwa mit der Hiattilgung auf - $d$ - im Hauptgebiet Mecklenburgs und auf - $g$ - in Vorpommern maiden versus maigen ,mähen..$^{10}$ Gundlach konstatiert damit eine Dreigliederung der Region: „Es staffelt sich also von Südwesten nach Osten meien/ meiden/meigen ". ${ }^{11}$ Allerdings sind diese Grenzen keineswegs fest und bereits in den Wenker-Übersetzungen aus der Region finden sich nach Ehlers fast zur Hälfte keinerlei Hiat-Tilgungen. ${ }^{12}$

4 Vgl. Schönfeld 1990, S. 117.

5 Sanders 1982, S. 74. Vgl. Niebaum 1986, S. 23.

6 Niebaum 1986, S. 30. Vgl. Foerste 1957, Sp. 1885.

7 Vgl. Kratz 1865; Foerste 1957, Sp. 1885.

8 Vgl. Schönfeld 1990, S. 97-98.

9 Vgl. Gundlach 1967, S. 181.

10 Vgl. Gundlach 1967, S. 181; Gernentz 1974, S. 223; Stellmacher 1980, S. 466; Schönfeld 1990, S. 98; Schröder 2004, S. 50; Kehrein 2012, S. 299. Zu Rügen vgl. HerrmannWinter 2013, S. 94.

11 Gundlach 1988, S. 427.

12 Vgl. den Beitrag von Ehlers in diesem Band. 
Die Entwicklung großräumiger sprachlicher Strukturen beginnt bereits früh: „In Mecklenburg entwickelte sich schon seit dem 16. Jh. die der Schriftsprache sehr angenäherte Umgangssprache, neben der seit Beginn des 18. Jh. ein stärker hochdeutsch durchsetztes Niederdeutsch, eine regionale Varietät des Hochdeutschen, das sogenannte Missingsch [...] gesprochen wurde, in dem ein von sozialem Prestigedenken gesteuertes Bemühen zum Hochdeutschsprechen bei bestimmten städtischen und dörflichen Bevölkerungsgruppen zum Ausdruck kam. "13

Schönfeld zeichnet die Entwicklung der Regiolekte im niederdeutschen Nordosten als eine regionale Ausbreitung städtischer Varietäten nach:

„Aus den Territorialdialekten bildeten sich - teilweise schon seit dem Mittelalter - ,Stadtdialekte heraus [...]. Von den großen Städten breitete sich in ihrem regionalen Einflußbereich die städtische Umgangssprache zuerst in die benachbarten Dörfer sowie in die mittleren und kleinen Städte aus, und zwar anfangs in den an der Großstadt orientierten Bevölkerungsgruppen, dann über das Bürgertum auf die übrigen Stadtbewohner und schließlich auf die Dorfbevölkerung einer größeren Region. Die städtische Umgangssprache wurde zur regionalen Umgangssprache. ${ }^{14}$

Ein „sehr frühes Zeugnis für den mündlichen Gebrauch des Hochdeutschen“ in Honoratiorenkreisen findet sich bei Kretschmer: Es wird vom Bürgermeister von Greifswald 1528 berichtet, im Rausch „wollt er nicht anders als hochteutisch reden“. ${ }^{15} 1639$ „klagt Joh. Micraelius, daß man in ganz Pommern fast keine pommerische Predigt mehr hören könne, weil es Alles muß hochdeutsch gebetet, geprediget, gesungen, geschrieben, geredet und verabscheidet werden" . ${ }^{16}$

Die landesfürstliche Kanzlei ging nach Böttcher in Mecklenburg 1502 zum Hochdeutschen über, die letzte niederdeutsche Urkunde wird für 1486 erwähnt; die städtischen Kanzleien begannen etwa zeitgleich, hochdeutsch zu urkunden, die letzten niederdeutschen Urkunden reichen jedoch bis ca. $1610 .{ }^{17}$ Schriftsprachlicher Übergang in städtischen Verordnungen wird für Stettin und Güstrow ab 1540, für Wolgast 1543, Schwerin ab 1548, Rostock 1558, Wismar ab 1560 berichtet. Dagegen hielt die Kirche länger am Niederdeutschen fest: Die Kirchenordnung war in Pommern bis 1661 nur niederdeutsch verfasst und bis 1731 zweisprachig, die letzten Lutherschen Gesangbücher auf Niederdeutsch sind für Stettin 1611, Rostock 1618, Greifswald 1626 verzeichnet. ${ }^{18}$

13 Protze 1997, S. 9.

14 Schönfeld 1989, S. 66.

15 Kretschmer 1918, S. 47.

16 Kretschmer 1918, S. 47, Hervorhebung im Original.

17 Böttcher 1922, S. 105.

18 Vgl. Sodmann 1983, S. 117-118, 124-125.

Birte Arendt, Andreas Bieberstedt and Klaas-Hinrich Ehlers - 978-3-631-71893-3 
Brandt berichtet von niederdeutsch-hochdeutschen Mischformen im „Rostocker Liederbuch" aus der zweiten Hälfte des 15. Jahrhunderts. ${ }^{19}$ Rösler zeichnet das Eindringen des Hochdeutschen in Schriften verschiedener Textsorten aus den Jahren 1550-1700 (aus dem Archiv Schwerin) nach. ${ }^{20}$ Sie findet kaum „rein“ niederdeutsche oder hochdeutsche Texte.

Im sprachlichen Ausgleich und der Ausbreitung städtischer Ausgleichsvarietäten ist die Wirkung des Dreißigjährigen Krieges nicht zu unterschätzen, der einen Hauptschauplatz in Mecklenburg und Pommern fand: Vor den marodierenden Heeren Flüchtende fanden - wenn überhaupt - nur Zuflucht in den stark befestigten Städten, aus denen sie nach Jahren in die entvölkerten ländlichen Räume zurückkehrten. Über Mecklenburg heißt es diesbezüglich: „Wie in den letzten Kriegsjahren Alles daniederlag, die Städte und Ortschaften theils verbrannt, [...] die Bewohner theils durch Schwert und Martern, theils durch Pest und Hunger umgekommen, zum kleineren Theile nach dem festen Rostock, besonders auch nach Lübeck und Hamburg geflohen. ${ }^{\text {"21 }}$

Die Überlebenden - der Bevölkerungsverlust wird mit ca. 70-80 \% in Mecklenburg angegeben - kehrten in verheerte Landstriche zurück, deren „weitgehende Entvölkerung des Landes zum Bauernlegen größten Ausmaßes, zum goldenen Zeitalter für die Gutsherrschaft “22 führte. Dies dürfte sowohl die Vereinheitlichung des mecklenburgisch-vorpommerschen Niederdeutsch als auch die Ausbreitung städtischer Ausgleichsvarietäten befördert haben.

In Mecklenburg bildeten sich städtische Varietäten insbesondere im 17. und 18. Jahrhundert heraus: ${ }^{23}$ Rostock, Wismar, Schwerin, Parchim, Grabow, Güstrow unterschieden sich etwa von der ländlichen Umgebung $u$. a. durch die Monophthonge $o$ : und $e$ : (Kooken,Kuchen', Been,Bein'), die im 19. Jahrhundert als feiner galten als die dialektalen Diphthonge au und ei (Kauken, Bein). ${ }^{24}$ In der Residenzstadt Schwerin (bis 1918) mit ihren Beamten und Offizieren galt das Niederdeutsche als „unfein“. ${ }^{25}$

Im östlichen Mecklenburg und besonders in Vorpommern machte sich in wachsendem Maße brandenburgischer, später preußischer Einfluss geltend. Seit der Schwedenzeit rang Brandenburg-Preußen um die Vorherrschaft in Vorpommern.

19 Brandt 2014, S. 66.

20 Rösler 1981, S. 68.

21 Balck 1903, S. 103.

22 Krüger 2005, S. 3.

23 Vgl. Schönfeld 1990, S. 125.

24 Vgl. Schönfeld 1989, S. 66.

25 Schönfeld 1989, S. 140.

Birte Arendt, Andreas Bieberstedt and Klaas-Hinrich Ehlers - 978-3-631-71893-3 
Im südlichen Vorpommern verstärkt sich die Orientierung nach Brandenburg mit dem Übergang „Altvorpommerns" (südlich der Peene) von Schweden an Preußen, der mit dem Stockholmer Frieden 1720 vollzogen wurde. Mit dem Wiener Kongress 1815 ging dann auch das restliche „Neuvorpommern“ an Preußen. In den südlichen und östlichen Landesteilen geriet der Regiolekt zunehmend unter den Einfluss Brandenburgs und mit wachsender Bedeutung der brandenburgischen (seit 1451), dann preußischen (seit 1701) Residenzstadt in den Bereich der „sprachlichen Strahlkraft Berlins". ${ }^{26}$ Diese Strahlkraft brachte hochdeutsches Sprachgut in der spezifischen berlinisch-brandenburgischen Form nach Norden. Standardsprachlicher Einfluss nahm seinen Weg seitdem von Berlin aus vor allem über die norddeutschen Städte: „Nun werden auf die später zum Hochdeutschen übergegangenen norddeutschen Städte kaum immer die ferneren hochdeutschen [sic!] sondern wohl mehr die ursprünglich niederdeutschen, jetzt hochdeutschen Städte (wie Berlin)

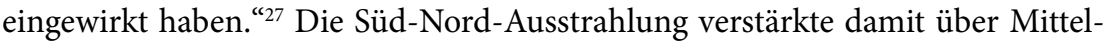
pommern auch die Ost-West-Differenzierung in Mecklenburg-Vorpommern, da der sprachliche Einfluss aus Brandenburg vor allem in den südöstlichen Landesteilen wirkte und seit dem 20. Jahrhundert auch Vorpommern erreichte. ${ }^{28}$

Diese Entwicklung betraf auch das Niederdeutsche. Ehlers zeigt, dass die Ersetzung des Plosivs / $\mathrm{k} /$ durch standarddeutschen Frikativ / $\mathrm{x} /$ in Wäk,Woche' und im Reflexivpronomen sik weit fortgeschritten ist: „Laut Mecklenburgischem Wörterbuch ist die hochdeutsche Verschiebung des Plosivs in Wäk bereits seit dem Ende des 18. Jahrhunderts belegt. " ${ }^{\text {"29 }}$ Bereits in den Wenker-Übersetzungen sei Wäk am Ende des 19. Jahrhunderts nur noch gering verbreitet gewesen. Nach HerrmannWinter werde Wäk ,jetzt von Woch verdrängt“. ${ }^{30}$ Der Lautwandel beim Reflexivpronomen sik ist deutlich jüngeren Ursprungs. Foerste stellt das Reflexivpronomen in den Kontext eines „Vorbruchs des Ostmitteldeutschen“ über Mittelpommern auf das mecklenburgisch-vorpommersche Gebiet. ${ }^{31}$ Diese Expansion finde nach Herrmann-Winter im Besonderen ,in Ost- und Südostmecklenburg sowie in Vorpommern seit Anfang des 20. Jh. s $^{\text {"32 }}$ statt und breite sich nach Lindow/Möhn/Niebaum/Stellmacher/Taubken/Wirrer ${ }^{33}$ im gesamten ostniederdeutschen Raum aus.

26 Protze 1997, S. 9.

27 Lasch [1911-12] 1979, S. 486.

28 Vgl. Schönfeld 1990, S. 99.

29 Ehlers (i. Vorber.).

30 Herrmann-Winter 1985, S. 373.

31 Foerste 1957, Sp. 1888.

32 Herrmann-Winter 2003, S. 207.

33 Lindow/Möhn/Niebaum/Stellmacher/Taubken/Wirrer 1998, S. 157.

Birte Arendt, Andreas Bieberstedt and Klaas-Hinrich Ehlers - 978-3-631-71893-3 
Dost $^{34}$ sieht die Landesgrenze zwischen Mecklenburg-Vorpommern und Brandenburg als Grenze zwischen zwei „umgangssprachlichen Haupttypen, der norddeutschen und der berlinischen Umgangssprache“, wobei im Übergangsgebiet (bei Röbel, Müritz) der mecklenburgisch-vorpommersche Regiolekt „Elemente der regionalen Umgangssprache Berlins aufgenommen" habe. ${ }^{35}$ Berliner Einfluss macht sich - seit dem 19. Jahrhundert mit erheblicher Intensität - bis an die Küste Mecklenburgs und besonders Vorpommerns geltend. ${ }^{36}$ Dieser wirkt offenbar seit Längerem vermittelt über Städte wie Rostock oder Wismar auch in die umgebenden ländlichen Räume. ${ }^{37}$ Der Prozess schließt im Kreis Greifswald, Vorpommern, auch die salienten berlinisch-brandenburgischen „Leitformen $i k$, det, wat “ $^{\text {" } i n . ~}{ }^{38}$

Im späten 19. Jahrhundert beschleunigte sich die institutionelle Verbreitung der Standardsprache auch in vergleichsweise siedlungsleeren Räumen: „Schule, Kirche, bis zum Ausgange des Weltkrieges auch die Kaserne, und vor allem das Zeitungswesen haben das Hd. bis in die entlegensten Winkel Niederdeutschlands eindringen lassen. ${ }^{\text {" }}$ "Andererseits besaß das Niederdeutsche als Primärsprache noch erhebliche Vitalität auch in höheren sozialen Schichten, wie sie etwa die am Schweriner Gymnasium Fridericianum herausgegebene „Hochdeutsche Grammatik mit Rücksicht auf die plattdeutsche Mundart zunächst für mecklenburgische Schulen bearbeitet" dokumentiert. ${ }^{40}$

\section{Die mecklenburgisch-vorpommersche Regionalsprache nach 1945}

Die Entwicklung der mecklenburgisch-vorpommerschen Regionalsprache nach 1945 lässt einen deutlichen Rückgang der ostniederdeutschen Basisdialekte erkennen: Regionales Sprechen findet seinen Ausdruck in wachsendem Maße im Regiolekt.

Nach Schönfeld war vor 1945 das Niederdeutsche in Mecklenburg-Vorpommern die dominierende Varietät auf dem Dorfe und in der Kleinstadt. ${ }^{41}$ „Ein Hort

\footnotetext{
34 Dost 1981, S. 121.
}

35 Dost 1981, S. 122.

36 Vgl. Gernentz 1974, S. 232; Herrmann-Winter 1979, S. 152; Schönfeld 1990, S. 98; Lauf 1996, S. 198; Mihm 2000, S. 2116; Kehrein 2012, S. 310-311.

37 Vgl. Gernentz 1980, S. 149.

38 Mihm 2000, S. 2114. Vgl. Herrmann-Winter 1979, S. 152.

39 Grimme 1922, S. 8.

40 Vgl. Möhn 1983, S. 639.

41 Schönfeld 1990, S. 128-129. 
des Plattdeutschen" blieben dabei lange vor allem die Kleinstädte. ${ }^{42}$ Dies änderte sich nach 1945: Nach 1945 verlor das Niederdeutsche in Mecklenburg erheblich an Bedeutung und Geltung, vor allem durch die Bevölkerungsmischung um 1945; an seine Stelle trat in wachsendem Maße der Regiolekt bzw. die Standardsprache. ${ }^{43}$ Auch Gernentz verweist auf die „nach 1945 veränderte [...] Bevölkerungsstruktur auf den Dörfern, vor allem in den Neubauerndörfern" und bezieht dies vor allem auf „das Seßhaftwerden der Umsiedler“. ${ }^{4}$

Die These des Dialektrückgangs angesichts der Zuwanderung von Flüchtlingen und Heimatvertriebenen, die örtlich bis zu $60 \%$ der Einwohnerschaft ausmachten, erweist sich allerdings im Lichte umfangreicher aktueller Studien als empirisch nicht zutreffend: ${ }^{45}$ Vor allem die nach 1945 zugewanderten Kinder und jüngeren Erwachsenen erwarben vielfach gute bis sehr gute Kompetenzen im niederdeutschen Basisdialekt und verwendeten den Dialekt über Jahre in ihrem neuen, noch stark niederdeutsch geprägten Lebens- und Arbeitsumfeld. Auch auf der Ebene des mecklenburgischen Regiolekts zeigen die Immigranten und ihre Nachkommen zu großen Teilen starke sprachliche Anpassungstendenzen, die mitunter dazu führen, dass die zweite (bereits in Mecklenburg-Vorpommern geborene) Generation in Vertriebenenfamilien bis heute eine deutlicher regional markierte Umgangssprache als ihre Altersgenossen aus autochthonen mecklenburgischen Familien spricht.

Gernentz betont den sozioökonomischen Wandel in der SBZ und DDR: „Das Gesicht dieses Gebietes war jahrhundertelang durch die Landwirtschaft geprägt", 46 was sich in einem landwirtschaftlichen Beschäftigtenanteil von 38,3 \% (1933), erheblichem Großgrundbesitz und einer geringen Bevölkerungsmobilität zeige. Durch die Bodenreform, die LPG-Zusammenlegung, den „Übergang zur industriemäßigen Produktion ${ }^{\text {“47 }}$ habe sich die sozioökonomische Struktur des Nordens der DDR fundamental verändert. Die Industrialisierung Rostocks, insbesondere was die Werftindustrie betraf, trug zu einem enormen Bevölkerungswachstum bei. Planmäßige Zuwanderung von Arbeitskräften sorgte dafür, dass „Industriearbeiter aus anderen Bezirken der DDR in die Dörfer umzogen und damit auch das Vordringen des Hochdeutschen auf dem Lande förderten“. ${ }^{48}$ Die durch die

42 Gundlach 1988, S. 436.

43 Vgl. Schönfeld 1989, S. 140; Schönfeld 1990, S. 128.

44 Gernentz 1974, S. 219.

45 Ehlers 2013.

46 Gernentz 1974, S. 216.

47 Gernentz 1974, S. 220.

48 Gernentz 1974, S. 220.

Birte Arendt, Andreas Bieberstedt and Klaas-Hinrich Ehlers - 978-3-631-71893-3 
Schulreform geschaffenen "ländlichen Zentralschulen “499 ließen die Vermittlung der hochdeutschen Standardsprache auch bei der ländlichen Jugend durchschlagende Wirkung erlangen, insbesondere seit den 1960er Jahren: ${ }^{50}$ Schönfeld ${ }^{51}$ berichtet von einer Studie von Beckmann und Gernentz unter Rostocker Schülern, die 1955 noch zu $36 \%$ eine aktive Niederdeutschkompetenz besaßen, 1962 jedoch nur noch zu $16 \%$ (die passive Kompetenz sank von $51 \%$ auf $33 \%$ ); auf dem Lande betrug ihr Anteil 20-26\% (passiv 38-46 \%). Noch 1923 war selbst in Rostock und Wismar von 64 \% bzw. 74 \% aller Volks- und Mittelschüler die Rede, die "durchweg in der niederdeutschen Mundart gesprochen" hätten. ${ }^{52}$

Niederdeutsch wurde im Zuge dieser Entwicklung zur Sprache der Familie und Arbeitswelt, zur Gruppensprache der manuell Arbeitenden, vor allem der Älteren, besonders der Männer. Durch den forcierten Werftausbau in den 1950er Jahren spielte das Niederdeutsche auch in der industriellen Arbeitswelt noch eine größere Rolle. Als Berufssprache der (männlichen) agrarischen, handwerklichen und industriellen Arbeitswelt wurde der Dialekt vielfach auch außerhalb der Familie erworben. ${ }^{53}$ Gundlach resümiert auf der Grundlage von Sprachaufnahmen in 61 Orten mit 245 Mundartsprechern dreier Altersgruppen aus den Jahren 1962/1963, dem „Plattdeutschen haftet, von den Frauen mehr empfunden als von den Männern, leicht der Makel einer gewissen Minderwertigkeit an". ${ }^{4}$

Mit Bezug auf Herrmann-Winters umfangreiche Untersuchungen in den Nordbezirken der DDR seit Beginn der 1970er Jahre konstatiert Gernentz: „Besonders in jüngeren Ehen spielt die Mundart kaum noch eine Rolle. Selbst wenn beide Elternteile in anderen Situationen häufig niederdeutsch sprechen, wird zumeist mit Rücksicht auf die heranwachsenden Kinder - zu Hause durchweg

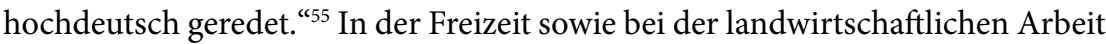
sei noch niederdeutsch gesprochen worden. Herrmann-Winter fasst die Entwicklung der Regionalsprache im „Norden der DDR“ zu Beginn der 1970er Jahre mit den Worten zusammen:

„Integration bedeutet für die Mundarten heute in erster Linie zweierlei: den schrittweisen Abbau lokaler mundartlicher Sonderformen zugunsten ausgeglichener, in Laut- und

49 Gernentz 1974, S. 220.

50 Vgl. Gernentz 1974, S. 240; Gundlach 1967, S. 175; Dost 1980, S. 149.

51 Schönfeld 1990, S. 128.

52 Gosselk 1924 nach Gernentz 1974, S. 218.

53 Vgl. Ehlers 2017.

54 Gundlach 1967, S. 174.

55 Gernentz 1974, S. 240-241.

Birte Arendt, Andreas Bieberstedt and Klaas-Hinrich Ehlers - 978-3-631-71893-3 
Formenbestand weitgehend einheitlicher großräumiger Verkehrsmundarten und die allmähliche Ablösung dieser Mundart durch eine landschaftlich geprägte hochdeutsche Umgangssprache. ${ }^{\text {“56 }}$

In Hinblick auf die soziale Verteilung und den pragmatischen Gebrauch regiolektaler Merkmale in dieser "hochdeutschen Umgangssprache“ sind insbesondere die Arbeiten aus den 1970er Jahren von Herrmann-Winter, Dahl und Gernentz hervorzuheben. Bei Herrmann-Winter bestanden die sozialen Gruppen, die die regiolektalen Merkmale besonders häufig verwenden, aus Industriearbeitern und Genossenschaftsbauern (auch mit Leitungsfunktionen); den geringsten Gebrauch wiesen die „Intelligenz" und die Schüler auf. ${ }^{57}$ Herrmann-Winter weist allerdings auf deren regionale Disparitäten hin, die im Küstenbereich noch eine höhere Kompetenz im Niederdeutschen als in den südlichen Landesteilen deutlich werden ließen..$^{58}$ Gernentz sieht pragmatische („Gesprächspartner, Gesprächsgegenstand und Gesprächssituation") gegenüber sozialen Steuerungsfaktoren als ausschlaggebend an, insgesamt weise die „Entwicklung eindeutig in Richtung der Ausbildung von großräumigen Umgangssprachen [...], keineswegs in Richtung auf sprachliche Uniformität“. ${ }^{59}$

Schönfeld erkennt für die 1970er Jahre drei soziolinguistische Bewertungstypen der Dialekt-Regiolekt-Konstellation im Norden der DDR: An der Küste werde der niederdeutsche Dialekt häufig verwendet und geschätzt, in den Übergangsgebieten (Altmark, Havelland, Prignitz, Uckermark) von Älteren und in lokalen und informellen Domänen, um Berlin und im Süden sei er aufgegeben oder besitze nur geringes Ansehen. ${ }^{60}$ Eine verbreitete Einstellung sei die Ablehnung des Dialektgebrauchs mit den Kindern, ${ }^{61}$ eine fast wortgleiche Formulierung, wie sie Mattheier für die Bundesrepublik fand. ${ }^{62}$ Der Regiolekt hingegen werde weitgehend verwendet und geschätzt, vor allem von Männern, Nichtdialektsprechern oder Jüngeren, ${ }^{63}$ mehr noch der mecklenburgische als der berlinische Regiolekt, am wenigsten der magdeburgische. ${ }^{64}$

56 Herrmann-Winter 1974, S. 173.

57 Herrmann-Winter 1979, S. 152-155.

58 Herrmann-Winter 1995, S. 186.

59 Gernentz 1974, S. 242-243.

60 Schönfeld 1981, S. 113-119.

61 Schönfeld 1981, S. 116.

62 Mattheier 1980, S. 50.

63 Schönfeld 1981, S. 117.

64 Schönfeld 1981, S. 118.

Birte Arendt, Andreas Bieberstedt and Klaas-Hinrich Ehlers - 978-3-631-71893-3 
Wenn auch das Niederdeutsche als „Volkssprache" phasenweise eine besondere Wertschätzung erfuhr, folgte die Schule in der DDR doch überall den gleichen Vorgaben der Erziehung zur deutschen „Literatursprache“. Hatte es im Lehrplan für Grund- und Oberschulen 1946 noch geheißen: „Die Mundart ist in die Sprachlehre einzubeziehen“, galt dies ab 1952 - ähnlich wie in der Bundesrepublik - nur noch für die Sprachbetrachtung: „Die Mundarten werden nur in der Sprachgeschichte berührt.“ Dialekte und Regiolekte galten in einer Empfehlung von 1955 als Problemquellen: „Der Lehrer stärkt den Willen der Schüler, ihre Muttersprache in Rede und Schrift immer sicherer zu beherrschen und Nachlässigkeiten abzulegen, die aus der Umgangssprache und der Mundart herrühren. “65 Wenn sich auch in den späten 1970er Jahren - wiederum ähnlich wie in der Bundesrepublik - die Berücksichtigung regionaler Sprache in der Bildungspolitik der DDR wieder erweiterte, so ist doch die Geschichte des Niederdeutschen in der DDR-Schule weitgehend eine Geschichte der Nichtbeachtung: Osnowski fasst „den Umgang mit Mundart und Umgangssprache in den Deutschplänen der DDR“ zusammen: „Die Mundart (wurde) in den Jahren zwischen 1946 und 1951 sehr stark einbezogen [...], von 1952 bis 1966 vorrangig abgelehnt, zwischen 1967 und 1980 fast völlig ignoriert und erst Ende der achtziger Jahre wieder verstärkt berücksichtigt." ${ }^{\circ 6}$

\section{Die mecklenburgisch-vorpommersche Regionalsprache seit 1989}

Die aktuelle regionalsprachliche Entwicklung in Mecklenburg-Vorpommern weist auch mit der deutschen Vereinigung in die Richtung einer zunehmenden Großräumigkeit: „Die Entwicklungen lassen deutlich eine Reduzierung auf wenige lokale Merkmale [...] in den städtischen Umgangssprachen erkennen. “67

Die soziodemographischen Besonderheiten des Bundeslandes MecklenburgVorpommern lösen dabei widersprüchliche strukturelle Prozesse aus. Eine gewisse Marginalisierung ist in Mecklenburg-Vorpommern in folgenden Faktoren erkennbar: Mecklenburg-Vorpommern ist seit alters ein ländlicher Raum geringer Bevölkerungsdichte. Das Bundesland hat die wenigsten Oberzentren

65 Osnowski 1998, S. 273-274. Vgl. Rösler 1998, S. 259; Brendel 2008, S. 37-46; Herrmann-Winter 1998.

66 Osnowski 1998, S. 275. Zur Einbeziehung von Dialekt und Regiolekt in MecklenburgVorpommern in verschiedenen öffentlichen Sprachdomänen vgl. Herrmann-Winter 1998 und Möller 2004.

67 Schönfeld 1989, S. 103.

Birte Arendt, Andreas Bieberstedt and Klaas-Hinrich Ehlers - 978-3-631-71893-3 
aller Flächenländer der Bundesrepublik (Rostock, Schwerin, Neubrandenburg, Greifswald/Stralsund). Die Bevölkerungsdichte macht nur ein Drittel des bundesdeutschen Durchschnitts aus und ist in den 2000er Jahren weiter abnehmend (von 74 Einwohner/ $\mathrm{km}^{2} 2005$ auf $69 \mathrm{im} \mathrm{Jahr} \mathrm{2011).} \mathrm{Sie} \mathrm{weist} \mathrm{überdies}$ ein West-Ost-Gefälle auf (von 73 in Westmecklenburg bis zu 61 im östlichen Vorpommern) ${ }^{68}$

Das Land ist ein strukturschwacher Raum und eine Abzugsregion: Die Arbeitslosenquote ist fast doppelt so hoch wie der bundesdeutsche Durchschnitt, die höchste in Deutschland, und liegt in Vorpommern noch höher. ${ }^{69}$ Die Entwicklung des Arbeitsmarkts zeigt im Zeitraum 2000-2009 (bis auf Greifswald und Wismar) überall einen Rückgang der sozialversicherungspflichtig Beschäftigten. ${ }^{70}$ Die Schülerzahlen haben sich im Zeitraum 2000-2009 in Vorpommern um 45-50 \%, in Mecklenburg um 35-40 \% reduziert. ${ }^{71}$

Parallel zu diesen Marginalisierungstendenzen ist eine wachsende Mobilität erkennbar: Der Pendleranteil an allen Arbeitnehmern beträgt in MecklenburgVorpommern $60 \%$. Der Anteil der Pendler mit mehr als $50 \mathrm{~km}$ Pendeldistanz an allen sozialversicherungspflichtig Beschäftigten am Arbeits- und Wohnort ist im Jahr 2009 in Mecklenburg-Vorpommern am höchsten in ganz Deutschland. Er liegt überwiegend bei über $17 \%$ und im Landesinneren bei über $22 \%$. PendlerZiele sind die genannten Oberzentren sowie in Westmecklenburg Hamburg und in den südlichsten Landesteilen Berlin. ${ }^{72}$ Die Tendenz ist gegenüber 2005 steigend. Nordwestmecklenburg und Ludwigslust-Parchim zählen zur „Metropolregion Hamburg“.

Gleichwohl erfreut sich regionale Sprache auch in Mecklenburg-Vorpommern einer weitgehend ungebrochenen Beliebtheit: In einer Umfrage des Instituts für niederdeutsche Sprache aus dem Jahr 2007 zur Frage „Wie gut können Sie Plattdeutsch sprechen?" antworteten in Mecklenburg-Vorpommern $23 \%$ mit sehr gut oder gut. $^{73}$ In einer Allensbach-Umfrage aus dem Jahr 2008 wird auf die Frage nach besonders gern gehörten Dialekten - neben "Norddeutschem Platt“ (29\%) - zusätzlich Mecklenburgisch (6 \%) und Pommerisch (2\%) genannt. ${ }^{74}$

68 Vgl. Statistisches Amt Mecklenburg-Vorpommern 2014.

69 Vgl. Bundesagentur für Arbeit 2012.

70 Vgl. Statistisches Amt Mecklenburg-Vorpommern 2009.

71 Vgl. Statistisches Amt Mecklenburg-Vorpommern 2009.

72 Bundesinstitut für Bau-, Stadt- und Raumforschung 2012, S. 79.

73 Möller 2008, S. 32.

74 Allensbacher Archiv: IfD-Umfrage 10016. Die Frage nach „Plattdeutsch“ bzw. „Norddeutschem Platt“ kann sich auf alle Formen regionaler Sprache beziehen. 
Die Beliebtheit regionaler Sprache in Mecklenburg-Vorpommern ist sicherlich einer der Gründe, weshalb die Förderung von „Niederdeutsch in der Schule“ Gegenstand einer Verwaltungsvorschrift des Ministeriums für Bildung, Wissenschaft und Kultur (2004) ist. Hierin wird festgestellt:

„Die niederdeutsche Sprache [...] ist ein wesentlicher Teil des Kulturgutes unseres Landes. Die Pflege des Niederdeutschen als lebendige Sprache des Alltags ist stark rückläufig. [...] Verbindliche Aufgabe der Schule [...] ist es, Kenntnisse über niederdeutsche Literatur, Kunst und Kultur zu vermitteln. Daneben soll die Schule zum Gebrauch der niederdeutschen Sprache ermuntern und insbesondere die Fähigkeit fördern, Niederdeutsch zu sprechen. [...] Niederdeutsch kann und soll nicht als eigenes Fach mit einem Stundenanteil ausgewiesen werden, muss aber an allen Schulen ein durchgängiges Unterrichtsprinzip sein. ${ }^{\text {"75 }}$

In den Rahmenplänen für Deutsch in der Sekundarstufe I wird vor allem auf die Sprachbetrachtung abgestellt. Lernziele sind, niederdeutsche Texte rezipieren, rezitieren und schreiben zu können, aber auch in niederdeutscher Sprache zu erzählen. Im Mecklenburg-Vorpommerschen Schulgesetz $\$ 2$ (3) wird festgelegt: „Die Verbundenheit der Schüler mit ihrer natürlichen, gesellschaftlichen und kulturellen Umwelt sowie die Pflege der niederdeutschen Sprache sind zu fördern.“

Wenn dies auch nur wenig über den Charakter der Förderung aussagt, so attestiert der Bericht der Bundesregierung zur Umsetzung der Europäischen Charta der Regional- oder Minderheitensprachen dem Bundesland doch einige Anstrengungen zur Förderung des Niederdeutschen in der Schule: „Lediglich in Schulen in Mecklenburg-Vorpommern wird Niederdeutsch in höherem Maße als eigenständiges Fach unterrichtet. ${ }^{\text {"16 }}$ Der die Umsetzung der Charta überprüfende EU-Expertenausschuss stellt jedoch fest, dass in der Primar- und Sekundarstufe die „Verpflichtung unbeschadet der in dieser Aufzählung enthaltenen von ihm sehr begrüßten Maßnahmen nur teilweise erfüllt" würden. ${ }^{77}$ Seit 2010 ist die Ent-

75 Ministerium für Bildung, Wissenschaft und Kultur 2004, S. 1. In seinem Landesprogramm „Meine Heimat - Mein modernes Mecklenburg-Vorpommern“ (2016, S. 7) sieht das Ministerium für Bildung, Wissenschaft und Kultur MecklenburgVorpommern als dritte Fördersäule „Plattdeutsch als freiwilliges reguläres Schulfach in den weiterführenden Schulen“ vor. Als Voraussetzung hierfür wird u. a. die Einrichtung eines Kompetenzzentrums Didaktik des Niederdeutschen in Greifswald angeregt (Landesprogramm "Meine Heimat - Mein modernes Mecklenburg-Vorpommern“ 2016, S. 8).

76 Vierter Bericht der Bundesrepublik Deutschland 2010, S. 2.

77 Vierter Bericht der Bundesrepublik Deutschland 2010, S. 10. 
wicklung rückläufig. „Nach Angaben von Vertretern der niederdeutschen Sprachgruppe ist das Niederdeutschangebot in den Vorschulen [...] sehr lückenhaft."78

An den Universitäten Greifswald (1992-2002) und Rostock (seit 1992/93) wurden Professuren für Niederdeutsche Philologie geschaffen bzw. wieder eingerichtet, in Greifswald ist der Lehrstuhl mittlerweile durch eine feste Lehrkraft (im „Schwerpunkt Niederdeutsch“) ersetzt worden. Niederdeutsch ist an beiden Universitäten möglicher Bestandteil der Studiengänge auf Bachelor- und Masterebene, auch im Lehramtsstudiengang. ${ }^{79}$ Als dringendste Maßnahme hält der Bericht fest: „Der entscheidende Schritt zu einem flächendeckenden, stetigen und ernsthaften Angebot zum Erlernen der niederdeutschen Sprache liegt in der Qualifikation der Lehrkräfte." ${ }^{80}$

In den 19 Interviews, die Birte Arendt mit 28 Gewährspersonen aus vier Familien und drei Generationen auf der Insel Usedom zu Spracheinstellungen gegenüber dem Niederdeutschen durchgeführt hat, wird deutlich, dass zur Niederdeutsch-Charakteristik bei den kompetenten Sprechern eine klare Unterscheidung in „richtiges“ und „falsches“ Niederdeutsch vorgenommen wird: „,falsches' Sprechen ist Sprachmischung ${ }^{\text {“ }}{ }^{\text {, }}$, die als mangelnde Kompetenz gewertet wird und vermieden werden sollte. Eine "Aufladung“ im Sinne einer sprachbezogenen „Identitätsinszenierung" finde sich „vermehrt bei semikompetenten Sprechern" ${ }^{82}$ Zusätzlich wurden Printmedien (Onlineausgabe der Ostsee-Zeitung 1999-2008) sowie sprachen- und bildungspolitische Dokumente untersucht. Wie Arendt resümierend feststellt, „erscheint Niederdeutsch als Sprache bei den Laien, als Freizeitgut in der Presse und als bedrohte Art und Kulturgut in der Politik“. Ein damit verbundener Effekt der „Musealisierung ${ }^{\text {“83 }}$ des Niederdeutschen sei dem Spracherhalt allerdings nicht förderlich.

Die aktuelle regionalsprachliche Entwicklung in Mecklenburg-Vorpommern lässt sich wie folgt resümieren: Strukturell lassen die Besonderheiten des Landes eine beschleunigte Aufgabe lokaler Sprachformen und einen weiteren Übergang zur Verwendung des Regiolekts erwarten. Eine sprachliche Reliktbildung in marginalen Räumen ist nicht absehbar, da die Region überaltert ist und die Jugend

78 Vierter Bericht der Bundesrepublik Deutschland 2010, S. 5.

79 Zum Niederdeutschstudium an der Universität Greifswald vgl. den Beitrag von Arendt in diesem Band. Vgl. auch Möller 2004, S. 290-292.

80 Vierter Bericht der Bundesrepublik Deutschland 2010, S. 10.

81 Arendt 2012, S. 109.

82 Arendt 2011, S. 74.

83 Arendt 2012, S. 115.

Birte Arendt, Andreas Bieberstedt and Klaas-Hinrich Ehlers - 978-3-631-71893-3 
in andere Regionen zieht, so dass die Weitergabe lokalsprachlicher Traditionen spätestens in der kommenden Generation eingeschränkt sein dürfte. Der Regiolekt hingegen genießt hohes Ansehen, ist weit verbreitet und besitzt regionale Eigenständigkeit im Bewusstsein seiner Sprecher.

\section{Merkmale des mecklenburgisch-vorpommerschen Regiolekts}

In sprachstruktureller Hinsicht ist der mecklenburgisch-vorpommersche Regiolekt der 1970er Jahre in den oben genannten Studien recht umfassend beschrieben worden. Hierbei wird meist zwischen obligatorischen und fakultativ auftretenden Sprachmerkmalen unterschieden. Herrmann-Winter findet in ihrer Studie mit 79 Gewährspersonen „charakteristische Besonderheiten gesprochener hochdeutscher Sprache im Raum nördlich der Linie Anklam - Demmin - Rostock “ ${ }^{\prime 84} \mathrm{~d}$. h. in Vorpommern und dem nordöstlichsten Mecklenburg, die „obligatorische“ Geltung haben. Dazu zählen die Hebung von langem ä (Meedchen), die Ersetzung der Affrikate $p f$ durch die Spirans $f$ (Fingsten), der [k]-Verschluss des $n g$-Lautes (Dink) und lexemspezifische Vokalkürzungen (Glass, Zuch).

Daneben treten „fakultativ“, je nach situativem Kontext, Alter und sozialer Gruppe, von den untersuchten 18 Varianten die folgenden für die Region charakteristischen Merkmale auf: ${ }^{85}$

- e-Apokope mit 92,4\% ([Iç kəm] ,ich komme', [Iç max], ich mache')

- $r$-Schwund nach langem $a$ mit 88,6\% ([ja:] ,Jahr', [ha:t] ,hart')

- stddt. <er> als [ $\varepsilon, \mathfrak{x}]$ mit 84,8 \% (['le:nən] ,lernen', [æ'tse:ln] ,erzählen')

- stddt. Affrikate $t s$ als Spirans $s$ mit 78,5 \% (['sukæ] ,Zucker')

- $t$-Apokope mit 75,9 \% ([nıç] ,nicht', [fas] ,fast')

- $r$-Elision nach Kurzvokal mit Vokalöffnung und -dehnung mit 73,4 \% (['ع:də] „Erde', ['fo:nə], vorne')

- wort- und silbenanlautende $g$-Spirantisierung mit 72,2 \% ([ja've:zn] ,gewesen', [jans] ,ganz', [fo:cjən] ,vorigen'): „, für den Norden noch ungewöhnlich“" ${ }^{\text {“6 }}$

- kurzes stddt. $a$ als [ $\varepsilon$, ə] mit 58,3\% ([d $\varepsilon$ t] ,das', [nə'he: $\varepsilon]$,nachher $\left.{ }^{c}\right)$

- Reduktion ['unze] für ,unser, unsere, unserer' mit 59,5\%

- stddt. $e$ als [ə] in Tonsilben mit 54,4\% (['rəbəra't $\left.t^{\mathrm{h}} \mathrm{u}^{\mathrm{\varepsilon}}\right]$, Reparatur')

84 Herrmann-Winter 1979, S. 173.

85 Herrmann-Winter 1979, S. 148-152.

86 Herrmann-Winter 1979, S. 152.

Birte Arendt, Andreas Bieberstedt and Klaas-Hinrich Ehlers - 978-3-631-71893-3 
- $t$ für stddt. $s$, vor allem bei Pronomen, mit 55,7 \% ([dat]/[dit] ,das '/,dies', [vat]

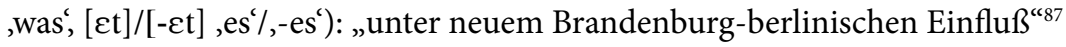

- Substitution von stddt. $r$ nach Kurzvokal durch $[\varepsilon]$ oder $[e]$ mit 50,6\% ([fo'et] ,fort')

- $a$-Velarisierung mit 49,4 \% (['nəmən] ,Namen', [gə'zə:xt ${ }^{\mathrm{h}}$ ], gesagt')

- l-Velarisierung mit 38,0 \% ([gett] ,Geld', [foł] ,voll')

- $r$-Elision bzw. schwache Vokalisierung nach Langvokal (außer /a:/) mit 29,1 \% ([hi: $]$, hier ${ }^{\mathrm{r}}$ )

- stddt. $-r g$ als $-x$ mit 10,1\% ([bu:x] ,Burg')

Am häufigsten waren diese Merkmale schon damals auf dem Lande vertreten, besonders bei der älteren Generation der vor dem Ersten Weltkrieg Geborenen sowie der „Werktätigen aus Industrie und Handwerk“, am wenigsten bei den Jüngeren, vor allem den Schülern, und der "Intelligenz “.88

Lauf beschreibt - auf der Grundlage von Aufnahmen u. a. des Pfeffer-Korpus (1961, Sprecher aus Greifswald und Rostock der Jahrgänge 1892-1947) - die Merkmale der „Mecklenburg-vorpommerschen Umgangssprache “. ${ }^{99}$ Neben „gemeinniederdeutschen" Merkmalen - das sind u. a. die bei Herrmann-Winter so bezeichneten „obligatorischen“ Varianten - benennt Lauf über die im weiteren nord- und nordostdeutschen Raum verbreiteten Merkmale hinaus einige regiolektale Besonderheiten: ${ }^{90}$

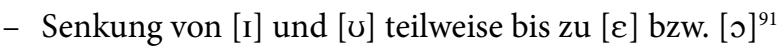

- vereinzelt anlautende $g$-Spirantisierung im Süden des Gebiets

- velarer Lateral [ł], vor allem auslautend, nach Süden hin abnehmend

- gelegentliche retroflexe Artikulation des $/ \mathrm{r} /{ }^{92}$

Auf morphologischer Ebene ist die spezifisch mecklenburgische Diminutivbildung auf -ing zu nennen, die aus dem Basisdialekt in den Regiolekt übernommen wurde und dort auch von nicht niederdeutsch kompetenten Sprechern mit pragmatischen Funktionen benutzt wird. ${ }^{93}$

In Hinblick auf die vertikale Sprachlagenschichtung unterscheidet Eva-Sophie Dahl für Rostock und Umgebung im regiolektalen Bereich des Spektrums zwei

87 Herrmann-Winter 1979, S. 152.

88 Herrmann-Winter 1979, S. 174.

89 Lauf 1996.

90 Lauf 1996, S. 200-205.

91 Vgl. Lauf 1996, S. 198.

92 Vgl. Lauf 1996, S. 204.

93 Vgl. Ehlers 2011. 
Schichten, eine „Umgangssprache“ (U) und eine „mundartnahe Umgangssprache" $\left(\mathrm{U}^{\mathrm{m}}=\right.$ hochdeutsch basierter Regiolekt mit starken niederdeutschen Interferenzen), die von der dem niederdeutschen Spektrum zugehörigen „umgangssprachenahen Mundart" $\left(\mathrm{M}^{\mathrm{u}}=\right.$ niederdeutscher Dialekt mit hochdeutschen Interferenzen) abgegrenzt werden. ${ }^{94}$

Eine ähnliche Unterscheidung findet sich auch bei Schönfeld: Als gemeinsame Merkmale der Sprachschichten „Umgangssprache“ (U) und „mundartnahe Umgangssprache“ $\left(\mathrm{U}^{\mathrm{m}}\right)$ nennt Schönfeld (basierend auf Sprachdaten aus den 1970er Jahren) Formen wie die Auslautspirans [tax] ,Tag', plosivisches [k] nach [n] [lank] ,lang', Vokalkürze in [glas] ,Glas', Öffnung in [عedə] ,Erde', Hebung in [ e:mən] ,schämen', Anlautspirans in [faefə] ,Pfeife' sowie Endungsreduktionen und $r$-Vokalisierung. ${ }^{95}$ Spezifisch für die Sprachschicht $\mathrm{U}^{\mathrm{m}}$ seien der $r$-Schwund vor Konsonant in [ha:t] ,hart', Anlautspirans in [sokæ] ,Zucker', $d$-Assimilation in [honæt] ,hundert', einzellexematische Rundung [rmæ] ,immer.

Kehreins Studie eröffnet die Möglichkeit, die ca. 40 bis 50 Jahre alten Beschreibungen des Sprachstands bei Herrmann-Winter, Dahl, Schönfeld und Lauf mit dem aktuellen Befund zu vergleichen. ${ }^{96}$ Grundlage bilden die Daten von vier Sprechern der Geburtsjahrgänge 1943, 1959, 1960 und 1990 aus Stralsund/ Vorpommern. Kehrein interpretiert die folgenden Merkmale als Elemente einer „Restarealität": :97

Vokalismus:

- /e:/, /ø:/, /o:/ als Diphthong(oid)e

- Rundung von $/ \mathrm{I} /$, besonders in labialer Umgebung

- Hebung von $/ \varepsilon: /$ und $/ \varepsilon /$, besonders vor vokalisiertem /r/-Allophon, mit Aufhebung der Opposition $/ \mathrm{e}: / \neq \mid \varepsilon: /$

- Das „häufigste und vielleicht auch auffälligste Merkmal“ ist die Realisierung von $<$ er $>$ als Affix oder Teil eines Affixes in ver-, zer-als $[\varepsilon, æ] .^{98}$

Selten bzw. nur vereinzelt treten in seinem Korpus folgende Varianten auf:

- Kurze Realisierung von stddt. /a:/ in unflektierten Einsilbern (Tag, Glas, Schlag)

- Senkung von stddt. /o:/ im betonten Präfix vor- ([fo:c-] z. B. in Vorpommern)

- [vf] für auf, [ke:n] ,kein, [glo:b] ,glaube, [o:x] , auch bei dem jüngsten Sprecher

94 Dahl 1974.

95 Schönfeld 1989, S. 71-72.

96 Kehrein 2012, S. 309-313.

97 Kehrein 2012, S. 309.

98 Kehrein 2012, S. 311.

Birte Arendt, Andreas Bieberstedt and Klaas-Hinrich Ehlers - 978-3-631-71893-3 


\section{Konsonantismus:}

- g-Spirantisierung im (Stamm-)Auslaut (Tag, gesagt, gelegt, weg)

- Lenisierung von inlautendem /t/ (Leute, hatte, Mittag)

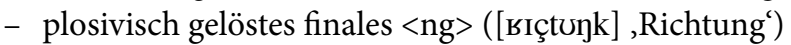

- Realisierung von (stamm-)anlautendem germ. $p$ als Spirans [f] ([gəfle:çtəs] ,gepflegtes')

- Ausfall von $d$ in der Lautverbindung [nd], auch über Wortgrenzen hinweg ([kınc] ,Kinder', [Ine] ,in der')

- Ausfall von $t$ in der Lautverbindung [st] im In- und Auslaut (in meistens, sonst)

- Erhalt der unverschobenen germ. Tenues (einzellexematisch in ich, das, was)

Selten nur finden sich die Varianten:

- $b$-Spirantisierung im Inlaut

- Spirantisierung von anlautendem /g/ im Präfix ge-

Nicht mehr belegt sind die Varianten:

- anlautendes /j/ als [3] oder [d3]

- -tion als [t $\int$ on, $\mathrm{t}$ [oun]

- Spirans [s] für anlautendes /ts/

- velares oder retroflexes /1/

- apikales [r]

Während $s$ für $t s$ bei Herrmann-Winter ${ }^{99}$ noch mit 78,5 \% belegt ist, velares $a$ mit $49,4 \%$, velares / $/$ / noch mit 38,0 \% und Dahl bzw. Gernentz ${ }^{100}$ alle drei - ebenso wie apikales $[\mathrm{r}]$ - noch als Merkmale der "mundartnahen Umgangssprache“ $\mathrm{U}^{\mathrm{m}}$ (s. o.) erwähnen, findet Kehrein für diese Formen keine Belege mehr. ${ }^{101}$ Die Sprachlage „mundartnahe Umgangssprache ${ }^{\text {“102 }}$ ist offenbar in den vergangenen 50 Jahren geschwunden.

Allerdings weist Ehlers nach, dass von 24 untersuchten autochthonen Sprechern der Vorkriegsgeneration im Raum Rostock apikales [r] noch zu über $90 \%$ im intendierten Niederdeutsch verwendet wird, ebenso wie von 13 zugewanderten Heimatvertriebenen (mit 80 \%), während sich der Gebrauch bei 1950-1970 Geborenen etwa halbiert. ${ }^{103}$ Wer in dieser Generation jedoch ausschließlich apikales [r] realisiert, behält dies auch in den standardnahen Sprachlagen bei: „Dafür ist aber

\footnotetext{
99 Herrmann-Winter 1979.

100 Dahl 1974; Gernentz 1974.

101 Kehrein 2012, S. 311.

102 Nach Dahl 1974.

103 Ehlers (i. Vorber.).
} 
bemerkenswert, dass die beiden einzigen Angehörigen der jüngeren Altersgruppe, die das $r$ im intendierten Niederdeutsch noch ausschließlich apikal bilden, auch in der regiolektalen Sprache, die sie im Interview verwenden, die prävokalischen $r$ als Zungenspitzen- $r$ artikulieren. ${ }^{\text {104 }}$

Kehrein kommt angesichts seines empirischen Befundes zu der Schlussfolgerung:

„Zwischen Dialekt und Regiolekt bildet sich [...] kein , mittlerer Bereich' aus [...] Schließlich lässt sich die Beobachtung, dass viele der nach Dahl (1974) für die Sprachschicht $\mathrm{U}^{\mathrm{m}}$, also der standardfernsten Varianten der hochdeutschen Umgangssprache, konstitutiven regionalsprachlichen Varianten in den standardorientierten Gesprächen meiner Sprecher nicht mehr enthalten sind, als ein diachroner Prozess der Standardannäherung deuten. ${ }^{\text {"105 }}$

Ältere Sprecher beherrschten noch Niederdeutsch, jedoch gebe es eine „große Zahl an Sprechern, die lediglich über eine monovarietäre Kompetenz des Regiolekts verfügen" ${ }^{106}$ Noch in der zwischen 1950 und 1970 geborenen Altersgruppe sind hingegen fundamentale Kompetenzen im Niederdeutschen durchaus vorhanden, die allerdings kaum einmal zu einem aktiven Gebrauch der Varietät genutzt werden. ${ }^{107}$

Übereinstimmend wird von Herrmann-Winter, Schönfeld und Lauf sowie Mihm und Kehrein auf Berliner Einfluss besonders in den südlichen und östlichen Gebieten Mecklenburg-Vorpommerns hingewiesen. ${ }^{108}$ Dieser mache sich in folgenden Merkmalen geltend:

- Zentralisierung von [I] und [ $\mathrm{\mho}]$ in von der Stadtsprache Berlins beeinflussten Räumen ${ }^{109}$

- [uf] für auf, [glo:b] ,glaube, [o:x] ,auch, die nach Kehrein eine „Orientierung am Berliner Dialekt/Regiolekt" und dessen „Vordringen nach Norden ${ }^{\text {“110 }}$ zeige

- anlautende $g$-Spirantisierung im Übergangsgebiet zum brandenburgischen Sprachraum, ${ }^{111}$ nach Kehrein ,ein Merkmal des südlichen MecklenburgischVorpommerschen“, das vermutlich „Berliner Einfluss“ entspringt ${ }^{112}$

104 Ehlers (i. Vorber.).

105 Kehrein 2012, S. 313.

106 Kehrein 2012, S. 313.

107 Vgl. Ehlers (i. Vorber.).

108 Herrmann-Winter 1979; Schönfeld 1981, 1989, 1990; Lauf 1996; Mihm 2000; Kehrein 2012.

109 Vgl. Lauf 1996, S. 198.

110 Kehrein 2012, S. 310. Vgl. Schönfeld 1989, S. 90.

111 Vgl. Lauf 1996, S. 204-205.

112 Kehrein 2012, S. 311.

Birte Arendt, Andreas Bieberstedt and Klaas-Hinrich Ehlers - 978-3-631-71893-3 
- „Markierungen wie dat, wat, et, Jejend, die auf neueren berlinischen Einfluß zurückgehen". ${ }^{113}$

Schönfeld ${ }^{114}$ sieht „zwischen der Berliner sowie der Rostocker und Greifswalder Umgangssprache mehrere Übereinstimmungen“": ${ }^{115}$

- Rundung vorderer Kurzvokale ([œlf], elf')

- $r$-Vokalisierung mit Ersatzdehnung des vorangehenden Vokals ([də:(ь)f] ,Dorf')

- g-Spirantisierung im absoluten und gedeckten Auslaut ([tax] ,Tag', [ve:ç] ,Weg', [busç] ,Burg', [za:xt] ,sagt', [fliiçt] ,fliegt')

- Plosiv $p$ statt stddt. Affrikate pf (Appel, Kopp, Strümpe)

- intervokalisch - tt-als - $d d$ - (Vadda)

- Assimilierung von $d$ in -nd- (hunnat, hundert')

Areal eingeschränkt auf die süd(öst)lichen Landesteile gelte dies für die Monophthongierung [be:n] ,Bein', die $g$-Spirantisierung anlautend im Präfix je- ${ }^{116}$ und intervokalisch [ve:jə], Wege', die $r$-Vokalisierung zu [a/r] [fered] ,Pferd', was weitgehend ,jüngeren Berliner Einflüssen auf die mecklenburgische Umgangssprache in ihren südlichen und östlichen Regionen "117 geschuldet ist.

Die arealen Gemeinsamkeiten zwischen den Regiolekten aus MecklenburgVorpommern und Berlin-Brandenburg werden von den Sprechern selbst deutlich wahrgenommen. Dies belegen die Erhebungen des Atlas der deutschen Alltagssprache (ADA), die eine deutlich empfundene Ähnlichkeit mit der „Alltagssprache" Berlins bis weit in den Norden zeigen. ${ }^{118}$ Die ADA-Karte „Ähnlichkeit Berlin“ („Wie stark unterscheidet sich nach Ihrer Einschätzung die Alltagssprache Berlins von der Alltagssprache Ihres Ortes?“; Antwortkategorien zwischen 1-2 „ziemlich ähnlich“ und 5-6 „sehr unähnlich“) zeigt die größte Ähnlichkeitsstufe (1-2) nach Norden bis Schwedt im Osten und Wittenberge im Westen Nordbrandenburgs, nördlich davon eine immer noch recht stark empfundene Ähnlichkeit (2-3) in einem 100-150-km-Radius nördlich Berlins von Prenzlau (Uckermark, Mittelpommersch) über Neubrandenburg und Waren im mittleren bis Ludwigslust im südwestlichen Mecklenburg. Nördlich davon wird eine größere Ähnlichkeit

113 Mihm 2000, S. 2116. Ähnlich bereits Herrmann-Winter 1979, S. 152.

114 Schönfeld 1989, S. 89-95.

115 Schönfeld 1989, S. 103.

116 Schönfeld 1989, S. 91.

117 Schönfeld 1989, S. 103.

118 Vgl. Elspass/Möller 2003 ff., 6. Runde, Frage 3. 
(1-2) mit Hamburg, Bremen und Kiel empfunden (ADA-Karten „Ähnlichkeit mit Bremen, Hamburg, Kiel“). Am geringsten ist das Empfinden sprachlicher Unterschiedlichkeit gegenüber Berlin in dieser Region im südöstlichen Vorpommern (ADA-Wabenkarte des „Anteils der nicht gemeinsamen Nennungen“ in benachbarten Orten).

\section{Ergebnisse des SiN-Projekts zum mecklenburgisch-vorpommerschen Regiolekt}

Die Daten des SiN-Projekts ${ }^{119}$ spiegeln die Eigenheiten des mecklenburgischvorpommerschen Regiolekts ebenso wie die oben beschriebene areale Gliederung in einer Reihe von Merkmalen wider:

- Die Lenisierung von stddt. $p, t, k$ im Inlaut (besonders $p$ und $t$ ) ebenso wie die Realisierung von $<$ er $>$ im Auslaut als $[\varepsilon]$ oder $[\varepsilon:]$ und mit geringer Frequenz auch die Diphthongierung von langem stddt. $e$, $o$, ö zeigen eine Gemeinsamkeit insbesondere mit dem nordniederdeutschen Raum, also mit den gemeinhin als ,dialektstarke Regionen“"120 geltenden Gebieten.

- Die $t$-Apokope ist in ganz Norddeutschland verbreitet, in den Wortarten Nomen und Adjektiv (Nacht, schlecht) ist sie in Mecklenburg ähnlich hoch wie im äußersten Norden, in Vorpommern dagegen niedriger als im Durchschnitt.

- Die Senkung von stddt. langem $e, o$, $\ddot{o}$ vor $r$ grenzt Mecklenburg-Vorpommern mit mittleren Frequenzen deutlich ab von Mittelpommern und Brandenburg; es geht hierin mit dem Norden und Westen des gesamtnorddeutschen Untersuchungsgebiets.

- Die Rundung von stddt. kurzem $i$ ist eine zugleich ost- und norddeutsche Erscheinung. Dies gilt ähnlich für die Assimilation von stddt. -nd-im Inlaut zu -nn- sowie - bei geringen Belegzahlen - für die Verwendung des Schwa statt des Vollvokals in der Endsilbe von Kaffee.

Der Erhalt von unverschobenem $t$ im Auslaut lässt die areale Ost-West-Gliederung des mecklenburgisch-vorpommerschen Regiolekts erkennen:

- - $t$ in stddt. das, was, es/-es findet sich regiolektal fast nur in Vorpommern, das hier mit Brandenburg und Mittelpommern übereinstimmt: Unverschobenes $-t$ in dat/dit (in allen syntaktischen Funktionen) erreicht ebenso wie in wat hohe Werte von 80-100\%, etwas geringere et ,es' (mit 60-80\% im

119 Vgl. Elmentaler/Rosenberg 2015.

120 Schröder 2015, S. 39.

Birte Arendt, Andreas Bieberstedt and Klaas-Hinrich Ehlers - 978-3-631-71893-3 
informellen Tischgespräch). Dies gilt allerdings nicht für das Morphem -et, das in Mecklenburg-Vorpommern fast nicht vorhanden ist. Die berlinischbrandenburgische Vokalhebung in dit/dis zeigt - ähnlich wie in Mittelpommern - nur geringen Einfluss in Mecklenburg-Vorpommern (nur 5-10 \% Vokalhebung in Vorpommern mit dit, in Mecklenburg mit dis).

- Auch die monophthongische Realisierung von stddt. Diphthongen ([o:x] ,auch') vereint das östliche Vorpommern - auf niedrigem Frequenzniveau mit Mittelpommern und dem Berlin-Brandenburger Raum.

- Die Kontraktion von so und unbestimmtem Artikel (sone) ist überall im norddeutschen Raum zu finden, die Vokalqualität zeigt hingegen die höchsten Anteile der offenen, kurzen Vokalrealisierungen im Nordosten unter Einschluss Vorpommerns.

- Der Erhalt von unverschobenem $p$ statt der stddt. Affrikate $p f$ findet sich vor allem im Osten des Untersuchungsgebiets.

- Das unverschobene $k$ im Lexem ich ist regiolektal auch im östlichen Mecklenburg-Vorpommern noch (mit fast $40 \%$ ) verbreitet.

- Kurzvokal statt standarddeutscher Länge ist in Vorpommern nur mit mittleren Prozentwerten (20-30 \%) vertreten: Während Lexeme auf -ag hohe Werte wie überall sonst in Norddeutschland zeigen, nimmt die Frequenz bei nach und noch deutlicher bei schon und über ab und geht bei wieder und Oma gegen Null.

- Die g-Spirantisierung im Wort- und Silbenanlaut ist in den SiN-Daten aus Mecklenburg-Vorpommern kaum präsent. Im Wort- und Morphemauslaut ist sie hochfrequent, höher als im berlinisch-brandenburgischen Raum und fast so hoch wie in der nordniederdeutschen Region, im Wortinlaut liegt sie ähnlich hoch wie in Berlin-Brandenburg. Scharioth ${ }^{121}$ weist anhand einer Auswahl von 27 SiN-Gewährspersonen aus Holstein, Mecklenburg-Vorpommern und Mittelpommern ebenfalls auf die deutliche Differenz zwischen MecklenburgVorpommern sowie Holstein einerseits und Mittelpommern andererseits hin, die sich u. a. im Gebrauch der $g$-Spirantisierung und den darauf bezogenen Sprechereinstellungen geltend mache. In Mecklenburg-Vorpommern und Holstein sei die Anlautspirantisierung kaum existent; sie komme nennenswert überhaupt nur in Vorpommern vor. Die Spirantisierung im Morphemauslaut ([za:xt] ,sagt', [mø:çlıç] ,möglich') zeige ein Ost-West-Gefälle von Holstein bis Mittelpommern: „In Mittelpommern wird in allen Situationen mehr spirantisiert als in Mecklenburg-Vorpommern, in Mecklenburg-Vorpommern mehr als in Hol-

121 Scharioth 2015. 
stein, d. h. die Spirantisierung des $g$ nimmt von Westen nach Osten zu. In allen drei Regionen sind die Werte höher als im restlichen norddeutschen Gebiet. “122

\section{Zusammenfassung}

Die mecklenburgisch-vorpommersche Regionalsprache erweist sich als eine in ihren Merkmalen markante und von den benachbarten Großräumen deutlich abgegrenzte regionalsprachliche Konfiguration. Der Basisdialekt und der Regiolekt zeigen eine Nord-Süd-Gliederung und eine gewisse Ost-WestDifferenzierung. Die regionalsprachliche Entwicklungsdynamik ist durch berlinisch-brandenburgischen regiolektalen Einfluss gekennzeichnet, der sich im Besonderen in den südlichen und östlichen Landesteilen bemerkbar macht. Dort, vor allem in Mittelpommern, findet sich durchaus eine Wertschätzung des Niederdeutschen bei allerdings stark abnehmendem Gebrauch und einer geringen Repräsentation des Niederdeutschen im Regionalbewusstsein, ${ }^{123}$ das eher vom Regiolekt getragen wird. In dieser Hinsicht schließt sich die südöstliche Region an die brandenburgische Entwicklung an. Die soziostrukturellen Gegebenheiten des Landes lassen eine sich fortsetzende Aufgabe lokaler Sprachformen und einen weiteren Übergang zur Verwendung des Regiolekts erwarten. Die Weitergabe lokalsprachlicher Traditionen dürfte angesichts der Bevölkerungsabwanderung weiter zurückgehen. Der Regiolekt hingegen genießt hohes Ansehen, ist weit verbreitet und besitzt regionale Eigenständigkeit im Bewusstsein seiner Sprecher.

\section{Literatur}

Allensbacher Archiv 2008: IfD-Umfrage 10016. Allensbach Februar 2008. [Online-Ressource: http://www.ifd-allensbach.de/uploads/tx_reportsndocs/ prd_0804.pdf (Stand: 10.12.2016)]

Arendt, Birte: Plattdeutsche Glokalisierung. Identitätsinszenierung auf subnationaler Ebene. In: Schiewe, Jürgen [u. a.] (Hrsg.): Kommunikation für Europa II. Sprache und Identität. Frankfurt a. M. [u. a.] 2011, S. 67-76.

Arendt, Birte: Niederdeutschdiskurse. Spracheinstellungen im Kontext von Laien, Printmedien und Politik. In: Niederdeutsches Jahrbuch 135 (2012), S. 101-117.

122 Scharioth 2015, S. 107.

123 Vgl. Scharioth 2015.

Birte Arendt, Andreas Bieberstedt and Klaas-Hinrich Ehlers - 978-3-631-71893-3 
Balck, Karl Wilhelm August: Mecklenburg im dreißigjährigen Kriege (Jahrbücher des Vereins für meklenburgische Geschichte und Alterthumskunde; 68). Schwerin 1903.

Böttcher, Kurt: Das Vordringen der hochdeutschen Sprache in den Urkunden des niederdeutschen Gebietes vom 13. bis 16. Jahrhundert. In: Zeitschrift für deutsche Mundarten 17 (1922), S. 97-108.

Brandt, Doreen: ,Nuwe mere und ,nige mere': Untersuchung zur hochdeutschniederdeutschen Sprachmischung im ,Rostocker Liederbuch. In: Niederdeutsches Jahrbuch 137 (2014), S. 59-79.

Brendel, Anja: Dialekte im Bildungssystem der DDR am Beispiel des niederdeutschen Sprachraums. Bachelorarbeit Frankfurt a. d. Oder 2008.

Bundesagentur für Arbeit 2012: Statistik. Statistik nach Regionen. [OnlineRessource: https://statistik.arbeitsagentur.de/Navigation/Statistik/Statistiknach-Regionen (Stand: 17.03.2015)]

Bundesinstitut für Bau-, Stadt- und Raumforschung Raumordnungsbericht 2011. Bonn 2012.

Dahl, Eva-Sophie: Interferenz und Alternanz - zwei Typen der Sprachschichtenmischung im Norden der Deutschen Demokratischen Republik. In: Ising, Gerhard (Hrsg.): Aktuelle Probleme der sprachlichen Kommunikation. Berlin 1974, S. 339-388.

Dost, Wolfgang: Zur Einwirkung der kommunikativen Bedingungen in der DDR auf die regionale Abgrenzung im Bereich der Umgangssprache und der Mundart, dargestellt an der Entwicklung im Raum Wittstock-Röbel. In: Das Niederdeutsche in Geschichte und Gegenwart. Hrsg. vom Zentralinstitut für Sprachwissenschaft der Akademie der Wissenschaften der DDR (Linguistische Studien. Reihe A: Arbeitsberichte; 75.2). Berlin 1981, S. 121-127.

Ehlers, Klaas-Hinrich: „Dürfen wir essing?“ Beobachtungen zur Übernahme des mecklenburgischen Diminutivs in das regionale Hochdeutsch. In: Niederdeutsches Jahrbuch 134 (2011), S. 79-92.

Ehlers, Klaas-Hinrich: Führte die Immigration der Heimatvertriebenen nach 1945 zu Dialektverlust und Nivellierung regionalsprachlicher Differenzen? Beobachtungen aus einer Untersuchungsregion in Mecklenburg. In: Niederdeutsches Jahrbuch 136 (2013), S. 97-116.

Ehlers, Klaas-Hinrich: Von der Sprachbiographie zur Sprachgebrauchsgeschichte: die Rekonstruktion des Varietätengebrauchs auf den Rostocker Werften. Erscheint in: Jürgens, Carolin/Schröder, Ingrid (Hrsg.): Sprachliche Variation in autobiographischen Interviews. Theoretische und methodische Zugänge. Frankfurt a. M. [u. a.] 2017, S. 143-165. 
Ehlers, Klaas-Hinrich: Meihen, meiden, meigen ,mähen ' - Zur Hiattilgung im mecklenburgischen Niederdeutsch. (in diesem Band)

Ehlers, Klaas-Hinrich: Geschichte der mecklenburgischen Regionalsprache seit dem Zweiten Weltkrieg. Varietätenkontakt zwischen Alteingesessenen und immigrierten Vertriebenen. (i. Vorber.)

Elmentaler, Michael/Rosenberg, Peter (Hrsg.): Norddeutscher Sprachatlas (NOSA). Bd. 1: Regiolektale Sprachlagen. Hildesheim/Zurich/New York 2015.

Elspass, Stephan/Möller, Robert (Hrsg.): Atlas der deutschen Alltagssprache. [ADA]. 2003 ff. [Online-Ressource: http://www.atlas-alltagssprache.de (Stand: 2.2.2014)]

Foerste, William: Die Geschichte der niederdeutschen Mundarten. In: Stammler, Wolfgang (Hrsg.): Deutsche Philologie im Aufriß. 1. Bd., 2., überarb. Aufl. Berlin 1957, Sp. 1729-1898.

Gernentz, Hans-Joachim: Die kommunikative Funktion der niederdeutschen Mundart und hochdeutschen Umgangssprache im Norden der DDR, unter besonderer Berücksichtigung der Interferenz und Alternanz zwischen diesen beiden sprachlichen Existenzformen. In: Studia Germanica Gandensia 15 (1974), S. 209-244.

Gernentz, Hans-Joachim: Niederdeutsch - gestern und heute. Beiträge zur Sprachsituation in den Nordbezirken der DDR in Geschichte und Gegenwart. 2. Aufl. Rostock 1980.

Grimme, Hubert: Plattdeutsche Mundarten (Sammlung Göschen; 461). 2. Aufl. Berlin/Leipzig 1922.

Gundlach, Jürgen: Plattdeutsch in Mecklenburg heute. Bericht über die Tonbandaufnahmen der mecklenburgischen Mundart 1962/63. In: Lachs, Johannes (Hrsg.): Rostocker Beiträge. Regionalgeschichtliches Jahrbuch der mecklenburgischen Seestädte. 1. Bd. Rostock 1967, S. 173-194.

Gundlach, Jürgen: Volkssprache. In: Bentzien, Ulrich/Neumann, Siegfried (Hrsg.): Mecklenburgische Volkskunde. Rostock 1988, S. 423-438.

Herrmann-Winter, Renate: Auswirkungen der sozialistischen Produktionsweise in der Landwirtschaft auf die sprachliche Kommunikation in den Nordbezirken der Deutschen Demokratischen Republik. In: Ising, Gerhard (Hrsg.): Aktuelle Probleme der sprachlichen Kommunikation. Soziolinguistische Studien zur sprachlichen Situation in der Deutschen Demokratischen Republik. Berlin 1974, S. 135-190.

Herrmann-Winter, Renate: Studien zur gesprochenen Sprache im Norden der DDR. Soziolinguistische Untersuchungen im Kreis Greifswald (Akademie der Wissenschaften der DDR. Zentralinstitut für Sprachwissenschaft: Reihe Sprache und Gesellschaft; 14). Berlin 1979.

Herrmann-Winter, Renate: Kleines plattdeutsches Wörterbuch für den mecklenburgisch-vorpommerschen Sprachraum. Rostock 1985. 
Herrmann-Winter, Renate: Sprache und Sprechen in Pommern. In: Niederdeutsches Jahrbuch 118 (1995), S. 165-187.

Herrmann-Winter, Renate: Neues hochdeutsch-plattdeutsches Wörterbuch für den mecklenburgisch-vorpommerschen Sprachraum: sinngleiche und sinnähnliche Wörter, Phrasen und Redensarten. 2. Aufl. Rostock 2003.

Herrmann-Winter, Renate: Sprachatlas für Rügen und die vorpommersche Küste. Kartographie Martin Hansen. Rostock 2013.

Herrmann-Winter, Renate (Hrsg.): Heimatsprache zwischen Ausgrenzung und ideologischer Einbindung. Niederdeutsch in der DDR. Frankfurt a. M. [u. a.] 1998.

Kehrein, Roland: Regionalsprachliche Spektren im Raum. Zur linguistischen Struktur der Vertikale (ZDL Beihefte; 152). Stuttgart 2012.

Kratz, Gustav: Die Städte der Provinz Pommern. Abriß ihrer Geschichte, zumeist nach Urkunden. Bearb. von Dr. Gustav Kratz, weiland zweitem Archivar am Königl. Provinzial-Archive zu Stettin. Berlin 1865.

Kretschmer, Paul: Wortgeographie der hochdeutschen Umgangssprache. Göttingen 1918.

Krüger, Renate: Mecklenburg. Wege eines Landes. 2005. [Online-Ressource: http:// www.dreikoenigswege.de/9._Der_Dreissigjahrige_Krieg_und_seine_Folgen.pdf (Stand: 17.03.2015)] (auch: Krüger, Renate: Mecklenburg. Wege eines Landes. Wismar 2007.)

Landesprogramm „Meine Heimat - Mein modernes Mecklenburg-Vorpommern. Ministerium für Bildung, Wissenschaft und Kultur Mecklenburg-Vorpommern. 2016 [Online-Ressource: www.regierung-mv.de/serviceassistent/_php/download. php?datei_id=1573248 (Stand: 3.11.2016)]

Lasch, Agathe: Die Berliner Volkssprache. In: Peters, Robert/Sodmann, Timothy (Hrsg.): Agathe Lasch. Ausgewählte Schriften zur niederdeutschen Philologie [1911-12]. Neumünster 1979, S. 471-486.

Lauf, Raphaela: ,Regional markiert'. Großräumliche Umgangssprache(n) im niederdeutschen Raum. In: Niederdeutsches Jahrbuch 119 (1996), S. 193-218.

Lindow, Wolfgang/Möhn, Dieter/Niebaum, Hermann/Stellmacher, Dieter/Taubken, Hans/Wirrer, Jan: Niederdeutsche Grammatik (Schriften des Instituts für niederdeutsche Sprache. Reihe Dokumentation; 20). Leer 1998.

Mattheier, Klaus J.: Pragmatik und Soziologie der Dialekte. Eine Einführung in die kommunikative Dialektologie des Deutschen. Heidelberg 1980.

Mihm, Arend: Die Rolle der Umgangssprachen seit der Mitte des 20. Jahrhunderts. In: Besch, Werner [u. a.] (Hrsg.): Sprachgeschichte. Ein Handbuch zur Geschichte der deutschen Sprache und ihrer Erforschung (Handbücher zur 
Sprach- und Kommunikationswissenschaft; 2.2). 2. Teilb., 2., vollständig neu bearb. und erw. Aufl. Berlin/New York 2000, Sp. 2106-2137.

Ministerium für Bildung, Wissenschaft und Kultur Mecklenburg-Vorpommern: Niederdeutsch in der Schule. Verwaltungsvorschrift des Ministeriums für Bildung, Wissenschaft und Kultur. Vom 9. März 2004. [Online-Ressource: http:// www.bildungmv.de/export/sites/bildungsserver/downloads/VerordnungNiederdeutsch-in-der-Schule.pdf (Stand: 10.12.206)]

Möhn, Dieter: Niederdeutsch in der Schule. In: Cordes, Gerhard/Möhn, Dieter (Hrsg.): Handbuch zur niederdeutschen Sprach- und Literaturwissenschaft. Berlin 1983, S. 631-659.

Möller, Frerk: Niederdeutsch: das sozio-kulturelle Umfeld. In: Stellmacher, Dieter (Hrsg.): Niederdeutsche Sprache und Literatur der Gegenwart (Germanistische Linguistik; 175/176). Hildesheim 2004, S. 281-358.

Möller, Frerk: Plattdeutsch im 21. Jahrhundert. Bestandsaufnahme und Perspektiven. Mit einem Aufsatz von Michael Windzio (Schriften des Instituts für Niederdeutsche Sprache; 34). Leer 2008.

Niebaum, Hermann: Niederdeutsch in Geschichte und Gegenwart. In: Schuppenhauer, Claus (Hrsg.): Niederdeutsch. Fünf Vorträge zur Einführung. Eine Gemeinschaftsveranstaltung von Universität Bremen und Institut für niederdeutsche Sprache. Wintersemester 1985/86 (Schriften des Instituts für niederdeutsche Sprache. Reihe Dokumentation; 12). Leer 1986, S. 7-41.

Osnowski, Stefan: Niederdeutsch in den Deutsch-Lehrplänen der DDR. In: Herrmann-Winter, Renate (Hrsg.): Heimatsprache zwischen Ausgrenzung und ideologischer Einbindung. Niederdeutsch in der DDR. Frankfurt a. M. [u. a.] 1998, S. 271-280.

Protze, Helmut: Wortatlas der städtischen Umgangssprache. Zur territorialen Differenzierung der Sprache der Länder Mecklenburg-Vorpommern, Brandenburg, Berlin, Sachsen-Anhalt und Thüringen. Köln [u. a.] 1997.

Rösler, Irmtraud: Untersuchungen zum Eindringen des Hochdeutschen im Norden des deutschen Sprachgebietes. In: Das Niederdeutsche in Geschichte und Gegenwart (Zentralinstitut fur Sprachwissenschaft. Reihe A: Arbeitsberichte; 75.1). Hrsg. vom Zentralinstitut für Sprachwissenschaft der Akademie der Wissenschaften der DDR, Berlin 1981, S. 64-74.

Rösler, Irmtraud: Niederdeutsch und Schule. In: Herrmann-Winter, Renate (Hrsg.): Heimatsprache zwischen Ausgrenzung und ideologischer Einbindung. Niederdeutsch in der DDR. Frankfurt a. M. [u. a.] 1998, S. 257-269.

Sanders, Willy: Sachsensprache, Hansesprache, Plattdeutsch. Sprachgeschichtliche Grundzüge des Niederdeutschen. Göttingen 1982. 
Scharioth, Claudia: Regionales Sprechen und Identität: Eine Studie zum Sprachgebrauch, zu Spracheinstellungen und Identitätskonstruktionen von Frauen in Schleswig-Holstein und Mecklenburg-Vorpommern (Deutsche Dialektgeographie; 120). Hildesheim/Zürich/New York 2015.

Schönfeld, Helmut: Gruppenspezifische Unterschiede bei der Verwendung und Bewertung des Niederdeutschen in der DDR. In: Das Niederdeutsche in Geschichte und Gegenwart (Zentralinstitut für Sprachwissenschaft. Reihe A: Arbeitsberichte; 75.2). Hrsg. vom Zentralinstitut für Sprachwissenschaft der Akademie der Wissenschaften der DDR, Berlin 1981, S. 112-120.

Schönfeld, Helmut: Sprache und Sprachvariation in der Stadt. Zu sprachlichen Entwicklungen und zur Sprachvariation in Berlin und anderen Städten im Nordteil der DDR. Berlin 1989.

Schönfeld, Helmut: East Low German. In: Russ, Charles V. J. (Hrsg.): Dialects of Modern German. A Linguistic Survey. London 1990, S. 91-135.

Schröder, Ingrid: Niederdeutsch in der Gegenwart: Sprachgebiet - Grammatisches - Binnendifferenzierung. In: Stellmacher, Dieter (Hrsg.): Niederdeutsche Sprache und Literatur der Gegenwart. Hildesheim/Zürich/New York 2004, S. 35-99.

Schröder, Ingrid: Zwischen Dialektologie und Regionalsprachenforschung - eine norddeutsche Perspektivierung. In: Elmentaler, Michael/Hundt, Markus/ Schmidt, Jürgen E. (Hrsg.): Deutsche Dialekte. Konzepte, Probleme, Handlungsfelder. Akten des 4. Kongresses der Internationalen Gesellschaft für Dialektologie des Deutschen (IGDD) in Kiel. Stuttgart 2015, S. 25-57.

Schulgesetz für das Land Mecklenburg-Vorpommern in der Fassung der Bekanntmachung vom 10. September 2010. Gesetz- und Verordnungsblatt für Mecklenburg-Vorpommern 2010, hrsg. vom Justizministerium MecklenburgVorpommern. Schwerin.

Sodmann, Timothy: Der Untergang des Mittelniederdeutschen als Schriftsprache. In: Goossens, Jan (Hrsg.): Niederdeutsch. Sprache und Literatur. Eine Einführung. Bd. 1: Sprache. 2., verb. und um einen bibliographischen Nachtrag erw. Aufl. Neumünster 1983, S. 116-129.

Statistisches Amt Mecklenburg-Vorpommern: Entwicklung des Arbeitsmarktes in Mecklenburg-Vorpommern 2000-2008/9; Entwicklung des Bildungssektors in Mecklenburg-Vorpommern 2000-2009. 2009. [Online-Ressource: http:// www.statistik-mv.de (Stand: 17.03.2015)]

Statistisches Amt Mecklenburg-Vorpommern: Bevölkerung, Haushalte, Familien, Fläche. Landesdaten im Überblick (2014). 2014. [Online-Ressource: http:// www.statistik-mv.de (17.03.2015)] 
Stellmacher, Dieter: Ostniederdeutsch. In: Althaus, Hans Peter/Henne, Helmut/ Wiegand, Herbert Ernst (Hrsg.): Lexikon der Germanistischen Linguistik. Berlin/New York, 1980, S. 464-468.

Verwaltungsvorschrift des Ministeriums für Bildung, Wissenschaft und Kultur Mecklenburg-Vorpommern: Niederdeutsch in der Schule. Verwaltungsvorschrift des Ministeriums für Bildung, Wissenschaft und Kultur vom 9. März 2004. In: Mecklenburg-Vorpommern. Mitteilungsblatt des Ministeriums für Bildung, Wissenschaft und Kultur 4/2004, S. 222-224.

Vierter Bericht der Bundesrepublik Deutschland gemäß Artikel 15 Absatz 1 der Europäischen Charta der Regional- oder Minderheitensprachen. Bonn 2010. 
Matthias Vollmer

\title{
Anmerkungen zu den sprachlichen Verhältnissen Vorpommerns in der ersten Hälfte des 19. Jahrhunderts
}

\begin{abstract}
This article will focus on linguistic properties of Low German and its importance as spoken language in Western Pomerania in the first half of the $19^{\text {th }}$ century. Archival documents collected between 1831 and 1833 form the base of this linguistic analysis. These archival documents are based upon an early language survey in Pomerania which covered topics like dialects and meta linguistic evaluations given by contemporaries.
\end{abstract}

\section{Einleitung}

Teilt man die Sprachgeschichte des niederdeutschen Raums in fünf Phasen ein, wie es Robert Peters vorgeschlagen hat, dann markiert die erste Hälfte des 19. Jahrhunderts das Ende der vierten Periode, die nach dem Schreibsprachenwechsel im deutschen Norden zu einer über zwei Jahrhunderte hinweg stabilen medialen Diglossie geführt hat. ${ }^{1}$ Für die gesprochene Alltagsprache hatte dieser Wechsel der Schreibsprache bis in das 19. Jahrhundert hinein aber für die verschiedenen sozialen Schichten unterschiedliche Konsequenzen. Der überwiegende Teil der Bevölkerung wurde nach wie vor niederdeutsch sozialisiert und blieb dem einheimischen Platt zeitlebens treu, während gehobene Schichten zweisprachig waren und je nach Situation und Gesprächspartner zwischen Hochdeutsch und Niederdeutsch wechseln konnten. Bei diesem gesprochenen Hochdeutsch handelte es sich zweifellos um eine hochdeutsche Umgangssprache auf niederdeutschem Substrat, die in Vorpommern nördlich der Peene im Wesentlichen mit den mecklenburgischen Verhältnissen übereinstimmte. ${ }^{2}$ Erst um die Mitte des 19. Jahrhunderts setzte dann ein auch maßgeblich durch schulische Bemühungen forcierter Sprachwandel im Bereich der Mündlichkeit ein, der das Niederdeutsche als gesprochene Sprache zunächst in bürgerlichen Kreisen und in den Städten zugunsten der hochdeutschen Umgangssprache verdrängte, bevor er spätestens seit den 30er Jahren des 20. Jahrhunderts auch die ländlichen Regionen wirksam erfasste.

1 Vgl. Peters 1998.

2 Vgl. Herrmann-Winter 1995, S. 184.

Birte Arendt, Andreas Bieberstedt and Klaas-Hinrich Ehlers - 978-3-631-71893-3 
Wie das gesprochene Niederdeutsch im mecklenburgisch-vorpommerschen Sprachraum um $1830 \mathrm{zu}$ charakterisieren ist, also noch vor dem Übergang zur fünften Phase, wie es von Zeitgenossen eingeschätzt wurde und welche Entwicklungstendenzen sich damals andeuteten, soll Thema dieses Beitrags sein. Dabei werden stellvertretend die sprachlichen Verhältnisse in Vorpommern im Mittelpunkt stehen, die wir in erster Linie durch metasprachliche Zeugnisse der damaligen Zeit rekonstruieren können, auch wenn solche Nachrichten leider recht spärlich gesät sind. Umso erfreulicher ist es, dass archivalische Unterlagen, die im Staatsarchiv Stettin aufbewahrt werden, aufschlussreiche Informationen zum Niederdeutschen und darüber hinaus zum gesamten damaligen Varietätenspektrum gesprochener Sprache in Vorpommern enthalten.

Dieses wichtige Quellenmaterial ist im Zusammenhang mit einem Aufruf der Gesellschaft für Pommersche Geschichte und Altertumskunde aus dem Jahr 1831 erhoben worden. Angeregt wurde die damalige Sammlung pommerscher Sprachproben durch den Stettiner Pädagogen und Historiker Wilhelm Böhmer, der aus den Einsendungen die erste, heute allerdings längst überholte lautgeographische Gliederung der pommerschen Dialekte ableitete. Als vergleichende Sprachprobe wählte Böhmer das biblische Gleichnis vom verlorenen Sohn, das schon seit dem Ende des 18. Jahrhunderts eine nicht unerhebliche Rolle als Vergleichstext für frühe sprachwissenschaftliche Erhebungen gespielt hatte. Das Vorbild dafür lag in Frankreich, erreichte dann mit den Forschungen Franz Joseph Stalders zu Beginn des 19. Jahrhunderts die deutschsprachige Schweiz und schließlich auch den niederdeutschen Sprachraum. ${ }^{3}$ Wenige Jahre vor Böhmer hatte schon der in Hinterpommern geborene Theologe Johann Christian Ludwig Haken eine ostpommersche und eine altmärkische Fassung dieses Textes gegenübergestellt, allerdings ohne sprachliche Gemeinsamkeiten und Unterschiede zu kommentieren. ${ }^{4}$ Wilhelm Böhmer bat 1831 aber nicht nur um die Übersetzung des Gleichnisses in den jeweiligen Ortsdialekt, sondern auch um andere Auskünfte, zu denen Fragen nach Zahl, Begrenzung, Vermischung, Eigentümlichkeit und lautlichen Besonderheiten der pommerschen Mundarten gehörten. ${ }^{5}$ Bei den erfragten Informationen handelt es sich also um eine Kombination aus objektsprachlichen und metasprachlichen Daten, die zusammen den besonderen Wert dieser Befragung in einer Zeit ausmachen, in der solche Mitteilungen rar gesät sind. Gerade die subjektiven Einschätzungen der Gewährspersonen zur sprach-

3 Vgl. Knoop 1982, S. 12.

4 Vgl. Haken 1823.

5 Vgl. Böhmer 1833, S. 139.

Birte Arendt, Andreas Bieberstedt and Klaas-Hinrich Ehlers - 978-3-631-71893-3 
lichen Situation des Niederdeutschen in Vorpommern sind von erheblichem Interesse und stehen deshalb zunächst im Mittelpunkt der vorliegenden Arbeit. Aufgrund offizieller kirchlicher Unterstützung des Aufrufs handelt es sich bei den meisten der damaligen Informanten übrigens um protestantische Geistliche.

\section{Niederdeutsch und Hochdeutsch}

Betrachten wir zunächst das Verhältnis zwischen Niederdeutsch und Hochdeutsch. Über die sprachliche Situation im abgelegenen Lieper Winkel auf der Insel Usedom berichtet der Theologiestudent Meinhold: „Da in der ganzen Umgegend platt gesprochen wird, so reden die Lieper Winkler gar kein Hochdeutsch, nur den Pastor und Küster ausgenommen, und in der Schule sind dann die Kinder

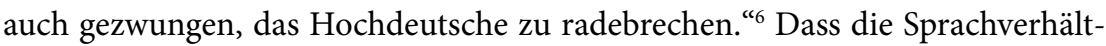
nisse in den Städten Vorpommerns bereits zu dieser Zeit anders aussahen, legen zahlreiche Einschätzungen anderer Gewährspersonen nahe. So äußerte sich Pastor Klöpper aus dem Greifswalder Vorort Weitenhagen wie folgt:

„Die plattdeutsche Sprache im Munde des jüngeren Geschlechts, so wie die Aussprache in den Städten, namentlich Greifswald, nähert sich, in Bezug auf die dem Platten eigenthümlichen Laute, immer mehr dem Hochdeutschen. [...] Es folgt daraus, daß das Plattdeutsche sich immer mehr dem Hochdeutschen namentlich in Städten und solchen Dörfern, die mehr bei den Städten liegen, anzubequemen sucht, wie es sich denn auch schon bedeutend abgeschliffen hat."

Die vor allem in den Städten zunehmende Verdrängung des Niederdeutschen thematisiert indirekt auch Johann Carl Balthasar, der damals als Pfarrer in Gützkow südlich von Greifswald tätig war. Balthasar, bei dem es sich um den Vater der namhaften niederdeutschen Lyrikerin Alwine Wuthenow (1820-1908) handelt, schrieb:

„dies ächte vorpommersche Plattdeutsch findet sich, natürlich in manchen kleinen Nuancen hier und da etwas abweichend fast nur noch in den von größeren Städten etwas entlegenen Bauer- und Fischerdörfern, besonders von alten Leuten gesprochen. Charakteristisch sind für diese Mundart das au für oo in Tau, gaud, [...] äu für öö in Fäute; $e i$ in $h e i, d e i[\ldots] .{ }^{\text {“s }}$

6 Staatsarchiv Stettin. Archiwum Panstwowe w Szczecinie. Rękopisy i spuścizny. Nr. 402 (Lieper Winkel).

7 Staatsarchiv Stettin. Archiwum Panstwowe w Szczecinie. Rękopisy i spuścizny. Nr. 402 (Weitenhagen).

8 Staatsarchiv Stettin. Archiwum Panstwowe w Szczecinie. Rękopisy i spuścizny. Nr. 402 (Gützkow). 
Wie sehr das Niederdeutsche aber noch im Mittelpunkt des sprachlichen Alltags in informellen Situationen stand, verdeutlicht eine Bemerkung des Superintendenten Ziemßen aus Hanshagen bei Greifswald:

„Gepredigt wird in hiesiger Gegend bekanntlich schon lange nicht mehr in dieser Mundart, auch bedient sich kein Geistlicher derselben mehr bei amtlichen Handlungen, und selbst in den Schulen ist sie nach und nach ganz außer Gebrauch gekommen. Es gehört zu den Seltenheiten, noch einen Lehrer in der Schule in dieser Mundart reden zu hören. Doch habe ich bei einem Schullehrer den Gebrauch gefunden, den Kindern etwas in plattdeutscher Mundart zu dictiren, was sie sogleich hochdeutsch niederschreiben müßen. Im außerbrieflichen Verkehr reden in dieser Gegend aber selbst die Prediger mit dem Volke und mit solchen Leuten, die im Hochdeutschen nicht geübt sind, gewöhnlich noch in der niederdeutschen Mundart, die auf dem Lande noch, selbst in manchen Priesterhäusern und in andern ähnlichen Familien die allgemeine Volkssprache, wenn gleich sich niemand derselben mehr zur schriftlichen Mitteilung bedient."

Dass die Rolle des Plattdeutschen als Unterrichtssprache um 1830 wenigstens in Teilen Vorpommerns noch erheblich stärker gewesen sein muss, als es bei Ziemßen durchklingt, ist einer Passage zu entnehmen, die sich in der Antwort des Superintendenten von Schubert aus Altenkirchen auf Rügen findet: „Plattdeutsch gepredigt wird nirgends, wohl aber in mehreren Schulen gelehrt“. ${ }^{10}$ Dazu passt die Einschätzung des evangelischen Pfarrers aus Gingst auf Rügen zur Sprache der geistlichen Unterrichtung: „Gepredigt wird in der plattdeutschen Mundart überall nicht mehr, weder auf Rügen noch in Neuvorpommern; jedoch im Unterrichte der Confirmanden und Präparanden muß der Geistliche, um manchen der Landeskinder verständlich zu werden, sich öfter des Plattdeutschen bedienen. "11 Ein zeitgenössisches Urteil des Historikers und Pädagogen Ludwig Giesebrecht im Rahmen einer 1826 veröffentlichten Verteidigungsschrift für das Plattdeutsche entspricht dieser Meinung: „Selbst in Stettin, mehr noch in den kleinen Städten unsrer Provinz, wird sehr viel Plattdeutsch gesprochen und ich habe das immer lieber gehört, als schlechtes Hochdeutsch, davon einem die Ohren weh thun. ${ }^{12}$ Drei Jahre zuvor hatte sich Giesebrecht bereits ähnlich über die Rolle des Niederdeutschen geäußert, wobei er von einer noch stärkeren Vitalität des

9 Staatsarchiv Stettin. Archiwum Panstwowe w Szczecinie. Rękopisy i spuścizny. Nr. 402 (Hanshagen).

10 Staatsarchiv Stettin. Archiwum Panstwowe w Szczecinie. Rękopisy i spuścizny. Nr. 402 (Altenkirchen).

11 Staatsarchiv Stettin. Archiwum Panstwowe w Szczecinie. Rękopisy i spuścizny. Nr. 402 (Gingst).

12 Giesebrecht 1826, S. 188-189.

Birte Arendt, Andreas Bieberstedt and Klaas-Hinrich Ehlers - 978-3-631-71893-3 
Plattdeutschen seiner mecklenburgischen Heimat in Relation zur pommerschen Situation ausging:

„In meinem Geburtslande hört man mehr, als ich in Pommern gefunden habe, auch von den Gebildeteren Plattdeutsch sprechen und es ist dort nicht ungewöhnlich, besonders in Frauengesellschaften, daß Hochdeutsch angefangen wird und, wenn die Herzen sich gegenseitig öffnen, geht man ins Plattdeutsche, als die traulichere Sprache über. ${ }^{\text {"13 }}$

Insgesamt kann deshalb der Einschätzung Wilhelm Böhmers zugestimmt werden, wenn er in seiner Auswertung der auf den Aufruf von 1831 eingegangenen Mitteilungen die sprachlichen Verhältnisse Vorpommerns wie folgt beurteilt: „In $\mathrm{Neu}=$ und zum Theil auch in Alt=Vorpommern lebt das Niederdeutsche [...] auch im Munde der Gebildeten noch in Städten und auf dem Lande als die trauliche, bequeme, geschmeidige Umgangssprache. ${ }^{\text {14 }}$

Die sprachliche Realität unterschied sich damit in Vorpommern um 1830 nicht wesentlich von der des mecklenburgischen Raums, über dessen Sprache des „gemeinen Mannes“ man schon 1794 in einem Zeitschriftenbeitrag des Theologen Ackermann folgende Äußerung lesen konnte: „Nur in den Unterrichtsjahren in der Schule hörte er ein dürftiges hochdeutsch, lernt es ein wenig beim Lesen, beim Auswendiglernen, hört in der Woche einmal hochdeutsch predigen, [...] alle andere Zeit spricht und hört er seine Leibsprache, sein liebes ächtes Meklenburgisch. ${ }^{\text {15 }}$

\section{Das Varietätenspektrum des Niederdeutschen}

Die zeitgenössischen Äußerungen machen deutlich, dass man um 1830 nicht nur Unterschiede zwischen den einheimischen niederdeutschen Dialekten zur Kenntnis genommen hat, sondern dass diese Differenzen zudem von den Sprechern auch in diastratischer Hinsicht interpretiert worden sind. Im Bewusstsein fast aller Informanten aus Vorpommern gab es im Spektrum gesprochener Varietäten eben nicht nur eine Dichotomie zwischen dem Hochdeutschen (d. h. einer standardnahen hochdeutschen Umgangssprache) und dem Niederdeutschen. Auch innerhalb des Plattdeutschen wurde eine Trennlinie zwischen einer prestigeträchtigeren Varietät der Städte mitsamt ihrem engeren Umfeld und der Basismundart der ländlichen Regionen gesehen. Außerdem wird ersichtlich, dass sich die

13 Giesebrecht 1823, S. 485.

14 Böhmer 1833, S. 157. Mit Neuvorpommern ist der Teil Vorpommerns nördlich der Peene gemeint, der noch bis 1815 unter schwedischer Verwaltung stand, während Altvorpommern südlich der Peene bereits 1720 an Preußen gefallen war.

15 Ackermann 1794, S. 27-28. 
jüngere Generation in den stadtnahen ländlichen Regionen mehrheitlich an dieser prestigereicheren niederdeutschen Varietät orientierte. Wesentliches Merkmal der frühen laiendialektologischen Unterscheidungen sind phonologische, nicht aber morphologische, lexikalische oder gar syntaktische Kriterien. Dabei galt besonders die auffällige Differenz in der Entwicklung der aus dem Mittelniederdeutschen ererbten langen $\hat{e}$ - und $\hat{o}$-Laute in Vorpommern als Indikator, an dem man diastratische Unterschiede im dialektalen Varietätengefüge festmachen konnte. Immer wieder wird nämlich der offenbar als salient empfundene Unterschied zwischen monophthongischer und diphthongischer Realisierung einiger dieser historischen Laute angesprochen. Betroffen sind dabei in Vorpommern mnd. $\hat{e}^{2 b}$ (z. B. Been versus Bein), $\hat{e}^{4}$ (z. B. Deef versus Deif), $\hat{o}^{1}$ (z. B. Koken versus Kauken) und der Umlaut von $\hat{o}^{1}$ (z. B. Föt versus Fäut).

Der Unterschied zwischen monophthongischen und diphthongischen Ortsdialekten ist nahezu jedem Einsender aus Vorpommern bekannt, wobei auch die Frage generationsspezifischer Variation am selben Ort angesprochen wird. Der aus Dortmund stammende Pastor Klöpper aus Weitenhagen bei Greifswald hat sein Antwortschreiben mit zahlreichen Anmerkungen versehen, die auf Besonderheiten des Plattdeutschen in der Region hinweisen. Auf den Vokalismus bezogen schreibt er:

„(1) däi; so klingt der Artikel noch im Munde älterer Leute hier im Dorfe, vornehmlich dem nahe gelegenen Behrenhoff. Das jüngere Geschlecht sagt einfach dä; (2) Deihl, so bei den älteren Bewohnern; die Jungen sagen Dehl; (3) taukümmt. Die jüngeren Einwohner, so wie die Greifswalder, sagen einfach: tokümmt, oder auch tokömmt; (4) Gaud, Gut, die Jüngeren schon Good; [...] (9) Schoh, so die Jüngeren, die alten Leute sagen noch hin und wieder Schau; so wie auch Fäute für das am meisten gebräuchliche Föte. ${ }^{\text {"16 }}$

Und etwas später fügt er hinzu:

„Wer nun im Sprechen den Vocalen und vornehmlich den Diphthongen das Breite nimmt und selbst das â oder ô, mehr als im Hochdeutschen wie a ausspricht, davon sagen die Alten: das soll schon etwas mehr sein, das ist höhere Sprache. [...] Es folgt daraus, daß das Plattdeutsche sich immer mehr dem Hochdeutschen namentlich in Städten und solchen Dörfern, die mehr bei den Städten liegen, anzubequemen sucht, wie es sich denn auch schon bedeutend abgeschliffen hat. ${ }^{17}$

Eine weitere Beurteilung stammt von Pastor Picht aus Gingst auf Rügen, der sich allerdings sehr kritisch zu der Stadtmundart von Stralsund äußert:

16 Staatsarchiv Stettin. Archiwum Panstwowe w Szczecinie. Rękopisy i spuścizny. Nr. 402 (Weitenhagen).

17 Staatsarchiv Stettin. Archiwum Panstwowe w Szczecinie. Rękopisy i spuścizny. Nr. 402 (Weitenhagen). 


\begin{abstract}
„Wenn die eigenthümlichen Mundarten einzelner Dörfer, Inseln und Gegenden sich allmählig immer mehr mit andern mischen werden, wozu hauptsächlich die vom Militär zurück kommenden jungen Leute beitragen; so ist das Plattdeutsch in Städten, besonders in Stralsund unter den Bürgern, und der dienenden weiblichen Classe schon sehr verfeinert. Wir Landleute, mit dem an die breitere Sprache des Landvolks gewohntem Ohr, finden den Dialect der Stralsunder, den wir den meßingenen zu nennen pflegen, sehr widerlich. “18
\end{abstract}

Bei dieser Einschätzung der Stralsunder Stadtmundart scheint es sich allerdings um ein in Vorpommern verbreitetes und langlebiges Stereotyp zu handeln, für das es bereits im 18. Jahrhundert überlieferte Zeugnisse gibt. Über das gesprochene Hochdeutsch der Stralsunder heißt es nämlich im Jahr 1786 unter Bezugnahme auf niederdeutsche Interferenzen: „Sie pronunciiren durchgängig triebe, flichtig, kennen, jelb statt trübe, flüchtig, können, gelb, und haben überhaupt eine so gezierte, kleinlich=süsse, wiederliche, von allen Pommern sie unterscheidende Aussprache, daß man sie nicht ohne Eckel hört. ${ }^{19}$ Als hervorstechendes lautliches Merkmal wird neben der angesprochenen Bewahrung alter Monophthonge nicht nur in der eben zitierten Beurteilung von 1786 die Tendenz zur Vokalentrundung in Stralsund genannt. Eine solche Zuschreibung findet sich sogar in bewusster Übertreibung in spöttischen Merksätzen, die offenbar in der Region sehr geläufig waren. ${ }^{20}$ Die Stigmatisierung des in Stralsund gesprochenen Niederdeutschen aus ländlicher Sicht und dessen Einschätzung als Missingsch ist jedoch vordergründig als gewöhnliche Form des Ortsspotts zu interpretieren und kann nicht darüber hinwegtäuschen, dass die städtischen Ausprägungen des Niederdeutschen im allgemeinen Bewusstsein der Bevölkerung und besonders bei der jüngeren Generation der damaligen Zeit höheres Prestige genossen haben.

Diese gesammelten Einschätzungen aus Laiensicht stimmen weitgehend mit den zeitgenössischen Stimmen der Sprachgelehrten überein. In Böhmers Auswertung der eingegangenen Sprachproben aus Pommern ist etwa die Rede von einer Mundart ohne alle Doppellaute (,runde Mundart'), die er von einer breiten Mundart mit zahlreichen Diphthongen unterscheidet. ${ }^{21}$ Für das angrenzende

18 Staatsarchiv Stettin. Archiwum Panstwowe w Szczecinie. Rękopisy i spuścizny. Nr. 402 (Gingst).

19 Reichenbach 1786, S. 164.

20 Die Stralsunder Dialektsprecher verspottete man z. B. mit folgendem Merksatz, der die Entrundung von $\ddot{u}$ herausstellt: De Miese sind bi 'n Grittbidel west (,die Mäuse sind bei dem Grützbeutel gewesen'). Vgl. auch Böhmer 1833, S. 162.

21 Böhmer 1833, S. 151. 
Mecklenburg hatte sich wenige Jahre zuvor Johann Jacob Mussäus in einer plattdeutschen Sprachlehre in ähnlicher Weise geäußert:

„Man nennt das Platte mit vielen Doppellauten das breite Platte, und dieses wird hier besonders berücksichtigt werden, weil es im Munde der Volksklasse sich findet, deren Aussprache am wenigsten durch Beimischungen verändert ward. Diese Beimischungen, vorzüglich aus dem Hochdeutschen, zeigen sich leider! mehr und weniger in den meisten plattdeutschen Schriften. [...] Die Landbewohner Mecklenburgs, an welche sich die östlichen Holsteiner und die Vorpommern anschließen, verwenden unzählige Doppellaute z. B. klein, ein, bleiw, Heu fast wie Heuj, häuden, mäuten [...]."22

Von diesem ländlichen Sprachgebrauch unterschied Mussäus deutlich den Dialekt der städtischen Bevölkerung: „Die Anwohner des Elbufers und die in den Städten von ganz Mecklenburg sprechen ohne viele starke Doppellaute und Dehnungen. " ${ }^{23}$

Insgesamt gesehen lassen die metasprachlichen Äußerungen die Hypothese zu, dass die damals gesprochenen Varietäten des Niederdeutschen im mecklenburgisch-vorpommerschen Sprachraum nicht nur in räumlicher Hinsicht, sondern auch sozial differenziert waren. Die Prestigevarietät des Niederdeutschen war die der Städte, während der Dialekt der ländlichen Regionen zwar als autochthon und ursprünglich galt, aber dennoch geringeres Ansehen genoss. Die jüngere Generation der stadtnahen ländlichen Bevölkerung orientierte sich offensichtlich an dieser städtischen Prestigevarietät, die nicht zuletzt durch vokalische Besonderheiten gekennzeichnet war, nämlich durch die Bewahrung monophthongischer Realisierungen gegenüber der sprachgeschichtlich jüngeren Entwicklung zur Diphthongierung der mnd. langen $e$ - und $o$-Laute. Dass diese lautlichen Eigenschaften aber keineswegs alleiniges Kriterium der Höherbewertung gewesen sein können, wird durch eine Bemerkung Wilhelm Böhmers ersichtlich, die sich besonders auf die Spracheinstellung der Sprecher des Mittelpommerschen westlich der Oder bezieht. Obwohl die langen $e$ - und $o$-Laute in dieser Region ebenfalls monophthongisch realisiert worden sind, unterschied sich offensichtlich die Wertschätzung der niederdeutschen Nachbarvarietäten, wobei das Mittelpommersche aus Sicht der eigenen Sprecher geringeren Stellenwert besaß: „Ja die Landleute westlich an der Oder [...] geben, wie uns Kundige mittheilen, der feineren Sprache des westlichen Vorpommerns vor der ihrigen ausdrücklich den Vorzug [...]. ${ }^{24}$

22 Mussäus 1829, S. V.

23 Mussäus 1829, S. IV.

24 Böhmer 1833, S. 159.

Birte Arendt, Andreas Bieberstedt and Klaas-Hinrich Ehlers - 978-3-631-71893-3 


\section{$4 \mathrm{Zu}$ sprachlichen Charakteristika des Niederdeutschen in Vorpommern um 1830}

Auf einige ausgewählte lautliche und morphologische Erscheinungen des Plattdeutschen in Vorpommern, die sich aus den Einsendungen der damaligen Gewährspersonen ableiten lassen, soll in den nächsten Abschnitten hingewiesen werden.

\subsection{Lautliche Realisierung der Endsilbe -er}

Wer heute als Fremder nach Vorpommern kommt, wird in der Regel rasch mit einer Eigenart der regionalen Umgangssprache konfrontiert, die die lautliche Realisierung der Endsilbe -er betrifft. Die meisten Sprecher vokalisieren auslautendes

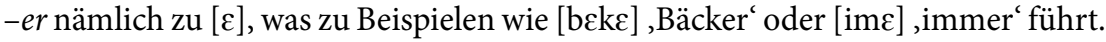
Ähnlich dominant ist die Realisierung dieser Lautverbindung in der Vorsilbe (z. B. [عlasən] ,erlassen'), die gleichfalls heute in Vorpommern zu hören ist. ${ }^{25}$ Dabei handelt es sich keinesfalls um einen sehr jungen sprachgeschichtlichen Vorgang, sondern um eine Eigenschaft, die sich in den mecklenburgisch-vorpommerschen Dialekten spätestens in der ersten Hälfte des 19. Jahrhunderts deutlich zeigt und später den Weg in die hochdeutsche Umgangssprache der Region gefunden hat. Sowohl die eingesandten Sprachproben der Erhebung von 1831 als auch die metasprachlichen Kommentare der Gewährspersonen bezeugen diese Charakteristik für die damaligen Dialekte Vorpommerns. So berichtet Pastor Klöpper aus Weitenhagen bei Greifswald über die Richtlinien seiner Transkription: „Die Endsilbe er in den Hauptwörtern, die so enden, ist mit ä bezeichnet, wie z. B. Akkä (der Acker), weil sich der Laut am meisten dem hochdeutschen ä nähert“ “26 Für den Lieper Winkel auf Usedom bezeugt der Theologiestudent Meinhold die Aussprache Vårä ,Vater', ujnä ,unter' sowie mit Nasalschwund vor $s$ die Varianten Fihstä und Feastä ,Fenster. ${ }^{27}$ Weitere Beispiele sind ävä ,aber in Gingst auf Rügen, werrä ,wieder' und Brorä ,Bruder' in der Antwort für den Ortspunkt Altenkirchen auf Rügen, Börgä ,Bürger ' und Vattä ,Vater ' in Dersekow westlich von

25 Die aktuelle Realisierung der Endsilbe wurde jüngst im Verbundprojekt „Sprachvariation in Norddeutschland“ (SiN) untersucht. Zu den Ergebnissen der rezenten Realisierung in regiolektalen Sprachlagen, die diesen Befund besonders für Mecklenburg und Vorpommern bestätigen, vgl. Elmentaler/Rosenberg 2015, S. 173-178.

26 Staatsarchiv Stettin. Archiwum Panstwowe w Szczecinie. Rękopisy i spuścizny. Nr. 402 (Weitenhagen).

27 Staatsarchiv Stettin. Archiwum Panstwowe w Szczecinie. Rękopisy i spuścizny. Nr. 402 (Lieper Winkel). 
Greifswald sowie heä ,her' für den Greifswalder Vorort Hinrichshagen. Ein gutes halbes Jahrhundert später wird der Deutsche Sprachatlas diesen Befund übrigens prinzipiell bestätigen, während der Greifswalder Sprachwissenschaftler Albert Hoefer 1851 in einem Aufsatz über die Lautverhältnisse im neuvorpommerschen Platt diese Eigenschaft überhaupt nicht anspricht. ${ }^{28}$ Auf den Wenkerbögen mehrerer vorpommerscher Orte sind entsprechende Beispiele aber vorhanden, auch wenn eine Vokalisierung von -er zwar grundsätzlich anzunehmen ist, die Aufhellung zu $[\varepsilon]$ aber keinesfalls auf allen Bögen deutlich wird. Auf dem Fragebogen für Greifswald ist dies jedoch der Fall, denn der erste Wenkersatz („Im Winter fliegen die trocknen Blätter durch die Luft herum.") wird dort wie folgt transkribiert: In’n Wintä flègen dē drōgn Blärä dörch dē Luft härüm. Dass diese dialektale Lautentwicklung schon wesentlich früher, in Mecklenburg bereits zu Beginn des 18. Jahrhunderts eingesetzt haben muss, legen Seltenheitsbelege nahe, die Hermann Teuchert in mecklenburgischen Hochzeitsgedichten entdeckt hat

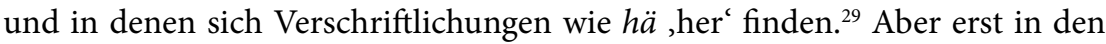
20er Jahren des 19. Jahrhunderts finden sich frühe grammatische Äußerungen, die immerhin ähnliche Vokalisierungen für auslautendes $-r$ (aber nicht für die Endsilbe -er) in Mecklenburg betreffen. Dazu gehören die 1824 belegten Formen

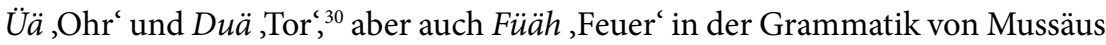
von 1829. ${ }^{31}$ Unabhängig davon ist die dialektale Herkunft dieser lautlichen Besonderheit standardnaher Umgangssprache in Vorpommern nicht zu bezweifeln, auch wenn sie selbst in jüngeren einschlägigen Veröffentlichungen nicht immer angesprochen wird. ${ }^{32}$

\subsection{Mouillierung}

$\mathrm{Zu}$ den in der Fachliteratur relativ wenig beachteten Eigenschaften der mecklenburgisch-vorpommerschen Dialekte gehören im Bereich des Konsonantismus Entwicklungen, die man früher verschiedentlich als Mouillierung

28 Vgl. Hoefer 1851.

29 Vgl. Teuchert 1959, S. 229.

30 Vgl. Teuchert 1959, S. 229.

31 Vgl. Mussäus 1829, S. 9.

32 Mihm 2000 erwähnt diese Eigenschaft nicht. Lauf bezieht sie explizit nur auf die Vorsilbe (vgl. Lauf 1996, S. 202). Ähnlich äußert sich Kehrein, der die für den mecklenburgisch-vorpommerschen Raum typische Variante am Beispiel von Stralsund erhoben hat (vgl. Kehrein 2012, S. 311). Deutlich früher wurde dieses Merkmal von Herrmann-Winter (1979, S. 147-148) für die hochdeutsche Umgangssprache in Mecklenburg und Vorpommern belegt. 
bezeichnet hat. ${ }^{33}$ Mittlerweile nur noch von historischer Bedeutung ist in dieser Hinsicht die Palatalisierung der Lautverbindung $n d, n t>n j$, jn, die Agathe Lasch für den niederdeutschen Sprachraum schon für das Jahr 1630 an verschiedenen Beispielen im Bereich der Niederelbe feststellen konnte. ${ }^{34}$ In Rostock ist dieser Lautwandel durch Belege wie Hujn ,Hunde' und Kijnnä ,Kinder' allerdings erst wesentlich später, nämlich im Jahr 1824 bezeugt, weitere Nachweise aus Mecklenburg sind zwar nicht überaus zahlreich, doch wenigstens in einem Umfang vorhanden, der etwaige Zweifel an der Relevanz dieser lautlichen Entwicklung auszuschließen vermag. ${ }^{35}$ Die Antworten der pommerschen Erhebung von 1831 enthalten aber bis auf eine Ausnahme keine Hinweise auf diese lautliche Charakteristik. Nur im abgelegenen Lieper Winkel auf Usedom ist diese spezielle Form der Mouillierung durch die damalige Datensammlung nachzuweisen. Beispiele dafür stellen folgende Laientranskriptionen dar: ${ }^{36}$

- dat ganze Lajnd, Land

- 'n Ring an sien Häjnd, Händen'

- väsüjnigt,versündigt'

- fujn'n, gefunden

- dat hei em sujnd, gesund' werrä heer

- heäw ick jug deijnt, gedient

- köjnt, gekonnt'

- ujnä,unter

Auf Besonderheiten der Sprache des Lieper Winkels hatte kurz vorher schon der auf Usedom geborene Theologe und Literat Wilhelm Meinhold hingewiesen. ${ }^{37}$ Meinhold verglich dabei den Dialekt des Lieper Winkels mit demjenigen der Halbinsel Mönchgut im Südosten Rügens und stellte eine enge Verwandtschaft fest, die er auch im Wortschatz vorzufinden glaubte: „hier wie dort ist die monotone gedehnte Aussprache des Plattdeutschen vorherrschend, und hier wie dort begegnet man zum Theil durchaus unverständlichen Idiotismen; z. B. et hönnickt: es schneit; da jünnern: dort hinten; däsen: tanzen; dampich: schwindsüchtig u. s. w. ${ }^{\text {“38 }}$ Eine solche Stigmatisierung ländlicher Basismundarten Vorpommerns

33 Vgl. Teuchert 1959, S. 212-214.

34 Vgl. Lasch 1920, S. 324.

35 Vgl. Teuchert 1959, S. 213.

36 Staatsarchiv Stettin. Archiwum Panstwowe w Szczecinie. Rękopisy i spuścizny. Nr. 402 (Lieper Winkel).

37 Wilhelm Meinhold (1797-1851) wurde vor allem durch eine 1843 publizierte Erzählung mit dem Titel Maria Schweidler, die Bernsteinhexe bekannt.

38 Meinhold 1830, S. 44-45.

Birte Arendt, Andreas Bieberstedt and Klaas-Hinrich Ehlers - 978-3-631-71893-3 
war im 19. Jahrhundert durchaus kein Einzelfall, sie findet sich auch schon in einem Reisebericht über die Insel Rügen aus dem Jahr 1805. Darin kommentiert der von der Insel stammende Heimatforscher Johann Jacob Grümbke die Sprache der Bewohner Mönchguts abfällig mit den Worten:

„Die hiesige Mundart ist mir fast eben so widerlich vorgekommen, als die der Hiddenseer. Wie diese pronunciiren die Eingeborenen ihr Plattdeutsch auch sehr langsam und bedächtlich, zuweilen halb singend, mischen Vocale ein und dehnen die Worte theils durch Anhängsel an den Endsylben, theils durch die Aussprache. ${ }^{{ }^{* 39}}$

Besser erkennbar als der Lautwandel von $n d, n t>n j$, jn wird durch die Einsendungen auf den Aufruf der Gesellschaft für Pommersche Geschichte und Altertumskunde von 1831 hingegen die dialektale Entwicklung von $l d, l t>l j$, $j l$. Dabei ist der mouillierende Einfluss des $d$ nach $l$ im mecklenburgisch-vorpommerschen Sprachraum, der zu einer Velarisierung des 1 zu [1] führt, schon für das 18. Jahrhundert bezeugt, ${ }^{40}$ doch hat sich diese Lautentwicklung offenbar nur in ländlichen Bereichen langfristig durchsetzen können. Vor allem nach hellen Vokalen (z. B. Gäljd ,Geld'), aber vereinzelt auch nach dunklen (z. B. Fulj ,Falte', ollj ,alt') Vokalen ist diese mundartliche Entwicklung mehrfach belegt, die im Mecklenburgischen Wörterbuch als veraltet charakterisiert wird. ${ }^{41}$ Pastor Balthasar aus Gützkow beschreibt die Mouillierung in seiner Antwort auf den Aufruf von 1831 mit den Worten: „Das i vor 1 gleicht in der Aussprache dem französischen l-mouillé, so in Uill (Oll). “Und für den Lieper Winkel zählt der Informant Meinhold folgende Beispiele auf, um die dortige dialektale Entwicklung von $l d, l t>j l$ zu verdeutlichen:

- dei Ojl deijlt dat Gaud, der Alte teilte das Gut

- hojllen, halten

- dei öjllst Söhn was upp’n Fejll ,der älteste Sohn war auf dem Feld

- Gäjld,Geld“

- lüjt Lü̈̈r ,kleine Leute

Anzumerken bleibt, dass sich die Velarisierung von $l$, bei der es sich um eine ursprünglich dialektale Entwicklung handelt, noch Ende der siebziger Jahre des 20. Jahrhunderts auch in der regionalen Umgangssprache MecklenburgVorpommerns gezeigt hat, wobei sie sich keineswegs auf die Lautverbindung $l d$ beschränkt hat, sondern sich nahezu auf jede postvokalische Position beziehen

39 Grümbke 1805, S. 227.

40 Vgl. Teuchert 1959, S. 214.

41 Vgl. Mecklenburgisches Wörterbuch. 4. Bd. 1965, Sp. 789-790. 
konnte. ${ }^{42}$ Anschließend ist jedoch ein deutlicher und rasch ablaufender Abbau dieses Merkmals in den regiolektalen Sprachlagen Mecklenburg-Vorpommerns zu verzeichnen, wie neuere Erhebungen dokumentieren konnten, die gleichzeitig darauf verweisen, dass Velarisierungen von $l$ wenigstens heute stärker im Südwesten des niederdeutschen Sprachraums nachzuweisen sind. ${ }^{43}$ Auch deswegen ist es kaum berechtigt, von einem „Ostsee-l“ zu sprechen, das besonders für Rügen und die mecklenburgische Küste relevant ist. ${ }^{44}$

\subsection{Das Diminutivsuffix -ing}

Als besonderes Charakteristikum für die mecklenburgisch-vorpommerschen Dialekte kann das sprachgeschichtlich noch junge Diminutivsuffix -ing gelten, über dessen Herkunft es jedoch unterschiedliche Auffassungen gibt. ${ }^{45}$ Der oben schon erwähnte Theologiestudent Meinhold berichtet von einer lautlichen Variante dieses vor allem kosende Funktion tragenden Suffixes, die meines Wissens bislang völlig unbeachtet geblieben ist. Meinhold gibt Auskunft über die Dörfer des Lieper Winkels auf Usedom, in denen nach seinen Angaben das Suffix in der Variante - ang unabhängig von der lautlichen Umgebung üblich gewesen ist. Er führt dafür zwei Beispiele, nämlich Kijnang (statt Kinning, Kindchen, Kindlein') und mien lüjttang (statt mien lütting, mein Kleines') an. ${ }^{46}$ Im Greifswalder Archiv des Pommerschen Wörterbuchs findet sich hierfür jedoch keinerlei Beleg, auch sonst ist mir kein Hinweis auf diese Variante bekannt. ${ }^{47}$ Offenbar hat sich diese Eigentümlichkeit der von den großen Verkehrswegen weit entfernten Region nicht bewahren können und ist vermutlich noch im 19. Jahrhundert aus der gesprochenen Sprache verschwunden. Die Wenkerbögen des Deutschen Sprachatlas aus den achtziger Jahren des 19. Jahrhunderts verzeichnen diese Variante für die beiden einzigen Schulorte des Lieper Winkels (Liepe und Warthe) jedenfalls nicht.

42 Vgl. Herrmann-Winter 1979, S. 146.

43 Vgl. Elmentaler/Rosenberg 2015, S. 323-328.

44 Vgl. Großes Wörterbuch der deutschen Aussprache, S. 133.

45 Vgl. Lasch 1912; Teuchert 1953; Schmuck 2009.

46 Staatsarchiv Stettin. Archiwum Panstwowe w Szczecinie. Rękopisy i spuścizny. Nr. 402 (Lieper Winkel).

47 Eine direkte Beziehung zum Diminutivsuffix - ang in den neiderländischen Dialekten Niederschlesiens ist wohl auszuschließen. Für den Hinweis auf diesen mitteldeutschen Befund danke ich Klaas-Hinrich Ehlers. 


\section{Fazit}

Die Bedeutung der sprachlichen Zeugnisse, die als Antwort auf den Aufruf der Gesellschaft für Pommersche Geschichte und Altertumskunde von 1831 schließlich ihren Weg in das Stettiner Staatsarchiv fanden, beruht vor allem auf zwei Gesichtspunkten. Einerseits werden durch die erhobenen Daten einige selten diskutierte lautliche und morphologische Charakteristika der niederdeutschen Dialekte Vorpommerns dieser Zeit bezeugt, auf der anderen Seite liefern die metasprachlichen Urteile der Zeitgenossen wichtige Hinweise für die Rolle und die Entwicklung niederdeutscher Varietäten im Spektrum gesprochener Sprache dieser Region.

In seiner Analyse der eingesandten Sprachproben hat Wilhelm Böhmer, der Initiator des Aufrufs von 1831, sich zwar in der Festlegung auf eine dialektgeographische Zweiteilung Pommerns geirrt, denn spätestens durch die Forschungen Robert Holstens zu Beginn des 20. Jahrhunderts konnte diese Annahme gründlich korrigiert werden. ${ }^{48}$ Böhmers Aussagen zur Variabilität des vorpommerschen Varietätenspektrums und besonders zur Etablierung einer gehobenen niederdeutschen Umgangssprache in den Städten kann aber fast uneingeschränkt zugestimmt werden, wobei er zudem einen Gegensatz zwischen den vorpommerschen und den mittel- und ostpommerschen Verhältnissen der damaligen Zeit herausstellt: „Näher der Oder und östlich derselben giebt es diese feinere Niederdeutsche Umgangssprache, - welche in Einerlei Form über den Volksmundarten schwebend, das Zerstückelte wohlthätig verbindet, -

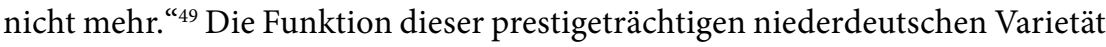
hat dann seit der zweiten Hälfte des 19. Jahrhunderts zunächst im städtischen Kontext und später auch im ländlichen Raum eine großräumigere hochdeutsche Umgangssprache auf niederdeutschem Substrat übernommen, die noch zu Beginn des 19. Jahrhunderts nur von einer kleinen gesellschaftlichen Elite gesprochen wurde. ${ }^{50} \mathrm{Um} 1830$ war die Vitalität des Niederdeutschen als gesprochene Sprache in Vorpommern jedoch nahezu ungebrochen. Besonders im Selbstverständnis der jungen Generation niederdeutscher Muttersprachler galt nicht die hochdeutsche Umgangssprache der sozialen Oberschicht als erstrebenswerte Alltagssprache, sondern das einheimische Niederdeutsch in einer städtisch-bürgerlichen Ausprägung.

48 Vgl. Herrmann-Winter 1995, S. 179.

49 Böhmer 1833, S. 157.

50 Vgl. Mihm 2000; Lauf 1996.

Birte Arendt, Andreas Bieberstedt and Klaas-Hinrich Ehlers - 978-3-631-71893-3 


\section{Literaturverzeichnis}

Ackermann, Georg Christian Benedikt: Ueber die Sprache des gemeinen Mannes in Meklenburg, mit Hinsicht auf Kanzelvortrag. In: Neue Monatsschrift von und für Mecklenburg 3 (1794), S. 25-31.

Böhmer, Wilhelm: Sammlung der Niederdeutschen Mundarten in Pommern. In: Baltische Studien, Alte Folge, Band 2/1 (1833), S. 139-172.

Ehlers, Klaas-Hinrich: „Dürfen wir essing?“ Beobachtungen zur Übernahme des mecklenburgischen ing-Diminutivs in das regionale Hochdeutsch. In: Niederdeutsches Jahrbuch 134 (2011), S. 79-92.

Elmentaler, Michael/Rosenberg, Peter (Hrsg.): Norddeutscher Sprachatlas (NOSA). Bd. 1: Regiolektale Sprachlagen. Hildesheim/Zürich/New York 2015.

Giesebrecht, Ludwig: Gedichte in niedersächsischer Mundart. In: Pommersche Provinzialblätter 5 (1823), S. 479-489.

Giesebrecht, Ludwig: Zu Gunsten des Plattdeutschen. In: Neue Pommersche Provinzialblätter 1 (1826), S. 188-192.

Großes Wörterbuch der deutschen Aussprache. Hrsg. von Krech, Eva-Maria, Leipzig 1982.

Grümbke, Johann Jacob: Streifzüge durch das Rügenland. In Briefen von Indigena. Altona/Leipzig 1805.

Haken, Johann Christian Ludwig: Sprach-Proben. In: Pommersche Provinzialblätter 5 (1823), S. 352-365.

Herrmann-Winter, Renate: Sprachen und Sprechen in Pommern. In: Niederdeutsches Jahrbuch 118 (1995), S. 165-187.

Herrmann-Winter, Renate: Studien zur gesprochenen Sprache im Norden der DDR. Berlin 1979.

Hoefer, Albert: Die neuniederdeutschen Lautverhältnisse besonders Neuvorpommerns. In: Zeitschrift für die Wissenschaft der Sprache 3 (1851), S. 375-396.

Kehrein, Roland: Regionalsprachliche Spektren im Raum. Zur linguistischen Struktur der Vertikale (ZDL Beihefte; 152). Stuttgart 2012.

Knoop, Ulrich: Das Interesse an den Mundarten und die Grundlegung der Dialektologie. In: Besch, Werner [u. a.] (Hrsg.): Dialektologie. Ein Handbuch zur deutschen und allgemeinen Dialektologieforschung (Handbücher zur Sprach- und Kommunikationswissenschaft; 1.1). 1. Halbbd. Berlin/NewYork 1982, S. 1-22.

Lasch, Agathe: Die Mundart in den nordniedersächsischen Zwischenspielen des 17. Jahrhunderts. In: Aufsätze zur Sprach- und Literatur-Geschichte. Wilhelm Braune zum 20. Februar 1920 dargebracht von Freunden und Schülern. Dortmund 1920, S. 299-351. 
Lasch, Agathe: Zur Deminutivbildung in der mecklenburgisch-vorpommerschen Mundart. In: Niederdeutsches Jahrbuch 38 (1912), S. 81-104.

Lauf, Raphaela: „Regional markiert“: Großräumliche Umgangssprache(n) im niederdeutschen Raum. In: Niederdeutsches Jahrbuch 119 (1996), S. 193-218.

Mecklenburgisches Wörterbuch. Hrsg. von der Sächsischen Akademie der Wissenschaften zu Leipzig. Aus den Sammlungen Richard Wossidlos und aus den Ergänzungen und nach der Anlage Hermann Teucherts. 7 Bde. Berlin 1942-1992. Unveränd., verkleinerter Nachdruck der Erstauflage von 1937-1992. Neumünster 1996. Rothe, Christian: Nachtrag und Index. Neumünster 1998.

Meinhold, Wilhelm: Miniaturgemälde von Rügen und Usedom. Greifswald 1830.

Mihm, Arend: Die Rolle der Umgangssprachen seit der Mitte des 20. Jahrhunderts. In: Besch, Werner [u. a.] (Hrsg.): Sprachgeschichte. Ein Handbuch zur Geschichte der deutschen Sprache und ihrer Erforschung (Handbücher zur Sprach- und Kommunikationswissenschaft; 2.2). 2. Teilb. 2., vollständig neu bearb. und erw. Aufl. Berlin/New York 2000, Sp. 2106-2137.

Mussäus, Johann Jacob: Versuch einer plattdeutschen Sprachlehre mit besonderer Berücksichtigung der mecklenburgischen Mundart. Neustrelitz/Neubrandenburg 1829.

Peters, Robert: Zur Sprachgeschichte des niederdeutschen Raumes. In: Zeitschrift für deutsche Philologie 117 (1998), S. 108-127.

Reichenbach, Johann David: Patriotische Beyträge zur Kenntnis und Aufnahme des Schwedischen Pommerns. Fünftes Stück. Stralsund 1786.

Schmuck, Mirjam: Personennamen als Quelle der Grammatikalisierung. Der -ingDiminutiv in Mecklenburg Vorpommern. In: Beiträge zur Namenforschung 44 (2009), S. 35-65.

Teuchert, Hermann: Beiträge zur Geschichte der mecklenburgischen Mundart. In: Niederdeutsches Jahrbuch 82 (1959), S. 207-236.

Teuchert, Hermann: Mudding „Mütterchen“: die Herkunft des mecklenburgischvorpommerschen -ing. In: Zeitschrift für Mundartforschung 21 (1953), S. 81-103.

\section{Quellenverzeichnis}

Staatsarchiv Stettin (Archiwum Panstwowe w Szczecinie). Rękopisy i spuścizny. Nr. 402. Teil 1 und 2: Neuvorpommern; Altvorpommern. 


\title{
André Köhncke \\ Regionale Unterschiede und Veränderungen im Wortschatz des Mecklenburgischen
}

\begin{abstract}
The article focuses on the lexical development of Low German in Mecklenburg over the last 50 years. It compares dialect recordings from the 1960's against new data from 2004-2012. Using lexical examples from different semantic fields (predominantly agriculture), the paper demonstrates the regional variation in the lexicon of the Mecklenburg dialect and shows the continuing disappearance of regional lexical differences as well as an ongoing lexical approximation of the Low German dialect towards the High German standard language. Lexical differences can not only be detected between the old dialect records from the 1960's and the new records from 2004-12, but also within the new records between the language samples of speakers from different generations.
\end{abstract}

\section{Einleitung}

\subsection{Frühere Untersuchungen zur Wortgeographie}

Der nachfolgende Beitrag fokussiert jüngere und rezente Entwicklungen im Bereich des Wortschatzes des Mecklenburgischen, insbesondere dessen wortgeographische Differenzierung und deren sukzessive Auflösung sowie die diachrone lexikalische Advergenz der mecklenburgischen Dialektvarietäten an die hochdeutsche Standardsprache.

Der Wortschatz des Mecklenburgisch-Vorpommerschen ist seit langer Zeit Untersuchungsgegenstand - sowohl von Laien als auch Wissenschaftlern. Schon im 16. Jahrhundert zeichnet der aus dem oberdeutschen Sprachgebiet stammende Rostocker Professor Nathan Chytraeus in seinem „Nomenclator latinosaxonicus" niederdeutsche Wörter auf, die er nach Sachgebieten (Religion, Wasser und Flüsse, Metalle, Körper, Krankheiten, Tiere usw.) ordnet, und zwar vom Allgemeinen zum Speziellen (z. B. Tiere allgemein, vierfüßige Tiere, Pferde, Decken und Zierrat der Pferde usw.). ${ }^{1}$ Das Buch erlebte seit seiner Erstausgabe im Jahre 1582 zahlreiche Auflagen in Norddeutschland (z. B. in Bremen, Hamburg, Lübeck, Lemgo) und wurde in einigen Abschnitten erweitert, wobei das

1 Chytraeus 1582 [1974], S. 4 (eigene Zählung).

Birte Arendt, Andreas Bieberstedt and Klaas-Hinrich Ehlers - 978-3-631-71893-3 
Verhältnis zwischen den Druckorten und dem darin enthaltenen Wortschatz bislang noch nicht vollständig geklärt worden ist. ${ }^{2}$

Bernhard Raupach führt in seiner 1704 erschienenen Dissertation sowohl Wörter als auch ganze Sätze an, um sie den hochdeutschen Entsprechungen gegenüberzustellen: „Se schmeten two̊lff schwarte Katten int Water; Mifnicè: Sie schmissen zwôlff schwartze Katzen ins Wasser. ${ }^{\text {“3 }}$ Hierbei kam es ihm aber darauf an, allgemein den Wert des Niederdeutschen zu betonen. ${ }^{4}$ Der Bützower Juraprofessor Ernst Johann Friedrich Mantzel behandelt 1757 in einer Dissertation und später seinen „Bützowschen Ruhestunden“5 vor allem „Idiotismen“ bzw. „Provinzial-Wörter“ und verzichtet deshalb auf Einträge, die „,on einem Hochteutschen, auch andern, bald zu errahten oder nachzurahmen [sic] " sind. ${ }^{6}$ Vielmehr konzentriert er sich auf Ausdrücke, „worauf ein mit der Meckl. geist- und weltlicher Rechtsgelahrtheit und Geschichte, auch Oeconomick, umgehender sonderlich achten muß“.7 Dabei geht er jedoch nicht auf lexikalische Unterschiede ein, die innerhalb des Landes herrschten. ${ }^{8}$ Auch nachfolgende Wörtersammlungen, die bis Anfang des 20. Jahrhunderts erschienen sind, sind eher allgemein gehalten und differenzieren nicht wortgeographisch: Friedrich Frehse und Carl Friedrich Müller erfassen den Wortschatz der Werke Fritz Reuters, ${ }^{9}$ verfolgten mithin das Ziel, seine Schriften Lesern verständlich zu machen, die des Niederdeutschen nicht mächtig sind. ${ }^{10}$ Sibeth erhebt mit seinem Wörterbuch den Anspruch, „tiefer [...] auf die Eigenthümlichkeiten und den Sprachschatz der mecklenburgischen Sprache " einzugehen, ${ }^{11}$ doch verzeichnet er allenfalls gelegentlich orthographische Abweichungen zwischen städtischem und

2 Chytraeus 1582 [1974], S. XII-XIII. Die Lemgoer Drucke weisen beispielsweise alemannisches, mecklenburgisches und ostwestfälisches Wörtermaterial auf, vgl. dazu Peters 2014 , S. 91-102.

3 Raupach 1704, S. 39.

4 Vgl. Raupach 1704, S. 70-71.

5 Mantzel 1757.

6 [Mantzel] 1761, S. 57.

7 [Mantzel] 1761, S. 58.

8 Wenn, dann differenziert er allenfalls einmal zwischen Gesellschaftsschichten in der Dissertation: „Minsch (plebeje Minsk)“ (Mantzel 1757, S. 19).

9 Frehse 1867; Müller [1904].

10 Frehse schreibt z. B. im Vorwort: „Wenn ich hiermit ein Glossar zu Reuter’s Schriften herausgebe, so glaube ich damit Denjenigen einen nicht unwichtigen Dienst zu erzeigen, welche der plattdeutschen Sprache nicht mächtig sind und dennoch nicht ausgeschlossen sein möchten von den literarischen Schätzen, welche der genannte große Dichter unserm Volke in seinen Werken darbietet." (Frehse 1867, Vorwort).

$11 \mathrm{Mi}$ [= Sibeth] 1876, Vorwort. 
ländlichem Dialekt, ${ }^{12}$ macht aber keine Angaben, ob beispielsweise Wörter mit gleicher Bedeutung einfach als Synonyme aufzufassen oder auf eine bestimmte Region beschränkt sind. So erfasst er für das hochdeutsche ,Geleise' Läus' und Trad; ob diese Wörter aber überall unterschiedslos oder spezifisch in verschiedenen Regionen gebraucht werden, lässt sich aus den Einträgen nicht erschließen. ${ }^{13}$

Erst im 20. Jahrhundert folgen Arbeiten, die sich mit regionalspezifischen Unterschieden im niederdeutschen Wortschatz beschäftigen: So untersucht Hugo Jacobs den südwestmecklenburgischen Raum. ${ }^{14}$ Im Vordergrund stehen in seiner Dissertation zwar die lautlichen Veränderungen seit mittelniederdeutscher Zeit. Jacobs widmet den lexikalischen Unterschieden in seiner Arbeit jedoch ein kleines Kapitel und zeigt besonders die Unterschiede zur Prignitz auf, behandelt aber auch diejenigen innerhalb seines Untersuchungsgebietes..$^{15}$ Eine ausschließlich auf die Lexik konzentrierte Arbeit legt 1933 Rudolf Blume vor, der das alte Stargarder Land, d.h. den Hauptteil des ehemaligen Mecklenburg-Strelitz, untersucht hat. Er geht dabei sowohl auf Unterschiede innerhalb des Strelitzer Gebietes als auch zu dem angrenzenden Schweriner und Brandenburger Umland ein. ${ }^{16}$ Ergänzend hierzu hat sich Hermann Teuchert dem Lautstand im südlichen Stargard gewidmet, aber auch den Wortschatz untersucht, wobei er wie schon Blume die Gemeinsamkeiten mit brandenburgischem Wortgut auf niederfränkische Siedler zurückführt. ${ }^{17}$

Im Zuge der Arbeit am Mecklenburgischen Wörterbuch hat Teuchert einige wortgeographische Aufsätze veröffentlicht. ${ }^{18}$ Das Material für das Wörterbuch fußt auf der Zettelsammlung Richard Wossidlos, daneben wurden jedoch auch Fragebogen versandt, um die räumlichen Grenzen des Gebrauchs von Wörtern, die dieselbe Bedeutung aufweisen, genauer bestimmen zu können. ${ }^{19}$ Die Einteilungen, die Teuchert aufgrund der zusammengetragenen Belege ermittelt hat, sind später von William Foerste und anderen Autoren übernommen worden. ${ }^{20}$

12 So notiert er beispielsweise „Blaud, (au-o), Blut“, aber ausschließlich „,bläukern, rauchen, sängen“ (Mi [= Sibeth] 1876, S. 9) und nicht auch blökern.

$13 \mathrm{Mi}$ [= Sibeth] 1876, S. 49 und 94. Lediglich der Bedeutungsumfang ist unterschiedlich bemessen, denn während Trad nach Sibeth ausschließlich,Geleise' bedeutet, umfasst Läus' daneben auch ,Furche' und ,Spur'.

14 Jacobs 1925/26; Jacobs 1926/27.

15 Jacobs 1926/27, S. 149-152.

16 Blume 1933.

17 Teuchert 1934, S. 21-31.

18 Als Beispiel sei hier nur genannt: Teuchert [1929].

19 Teuchert 1942.

20 Foerste 1954, speziell zum Mecklenburgisch-Vorpommerschen, Sp. 2040-2043. Vgl. auch Niebaum 2004. 
Eine der Fragen, denen dieser Beitrag nachgehen wird, ist daher, inwieweit diese Ergebnisse heute noch gültig sind, da das Material heute über achtzig Jahre alt ist und andererseits bereits damals in einer wenig beachteten kurzen Rezension aus dem Jahre 1929 einige Aussagen Teucherts präzisiert worden sind. ${ }^{21}$

Ein weiterer Aspekt, den dieser Aufsatz untersuchen möchte, ist die aktuelle Entwicklung des Wortschatzes, d. h. die Frage, inwieweit die deutsche Standardvarietät auf die mundartliche Lexik wirkt und wie Dialektsprecher mit neuen Entwicklungen im hochdeutschen Wortschatz und in der Sachkultur umgehen. Dieser Frage ist 1964 bereits Ulrich Bentzien nachgegangen, der dafür besonders den landwirtschaftlichen Sektor untersucht hat. ${ }^{22}$ Die Arbeit wird dazu an seine Ergebnisse anknüpfen.

\subsection{Materialgrundlage}

Um die Entwicklung des Wortschatzes seit der Zeit seiner Erhebung durch Wossidlo und Teuchert bestimmen und die damaligen Untersuchungsergebnisse gegebenenfalls präzisieren zu können, müssen neuere Sprachdaten herangezogen werden. Eine wichtige Grundlage für die nachfolgenden Ausführungen bilden Tonbandaufnahmen, die Jürgen Gundlach 1962/63 in Mecklenburg durchgeführt hat. Dazu befragte er in 61 Orten insgesamt 245 Probanden. In jedem Ort wurde mindestens ein Sprecher der ältesten, mittleren und jüngeren Generation aufgenommen. ${ }^{23}$ Das bedeutet natürlich, dass auch diese Belege inzwischen schon recht alt sind. Neuere flächendeckende Aufnahmen existieren aber nicht, so dass die Aufnahmen Gundlachs nach wie vor unverzichtbar sind. Außerdem sind die meisten Sprecher, die Niederdeutsch noch als Erstsprache erlernt haben, heute mindestens 70 Jahre alt, so dass die damals noch vorhandene Möglichkeit, mehrere Generationen von Mundartsprechern aufzunehmen,

21 Buddin 1929, S. 45. Buddin war kein Sprachwissenschaftler, kannte sich als nativer Mundartsprecher aber sehr gut mit dem im Fürstentum Ratzeburg gesprochenen Dialekt aus. Teuchert stellt beispielsweise fest, dass für die Bezeichnung der Stirnseite eines Feldes, wo der Pflug gewendet wird, im Großteil des Landes Anwenn ‘ und damit zusammenhängende Formen gebraucht werden, sich das Land Ratzeburg jedoch durch das Wort Vörjord davon absondere (Vgl. Mecklenburgisches Wörterbuch, 1. Bd., Sp. 400). Buddin meint, dass neben diesem Wort dort aber , auch noch häufig Anwenn, Awenn oder kurzweg Wenn“" zu hören seien (Buddin 1929, S. 45).

22 Bentzien 1964.

23 Gundlach 1967. Das Aufnahme-Korpus, an dem Gundlach mitgearbeitet hat, ist im Internet zugänglich als IDS-Korpus: Datenbank Gesprochenes Deutsch, „DGD/Deutsche Mundarten - DDR“ (http://dgd.ids-mannheim.de). 
für neue Erfassungen oftmals gar nicht mehr gegeben ist. Probanden, die heute befragt werden können, gehörten am Beginn der 1960er Jahre größtenteils der jüngeren Generation an, sind aber in der Gegenwart trotz ihres hohen Alters zugleich zumeist die letzten, die das Niederdeutsche noch als Erstsprache erlernten. ${ }^{24}$ Die Anzahl derjenigen, die nach 1950 geboren sind und das Mecklenburgische noch auf muttersprachlichem Niveau beherrschen, ist dagegen recht gering. Zwar wird in Kindergärten und Schulen zum Teil Niederdeutsch gelehrt; dieses ist jedoch eine Variante, die die Besonderheiten der Ortsdialekte gar nicht mehr erfasst. Der in dieser Arbeit dargestellte Zustand stellt also eine Momentaufnahme dar, die aufgrund der weiter schwindenden Sprecherzahlen bald nicht mehr aktuell sein wird. Die dargelegten Unterschiede werden dann nur noch vereinzelt - wenn überhaupt - in der hochdeutschen Umgangssprache fassbar sein. ${ }^{25}$ Ferner ermöglichen es die Aufnahmen Gundlachs, einen Vergleich zwischen den Generationen von Sprechern an einem Ort zu ziehen, der heute ebenfalls kaum noch zu realisieren ist. Die Veränderungen im Wortschatz können so besser nachvollzogen werden, als wenn lediglich noch eine Sprechergeneration pro Ort untersucht werden kann.

Als eine bis an die Gegenwart heranführende Ergänzung und Vergleichsgrundlage zu den Aufnahmen Gundlachs dienen in meiner Studie neuere Aufnahmen, die zwischen 2004 und 2012 angefertigt wurden. ${ }^{26}$ Zur Unterscheidung von Gundlachs „alten Aufnahmen“ der frühen 1960er Jahre werden meine eigenen Aufnahmen im folgenden Text als „,neue Aufnahmen“ bezeichnet. Der Forderung aus den 1960er Jahren, dass die Sprecher in dem jeweiligen „Aufnahmeort geboren, in ihm aufgewachsen und nicht lange von ihm abwesend gewesen sein" sollten, ${ }^{27}$ ließ sich für die neuen Aufnahmen nicht immer erfüllen, da einige Gewährspersonen ihren Wohnort gewechselt haben. Insgesamt wurden 20 Probanden aus zehn Orten erfasst, deren geographische Lage ermöglichen sollte, die Aussagen der älteren Forschungsliteratur zur Wortgeographie Mecklenburg-Vorpommerns zu überprüfen. Diese Orte befinden sich

24 Der jüngste Sprecher wurde in den 1950er Jahren geboren, der zweitjüngste 1948, alle anderen in den 1920ern und 1930ern; der älteste, eine Frau, 1913.

25 Als Beispiel sei hier das Wort Ameisen genannt, die von Kindern in Demen in den 1980er Jahren umgangssprachlich noch mit dem dort üblichen Mundartwort als Pissaminken bezeichnet wurden, d.h., das Wort ist heute in dem Ort selbst bei jüngeren Sprechern noch bekannt, die das Niederdeutsche nicht beherrschen.

26 Die Aufnahmen wurden im Rahmen meines Dissertationsprojekts begonnen, vgl. Köhncke 2010.

27 Gundlach 1967, S. 174.

Birte Arendt, Andreas Bieberstedt and Klaas-Hinrich Ehlers - 978-3-631-71893-3 
hauptsächlich im heutigen Kreis Ludwigslust-Parchim bzw. den früheren DDRKreisen Schwerin-Land (Banzkow, Demen, Rastow, Sukow, Tramm), Parchim (Kossebade) und Ludwigslust (Menkendorf). Um einen Ausblick auf das weitere Umfeld der zentralmecklenburgischen Verhältnisse zu ermöglichen, wurden auch einzelne weiter entfernte Orte in die Betrachtung einbezogen (z. B. Bützow, Rostock, Wismar).

Der Großteil meiner Probanden ist am jeweiligen Aufnahmeort geboren und aufgewachsen. Das trifft insbesondere zu für die Aufzeichnungen aus Demen, Kossebade, Tramm und Menkendorf. Weiterhin erfasst wurden die Orte Bützow, Rastow, Rostock, Banzkow und Wismar, allerdings gelten hier Einschränkungen: Die Sprecherin aus Bützow lebt heute in Demen, der Wismarer in Crivitz, die beiden in Rostock aufgezeichneten Personen sind in Gnoien bzw. Bad Doberan aufgewachsen und sprechen deshalb auch nicht die Rostocker Mundart, der Rastower Sprecher kommt ursprünglich aus Goldenstädt, das aber in der Nähe des Aufnahmeortes liegt, und der Banzkower lebt seit fünfzig Jahren in Sukow, woher seine ebenfalls aufgezeichnete Ehefrau stammt. Dadurch kann also nicht in allen Fällen die übliche Ortsmundart erfasst werden, allerdings lassen sich durch die Ortswechsel Aussagen darüber treffen, inwieweit die Umzüge diese Probanden in ihrem Sprachverhalten beeinflusst haben. Ergänzend zu den genannten Aufnahmen wurden zwei Personen aufgezeichnet, die an der westlichen Grenze des mecklenburgischen Dialektgebietes leben, wobei eine aus dem Lübecker Stadtteil Schlutup stammt und auch dort ihr gesamtes Leben verbracht hat, die andere im niedersächsischen Woltersdorf (Kreis Herzogtum Lauenburg) lebt und in der Nähe zur mecklenburgischen Grenze aufgewachsen ist.

Die Gundlachschen Aufnahmen beinhalten einen hochdeutschen „Festen Text“ (FT), den die Sprecher in ihre Ortsmundart zu übersetzen hatten, sowie eine „Freie Erzählung“ (FE), in der frei mit dem Interviewer auf Niederdeutsch gesprochen wurde. Ergänzend dazu hat Gundlach Sätze für das Mecklenburgische Wörterbuch abgefragt, die sich jedoch von Region zu Region unterschieden. ${ }^{28}$ Für die neuen Aufnahmen wurde, um Einheitlichkeit zu gewährleisten, genauso verfahren, das heißt, nach einem freien niederdeutschen Gespräch wurden die Probanden gebeten, den selben Text und die Wörterbuchsätze zu übersetzen wie die Probanden Gundlachs vor gut 40 Jahren.

28 Die Texte sind abgedruckt bei Gundlach 1967, S. 189-190.

Birte Arendt, Andreas Bieberstedt and Klaas-Hinrich Ehlers - 978-3-631-71893-3 


\section{Regionale Unterschiede im Wortgebrauch}

\subsection{Wortgeographische Binnengliederungen des Mecklenburgischen}

Für die dialektgeographische Binnengliederung des Mecklenburgisch-Vorpommerschen werden von der Forschung neben lautlichen Unterschieden ${ }^{29}$ auch die Lexik herangezogen. Von den dabei üblicherweise zugrundgelegten Wörtern sind jedoch nicht alle auf den Aufnahmen der 1960er Jahre zu hören, so dass hier eine Auswahl getroffen wird, die Vergleiche zwischen den verschiedenen Zeitstufen ermöglicht. Dabei ist zu überprüfen, ob die gängigen Angaben in der Fachliteratur noch gültig sind.

Für Dieter Stellmacher ist das Mecklenburgische charakterisiert durch einen „Nord-Süd-Gegensatz, den die Linie Wittenburg-Crivitz-Goldberg-Friedland festlegt. Sie trennt die nördlichen Kütik, Tram, Trad, Arnbier von den südlichen Harrik, Sprat, Lois, Austkost ,Hederich, Leitersprosse, Wagenspur, Erntefest'. "30 Auf den neuen Aufnahmen und weiteren Gesprächen ist daher gezielt nach diesen Wörtern gefragt worden oder sie bildeten z. T. das Gesprächsthema (z. B. das Erntefest), auf den alten Aufnahmen Gundlachs sind sie zumindest in einigen Orten abgefragt oder verwendet worden.

Teuchert unterscheidet hingegen zwischen einem „West-Ost-Gefälle“, worunter die Wörter Wädik/Arpel,Enterich' und Äwer/Bir ,Eber ' fielen, und dem von Stellmacher ausgemachten „Nord-Süd-Gegensatz“, wozu neben „Kütik Ackersenf, sinapis arvensis / Haddik, Harrik Hederich, raphanistrum arvense ${ }^{\text {“31 }}$ und „Trad'/ Leus' Wagenspur, Geleise, Tram/Sprat Leitersprosse“ auch „Band/Seil Strohseil zum Binden des Strohbundes“ gehörten. ${ }^{32}$ Hans Joachim Gernentz stellt ebenfalls

29 Hier wären z. B. zu nennen: der Verlauf der Vokalerhöhung, die beispielsweise noch heute im Eldewinkel nicht durchgeführt worden ist; der Gegensatz veier/vier,vier; fäuhren/führen, fahren, führen', wobei die diphthongische Aussprache im Westen üblich, allerdings stark rückläufig ist, sowie das monophthongische Koken im Strelitzer Landesteil gegenüber Kauken, das im Großteil Mecklenburgs üblich ist (vgl. Teuchert 1942, S. VII sowie S. IX). Meine Untersuchung konzentriert sich allerdings allein auf Mecklenburg und lässt die vorpommersche Wortgeographie außer Betracht.

30 Stellmacher 2000, S. 147.

31 Kütik und Haddik sind die beiden areal unterschiedlich verbreiteten niederdeutschen Äquivalente, mit denen im Übersetzungstest die hochdeutsche Bezeichnung Hederich übertragen wurde. Es ist davon auszugehen, dass die Laienbezeichnungen keinen Unterschied zwischen den äußerlich sehr ähnlichen Pflanzen sinapis arvensis und raphanistrum arvense geltend machen, die hochdeutsch heute mitunter auch als Ackersenf bzw. Ackerettich benannt werden.

32 Teuchert 1942, S. VIII.

Birte Arendt, Andreas Bieberstedt and Klaas-Hinrich Ehlers - 978-3-631-71893-3 
"- besonders deutlich im Wortschatz - Unterschiede zwischen dem Norden und dem Süden des Dialektgebietes" fest, wobei er die bereits genannten Beispiele Teucherts übernimmt. ${ }^{33}$ Die Gruppe der in den neuen Aufnahmen erfassten Orte in Mecklenburg ist so gewählt, dass sie sowohl von den wortgeographischen NordSüd-Isoglossen als auch von den genannten West-Ost-Gegensätzen durchquert werden, die die ältere Dialektgeographie festgestellt hatte.

\subsubsection{Ernte}

Zunächst soll der wortgeographische Nord-Süd-Gegensatz untersucht werden. Die Beispiele hierfür gleichen sich bis auf ein Wortpaar, das nur Stellmacher nennt, und zwar die Bezeichnung für das Erntefest. Da die Ernte in einer ländlich geprägten Region wie Mecklenburg eine große Rolle spielte, soll auch die Bezeichnung dafür hier Untersuchungsgegenstand sein. Die beiden von Stellmacher angeführten Komposita Arnbier und Austkost unterscheiden sich in beiden Gliedern, das Bestimmungswort für „Ernte“ lautet demnach im Norden Arn, im Süden Aust. Das Wort ist dabei mittels der vorgegebenen Sätze für das Mecklenburgische Wörterbuch abgefragt worden. Auf den neuen Aufnahmen verwenden die Probanden fast ausschließlich Aust. Lediglich der Rastower und der Banzkower Sprecher sagen Oorn. ${ }^{34}$ Anzumerken ist, dass der Proband aus Banzkow schon seit über fünfzig Jahren im nordöstlicher gelegenen Nachbarort Sukow lebt. Seine Frau und seine Schwiegermutter sagen aber beide Aust. Er ist deshalb auch unsicher, als im Gespräch nochmals nachgefragt wird und wägt ab: „Oorn oder Aust. Nu geht't inne Aust. Ja, un in de, inne Oorn. Ja." Schließlich entscheidet er sich für das von ihm bereits in den übersetzten Sätzen vorgetragene Wort Oorn. Die Unsicherheit ist demnach wohl auf den Umzug zurückzuführen. Dennoch scheint er das in Sukow gebräuchliche Wort jedenfalls nicht vollständig übernommen zu haben. Bereits diese Belege zeigen, dass die Verbreitung des Bestimmungswortes nicht mit der von Stellmacher angegebenen Isoglosse übereinstimmt, denn Demen liegt beispielsweise nördlich der Linie, dort verwenden die Probanden aber noch das von Stellmacher im Süden lokalisierte Aust. Auf den alten Aufnahmen dominiert dieses Wort ebenfalls großräumig. Es herrscht

33 Gernentz 1980, S. 28.

34 Im Folgenden wird darauf verzichtet, etwaige lautliche Unterschiede bei Oorn zu markieren. So schieben einige Sprecher ein /d/ ein [ordṇ] oder das /o/ wird dem /u/ angenähert [ur(d)ṇ], was besonders im Südwesten üblich ist, vgl. z. B. die Aussprache [du:e] ,da, dort' eines Sprechers aus Lüttow sowie Jacobs 1926/27, S. 130 und Teuchert 1959, S. 234, für den $d$-Einschub vor /n/ siehe dort S. 225. 
im Osten und der Landesmitte Mecklenburgs ausschließlich vor. Lediglich im Westen ist auf den Aufnahmen von 1962/63 Oorn zu hören. Östlichste Orte bilden hierbei Züsow, Pinnow und das später erfasste Banzkow. Allerdings schwanken die Sprecher zwischen beiden Bezeichnungen. Das ist auch in vielen anderen noch westlicher gelegenen Aufnahmeorten so. Stellvertretend sei hier ein Proband Gundlachs aus Hoben genannt, der im freien Gespräch immer wieder wechselt: „Un nåhher tau de Aust, tau de Oorn, denn säd“ wi: ,Ach, nu låt all een poor Dååg, acht Dååg, denn geht wedder bi de Aust, denn is dat Fischen wedder vörbi.“ Selbst in den westlichsten mecklenburgischen Aufnahmeorten Selmsdorf, Lüttow und Bennin lässt sich neben Oorn auch Aust nachweisen. Teilweise wechseln die einzelnen Sprecher zwischen beiden Wörtern, teilweise bevorzugt am selben Ort der eine Sprecher Aust der andere Oorn. Ausschließlich verwendet wird Oorn in den westlichen Grenzorten Schlagsdorf und Zweedorf, des Weiteren in Möllin und Woez. In Selmsdorf, Hoben, Alt Meteln und Pinnow überwiegt es, während Aust in Lüttow, Bennin und Pritzier dominiert. Im Südwesten ist dagegen in den alten Aufnahmen ausschließlich Aust zu hören, d. h. in Alt Jabel, Glaisin, Eldena, Lüblow und Prislich. Auch die Menkendorfer Probanden verwenden auf den 2012 angefertigten Aufzeichnungen dieses Wort. Die Sprecher in den an Mecklenburg angrenzenden holsteinischen bzw. lauenburgischen Orten Schlutup und Woltersdorf sagen in den neuen Aufnahmen Oorn. In Sumte, das von Gundlach ebenfalls erfasst worden ist, da es damals zur DDR gehörte, dominiert Aust, wenngleich auch Oorn vorkommt.

Während das Genus von Oorn stets Femininum ist, gibt das Mecklenburgische Wörterbuch für Aust an, es werde ,heute häufig f[eminin]. gebraucht, wohl in Anlehnung an Oorn, [...], sonst meist m[askulin] “. ${ }^{35}$ Auf den alten Aufnahmen überwiegt das Femininum. Es zeigt sich jedoch eine räumliche Verteilung für beide Genera: Das Maskulinum ist fast ausschließlich in Orten nachweisbar, die in Mecklenburg-Strelitz liegen. In Klein Trebbow, Triepkendorf und Peetsch übersetzen alle Probanden „nach der Ernte“ mit „nåh den Aust", in Granzin und Röbel, das als einzige Ortschaft außerhalb des alten Stargarder Landes liegt, überwiegt es gegenüber femininem „,nåh de Aust“, in Warlin, Cammin und Weisdin dominiert eben diese Form gegenüber dem Maskulinum. Diese Verteilung könnte mit der Verbreitung des Wortes Aust zusammenhängen: Da es von Südosten aus Oorn verdrängt hat und mittlerweile auch im Westen verwendet wird, haben die Sprecher offenbar zwar das Wort übernommen, aber nicht dessen Genus. In

35 Mecklenburgisches Wörterbuch, 1. Bd., Sp. 490, Lemma „Aust“. 
Mecklenburg-Strelitz, wo es schon länger verwendet wird, hat sich dagegen das ursprüngliche Maskulinum halten können.

Für das Wort „Ernte“ lässt sich somit feststellen: Noch in den 1960er Jahren ließ sich ein West-Ost-Gegensatz zwischen beiden Bezeichnungen ausmachen, der aber bereits damals zusehends schwand. Aust dominiert im Untersuchungsgebiet sowohl auf den alten Aufnahmen wie auch auf den neueren Erhebungen, lediglich im Westen Mecklenburgs ist noch Oorn im Gebrauch, so dass sich dieser Landesteil wortgeographisch vom Rest abhebt. Auf den neueren Aufzeichnungen verwendet dieses Wort nur noch ein aus Banzkow stammender Sprecher. In Menkendorf, das den westlichsten Ort der neueren Erhebungen darstellt, gilt bereits Aust, wobei die Probanden des in Nachbarschaft gelegenen Eldena bereits in den 1960er Jahren ebenfalls ausschließlich dieses Wort nutzten. Beide Orte liegen auf dem südlichsten Planquadrat der Erhebung Gundlachs und setzen sich von den nördlicheren Orten z. B. auch in der Aussprache von „mähen“ und „zehn“ ab. ${ }^{36} \mathrm{Im}$ gesamten Westen war allerdings schon in den 1960er Jahren Aust bereits üblich. Das dominierende Genus für Oorn und Aust ist das Femininum. Eine maskuline Form existiert nur für Aust, die aber wiederum nur in Mecklenburg-Strelitz und Röbel zu hören ist.

\subsubsection{Erntefest}

Es wäre nur folgerichtig, dass die Verteilung von Aust und Oorn auch für das Ernte-fest gilt. Es zeigt sich aber, dass das Bestimmungswort Oorn- weiter verbreitet ist als das Simplex. Auf den alten Aufnahmen ist es in Züsow, Pinnow, Zahrensdorf und Mestlin zu hören, auf den neueren in Banzkow Demen, und Kossebade, das heißt, es kommt auch in Orten vor, die westlich von Schwerin liegen, was beim Simplex „Oorn“ nicht der Fall war. In diesem Gebiet herrscht

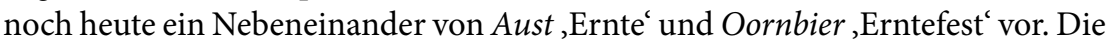
östlichste Ortschaft, in der das Erstglied Oorn- nachgewiesen werden kann, ist auf den alten Aufnahmen Satow, das östlich des Plauer Sees liegt. Es kommt in den oben genannten Orten allerdings nur in diesem einen Wort vor, andere Komposita mit ,Ernte' als Bestimmungswort werden dagegen mit Aust gebildet. So heißt die ,Kartoffelernte in Demen Tüffelaust. Das Bestimmungswort in ,Ernte-

36 In beiden Orten werden die Wörter mit Diphthong artikuliert: [mardṇ] ,mähen und [tardṇ] ,zehn, wohingegen z. B. im nördlich von Menkendorf gelegenen Leussow nach Auskunft eines Menkendorfer Probanden Monophthong verwendet werde: [medn]] und [tedn]. 
fest' ist also erstarrt und nicht mehr produktiv - ganz im Gegensatz zum Westen, wo Oorn nach wie vor auch für Wortbildungen verwendet wird.

Im Norden Mecklenburgs ist Aust- schon weiter westlich belegt und begegnet in Retschow und Letschow als Austbier. Im Osten wechselt das Grundwort und lautet in Jördenstorf, Bansow, Nossentiner Hütte, Carolinenhof und Peetsch Austköst. In den anderen Strelitzer Orten sowie Kölzow und Eldena verwenden die Sprecher bereits auf den alten Aufnahmen das hochdeutsche Wort Erntefest. Für den Südwesten wird auf den alten Aufnahmen bis auf Eldena kein Wort für das Erntefest genannt. In Menkendorf gibt der Proband an, dass man Erntefest sage oder Austfest, wobei ihm aber auch Oornbier geläufig ist, das aber im Ort nicht gesagt werde. ${ }^{37}$

Anhand der Befunde lässt sich feststellen, dass bei ,Erntefest ${ }^{ }$kein Nord-SüdGegensatz vorliegt, da Oornbier auch in nördlichen Orten zu finden ist, z. B. in den 1960er Jahren in Welzin, Züsow, Zahrensdorf und noch heute in Demen, während im südöstlichen Peetsch Austköst üblich ist. Vielmehr ist wie bei Aust/Oorn ein Unterschied zwischen Ost und West auszumachen, wie er - allerdings nur sehr grob - bereits in einer Karte im Mecklenburgischen Wörterbuch dargestellt ist. ${ }^{38}$ Die neueren Befunde zeigen einen Wechsel von Oornbier zu Austbier, das schließlich zu Ausköst übergeht, im Süden folgt auf Oornbier Austköst. ${ }^{39}$ Die Variante Oornklaatsch, die als Streubeleg im Norden auf der besagten Karte des Mecklenburgischen Wörterbuchs angegeben wird, war auf keiner der alten und neuen Aufnahmen mehr zu hören.

\subsubsection{Hederich, Leitersprosse und Strohband}

Über die anderen in der Literatur genannten Wörter können keine präzisen Angaben zur regionalen Verbreitung gemacht werden, da diese nur in wenigen Ortschaften abgefragt und kaum im freien Gespräch verwendet wurden: Hochdeutsch Hederich (Ackersenf, Ackerrettich) erscheint in der überwiegenden Zahl der Aufnahmeorte Gundlachs als Harrick, die umgelautete Form Härrick ist in den südwestlichen Orten Alt Jabel, Glaisin und Eldena zu hören. Die Probanden auf

37 Der Proband erwähnt das Wort von sich aus. Darauf angesprochen, ob er „Aust“ oder „Oorn“ sage, meint er: „Wi seggen Aust. Wo de annern, de seggen Oorn oder Oornbier, denn da, dann daher dat Erntefest is Oornbier.“ Für den Ort gibt er jedoch an: „Wi seggen einfach Erntefest. Oder Austfest.“

38 Mecklenburgisches Wörterbuch, 1. Bd., Sp. 499-500.

39 So heißt es im südöstlichen Satow Oornbier, im nördlicheren Nossentiner Hütte Ausköst.

Birte Arendt, Andreas Bieberstedt and Klaas-Hinrich Ehlers - 978-3-631-71893-3 
den neuen Aufnahmen kennen das Wort teilweise gar nicht mehr und weichen entweder auf andere Bezeichnungen aus (z.B. in Demen Quäck und Unkruut) oder verwenden das Wort der hochdeutschen Vorlage Hederich. In Kossebade lautet das Wort Harrick, in Demen war dieses Wort nur auf Nachfrage hin bekannt, in Menkendorf verwendet der Sprecher wie in den auf den alten Aufnahmen erfassten Nachbardörfern Härrick. Das Wort Kütik, das laut Stellmacher nördlich der Linie Crivitz-Goldberg für Hederich genutzt werde, war im nördlich davon gelegenen Demen gänzlich unbekannt; der einzige neuere Nachweis für dieses Wort stammt von einem Sprecher aus Rostock, der jedoch ursprünglich aus Bad Doberan kommt. Die altdialektalen Lexeme Kütik und Harrick/Härrick scheinen seit der Befragung Gundlachs weiter aus dem mecklenburgischen Wortschatz zu schwinden, entsprechend beginnen hier auch die regionalen Differenzen zu verschwimmen.

Die Leitersprosse wurde auf den alten Aufnahmen lediglich im Osten Mecklenburgs abgefragt, daher lassen sich für das übrige Untersuchungsgebiet nur bedingt Aussagen machen. In Bansow, Bristow, Brudersdorf und Jördenstorf übersetzen die Probanden Gundlachs das Wort mit Leddertråm bzw. -trån. Die Sprecher im südlicher gelegenen Carolinenhof schwanken, hier verwendet eine ältere Frau Tråm, ein anderer Proband jedoch Ledderspråt. Letzteres gilt ausschließlich in Mecklenburg-Strelitz und in den südlichen Orten Ostmecklenburgs. Daneben nutzen vor allem jüngere Sprecher bereits in den 1960er Jahren halbhochdeutsches Ledderspross, so z. B. in Bansow, Bristow, Carolinenhof, Granzin, Nossentiner Hütte, Peetsch, Triepkendorf und Weisdin. Diese Bezeichnung verdrängt damit sowohl nördliches Tråm als auch südmecklenburgisches Språt. Diese Tendenz setzt sich auf den neuen Aufnahmen fort: In Demen, Kossebade und Rastow wird diese Form ausschließlich benutzt, wobei Språt zumindest noch bekannt ist. Die rein niederdeutsche Bezeichnung ist noch in Menkendorf und Tramm zu hören. Für Tråm gibt es in den neuen Aufnahmen auch in nördlichen Teilregionen keinen Nachweis mehr. Das hochdeutschnähere Språt (vgl. Sprosse) scheint abbauresistenter zu sein als Tråm.

Über die Verbreitung von Trad/Läus',Geleise, Wagenspur' und Band/Seil (zum Binden von Stroh), für die die frühere Wortgeographie jeweils eine nord-südliche Raumgliederung annahm, können kaum aktuellere Aussagen getroffen werden, da diese Wörter auf den Aufnahmen der 1960er Jahre nicht abgefragt wurden. Läus' ist auf keiner der neuen Aufnahmen zu hören, der Sprecher aus dem recht weit südlich gelegenen Rastrow sagt im freien Gespräch Trad. Diese Bezeichnung herrscht auch in Demen und Kossebade vor. Neben der geringen Belegdichte ergibt sich bei Band/Seil eine zweite Schwierigkeit, da die Probanden auf den neuen 
Aufnahmen beide Bezeichnungen verwenden, es sich dabei jedoch offensichtlich nicht um Synonyme handelt. Seil benutzen sie, um darzustellen, dass die Garben mittels Strohhalmen gebunden wurden, später nutzte man dazu das künstliche Band. Hier ist zwischen den ehemals regionalspezifischen Wörtern offensichtlich eine semantische Differenzierung eingetreten.

Trotz der teilweise dünnen Belegdichte kann festgehalten werden, dass der von Stellmacher beschriebene „Nord-Süd-Gegensatz“ für Ornbier/Austköst empirisch nicht bestätigt werden kann. Für die anderen bei Stellmacher in nord-südlicher Dimension gegliederten Wörter ist diese wortgeographische Linie für heutige Verhältnisse zumindest zu ungenau, da die nördlichen Kütik und Tråm im nördlich gelegenen Demen unbekannt sind und dort stattdessen die für den Süden angegebenen Bezeichnungen noch gelten. Das nördlich der Isoglosse lokalisierte Tråd wird hingegen selbst noch in den viel südlicher gelegenen Ortschaften Rastow oder Kossebade verwendet. Meine neuen Aufnahmen deuten darauf hin, dass bei allen genannten Belegwörtern für einen wortgeographischen „Nord-SüdGegensatz" ein Abbauprozess eingesetzt hat, mit dem die altdialektalen Lexeme fortschreitend außer Gebrauch kommen.

\subsection{Tier- und Pflanzenbezeichnungen}

\subsubsection{Enterich und Eber}

Nach Teuchert existiert im Mecklenburgischen auch ein „West-Ost-Gefälle“, wofür er u. a. unterschiedliche Bezeichnungen für den Enterich anführt. Daher soll im Folgenden die Verbreitung der niederdeutschen Äquivalente Wädik und Arpel untersucht, aber auch auf die regionale Gliederung anderer Tier- und Pflanzenbezeichnungen eingegangen werden.

Die westliche Bezeichnung Wädik ist auf den alten Aufnahmen Gundlachs noch in den westmecklenburgischen Orten Alt Meteln, Boldela, Groß Lantow, Hoben, Möllin, Warnow und Zahrensdorf zu hören; in Boldela, Groß Lantow und Lüblow erscheint sie auch als Wäding. Arpel und Erpel dominieren im Osten, sind aber auch in der Landesmitte nachweisbar. Besonders hochdeutsches Erpel dringt dabei immer weiter nach Westen vor, wie bereits die Aufnahmen aus den 1960er Jahren belegen, da das Wort damals bereits in Selmsdorf, Möllin, Alt Jabel, Prislich, Warnow und Zahrensdorf vorkommt. Auf den neuen Aufnahmen seit 2004 ist Erpel im gesamten Untersuchungsgebiet fast ausschließlich zu hören. In Kossebade ist Wädik auf Nachfrage hin noch bekannt und wurde dem Vernehmen nach noch in den 1960er Jahren von den Eltern der beiden Sprecherinnen genutzt, in Tramm korrigiert der ältere Sprecher Erpel zu Wäding. In Demen gilt ausschließlich Erpel, Wädik hingegen ist völlig unbekannt, ein Sprecher meint sogar, 
dass es nicht Mecklenburgisch sei. Wossidlo stellte bereits Ende des 19. Jahrhunderts im Warener Raum fest, dass diese Bezeichnung einigen Gewährsleuten nicht bekannt war. ${ }^{40}$ Weiterhin stellte er fest, „, [b]eide Namen [d.h. Wädik und Arpel, A. K.] gehen durcheinander in der Gegend Lübz-Parchim-Crivitz, und DargunGnoien-Laage-Tessin-Rostock“. ${ }^{41}$ Das erklärt auch, warum Wädik in Kossebade noch bekannt ist, in Demen aber nicht. Offenbar ist Erpel in der letztgenannten Ortschaft schon länger in Gebrauch.

Im Südwesten Mecklenburgs heißt der Enterich laut Mecklenburgischem Wörterbuch hauptsächlich Wänker. ${ }^{42}$ Diese Bezeichnung gilt den Aufnahmen Gundlachs zufolge noch im Südwesten in Alt Jabel, Eldena, Menkendorf, Glaisin und Prislich, allerdings dringt auch hier schon in den 1960er Jahren wie oben erwähnt Erpel vor. Die Sprecher in den nordwestlichsten Aufnahmeorten Schlagsdorf und Selmsdorf verwenden die aus Wädik gekürzte Form Wäät. Das gilt auch für das außermecklenburgische Sumte. Im holsteinischen Schlutup ist in den neuen Aufnahmen Enterich zu hören, der Woltersdorfer Sprecher verwendet bereits Erpel.

Eine klare West-Ost-Verteilung von Wädik/Erpel bzw. Arpel ist also nicht mehr vorhanden. Die entsprechende Karte im Mecklenburgischen Wörterbuch ist damit nicht mehr aktuell. ${ }^{43}$ Allerdings handelte es sich schon in der Entstehungszeit des Wörterbuch offenbar um keine strikte regionale Unterscheidung, weil auf der genannten Karte ein geschlossenes Wädik-Gebiet dargestellt wird, dessen Ostgrenze sich über Laage und Güstrow erstreckt, wobei aber auch im östlichen Arpel-Territorium noch Streubelege für die Bezeichnung Wädik verzeichnet wurden. Wossidlo hat andererseits bereits für einige Gegenden innerhalb des westlichen Wädik-Gebietes ein Durcheinander beider Formen festgestellt, so dass die Karte auch dort zumindest Streubelege für Arpel aufweisen müsste.

Auch der von Teuchert angeführte West-Ost-Unterschied zwischen Bir und Äwer ,Eber' schwindet zusehends. Das Wort wurde auf den alten Aufnahmen allerdings nur im Südwesten abgefragt, jedoch lässt sich anhand der neuen Aufnahmen die Tendenz zum Abbau des West-Ost-Gegensatzes aufzeigen. Laut Karte im Mecklenburgischen Wörterbuch gilt im Großteil Mecklenburg-Vorpommerns

40 So schreibt er: „Wi seggen arpel, wädick sall wol mihr hoochdüütsch sien: hörte ich in diesen Gegenden öfter sagen. Meist ist wädick den Leuten völlig unbekannt. "Wossidlo 1899, S. 378.

41 Wossidlo 1899, S. 378.

42 Mecklenburgisches Wörterbuch, 1. Bd., Sp. 441-442.

43 Mecklenburgisches Wörterbuch, 1. Bd., Sp. 441-442.

Birte Arendt, Andreas Bieberstedt and Klaas-Hinrich Ehlers - 978-3-631-71893-3 
Bir,${ }^{44}$ im Westen Mecklenburgs Bir und Äwer nebeneinander, im Südwesten von der Gegend um Lübtheen bis nach Röbel die Entsprechung Kemp. ${ }^{45}$ Das letztgenannte Wort ist in Alt Jabel, Eldena, Glaisin, Lüblow, Marnitz und Prislich in den alten Aufnahmen noch zu hören, allerdings nur von den älteren Sprechern. Die jüngeren nutzen bereits in den 1960er Jahren das der Standardsprache nähere Äwer. Auf den neuen Aufnahmen dominiert diese Bezeichnung bereits. In Tramm bevorzugen es beide Sprecher, der ältere kennt Kemp aber zumindest noch. In Demen und Kossebade übersetzen alle Sprecher „Eber“ mit Äwer, wenngleich Bir wiederum noch allen bekannt ist. Der Wismarer Sprecher und der aus Gnoien stammende Rostocker kennen nur Äwer. Lediglich zwei Probanden verwenden dieses Wort nicht: Im südwestlichen Menkendorf nutzt der Sprecher Kemp, der aus Bad Doberan stammende Rostocker Bir. Das laut Mecklenburgischem Wörterbuch im Untersuchungsgebiet dominierende Bir ist demnach heute zumindest in der Gegend Crivitz - Parchim größtenteils nur noch im passiven Wortschatz vorhanden, das heißt, das westliche Äwer setzt sich auch in östlichen Regionen Mecklenburgs immer weiter durch und verdrängt die beiden anderen Bezeichnungen Bir und Kemp.

\subsubsection{Ameise, Frosch/Kröte und Regenwurm}

Ausgleichstendenzen lassen sich auch für die Bezeichnungen für Ameise, Frosch/ Kröte und Regenwurm feststellen, allerdings herrscht hier teilweise noch eine recht große Formenvielfalt, die im Folgenden dokumentiert werden soll.

Für die Ameise werden im Mecklenburgischen drei verschiedene Grundwörter genutzt: Eemk, Mirr und Mink. Diese können sowohl als Simplex aber auch als Kompositum auftreten. Mink wird als einfaches Wort jedoch nur vom Banzkower Probanden verwendet. Die nachfolgenden Angaben gelten für den Osten und die Mitte Mecklenburgs, für den Westen und Südwesten konnten lediglich die neuen Aufnahmen herangezogen werden, da das Wort von Gundlach nicht abgefragt worden ist. Eemk und Mirr treten in zahlreichen Varianten auf: Ä̈̈nken (Nossentiner Hütte), Eenken (Nossentiner Hütte), Inken (Broock, Nossentiner Hütte, Satow) bzw. Määrden (Granzin), Miern (Carolinenhof, Granzin). Häufig werden sie mit den Verben miegen oder pissen ,urinieren' verbunden, wobei einige Sprecher Eemk kürzen: Miechäämken (Jördenstorf), Miecheemken

44 Vgl. für Vorpommern die Karte „männl. Schwein“ in Herrmann-Winter 2013, S. 41, die für das vorpommersche Festland Bier/Biern und für Rügen die Sonderform Höcker nachweist.

45 Mecklenburgisches Wörterbuch, Bd. 1, Sp. 887-888.

Birte Arendt, Andreas Bieberstedt and Klaas-Hinrich Ehlers - 978-3-631-71893-3 
(Bansow), Miecheem (Jördenstorf), Miecheenken (Bansow), Pisseemk (Rostock [Gnoien]), Pissmirrn (Warlin). Mink erscheint fast ausschließlich als Kompositum Pisseminken (Demen, Kossebade, Nossentiner Hütte, Rostock [Bad Doberan], Satow, Tramm sowie im außermecklenburgischen Woltersdorf). In Bansow, Bruderstorf und Jördenstorf wird Eemk als Grundwort schließlich zu -imm gekürzt: Miechimm. Damit ergibt sich ein Anschluss an Imm ,Biene‘. So ist wohl auch die Übersetzung Miechhäuhner zu erklären, die in Bruderstorf zu hören ist, d.h., das Grundwort wird in Anlehnung an Imm weiter variiert. ${ }^{46}$ Ein von dieser Bildungsweise abweichendes Kompositum benutzt der Menkendorfer Sprecher: Er übersetzt das Wort mit Sprockeemk.

Für die beiden Grundwörter Eemk und Mirr gilt dabei nach den Befunden der älteren und neueren Aufnahmen folgende geographische Verteilung: Letztgenanntes tritt ausschließlich in Mecklenburg-Strelitz auf und wird auch noch in Kieve, Röbel und Carolinenhof verwendet, im übrigen Untersuchungsgebiet gilt Eemk bzw. Mink/Pissemink. Daneben treten weitere Mischformen auf, die vor allem auf hochdeutschen Einfluss zurückzuführen sind: Aminken (Rastow, Nossentiner Hütte), Emsen (Wismar, lautlich [emzn]]) sowie das standardsprachliche Wort selbst. Dieses lässt sich bereits auf den alten Aufnahmen in fast allen Ortschaften nachweisen, ${ }^{47}$ auf den neuen Aufnahmen in allen Untersuchungsorten. Ameise wird von den Sprechern als „feiner" empfunden, das mundartliche Wort gilt - gerade wegen des Erstgliedes Miech-/Piss- - teilweise als anstößig, so dass es bei den Übersetzungen auf den neuen Aufnahmen zumindest von einigen Probanden bewusst gemieden worden ist. ${ }^{48}$

Auch „Frosch“ und „Kröte“ werden schon gelegentlich mit den hochdeutschen Wörtern wiedergegeben. Daneben sind aber auch die mundartlichen Lexeme noch geläufig. Wie schon bei Ameise werden unterschiedliche Grundformen ge-

46 Mecklenburgisches Wörterbuch. 2. Bd., Sp. 662, Lemma „Eemk“. Der Übergang von Eemk zum Imm, das lautlich mit der Bezeichnung für Biene zusammenfällt, scheint zu weiteren, und zwar freieren Wortbildungen mit anderen Tieren, z. B. Häuhner ,Hühner, geführt zu haben.

47 Mindestens einmal kommt das standarddeutsche Wort vor in Bansow, Bristow, Broock, Cammin, Carolinenhof, Granzin, Kieve, Nossentiner Hütte, Satow, Schönbeck, Triepkendorf, Warlin und Weisdin.

48 So sagt die jüngere Sprecherin aus Kossebade: „Pisseminken. Hemm s’ ok ümmer seggt, Pisseminken. Ach, dat segg bloß nich den Liehrer! Wat ick seggt heff mit Pisseminken." In Demen bemerkt ein Proband: „Ja, dat seggt man wenn, dat is wat fiener, Ameisen is fiener, Pisseminken is so gewöhnlich, ja." Dort wurde das Wort aber zumindest in den 1980er Jahren selbst von Kindern noch in der hochdeutschen Umgangssprache verwendet. 
nutzt, die dann ihrerseits noch variiert werden. Als Simplex kommen den Aufnahmen Gundlachs zufolge in Kieve zum Beispiel Pogg und Hücks vor. Letzteres wird andernorts als nicht mehr erkennbares Kompositum in Verbindung mit quad ,böse immer wieder abgewandelt. Als Beispiele seien genannt: Quaducks (Lüttow, Glaisin, Selmsdorf), in Demen lautmalerisch erweitert zu Quaggadux, in Nossentiner Hütte zu Quaddahucks und Quarraducks. Stärker abgewandelt findet es sich als Quollducks in Menkendorf und Kollducks in Bristow, Jördenstorf, Kossebade, Sukow und Tramm bzw. Kollduck und Kullhücks in Jördenstorf sowie Kullhucks in Bansow. Des Weiteren wird das Grundwort mit Padde ,Frosch verbunden, so dass wiederum verschiedene Komposita entstehen: Parraducks (Broock, Satow), Parucks (Broock), Parrahucks (Satow), Pårrahücks (Röbel). Das in Zweedorf belegte Quedütschen setzt sich aus quad und dem nicht als Simplex belegten Ütze zusammen..$^{49}$ Pogg wird ebenfalls in Verbindung mit anderen Wörtern als Kompositum gebraucht: Schottspogg (Jördenstorf), Rietpogg (Bansow, Jördenstorf) und Irdpogg (Bansow).

Die Bedeutung der beiden Grundwörter schwankt, d.h., sie bedeuten nicht überall ausschließlich ,Frosch oder ,Kröte‘. Ersterer heißt in Bansow beispielsweise Kollducks/Kullhucks, letztere Irdpogg bzw. Rietpogg. Auch in Bristow und Jördenstorf verwenden die Sprecher für den Frosch Varianten von Kollducks, die Kröte heißt dagegen Rietpogg, Schottpogg bzw. einfach Pogg. In den südlicheren Orten Röbel und Granzin ist mit dem Simplex Pogg aber der Frosch gemeint, während die Kröte als Kröt oder Pårrahücks bezeichnet wird. Anhand der Belege lässt sich folgende wortgeographische Einteilung gewinnen: Im Nordosten Mecklenburgs nennen die Probanden den Frosch Kollducks (das aber in unterschiedlichen Varianten auftreten kann), die Kröte Pogg, oder sie bilden mit diesem als Grundwort ein Kompositum. Im übrigen Mecklenburg ist Pogg häufig der Name des Frosches, die zahlreichen Komposita mit Hücks, mitunter auch das Simplex, bezeichnen die Kröte. Die Unterscheidung zwischen beiden Tieren ist schon aufgrund der gewissen Ähnlichkeit nicht immer konsequent, ${ }^{50}$ teilweise sind die Sprecher auf den neuen Aufnahmen auch unsicher. ${ }^{51}$

Der Regenwurm wird häufig schon mit dem der Standardsprache nahestehenden Wort Rägenworm bezeichnet, so u. a. in den alten Aufnahmen bereits in Eldena, Alt Jabel, Lancken und durchgängig auf den neuen Aufnahmen aus den

49 Wossidlo 1899, S. 334.

50 Mecklenburgisches Wörterbuch, 4. Bd., Sp. 511, Lemma „Kollducks“.

51 Die jüngere Kossebaderin meint etwa: „Frosch, Frosch is ok Kollducks. Dat is woll, dat is woll beides, äh, wohl Kollducks is wohl un, un de Frosch ook. Ick weit't œwer nich genau." 
Jahren nach 2004. Die mundartlichen Bezeichnungen sind am Beginn der 1960er Jahre allerdings in all diesen Orten noch bekannt gewesen. Gundlach hat das Wort nur im Westen abgefragt, so dass für den Osten und Mecklenburg-Strelitz keine Belege aus den 1960er Jahren vorliegen. Der am weitesten verbreitete dialektale Ausdruck ist in den Aufnahmen Gundlachs noch Maddik, der in Alt Meteln, Boldela, Groß Lantow, Hoben, Pinnow, Warnow und Zahrensdorf als [marrk] artikuliert wird, in den neueren Erhebungen ist er noch zu hören in Kossebade; in Demen und Tramm erscheint er als [marınk]. Diese Ausspache ist auch einmal auf den Gundlach'schen Aufnahmen in Boldela zu hören. ${ }^{52}$ Im Südwesten herrscht 1962/1963 Mett (bzw. als flektierte Form Metten) in fast allen Orten vor, so auch im lauenburgischen Woltersdorf; in Eldena findet sich leicht abgewandeltes Metk. Die Sprecher in Glaisin übersetzen das Tier mit Melger, in Lüblow ist die nicht umgelautete Form Matt üblich. In Spornitz heißt es Måd [mo:]. Die aus der Prignitz stammende Bezeichnung Piermåd erscheint in Marnitz und Prislich an der Südgrenze Mecklenburgs. ${ }^{53}$ All diese Bezeichnungen werden aber, wie oben dargestellt, durch standardnahes Rägenworm verdrängt.

\section{Entwicklungstendenzen im mecklenburgischen Wortschatz}

\subsection{Wortneubildungen}

Ulrich Bentzien hat bereits in den 1960er Jahren den Einfluss der modernen Technik auf den Wortschatz des Mecklenburgischen untersucht und drei Möglichkeiten der Bildung bzw. der Übernahme neuer Wörter aus dem Hochdeutschen ermittelt:

1. „Volkssprachliche Eigenbewältigungen“, 54

2. Verkürzungen, „Verniederdeutschungen“ und Übersetzungen der standardsprachlichen Bezeichnung ${ }^{55}$ sowie

3. einfache Übernahme des „offiziellen“ Begriffs ${ }^{56}$

52 Dabei ist auffällig, dass der Sprecher aus Tramm sowohl bei ,Erpel' (Wäding) als auch bei ,Regenwurm' (Marring) -ing als Endung bevorzugt. In Demen korrelieren offenbar Marring und Pürring ,Fleischmade‘, in Kossebade Wädik, Marrik und Pürrik.

53 Mackel 1905, S. 111 sowie Jacobs 1926/27, S. 146. Die Angaben von Jacobs für Südwestmecklenburg gelten heute wohl nur noch eingeschränkt. Für Tramm gibt er beispielsweise Mak an. Diese Form konnte jedoch nicht mehr nachgewiesen werden.

54 Bentzien 1964, S. 88.

55 Bentzien 1964, S. 97.

56 Bentzien 1964, S. 102.

Birte Arendt, Andreas Bieberstedt and Klaas-Hinrich Ehlers - 978-3-631-71893-3 
Neue Wortschöpfungen, die unabhängig von der Standardsprache gebildet werden, kommen im Niederdeutschen heute nur noch selten vor und sind zumeist humorige oder literarische Schöpfungen wie z. B. Ackersnacker ,Handy', Kiekschapp ,Fernseher' oder Hulbessen ,Staubsauger ${ }^{67}{ }^{57}$ Sie haben nach Gernentz kaum Aussicht, sich dauerhaft im alltäglichen Sprachgebrauch durchzusetzen. ${ }^{58}$ Größere Verbreitung hat lediglich Rucksackbull ,Besamungstechniker' erfahren.

Weitaus gebräuchlicher sind Verkürzungen, Verniederdeutschungen und Übersetzungen der standardsprachlichen Ausdrücke. Die im Folgenden gewählten Beispiele stammen aus den neuen wie alten Aufnahmen. Aufgrund des technischen Fortschritts veralten viele Maschinen und damit auch die dazugehörigen Bezeichnungen sehr schnell, doch sollen auch sie hier erwähnt werden, um die lexikalischen Bildungsweisen zu verdeutlichen.

Oftmals übernehmen die Sprecher einfach die Wörter der Standardsprache, passen sie aber lautlich an die Mundart an. Ein Mittel der phonetischen Adaption ist dabei das Fortlassen des Schwas am Ende des Wortes, z. B. Maschin, EAnlåg, Grenz oder Thermosflasch. Dies ermöglicht eine schnelle Übernahme neuer Wörter ins Niederdeutsche. Daneben werden besonders bei Komposita Teile oder alle Bestandteile ins Niederdeutsche übertragen: Gewerbeschaul,Gewerbeschule, Zentrålschaul ,Zentralschule', Fischgrädenmelkhus ,Fischgrätenmelkstand', Fohrerlaubnis ,Fahrerlaubnis', Fohrradbereifung ,Fahrradbereifung. Teilweise ist nicht $\mathrm{zu}$ entscheiden, ob es sich um eine rein lautliche Verniederdeutschung oder um eine Übersetzung handelt, d.h. ob einfach Laut für Laut in das niederdeutsche Äquivalent umgesetzt wird oder aber die standardsprachlichen Wörter ausschlaggebend sind und es sich damit um eine Wort-für-Wort-Übersetzung handelt. Bentzien führt z. B. Dampkätel als Laut-für-Laut-Übertragung an, aber es könnte sich dabei auch um eine wörtliche Übersetzung handeln, ${ }^{59}$ da die Formative in diesem Fall in Mundart und Hochsprache übereinstimmen.

Schaut man sich jedoch die Neubildungen an, dann scheinen die Sprecher zumeist die Wortbestandteile in die Mundart zu übertragen, d.h., sie wenden keine phonologischen Strategien an, sondern lexikalische: Fohrrad [forrat], ${ }^{60}$ Utpuff

57 Vgl. Gundlach 1984, S. 36 und Niebaum 2004, S. 174.

58 Gernentz 1980, S. 144.

59 Bentzien 1964, S. 98.

60 Bentzien meint, es heiße „Fahrrad oder Rad“ (Bentzien 1964, S. 105), doch ist auf den Aufnahmen die oben angegebene niederdeutsche Variante zu hören. Sie wird auch in Demen und Kossebade ausschließlich verwendet, wenngleich die gekürzte Variante Rad häufiger ist. Diese wird aber wie im Mecklenburgischen üblich mit kurzem /a/ realisiert. Der Plural lautet dementsprechend Fohrrohr bzw. Roehr. 
,Auspuff', Fautball ,Fußball'. Deutlich wird das an der Bezeichnung für den Mähdrescher, bei der drei Varianten zu hören sind: das standardsprachliche und somit unveränderte Wort Mähdrescher, das halbniederdeutsche Meihdrescher sowie das seltener vorkommende Meihdöscher. ${ }^{61}$ Die unterschiedlichen Bezeichnungen resultieren in dem Fall daraus, wie konsequent der Sprecher das Kompositum übersetzt hat. Dabei zeigt sich an den unterschiedlichen Stufen, dass Wort für Wort vorgegangen wird, wobei hochsprachliche Bestandteile entweder einfach integriert oder übersetzt werden. Deutlich wird das auch an Fernsehkieken, das Fernsehprogramm schauen' und Fernsehkieker ,Fernsehzuschauer. ${ }^{6} 2$ Diese Strategie ist deshalb nur eindeutig erkennbar, wenn Bestandteile des Kompositums vom standardsprachlichen Formativ abweichen, z. B. Käuhlschapp ,Kühlschrank und Trüggslag,Rückstoß beim Gewehr. Beispiele für solche Wortbildungen sind: Borgeld ,Bargeld', Fohrliehrer, Fahrlehrer', Kinnergorden ,Kindergarten', Schlagtüg ,Schlagzeug' und Stoppstråt ,Stoppstraße'. Dabei treten gelegentlich auch Wortneuschöpfungen auf: Ein Niendorfer berichtet z. B. über die Vorzüge tschechischer Motorräder: „Se warden ja nich mehr handperrt“ (,sie werden nicht mehr durch das Treten des Motors gestartet').

Häufig übernehmen die Sprecher aber auch einfach das standardsprachliche Wort unverändert in Niederdeutsche, z. B. Anlasser, Anreiz, Fernsehen, Geigerzähler, Gerichtsvollzieher, Hydraulik, Schwermaschinenbau, Staubsauger, Tagung, Wirkungsbereich. Das trifft auch für die schon in der Standardvarietät einfach aus dem Englischen übernommenen Wörter wie Computer zu. ${ }^{63}$

\subsection{Einfluss der hochdeutschen Standardvarietät auf den mecklenburgischen Wortschatz}

Bereits bei der Untersuchung der regionalen Unterschiede im Wortschatz fiel auf, dass hier nicht nur ein regionaler Ausgleich stattfindet, sondern dass dieser gerade auch durch das Einwirken der Standardvarietät forciert wird. Regionalspezifische Lexeme des Dialekts werden dabei zunehmend durch standardnahe oder stan-

61 So auch Bentzien 1964, S. 104.

62 Das Wort Fernsehen, das nur auf den neueren Aufnahmen belegt ist, wird von meinen Probanden grundsätzlich in hochdeutscher Lautform artikuliert. Vgl. dazu Ehlers (i. Vorber.): In einem Übersetzungstest mit 47 niederdeutschkompetenten Personen aus der Vorkriegs- und der Nachkriegsgeneration übersetzten im Raum Rostock das Wort fernsehen 74,5 \% völlig standardidentisch bzw. mit leicht regiolektaler Färbung (fäänsehn) ins Niederdeutsche, lautliche bzw. lexikalische Adaptionen ans Niederdeutsche wie feernsekiken, feernsain, firnkiken, firnsain blieben seltene Ausnahmen.

63 Alle Wortbeispiele stammen aus meinen Aufnahmen im Untersuchungsgebiet. 
dardidentische Wörter ersetzt, die überregional in Gebrauch sind. Begünstigt wird dieser Prozess durch den immer häufigeren Gebrauch der hochdeutschen Umgangssprache, den die Sprecher z. T. selbst herbeigeführt haben, indem sie mit ihren Kindern nicht mehr Niederdeutsch sprachen, damit diese es leichter in der Schule hätten. Der bereits angesprochene wissenschaftlich-technologische Fortschritt wirkte ebenfalls stark auf den mecklenburgischen Wortschatz ein. Dabei wurden neue hochdeutsche Wörter und Termini vielfach in den Dialekt übernommen.

Inwieweit die Fluchtbewegung nach dem Zweiten Weltkrieg nachhaltige Wirkung auf den regionalen Wortschatz zeigt, lässt sich nur schwer einschätzen, da hier viele Faktoren eine Rolle spielen. So lernte z. B. der Ehemann meiner jüngeren Probandin aus Kossebade das Mecklenburgische und sprach es auch innerhalb der Familie; der zugewanderte Ehemann der älteren Probandin aus Kossebade verblieb jedoch beim Hochdeutschen, obwohl beide Männer vom Alter her sehr jung waren, als sie nach Mecklenburg kamen. Auf die Dialektkompetenz beider Sprecherinnen hatte das Sprachverhalten der Ehepartner offenbar keinen Einfluss, da diese sich hierin nicht unterscheiden. Die Entscheidung, nicht mehr Niederdeutsch mit den Kindern zu sprechen ${ }^{64}$ scheint jedenfalls einschneidender für den Rückgang des mecklenburgischen Dialektgebrauchs gewesen zu sein als das Sprachverhalten der Flüchtlinge und Vertriebenen, zumal diejenigen Zuwanderer, die das Niederdeutsche erlernt hatten, die Sprecheranzahl des Dialekts sogar vergrößert haben. ${ }^{65}$

64 Paul Beckmann konstatiert z. B. für die Zeit kurz nach dem Kriegsende zunächst einen starken Rückgang des Niederdeutschen und vermerkt, dass es in der Landwirtschaft nicht mehr allein bestimmend gewesen sei, aber seit der Mitte der 1950er Jahre wieder die führende Rolle in denjenigen Dörfern und Produktionsgemeinschaften spiele, in denen Flüchtlinge aus Ostpommern stammten. Beckmann 1954/55, S. 132-133. In Möllin meint eine Sprecherin Anfang der 1960er Jahre, dass dort durchschnittlich mehr Hochdeutsch gesprochen werde, auch in der LPG. Als Grund dafür gibt sie an: „Weil de vä, meisten un väle ok nich mehr von hier sünd. Ostpreußen un wo se denn all her sünd, nich, Pommern un..." Die Erklärung für den Rückgang ist jedoch nicht so einfach, denn auf die Kinder angesprochen, erklärt sie, dass diese auch bereits mehrheitlich Hochdeutsch sprächen. Das schließt ihre eigene Tochter mit ein: „Mien Dochder? Jå, de kann œwerhaupt nich richtig Platt. De versteht uns je noch nich ma richtig. Wenn wi richtig Platt spräken, denn versteht se manches nich.“ Zwar begründet sie das damit, dass ihre Tochter mehrheitlich mit „Hochdeutschen“ aufwachse, die auch in ihrem Haus lebten, doch zeigt sich hier eben auch die Einstellung, dass mit den Kindern Hochdeutsch geredet wird und nicht Niederdeutsch, denn nur so ließe sich erklären, warum die Tochter laut Sprecherin selbst das zum Basiswortschatz gehörige Wort Schapp ,Schrank' nicht mehr versteht.

65 Vgl. Ehlers 2013, S. 106-108.

Birte Arendt, Andreas Bieberstedt and Klaas-Hinrich Ehlers - 978-3-631-71893-3 
Die alten wie die neuen Aufnahmen zeigen, dass jüngere Sprecher tendenziell der Standardsprache näherstehende Formative bevorzugen. Davon zeugt der Rückgang regionalspezifischer Mundartausdrücke, die zum Teil sogar direkt durch die standarddeutschen Wörter verdrängt werden. Nur die älteste Generation hat auf den alten Aufnahmen noch vermehrt die für ihre Region typischen niederdeutschen Bezeichnungen verwendet. Die skizzierte Entwicklung hängt zum einen damit zusammen, dass die hochdeutschen Wörter ein höheres Prestige besitzen, sehr deutlich z. B. bei Ameise, zum anderen lässt sich in einer Sprachumgebung, in der der Sprecher zwischen mehreren Varietäten umschalten muss, der Wechsel einfacher realisieren. Es kommt zur hochdeutsch-niederdeutschen "Diasystematisierung" auf der Ebene des Wortschatzes. ${ }^{66}$ Deutlich wird das an den Numeralien, die häufig hochdeutsch ausgesprochen werden, besonders wenn der Sprecher zwischen Mundart und Hochdeutsch im Gespräch wechseln muss. Nur in einem längeren auf Niederdeutsch gehaltenen Gespräch erfolgt dann der dauerhafte Wechsel auf die mundartlichen Bezeichnungen. Zwar beeinflusst der Dialekt auch die hochdeutsche Umgangssprache, ${ }^{67}$ doch wirken sowohl Standardvarietät als auch die regionale Umgangssprache stärker auf die Mundart ein. Daher werden zunehmend mundartliche durch hochsprachliche Wörter verdrängt bzw. stehen neben diesen („lexikalische Parallelschaltung ${ }^{“ 68}$ ), z. B. heizen statt bäuten, zeigen statt wiesen, Schrank statt Schapp. Diese Entwicklung ist jedoch von Ort zu Ort und Sprecher zu Sprecher verschieden. So wird in Demen heute bereits stricken gesagt, im etwa 20 Kilometer entfernten Kossebade ist dafür noch knütten gebräuchlich. Im Großteil Mecklenburgs wird die Lerche heute bereits standardnahe als Lerch bezeichnet, in Menkendorf herrscht noch Leink vor. ${ }^{69}$ In Mecklenburg-Strelitz sagen die Sprecher dreißig, vierzig, fuffzig,

66 Höder 2011.

$67 \mathrm{Zu}$ nennen wären hier z. B. Wörter wie Wrucke,Steckrübe', perren, treten, ausverschämt (gebildet aus utverschamt), unverschämt', die in der mecklenburgische standardnahe Umgangssprache verwendet werden, oder die Übernahme des Diminutivsuffix -ing, vgl. dazu Ehlers 2011, besonders S. 85-91. Besonders häufig höre ich die Verabschiedung Tschüssing [ $\left.\mathrm{t} \int \mathrm{y}: \mathrm{sin}\right]$, aber auch den Ausruf Ach Gotting! Mitunter verwenden Hochdeutschsprecher - hier aber besonders diejenigen der Generation der um 1950 Geborenen - das Suffix -sch(e), um weibliche Personen zu kennzeichnen, z. B. die Doktorsche.

68 Menke 1998, S. 180.

69 Jacobs gibt Mitte der 1920er Jahre nur noch ein recht kleines Gebiet um Dütschow, Spornitz, Karrenzin, Möllenbeck, Stolpe und Brenz an, in dem „Leirk“/„Leink“ gesagt werde, sonst gelte hochdeutsches „le·zx“ (Jacobs 1926/27, S. 151). Menkendorf liegt allerdings außerhalb dieses Gebietes. 
während die Einerstelle der Zahl weiterhin niederdeutsch bleibt, z. B. fiefundreißig. Das ehemals nur vorpommersche Reflexivpronomen sik wird im Osten Mecklenburgs durch das hochdeutsche sich verdrängt. ${ }^{70}$ In Mecklenburg-Strelitz gilt letzteres ausschließlich, in Brudersdorf, Carolinenhof, Granzin und Kölzow dominiert es auf den Aufnahmen aus den 1960er Jahren bereits. Für das Erntefest wird ebenfalls vielfach schon das standardsprachliche Wort verwendet, das gilt auch für das Simplex Ernte, das teilweise lautlich an die Mundart angepasst wird..$^{71}$

\section{Fazit}

Die Untersuchungen haben gezeigt, dass die regionalen Unterschiede im niederdeutschen Wortschatz Mecklenburgs zwar noch greifbar sind, aber zusehends verschwinden. Dieser Prozess wurde beim Vergleich der Wortschatzerhebungen Wossidlos und Teucherts zum Mecklenburgischen Wörterbuch mit den Aufnahmen der 1960er Jahre und der Jahre nach 2004 bzw. besonders auch im Vergleich der Sprechergenerationen an den Untersuchungsorten immer wieder erkennbar. Vielfach werden die regionalspezifischen Wörter durch die hochdeutsche Bezeichnung ersetzt (Frosch, Kröt, Ameisen) oder zumindest eine der Standardsprache lautlich relativ nahestehende Variante gewählt ( $\ddot{w} w e r$, Rägenworm). Diese lexikalische Annäherung des mecklenburgischen Niederdeutsch an die überregionale Standardvarietät führt zu einer fortschreitenden Entregionalisierung des mecklenburgischen Wortschatzes, sodass auch wortgeographische Grenzen innerhalb des Mecklenburgisch-Vorpommerschen zunehmend verblassen.

Da einige der Wortbelege in Gundlachs und in meiner Erhebung mittels hochdeutscher Sätze abgefragt wurden, kann nicht immer zweifelsfrei geklärt werden, inwieweit die Vorlage die Probanden beeinflusst hat. Hierbei sind zwei Richtungen der Beeinflussung durch die Übersetzungsvorlage möglich: In Demen übersetzt ein Sprecher z. B. ,Regenwurm' mit Rägenworm. Auf die Form Maddik angesprochen, meint dessen Frau, dass er dieses Wort im täglichen Gespräch noch verwende. Hier könnte der Proband also - gegen seine alltägliche Sprachgewohnheit - im Test das der hochdeutschen Textvorlage näherstehende Lexem verwendet haben. Andererseits verlangt ein Übersetzungstest generell den bewussten Umgang mit der eigenen Sprache, so dass zumindest einige Probanden Formen

70 Vgl. die auf den Sprachatlas Wenkers zurückgehende Karte zu sik/sich in Foerste 1954, Sp. 2015-2052.

71 So z. B. in Schönbeck (Mecklenburg-Strelitz), wo ein Sprecher das /e/ zu Irnt erhöht. 
wählen, die „plattdeutscher“ (= standardferner) klingen, d.h., Ausdrücke wählen, die sie in der täglichen niederdeutschen Konversation selten oder gar nicht mehr verwenden. Ungeachtet dieser Problematik der Übersetzungstests lässt sich die oben dargestellte Entwicklungstendenz auch in den freien Gesprächen immer wieder feststellen.

Bei der Übernahme neuer Begrifflichkeiten für neue technische und gesellschaftliche Entwicklungen ist der Einfluss der Standardsprache ohnehin unverkennbar. Selbst die hierfür neu geschaffenen niederdeutschen Wörter basieren in der Regel als Lehnübersetzungen auf den hochdeutschen Vorbildern. Durch den häufigen Gebrauch der hochdeutschen Umgangssprache fällt es so auch leicht, standardsprachliche Wörter einfach mit lediglich kleinen Anpassungen in den Wortschatz zu übernehmen. Bentzien nennt als Grund für die Adaption bzw. Übernahme hochdeutscher technischer Wörter neben der kurzen zeitlichen Spanne gegenüber der Entstehung bzw. Entwicklung der technischen Neuerung die „Kompliziertheit der betreffenden Sache“. ${ }^{72}$ Schaut man sich jedoch den Aufbau älterer z. B. landwirtschaftlicher Gerätschaften und Fahrzeuge an, so zeigt sich auch darin eine große technische Komplexität. Größere Komplexität neuere technischer Geräte hat wohl kaum den Ausschlag dafür gegeben, warum z. B. die Teile des Motors heute ausschließlich, die des Autos zumindest überwiegend hochdeutsch erscheinen. ${ }^{73}$ Viel wichtiger scheinen die zeitliche Spanne, die Vielzahl neuer Wörter sowie der Einfluss der Standardvarietät und der hochdeutschen Umgangssprache zu sein. Teile technischer Geräte, für die es aus anderen Sachgebieten bereits niederdeutsche Entsprechungen gibt, werden einfach mit diesen benannt, z. B. Dör ,Tür' und Finster ,Fenster ' beim Auto, teilweise werden einige Wörter übersetzt, wenn sie häufig vorkommen, wie z. B. Kufferruum ,Kofferraum oder Utpuff,Auspuff‘. Technische Fachbegriffe, die sonst nicht so häufig verwendet werden, bleiben dagegen hochdeutsch, so z. B. der Kühler. Hier spielt weniger die Komplexität des Bezeichneten eine Rolle als die Häufigkeit der Wortverwendung. Außerdem besteht oftmals für die Niederdeutschsprecher gar kein Zwang, die Wörter zu übersetzen, da sie verstanden werden und zudem den Wechsel in eine andere Varietät erleichtern. Ähnliche Beobachtungen lassen sich heute auch für die Standardsprache machen, in der Fachbegriffe aus dem Englischen einfach übernommen werden.

72 Bentzien 1964, S. 105.

73 Bentzien 1964, S. 104.

Birte Arendt, Andreas Bieberstedt and Klaas-Hinrich Ehlers - 978-3-631-71893-3 


\section{Literatur}

Beckmann, Paul: Die Weiterentwicklung der Mecklenburger Mundart nach dem Tode Fritz Reuters. In: Wissenschaftliche Zeitschrift der Universität Rostock. Gesellschafts- und sprachwissenschaftliche Reihe 4 (1954/55), S. 129-133.

Bentzien, Ulrich: Wörter der modernen Technik in der mecklenburgischen Mundart. In: Niederdeutsches Jahrbuch 87 (1964), S. 87-106.

Blume, Rudolf: Wortgeographie des Landes Stargard. In: Teuthonista 9 (1933), S. 1-33, S. 65-89, S. 129-143, S. 193-207.

Buddin, Fr[itz]: Der mecklenburgische Sprachraum. Von Hermann Teuchert. In: Mitteilungen des Heimatbundes für das Fürstentum Ratzeburg 11, Nr. 3 (1929), S. 45.

Chytraeus, Nathan: Nomenclator latinosaxonicus. Mit einem Vorwort von Gilbert de Smet. Hildesheim, New York 1974. (Nachdruck der Ausgabe Rostock 1582)

Ehlers, Klaas-Hinrich: „Dürfen wir essing?“ Beobachtungen zur Übernahme des mecklenburgischen -ing-Diminutivs in das regionale Hochdeutsch. In: Niederdeutsches Jahrbuch 134 (2011), S. 79-92.

Ehlers, Klaas-Hinrich: Führte die Immigration der Heimatvertriebenen nach $1945 \mathrm{zu}$ Dialektverlust und Nivellierung regionalsprachlicher Differenzen? Beobachtungen aus einer Untersuchungsregion in Mecklenburg. In: Niederdeutsches Jahrbuch 136 (2013), S. 97-116.

Ehlers, Klaas-Hinrich: Geschichte der mecklenburgischen Regionalsprache seit dem Zweiten Weltkrieg. Varietätenkontakte zwischen Alteingesessenen und zugewanderten Vertriebenen. (in Vorbereitung)

Foerste, William: Die Geschichte der niederdeutschen Mundarten. In: Stammler, Wolfgang (Hrsg.): Deutsche Philologie im Aufriß. 1. Bd., 2., überarb. Aufl. Berlin 1957, Sp. 1729-1898.

Frehse, Fr[iedrich]: Wörterbuch zu Fritz Reuter's sämmtlichen Werken. Ludwigslust/ Rostock/Wismar 1867.

Gundlach, Jürgen: Plattdeutsch in Mecklenburg heute. Bericht über die Tonbandaufnahmen der mecklenburgischen Mundart 1962/63. In: Rostocker Beiträge. Regionalgeschichtliches Jahrbuch der mecklenburgischen Seestädte 1 (1966), S. $173-194$.

Gundlach, Jürgen: Von Aant bis Zäg'. Plattdeutsche Wörter und Wendungen. 2. Aufl. Leipzig 1984.

Gernentz, Hans Joachim: Niederdeutsch - gestern und heute. Beiträge zur Sprachsituation in den Nordbezirken der Deutschen Demokratischen Republik in Geschichte und Gegenwart. Rostock 1980. 
Herrmann-Winter: Sprachatlas für Rügen und die vorpommersche Küste. Kartographie Martin Hansen. Rostock 2013.

Höder, Steffen: Niederdeutsch und Hochdeutsch - ein Fall von Diasystematisierung. In: Niederdeutsches Jahrbuch 134 (2011), S. 113-136.

Jacobs, Hugo: Dialektgeographie Südmecklenburgs zwischen Lübz und Hagenow. In: Teuthonista $2(1925 / 26)$, S. 46-55; S. 107-133 sowie Teuthonista 3 (1926/27), S. 119-152; S. 241-262.

Köhncke, André: Mecklenburgisch heute - Bestandsaufnahme eines niederdeutschen Dialekts. Rostock 2010. [Online-Ressource: urn:nbn:de:gbv:28diss2011-0137-3 (Stand: 24.11.2016)]

Mackel, E[mil]: Die Mundart der Prignitz. In: Jahrbuch des Vereins für niederdeutsche Sprachforschung 31 (1905), S. 65-164.

Mantzel, Ernst Johann Friedrich: Dissertatio, continens, idiotici, Mecklenbvrgensis, ivridico-pragmatici, specimen primum [...], defendit, Hans Carl Larson. Rostock 1757.

Mantzel, Ernst Johann Friedrich: Bützowsche Ruhestunden, gesucht, in Mecklenburgschen, vielentheils, bisher noch ungedruckten zur Geschichte und Rechtsgelahrtheit vornehmlich gehörigen Sachen. 1. Teil. Bützow 1761.

Mecklenburgisches Wörterbuch. Hrsg. von der Sächsischen Akademie der Wissenschaften zu Leipzig. Aus den Sammlungen Richard Wossidlos und aus den Ergänzungen und nach der Anlage Hermann Teucherts. 7 Bde. Berlin 1942-1992. Unveränd., verkleinerter Nachdruck der Erstauflage von 1937-1992. Neumünster 1996.

Menke, Hubertus: Niederdeutsch: Eigenständige Sprache oder Varietät einer Sprache? In: Hartel, Nina/Meurer, Barbara/Schmitsdorf, Eva (Hrsg.): Lingua Germanica. Studien zur deutschen Philologie. Jochen Splett zum 60. Geburtstag. Berlin [u. a.] 1998, S. 171-184.

Mi [= Sibeth, Friedrich Georg]: Wörterbuch der Mecklenburgisch-Vorpommerschen Mundart. Leipzig 1876.

Müller, Carl Friedrich: Reuter-Lexikon. Der plattdeutsche Sprachschatz in Fritz Reuters Schriften. Leipzig [1904].

Niebaum, Hermann: Zur Lexik und Lexikographie des Niederdeutschen. In: Stellmacher, Dieter (Hrsg.): Niederdeutsche Sprache und Literatur der Gegenwart. Hildesheim/Zürich/New York, S. 149-189.

Peters, Robert: Die Lemgoer Drucke des 'Nomenclator latinosaxonicus': eine Quelle für den ostwestfälischen Wortschatz des späten 16. Jahrhunderts? Gilbert de Smet (1921-2003) zum Andenken. In: Niederdeutsches Jahrbuch 137 (2014), S. 81-104. 
Raupach, Bernhardus: Exercitationem Academicam, De Lingvæ Saxoniæ Inferioris Neglectu atq[ue] Contemtu Injusto, Von Unbilliger Verachtung Der PlatTeutschen Sprache [...]. Rostock 1704.

Reuter, Fritz: Sämmtliche Werke. Volks-Ausgabe in 7 Bänden. Ludwigslust/Rostock/Wismar 1877/1878.

Stellmacher, Dieter: Niederdeutsche Sprache. Eine Einführung (Germanistische Lehrbuchsammlung; 26). 2., überarb. Aufl. Berlin 2000.

Teuchert, Hermann: Der mecklenburgische Sprachraum. Rostock [1929] (Sonderdruck aus dem Jahresbericht der Mecklenburgischen Landes-UniversitätsGesellschaft).

Teuchert, Hermann: Der Lautstand im Südteil des Landes Stargard. In: Teuthonista 10 (1934), S. 2-34.

Teuchert, Hermann: Einleitung zum 1. Bande. In: Mecklenburgisches Wörterbuch. Hrsg. von der Sächsischen Akademie der Wissenschaften zu Leipzig. Aus den Sammlungen Richard Wossidlos und aus den Ergänzungen und nach der Anlage Hermann Teucherts. 1. Bd. Neumünster 1942, S. XII-XV.

Teuchert, Hermann: Beiträge zur Geschichte der mecklenburgischen Mundart. In: Niederdeutsches Jahrbuch 82 (1959), S. 207-236.

Wossidlo, Richard: Mecklenburgische Volksüberlieferungen. Im Auftrage des Vereins für mecklenburgische Geschichte und Altertumskunde gesammelt und herausgegeben. 2. Bd.: Die Tiere im Munde des Volkes. Erster Teil. Wismar 1899. 
Birte Arendt, Andreas Bieberstedt and Klaas-Hinrich Ehlers - 978-3-631-71893-3

Downloaded from PubFactory at 01/11/2019 10:11:06AM

via free access 


\title{
Klaas-Hinrich Ehlers
}

\section{Meihen, meiden, meigen , mähen' - Zur Hiattilgung im mecklenburgischen Niederdeutsch}

\begin{abstract}
The different forms of hiatus-elimination are considered to be one of the few phonetic features that distinguish Mecklenburg and Western Pomeranian Low German. However, recent surveys show that this characteristic feature, which is often used to discriminate different regional varieties of the Mecklenburg dialect, has nowadays lost much of its importance. The article presents results of a recent study that compares language data in form of dialect translations from the $19^{\text {th }}$ century with new translations made by dialect speakers from two different generations ('Wenkersätze'). It illuminates the diachronic development of this dialect feature and thus exemplifies both a structural approximation of the Mecklenburg Low German dialect to the High German standard language as well as an equalization of specific regional dialect features.
\end{abstract}

\section{Forschungsstand und Problemstellung}

In der Grammatik der „Mecklenburgisch-Vorpommerschen Mundart“ von Julius Wiggers hieß es 1857: „Wenn von zwei Silben die erste auf einen langen Vocal auslautet, die zweite mit kurzem oder stillen e beginnt, so tritt gewöhnlich zwischen beide ein die Stelle eines Hauchlautes (h, ch, w) vertretendes g. " 1 Anstelle eines Frikativs in buhen oder buwen ,bauen' tritt nach Wiggers also „das vocaltrennende g“ auf. Nur zwölf Jahre später wies die mecklenburgische Grammatik von Karl Nerger auf eine weitere Form des Konsonanteneinschubs im Vokal-Hiat (= Hiattilgung) hin, die ausdrücklich als eine neue Entwicklung betrachtet wurde: „In jüngster Zeit wird neben g ein schwaches d häufig als Vertreter der Fricativen gebraucht, z. B. teiden decem, neiden suere u. a. m. “2

1 Wiggers 1857, S. 5.

2 Nerger 1869, S. 151. Teuchert (1959, S. 226) bestätigt „das verschiedene Alter dieser beiden Übergangslaute" und führt sie auf Lautentwicklungen im Wortkontext zurück: „Es dürfte sich - $g$ - als ein hiattilgender Laut zwischen Vokalen erklären, das gälte auch vor der Endung - en, dagegen wäre - $d$ - erst nötig geworden, nachdem der Vokal der Endung -en geschwunden war. Der Erstbeleg bett Teiden ,bis zehn Uhr' stammt [...] aus dem Jahr 1790 [...] aber teigen ist schon 1734 [...] belegt. “ Über den (historischen) Ursprung der Hiattilgung sind sich Nerger und Wiggers uneins; während Wiggers den Ursprung 
Die noch einmal zehn Jahre später erfolgende Fragebogenerhebung Georg Wenkers in Norddeutschland (1879-1880) ergab für die Region MecklenburgVorpommern eine recht deutliche areale Begrenzung des Merkmals. Dies lässt sich beispielsweise an der Wortschatzkarte für nähen des „Sprachatlas des Deutschen Reiches" von Georg Wenker ablesen. ${ }^{3}$ Im Westen Mecklenburgs wird als Entsprechung für nähen die niederdeutsche Leitform neid-en verzeichnet, bei welcher ein $d$ im Vokalhiat auftritt. Für Südostmecklenburg und Vorpommern verzeichnet der Sprachatlas dagegen eine Leitform mit $g$ (neig-en). Die eingezeichnete Isoglosse für die jeweilige Leitform ergibt also eine west-östliche Zweiteilung Mecklenburg-Vorpommerns entlang einer stark mäandernden Grenze vom Darß im Norden zum Gebiet östlich des Müritzsees im Süden. Diese setzt sich auch ins östlich benachbarte mittelpommersche Dialektgebiet fort (dort ohne Diphthongierung: näg-en), während im äußersten Südwesten Mecklenburgs und den angrenzenden Dialektregionen Holstein und Brandenburg keine Hiattilgung kartiert wird.

Schon Teuchert sieht in der unterschiedlichen Realisierung der Hiattilgung eines der wenigen lautlichen Merkmale, die die ansonsten sprachlich sehr homogene Großregion Mecklenburg-Vorpommern in einen mecklenburgischen und einen vorpommerschen Bereich teilen. ${ }^{4}$ Sein Mecklenburgisches Wörterbuch weist allerdings für eine Reihe von Lexemen mit Vokal-Hiat drei Realisierungsformen nach, z. B. „meihen, meiden, meigen“ ,mähen', „schrien (lautl. šrīon), schriden,

des „vocaltrennenden g“ eher in kontaktphonetische Bedingungen legt, führt Nerger die Hiattilgung bis auf wenige Ausnahmefälle ,auf älterer [sic] Fricativa“ zurück (Nerger 1869, S. 151 und 138-139). Auf die frühe Sprachgeschichte der Hiattilgung und ihren allgemein angenommenen Import über Siedler aus Westfalen in das ostniederdeutsche Kolonialgebiet soll hier nicht eingegangen werden, vgl. dazu Ehlers im Druck. Ich beschränke die Betrachtung hier nur auf die mecklenburgischen Verhältnisse seit 1880.

3 Vgl. Digitaler Wenker-Atlas: www.regionalsprache.de/SprachGiS/Map/aspx, Lautkarte/Wortschatzkarte WA 260 (Stand: 11.12.2016). Der Rostocker Studienrat Paul Beckmann (1954/55, S. 131-132) datiert die Ausbreitung der Hiattilgung auf $d$ in meinem Untersuchungsgebiet aus eigener Anschauung deutlich später. Demnach habe sich „,vom nördlichen Westen her [...] etwa seit 1890 der Gleitlaut d durchzusetzen“ begonnen, aber bis 1920 Rostock kaum erreicht und erst nach dem Zweiten Weltkrieg dort „vor allem in der jungen Generation“ das „Übergewicht“ über den $g$-Klusil gewonnen. Bemerkenswert bleibt an diesen subjektiven Einschätzungen, dass ältere Sprachteilnehmer aus der Großstadt Rostock die Hiattilgung auf $d$ offenbar noch in den 1920er Jahren als ,neue und ,von außen kommende` Entwicklung empfunden haben.

4 Teuchert 1942, S. IX. 
schrigen“,schreien'. Neben den beiden Varianten mit konsonantischer Hiattilgung verzeichnet das Mecklenburgische Wörterbuch also auch Formen, bei denen der Vokal-Hiat gar nicht getrennt ist. Dabei kennzeichnen die Wörterbuchartikel zu einzelnen Lemmata den Einschubkonsonanten $g$ als östlich, eingeschobenes $d$ dagegen als westlich. Die fehlende Hiattilgung wird offenbar nicht eindeutig areal begrenzt: „schneien; neben snien gilt snigen im Osten, sniden im Westen“. ${ }^{\text {" }}$

Auch die spätere Fachliteratur nutzt im Gefolge von Teuchert und Foerste ${ }^{6}$ die unterschiedlichen Realisierungsformen des Vokal-Hiats mit großer Übereinstimmung als ein Leitmerkmal für die interne areale Gliederung des ostniederdeutschen Dialekts. Nach Stellmacher „unterscheidet sich das Vp. [Vorpommersche] vom M. [Mecklenburgischen] durch die Bewahrung eines im Hiat entwickelten Klusils: m. mei(d)en wird von vp. meigen durch eine Linie getrennt, die vom Darß über Malchin Stavenhagen bis Penzlin verläuft".7 Eine beigegebene Karte veranschaulicht diese Isoglosse zwischen mei(d)en und meigen mit dem ausdrücklichen Zeitbezug „Stand 1979“. 8 Auch Gernentz, Schönfeld und Schröder führen die Hiattilgung auf $g$ und auf $d$ als regionale Kennzeichen an, anhand derer der vorpommersche vom mecklenburgischen Dialektraum abzugrenzen sei. ${ }^{9}$ Gundlach kommt unter Einbeziehung der Formen ohne Hiattilgung zu einer Dreigliederung der Region. Demnach

„steht mecklenburgisches sniden (,schneien') vorpommerschem schnigen gegenüber, wobei die vorpommersche Form auch noch im Osten und Südosten Mecklenburgs einschließlich Stavenhagen gilt. [...] Der äußerste Südwesten des Landes hat keinen Hiateinschub. Es staffelt sich also von Südwesten nach Osten meien/meiden/meigen. "10

Die grundlegende West-Ost-Gliederung der Dialektlandschaft wäre aber auch nach Gundlach nur im „,äußersten Südwesten“ feiner auszudifferenzieren.

5 Mecklenburgisches Wörterbuch 6 (1976), S. 466, vgl. etwa zu Krähen (Plur.): „die westl. Form Kreiden reicht bis in die Rostocker Heide" (Mecklenburgisches Wörterbuch 4 (1965), S. 648).

6 Foerste 1954, Sp. 2042.

7 Stellmacher 1980, S. 466.

8 Stellmacher 1980, S. 465. Die ausdrücklich für den „Stand: 1979“ gezeichnete Isoglosse der Hiatrealisierung bei Stellmacher unterscheidet sich in ihrem Verlauf allerdings kaum von der maigen/maiden-Isoglosse, die schon Foerste auf der Basis des Sprachatlas kartiert (vgl. Foerste 1954, Sp. 2039-2040). Teepe bringt später eine Karte zur Realisierung des Hiats in den niederdeutschen Entsprechungen für neue, auf welcher bei ganz ähnlichem Isoglossenverlauf die getilgte Form nig- in Vorpommern einer mecklenburgischen Form nī- gegenübersteht (Teepe 1983, Kartenanhang S. VIII).

9 Gernentz 1980, S. 89; Schönfeld 1990, S. 98; Schröder 2004, S. 50.

10 Gundlach 1988, S. 427. 
Diese scheinbar so klare räumliche Verteilung des phonetischen Leitmerkmals finde ich allerdings bei aktuellen Sprachaufnahmen in meinem zentralmecklenburgischen Untersuchungsgebiet südwestlich von Rostock kaum bestätigt. Statt der erwartbaren Dominanz der Hiattilgung auf $d$ stehen hier Tilgungsvarianten auf $d$ und auf $g$ in Konkurrenz nebeneinander, es finden sich aber auch sehr zahlreiche Belege ohne getilgten Vokal-Hiat. Ein Vergleich von niederdeutschen Aufnahmen von Probanden verschiedener Altersgruppen deutet zudem auf eine starke Abbaudynamik des Merkmals hin. Ich möchte in meinem Beitrag deshalb die verbreitete Annahme einer klaren arealen Verteilung des Merkmals im Mecklenburgisch-Vorpommerschen einmal empirisch überprüfen und versuchen, aus der Zusammenführung verschiedener Sprachdaten aus meiner Erhebungsregion ein Bild von der Entwicklung und vom gegenwärtigen Stand der arealen Verbreitung der Hiattilgung zu gewinnen.

Empirische Grundlage meiner Beobachtungen sind vor allem Sprachtests, die ich zwischen 2010 und 2013 mit Unterstützung der Deutschen Forschungsgemeinschaft im Erhebungsgebiet durchgeführt und aufgezeichnet habe. ${ }^{11} \mathrm{Im}$ Fokus der Untersuchung stehen die Großstadt Rostock, die etwa 25 Kilometer südlich davon gelegene Kleinstadt Schwaan, die westlich dieser beiden Orte gelegenen Dörfer Satow, Jürgenshagen und Ostseebad Nienhagen sowie unmittelbar benachbarte kleine Ortschaften. Im Zentrum der Überlegungen zur Hiattilgung sollen vor allem die niederdeutschen Übersetzungen der 40 klassischen sogenannten ,Wenkersätze stehen, die Georg Wenker bei seiner großangelegten Fragebogenerhebung um 1880 als Übersetzungsvorlagen zur Übertragung in die jeweiligen Ortsdialekt verschickt hatte. Diese Wenkersätze werden in meiner Untersuchung um fünf weitere hochdeutsche Sätze zur Elizitierung speziell mecklenburgischer Merkmale ergänzt. Neben 25 historischen Wenkerbögen aus der Untersuchungsregion wurden insgesamt 53 Wenkerübersetzungen von Gewährsleuten mit guter und sehr guter Dialektkompetenz ausgewertet. ${ }^{12}$ Diese Probanden gruppieren sich in zwei Alterskohorten: in die vor 1940 geborenen Personen

11 Im Rahmen meines DFG-Projekts „Kontaktlinguistische Untersuchungen zur sprachliche Akkulturation Heimatvertriebener in Mecklenburg" habe ich im genannten Zeitraum mit 86 Zeitzeugen (sprach)biografische Interviews und verschiedene Sprachtests durchgeführt, die später um weitere vier Interviews ergänzt wurden.

12 Um die schwierige Beurteilung der Niederdeutschkompetenz nicht allein auf Selbsteinschätzungen der Probanden zu stützen, wurden die Wenkerübersetzungen einer Referenzgruppe von zehn alteingesessenen MecklenburgerInnen der Vorkriegsgeneration, die primär niederdeutsch sozialisiert wurden, als relative Bezugsgröße gewählt. Nur Probanden, die in ihren Übersetzungen dreier Testsätze mindestens 80 \% der 44 
(,Vorkriegsgeneration') und in Personen, die zwischen 1950 und 1969 geboren wurden (,Nachkriegsgeneration'). Hierbei geht es zum einen um ortsansässige Mecklenburger, die ich im Folgenden als, Alteingesessene' bezeichne, wenn beide Elternteile in der Untersuchungsregion geboren wurden. Da Niederdeutsch im Erhebungsgebiet aber durchaus nicht nur von autochthonen Mecklenburgern gesprochen wurde bzw. wird, berücksichtigt meine Untersuchung zum anderen auch niederdeutschkompetente Gewährspersonen, die nach 1945 aus mittel- und oberdeutschen Dialektregionen Schlesiens und der Tschechoslowakei vertrieben wurden und seitdem dauerhaft im Erhebungsgebiet leben, sowie deren in Mecklenburg geborenen Nachkommen, soweit sie über gute Niederdeutschkompetenz verfügen. ${ }^{13}$

Abbildung 1: Übersicht über das Untersuchungssample: Getestete Gewährspersonen aus verschiedenen Bevölkerungsgruppen bzw. Altersgruppen und historische Wenkerübersetzungen

\begin{tabular}{|l|c|c|c|}
\cline { 2 - 4 } \multicolumn{1}{c|}{} & \multicolumn{1}{c|}{$\mathbf{1 8 8 0}$} & $\begin{array}{c}\text { Vorkriegsgeneration } \\
\text { geb. 1920-1939 }\end{array}$ & $\begin{array}{c}\text { Nachkriegsgeneration } \\
\text { geb. 1950-1969 }\end{array}$ \\
\hline Alteingesessene & 25 Wenkerbögen & 24 Gewährspersonen & 9 Gewährspersonen \\
\hline $\begin{array}{l}\text { Vertriebene/ } \\
\text { Nachkommen }\end{array}$ & & 13 Gewährspersonen & 7 Gewährspersonen \\
\hline
\end{tabular}

Das Korpus von akustisch aufgezeichnetem intendiertem Niederdeutsch der Wenkerübersetzungen wird einerseits durch einzelne freie niederdeutsche Dialekterzählungen der Gewährspersonen und andererseits durch historische Sprachdaten ergänzt: Hier sind vor allem die schon erwähnten 25 historische Wenkerbögen aus der Erhebungsregion zu nennen, ${ }^{14}$ berücksichtigt wurden

niederdeutschen Merkmale realisierten, in denen die Übertragungen der zehn Referenzpersonen völlig übereinstimmten, wurden in diese Untersuchung als gute bzw. sehr gute Niederdeutschsprecher aufgenommen.

13 Vgl. Ehlers 2013.

14 Die 25 ausgewählten historischen Wenkerbögen stammen aus den genannten fünf Fokusorten bzw. aus deren näherer Umgebung: Berendshagen (Bogennummer 48752), Bernitt 48927, Biestow 48592, Börgerende 48528, Damm 48601, Heiligenhagen 48580, Letschow 48762, Neu-Bernitt 48926, Niendorf 48764, Nienhagen 48537, Rederank 48578, Reinshagen 48579, Rethwisch 48530, Retschow 48576, (Rostock) Barnsdorf 48547, (Rostock) Gehlsdorf 48549, (Rostock) Lichtenhagen 48540, (Rostock) Riekdahl 48553, Satow 48755, Schwaan 1 48763, Schwaan 2 53091, Schwaan 3 53092, Vorbeck 48936, Warnemünde 48518, Wiendorf 48765. 
aber auch einige historische Sprachaufnahmen, die in den 1960er und 1990er Jahren in der Region aufgezeichnet wurden. ${ }^{15}$ Ein kleines Vergleichskorpus von aufgezeichneten Wenkerübersetzungen aus der vorpommerschen Kleinstadt Gützkow (sechs Probanden) soll die areale Dimension des Merkmals deutlicher konturieren helfen.

Bevor auf der Basis dieser Sprachdaten nach Stand und Entwicklung der Hiattilgung im mecklenburgischen Niederdeutsch gefragt werden soll, sei darauf hingewiesen, dass sich auch in der Fachliteratur gelegentlich Hinweise finden, die Zweifel an einer (noch) fest abgegrenzten arealen Verteilung des ,mecklenburgischen' $d$ und des ,vorpommerschen' $g$ aufkommen lassen. Schon HerrmannWinter konstatiert, die Lautgrenze „schnigen/schniden zwischen Vorpommern und Mecklenburg [sei] heute nicht mehr fest ${ }^{\text {" }}{ }^{16}$ Auch nach Gernentz treten hier „an die Stelle ehemals fester Lautgrenzen [...] heute breite Übergangszonen“. ${ }^{17}$ Expansionsbewegungen in beide Richtungen werden festgestellt. So setzten sich nach Gernentz als Folge der Verbreitung der Schriften Fritz Reuters ,in neuerer Zeit auch im Westen des Mundartgebietes die g-Formen durch" ${ }^{18}{ }^{18}$ Gegenläufig dazu breite sich nach Gundlach „der d-Einschub neuerdings immer mehr nach Osten hin aus “ ${ }^{19}$ Gernentz beobachtet andererseits auch „das Fortlassen des erst im 19. Jahrhundert aufgekommenen, Gleitlauts' -g- (oder -d-) zwischen Vokalen“. Diese Gebrauchsveränderung sei derart häufig, „dass man den Beginn eines allgemeinen Lautwandels erwägen muß $3^{\text {“ }}{ }^{20}$ Hermann-Winter bezieht dieses „Fortlassen“ offenbar vor allem auf den Plosiveinschub $d$ : „Da in den konjugierten Formen dieser Lautunterschied [zwischen schniegen und schnieden] weitgehend aufgehoben ist, gilt heute weiträumig schnien und schniegen. " Auch hier wird der Bestand der „ursprünglich“ durch den $g / d$-Gegensatz markierten sprachlichen "Grenze zwischen Mecklenburg und Vorpommern" fraglich. ${ }^{21}$

15 Zwei Aufnahmen der Wenkerübersetzungen von 1990 aus Jürgenshagen (Korpus: Phonetischer Atlas von Deutschland (MR PAD), Siglen WEG 55AW1, WEG 56AW1, Aufnahmejahr 1990, www.regionalsprache.de, Audiokatalog) und sechs Aufnahmen aus Retschow und Letschow von 1962 (je drei Übersetzungen und freie Erzählungen) aus dem IDS-Korpus „Archiv für Gesprochenes Deutsch AGD / Deutsche Mundarten - DDR“.

16 Herrmann-Winter 1974, S. 174.

17 Gernentz 1974, S. 223.

18 Gernentz 1980, S. 89.

19 Gundlach 1988, S. 427.

20 Gernentz 1974, S. 226.

21 Hermann-Winter 2003, S. 198.

Birte Arendt, Andreas Bieberstedt and Klaas-Hinrich Ehlers - 978-3-631-71893-3 
Die Aussagen der Forschungsliteratur zur Hiattilgung in der mecklenburgischen Dialektlandschaft lassen sich demnach wie folgt zusammenfassen: Die verschiedenen Autoren sind sich sehr weitgehend einig, dass es zumindest ehemals eine recht klare areale Verteilung der unterschiedlichen Realisierungsformen des Vokal-Hiats im mecklenburgisch-vorpommerschen Niederdeutsch gegeben habe. Für die jüngere Vergangenheit gehen die Aussagen zur bestehenden Stabilität dieser arealen Struktur bzw. die Befunde über eine einsetzende Entwicklungsdynamik und auch über die Richtung dieser Entwicklung aber zum Teil weit auseinander.

\section{Die Hiattilgung im Niederdeutsch alteingesessener Mecklenburger}

\subsection{Die Hiatrealisierung in ihrer historischen Entwicklung im Untersuchungsgebiet}

Lässt sich wenigstens für mein räumlich sehr begrenztes Untersuchungsgebiet südwestlich von Rostock ein klares Bild der Verhältnisse gewinnen? Ein Blick auf die 25 historischen Wenkerbögen aus meiner zentralmecklenburgischen Erhebungsregion legt nahe, dass die arealen Abgrenzungen schon im ausgehenden 19. Jahrhundert keineswegs so eindeutig waren, wie die Forschungsliteratur großenteils nahe legt. Die historischen Wenkersatzvorlagen enthalten jeweils neun Wörter, bei deren Übertragung ins Niederdeutsche ein Vokal-Hiat bzw. dessen Tilgung auftreten kann: schneien, nähen, rein, neue (2x vertreten), schreien, Kindereien, bauen, mähen. ${ }^{22}$ Statt der erwartbar deutlichen Dominanz einer ,westmecklenburgischen' Tilgungsvariante auf Plosiv $d$ sind die Zahlenverhältnisse bei der niederdeutschen Übertragung dieser neun überprüften Belegwörter in der schriftlichen Erhebung Georg Wenkers nahezu ausgewogen: Nur 29,8 \% der insgesamt gewerteten 178 Belege wurden mit intermittiertem $d$ übersetzt, $27 \%$ mit einer Hiattilgung auf $g$ (vgl. Abb. 2). Schon im ausgehenden 19. Jahrhundert finden sich also weit westlich der allgemein angenommenen schniden/ schnigen-Isoglosse zahlreiche Belege für die mutmaßlich ,vorpommersche’ Form der Hiattilgung. An keinem der überprüften Wenker-Orte wurde dabei auf dem

22 Kontexte in den historischen Wenkersätzen, WS 2: „... hört auf zu schneien...“; WS 17 „... Kleider ... fertig nähen ...“; WS 17: „... mit der Bürste rein machen.“; WS 21: „.. die neue Geschichte erzählt ...“; WS 22: „Man muss laut schreien ...“; WS 28: „... solche Kindereien treiben ...“; WS 33: „... schöne neue Häuser ...“; WS 33: „... Häuser in euren Garten bauen ...“; WS 38: „Die Leute sind draußen ... und mähen ...“. 
historischen Bogen ausschließlich eine einzige Hiattilgungsform favorisiert, auf allen Bögen wurde vielmehr auf mindestens zwei der drei möglichen Hiatrealisierungen ( $g, d$, ohne Tilgung) zurückgegriffen. Auf der Hälfte aller historischen Bögen der Schulorte finden sich sowohl Tilgungen auf $d$ als auch solche auf $g$ neben Testwörtern mit nichtgetilgtem Hiat. Die beiden konsonantischen Varianten sind also in der historischen Sprachlandschaft Zentralmecklenburgs nebeneinander nachweisbar. Und die Dominanz der von Wenker als areale Leitform gewählten Hiattilgung auf $d$ war in meinem Untersuchungsgebiet um 1880 allenfalls schwach ausgeprägt.

Bemerkenswert ist auch, wie oft die Gewährsleute Wenkers die Belege mit Vokal-Hiat ohne Tilgung belassen. Ganze 43,3 \% der historischen WenkerÜbersetzungen lassen den jeweiligen Vokal-Hiat ganz ohne Einschub (vgl. Abb. 2). Abgesehen von bauen, das ausschließlich als bugen, bug'n o. ä. übertragen wurde ( $100 \%, \mathrm{~N}=25)$, erreichen die Häufigkeiten für eine der beiden konsonantischen Tilgungsvarianten bei den einzelnen Testwörtern damit kaum jemals mehr als $50 \%$ der Belege. Auch dieser Befund muss angesichts der Forschungsliteratur überraschen, die Formen ohne Hiattilgung ja ursprünglich vor allem im „äußersten Südwesten des Landes“ erwarten lässt.

Soweit der Stand der schriftlichen Erhebung Georg Wenkers. Wie stellen sich die Verhältnisse nun im rezenten Dialekt des Untersuchungsgebietes dar? Hier soll zunächst das intendierte Niederdeutsch meiner älteren Gewährspersonen betrachtet werden, die in den 1920er und 1930er Jahren, also spätestens 60 Jahre nach der Wenkererhebung in meinem Untersuchungsgebiet geboren wurden. Überraschenderweise ist die Frequenz der Hiattilgungen in den spontanen und mündlichen Übersetzungen der Wenker-Vorlage durch die 24 Angehörigen der Vorkriegsgeneration alteingesessener Mecklenburger viel höher als auf den schriftlichen Wenkerbögen von 1880. Freilich mögen die Zahlendifferenzen zu guten Teilen der schriftlichen und indirekten Erhebungsmethode des Sprachatlas des deutschen Reiches geschuldet sein. Die Lehrer, die Wenkers historische Fragebögen stellvertretend ausgefüllt haben, könnten einen intervokalischen Übergangslaut vielfach ,überhört' bzw. für irrelevant gehalten haben. Die starke Norm-Nähe der Schriftsprache wird die Tendenz zu einer standardnäheren, also tilgungslosen Schreibweise in ihren Wenker-Übersetzungen zusätzlich bestärkt haben. ${ }^{23}$ Jedenfalls wählen die älteren Probanden in den aktuellen akustischen

23 Dies zeigt sich etwa bei der Übertragung des Testwortes Kindereien, das eine Reihe von Gewährsleuten des mündlichen Übersetzungstests nicht als direkt ins Niederdeutsche umlautbar empfunden hat und durch Ersatzlexeme (Kinnerkråm o. ä.) ersetzt hat. 
Aufzeichnungen nicht nur insgesamt häufiger eine konsonantische Hiattilgung als die Gewährsleute Wenkers, sondern gerade auch die Hiattilgungen auf $d$ treten in den aktuellen Aufnahmen deutlich häufiger auf. Von 164 Belegen für die oben angegebenen neun Testwörter werden von der Vorkriegsgeneration $48,8 \%$ mit Hiattilgung auf $d, 34,2 \%$ mit einer Tilgung auf Plosiv $g$ und nur noch $17,1 \%$ ohne konsonantische Tilgung ins intendierte Niederdeutsch übertragen. ${ }^{24}$ Von einer ausschließlichen Dominanz eines ,westmecklenburgischen’ $d$ kann auch bei diesen viel höheren Frequenzen plosivischer Tilgung allerdings kaum gesprochen werden. Die Häufigkeiten der Tilgungen auf $g$ bleiben auch in den Übersetzungstests der Vorkriegsgeneration mit mehr als einem Drittel aller Belege erheblich. Es ist für mein Untersuchungsgebiet also auch in der Gegenwart eher ein Nebeneinander beider Varianten zu konstatieren, wobei die alteingesessenen Probanden der Vorkriegsgeneration freilich mit recht deutlicher Präferenz Formen auf $d$ wählen. ${ }^{25}$

Wo die schriftlichen Wenkerübersetzungen dennoch eine wörtliche Übertragung ins Niederdeutsche vornehmen, orientieren sie sich meist strikt an der hochdeutschen Vorlage und übertragen sie dann zu $95 \%(\mathrm{~N}=20)$ standardnah ohne Hiattilgung als Kinneri-en, Kinnerin o. ä.

24 Für den diachronischen Vergleich werden dieselben neun Testwörter zugrunde gelegt, die auch in der historischen Wenkervorlage verwendet wurden. In den schriftlichen Übersetzungen der historischen Bögen kann natürlich nicht mit Sicherheit entschieden werden, ob der geschriebene Tilgungskonsonant $g$ plosivisch oder spirantisch gesprochen wurde. In den aktuellen Aufnahmen meines Erhebungsgebiets wird die Hiattilgung auf $g$ fast ausschließlich plosivisch realisiert. Die äußerst seltenen spirantischen Tilgungen sind aus der Auswertung ausgeschlossen worden.

25 Ein Nebeneinander beider Tilgungsvarianten findet sich auch in den 1990 aufgezeichneten Wenkerübersetzungen von zwei Angehörigen derselben Altersgruppe aus Jürgenshagen. Dort war bei insgesamt geringer Belegzahl die Präferenz für den Tilgungskonsonanten $d$ etwas ausgeprägter: Von den nur 13 Belegen wurden 61,5\% Tilgungen auf $d, 30,8 \%$ Tilgungen auf $g$ gewählt, 7,7 \% der Belege blieben ohne plosivische Hiattilgung, vgl. Korpus: Phonetischer Atlas von Deutschland (PAD), Signaturen WEG55AW1, WEG56AW1. Aufnahmejahr 1990, www.regionalsprache.de, Audiokatalog. Die sechs historischen Übersetzungs-Aufnahmen aus dem IDS-Korpus „Deutsche Mundarten - DDR“ aus Letschow und Retschow enthalten leider keine geeigneten Belegwörter. 
Abbildung 2: Formen der Hiatrealisierung bei Alteingesessenen

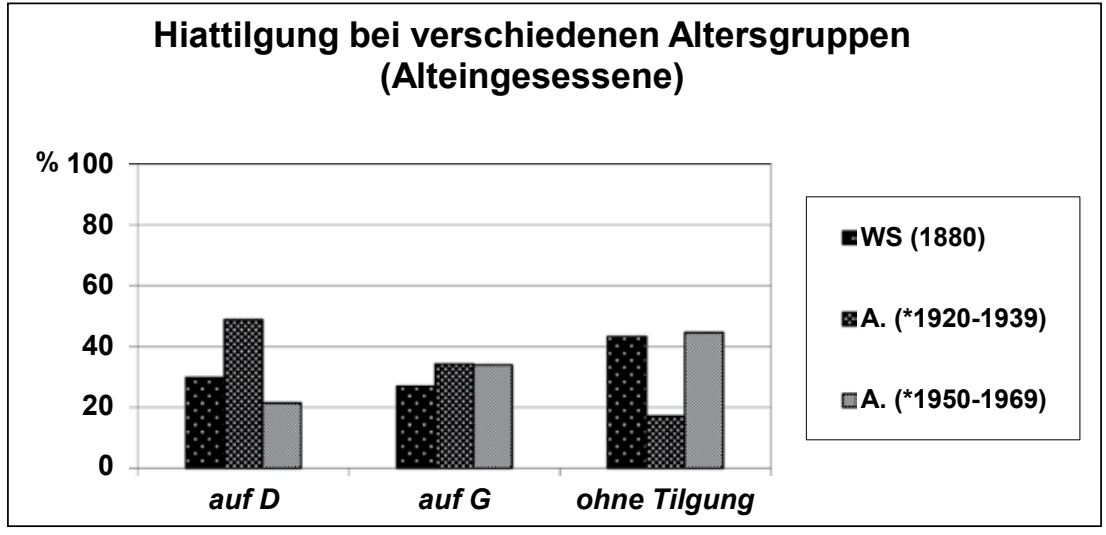

Wie sind nun die Befunde in den Wenker-Übersetzungen der jüngeren Generation? Die neun niederdeutschkompetenten Alteingesessen, die in den Jahren zwischen 1950 bis 1969 geboren wurden ${ }^{26}$ realisieren im Übersetzungstest der neun Testwörter die plosivischen Hiattilgungen viel seltener als die Angehörigen ihrer Elterngeneration. In nahezu der Hälfte der 56 niederdeutschen Belege mit Vokal-Hiat belässt es diese Gruppe bei Realisierungen ohne Hiattilgung (44,6 \%). Die Frequenzen für intermittiertes $d$ liegen dabei sogar unter denen für intermittiertes $g: 21,4 \%$ Varianten mit $d$ stehen 33,9 \% Varianten mit $g$ gegenüber. Da die Übersetzungsdaten in den beiden Altersgruppen dialektkompetenter Alteingesessener identisch erhoben worden sind, deuten die Unterschiede im intendierten Niederdeutsch beider Probandengruppen auf Entwicklungen im regionalen Niederdeutsch hin. Demnach gibt es deutliche Anzeichen, dass sich auch im Kontext des Vokal-Hiats eine Tendenz zur Annäherung an das Standarddeutsche geltend macht und das typisch mecklenburgische Merkmal sich insgesamt im Abbau befindet. Bemerkenswert ist dabei, dass offenbar gerade die Hiattilgung auf $d$ besonders stark in diesen Prozess gerät, während die Verwendung der Hiattilgung auf $g$ recht stabil bleibt (vgl. Abb. 2).

26 Die niedrige Zahl der Gewährspersonen aus der Nachkriegsgeneration spiegelt die Schwierigkeiten, in dieser Altersgruppe im Untersuchungsgebiet noch Probanden zu finden, die die vorausgesetzte Niederdeutschkompetenz haben. 


\subsection{Die Hiatrealisierung im phonetischen Kontext}

Diese Beobachtungen bleiben unscharf, solange alle neun Testwörter der historischen Wenkerbögen gemeinsam betrachtet werden. In der neueren Forschung ist es erst Köhncke, der darauf hinweist, dass die Hiatrealisierung nicht allein mit arealen Faktoren korreliert, sondern mitunter auch „lexemabhängig ${ }^{\text {“27 }}$ bzw. kontextbezogen gebildet wird. Um die Belegzahlen zu erhöhen, wurden in die folgenden Untersuchungen zusätzliche Lexeme aus der erweiterten WenkersatzVorlage einbezogen, die ich meinen Gewährspersonen zur mündlichen Übersetzung ins Niederdeutsche vorgelegt habe. Es handelt sich in der erweiterten Übersetzungsvorlage um die folgenden zehn bzw. zwölf Testwörter: schneien (2x vertreten), schreien, Kindereien, nähen, mähen, das Krähen, blühen, bauen, hauen, neue ( $2 \mathrm{x}$ vertreten) ${ }^{28}$ Anhand dieser Testwörter sollte geprüft werden, ob die Hiattilgung in bestimmten phonetischen Umgebungen oder bei einzelnen Lexemen besonders häufig auftritt. Die niederdeutschen Übersetzungen dieser Wörter lassen sich zu den folgenden Lautkontexten im Hiat gruppieren: [i:-ən] (schni-en, schri-en, Kinneri-en); [ae-ən] (nai-en, mai-en, dat Krai-en); [əø-ən] (bloi-en); [u:-ən] (bu-en); [ao-ən] (hau-en) und [i:-ə\#] (ni-e).

Das folgende Diagramm (Abb. 3) veranschaulicht, dass von den niederdeutschkompetenten Angehörigen der Vorkriegsgeneration aus meiner Erhebungsregion je nach Lautkontext im Hiat unterschiedliche Tilgungskonsonaten präferiert werden..$^{29}$ Für die auf -en bzw. bei Schwa-Ausfall auf bloßen Nasal auslautenden Testwörter (mai-n) scheint diese Präferenz mit der Qualität des Erstvokals im Hiat zusammenzuhängen. Nach Vordervokal, sei es monophthongisch als Langvokal [i:] oder sei es im upglide der vorangehenden Diphthonge [ae] und [॰ø], schließen die Probanden aus der Erhebungsregion in den weitaus meisten Fällen mit dem vorderen Plosiv $d$ an. Vor demselben Folgekontext -(e) $n$ wählen sie ausschließlich

27 Köhncke 2006, S. 171. Im Zusammenhang seiner Überlegungen zur Entstehung der beiden plosivischen Formen erörtert allerdings schon Teuchert (1959, S. 223-226) die Abhängigkeit des Merkmals von verschiedenen Lautkontexten.

28 Das mögliche Testwort rein (rai-en) wurde aus der Betrachtung ausgeschlossen, weil es im Kontext der Vorlage (mit der Bürste rein machen) offenbar als nicht wörtlich ins Niederdeutsche umsetzbar empfunden wurde, vielfach durch Ersatzbildungen (sauwer måken, affbösten) übertragen und im Übrigen in den mündlichen Übersetzungen fast nie mit plosivischer Hiattilgung gebildet wurde und so die im Übrigen sehr kohärenten Ergebnisse für den Lautkontext [ae-ən] stark verzerrt hätte.

29 In den folgenden Diagrammen wird der Gegensatz der beiden Lautkontexte durch eine vereinfachte Verschriftlichung der Diphthonge hervorgehoben: Vordervokal vor Hiat (i:, ai, oi) versus Hintervokal vor Hiat (u:, au). 
den hinteren Plosiv $g$ als Tilgungselement, wenn schon der vorausgehende Vokal bzw. die zweite Diphthongphase hinten artikuliert wird ([u:], [ao]). Das oben beobachtete areale Nebeneinander des, vorpommerschen' $g$-Einschubs und des ,mecklenburgischen' $d$-Einschubs im zentralmecklenburgischen Erhebungsgebiet ist also weitgehend an bestimmte Lexemgruppen gebunden und lässt sich wohl in vielen Fällen auf Koartikulationsphänomene zurückführen.

Abbildung 3: Formen der Hiatrealisierung in verschiedenen phonetischen Kontexten (bei Alteingesessenen der Vorkriegsgeneration)

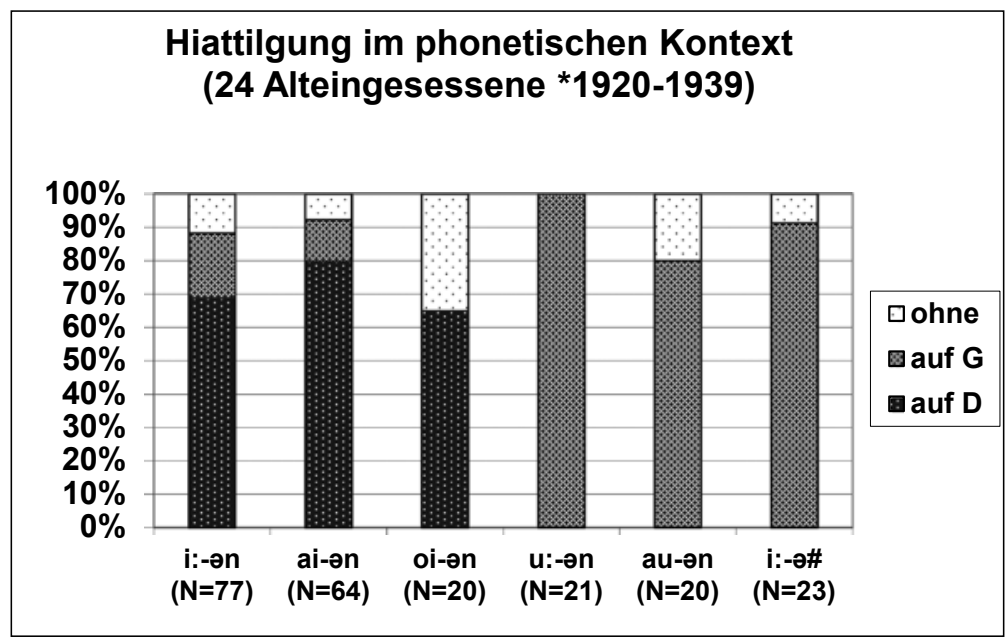

Anders als in den Testwörtern mit Folgekontext - (e)n fällt die Hiatrealisierung im niederdeutschen Adjektiv ni-e (,neue') aus, das auf Schwa auslautet. ${ }^{30}$ Hier wählen die Probanden der Altersgruppe ausschließlich den hinteren Einschubkonsonanten, obwohl ein langer Vordervokal vorangeht. Köhncke kommt bei Aufnahmen in seinem weiter südlich gelegenen mecklenburgischen Untersuchungsgebiet (Dörfer südlich von Sternberg) zu ähnlichen Distributionsverhältnissen für die beiden plosivischen Hiattilgungen:

30 Obwohl das Testwort neue zweimal in der Übersetzungsvorlage vorkam (WS 21: „neue Häuser“; WS 33: „die neue Geschichte“), bleibt die Gesamtbelegzahl vergleichsweise gering, weil die Probanden hier vor Anlaut $g$ im Folgewort Geschichte meist suffixlos übersetzten (de ni Geschicht versus nige Hüser) und dieses daher nicht als Beleg für den Hiat gewertet werden konnte. Auch die mehrfach elizitierte Übersetzungsvariante de niich Geschicht wurde hier nicht als Hiattilgung gewertet, zu derartigen Formenbildungen siehe unten. 
„Über die Distribution beider Konsonanten lässt sich nur soviel sagen, dass $d$ im Untersuchungsgebiet häufiger ist. $g$ hingegen kommt nur bei bestimmten Wörtern vor, zudem ist es auf $u$ und $i$ beschränkt. [...] Dagegen ist mir kein Fall bekannt, bei dem das $d$ dem $u$ als Hiatustilger folgt. Bei $i$ können [...] beide Konsonanten auftreten. Es gibt also Tendenzen, wann welcher Laut favorisiert wird, dennoch ist die Verteilung nicht eindeutig und nicht immer vom vorangehenden Vokal abhängig. ${ }^{{ }^{31}}$

$\mathrm{Zu}$ ergänzen wäre, dass das $d$ offensichtlich auch insofern distributionsbeschränkt ist, als es weder in den 25 historischen Wenkerbögen meiner Erhebungsregion noch in den mündlichen Übersetzungsaufzeichnungen jemals im Kontext [i:-ə\#] auftritt. „Neue“ wird hier ausschließlich mit Hiattilgung auf $g$ (nige) übertragen. Die ,vorpommersche' Tilgung auf $g$ ist also breiter distribuierbar als das ,mecklenburgische' $d$.

Die Bindung der Hiatrealisierung an bestimmte Lautkontexte bestätigt sich auch bei den niederdeutschen Übersetzungen der Belegwörter durch die Nachkriegsgeneration alteingesessener Mecklenburger. Auch bei diesen neun niederdeutschkompetenten Probanden ist das Nebeneinander der Formen auf verschiedene Lautkontexte bzw. Lexemgruppen verteilt (vgl. Abb. 4).

Abbildung 4: Formen der Hiatrealisierung in verschiedenen phonetischen Kontexten (bei Alteingesessenen der Nachkriegsgeneration)

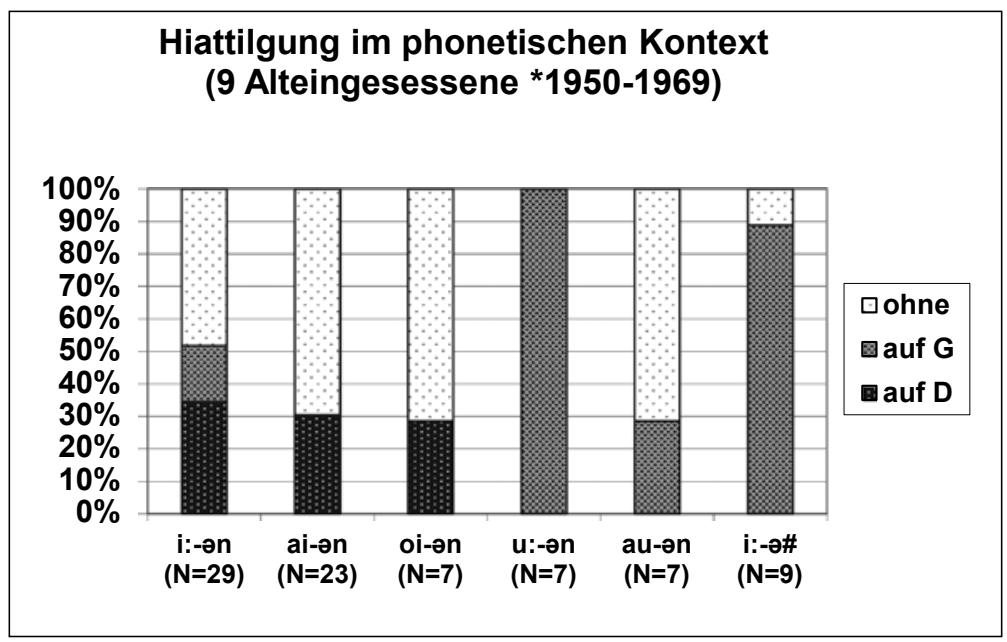

31 Köhncke 2006, S. 170. 
Im Vergleich zur Elterngeneration ist dabei zugleich ein deutlich häufigeres „Fortlassen des erst im 19. Jahrhundert aufgekommenen ,Gleitlauts' -g- (oder -d-) zwischen Vokalen“ zu bemerken, das schon Gernentz beobachtet hatte. ${ }^{32}$ Dieser zurückgehende Gebrauch im intendierten Niederdeutsch der jüngeren Generation betrifft, wie schon gesagt, in besonderem Maße die Hiattilgung auf $d$, die in ihren angestammten Lautkontexten auf Realisierungsfrequenzen von ca. $30 \%$ der Belege zurückfällt. In den Übersetzungen derselben Testwörter durch die Elterngeneration hatten die Anteile noch zwischen 79,7 \% und $65 \%$ der Belege gelegen. Auch der Rückgang im Gebrauch des $g$-Einschubs ist für einen der Kontexte drastisch (hauen), ${ }^{33}$ die Realisierung der Hiattilgung bleibt aber im Falle von bugen und nige bemerkenswert stabil. Bugen ist sogar über alle untersuchten Zeitstufen seit der historischen Wenker-Befragung von 1880 hinweg überhaupt die einzige gewählte Übertragung für die standarddeutsche Übersetzungsvorlage bauen.

Wenn man die Unterschiede in den niederdeutschen Übersetzungen der Vorkriegsgeneration und der kleinen, zwischen 1950 und 1969 geborenen Probandengruppe als weiteres Indiz für „den Beginn eines allgemeinen Lautwandels ${ }^{\text {“34 }}$ interpretieren möchte, so dürften die Formen der plosiven Hiattilgung in $\mathrm{Zu}$ kunft wohl weiter zurückgehen und sich nur der $g$-Einschub mit zunehmend ausschließlicher Bindung an einzelne Lexeme dauerhafter erhalten.

\subsection{Stadt-Land Unterschiede in der Hiatrealisierung}

Mein zentralmecklenburgisches Untersuchungsgebiet, das nur einen Durchmesser von etwa 30 Kilometern umspannt, ist wohl zu klein, als dass sich dort deutliche areale Binnengliederungen abzeichnen dürften. In den Aufnahmen aus diesem Gebiet deuten sich aber doch Binnendifferenzierungen an, die allerdings eher sozial als areal zu nennen wären. Vergleicht man nämlich die Wenkerübersetzungen von ortsfesten Alteingesessenen der Vorkriegsgeneration aus Rostock mit denen von entsprechenden Gewährsleuten aus der Kleinstadt Schwaan und den Dörfern Satow und Jürgenhagen, deutet sich eine Korrelation der Hiatrealisierung und der Wohnortgröße an (vgl. Abb. 5). Die fünf Gewährspersonen, die ihr Leben ohne größere Unterbrechung in der Großstadt Rostock bzw. seiner damals noch nicht eingemeindeten unmittelbaren Umgebung (Dierkow, Biestow, Papendorf) verbracht haben, tendieren eher zu Hiattilgungen auf $g$ als ortsfeste Kleinstädter aus Schwaan. Und die Schwaaner Gewährs-

32 Gernentz 1974, S. 226.

33 Für den besonders raschen Abbau der Hiattilgung in haugen könnte eine Rolle spielen, dass das Lexem anders als bugen ohne die Hiattilgung völlig standardidentisch klingt.

34 Herrmann-Winter 2003, S. 198. 
personen dieser Altersgruppe realisieren die Hiattilgung auf $g$ ihrerseits insgesamt häufiger als ortsfeste Bewohner der Dörfer Satow, Jürgenshagen und unmittelbarer Nachbarorte (Püschow). Die Frequenz der Hiattilgungen auf $g$ nimmt (wie die Zahl der tilgungslosen Realisierungen) mit steigender Wohnortgröße im intendierten Niederdeutsch der Alteingesessenen zu, umgekehrt proportional werden Hiatrealisierungen auf $d$ mit steigender Wohnortgröße seltener gewählt. ${ }^{35}$ Vor dem Hintergrund dieser Befunde könnte die Hiattilgung auf $d$ in meinem Erhebungsgebiet als ländliche Reliktform angesehen werden, die im Untersuchungszeitraum einem besonders starken Abbau unterliegt. Erweitert man den historischen Fokus der Betrachtung auf das frühere 19. Jahrhundert, in dem der Tilgungskonsonant $d$ erst als jüngere Form neben das ältere $g$ trat und sich offenbar vor allem im ländlichen Raum gegen die konservativeren Städte durchsetzte, ${ }^{36}$ dann erscheinen die feststellbaren Stadt-Land-Differenzen bei der Vorkriegsgeneration als Nachwirkungen einer ursprünglich ländlichen Innovation, die in der jüngeren Generation dann an ihr Ende kommen wird.

Abbildung 5: Hiattilgung in Wohnorten unterschiedlicher Größe (ortsfeste Alteingesessene, geb. vor 1940)

\begin{tabular}{|l|c|c|c|c|}
\cline { 2 - 5 } \multicolumn{1}{c|}{} & Hiattilgung auf d & Hiattilgung aufg & Keine Hiattilgung & Gesamtbelege \\
\hline $\begin{array}{l}\text { Dorf } \\
\text { (5 Personen) }\end{array}$ & $66,7 \%$ & $24,6 \%$ & $8,8 \%$ & $\mathrm{~N}=57$ \\
\hline $\begin{array}{l}\text { Kleinstadt } \\
\text { (6 Personen) }\end{array}$ & $46,3 \%$ & $35,2 \%$ & $18,5 \%$ & $\mathrm{~N}=54$ \\
\hline $\begin{array}{l}\text { Großstadt } \\
\text { (5 Personen) }\end{array}$ & $34,6 \%$ & $43,6 \%$ & $21,8 \%$ & $\mathrm{~N}=55$ \\
\hline
\end{tabular}

35 Dabei sind die Variationsbreiten in der großstädtischen und in der dörflichen Vergleichsgruppe interpersonell bemerkenswert gering: Die Frequenzen der Hiattilgungen auf $g$ variieren bei den fünf Rostocker Probanden nur zwischen $36,7 \%$ und $45,5 \%$, bei den fünf Probanden aus Satow, Jürgenshagen und Püschow nur zwischen 22,2 \% und $27,3 \%$. Die Übersetzungen der sechs Probanden aus Schwaan fallen hinsichtlich des Merkmals erheblich heterogener aus, hier reichen die Frequenzen der Hiattilgungen auf $g$ von $0 \%$ bis $69,2 \%$, der Mittelwert für die gesamte kleinstädtische Gruppe ist also deutlich weniger aussagekräftig.

36 Für sein lauenburgisches Untersuchungsgebiet konstatiert Heigener (1937, S. 31, 53, 63) in seiner Landschaftsgrammatik, dass die „ganz jung[e]“ Entwicklung des „Gleitlauts" $d$ von den konservativen Städten (Ratzeburg, Mölln, Lauenburg) nicht übernommen wurde. Vgl. den Rostocker Beckmann (1954/55), der den Gebrauch der Hiattilgung auf $d$ als eine aus dem Westen kommende Entwicklung bestimmt, die erst spät in den großstädtischen Sprachusus überging. 


\section{Die Hiattilgung im Lerner-Niederdeutsch der immigrierten Vertriebenen}

Bislang bin ich nur auf das intendierte Niederdeutsch von Probanden aus alteingesessenen mecklenburgischen Familien eingegangen. Untersuchungen zum Sprachwandel in Mecklenburg-Vorpommern sollten aber grundsätzlich die außerordentlich hohe Zahl von immigrierten Flüchtlingen und Vertriebenen in Rechnung stellen, die sich hier in der Nachkriegszeit angesiedelt haben. Meine Untersuchungen zeigen, dass ein beträchtlicher Teil der heute noch befragbaren Immigranten der Vorkriegsgeneration hohe produktive Kompetenzen im Niederdeutschen erworben hat und auch die Nachkommen dieser Immigranten zum Teil bemerkenswert hohe Niederdeutschkompetenzen aufweisen. ${ }^{37}$ Das von vielen dieser Immigranten ungesteuert erworbene Niederdeutsch ${ }^{38}$ könnte einerseits den Sprachstand des autochthonen Niederdeutsch der Nachkriegsjahre abbilden und andererseits in der wechselseitigen Kommunikation mit den alteingesessenen Mecklenburgern sogar Einfluss auf dessen weitere Entwicklung genommen haben. Es zeigt sich, dass die Hiattilgung in den Wenker-Übersetzungen der niederdeutschkompetenten Zuwanderer über die Generationsfolge eine ganz ähnliche Entwicklungsdynamik aufweist wie bei den alteingesessenen Niederdeutschsprechern. Auch hier bleibt der Anteil der Übersetzungsvarianten mit einer Hiattilgung auf $g$ in den beiden Generationen von Vertriebenen auffallend stabil: $25,2 \%(\mathrm{~N}=131)$ im intendierten Niederdeutsch der dreizehn befragten Zuwanderer aus der Vorkriegsgeneration und 27,8 \% $(\mathrm{N}=72)$ bei den sieben Angehörigen der Nachkriegsgeneration (vgl. Abb. 6). Dagegen findet auch hier ein Rückgang der Hiattilgung auf $d$ statt. Dieser Abbau, der sich bereits im Übersetzungstest der alteingesessenen Mecklenburger abgezeichnet hat, wird vom Lerner-Niederdeutsch der Vertriebenen und ihrer Nachkommen gewissermaßen vorweggenommen und verstärkt. Die im Sprachgebrauch der alteingesessenen Ortsansässigen immerhin noch erkennbare Dominanz der $d$-Formen unter den nebeneinander gebräuchlichen Hiatrealisierungen wird von den immigrierten Vertriebenen beim sekundären Dialekterwerb nicht reproduziert. Hiattilgungen auf $d$ treten im intendierten Niederdeutsch der älteren Vertriebenengeneration

37 Ehlers 2013.

38 Es wurden nur Vertriebene in die Untersuchung einbezogen, die vor ihrer Vertreibung keinen Kontakt mit dem Niederdeutschen gehabt haben konnten, weil sie aus mitteloder oberdeutschen Dialektregionen zugewandert sind. Diese Personen haben ihre Niederdeutschkompetenz gegebenenfalls erst in ihrem neuen mecklenburgischen Lebensumfeld erworben. 
(geb. vor 1940, seit 1945/1946 im Untersuchungsgebiet ansässig) nur in 24,4 \% der Belege auf $(\mathrm{N}=131)$. Die Nachkriegsgeneration der Vertriebenenfamilien kennt diese Variante dann offensichtlich kaum noch, hier fällt die Verwendungsfrequenz auf nur noch 14,8 \% $(\mathrm{N}=72)$ ab. Entsprechend steigt der Anteil von Hiatrealisierungen ohne intermittierten Konsonanten bei den Immigranten früher und stärker an als bei den jeweiligen Mecklenburger Altersgenossen an. Die Tendenz zum Abbau der , mecklenburgischen' Hiatattilgung ist bei den Zuwanderern und ihren Familien deutlicher ausgebildet als bei den Alteingesessenen, die stärker an einzelne Lautkontexte bzw. Lexeme gebundene Hiattilgung auf $g$ wird jedoch von den Alteingesessenen übernommen. Falls das L2-Niederdeutsche der Immigranten also Rückwirkungen auf das Niederdeutsch der alteingesessenen Gesprächspartner gehabt haben sollte, dann hätte es den Abbau der Hiattilgung auf $d$ tendenziell beschleunigt.

Abbildung 6: Hiattilgung bei Alteingesessenen und Vertriebenen im diachronischen Vergleich (A: Alteingesessene zweier Generationen, V: Vertriebene und ihre Nachkommen)

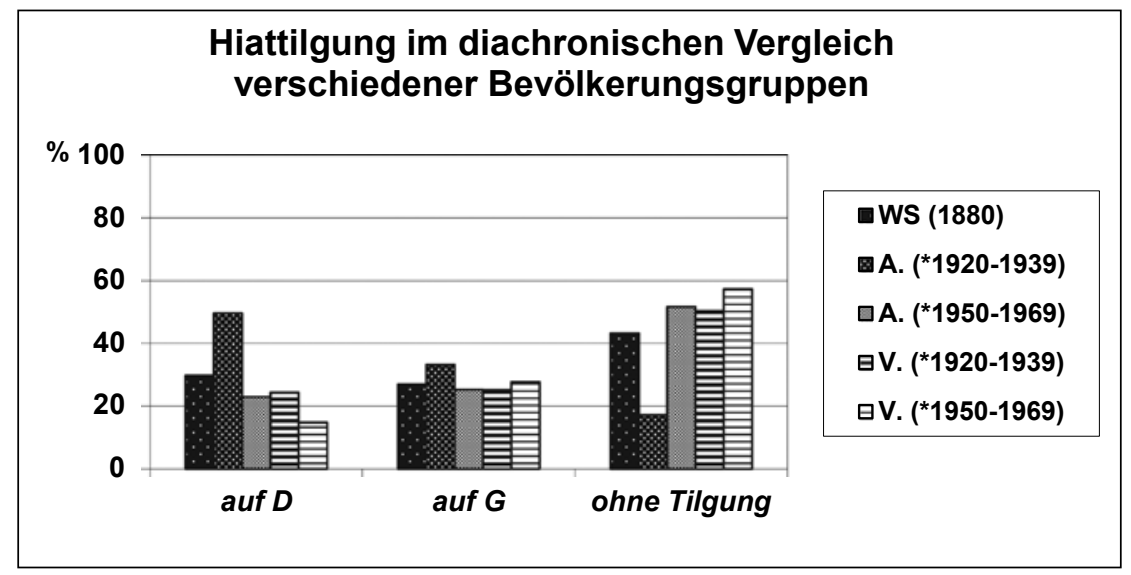

\section{Ein Seitenblick nach Vorpommern}

Die hier bislang getroffenen Aussagen zur Entwicklungsdynamik der Hiattilgung und zu ihrer Distribution in lautlichen Kontexten müssen in ihrer Gültigkeit auf mein zentralmecklenburgisches Untersuchungsgebiet beschränkt werden. Dies zeigt ein Blick auf eine kleine Vergleichsstichprobe aus Vorpommern. In der etwa 30 Kilometer südlich von Greifswald gelegenen Kleinstadt Gützkow habe ich im 
Jahr 2008 ebenfalls Wenkerübersetzungen aufgenommen. ${ }^{39}$ Es handelt sich um drei Aufnahmen von Alteingesessenen aus der Vorkriegsgeneration und drei Aufnahmen von alteingesessenen Gützkowern, die zwischen 1948 und 1956 geboren wurden. Die Realisierung der Hiattilgung in diesem kleinen vorpommerschen Vergleichskorpus weist bemerkenswerte Differenzen zu den zentralmecklenburgischen Wenkerübersetzungen auf. Überprüft wurden die Dialektübertragungen der auch für Mecklenburg zugrunde gelegten Testwörter für den Vokal-Hiat bei sechs Gewährspersonen aus Gützkow. Von insgesamt 63 Belegen weisen $92 \%$ eine Hiattilgung mit dem Plosiv $g$ auf. Nur 6,4 \% der Belegübersetzungen wurden ganz ohne Hiattilgung realisiert. In allen vier Einzelfällen handelt es sich um Übertragungen der beiden Wörter rein und Kindereien, die auch von den zentralmecklenburgischen Gewährsleuten besonders häufig ohne Hiattilgung übersetzt wurden. Der einzige Beleg für ein intermittiertes $d$ (Kraid'n) stammt von einem Angehörigen der jüngeren Generation. Dies ist auch der einzige Unterschied zwischen den Aufnahmen der jüngeren und der älteren Generation, von welcher Hiattilgungen auf $d$ gar nicht verwendet wurden. Das intendierte Niederdeutsch beider Altersgruppen aus Gützkow stimmt also bis auf diesen minimalen Unterschied im Hinblick auf die Hiatrealisierung vollständig überein. Für eine diachronische Entwicklungsdynamik, wie sie sich im zentralmecklenburgischen Korpus abzeichnet, gibt es in Gützkow keine Hinweise. Anders als in Zentralmecklenburg lassen sich für die Hiattilgung auf $g$ auch keine Präferenzen für spezifische phonetische Kontexte ausmachen. Die Variante auf $g$ ist vielmehr über sämtliche Testwörter mit Ausnahme des Lexems Kindereien ${ }^{40}$ gleichmäßig distribuiert. Die These einer Ausbreitung des ,mecklenburgischen' $d$-Einschubs nach Osten findet jedenfalls in Gützkow keine Bestätigung. ${ }^{41}$ Hier dominiert mit hohen Frequenzzahlen und großer intergenerationeller Stabilität in allen getesteten phonetischen Kontexten die Hiattilgung auf $g$ im intendierten Niederdeutsch. So gesehen kann hier weiterhin von einem ,vorpommerschen' Dialektmerkmal gesprochen werden. Der areale Faktor ist hier nicht wie in Westmecklenburg mit zusätzlichen kontaktphonetischen Bedingungen verschränkt.

39 Die Aufnahmen entstanden im Zusammenhang mit Recherchen für das DFG-Projekt „Sprachvariation in Norddeutschland (SiN)“, sind aber nur zu einem Teil in das SiNKorpus eingegangen, das sich auf Frauen der jüngeren Altersgruppe beschränkte.

40 Kindereien wurde meist mit anderer lexikalischer Grundlage (z. B. Görenstraik) oder ohne plosivische Tilgung ins Niederdeutsche übersetzt.

41 Gundlach 1988, S. 427.

Birte Arendt, Andreas Bieberstedt and Klaas-Hinrich Ehlers - 978-3-631-71893-3 


\section{Die „Adoption“ der Hiattilgung in flektierte Wortformen}

Abschließend möchte ich noch auf eine eigentümliche Transformation der Hiattilgung hinweisen, die in den niederdeutschen Aufnahmen der Erhebungsregion immer wieder zu hören ist, und die schon Schirmunski für mitteldeutsche Dialekte als „Adoption“42 der Tilgungskonsonanten in Flexionsformen der betreffenden Lexeme beschrieben hat. Gemeint sind Fälle wie Fruch ,Frau' oder umbuucht ,umgebaut', bei denen der Tilgungsplosiv aus Formen mit VokalHiat (Frugen, bugen) auch auf Flexionsformen übertragen wird, in denen auf den langen Stammvokal des Lexems kein zweiter Vokal (Schwa) folgt, also gar kein Vokal-Hiat vorliegt. Im Falle von Fruch wird das Tilgungs- $g$ aus dem Plural Frugen in den Singular übertragen, wo es im absoluten Auslaut stellungsbedingt spirantisiert wird (Frug- > Fruch). Fruch ist in aktuellen und historischen Tonaufnahmen der Wenker-Übersetzungen nur selten zu verzeichnen. ${ }^{43}$ In den 25 historischen Wenkerbögen der Untersuchungsregion treffen wir die Übernahme des Tilgungsplosivs in die Übersetzung von Singular Frau, als Frug verschriftlicht, aber noch recht häufig an $(32 \%, \mathrm{~N}=25)$.

Der entsprechende Mechanismus findet sich bei flektierten Verbformen, in die der Tilgungsplosiv aus der Grundform übertragen und dort vor $-t$ stellungsbedingt ebenfalls spirantisiert wird (umbuug- $t>$ umbuucht). In aktuellen und historischen Aufnahmen niederdeutscher Interviews und freier Dialekterzählungen findet sich das Phänomen besonders häufig bei Partizipien von Derivationsformen des Verbs bugen, bauen': etwa als umbuucht oder utbuucht, inbuucht. ${ }^{44}$ Oder als upbuucht, ümbuucht im niederdeutschen Interview einer Alteingesessenen aus Heiligenhagen ${ }^{45}$ oder als upbuucht $(2 \mathrm{x})$, anbuucht in der niederdeutschen Dialekterzählung eines Vertriebenen aus Schwaan. ${ }^{46}$ Wir finden aber auch Belege

42 Schirmunski 2010 [1961], S. 430-431.

43 „Bi de Fruch“ (in zwei Aufnahmen von 1962, IDS-Korpus „AGD/Deutsche Mundarten - DDR“: Mann, geb. 1890 in Retschow (DR933) und Mann, geb. 1930 in Letschow (DR928) und in einer Aufnahme von 1990 aus dem Korpus des Digitalen Wenkeratlas (Korpus: MR PAD, www.regionalsprache.de, Audiokatalog, Mann, geb. 1924 in Jürgenshagen, WEG56AW2) sowie bei einer Vertriebenen aus meinem Korpus, Frau 19, geb. 1923 in Böhmen).

44 Zwei Interviewaufnahmen von 1962 aus dem IDS-Korpus „AGD/Deutsche Mundarten - DDR“: Mann, geb. 1929 in Retschow (DR934) und Mann, geb. 1930 in Letschow (DR928).

45 Frau 85, geb. 1954 in Mecklenburg.

46 Herr 1, geb. 1932 in Schlesien.

Birte Arendt, Andreas Bieberstedt and Klaas-Hinrich Ehlers - 978-3-631-71893-3 
wie affhaucht , abgehauen' ${ }^{37}$ oder in der 3. Pers. Präsens ,dei verstait alls œewer [...] druucht sich nich" , die versteht alles, aber traut sich nicht.$^{48}$ In all den genannten Fällen handelt es sich um phonetische Kontexte nach hinterem Stammvokal [u: oder $a o$ ], also um Kontexte, in denen bei der Hiattilgung auch in Zentralmecklenburg der Plosiv $g$ präferiert wird. Für Kontexte nach Vordervokal im Stammmorphem habe ich bislang nur in einer historischen Tonaufnahme aus Letschow einen Beleg für den Transfer des Tilgungs- $g$ gefunden: vörglöicht ,vorgeglüht, beim Dieselmotor. ${ }^{4} 9$

Das phonetische Kontaktphänomen der Hiattilgung wird von den Sprecherinnen und Sprechern offenbar häufig als plosiver Auslaut des Stammmorphems interpretiert und entsprechend in die Bildung von Flexionsformen der entsprechenden Lexeme , adoptiert'. Man könnte hier von einer Morphologisierung bzw. Lexematisierung der Hiattilgung sprechen.

Dieser Mechanismus scheint allerdings ausschließlich auf Hiattilgungen mit dem Plosiv $g$ beschränkt zu sein. Ein Transfer des Tilgungsplosivs $d$ in flektierte Verbformen, in der dann zwei dentale Plosive aufeinanderträfen, wäre wohl artikularisch kaum realisierbar $\left({ }^{\star}\right.$ vörglöid- $t$ ). Entsprechend realisiert eine Gewährsperson aus Schwaan den verbalen Präsens Plural des Verbs freuen im niederdeutschen Interview an einer Belegstelle mit Tilgungs- $d$ („,dei froiden sich“, ,die freuen sich'), bildet das Partizip des Verbs im selben Interview aber ohne hörbar intermittiertes $d$ : „hai het sich froit“ ,er hat sich gefreut'. ${ }^{50}$ Die Möglichkeit, die Hiattilgung auf $g$ in spirantisierter Form in die besonders frequente dritte Person Singular des Präsens oder in das Präteritum der entsprechenden Verben zu übertragen und auf diese Weise als Morphemauslaut zu interpretieren (schriecht, schriechte $(n / s t))$, dürfte die $g$-Tilgungen im Sprachgebrauch stabilisieren, während bei der Hiattilgung auf $d$ ein vergleichbarer Mechanismus aus artikulatorischen Gründen weitgehend ausgeschlossen ist (?schried-t, schried-te(n/st)). Hier liegt vermutlich eine zusätzliche Erklärung, wieso bei einer generellen Tendenz zum Abbau standardferner Varianten des Niederdeutschen die Hiattilgung auf $g$ stärkere Resistenz zeigt als der Tilgungsplosiv $d .^{51}$

47 IDS-Korpus „AGD/Deutsche Mundarten - DDR“: Mann, geb. 1929 in Retschow (DR934).

48 Herr 58, geb. 1950 in Mecklenburg.

49 IDS-Korpus „AGD/Deutsche Mundarten - DDR“: Mann, geb. 1930 in Letschow (DR928).

50 Herr 58, geb. 1950 in Mecklenburg.

51 Einen Zusammenhang zwischen der Bildung der Flexionsformen und der diachronischen Entwicklung der Hiattilgung deutet auch Herrmann-Winter (2003, S. 198) in 


\section{Zusammenfassung}

Die strikte Gegenüberstellung einer westlichen, mecklenburgischen und einer ostmecklenburgisch bzw. vorpommerschen Realisierung der Hiattilgung, von der die Literatur zum mecklenburgischen Niederdeutsch bis heute meist ausgeht, lässt sich in meinem zentralmecklenburgischen Untersuchungsgebiet nicht bestätigen. Vielmehr stehen die Hiattilgung auf $d$ und die auf $g$ im Untersuchungsgebiet in starker Konkurrenz - und standen es auch schon zur Zeit von Georg Wenkers Fragebogenerhebung. Neben der arealen Verteilung ist die Wahl des Tilgungskonsonanten in Zentrum Mecklenburgs recht deutlich auch von phonotaktischen Faktoren bestimmt bzw. bestimmt gewesen. Unter heutigen Niederdeutschsprechern der Region sind konsonantische Hiattilgungen insgesamt stark rückläufig, hier zeichnet sich eine Strukturadvergenz an das Standarddeutsche ab, das keine Hiattilgungen kennt. Dominierte die Hiattilgung auf $d$ im intendierten Niederdeutsch der Vorkriegsgeneration insbesondere bei Dorfbewohnern noch deutlich, so wird gerade der Tilgungskonsonant $d$ im Zuge eines allgemeinen Übergangs zu ungetilgten Hiatrealisierungen besonders stark abgebaut. Das Lernerniederdeutsch der in das Untersuchungsgebiet immigrierten Heimatvertriebenen dürfte die Abbaudynamik des Merkmals noch verstärkt haben. Der Tilgungskonsonant $g$ erweist sich im Untersuchungsgebiet bei einigen Lexemen und Lautkontexten als recht resistent. Er bleibt, wie ein stichprobenartiger Vergleich mit Sprachaufnahmen aus Gützkow nahelegt, auch in Vorpommern stabil, wo er keine nennenswerte Konkurrenz durch den Tilgungskonsonant $d$ bekommt.

einer Zusatzbemerkung zum Lemma schneien in ihrem Wörterbuch an: „Ursprünglich markierten die unterschiedlichen Formen schniegen (östlich) und schnieden (westlich) die Grenze zwischen Mecklenburg und Vorpommern. Da in konjugierten Formen dieser Lautunterschied aufgehoben ist, gilt heute weiträumig schnien und schniegen." Dieser Darstellung ist mit der Einschränkung zuzustimmen, dass in den konjugierten Formen der Lautunterschied der Tilgungskonsonanten $g$ - $d$ keineswegs aufgehoben wird, sondern sich auf einen Kontrast von [ç] und Nullmorphem verschiebt: schniecht versus schniet. Die Hiattilgung auf $d$ hat keine morphologisierende Stütze in den konjugierten Formen.

Birte Arendt, Andreas Bieberstedt and Klaas-Hinrich Ehlers - 978-3-631-71893-3 


\section{Literatur}

Beckmann, Paul: Die Weiterentwicklung der mecklenburger Mundart nach dem Tode Fritz Reuters. In: Wissenschaftliche Zeitschrift der Universität Rostock, Gesellschafts- und sprachwissenschaftliche Reihe 4 (1954/55), S. 129-133.

Ehlers, Klaas-Hinrich: Führte die Immigration der Heimatvertriebenen nach $1945 \mathrm{zu}$ Dialektverlust und Nivellierung regionalsprachlicher Differenzen? Beobachtungen aus einer Untersuchungsregion in Mecklenburg. In: Niederdeutsches Jahrbuch 136 (2013), S. 97-116.

Ehlers, Klaas-Hinrich: Aufhebung der Hiattilgung. Erscheint in: Elmentaler, Michael/Rosenberg, Peter: Norddeutscher Sprachatlas. Bd. 2: Niederdeutsche Sprachlagen. Hildesheim. (im Druck)

Foerste, William: Geschichte der niederdeutschen Mundarten. In: Stammler, Wolfgang (Hrsg.): Deutsche Philologie im Aufriß. 2. Bd. Berlin 1954, Sp. 1905-2062.

Gernentz, Hans-Joachim: Die kommunikative Funktion der niederdeutschen Mundart und hochdeutschen Umgangssprache im Norden der Deutschen Demokratischen Republik unter besonderer Berücksichtigung der Interferenz und der Alternanz zwischen diesen beiden sprachlichen Existenzformen. In: Studia Germanica Gandensia 15 (1974), S. 209-244.

Gernentz, Hans-Joachim: Niederdeutsch gestern und heute. Beiträge zur Sprachsituation in den Nordbezirken der Deutschen Demokratischen Republik in Geschichte und Gegenwart. Rostock 1980.

Gundlach, Jürgen: Volkssprache. In: Bentzin, Ulrich/Neumann, Siegfried (Hrsg): Mecklenburgische Volkskunde. Rostock 1988, S. 423-438.

Heigener, Hans: Niederdeutsche Mundarten im Kreis Herzogtum Lauenburg. Hamburg 1937.

Herrmann-Winter, Renate: Auswirkungen der sozialistischen Produktionsweise in der Landwirtschaft auf die sprachliche Kommunikation in den Nordbezirken der Deutschen Demokratischen Republik. In: Ising, Gerhard (Hrsg.): Aktuelle Probleme der sprachlichen Kommunikation. Soziolinguistische Studien zur sprachlichen Situation in der Deutschen Demokratischen Republik. Berlin 1974, S. 135-190.

Herrmann-Winter, Renate: Neues hochdeutsch-plattdeutsches Wörterbuch für den mecklenburgisch-vorpommerschen Sprachraum. 2. Aufl. Rostock 2003.

Köhncke, André: Untersuchungen zur gegenwärtigen mecklenburgischen Mundart. In: Niederdeutsches Jahrbuch 129 (2006), S. 157-184.

Mecklenburgisches Wörterbuch. Hrsg. von der Sächsischen Akademie der Wissenschaften zu Leipzig. Aus den Sammlungen Richard Wossidlos und aus 
den Ergänzungen und nach der Anlage Hermann Teucherts. 7 Bde. Berlin 1942-1992. Unveränderter, verkleinerter Nachdruck der Erstauflage von 1937-1992. Neumünster 1996. Nachtrag und Index. Neumünster 1998.

Nerger, Karl: Grammatik des meklenburgischen Dialektes älterer und neuerer Zeit. Laut- und Flexionslehre. Leipzig 1869.

Schirmunski, Viktor M.: Deutsche Mundartkunde. Hrsg. und kommentiert von Larissa Naiditsch. Unter Mitarbeit von Peter Wiesinger. Übersetzt von Wolfgang Fleischer. Frankfurt a. M. [u. a.] 2010 [deutsche Erstausgabe 1961].

Schönfeld, Helmut: East Low German. In: Russ, Charles (Hrsg.): Dialects of Modern German. A linguistic Survey. London 1990, S. 91-135.

Schröder, Ingrid: Niederdeutsch in der Gegenwart. Sprachgebiet - Grammatisches - Binnendifferenzierung. In: Stellmacher, Dieter (Hrsg.): Niederdeutsche Sprache und Literatur der Gegenwart. Hildesheim 2004, S. 35-97.

Stellmacher, Dieter: Ostniederdeutsch. In: Lexikon der Germanistischen Linguistik. 3. Bd., 2. Aufl. Tübingen 1980, S. 464-468.

Teepe, Paul: Zur Lautgeographie. In: Goossens, Jan (Hrsg.): Niederdeutsch. Sprache und Literatur. 1. Bd. Neumünster 1983, S. 138-157 und Kartenanhang, S. VIII.

Teuchert, Hermann: Einleitung zum 1. Bande. In: Mecklenburgisches Wörterbuch. Hrsg. von der Sächsischen Akademie der Wissenschaften zu Leipzig. Aus den Sammlungen Richard Wossidlos und aus den Ergänzungen und nach der Anlage Hermann Teucherts. 1. Bd. Neumünster 1942, S. VII-XV.

Teuchert, Hermann: Beiträge zur Geschichte der mecklenburgischen Mundart. In: Niederdeutsches Jahrbuch 82 (1959), S. 207-236.

Wiggers, Julius: Grammatik der plattdeutschen Sprache. In Grundlage der Mecklenburgisch-Vorpommerschen Mundart. Leipzig 1857. 
Birte Arendt, Andreas Bieberstedt and Klaas-Hinrich Ehlers - 978-3-631-71893-3

Downloaded from PubFactory at 01/11/2019 10:11:06AM

via free access 


\title{
Zum Wandel des Niederdeutschen auf der Insel Rügen zwischen dem 19. und 21. Jahrhundert - Ein diachronischer Vergleich anhand ausgewählter Sprachmerkmale
}

\begin{abstract}
Since the 2 nd half of the $19^{\text {th }}$ century a reinforced and nowadays still continuing decline in the use of Low German - in favor of Standard German language - is detectable in the everyday communication in northern Germany. This also applies to the isle of Rügen, located in the northeast of the German federal state of Mecklenburg-Western Pomerania. The article illustrates - based on selected speech features - the development of the Mecklenburg-Western Pomeranian dialect in the language area of Rügen. The empirical basis are local dialect translations of the 40 Wenker sentences that were surveyed by Georg Wenker in 1880. For this study I compared the transliterated 40 Wenker sentences with audio material surveyed by Renate Herrmann-Winter in 1962/63 and 2006/07 in similar locations. Based on this comparison and the analysis of certain language variables, significant evidences can be found that characterize the diachronic development of this Low German dialect over two centuries.

The aim of this study is to show in six examples how the linguistic approximation of Low German to Standard German occurs and how these changes can be described appropriately. In order to make the process of approximation understandable I will identify so-called standard-close dialect variants and standard-identical variants in phonetics-phonology, morphosyntax and lexicon.

The interpretation of the results is orientated on the concepts of "language dynamics" and "synchronization", developed by Schmidt/Herrgen. Based on these concepts language variation can be understood as a necessary consequence of the communicative interaction between Low German and Standard German speakers. Besides an approximation of the Low German language system towards the Standard German language system (hypothesis I), these contact-induced variations lead to a decrease of the speaker's dialect competence (hypothesis II). In connection with that an increase of variants and non-dialect elements is to be noticed (hypothesis III). In the analysis these crucial effects can be linked with the socio-communicative factor of language acquisition that changed during the observed time: from a primary Low German to a primary Standard German acquisition.
\end{abstract}

\section{Einleitung}

Die niederdeutsche Sprache befindet sich seit rund 500 Jahren in einer mehr oder weniger dauerhaften Konkurrenzsituation zur hochdeutschen Standardsprache. Seit der zweiten Hälfte des 19. Jahrhunderts ist in der alltäglichen Kommunikation 
im Norden Deutschlands ein verstärkter und bis heute kontinuierlicher Rückgang im Gebrauch des Niederdeutschen zugunsten des hochsprachlichen Sprechens feststellbar. In dem jahrhundertelangen Nebeneinander von standarddeutscher Prestigesprache und niederdeutscher Volkssprache kam es zu zahlreichen wechselseitigen Beeinflussungen auf sprachlicher Ebene.

Der folgende Beitrag beschäftigt sich explizit mit der diachronischen Entwicklung der niederdeutschen Sprache auf dem relativ abgeschlossenen und kleinräumigen Gebiet der Insel Rügen im Zeitraum zwischen dem Ende des 19. und dem Anfang des 21. Jahrhunderts. Vorwiegendes Interesse der Studie ist es, anhand einzelner dialektaler Sprachmerkmale die sprachliche Annäherung des Niederdeutschen an das Hochdeutsche exemplarisch zu beschreiben, und darzulegen, wie sich eine sprachsystemische Angleichung auf den Sprachebenen der Lexik, Morphosyntax und Phonetik-Phonologie vollzogen hat.

Zunächst erfolgt eine kurze Charakterisierung des mecklenburgisch-vorpommerschen Niederdeutsch unter Einbezug relevanter Forschungsresultate zu Sprachwandeltendenzen. Eine kurze Einführung zum Niederdeutschen auf Rügen nebst Vorstellung der Untersuchungshypothesen schließt sich an. Danach werden die zugrundeliegenden Sprachdaten und die Analysemethode erläutert. Im empirischen Teil werden die Resultate der Sprachvariablenanalyse vorgestellt und diskutiert sowie in einem Resümee abschließend zusammengefasst.

\section{Niederdeutsch in Mecklenburg-Vorpommern}

Sprachlich gehört das Plattdeutsche auf Rügen zur mecklenburgisch-vorpommerschen Mundart, die dem Ostniederdeutschen zuzuordnen ist. Als typische Besonderheiten des mecklenburgisch-vorpommerschen Sprachgebietes gelten die Vokalerhöhung $o>u$ und $e>i$ vor $r$ (Uhr,Ohr', mihr , mehr'), die Diminutivendung -ing, sowie die Monophthongierung des Diphthongs /âi/ (steet, steht', deet ,tut'). ${ }^{1}$ Das Mecklenburgisch-Vorpommersche kennzeichnet ein relativ einheitliches Laut- und Formensystem. Als lange Zeit grenzbildendes Unterscheidungsmerkmal galt für Vorpommern, d. h. auch für Rügen, der $g$-Einschub im Hiat bei niederdeutschen Verben wie schniegen, schneien' und dreigen ,drehen. Auf mecklenburgischer Seite wurde dagegen $d$ gesprochen: schnieden, dreiden. Diese sprachgeographische Differenzierung kann heutzutage allerdings nicht mehr als feste Lautgrenze zwischen beiden Regionen bezeichnet werden. ${ }^{2}$ Die wohl mar-

1 Vgl. Schröder 2004, S. 50.

2 Vgl. den Artikel zur Hiattilgung im Mecklenburgischen von Klaas-Hinrich Ehlers in diesem Band. 
kanteste Sprachgrenze innerhalb des pommerschen Sprachraums bildet die Linie Landgraben-Zarow, die den nördlichen vorpommerschen vom südlichen mittelpommerschen Sprachraum trennt. Noch bis heute auffällige Sprachmerkmale im gesprochenen Nieder- und Hochdeutschen des mittelpommerschen Gebietes sind beispielsweise die Auslautveränderung -er $>-a$ (Mutter $>$ Mutta) und die Spirantisierung $g>j$ (ganz $>$ janz). Sprachliche Differenzen zwischen dem vorpommerschen Niederdeutsch und dem gesprochenem Dialekt auf Rügen werden in Kapitel 3 thematisiert.

Neuere Sprachwandelphänomene im mecklenburgisch-vorpommerschen Dialekt wurden seinerzeit von Dahl (1974), Herrmann-Winter (1974) und Gernentz (1975) beschrieben. Danach lassen sich unter dem Oberbegriff „Regionalisierung" drei maßgebliche Sprachwandeltendenzen subsumieren, die im 130-jährigen Untersuchungszeitraum auch für das Niederdeutsche auf Rügen gelten können:

1. Der schrittweise Abbau kleinräumiger lexikalischer, morphologischer und phonetisch-phonologischer Dialektmerkmale zugunsten einheitlicher großregionaler Dialektformen.

2. Die Integration hochdeutscher Sprachelemente in das niederdeutsche Sprachsystem.

3. Die Übernahme niederdeutscher Sprachmerkmale in die Umgangssprache/ den Regiolekt. ${ }^{3}$

Der unter 2. angesprochene und hier vorrangig im Fokus stehende Sprachkontakt zwischen den beiden Varietäten wird von den genannten Autoren anhand synchron beobachteter Sprachphänomene beschrieben. ${ }^{4}$ Die komplexen Vorgänge, die beim Aufeinandertreffen beider Sprachsysteme mit Hinzunahme einer diachronischen Perspektive eine Rolle spielen, werden deutlicher verständlich mit dem von Schmidt/Herrgen entwickelten sprachdynamischen Grundprinzip der „Synchronisierung". Ausgangspunkt dieses theoretischen Ansatzes ist der per se heterogene Charakter von gesprochener Sprache, der aus der Tatsache resultiert, dass lebenslang verschiedenste Situationen von Sprechern sprachlich bewältigt werden und Differenzen im individuellen Sprachwissen der Einzelsprecher verursachen. Im kommunikativen Austausch finden

3 Vgl. zum Regiolekt auf Rügen den Artikel von Lars Vorberger in diesem Band.

4 Je nach der im Zentrum stehenden Fragestellung wird der niederdeutsch-hochdeutsche Sprachkontakt von Dahl 1974, Herrmann-Winter 1974 und Gernentz 1974 in ihren Beiträgen mit den Begriffen „Integration“, „Transferenz“, „Interferenz“ und „Alternanz“ beschrieben.

5 Schmidt/Herrgen 2011. 
ständig stabilisierende bzw. modifizierende Synchronisierungsprozesse mit den Sprachkompetenzen anderer Sprecher statt, die längerfristig zu Umstrukturierungen im Sprachwissen einer Sprachgemeinschaft führen können und damit Sprachwandel ermöglichen. ${ }^{6}$

Der im Untersuchungszeitraum auf Rügen stattgefundene Sprachkontakt wird als Resultat solcher Abgleichungsprozesse verstanden, die durch sprachkognitivindividuelle sowie kommunikativ-soziale Faktoren in Gang gesetzt wurden, um gegenseitiges Sprachverständnis zu gewährleisten. Mit Bezug auf diese Faktoren soll in der Sprachvariablenanalyse deutlich werden, inwieweit sich das niederdeutsche Sprachsystem als flexibel genug erweist, hochdeutsche Sprachmerkmale zu integrieren bzw. vollständig zu übernehmen oder inwieweit hochdeutsche Merkmale gar nicht integriert werden. In einer neueren empirischen Arbeit zur diachronischen Entwicklung des Niederdeutschen in Schleswig kann Viola Wilcken am Beispiel einiger Sprachvariablen ebenfalls einen zunehmenden hochdeutschen Spracheinfluss nachweisen. ${ }^{7}$ Weil im Untersuchungszeitraum im Sprachgebiet der Insel Rügen ebenfalls die Synchronisierungsprozesse mit der hochdeutschen Standardsprache dominieren, spielen die sogenannten „standardnahen niederdeutschen Dialektvarianten“ und die „standardidentischen Varianten“ eine entscheidende Rolle. Sie sollen dabei helfen zu verdeutlichen, wie sich Sprachwandel vollzieht.

\section{Niederdeutsch auf Rügen}

Zur regionalen Spezifik des Niederdeutschen auf der Insel Rügen, aber auch zu allgemeinen Sprachwandeltendenzen im Dialekt Norddeutschlands, gibt der 2013 erschienene Sprachatlas für Rügen und die vorpommersche Küste auf 50 Sprachkarten anschaulich und detailliert Auskunft. ${ }^{8}$ In der diachronen Dokumentation von 180 Jahren Niederdeutsch auf Deutschlands größter Insel wird in Einzelkarten, aber ebenso in der Gesamtschau deutlich, dass der Sprachraum auch durch die geografische Vielgliedrigkeit der Insel selbst und der zugehöri-

6 Vgl. dazu besonders Schmidt/Herrgen 2011, S. 28-33.

7 Wilcken kann bei der Übertragung der Wenkersätze ins regionale Niederdeutsch bei ihren Probanden mehrfach eine Zunahme im Gebrauch standarddeutscher Sprachelemente feststellen. Beispielsweise wird besonders bei den jüngeren Sprechern, die vor 1977 geboren wurden, eine sprachliche Angleichung an standarddeutsche Konjunktionen deutlich sowie auf Lautebene eine Annäherung an den hochdeutschen Vokalismus erkennbar. Vgl. Wilcken 2013, S. 24 und S. 32.

8 Herrmann-Winter 2013.

Birte Arendt, Andreas Bieberstedt and Klaas-Hinrich Ehlers - 978-3-631-71893-3 
gen Halbinseln und Inseln geprägt ist, und das zum Teil bis heute. Auffälligstes regionalspezifisches Sprachmerkmal ist wahrscheinlich die Entwicklung der mittelniederdeutschen Langvokale $e^{2}$ und $o^{1}$. Im rügenschen Kernland wird bis heute der Monophthong in Schnee/Koken gegenüber dem vorpommerschen Festland mit Schnei/Kauken gesprochen. Die diphthongische Artikulation ist, möglicherweise geprägt durch die frühe deutsche Besiedlung und durch kirchliche Einflussnahme, bis heute auf der Insel Hiddensee, Ummanz und Mönchgut stabil. ${ }^{9}$ Der Sprachatlas veranschaulicht eine Reihe weiterer lexikalischer, aber auch morphologischer Sprachspezifika Rügens, die teils im deutlichen Kontrast zur Merkmalsverbreitung auf dem vorpommerschen Festland stehen. ${ }^{10}$ Bei den hier untersuchten Sprachvariablen sind rügenspezifische Sprachmerkmale und ein, wie auch immer gearteter Inselcharakter, allerdings kein explizites Thema. Daher bleibt auch unberücksichtigt, ob die niederdeutsche Sprache im groben Einzugsgebiet Rügens als beständiger oder intakter gegenüber anderen Regionen bezeichnet werden kann.

Als Ergebnis der oben erwähnten Forschungsbeiträge von Dahl, HerrmannWinter und Gernentz können unter Zuhilfenahme des sprachdynamischen Ansatzes folgende Leithypothesen formuliert werden, die in der folgenden Untersuchung ausgewählter Sprachvariablen überprüft werden:

Hypothese I: Stetige Abnahme der niederdeutschen Sprachkompetenz.

Im Untersuchungszeitraum vom 19. bis 21. Jahrhundert nimmt die Bedeutung des niederdeutschen Dialekts als gesprochene Alltagssprache kontinuierlich ab. Unmittelbare Folge ist ein beständiger Abbau der dialektalen Sprachkompetenz, die an einer über die Sprechergenerationen hinweg abnehmenden Verwendung niederdeutscher Sprachmerkmale deutlich wird. Die Reduktion des individuellen niederdeutschen Sprachwissens der Dialektsprecher ist auf allen Sprachebenen nachweisbar.

9 Vgl. Herrmann-Winter 2013, Karte 32, 35. 1252 wurde der größte Teil der Halbinsel Mönchgut dem Kloster Eldena bei Greifswald übergeben. Ab 1296 gehörte die Insel Hiddensee zum Kloster Neuenkamp bei Stralsund. Die Insel Ummanz zählt ab 1341 zum Besitz des Heiliggeisthospitals zu Stralsund. Es kann vermutet werden, dass das charakteristische diphthongische Sprechen in diesen Sprachgebieten durch die vom vorpommerschen Festland ausgehende Besiedlung begründet wurde. Möglicherweise begünstigte die geographische Randposition und Isoliertheit der Inseln und Halbinseln zusätzlich die jahrhundertelange Tradierung dieser Sprechweise, die im starken Gegensatz zur monophthongischen Artikulation im Kerngebiet Rügens steht.

10 Vgl. Herrmann-Winter 2013, Karte 1, 2, 3, 4, 6, 8, 9, 10, 11, 12. 
Hypothese II: Sprachliche Annäherung an das Hochdeutsche.

Der bestimmende Faktor für die meisten sprachlichen Veränderungen im gesprochenen Niederdeutsch auf Rügen ist der Einfluss der hochdeutschen Sprache. Auf lexikalischer, morphologisch-syntaktischer und auf phonetisch-phonologischer Ebene vollziehen sich im Untersuchungszeitraum sprachliche Angleichungsprozesse („Synchronisierungen“) zwischen Mundart und Standardsprache. Die sprachstrukturelle Annäherung ist im Niederdeutschen am zunehmenden Gebrauch „standardnaher niederdeutscher Dialektvarianten“ bzw. „standardidentischer Varianten“ zu erkennen.

Hypothese III: Zunahme des Variantenspektrums und der nicht-niederdeutschen Sprachelemente.

Die geringer werdende dialektale Sprachkompetenz verursacht vor allem bei den sekundär im Dialekt sozialisierten Mundartsprechern eine zunehmende Unsicherheit in der Realisierung basisdialektaler Sprachelemente. In der jüngeren Sprechergeneration ist deshalb eine rückläufige Tendenz in der Verwendung herkömmlicher niederdeutscher Lexeme, Flexionsformen und Lautmerkmale festzustellen. Bei den primär in der Standardsprache sozialisierten Sprechern ist allgemein eine Zunahme von Umsetzungsvarianten nachweisbar, ihr Niederdeutsch weist eine größere Vielzahl von Varianten auf, die meist am Standarddeutschen orientiert sind.

\section{Sprachkorpus und Methodik}

Die hier vorgestellte Untersuchung ist entstanden innerhalb des Projekts „Sprachatlas für Rügen und die vorpommersche Küste“, das von 2006 bis 2013 vom Pommerschen Wörterbuch der Universität Greifswald von Renate Herrmann-Winter geleitet wurde. Als eigenständige Studie wurde sie im Rahmen meiner Masterarbeit 2009 durchgeführt und legt für das Untersuchungsgebiet Rügen dieselben Sprachkorpora zugrunde wie das Gesamtprojekt. Im Fokus standen aber andere Sprachvariablen, die gezielt in Hinsicht auf eine diachrone Sprachvariablenanalyse ausgewählt wurden. Untersuchungsgrundlage sind die 40 standarddeutschen Abfragesätze von Georg Wenker, die 1879/1880 an alle Schulen des damaligen Deutschen Reichs mit der Aufforderung verschickt wurden, sie in den Ortsdialekt zu übertragen. Hinzu kommt Tonbandmaterial aus dem Pommerschen Wörterbuch Greifswald, das 1962/63 von Herrmann-Winter, mit leicht veränderten Ab- 
fragesätzen, erhoben wurde. ${ }^{11}$ Die vollständigen Wenkersätze wurden 2006/07 von Herrmann-Winter und mir erneut auf Rügen abgefragt. Qualitative und quantitative Vergleichsbasis meiner Untersuchung sind also die dialektalen Übertragungen der Abfragesätze aus zumeist gleichen Rügen-Erhebungsorten aus drei Zeitstufen im Zeitraum von 130 Jahren:

- 1880 („WB“ - Wenkerbögen): 20 verschriftete Wenkerbögen von 20 Orten auf Rügen.

- 1962/63 („MA“ - Mittlere Aufnahmen): 10 Orte mit jeweils 15 Personen, die vor 1910 (,alte Generation“), und 15 Personen, die zwischen 1910 und 1940 („mittlere Generation“) geboren wurden.

- 2006/07 („NA“ - Neue Aufnahmen): 15 Orte mit jeweils 15 Personen, die vor 1942 („alte Generation“), und 15 Personen, die zwischen 1942 und 1972 („mittlere Generation“) geboren wurden.

Im Folgenden werden sechs von insgesamt zwölf untersuchten Sprachvariablen präsentiert. Die zur jeweiligen Variable gehörenden Ausgangsvarianten werden im betreffenden Abschnitt eingangs vorgestellt. In einem Diagramm ist die relative Häufigkeit der Varianten in den Abfragesätzen gestaffelt nach den Erhebungszeiträumen veranschaulicht. Bei den lexikalischen Variablen 1, 2 und 3 und der morphosyntaktischen Sprachvariable 4 ist an der linken Achse des zugehörigen Diagramms die Anzahl der durchgesehenen WB bzw. der untersuchten Sprecher verzeichnet (NA $30=$ Neue Aufnahmen mit 30 Sprechern). Rechts unterhalb des Diagramms ist die Häufigkeit des Auftretens der Sprachvariable in den Einzelerhebungen angegeben (WB (1) = ein Beleg im Wenkerbogen des jeweiligen Ortes).

Bei den phonetisch-phonologischen Sprachvariablen 5 und 6 ist die relative Häufigkeit der Varianten nach zugrundeliegender Erhebung zusätzlich nach den in den Aufnahmen vertretenden Sprechergenerationen (alte Generation, mittlere Generation) differenziert (MA-aG = alte Generation der Mittleren Aufnahmen, NA-mG $=$ mittlere Generation der Neuen Aufnahmen). Zudem sind die Kennwörter der hochdeutschen Standardsätze, durch welche die dialektalen Phoneme in vergleichbarer Lautumgebung untersucht werden konnten, unterhalb des Diagramms verzeichnet. Ebenfalls ist angegeben, wie oft die Wörter in den jeweiligen Übersetzungsvorlagen auftraten. Anschließend wird auf die auffälligsten Veränderungen in den Verteilungsverhältnissen der Varianten hingewiesen und Erklärungsansätze diskutiert.

11 Genaue Informationen zum Hintergrund der Einzelerhebungen sowie den Erhebungsorten finden sich im Sprachatlas, vgl. Herrmann-Winter 2013, S. 15-16. 


\section{Sprachvariablenanalyse}

\subsection{Lexikalische Sprachvariablen}

Variable 1: Adverb all versus schon

In der diachronen Untersuchung werden die Gebrauchsanteile für die dialektale Umsetzung des standarddeutschen Adverbs ,schon/bereits' dargestellt. In den niederdeutschen Übersetzungen der Gewährspersonen stehen sich die Lexeme all und schon gegenüber.

Abbildung 1: all versus schon

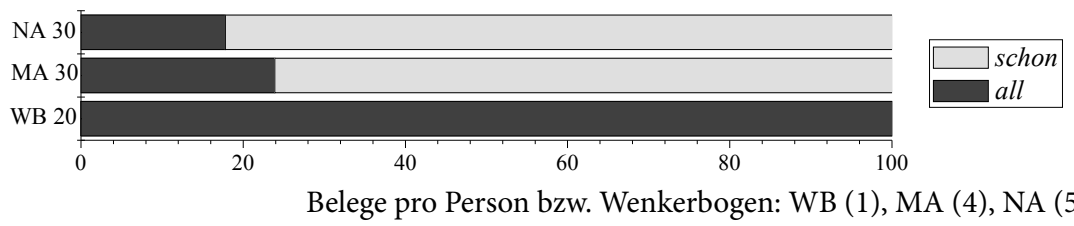

In den Wenkerbögen (WB) wird ausnahmslos die Variante all verwendet. Dementsprechend kann davon ausgegangen werden, dass all in der Funktion als temporales Adverb am Ende des 19. Jahrhunderts das gebräuchliche dialektale Lexem ist. In den Mittleren Aufnahmen (MA) sinkt der Anteil für all auf etwa $25 \%$, während schon zu $75 \%$ nachweisbar ist. In den Neuen Aufnahmen (NA) nimmt der Wert für schon nochmals zu, wohingegen sich der all-Anteil weiter verringert. Ein tendenzieller Unterschied zwischen den Sprechergenerationen innerhalb der MA und NA ist nicht feststellbar. Erwähnenswert ist die Tatsache, dass bei den Informanten aus den Untersuchungsregionen Hiddensee, Ummanz und Mönchgut zumeist all nachgewiesen wird.

Aus dem diachronen Vergleich geht hervor, dass die Anteile für all in den MA und NA deutlich abnehmen. Die Dominanz des Lexems in den WB bestätigt den basisdialektalen Status des niederdeutschen Adverbs. Die signifikante Reduktion des Lexems hängt sehr wahrscheinlich mit dem hochdeutschen Einfluss zusammen. Die rasche Zunahme der Anteile für die standardidentische Variante schon, d.h. die vollständige Übernahme des hochdeutschen Lexems in das Niederdeutsche, ist wohl auch darauf zurückzuführen, dass eine schrittweise Umphonologisierung des hochdeutschen Lexems in eine standardnahe Dialektvariante nicht angemessen möglich ist (vgl. Sprachvariable 2). Obwohl das sprachstrukturelle Angleichungspotenzial an das dialektale Lautsystem bei schon nicht vorhanden ist, wird das Lexem problemlos in die niederdeutsche Kommunikation 
integriert. Der Grund ist in der im Untersuchungszeitraum zunehmenden Synchronisierung mit der Standardsprache zu vermuten. So wird bei einer Vielzahl von Sprechern aus Hiddensee, Mönchgut und Ummanz noch all nachgewiesen. ${ }^{12}$

Zusammenfassend kann in der diachronen Untersuchung eine deutliche Reduktion im Gebrauch des basisdialektalen Adverbs all nachgewiesen werden. Entsprechend ist für den Gebrauch des Lexems eine Abnahme im dialektalen Sprachwissen zu konstatieren (Hypothese I). Die direkte Übernahme und der hohe Gebrauchsanteil für die standardsprachliche Alternative schon belegen die lexikalische Annäherung der niederdeutschen Varietät an die hochdeutsche Standardvarietät (Hypothese II).

Variable 2: Interrogativadverb $w u(r)$ väl versus wieviel

Für die Realisierung des Interrogativadverbs $w u(r) v a ̈ l / w o v a ̈ l$, wieviel, wieviele wird dagegen in der Gegenüberstellung von Sprachdaten aus den Jahren 1880 und 2006 deutlich, dass sich die Annäherung des niederdeutschen Frageadverbs an die standardsprachliche Entsprechung, in einer schrittweisen Umphonologisierung an das hochdeutsche Lexem vollzieht. ${ }^{13}$

Abbildung 2: Interrogativadverb wu(r)väl versus wieviel

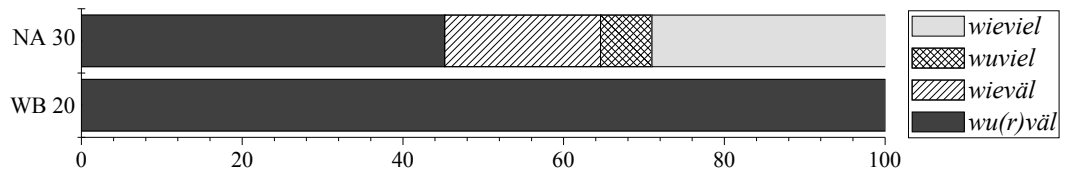

Belege pro Person bzw. Wenkerbogen: WB (1), NA (1)

In den WB ist ausschließlich die Variante $w u(r) v a ̈ l$ verzeichnet. Dadurch wird der basisdialektale Status des Adverbs bzw. der Konjunktion wur , wie gegenüber der hochdeutschen Lautform wo ,wie bestätigt. $^{14}$ In den NA beträgt der Anteil

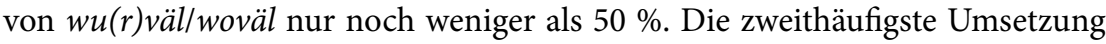
erfolgt mit der hochdeutschen Entsprechung wieviel. Weiterhin werden die Lautvarianten wieväl und wuviel nachgewiesen.

12 Möglicherweise erweist sich die niederdeutsche Sprachkompetenz dieser Informanten durch intaktere dialektale Sprachkollektive resistenter gegenüber vollständigen Übernahmen aus dem Hochdeutschen.

13 In der Übersetzungsvorlage der MA kam das Adverb wieviel nicht vor, deshalb werden hier nur die WB und NA miteinander verglichen.

14 Vgl. MWB, Bd. 7, S. 1580 und Herrmann-Winter 2003, S. 391. 
Maßgebliche Ursache für die Abnahme der Anteile des niederdeutschen In-

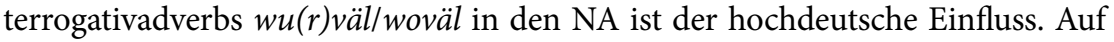
die ausschließliche Realisierung des niederdeutschen Lexems in den WB folgen in den NA Lautvarianten, die eine sich schrittweise vollziehende Lautassimilation an das hochdeutsche Äquivalent erkennen lassen (Hypothese II). Die progressive Lautangleichung vollzieht sich in der ersten Silbe von wur- $>$ wu- $>$ wo- $>$ wieund in der zweiten Silbe von -väl > -viel. In dieser Abfolge wird die lexikalische Annäherung des dialektalen Lexems an die hochdeutsche Entsprechung deutlich. Die nachgewiesenen Lautvarianten wieväl und wuviel können als standardnahe lexikalischen Dialektvarianten bezeichnet werden, wohingegen wieviel das standardidentische Frageadverb darstellt. Die Zunahme von lexikalischen Lautvarianten ist ein Beleg für die stärkere Verunsicherung der Informanten beim Gebrauch bestimmter niederdeutscher Lexeme (Hypothese III). Aufgrund der Veränderungstendenzen in der Artikulation des Interrogativadverbs kann von einer Abnahme in der lexikalischen Sprachkompetenz ausgegangen werden (Hypothese I).

Variable 3: Pronomen dat versus es

In der diachronen Gegenüberstellung der niederdeutschen Übersetzungen aus den drei Zeitstufen werden die Anteile für die niederdeutschen Umsetzungen des standarddeutschen Pronomens ,es' miteinander verglichen. Das Pronomen tritt in der 3. Person Singular im Nominativ bzw. Objektkasus auf, d.h. ,es' übernimmt in den hochdeutschen Abfragesätzen die Rolle des Subjekts oder des Dativ-/ Akkusativobjekts. Für gewöhnlich erfolgt die Übertragung ins Niederdeutsche mithilfe von dat. Vor dem Ausgang des 18. Jahrhunderts sind auch die Varianten it/et belegt. ${ }^{15}$ Das Pronomen befindet sich bei fünf der untersuchten acht Erhebungssätze der NA am Satzanfang.

Abbildung 3: dat versus, es

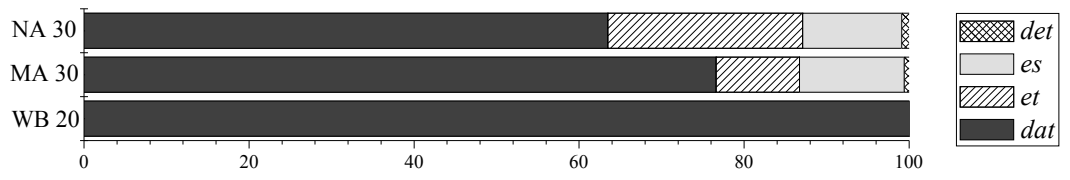

Belege pro Person bzw. Wenkerbogen: WB (3), MA (5), NA (8)

15 Lasch verweist auf die Doppelformen it (et) des Personalpronomens im Nominativ und Akkusativ Singular Neutrum, Lasch 1914, S. 217. Im MWB ist verzeichnet, dass „et, it, ' $t$ nur in älterer Sprache noch voll gebräuchlich [ist]“, denn die pronominalen Flexionsvarianten wurden „seit dem Ausgange des 18. Jahrhunderts, allmählich durch dat verdrängt.“ MWB, Bd. 2, S. 764. 
In den WB ist die ausnahmslose Realisation mit dat belegt. Die Flexionsvarianten es und et werden in den MA mit einem Anteil von annähernd $11 \%$ nachgewiesen. Entsprechend verringert sich der dat-Anteil. Von einem Sprecher wird det realisiert. In den NA reduziert sich der Anteil für dat weiter. Er beträgt nunmehr $63 \%$. Während der Gebrauchsanteil für es in den NA konstant bleibt, nimmt der Wert für et zu. Er beträgt in den NA nahezu $25 \%$. Die Variante det wird dabei von zwei Sprechern realisiert.

In der Variablenuntersuchung wird der tendenzielle Rückgang im Gebrauch des Lexems dat, in der pronominalen Verwendung von ,es' deutlich. Die Ergebnisse der WB belegen den ausschließlichen Gebrauch für das ausgehende 19. Jahrhundert im rügenschen Sprachgebiet. In der Funktion als Pronomen, es' kann dat daher als basisdialektale Variante in diesem Zeitraum gelten. Zentrale Ursache für den Anteilsrückgang von dat ist der hochdeutsche Spracheinfluss. Die belegten Varianten det und et sind standardnahe Dialektvarianten. ${ }^{16} \mathrm{Ihr}$ sprachstrukturelles Angleichungspotenzial wird deutlich in der lautlichen Annäherung an das hochdeutsche Äquivalent, es'. Die Realisierung von det könnte zudem gestützt sein durch den Einfluss des berlin-brandenburgischen Regiolekts. Die lexikalische Annäherung des Niederdeutschen an das Hochdeutsche wird daran deutlich, dass sich die Anteile für beide Flexionsvarianten in den MA und NA vergrößern (Hypothese II). Durch den abnehmenden pronominalen Gebrauch von dat wird ein Abbau in der dialektalen Sprachkompetenz nachgewiesen (Hypothese I). Der Verlust an Sprachwissen zeigt sich besonders in der dialektalen Umsetzung des Pronomens am Satzanfang. Bei den Sprechern der MA und noch mehr bei denen der NA bezeugt zusätzlich das zögerliche Übersetzen und die allgemeine Variantenzunahme die Unsicherheit der Informanten (Hypothese III). Anstatt der Übertragung mit dat ist öfter et bzw. es nachweisbar. In beiden neueren Erhebungen bleibt der Anteil für die standardidentische Variante es konstant. Zuständig für den gleichen Beleganteil von es in den NA sind der hochdeutsche Einfluss und die geringere Dialektkompetenz dieser Informanten. Das Hochdeutsche ist vermutlich auch Ursache für die Verdoppelung der Anteile der standardnahen Dialektvariante et. Die Variante entstand wahrscheinlich durch Angleichung des

16 In diesem Zusammenhang sei auf die aufschlussreiche Darstellung von Michael Elmentaler zum Übergang von et zu dat im Schleswig-Holsteinischen Raum verwiesen. Hier wird in vergleichbarer Weise und anhand schriftlicher und mündlicher Sprachbelege fundiert deutlich, dass et als veraltete Dialektform in Schleswig-Holstein im Laufe des 19. Jahrhunderts zugunsten von dat verdrängt wird, und ab Mitte der zweiten Hälfte des 20. Jahrhunderts bis heute als Analogiebildung zu standarddeutschem es von Niederdeutschsprechern vereinzelt realisiert wird. Vgl. Elmentaler 2015, S. 304 und S. 321. 
hochdeutschen es an den niederdeutschen Lautstand. Ausschlaggebend für die Umphonologisierung ist womöglich das latente Sprachwissen um die markante Lautkorrespondenz zwischen niederdeutsch $t$ und hochdeutsch $s$, das vor allem bei den bivarietären Sprechern der MA und NA, die nach 1945 geboren wurden, zu vermuten ist. Außerdem sind sprachökonomische Motive für den Anstieg der et-Anteile anzuführen. Durch den Wegfall des anlautenden Dentals $d$ kann die Zungenbewegung zum oberen Zahndamm entfallen, wobei die morphologische Distinktionsleistung nicht verringert wird. Zudem wird durch die Variante et eine einfachere Koartikulation mit vorangehenden Lauteinheiten anderer Lexeme möglich. So erfolgt beispielsweise in Wenkersatz 10 „Ik will'et ... “in den MA und NA recht häufig die Umsetzung mit $e t$, weil dadurch eine effektive lautliche $\mathrm{Zu}$ sammenziehung mit vorausgehenden will realisiert werden kann. Die geringanteilig belegte standardnahe Dialektvariante det kann ebenfalls als Lautangleichung an das hochdeutsche Pronomen gelten.

\subsection{Morphologisch-Syntaktische Sprachvariablen}

Variable 4: 3. Person Singular Präteritum von daun/doon ,tun`

Untersucht werden die Flexionsvarianten für die Konjugation des starken Verbs daun/doon in der 3. Person Singular Präteritum. Im Niederdeutschen besitzt daun/doon aufgrund seiner vielfältigen Funktion als Hilfs-, Modal- oder Vollverb einen besonderen Status.

Abbildung 4: 3. Pers. Sg. Prät. daun/doon

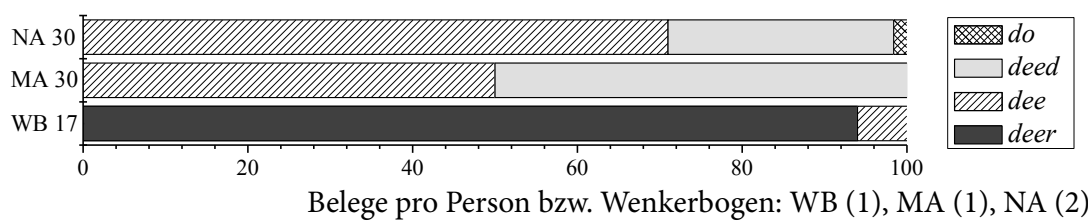

Die Präteritumsform deer ist in den WB am häufigsten belegt und demnach fester Bestandteil der morphosyntaktischen Sprachwissensstruktur der Informanten des 19. Jahrhunderts. Als entsprechende niederdeutsche Präteritalbildung für das unregelmäßige Verb ,tun wird von Wiggers 1858 noch deed angeführt. ${ }^{17}$ Die Flexionsvariante entwickelte sich durch Abfall des auslautenden $e$ in mnd. dede. Weil auslautendes $-d$ beim Sprechen starken Veränderungen unterliegt (Rhotazismus

17 Wiggers 1858, S. 79.

Birte Arendt, Andreas Bieberstedt and Klaas-Hinrich Ehlers - 978-3-631-71893-3 
d > r) kann in den Wenkerbögen von 1880 großanteilig deer nachgewiesen werden. Zu geringen Anteilen wird in den WB die Umsetzungen dee realisiert. Diese Präteritumsvariante ist die weiterentwickelte Flexionsform mit weggefallenem $r$ im Auslaut. ${ }^{18}$ In den MA ist dee bereits zu einem Anteil von $50 \%$ nachweisbar. Die restliche Realisierung erfolgt durch deed. Beide Präteritumsvarianten sind auch in den NA belegt. Während sich der dee-Anteil geringfügig steigert, sinken die Belege für deed in geringem Maße. Zusätzlich wird bei einem Sprecher die Variante do nachgewiesen.

Die Untersuchung macht deutlich, dass sich in der 3. Person Singular Präteritum von daun/doon mehrere sprachliche Veränderungen vollzogen haben. Hervorzuheben ist, dass die in den WB vorherrschende Flexionsvariante deer in den MA und NA nicht mehr realisiert wird. Ursachen hierfür sind vorrangig in der abnehmenden Dialektkompetenz der Informanten und in sprachökonomischen Entwicklungen zu suchen. Durch sprachartikulatorische Vereinfachung fällt der Endkonsonant $r$ in deer weg. Die Auslautreduktion führt zur suffixlosen Flexionsform dee, die geringanteilig bereits in den WB belegt ist und in den MA die Hälfte der Resultate ausmacht. Für die anderen Anteile in den MA ist die Vollform deed belegt. Ein bewusster Rückbezug der Sprecher auf die alte niederdeutsche Vergangenheitsform, die bei Wiggers dokumentiert wird, kann wohl ausgeschlossen werden. Vielmehr ist die Präteritalvariante mit auslautendem $-d$ als eine lautliche Weiterentwicklung der unmarkierten Flexionsform dee zu interpretieren, die sich wahrscheinlich durch Synchronisierung mit dem standardsprachlichen Äquivalent , tat' entwickelte. Der Schlusskonsonant $-d$ stellt demnach eine lenisierte Lautsynchronisierung zum auslautenden - $t$ in der hochdeutschen Flexionsvariante dar. Die phonetische Ähnlichkeit der beiden Abschlussdentale hat vermutlich bei den Informanten der MA und NA dazu geführt, deed so häufig zu realisieren. Vermutlich durch eine solche Lautangleichung mit der hochdeutschen Entsprechung entstanden, kann diese niederdeutsche Präteritumsform demnach auch als standardnahe Dialektvariante gelten, und ist dann ein Indiz für die sprachstrukturelle Annäherung an das Hochdeutsche (Hypothese II).

Die Anteile für die Präteritalform dee nehmen im gesamten Untersuchungszeitraum konstant zu. Dies könnte auf die sprachökonomische Attraktivität der Flexionsform ohne spezifischen Abschlusslaut zurückgeführt werden. Ungeklärt bleibt die von einem Sprecher in den NA artikulierte ungewöhnliche Umsetzung do. Im Mittelniederdeutschen ist dô die 1. Person Singular Präsens Indikativ. ${ }^{19}$ Die

18 Vgl. dazu Lasch 1914, S. 246 und Wiggers 1858, S. 79.

19 Vgl. Lasch 1914, S. 246.

Birte Arendt, Andreas Bieberstedt and Klaas-Hinrich Ehlers - 978-3-631-71893-3 
Resultate der MA und NA für die 3. Person Singular Präteritum von daun/doon, und vor allem der Verlust der basisdialektalen deer-Realisation, sind Zeichen der geringeren dialektalen Sprachkompetenz der jüngeren Sprechergeneration (Hypothese I). Die Variantenzunahme für das Präteritum des im Gebrauch sehr frequenten niederdeutschen Verbs ist ein Anzeichen für die größere Unsicherheit der Sprecher bei der Bildung basisdialektaler Flexionsformen (Hypothese III).

\subsection{Phonetisch-phonologische Sprachvariablen}

Variable 5: Rundung von kurzem /I/

Die Rundung von kurzem /I/ ist ein typisches Kennzeichen im Vokalismus der niederdeutschen Mundart. Wahrscheinlich war dieses Sprachmerkmal tendenziell bereits in mittelniederdeutscher Zeit vorzufinden. Die Rundung ist gebunden an die lautliche Umgebung labialer Konsonanten. Schirmunski benennt als konkreten Lautkontext die „Umgebung von Lippenlauten und l, bisweilen vor n. ${ }^{\text {“20 }}$ Die Rundung des Vokals wird hier anhand des Verbs bün ,bin' untersucht. In der Laienverschriftung der WB konnte eine ausnahmslose Umsetzung der Übersetzungsvorlage bin mit $<\ddot{u}>$ nachgewiesen werden, was auf eine gerundete Aussprache hindeutet. In den mündlichen niederdeutschen Umsetzungen von bin in den MA und NA stehen gerundete Realisierungen ([byn]) neben ungerundeten ([bin]).

Abbildung 5: Rundung kurzes/I/

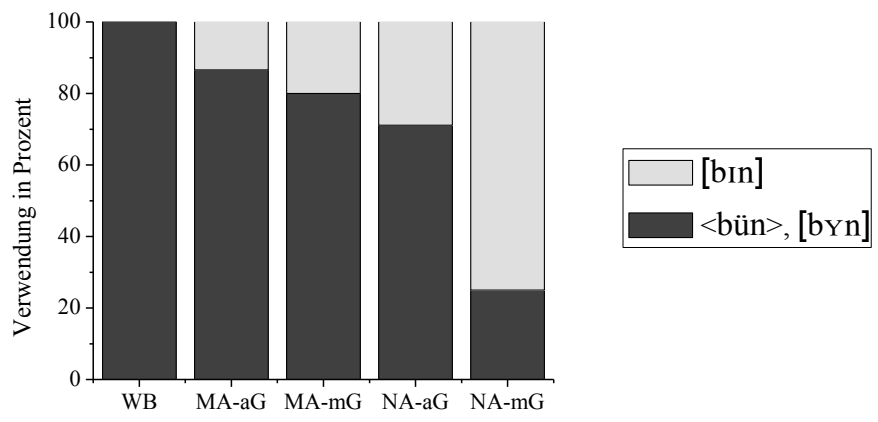

Kennwort ,bin': Belege pro Person bzw. Wenkerbogen: WB (2), MA (1), NA (3) 
Im diachronen Vergleich der gerundeten niederdeutschen Lautvarianten für hochdeutsch ,bin' wird die stetige Abnahme im Gebrauch dieses dialektalen Sprachmerkmals nachgewiesen. Anhand der WB wird deutlich, dass die Rundung von /I/ in labialer Umgebung um 1880 ein verbindliches Merkmal im Niederdeutschen der Insel Rügen war. In beiden Sprechergenerationen der MA bis zur alten Generation der NA sinkt der Anteil der gerundeten Formen stetig von $86 \%$ zu $80 \%$ auf $71 \%$. Eine Zäsur in der gleichmäßig verlaufenden Reduktion der [Y]-Artikulation erfolgt in der mittleren Generation der NA. Der Rundungsanteil verringert sich bei diesen jüngsten Informanten um nahezu die Hälfte, d.h. die im Erhebungszeitraum unter 55-jährigen Niederdeutschsprecher realisieren zu etwa $75 \%$ das ungerundete [I].

Die Gründe für den Abbau dieses Lautmerkmals sind in der Hauptsache auf den Einfluss des Hochdeutschen zurückzuführen. Das sprachstrukturelle Angleichungspotenzial ist dadurch gegeben, dass die ungerundete Lautvariante [bin] mit der hochdeutschen Verbform übereinstimmt und so problemlos in die niederdeutsche Kommunikation integriert werden kann. Die vermehrte Häufigkeit der ungerundeten Lautvariante kann also mit der im Untersuchungszeitraum zunehmenden hochdeutschen Primärsprachsozialisation und dem geringeren Dialektgebrauch in Zusammenhang gebracht werden. Bei den Sprechern der MA ist zu vermuten, dass durch "passiv“ gehörtes und aktiv gesprochenes Hochdeutsch bewusste und unbewusste Synchronisierungsprozesse mit der ungerundeten Variante [bIn] stattgefunden haben. Beim zumeist sekundären Dialekterwerb der Folgegeneration konnte dann die einfache Übernahme der bereits aus der hochdeutschen Primärstruktur bekannten ungerundeten Lautvariante erfolgen. Weil durch diesen Verlust an dialektaler Kompetenz bei der mittleren Generation der NA die gerundete Variante [byn] vermutlich gar nicht mehr als eigentliche niederdeutsche Variante wahrgenommen wird und im Sprachgebrauch von [bin] verdrängt wird, sinkt der Anteil des gerundeten /I/ bei der Sprechergeneration entschieden.

Es ist davon auszugehen, dass die Abnahme des niederdeutschen Sprachmerkmals zusätzlich begünstigt wird durch einen sprachökonomischen Vorteil der ungerundeten, offeneren Lautvariante. Im Gegensatz zu [byn] kann bei [bin] nach der Artikulation des bilabialen Plosivs die Zungenposition konstant gehalten werden. Weil die Zungenposition bei [I] etwas höher als bei [Y] liegt, kann im sofortigen Anschluss der Laut [n] gebildet werden. Der geringer werdende Anteil der gesprochenen niederdeutschen Phonem/Lexem-Zuordnung von $[\mathrm{Y}]$ in bün wird durch die einfachere Artikulation der standardidentischen Lautvariante bin gestützt. 
Im Untersuchungszeitraum wandelt sich das Untersuchungsmerkmal der /I/Rundung in labialer Umgebung von einem festen, obligatorischen zu einem fakultativen niederdeutschen Sprachmerkmal im Sprachgebiet Rügens. In der diachronen Untersuchung konnte eine Annäherung an das hochdeutsche Lautsystem festgestellt werden (Hypothese II). Die zunehmende Tendenz zur Entrundung von $[\mathrm{Y}]$ lässt auf eine Abnahme der dialektalen Sprachkompetenz im Fundamentalbereich des niederdeutschen Lautsystems schließen (Hypothese I).

Variable 6: phonetische Realisierungen von /r/ in prävokalischer Stellung

Ein typisches Lautmerkmal des niederdeutschen Konsonantismus ist das gerollte Zungenspitzen- $r$ (phonetisch: [r]). Der im prävokalischen Lautkontext artikulierte Konsonant ist in der standarddeutschen Aussprache nicht obligatorisch. Es wird an den Lexemen Broder, Fru, torüch/retour, räd und öwrigens untersucht, ob und inwieweit sich [ $\mathrm{r}$ ] als typisches Merkmal des niederdeutschen Lautsystems gegenüber dem, vor allem im Standard, aber auch im Dialekt gesprochenen Reibe- $r$ (phonetisch: [в]), behaupten konnte. Da die schriftlichen Umsetzungen der WB für die Artikulation von /r/ keine Rückschlüsse erlauben, können sie nicht in den diachronen Vergleich einbezogen werden.

\section{Abbildung 6: Lautrealisierungen prävokalisches $/ r /$}

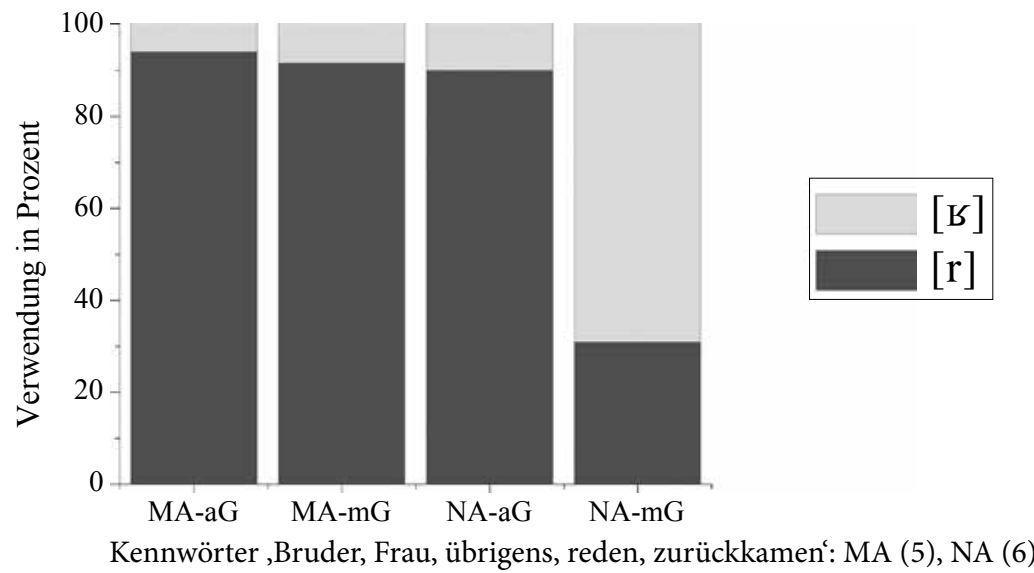

In den ersten drei aufgenommenen Sprechergenerationen liegt der Anteil für die Artikulation des Zungenspitzen- $r$ konstant bei nahezu $90 \%$. Trotz der nicht vorhandenen Sprachdaten in den WB kann die Aussprache von prävokalischem $r$ als $[r]$ auch in diesem Erhebungszeitraum als sicheres Kennzeichen des nieder- 
deutschen Lautsystems gelten. Das diachron konstante Sprachmerkmal wird in den ersten drei Altersgruppen von annähernd allen Informanten realisiert. Die geringen Anteile der [в]-Artikulation sind vornehmlich bei räd und Fru belegt. Die bemerkenswerteste Entwicklung des Sprachmerkmals wird in den Resultaten der mittleren Generation der NA deutlich: Die Reduzierung der typisch niederdeutschen $/ \mathrm{r}$-Artikulation um fast $60 \%$. Die zumeist sekundär niederdeutsch sozialisierten Informanten sprechen das gerollte Zungenspitzen- $r$ nur noch mit einem Anteil von $30 \%$.

Bei den älteren Sprechergenerationen fällt auf den ersten Blick die gleichbleibend hochfrequente und verlässliche [r]-Aussprache auf. Die Artikulation des alveolaren Vibranten scheint bei den primär im Dialekt sozialisierten Sprechern fest in der lautsystemischen Dialektkompetenz verankert. Die Konstanz in der Phonem/Lexem-Zuordnung mit $[r]$ ist in den drei Sprechergenerationen noch weitestgehend vorhanden. Nur unwesentlich unterbrochen wird die gemeinhin lexemunabhängige Stabilität des Lautmerkmals durch die erwähnte, geringanteilige Umsetzung [ Beide Lautvarianten sind vermutlich das Resultat von Synchronisierungen mit den hochdeutschen Lexemen.

Augenscheinlich wird der hochdeutsche Einfluss bei der Folgegeneration, der mittleren Generation der NA. Die überwiegend uvulare Artikulation von /r/ ist zurückzuführen auf die Standardsprache. Das sprachstrukturelle Angleichungspotenzial von [в] ist unmittelbar gegeben, weil das Reibe- $r$ in den Lautsystemen beider Varietäten vorhanden ist. Die standardidentische dialektale Lautvariante wird von dieser Sprechergeneration nicht nur aufgrund des allgemein geringen Dialektgebrauchs bevorzugt. Zusätzlich ist die Aussprache des stimmhaften Frikativs einfacher für die primär in der Standardsprache sozialisierten Sprecher, weil der physiologische Lautbildungsvorgang aus dem Hochdeutschen bereits bekannt ist. Schwieriger zu klären ist die Frage, warum die Erhebungsanteile für das niederdeutsche Lautmerkmal innerhalb von nur einer Sprechergeneration so deutlich gesunken sind. Wahrscheinlich wurde die Weitergabe von [r] zwischen den beiden Sprechergenerationen nicht nur durch die zwangsläufigen Synchronisierungen mit dem allgegenwärtigen Hochdeutsch gestört, sondern möglicherweise, weil ein Korrigieren der mittleren Generation durch die alte Generation nicht explizit stattgefunden hat. Denkbar ist, dass die alte Generation eine lautliche Berichtigung der mittleren Generation schlichtweg nicht für notwendig ansah, denn die mundartliche und standardsprachliche Verständigung ist durch die unterschiedliche Realisierung der /r/-Artikulation nicht beeinträchtigt. Im Umkehrschluss ist es demnach auch für die mittlere Generation nicht notwendig, in das primär erworbene hochsprach- 
liche Lautsystem ein niederdeutsches Lautmerkmal zu integrieren, das zudem nur in einem speziellen Lautkontext realisiert wird.

Zusammenfassend kann in der diachronen Sprachmerkmalsanalyse eine deutliche Veränderung im Lautsystem des Niederdeutschen festgestellt werden. Bei den bivarietären Dialektsprechern ist in der prävokalischen / $\mathrm{r}$-Artikulation ein ausgeprägter Lautwandel von $[\mathrm{r}] \mathrm{zu}[\mathbf{~} \mathrm{B}]$ nachweisbar. Wilcken kann für die jüngeren Sprecher, die nach 1977 in Schleswig geboren wurden, dieselben Resultate feststellen. ${ }^{21}$ In Bezug auf das Lautmerkmal ist in dieser Sprechergeneration ein Verlust an Sprachkompetenz im Fundamentalbereich des niederdeutschen Lautsystems dokumentierbar (Hypothese I). Die Lautentwicklung geht einher mit einer sprachsystemischen Annäherung des dialektalen an das standardsprachliche Lautsystem (Hypothese II). Als maßgeblicher Beweggrund für den sehr raschen und deutlichen Abbau des niederdeutschen Sprachmerkmals in der mittleren Generation werden der in dieser Sprechergeneration nicht mehr stattfindende orale niederdeutsche Primärspracherwerb und die stattdessen vorherrschenden Synchronisierungsakte mit der Hochsprache angeführt. Das Zungenspitzen- $r$ vor einem Vokal gilt nicht mehr unbedingt als ein offensichtliches und markantes Lautmerkmal des niederdeutschen Lautsystems. Der Vergleich der Tonaufnahmen unterschiedlicher Zeitstufen verdeutlicht, dass prävokalisch [r] bzw. [в] zumindest auf der Insel Rügen als recht sicheres distinktives Merkmal in der Unterscheidung zwischen primär und sekundär sozialisierten Mundartsprechern gelten kann. ${ }^{22}$

\section{Zusammenfassung}

In der Untersuchung konnte gezeigt werden, dass innerhalb des fast 130-jährigen Untersuchungszeitraums auf allen Sprachebenen des niederdeutschen Dialekts der Insel Rügen sprachliche Veränderungen stattgefunden haben. Die eingangs formulierten Hypothesen wurden dabei grundsätzlich bestätigt.

Die sprachliche Annäherung an das Hochdeutsche wird im diachronen Vergleich ausgewählter niederdeutscher Sprachvariablen als vorherrschende Sprachentwicklungstendenz deutlich (Hypothese II). Als unmittelbare Folge wird ein zunehmendes Variantenspektrum in den untersuchten Sprachvariablen beobacht-

21 Vgl. Wilcken 2013, S. 32-33.

22 Diese Annahme wird gestützt durch Vorbergers Untersuchung zum „Hochdeutsch auf Rügen" in diesem Band. Dort realisiert ein 1971 geborener, primär im Dialekt sprachsozialisierter Proband (RUEGALT2) aus Bergen in allen Erhebungssituationen des REDE-Projekts durchweg apikale/r/-Varianten. 
bar, das die größer werdende sprachliche Unsicherheit der Informanten in der Bildung lautlicher, morphologischer und lexikalischer niederdeutscher Sprachelemente offenbart (Hypothese III). Neben den höheren Anteilen standardnaher Dialektvarianten werden zunehmend standardidentische Varianten, wie zum Beispiel schon statt all, wieviel statt wu(r)väl, bin statt bün, es statt dat nachgewiesen. Ein sprachstrukturelles Angleichungspotenzial in Richtung Standardsprache wird in den belegten Varianten wieväl und wuviel (Sprachvariable 2), det und et (Sprachvariable 3) sowie deed (Sprachvariable 4) offenbar. Die Möglichkeit einer schrittweisen Annäherung an die Standardsprache ist bei Sprachvariable 1 nicht gegeben, und folglich verdrängt schon die basisdialektale Variante rapide.

Eine vollständige Reduktion von basisdialektal deer als Präteritalvariante von daun/doon, wird in den Aufnahmen von 2006 nachgewiesen. Der rasche Verlust basisdialektaler Sprachvarianten in den neueren Aufnahmen wird mit dem vorwiegend sekundärsprachlichen Dialekterwerb der untersuchten mittleren Generationen der NA in Zusammenhang gebracht, und kann anhand zweier Lautmerkmale bestätigt werden. Sowohl bei der Rundung des kurzen /I/ (Sprachvariable 5) als auch bei der Artikulation von /r/ in prävokalischer Stellung (Sprachvariable 6) kann eine lautliche Annäherung an die phonetisch-phonologische Struktur des Hochdeutschen aufgezeigt werden. Die Resultate auf lautlicher Ebene verdeutlichen, dass eine generationelle Weitergabe der beiden niederdeutschen Lautmerkmale nur unzureichend stattgefunden hat. Als unmittelbarer sozio-kommunikativer Faktor spielt dabei wahrscheinlich der Verlust des erstsprachlichen Niederdeutscherwerbs der Generation, die nach 1945 geboren wurde, eine entscheidende Rolle.

Insgesamt verdeutlichen die Untersuchungsresultate eine kontinuierliche Abnahme in der dialektalen Sprachkompetenz der rügenschen Dialektsprecher (Hypothese I). Im Untersuchungszeitraum wurde demnach die Kommunikation im niederdeutschen Dialekt, also der ständige Abgleich von dialektalem Sprachwissensbeständen zwischen den Plattsprechern auf Rügen, längerfristig durch die dominierende Hochsprache „gestört“. Der niederdeutsche Dialekt ist als zuverlässiges und probates Mittel zur sprachlichen Bewältigung der täglichen Lebenswelt der Rüganer merklich von der Hochsprache zurückgedrängt worden.

Es wird zu zeigen sein, ob sich ähnliche Resultate zur Sprachkompetenz auch in anderen Untersuchungsgebieten Norddeutschlands sowie in Studien zum frei gesprochen Niederdeutsch finden lassen. Dabei scheint es, neben den hier dargestellten Beispielen zur sprachsystemischen Annäherung an das Hochdeutsche, ebenfalls von Interesse aufzuzeigen, in welcher Weise sich welche Sprachmerkmalen des Niederdeutschen als besonders resistent gegenüber hochsprachlichen Einflüssen verhalten. 


\section{Literatur}

Dahl, Eva Sophie: Interferenz und Alternanz - zwei Typen der Sprachschichtenmischung im Norden der Deutschen Demokratischen Republik. In: Ising, Gerhard (Hrsg.): Aktuelle Probleme der sprachlichen Kommunikation. Soziolinguistische Studien zur sprachlichen Situation in der Deutschen Demokratischen Republik. Berlin 1974, S. 339-387.

Ehlers, Klaas-Hinrich: Meihen, meiden, meigen ,mähen ' - Zur Hiattilgung im mecklenburgischen Niederdeutsch. In diesem Band.

Elmentaler, Michael: Dialektaler Wandel in Schleswig-Holstein. Die wechselvolle Geschichte von et und dat. In: Langhanke, Robert (Hrsg.): Sprache, Literatur, Raum. Festgabe für Willy Diercks. Bielefeld 2015, S. 301-351.

Gernentz, Hans Joachim: Die kommunikative Funktion der niederdeutschen Mundart und hochdeutschen Umgangssprache im Norden der Deutschen Demokratischen Republik, unter besonderer Berücksichtigung der Interferenz und der Alternanz zwischen diesen beiden sprachlichen Existenzformen. In: Studia Germanica Gandensia 15 (1974), S. 209-244.

Herrmann-Winter, Renate: Auswirkungen der sozialistischen Produktionsweise in der Landwirtschaft auf die sprachliche Kommunikation in den Nordbezirken der DDR. Unter Mitarbeit v. Christa Baufeld, Irmgard Rosenthal, Max Groth und Jürgen Gundlach. In: Ising, Gerhard (Hrsg.): Aktuelle Probleme der sprachlichen Kommunikation. Soziologische Studien zur sprachlichen Situation in der DDR (Veröffentlichungen des Zentralinstituts für Sprachwissenschaft der Akademie der Wissenschaften der DDR, Reihe Sprache und Gesellschaft; 2). Berlin 1974, S. 135-190.

Hermann-Winter, Renate: Plattdeutsch-hochdeutsches Wörterbuch für den mecklenburgisch-vorpommerschen Sprachraum. 5. Aufl. Rostock 2003.

Herrmann-Winter, Renate: Sprachatlas für Rügen und die vorpommersche Küste. Kartographie Martin Hansen. Rostock 2013.

Lasch, Agathe: Mittelniederdeutsche Grammatik (Sammlung kurzer Grammatiken germanischer Dialekte; 9). Halle 1914.

MWB = Mecklenburgisches Wörterbuch. Hrsg. von der Sächsischen Akademie der Wissenschaften zu Leipzig. Aus den Sammlungen Richard Wossidlos und aus den Ergänzungen und nach der Anlage Hermann Teucherts. 7 Bde. Berlin 1942-1992. Unveränd., verkleinerter Nachdruck der Erstauflage von 1937-1992. Neumünster 1996. Rothe, Christian: Nachtrag und Index. Neumünster 1998. 
Schirmunski, Viktor M.: Deutsche Mundartkunde. Vergleichende Laut- und Formenlehre der deutschen Mundarten (Deutsche Akademie der Wissenschaften zu Berlin. Veröffentlichungen des Instituts für deutsche Sprache und Literatur; 25). Aus dem Russischen übersetzt von Wolfgang Fleischer. Berlin 1962.

Schmidt, Jürgen Erich/Herrgen, Joachim: Sprachdynamik. Eine Einführung in die moderne Regionalsprachenforschung (Grundlagen der Germanistik; 49). Berlin 2011.

Schröder, Ingrid (2004): Niederdeutsch in der Gegenwart. Sprachgebiet - Grammatisches - Binnendifferenzierung. In: Stellmacher, Dieter (Hrsg.): Niederdeutsche Sprache und Literatur der Gegenwart. Hildesheim 2004, S. 35-97.

Vorberger, Lars: Hochdeutsch auf Rügen. Eine Untersuchung zum Regiolekt in Bergen auf Rügen. In diesem Band.

Wiggers, Julius (1858): Grammatik der plattdeutschen Sprache. In Grundlage der Mecklenburgisch-Vorpommerschen Mundart, 2. Aufl. Hamburg 1858.

Wilcken, Viola (2013): Wandeltendenzen im Nordniederdeutschen. Dialektproben im diachronen Vergleich. In: Hettler, Yvonne/Jürgens, Carolin/Langhanke, Robert/Purschke, Christoph (Hrsg.): Variation, Wandel, Wissen. Studien zum Hochdeutschen und Niederdeutschen. Frankfurt a. M. [u. a.], S. 15-36. 
Birte Arendt, Andreas Bieberstedt and Klaas-Hinrich Ehlers - 978-3-631-71893-3

Downloaded from PubFactory at 01/11/2019 10:11:06AM

via free access 


\title{
Lars Vorberger
}

\section{Hochdeutsch auf Rügen - Eine Untersuchung zum Regiolekt in Bergen auf Rügen}

\begin{abstract}
This paper presents a synchronous study of the linguistic behavior of three speakers from different generations from the town Bergen on Rügen. Different records of the competence and the usage of High German are analyzed. It can be shown that High German-based talk on Rügen is presumably more regionally marked than in other regions of northern Germany. Furthermore, two different linguistic styles are identified due to the quantity and quality of regional features. Moreover two different patterns of linguistic behavior can be presented that could be attributed to the linguistic primary socialization.
\end{abstract}

\section{Hinführung}

Die Insel Rügen nimmt innerhalb des mecklenburgisch-vorpommerschen Dialektverbandes, für den gemeinhin eine große Einheitlichkeit angenommen wird, ${ }^{1}$ eine sprachliche Sonderstellung ein. ${ }^{2}$ Diese äußert sich in vielerlei Hinsicht und war wie das Niederdeutsche der Insel insgesamt bisher Gegenstand zahlreicher Untersuchungen. ${ }^{3}$ Hansen stellt für das letzte Jahrhundert einen Wandel des Gebrauchs im Niederdeutschen fest, mit der Folge, dass die niederdeutschen Basisdialekte für den kommunikativen Alltag der Inselbewohner - mit Ausnahmen - an Bedeutung verlieren. ${ }^{4}$ Hochdeutsch basierte Sprechlagen ${ }^{5}$ gewinnen demgegenüber an Bedeutung. Über dieses standardnähere, dennoch regional geprägte Sprechen liegen wenige Erkenntnisse vor. ${ }^{6}$ Bisherige Studien zum hochdeutsch basierten Teil des regionalsprachlichen Spektrums im niederdeutschen

1 Vgl. Schröder 2004; Wiesinger 1983; Gernentz 1980.

2 Vgl. u. a. Hansen 2009.

3 Vgl. u. a. Herrmann-Winter 2013; Hansen 2009 siehe auch den Beitrag von Hansen in diesem Band.

4 Hansen 2009, S. 42. Vgl. auch Gernentz 1974.

5 Hochdeutsch ist hier nicht als subjektiver Begriff für die Standardvarietät zu verstehen, sondern im Sinne einer binären Gegenüberstellung als struktureller und sprachgeografischer Gegensatz zum Niederdeutschen.

6 Mit Stellmacher 1977 setzt die Beschäftigung mit dem nichtdialektalen Bereich des regionalsprachlichen Spektrums im norddeutschen Raum ein. Die Erforschung des gesamten Spektrums als neues Paradigma der Variationslinguistik/Dialektologie setzt sich in den 2000er Jahren durch (vgl. Schmidt 1998; Schmidt/Herrgen 2011; Kehrein 2012). 
Raum konnten einen recht standardnahen Regiolekt aufzeigen. ${ }^{7}$ Es ist zu fragen, ob diese Ergebnisse vor dem spezifischen sprachlichen (sowie geografischen) Hintergrund auch für Rügen gelten. Der vorliegende Beitrag wird dieser Frage nachgehen und den Regiolekt ${ }^{8}$ für einen Untersuchungsort auf Rügen erhellen. $\mathrm{Zu}$ diesem Zweck werden für verschiedene Situationen (intendierte Standardsprache und freies Sprechen verschiedenen Formalitätsgrades) die phonetischphonologischen Merkmale samt deren Variation analysiert.

Hierfür wird im nächsten Abschnitt ein kurzer Abriss der bisherigen Erkenntnisse zum Regiolekt in Norddeutschland präsentiert. Darauf folgt die Vorstellung der verwendeten Daten und der angewendeten Methoden. In Abschnitt 4 werden die Ergebnisse der Analyse besprochen, systematisiert und ein Bezug zu bisherigen Studien hergestellt, um abschließend ein Resümee zu fassen.

\section{Regiolekt in Norddeutschland}

Zum Regiolekt in Norddeutschland, d. h. dem niederdeutschen Sprachraum, liegen recht wenige Beschreibungen der auftretenden Merkmale - teilweise im Sinne niederdeutsch bedingter Standarddifferenzen - vor. ${ }^{9}$ König unternimmt eine umfassende Untersuchung der Vorleseaussprache und der dort auftretenden Regionalismen. ${ }^{10}$ Sein Untersuchungsraum ist die ehemalige BRD, sodass zwar Erkenntnisse für den westlichen Teil Norddeutschlands vorliegen, nicht jedoch für den hier untersuchten Raum. ${ }^{11}$ Für den mecklenburgisch-vorpommerschen Raum im Speziellen liegen folgende Erkenntnisse vor:

7 Vgl. beispielsweise Kehrein 2012 und Vorberger/Schröder 2011.

8 Zum Begriff Regiolekt vgl. Schmidt/Herrgen 2011, S. 66-67. Regiolekt wird hier stets als hochdeutsch basierter Regiolekt verstanden. Die besondere sprachliche Situation im niederdeutschen Raum bedingt Unterschiede im Aufbau des regionalsprachlichen Spektrums sowie in der Genese des Regiolektes im Vergleich zum Mittel- und Oberdeutschen. Hier konnte bisher kein dialektnaher (und somit gegebenenfalls niederdeutsch basierter) Regiolekt im Sinne einer Ausgleichvarietät aufgezeigt werden (vgl. u. a. Kehrein 2012). Kehrein 2012 konnte für zwei Erhebungsorte im niederdeutschen Raum empirisch nachweisen, dass der Regiolekt ausschließlich hochdeutsch basierte Sprechlagen umfasst. Mischprodukte lassen sich zwar zeigen, sind jedoch nicht als Regiolekt zu beschreiben, sondern als alternierende hochdeutsche und niederdeutsche Passagen (vgl. Kehrein 2012, S. 305-309).

9 Vgl. u. a. Martens/Martens 1988; Trenschel 1997. Oft bleiben jedoch die genauen (empirischen) Grundlagen und Informationen zur möglichen Variation der Merkmale unklar.

10 Vgl. König 1987.

11 Stellmacher 1977; Lameli 2004 und Vorberger/Schröder 2011 beschäftigen sich auch mit dem norddeutschen Regiolekt (beziehen sich dabei wiederum auf den westlichen 
Dahl unterscheidet in ihrer Beschreibung der Sprachvariation im Norden der ehemaligen DDR fünf verschiedene Sprachschichten: (1) hochsprachliches Hochdeutsch, (2) hochsprachenahes (umgangssprachliches) Hochdeutsch, (3) Hochdeutsch mit niederdeutscher Beimischung (mundartnahe Umgangssprache), (4) Niederdeutsch mit hochdeutscher (vor allem umgangssprachlicher) Beimischung und (5) niederdeutsche Mundart. ${ }^{12}$ Den bisherigen Erkenntnissen zufolge können daher die Sprachschichten 2 und 3 dem Regiolekt zugeordnet werden. Somit lassen sich hier erste Hinweise auf eine Variation und somit gegebenenfalls auch auf die Struktur innerhalb des Regiolekts für den mecklenburgisch-vorpommerschen Raum finden. Dahl zählt dann auch die sprachlichen Merkmale auf, die sowohl im hochsprachenahen Hochdeutsch als auch im Hochdeutsch mit niederdeutscher Beimischung vorkommen sowie jene, die nur in Sprachschicht 3 auftreten.

Lauf beschreibt ausgehend von der Datenbank regionaler Umgangssprachen die Merkmale der einzelnen Regiolekte. ${ }^{13}$ Neben gemeinsamen Varianten der norddeutschen regionalen Umgangssprachen - wie beispielsweise der Zusammenfall von /e:/ und / $\varepsilon: / z u$ /e:/ oder der auslautenden Spirantisierung von /g/ - charakterisiert sie die Besonderheiten der Regiolekte der einzelnen dialektalen Großräume des Niederdeutschen. Dem Regiolekt des Mecklenburgisch-Vorpommerschen attestiert sie „so viele Gemeinsamkeiten mit der nordniedersächsischen Umgangssprache ${ }^{\text {"14 }}$, dass sie auf deren Auflistung verweist. Unterschiede könnten jedoch

Teil) und dessen Merkmale, doch liegt der Fokus dieser Untersuchungen auf der Diachronie (apparant- und real-time-Studien). Da die vorliegende Untersuchung synchron ist und mögliche diachrone Aspekte nur streift, sei an dieser Stelle lediglich auf die Studien verwiesen. Der Norddeutsche Sprachatlas (NOSA) von Elmentaler/ Rosenberg 2015 erschien erst nach Abschluss des Manuskripts und konnte hier nicht mehr berücksichtigt werden.

12 Vgl. Dahl 1974, S. 343-345. Datengrundlage für die Untersuchung sind Aufnahmen und Texte aus dem Raum Rostock (vgl. Dahl 1974, S. 345; S. 382-387).

13 Vgl. Lauf 1994; 1996. Lauf 1994, S. 2-5; 1996, S. 193-197 definiert regionale Umgangssprache als regional bedingte Abweichungen von der Norm der deutschen Standardsprache - mindestens auf der lautlichen Ebene -, deren Verwendung räumlich eindeutig begrenzt ist. Dies entspricht in gewisser Weise in der Konzeption der modernen Regionalsprachenforschung dem Regiolekt. Datengrundlage Laufs sind umgangssprachliche Aufnahmen aus verschiedenen Korpora, die sie nach den Kriterien hoher regionaler Prägung, guter technischer Qualität und hoher Repräsentativität der Sprecher ausgewählt hat (vgl. Lauf 1994, S. 8-11). Für den mecklenburgischvorpommerschen Regiolekt wurden Aufnahmen aus Rostock, Greifswald und Malchin verwendet (vgl. Lauf 1994, S. 55).

14 Lauf 1994, S. 55. 
in der Frequenz bestehen; qualitativ unterscheidet sich die mecklenburgischvorpommersche Umgangssprache von der nordniedersächsischen durch velares /1/, den Ersatz des palatalen stl. Frikativs durch den velaren und die Lenisierung inlautender, stl. Frikative. ${ }^{15}$ Bei Lauf findet sich somit eine detaillierte empirische Beschreibung der Merkmale des mecklenburgisch-vorpommerschen Regiolekts.

Die Charakteristika des mecklenburgisch-vorpommerschen Regiolekts sind demnach - neben den bereits genannten - die Spirantisierung von inlautend /b/, Vokalkürze in verschiedenen Kontexten, Zentralisierungs- und Rundungstendenzen von /I/, Zusammenfall von /I, Y, $/$ und /e, ø, o/, Diphthongierung von /e:, ø:, $\mathrm{o}: /$, Vorverlagerung/Hebung von /a/ / sowie Verdumpfung von /a/, Elision von /t, d/ in diversen Kontexten, Vorverlagerung und Hebung von Tiefschwa, Realisierung von Affrikaten als Frikative, Ausfall von /r/ ohne Ersatzdehnung, oraler Verschluss von $<-$ ng $>$, Hebung von $/ \varepsilon: /$, verschiedene Assimilationserscheinungen, Nasalität (v. a. /a /, Systematik unsicher), /l/-Vokalisierung und unverschobene Formen in bestimmten Lexemen (dat, ik usw.). ${ }^{16}$

Kehrein bezieht in seiner Studie zur Vertikale in den deutschen Regionalsprachen auch den ostniederdeutschen Sprachraum ein. ${ }^{17}$ Am Untersuchungsort Stralsund analysiert er das variationslinguistische Spektrum. Für den hochdeutsch basierten Teil des Spektrums kommt er zu folgendem Ergebnis: Sämtliche Aufnahmen außer die Dialektkompetenzerhebungen aller Sprecher und das Freundesgespräch des alten Sprechers ${ }^{18}$ ordnet er dem Regiolekt Stralsunds zu. Diesen beschreibt er als „relativ standardnah ${ }^{\text {“19, }}$, was den phonetischen Abstandswerten

15 Vgl. Lauf 1994, S. 55.

16 Vgl. Dahl 1974; Lauf 1994; Trenschel 1997. Die Auflistung ist eine Zusammenstellung und Zusammenfassung der beschriebenen Merkmale. Zur genauen Beschreibung der Merkmale, deren Auftretenskontext und Beispielen vgl. Dahl 1974, S. 346-349; Trenschel 1997, S. 207-208 und Lauf 1994, S. 42-46; 55. Für das Hochdeutsch mit niederdeutscher Beimischung beschreibt Dahl 1974, S. 348-353 neben den oben bereits genannten Merkmalen der Diphthongierung, der /âa/-Verdumpfung, des /r/-Ausfalls, der /d/-Elision, der Aussprache von / $\widehat{\text { ts}} /$ als [s] zusätzlich die Aussprache von anlautend $/ \mathrm{j} /$ als $[3, \widehat{\mathrm{d}}]$ ], die Bewahrung des niederdeutschen Kurzvokals wie in [dis] dies(es) und die Bewahrung des niederdeutschen Vokalismus wie in [Yme] immer.

17 Vgl. Kehrein 2012.

$18 \mathrm{Zu}$ den Erhebungsmethoden des REDE-Projekts, an das sich auch Kehreins Analyse anschließt, vgl. Abschnitt 3. Die Dialektkompetenzerhebung von HST1 stellt eine Ausnahme dar, da der Sprecher hier fast „,ausschließlich standardnahe Formen“ (Kehrein 2012, S. 305) produziert und diese daher auch dem Regiolekt zugeordnet werden kann.

19 Kehrein 2012, S. 307. 
von 0,4 bis $0,8 \mathrm{zu}$ entnehmen ist..$^{20}$ Der Regiolekt zeichnet sich insgesamt durch eine „sehr geringe individuelle Variationsbreite“21 aus, sodass Kehrein keine Differenzierung in Sprechlagen im Sinne von Verdichtungsbereichen variativer Sprachverwendung möglich erscheint. Für den Regiolekt im norddeutschen Raum hält er fest, dass dieser zwar zahlreiche regionale Abweichungen von der Standardsprache aufweist, diese jedoch meist tendenziell sind. Als remanente Merkmale, das heißt Merkmale, die in den standardintendierten Sprechlagen erhalten bleiben und somit nicht kontrolliert werden können, beschreibt er folgende: Diphthongierung von /e:, ø:, o:/, Rundung von /I/, Hebung von / $\varepsilon: /$, auslautende /g/-Spirantisierung, Lenisierung von inlautend / $\mathrm{t} /$, oraler Verschluss von <-ng>, Realisierung von anlautend germ. $\mathrm{p}$ als [f], Ausfall von /d/ in /nd/ und $/ \mathrm{t} / \mathrm{in} / \mathrm{st} / \mathrm{im}$ In- und Auslaut, Erhalt von unverschobenen Tenues (nur in Kennwörtern ich, das, was), vereinzelt /b/-Spirantisierung, Schwächung von /e/ in nebenbetonten Stammsilben und Vorverlagerung/Hebung von Tiefschwa. ${ }^{22}$

\section{Daten und Methoden}

Die Daten der vorliegenden Untersuchung sind dem Forschungsprojekt Regionalsprache.de (REDE) entnommen. Dieses wird durchgeführt am Forschungszentrum Deutscher Sprachatlas der Philipps-Universität Marburg und ist gefördert durch die Akademie der Wissenschaften und der Literatur in Mainz. Projektbeginn war 2008 und die Laufzeit beträgt insgesamt 19 Jahre. Eines der großen Teilziele des Projekts ist die Ersterhebung und Analyse der linguistischen Struktur der modernen Regionalsprachen des Deutschen. Dazu wurden in 150 Orten der Bundesrepublik Deutschland Aufnahmen mit Sprechern aus drei Generationen in fünf bis sechs Erhebungssituationen durchgeführt. ${ }^{23}$

Einer der 150 Erhebungsorte des REDE-Projekts ist Bergen auf Rügen. Die ehemalige Kreisstadt hat ca. 13.500 Einwohner und ist heute eines der Mittelzentren im Bundesland Mecklenburg-Vorpommern. Für die Insel Rügen nimmt sie die Funktion eines Geschäfts-, Verwaltungs- und Verkehrszentrums ein (vgl. Bergen-I). Für die vorliegende Untersuchung wurden von den fünf erhobenen

20 Auch zur Methode der phonetischen Abstandsmessung und den jeweiligen Werten vgl. Abschnitt 3.

21 Kehrein 2012, S. 303.

22 Vgl. Kehrein 2012, S. 309-312.

23 Vgl. für genauere Informationen zum Projekt und dessen Zielen sowie zu den Erhebungsorten, den Sprechern und den Erhebungssituationen Schmidt/Herrgen/Kehrein 2008 ff. und zusammenfassend Kehrein 2012, S. 66-71. 
Sprechern drei ausgewählt - jeweils einer pro Generation. Die Informanten der älteren Generation sind über 65-jährige, männliche Sprecher, die (meist) einer handwerklichen oder landwirtschaftlichen Tätigkeit nachgegangen sind. Mit der mittleren Generation werden 45- bis 55-jährige Polizisten aufgezeichnet und schließlich in der jüngsten Generation 18- bis 23-jährige Abiturienten aus dem jeweiligen Ort. ${ }^{24}$ Der Sprecher RUEGALT2 ${ }^{25}$ war zum Zeitpunkt der Aufnahme 71 Jahre alt und hat zunächst als Hochseefischer und dann in der Fischereiaufsicht gearbeitet. Er hat sein ganzes Leben in Bergen auf Rügen verbracht, wohnt jedoch seit sieben Jahren in Saßnitz. RUEG1 war 49 Jahre alt, als die Aufnahmen gemacht wurden. Er hat vor seiner Tätigkeit als Polizist eine mechanische Ausbildung absolviert. Auch er ist als ortsfest zu klassifizieren. RUEGJUNG1 war 20 Jahre alt zum Aufnahmezeitpunkt. Er absolvierte zu dieser Zeit eine Lehre als Bankkaufmann.

Mit allen Sprechern wurden im Zeitraum von 2012-2013 Aufnahmen in verschiedenen Erhebungssituationen durchgeführt. Ausgewertet wurden die Freundesgespräche, die Interviews, die Abfrage der Standardsprache und die Vorleseaussprache der drei Sprecher. ${ }^{26}$ Beim Freundesgespräch handelt es sich um ein freies, halbstündiges Gespräch in informeller Atmosphäre. Die Informanten werden gebeten, sich mit einem ihnen vertrauten Gesprächspartner ohne Beisein Dritter zu unterhalten. Dabei werden sie mit kleinen Funkmikrofonen aufgezeichnet. Das Interview stellt eine formellere Situation dar. Hier führt eine Mitarbeiterin/ein Mitarbeiter des Projekts in gehobener Kleidung ein sprachbiografisches Interview mit dem Informanten. Bei der Abfrage der Standardsprache handelt es sich um die Übertragung der Wenkersätze von einer dialektalen Vorlage, die dem Ortsdialekt möglichst genau entspricht, in das individuell beste Hochdeutsch der Informanten (d. h. intendierter Standard), weswegen sie auch Standardübersetzung genannt wird. Die Vorleseaussprache - auch im Sinne einer Standardkompetenzerhebung - wird während des Vorlesens des Textes „Nordwind und Sonne“ aufgezeichnet. Bei den Situationen Freundesgespräch und Interview handelt es sich somit um Performanzsituationen (d. h. freie Gespräche). Durch den zunehmenden

24 Für weitere Informationen zu den Sprechern, den Auswahlkriterien, den Hintergründen usw. vgl. Schmidt/Herrgen 2011, S. 378-380 sowie Kehrein 2012, S. 74-78.

25 Zur Nomenklatur, die hier beibehalten wird, um Bezüge zu weiteren Beiträgen und zum REDE-System zu ermöglichen: RUEG = Bergen auf Rügen; ALT = ältere Generation, JUNG = jüngere Generation, keine Angabe = mittlere Generation; Ziffer = projektinterne Nummerierung der Sprecher.

26 Da die Untersuchung den hochdeutsch basierten Teil des regionalsprachlichen Spektrums fokussiert, wurde die Erhebung der Dialektkompetenz von den Analysen hier ausgeschlossen. 
Formalitätsgrad vom Freundesgespräch zum Interview wird versucht, eine zunehmende Orientierung an der Standardsprache zu evozieren. Bei der Abfrage der Standardsprache und der Vorleseaussprache wird wie gesagt versucht, die Standardkompetenz der Informanten in zwei verschiedenen Situationen zu erheben. ${ }^{27}$

Zur Auswertung wurde eine Kombination aus quantitativen und qualitativen Methoden gewählt. Zunächst wurde der Dialektalitätswert im Sinne des phonetischen Abstands zur Standardsprache der Sprachproben bestimmt, in einem zweiten Schritt wurden danach die standarddifferenten Varianten der einzelnen Proben ermittelt.

Die Messung des Dialektalitätswerts ist ein Verfahren zur Quantifizierung des phonetischen Abstands einer standarddifferenten sprachlichen Äußerung zu einem standardsprachlichen Bezugssystem. ${ }^{28}$ Dies bedeutet, dass die tatsächlich realisierte Äußerung, die phonetisch transkribiert wurde, segmentweise mit der normierten Standardlautung verglichen wird und die Abweichungen von dieser Norm - im Sinne eines Abstands - nach einem festen Punktesystem bewertet werden. Der sich daraus ergebende Rohwert wird zuletzt durch die Anzahl der gemessenen Wörter dividiert. Ein Wert von beispielsweise 1,0 bedeutet somit, dass in der gesamten Sprachprobe durchschnittlich in jedem Wort ein regionales Merkmal vorhanden ist. ${ }^{29}$ Die Messungen für den vorliegenden Beitrag wurden mithilfe des halbautomatischen Messprogramms PAM $^{30}$ durchgeführt, jedoch im Anschluss nochmals manuell korrigiert.

Um die den Dialektalitätswert bedingenden Merkmale zu ermitteln, wurden die standarddifferenten, regionalen Merkmale in den jeweiligen Aufnahmen (exhaustiv) erfasst. Eine herkömmliche Variablenanalyse ${ }^{31}$ mit Bestimmung der Frequenzen wurde nicht gewählt, da ohne Vorannahmen alle auftretenden Merkmale des Regiolekts beschrieben werden sollten. Auch nach der Bestimmung der Varianten erschien eine Variablenanalyse nicht sinnvoll, da für die meisten der

27 Vgl. hierzu auch Schmidt/Herrgen 2011, S. 379-380 sowie Kehrein 2012, S. 75-76.

28 Vgl. Herrgen/Lameli/Rabanus/Schmidt 2001. Das Verfahren wurde von Herrgen/ Schmidt 1989 entwickelt und von Lameli 2004 weiterentwickelt. Zur genauen Beschreibung des Verfahrens vgl. Herrgen/Lameli/Rabanus/Schmidt 2001; Lameli 2004 und zuletzt Kehrein 2012.

29 Es handelt sich bei diesem Abstandswert tatsächlich um einen Dialektalitätswert, da Abweichungen von der normierten Standardlautung, die idiolektal oder sprechsprachlich/realisationsphonetisch bedingt sind, aus der Messung ausgeschlossen werden und somit nur regional bedingte Abweichungen vermessen werden.

30 Vgl. Lüders 2013.

31 Vgl. etwa Stellmacher 1977 oder zuletzt Bieberstedt/Ruge/Schröder 2016. 
auftretenden Phänomene keine ausreichende Anzahl der Beleg für die möglichen Variablen zu finden war. ${ }^{32}$ Deshalb werden für die jeweiligen Sprachproben der Sprecher die auftretenden Regionalismen qualitativ bestimmt und systematisiert dargestellt. Mögliche Auffälligkeiten in der Frequenz, wie z. B. einmaliges Vorkommen, werden jedoch berücksichtigt und gegebenenfalls kommentiert.

\section{Analyse}

In Abbildung 1 sind die ermittelten durchschnittlichen phonetischen Abstandswerte für die jeweilige Sprachaufnahme der drei Sprecher zu sehen. ${ }^{33}$

Abbildung 1: Phonetische Abstandswerte der drei Sprecher aus Bergen

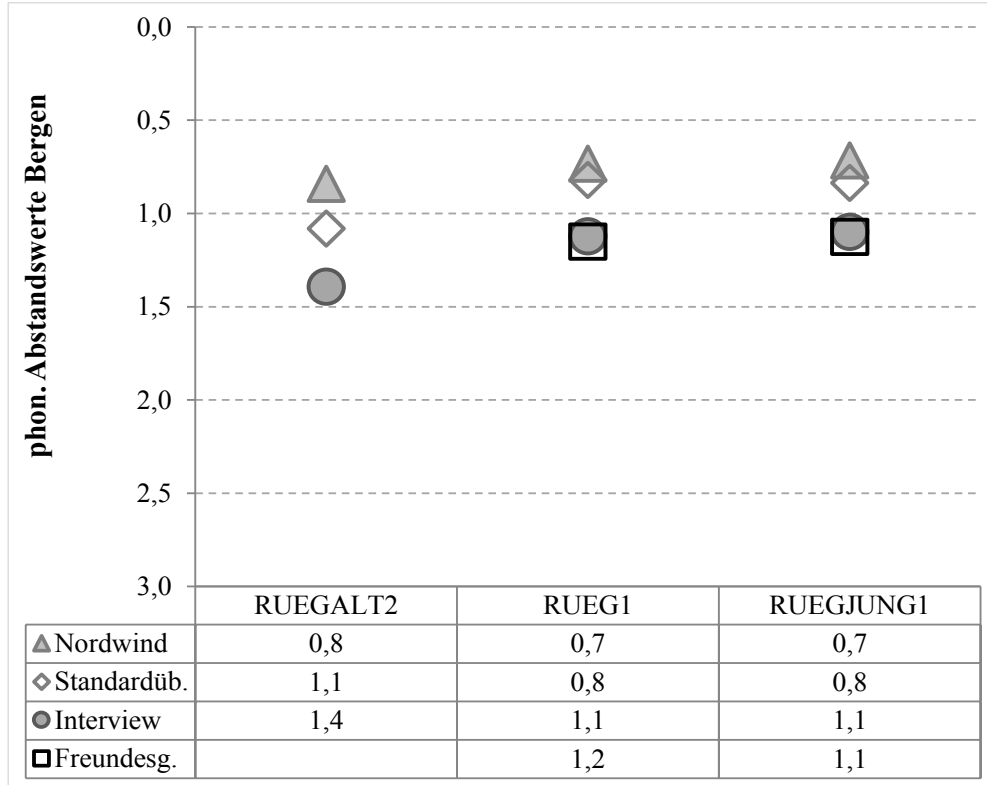

32 Zugrunde gelegt wurde die phonetische Transkription im Umfang von jeweils 250-300 Wörtern, die auch Grundlage der phonetischen Abstandsmessung war. Kehrein 2012, S. 86 setzt für valide Ergebnisse ein Minimum von zehn Belegen der Variable pro Sprachprobe fest. Mögliche Variablen wie beispielsweise germ. $p$ oder die Lautfolge [nd] treten in allen Sprachproben zu selten auf, um Frequenzen zu bestimmen.

33 Die Skala mit einem Maximum von 3 wurde trotz der geringeren Werte in dieser Analyse beibehalten, um eine bessere Vergleichbarkeit mit anderen Studien, die dasselbe Verfahren anwenden, zu gewährleisten. 
Das Freundesgespräch von RUEGALT2 fehlt in Abbildung 1 und in der Analyse, da es sich um ein niederdeutsches Gespräch handelt. Die Analyse hat ergeben, dass es dem Dialekt und nicht dem Regiolekt zugeordnet werden kann. Alle anderen Aufnahmen können dem Regiolekt zugeordnet werden (vgl. auch Merkmalsbeschreibung weiter unten). Bei der Betrachtung der Abstandswerte fällt zunächst auf, dass alle Sprachproben - und somit auch der Regiolekt - mit Werten zwischen 0,7 und 1,4 als nicht sehr standardnah zu charakterisieren sind. Auch die Variationsbreite innerhalb des Regiolekts (Umfang von 0,7) ist zwar nicht sehr ausgeprägt, doch auch nicht derart gering, wie für den norddeutschen Raum angenommen. Außerdem lässt sich festhalten, dass die jeweiligen $\mathrm{Ab}$ standswerte von RUEG1 und RUEGJUNG1 sehr ähnlich sind, beide also ein ähnliches Variationsmuster zeigen, wohingegen Unterschiede zu den Werten und dem Sprachverhalten von RUEGALT2 bestehen.

Bevor die Gesamtergebnisse für den Regiolekt und die Sprecher weiter analysiert werden, folgt eine Charakterisierung der einzelnen Aufnahmen.

\subsection{RUEGALT2}

Einer der hauptsächlichen Unterschiede zwischen den Sprachproben von RUEGALT2 und den beiden anderen Sprechern besteht in der durchgehenden Verwendung apikaler/r/-Varianten in allen Erhebungssituationen von RUEGALT2. Dies ist auch ein Grund, weshalb seine Dialektalitätswerte insgesamt höher sind als die der anderen Sprecher.

In seiner Vorleseaussprache treten folgende Merkmale auf: Rundungstendenzen [I] (bzw. Zentralisierungstendenzen) wie in mit [mİd], (tendenzielle) Lenisierung wie in stritten [ $\int \mathrm{tte}^{\mathrm{e}} \mathrm{d}_{\mathrm{n}}$ ], Hebungstendenzen von [e:, o:], z. B. in wenigen

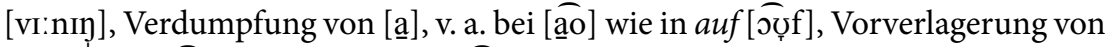
[a] ], v. a. bei [âa] ], Beispiel seinen [zָæên], Tiefschwavorverlagerung und Elision von $[\mathrm{d}, \mathrm{t}]$ nach [n] wie in Wanderer [vanəre:] und Spirantisierung von [b] bspw. in aber [pve]. Die Realisierung von [ $\widetilde{\mathrm{ts}}]$ als Frikativ sowie die Elision von auslautend [t] tritt jeweils nur einmal auf.

In der Übertragung der Wenkersätze in den Standard treten zu diesen

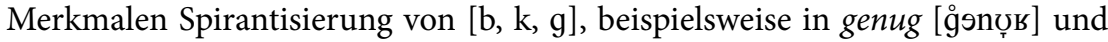
über [Yvع], Realisierung der Affrikaten [ $\widehat{\mathrm{ts}}, \widehat{\mathrm{pf}}$ ] als Frikative wie in Pferd [feзd] und $z u$ [zỡ], vermehrte Elision von [t] (wortfinal), Beispiel anfängt [ânfen], lVokalisierungstendenzen wie in gefallen [gofọln] lautenden Frikativen wie in noch [nọ]] und einmal eine Diphthongierungstendenz von [o:] in Brot [broed]]. 
Im Interview lassen sich folgende Varianten zusätzlich beschreiben: wort-

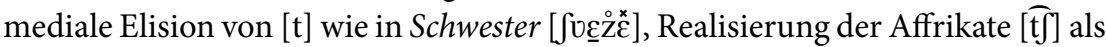
Frikativ und Monophthongierungstendenzen in Plattdeutsch [p'lædœ' $]$ ], 1-Elision bspw. in weil [৩ọ̊] ], vermehrte Elision von auslautenden Frikativen, Beispiel nicht [nĨ̃], Assimilationen wie werden [vદ̨:n] und unverschobene Formen für ich [ẹğ]

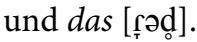

\subsection{RUEG1}

In der Vorleseaussprache des Sprechers kommen als regionale Varianten vor: Rundungstendenzen [I] (bzw. Zentralisierungstendenzen) wie in Nordwind

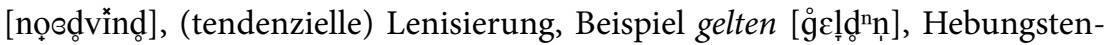
denzen von [e:, o:], beispielsweise in wohl [vv:l], Verdumpfung von [a] ], v. a. bei

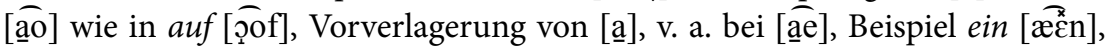
Tiefschwavorverlagerung, Beispiel fester [fㅌst $\varepsilon]$, und Elision von $[\mathrm{d}, \mathrm{t}]$ nach $[\mathrm{n}]$

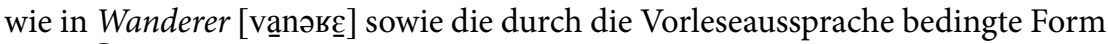

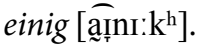

Bei seiner Standardübertragung der Wenkersätze lassen sich zusätzlich zu den beschriebenen Merkmalen folgende beobachten: vereinzelt Spirantisierungen

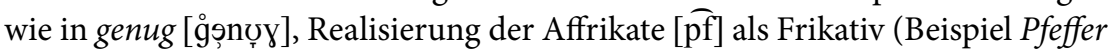

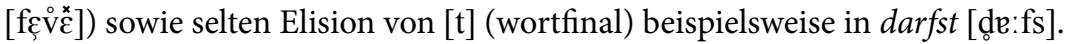

Im Interview können ferner folgende Varianten bestimmt werden: Elision von

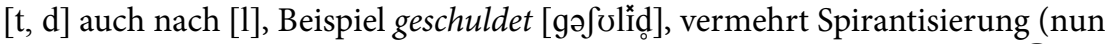

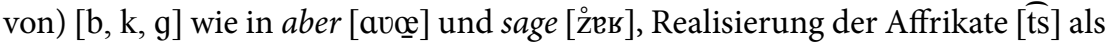

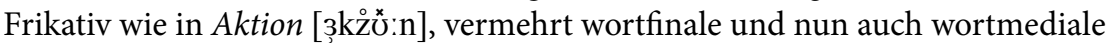
Elision von [t], beispielsweise in Ostpreußen [эsp

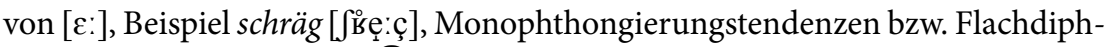

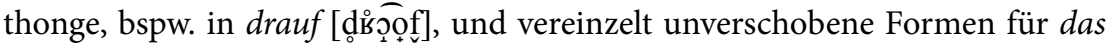
[red].

Darüber hinaus treten im Freundesgespräch auf: vermehrt unverschobene Formen nun für das, dass, es, Beispiel dass [rз̧d] und es [ot], Elision von auslautenden Frikativen wie in noch [nọ], Assimilationen wie an der [enə], Hebung und Vorverlagerung bei dann [d트] und vereinzelt apikale /r/-Varianten, bspw. in Richtung [riçdỡu]].

\subsection{RUEGJUNG1}

Während des Vorlesens von „Nordwind und Sonne“ treten bei RUEGJUNG1 folgende Regionalismen auf: Rundungstendenzen [I] (bzw. Zentralisierungstenden-

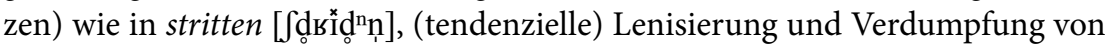




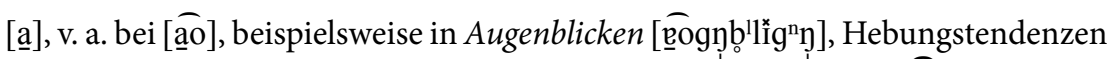
von [e:, o:] wie in Weges [vr:gəs], Vorverlagerung von [a] , v. a. bei [âe] wie in einst [æ๕घnst], Tiefschwavorverlagerung, Beispiel aller [alẹ], Elision von [d, t] nach [n] wie in Wanderer [vænзڤ̊ง] sowie zwei Mal eine Monophthongierung bei seinen [zenə̆m], die jedoch auch - gerade da es sich um einen Kurzvokal handelt - durch erhöhtes Sprechtempo (während der gesamten Vorleseaussprache) bedingt sein kann.

In den Wenkersätzen der Standardübertragung kommen hinzu: Spirantisierung von [b, k], beispielsweise in über [Y৩९̣] und genug [g̊’nọx], Realisierung der Affrikate [ $\widetilde{p f}]$ als Frikativ, Beispiel Pfeffer [fㅌfe], sowie Elision von [t] (wortfinal) wie in hast [hes].

Im Interview des Sprechers können darüber hinaus diese Phänomene bestimmt werden: Elision von [t, d] auch nach [1] bspw. in Weltkrieg [vẹskii:g̊] hier in Kombination mit weiteren Assimilationen, vermehrt Spirantisierung (nun von) [b, k, g] wie in sage [zев] und habe [hạ:vỸ], Realisierung der Affrikate $[\widehat{\mathrm{ts}}]$ als Frikativ, Beispiel zwei [svæe] , wortfinale und jetzt auch wortmediale Elision von [t] wie in eigentlich [æe-nij], Monophthongierungstendenzen bzw.

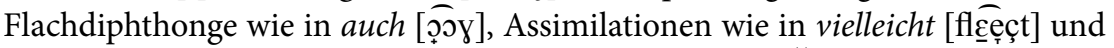

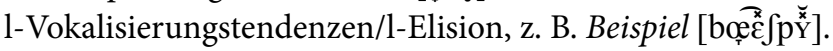

Im Freundesgespräch kommen zusätzlich folgende regionale Varianten vor: Elision von auslautenden Frikativen wie in noch [nọ], Hebung von [ $\varepsilon$ :], bspw. in dämlich [de:miç], Hebung und Vorverlagerung bei dann [dę̆n] und unverschobene Formen für das, was [dəd, ved].

\subsection{Zusammenführung}

Für die einzelnen Erhebungssituationen der Sprecher scheint es, bei genauerer Betrachtung der qualitativen Auswertung, charakteristische Merkmale zu geben. Auffallend ist jedoch, dass sich bei einer Systematisierung der Merkmale zwei Gruppen abzeichnen.

Zunächst gibt es Varianten, die in allen Sprachproben auftreten und nur in ihrer Frequenz zwischen den verschiedenen Situationen variieren. Hierzu zählen:

- Rundungstendenzen (bzw. Zentralisierungstendenzen) von [I]

- (tendenzielle) Lenisierung

- Hebungstendenzen von [e:, o: ]

- Verdumpfung von [a] $]$ v. a. bei [âa] $]$

- Vorverlagerung von [a] $]$, v. a. bei [âe]

- Tiefschwavorverlagerung/-hebung

- Elision von $[\mathrm{d}, \mathrm{t}]$ nach $[\mathrm{n}]$ 
Da diese auch in der Vorleseaussprache sowie der Standardübertragung der Wenkersätze - also im intendierten Standard - aller Informanten vorkommen, mit anderen Worten erhalten bleiben, können sie als remanente Merkmale bzw. Restarealität des Regiolekts auf Rügen definiert werden.

Die zweite Gruppe der Varianten zeichnet sich dadurch aus, dass diese erst in den freien Gesprächen (Interview und Freundesgespräch) der Informanten vermehrt und systematisch auftreten und somit charakteristisch für diese Situationen zu sein scheinen. Hierzu zählen:

- Spirantisierung von $[\mathrm{b}, \mathrm{k}, \mathrm{g}]$

- Elision von [t, d] nach [1]

- Realisierung der Affrikaten [ [ts, $\widehat{\mathrm{pf}}]$ als Frikative

- Elision von [t] (wortmedial und -final)

- unverschobene Formen (das, dass, es)

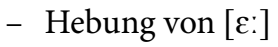

- Assimilationen

- Monophthongierungstendenzen bzw. Flachdiphthonge

- Elision von auslautenden Frikativen

- Hebung und Vorverlagerung bei dann

Einige dieser Merkmale treten bereits in der Übertragung der dialektalen Wenkersätze in die Standardsprache auf, dies aber nur vereinzelt und unsystematisch. Auch gibt es geringe Unterschiede der Variantenverteilung zwischen den beiden freien Gesprächen, so tritt die Verwendung von [den] als dann erst in den Freundesgesprächen auf und auch die unverschobenen Formen für das, was und es häufen sich vor allem in den Freundesgesprächen. ${ }^{34}$ Ein deutlicher Unterschied sowohl in qualitativer Sicht, d. h. im Auftreten neuer Merkmale und neuer Auftretenskontexte, und in quantitativer Sicht, d. h. in der Frequenz des Vorkommens, besteht aber zwischen den Standardkompetenzerhebungen der Sprecher und ihren freien Gesprächen, sodass die obige Zusammenfassung der Varianten sinnvoll erscheint. Dies wiederum lässt den Schluss zu, dass der hier untersuchte Regiolekt eine zweiteilige Struktur aufweist, d. h. sich zwei verschiedene Sprechlagen innerhalb des Regiolektes abgrenzen lassen. Sprechlagen sind definiert als „Verdichtungsbereiche variativer Sprachverwendung [...], für die sich - empirisch signifikant - differente sprachliche Gruppenkonventionen nachweisen lassen“. ${ }^{\text {“ }}{ }^{3}$

34 Gerade bei den lexikalisch beschränkten Phänomenen - aber nicht ausschließlich bei diesen - kann auch die Größe des Korpus ausschlaggebend für das Auftreten bzw. Nichtauftreten eines Merkmals sein.

35 Schmidt/Herrgen 2011, S. 52.

Birte Arendt, Andreas Bieberstedt and Klaas-Hinrich Ehlers - 978-3-631-71893-3 
Weitere empirische (v. a. statistische) Analysen stehen noch aus, um die Ergebnisse zu verifizieren, doch kann die vorliegende empirische Untersuchung anhand der Bestimmung der Abstandswerte und der diese bedingenden Varianten (in ihrer qualitativen wie quantitativen Verteilung) zeigen, dass es zwei Verdichtungsbereiche zu geben scheint, für die auch Gruppenkonventionen der Situationsadäquatheit zu bestehen scheinen: Die Verwendung des intendierten Standards kann dem Regionalakzent zugeordnet werden (Abstandwerte ungefähr zwischen 0,7 und 1,05), die (freien) Performanzsituationen wiederum dem unteren Bereich des Regiolekts ${ }^{36}$ (Abstandwerte ungefähr zwischen 1,05 und 1,4).

Dies bedeutet, dass die Vorleseaussprache aller drei Sprecher sowie die Standardübertragung der Wenkersätze von RUEG1 und RUEGJUNG1 dem Regionalakzent zugeordnet werden können und die Interviews der drei Sprecher sowie wiederum die Freundesgespräche von RUEG1 und RUEGJUNG1 dem unteren Regiolekt. Problematisch ist die Zuordnung der Wenkersätze von RUEGALT2. Diese Sprachprobe befindet sich nicht nur hinsichtlich des Abstandswerts $(1,08)$ in einem mittleren Bereich zwischen den ermittelten Sprechlagen. Sowohl die Frequenzen der Varianten als auch das Vorkommen einzelner Varianten ähneln zum Teil dem Sprachverhalten der anderen Sprecher in den freien Gesprächen. So treten bei diesem Sprecher bereits vereinzelt die Realisierung der Affrikate [ $\widetilde{\mathrm{ts}}]$ als Frikativ, l-Vokalisierungen und Elision auslautender Frikative auf, was bei den anderen Sprechern erst im Interview bzw. Freundesgespräch zu beobachten ist. Die Sprachprobe ist daher sowohl qualitativ als auch quantitativ nicht eindeutig einer Sprechlage zuzuordnen. Hierfür bedarf es weiterer Analysen.

\subsection{Sprachverhaltensmuster}

Wie aus den bisherigen Ausführungen hervorgegangen ist, unterscheidet sich der Sprecher RUEGALT2 von den anderen beiden Sprechern - es lässt sich daher von zwei verschiedenen Sprachverhaltensmustern sprechen. Die Analyse der Übertragung der Wenkersätze in den tiefst möglichen Dialekt durch RUEGALT2 hat ergeben, dass er - bis auf sehr wenige Ausnahmen - den alten niederdeutschen Basisdialekt von Bergen aktiv beherrscht. Die Kompetenz im Niederdeutschen setzt er auch im Alltag um - im Freundesgespräch (vgl. Kap. 4) verwendet er seinen niederdeutschen Dialekt. Auch sonst spricht er laut eigener Aussage recht häufig im Alltag Niederdeutsch. Er wechselt erst die Varietät, wenn er mit Frem-

36 Zur Terminologie vgl. Schmidt/Herrgen 2011. Die zweite Sprechlage könnte als mittlerer oder nur auf das vorliegende Spektrum bezogen unterer Regiolekt bezeichnet werden. 
den spricht (vgl. Interview), bleibt dabei aber im unteren Bereich des Regiolekts. Eine weitere Annäherung an die Standardvarietät lässt sich beobachten, wenn er gebeten wird, sein bestes Hochdeutsch zu sprechen (vgl. Wenkersätze Standard), bleibt aber auch hier in einem mittleren Bereich des Regiolekts (s. o.). Eine nochmalige Annäherung an die Standardvarietät (und gegebenenfalls ein Wechsel der Sprechlage) zeigt sich, wenn er einen Text laut vorliest. Hier erreicht er seine maximale Annäherung an die Standardsprache, verwendet aber auch hier durchschnittlich in fast jedem Wort ein regionales Merkmal (vgl. Abbildung 1). Dieses Sprachverhaltensmuster (Verwendung des unteren Regiolekts in gewissen Situationen u. a. im Gespräch mit Fremden, Annäherung an die Standardvarietät bei freier Produktion des intendierten Standards und nochmalige Annäherung bei der Vorleseaussprache) lässt sich mit der Sprachbiografie des Sprechers erklären. Die Muttersprache von RUEGALT2 ist Niederdeutsch, Hochdeutsch hat er erst in der Schule erlernt. Dem Niederdeutschen kommt also für seine Sprachbiografie, aber auch für den kommunikativen Alltag hohe Bedeutung zu. Kompetenz im Hochdeutschen hat er sekundär erworben, die Bedeutung für seinen kommunikativen Alltag (Verwendung während der Tätigkeit in der Fischereiaufsicht, mit immer mehr Nichtniederdeutschsprechern auf der Insel und mit seiner Familie) hat zwar zugenommen, doch ist die Verwendung seines Hochdeutsch in freien Gesprächen stark regional geprägt, was zum Teil niederdeutsch bedingt ist und in diesem Falle sprachbiografisch erklärt werden kann. Durch diesen Spracherwerb ist auch seine Standardkompetenz weniger ausgeprägt als bei den anderen Sprechern und erreicht ihr Maximum erst mit schriftlicher Vorlage. ${ }^{37}$

RUEG1 und RUEGJUNG1 zeigen im Regiolekt ein sehr ähnliches Sprachverhalten mit nur geringfügigen quantitativen und qualitativen Unterschieden. Ein weiterer Unterschied zwischen den Sprechern besteht darin, dass RUEG1 in seiner Kindheit Niederdeutsch passiv erworben hat - er überträgt die Wenkersätze zwar ins Niederdeutsche, doch ist dieses sehr stark hochdeutsch ${ }^{38}$ interferiert. RUEGJUNG1 hat kein Niederdeutsch erworben und weist somit keine aktive Kompetenz mehr auf - bei der Übertragung der Wenkersätze in den tiefst möglichen Dialekt produziert er lediglich einige niederdeutsche Lexeme; zahlreiche Hyperdialektalismen bekräftigen die nicht vorhandene aktive Kompetenz. Für beide

37 Kehrein 2012, S. 350 bezeichnet diesen Sprechertypen als Sprecher mit bivarietärer Kompetenz. Allerdings tritt die beschriebene Varietätenmischung bei RUEGALT2 nicht auf.

38 Hochdeutsch ist auch hier als struktureller Gegensatz zum Niederdeutschen zu verstehen. Die auftretenden Interferenzen sind sowohl regiolektalen als auch standardsprachlichen Ursprungs. 
Sprecher spielt das Niederdeutsche laut eigener Aussage im kommunikativen Alltag keine Rolle. Die beiden Performanzsituationen der Sprecher lassen sich dem unteren Bereich des Regiolekts zuordnen (s. o.) - diese Sprechlage ist somit bestimmend für ihren sprachlichen Alltag. Ein bedeutender Unterschied im sprachlichen Verhalten in formellen und informellen Situationen lässt sich nicht erkennen, weswegen beide derselben Sprechlage zugeordnet werden können. ${ }^{39}$ Ein Shiften innerhalb der Regiolekts ${ }^{40}$ und somit eine Annäherung an die Standardvarietät findet erst statt, wenn beide ihr individuell bestes Hochdeutsch produzieren sollen. Die entsprechenden Sprachproben konnten dem Regionalakzent zugeordnet werden. Auch zwischen ihnen konnte kein gewichtiger Unterschied festgestellt werden. Zu erklären ist auch dieses Sprachverhaltensmuster (Verwendung des unteren Regiolekts im gesamten kommunikativen Alltag und Verwendung des Regionalakzents im Bemühen, die Standardvarietät zu realisieren) mit der Sprachbiografie der Sprecher. Beide haben entweder kein Niederdeutsch oder dieses nur passiv erworben - ihre sprachliche Sozialisation fand im Regiolekt der Insel - so, wie die ältere Generation mit ihnen auf hochdeutsch sprach (vgl. RUEGALT2) - statt. Durch die Tatsache, dass Hochdeutsch ihre Muttersprache ist und sie diese durch eine andere Schulbildung als RUEGALT2 intensiver erworben haben, weisen sie auch eine höhere Standardkompetenz auf, was sich in den entsprechenden Kompetenzerhebungen zeigt. Doch bleiben auch sie dabei im Regiolekt. Ausschlaggebend für die Variation (also die Ausprägung zweier Sprachvariationsmuster) scheint somit weniger das Alter - zwar unterscheidet sich das Sprachverhalten des ältesten Sprechers von dem der beiden anderen, doch gleicht sich das dieser beiden Sprecher aus unterschiedlichen Generationen wiederum - als vielmehr die sprachliche Primärsozialisierung (Niederdeutsch versus Hochdeutsch) und damit einhergehend die Verwendung des Niederdeutschen. ${ }^{41}$

39 Die Unterschiede zwischen den hier untersuchten Situationen der beiden Sprecher (Interview und Freundesgespräch) ergeben sich aus qualitativer Sicht hauptsächlich aus der Verwendung von [den] für dann und der fast ausschließlichen Verwendung unverschobener Formen für die Kennwörter das, was und ich im Freundesgespräch. Für beide Phänomene kann von einer Lexikalisierung ausgegangen werden. Die Verwendung im Freundesgespräch deutet gegebenenfalls auf eine soziale Funktion der Identitätsstiftung für diese Gespräche hin.

40 Vgl. Schmidt/Herrgen 2011, S. 52.

41 Vgl. hierzu auch Kehrein 2012, S. 355-361. Im Sinne einer apparant-time-Untersuchung (vgl. Fußnote 11) ließe sich hier auch nur bedingt von einer Standardadvergenz (zum Begriff vgl. Mattheier 1996, S. 34) sprechen - zwar nähert sich das am Standard orientierte Sprechen der Informanten der mittleren und jüngeren Generation der Stan- 


\subsection{Bezüge zu anderen Studien}

Alle für den mecklenburgisch-vorpommerschen Regiolekt beschriebenen Merkmale (s. o.) sind in den untersuchten Sprachproben enthalten. Dies kann als empirischer Beleg für diese Merkmale im Bergener/Rügener Regiolekt gelten. Vorteil der vorliegenden Untersuchung ist, dass die Merkmale nun verschiedenen Bereichen des Regiolekts subsumiert werden können und remanente Merkmale klar herausgestellt werden können. Teil der bisherigen Beschreibungen sind auch die Vorverlagerung/Hebung von /â/ sowie Verdumpfung von /a/, die auch in den vorliegenden Sprachproben vorkommen. Doch tritt die Vorverlagerung/Hebung hauptsächlich im Diphthong [âa $]$ auf und die Verdumpfung in [a $\underline{a} 0]$. Dies betrifft bei den drei Sprechern nahezu alle Diphthonge (es lässt sich auch keine systematische intersituative Variation erkennen), sodass die fast ausnahmslose standarddifferente Realisierung (Vorverlagerung/Hebung sowie Verdumpfung der ersten Diphthongkomponente) der Diphthonge [âa] und [āo ] - explizit - als Merkmal der Regiolekts auf Rügen herausgestellt werden kann.

In den Aufnahmen waren folgende Merkmale nicht zu finden: velares /1/, Ersatz des palatalen stl. Frikativs durch den velaren, Diphthongierung von /e:, ø:, $\mathrm{o}: /^{42}$, Aussprache von anlautend $/ \mathrm{j} /$ als $[3, \widehat{\mathrm{d}}]$ ], Bewahrung des niederdeutschen Vokalismus, Ausfall von /r/ ohne Ersatzdehnung, oraler Verschluss von <-ng> und Vokalkürze. Für die ersten sechs Merkmale ist anzunehmen, dass diese abgebaut wurden und daher nicht mehr im Regiolekt von Rügen/MecklenburgVorpommern zu finden sind. ${ }^{43}$ Bei den anderen Phänomenen ist das Ausbleiben eher darauf zurückzuführen, dass kaum bzw. keine Lexeme in den untersuchten Sprachproben enthalten waren, in denen das Merkmal hätte auftreten können.

Dahl unterscheidet - wie oben ausgeführt - für den regiolektalen Bereich zwei Schichten: hochsprachenahes (umgangssprachliches) Hochdeutsch und Hochdeutsch mit niederdeutscher Beimischung (mundartnahe Umgangssprache). Dies lässt sich in Verbindung mit den hier empirisch ermittelten Sprechlagen bringen. Ein Unterschied besteht jedoch in der Konstitution (Konzeption) der Bereiche,

dardsprache im Vergleich zum älteren Sprecher an, doch lässt sich zwischen diesen beiden Generationen kein bedeutsamer Unterschied feststellen.

42 Bis auf einmaliges Auftreten in der Standardübertragung der Wenkersätze bei RUEGALT2.

43 Da Lauf 1994, S. 55 diese teilweise als Besonderheiten des mecklenburgischvorpommerschen Regiolekts herausstellt und diese nun bereits abgebaut wurden, lässt sich annehmen, dass sich der mecklenburgisch-vorpommersche Regiolekt - zumindest - qualitativ noch weiter dem des nordniederdeutschen Raums angenähert hat. 
wie die unterschiedliche Terminologie bereits andeutet. Können hochsprachenahes (umgangssprachliches) Hochdeutsch und Regionalakzent relativ gut in Einklang gebracht werden, fällt dies beim Hochdeutsch mit niederdeutscher Beimischung (mundartnahe Umgangssprache) und dem mittleren/unteren Regiolekt schwerer. Diese Sprechlage als mundartnah zu klassifizieren, scheint irreführend, da sie in den vorliegenden Untersuchung klar vom niederdeutschen Dialekt zu unterscheiden ist und - wenn auch im unteren Bereich des Regiolekts lokalisiert eine große Distanz zu diesem aufweist. ${ }^{44}$ Auch qualitativ handelt es sich bei den Merkmalen - außer bei der Verwendung unverschobener Formen (für das, was, es) - nicht um niederdeutsche Beimischungen, sondern allenfalls um niederdeutsch bedingte Merkmale - bei anderen Regionalismen ist der Ursprung noch zu diskutieren. Auch in eben jenen für die jeweiligen Bereiche beschriebenen Merkmalen unterscheiden sich die vorliegenden Ergebnisse teilweise von den Ausführungen von Dahl - gerade für den unteren Bereich des Regiolekts. Die auf den ersten Blick erscheinende Nähe der Differenzierung des mecklenburgischvorpommerschen Regiolekts bestätigt sich - zumindest auf Grundlage der vorliegenden Ergebnisse - für Bergen auf Rügen nur bedingt, dennoch können die Hinweise auf eine mögliche Differenzierung bei Dahl hier - wenn auch modifiziert - bekräftigt werden.

Im Vergleich zu Kehreins Untersuchung in Stralsund ergeben sich - bei Bestätigung vieler Ergebnisse - zwei Unterschiede. Die Dialektalitätswerte der Sprachproben, die dem Regiolekt zugeordnet werden konnten, sind allgemein etwas höher, d. h. mit anderen Worten der Regiolekt ist nicht derart standardnah wie in Stralsund. Außerdem ist die Variationsbreite $(0,7)$ des Regiolekts höher als in Stralsund, sodass sich innerhalb des Regiolekts eine Differenzierung zweier Sprechlagen abzeichnet, die Kehrein in Stralsund nicht beobachten konnte. ${ }^{45}$ Erklärt werden könnten diese Unterschiede - unter Berücksichtigung der metho-

44 Diese Distanz ergibt sich aus den angesprochenen Unterschieden in der Genese der norddeutschen Regionalsprachen (s. o.). Ein Vergleich zu anderen dialektalen Räumen (vgl. Kehrein 2012) zeigt diese sehr deutlich. Die phonetischen Abstandswerte für den niederdeutschen Dialekt in Bergen liegen im Bereich von 2,8.

45 Vgl. Kehrein 2012, S. 308. Durch die unterschiedlichen Messverfahren (manuelle versus halbautomatische Messung) sind die Werte nicht direkt aufeinander beziehbar. Die qualitative Analyse stützt jedoch die Annahme, dass die Sprachproben aus Bergen auf Rügen regionaler geprägt sind als in Stralsund. Direkt vergleichbar ist jedoch das jeweilige Verhältnis der D-Werte der Sprachproben untereinander (also die Strukturierung des Spektrums). 
dischen Überlegungen ${ }^{46}$ - mit den unterschiedlichen Sprechern und der sprachlichen Situation auf Rügen. Im Gegensatz zum älteren Sprecher aus Stralsund (HSTALT1), der zwar die Wenkersätze in das ortsübliche Niederdeutsch überträgt, jedoch im Freundesgespräch schon niederdeutsche Passagen mit hochdeutschen mischt ${ }^{47}$ verwendet RUEGALT2 sein Niederdeutsch fast interferenzfrei im kommunikativen Alltag. Es kann allgemein auf Grundlage der Aussagen der Sprecher angenommen werden, dass Niederdeutsch in Bergen (und auf Rügen) häufiger gesprochen wird als in der (größeren) Stadt Stralsund. Auf Rügen gab und gibt es also eine eher geschlossenere - durch die Insellage bedingte - Sprachgemeinschaft mit Niederdeutschsprechern, die einen für norddeutsche Verhältnisse eher standardferneren Regiolekt verwenden, wenn sie Hochdeutsch sprechen wollen/ sollen (vgl. RUEGALT2). Durch die Primärsozialisierung der darauffolgenden Generationen in diesem Regiolekt und die Kommunikation auf der Insel konnte sich dieser Regiolekt im Laufe der Zeit etablieren und ist bestimmend für den rezenten kommunikativen Alltag in Bergen. Dadurch dass dieser Regiolekt bzw. die Sprechlage des unteren Regiolekts etwas standardferner ist, können v. a. die Nichtniederdeutschsprecher bei Bedarf (vgl. Standardkompetenzerhebung) in eine standardnähere Sprechlage shiften, was die interne Strukturierung erklären kann.

\section{Fazit}

Der vorliegende Beitrag präsentierte eine synchrone Untersuchung des Sprachverhaltens dreier Sprecher aus unterschiedlichen Generationen aus Bergen auf Rügen. Analysiert wurden Sprachproben des hochdeutsch basierten Regiolekts aus verschiedenen Aufnahmesituationen mit den Methoden der modernen Regionalsprachenforschung.

Es konnte gezeigt werden, dass der Regiolekt in Bergen auf Rügen insgesamt standardferner ist als in den bisher erforschten Untersuchungsorten, mit anderen Worten das hochdeutsch basierte Sprechen in Rügen ist regionaler geprägt als in anderen Regionen Norddeutschlands. Ferner ließen sich aufgrund der Quantität und Qualität der auftretenden regionalen Merkmale zwei verschiedene Sprechlagen innerhalb dieses Regiolekts aufzeigen: ein Regionalakzent, dem die Sprachproben der Standardkompetenzerhebungen zugeordnet werden konnten, und ein mittlerer/unterer Regiolekt, der in den freien Gesprächen Verwendung findet. Für die einzelnen Sprechlagen wurden die prägenden Merkmale genau

46 Vgl. Fußnote 45.

47 Vgl. zum Thema Alternanz Kehrein 2012, S. 307 und Dahl 1974.

Birte Arendt, Andreas Bieberstedt and Klaas-Hinrich Ehlers - 978-3-631-71893-3 
beschrieben, was auch bedeutet, dass die remanenten Merkmale (= Restarealität) für die Sprecher aus Bergen bestimmt werden konnten. Außerdem zeigten sich in den Analysen zwei verschiedene Sprachverhaltensmuster, die auf die sprachliche Primärsozialisierung zurückgeführt werden konnten.

Es scheint also, dass sich Bergen und somit auch Rügen - aus sprachlicher Sicht - auch im hochdeutschen Bereich des regionalsprachlichen Spektrums vom übrigen norddeutschen Raum (v. a. in der Frequenz der Merkmale und der Strukturierung des Regiolekts) etwas unterscheiden. Weitere Studien, die die Analysen um weitere Sprecher, eine größere Datengrundlage, weitere Methoden (v. a. quantitativer Art) und weitere Aufnahmesituationen (v. a. Niederdeutsch) ergänzen, müssen diese Ergebnisse bestätigen.

\section{Literatur}

Bergen-I = Internetseite des Stadt Bergen auf Rügen. [Online-Ressource: http:// www.stadt-bergen-auf-ruegen.de. (Stand: 13.05.2015)]

Bieberstedt, Andreas/Ruge, Jürgen/Schröder, Ingrid: Kontaktinduzierte sprachliche Variation in der Hamburger Peripherie. Ein Modell zur Messung sprachlicher Konvergenz. In: Bieberstedt, Andreas/Ruge, Jürgen/Schröder, Ingrid (Hrsg.): Hamburgisch. Struktur, Gebrauch, Wahrnehmung der Regionalsprache im urbanen Raum (Sprache in der Gesellschaft; 34). Frankfurt a. M. [u. a.] 2016, S. 21-66.

Dahl, Eva-Sophie: Interferenz und Alternanz - Zwei Typen der Sprachschichtenmischung im Norden der Deutschen Demokratischen Republik. In: Ising, Gerhard (Red.): Aktuelle Probleme der sprachlichen Kommunikation - soziolinguistische Studien zur sprachlichen Situation in der Deutschen Demokratischen Republik. Berlin 1974, S. 339-387.

Elmentaler, Michael/Rosenberg, Peter: Norddeutscher Sprachatlas (NOSA). Bd. 1: Regiolektale Sprachlagen (Deutsche Dialektgeographie; 133.1). Hildesheim/ Zürich/New York 2015.

Gernentz, Hans Joachim: Die kommunikative Funktion der niederdeutschen Mundart und hochdeutschen Umgangssprache im Norden der Deutschen Demokratischen Republik, unter besonderer Berücksichtigung der Interferenz und Alternanz zwischen beiden sprachlichen Existenzformen. In: Studia Germanica Gandensia 15 (1974), S. 209-244.

Gernentz, Hans-Joachim: Niederdeutsch - gestern und heute. Beiträge zur Sprachsituation in den Nordbezirken der DDR in Geschichte und Gegenwart. Rostock 1980. 
Hansen, Martin: Zum Wandel des Niederdeutschen auf der Insel Rügen zwischen dem 19. und 21. Jahrhundert. Ein diachronischer Vergleich anhand ausgewählter Sprachmerkmale. Masterarbeit Greifswald 2009.

Herrmann-Winter, Renate: Sprachatlas für Rügen und die vorpommersche Küste. Kartographie Martin Hansen. Rostock 2013.

Herrgen, Joachim/Schmidt, Jürgen Erich: Dialektalitätsareale und Dialektabbau. In: Putschke, Wolfgang/Veith, Werner H./Wiesinger, Peter (Hrsg.): Dialektgeographie und Dialektologie. Günter Bellmann zum 60. Geburtstag von seinen Schülern und Freunden. Marburg 1989, S. 304-346.

Herrgen, Joachim/Lameli, Alfred/Rabanus, Stefan/Schmidt Jürgen Erich: Dialektalität als phonetische Distanz. Ein Verfahren zur Messung standarddivergenter Sprechformen. Onlinepublikation 2001. [Online-Ressource: www. sprachatlas.de. (Stand: 12.11.2016)]

Kehrein, Roland: Regionalsprachliche Spektren im Raum. Zur linguistischen Struktur der Vertikale (ZDL Beihefte; 152). Stuttgart 2012.

König, Werner: Atlas zur Aussprache des Schriftdeutschen in der Bundesrepublik Deutschland. 2 Bde. Ismaning 1987.

Lameli, Alfred: Standard und Substandard. Regionalismen im diachronen Längsschnitt (ZDL Beihefte; 128). Stuttgart 2004.

Lauf, Raphaela (Bearb.): Datenbank regionaler Umgangssprachen des Deutschen. DRUGS. Abschlussbericht. Marburg 1994.

Lauf, Raphaela: „Regional markiert“: großräumliche Umgangssprachen im niederdeutschen Raum. In: Niederdeutsches Jahrbuch 119 (1996), S. 193-218.

Lüders, Björn: PAM - Ein Programm zur phonetischen Abstandsmessung. Version 0.3.12, Onlinepublikation 2013. [Online-Ressource: www.mpiorn.de/pam. (Stand: 26.02.2015)]

Martens, Hans/Martens, Hiltrud: Niederdeutsch bedingte Abweichungen von der hochdeutschen Standard-Aussprache. In: Behme, Helma (Hrsg.): Angewandte Sprechwissenschaft. Interdisziplinäre Beiträge zur mündlichen Kommunikation. Stuttgart 1988, S. 123-137.

Mattheier, Klaus: Varietätenkonvergenz. Überlegungen zu einem Baustein einer Theorie der Sprachvariation. In: Ammon, Ulrich/Mattheier, Klaus J./Nelde, Peter (Hrsg.): Konvergenz und Divergenz von Dialekten in Europa (Sociolinguistica: internationales Jahrbuch für europäische Soziolinguistik; 10). Tübingen 1996, S. 31-52.

Schmidt, Jürgen Erich: Moderne Dialektologie und regionale Sprachgeschichte. In: Zeitschrift für deutsche Philologie, Sonderheft 117 (1998), S. 163-179. 
Schmidt, Jürgen Erich/Herrgen, Joachim/Kehrein, Roland (Hrsg.): Regionalsprache.de (REDE). Forschungsplattform zu den modernen Regionalsprachen des Deutschen. Marburg: Forschungszentrum Deutscher Sprachatlas $2008 \mathrm{ff}$. [Online-Ressource]

Schmidt, Jürgen Erich/Herrgen, Joachim: Sprachdynamik. Eine Einführung in die moderne Regionalsprachenforschung (Grundlagen der Germanistik; 49). Berlin 2011.

Schröder, Ingrid: Niederdeutsch in der Gegenwart: Sprachgebiet - Grammatisches - Binnendifferenzierung. In: Stellmacher, Dieter (Hrsg.): Niederdeutsche Sprache und Literatur der Gegenwart. Hildesheim 2004, S. 35-97.

Stellmacher, Dieter: Studien zur gesprochenen Sprache in Niedersachsen: eine soziolinguistische Untersuchung. Marburg 1977.

Trenschel, Walter: Die Standardaussprache und phonetische Haupteigenheiten im Norddeutschen. In: Haase, Martina/Meyer, Dirk (Hrsg.): Von Sprechkunst und Normphonetik. Festschrift zum 65. Geburtstag von Eva-Marie Krech am 6. November 1997. Hanau 1997, S. 205-209.

Vorberger, Lars/Schröder, Ingrid: Standardadvergenz im nördlichen Niedersachsen? In: Niederdeutsches Jahrbuch 134 (2011), S. 137-147.

Wiesinger, Peter: Die Einteilung der deutschen Dialekte. In: Besch, Werner [u. a.] (Hrsg.): Dialektologie. Ein Handbuch zur deutschen und allgemeinen Dialektforschung (Handbücher zur Sprach- und Kommunikationswissenschaft; 1.2). 2. Halbbd. Berlin/New York 1983, S. 807-900. 
Birte Arendt, Andreas Bieberstedt and Klaas-Hinrich Ehlers - 978-3-631-71893-3

Downloaded from PubFactory at 01/11/2019 10:11:06AM

via free access 


\title{
„Wir haben's zwar gehört, konnten's verstehen und... aber selbst so richtig benutzt haben wir's auch nicht." Eine Fallstudie zum Wandel des Dialektgebrauchs in der Generationsfolge in einer mecklenburgischen Gemeinde
}

\begin{abstract}
This article discusses aspects of dialect change in Mecklenburg-Western Pomerania over the last seven decades from a biographical point of view. It presents results of a biographical study with Low German dialect speakers of three generations from the community of Satow near Rostock. The participants of this study belong to the old, middle and younger speaker generation who have aquired their first and second language in a period in which the Low German dialect underwent a severe change with regard to both the number of its speakers and the patterns of its acquisition and usage. The article discusses autobiographical statements about the participants' linguistic socialization and language experiences and in that way it reveals individual and collective concepts about the usage and change of the Low German dialect in Mecklenburg on a sociological micro-, meso- and macro-level.
\end{abstract}

\section{Einleitung}

Dieser Beitrag befasst sich mit dem Gebrauchswandel der mecklenburgischen Mundart der letzten 60 Jahre. Präsentiert werden Ergebnisse sprachbiografischer Tiefeninterviews mit Dialektsprechern verschiedener Generationen aus der Gemeinde Satow im Landkreis Rostock. Vergleicht man die individuellen Sprachbiografien der Probanden, lassen sich wiederkehrende Prozessstrukturen erkennen, die diesen Wandel begünstigt haben. Diese umfassen unterschiedliche gesellschaftliche Ebenen, die regionale Ebene bzw. regionale Sprachgemeinschaft (Makroebene) ebenso wie die lokale Ebene oder örtliche Sprachgemeinschaft (Mesoebene). Aber auch im kleinen gesellschaftlichen Rahmen, der Familie (Mikroebene), lassen sich wesentliche regionalsprachliche Entwicklungstendenzen aufzeigen.

Ziel dieser Fallstudie ist es, ausgehend von der Mikroebene den Wandel in der Sprachvermittlung und des Sprachgebrauchs bezüglich der mecklenburgischen Mundart in verschiedenen sozialen Kontexten aufzuzeigen. Die Frage ist, wie die Bewohner meines Untersuchungsortes den Sprachwandel der vergangenen sechs Jahrzehnte beschreiben und welche sozialen Faktoren sie dafür verantwortlich 
machen. Hierfür bedient sich die Untersuchung eines qualitativen sprachbiografischen Ansatzes, indem die subjektiven Erfahrungen der Sprachteilnehmer als Erkenntnisquelle in den Mittelpunkt gestellt werden.

\section{Vorüberlegungen}

\subsection{Problemaufriss}

Während ihrer Entstehungs- und Entwicklungsgeschichte unterlag die mecklenburgisch-vorpommersche Mundart permanenten Veränderungen. Der Wandelprozess des Mecklenburgischen in Bezug auf seine Struktur, seine Sprecher, den Erwerb und die Verwendung sowie hinsichtlich der Einstellungen ihm gegenüber erfuhr besonders in der jüngeren Vergangenheit des 19. und 20. Jahrhunderts eine zunehmende Dynamik und veränderte die sprachliche Situation in Mecklenburg-Vorpommern gerade in den vergangenen sechs Jahrzehnten grundlegend. Schon die soziolinguistische Forschung der 1970er Jahre zeigte, dass sich im regionalen Varietätenspektrum Mecklenburg-Vorpommerns deutliche Konvergenzerscheinungen erkennen lassen, ${ }^{1} \mathrm{~d}$. h. dass der Basisdialekt ${ }^{2}$ strukturell und funktional fortlaufend abgebaut wird. ${ }^{3}$ Das Niederdeutsche nähert sich über Interferenzerscheinungen einerseits strukturell dem Standarddeutschen an und wird andererseits auch in der Kommunikation immer weiter von regiolektalen bzw. standardnahen Sprechlagen ersetzt. Dabei nehmen die Anzahl der Dialektsprecher sowie deren dialektale Kompetenzen stetig ab. ${ }^{4}$ Als Konsequenz verschwindet die Mundart aus den relevanten Kommunikationsbereichen, z. B. aus dem Arbeitsleben oder dem Familien- und Freundeskreis, und verliert damit ihre Funktion als Mittel der Alltagskommunikation.

Auch der Regiolekt in Mecklenburg-Vorpommern weist Abbauprozesse und eine wachsende Nähe zum standardsprachlichen Pol auf. ${ }^{5}$ So konstatiert etwa

1 Erscheinungen von Interferenz und Alternanz im Dialekt werden von Dahl bereits in den 1970er Jahren konstatiert, vgl. Dahl 1974. Ähnlich u. a. Gernentz 1974.

2 Unter Basisdialekt ist mit Schmidt 1998, S. 163 die „standardfernste Sprechlage der jeweils ältesten immobilen Sprecher“ zu verstehen.

3 Lenz 2005, S. 85 unterscheidet Prozesse „eines strukturellen Dialektwandels (,Dialektabbau')“, die auf „systemische Veränderungen des Dialekts“ hinauslaufen, von Prozessen „eines funktionalen Dialektwandels [...] (,Dialektabnahme`)", die sich in einer „Abnahme der Verwendungshäufigkeit des Dialekts“ niederschlagen.

4 Vgl. dazu etwa die Ergebnisse des Sprachzensus von 2007 in Möller 2008 und Möller 2010.

5 So bereits Herrmann-Winter 1974, S. 173.

Birte Arendt, Andreas Bieberstedt and Klaas-Hinrich Ehlers - 978-3-631-71893-3 
Kehrein in seiner aktuellen Studie zu den regionalsprachlichen Spektren im Raum Stralsund unter Verweis auf Dahls frühere Untersuchungen zur „mundartnahen Umgangssprache“ $\left(U^{m}\right)$ im Raum Rostock ${ }^{6}$, dass viele der für diese „standardfernste Sprachschicht der hochdeutschen Umgangssprache konstitutiven regionalsprachlichen Varianten“ in den Sprachproben der von ihm beobachteten Probanden nicht mehr nachweisbar seien. ${ }^{7}$ Der hier umrissene Wandelprozess beeinflusst also den Aufbau des Gefüges regionaler Varietäten und Sprechlagen insgesamt ebenso wie die sprachliche Struktur der einzelnen Varietäten.

Versucht man diese Entwicklungsprozesse modellhaft abzubilden, bietet sich der Vorschlag von Schmidt an, der die Entwicklung der regionalen Varietäten und Sprechlagen zwischen den Polen Basisdialekt und Standardsprache im deutschen Sprachraum in einem mehrstufigen Ablaufmodell darstellt. ${ }^{8}$ Basis ist die Annahme ursprünglich diglossischer, hochdeutsch-dialektaler Sprachverhältnisse und deren diachrone Entwicklung hin zu monoglossischen Verhältnissen durch die Auflösung des dialektalen Anteils am Varietätenspektrum. ${ }^{9}$ Die erste Entwicklungsphase, die vom Basisdialekt als hauptsächlichem Kommunikationsmittel wegführt, ist demnach von einer klassischen Diglossie geprägt. Zwischen Mundart und Standardsprache gibt es eine klare, sich stetig verfestigende Domänenverteilung. Der Dialekt wird beispielsweise in der Familie bzw. im Privaten gebraucht, während die Standardsprache im Berufsleben oder in Behörden verwendet wird. In der zweiten Entwicklungsphase konstituieren sich neben Dialekt und Standardsprache weitere Schichten der gesprochenen Sprache. In der folgenden Phase stellt Schmidt eine „Entdiglossierung“ zwischen Dialekt und Standardsprache fest. In der Kommunikation ist ein breites Spektrum im mittleren Varietätenbereich zu finden, das weder den basisdialektalen noch den standardsprachlichen Pol erreicht. ${ }^{10}$ Sprachvariation innerhalb dieses mittleren Spektrums besteht nicht mehr im Wechsel zwischen Varietäten, die mehr oder weniger fest an soziale oder situative Gegebenheiten gebunden sind, sondern beläuft sich auf graduelle Verschiebungen zwischen „Sprech-

6 Vgl. Dahl 1974.

7 Kehrein 2012, S. 313. Zum Regiolekt in Mecklenburg-Vorpommern vgl. auch die aktuellen Daten aus dem Norddeutschen Sprachatlas, Elmentaler/Rosenberg 2015.

8 Vgl. Schmidt, 1998, S. 163-179.

9 Für den norddeutschen Sprachraum umfasst dies in etwa die Phase des Neuniederdeutschen seit dem 17. Jahrhundert bis zur Gegenwart. Vgl. dazu auch das ähnlich gelagerte sprachdynamische Modell zur Genese der modernen Regionalsprachen seit 1700 in Schmidt/Herrgen 2011, S. 63-67.

10 Hierzu gehört in Norddeutschland etwa das sogenannte „Missingsch“ (als inzwischen historische Varietät), vgl. Möhn 2004.

Birte Arendt, Andreas Bieberstedt and Klaas-Hinrich Ehlers - 978-3-631-71893-3 
lagenschwerpunkten“, die situationsbezogen als Stilmittel eingesetzt werden. In der vierten Entwicklungsphase sind die Dialekte, bis auf einige Dialektreste, aus der Kommunikation verschwunden. Es herrscht eine standardsprachliche Monoglossie, die nur noch wenige regionalsprachliche Merkmale enthält. Diese Stufe stellt nach Schmidt den Endpunkt der Dialektentwicklung dar, die von einer Auflösung des dialektalen Spektrums gekennzeichnet ist.

Im norddeutschen (und damit auch im mecklenburgisch-vorpommerschen) Sprachraum ist Schmidt zufolge dieser Endpunkt der dialektalen Entwicklung in der Gegenwart bereits erreicht. Die hier verwendete Alltagssprache entspreche weitgehend der Standardsprache, die lediglich einige norddeutsche Aussprachevarianten enthält. Allerdings verweist Schmidt gleichzeitig auf Menke und Stellmacher, die für den norddeutschen Sprachraum in einigen (ruralen) dialektstarken Regionen weiterhin eine niederdeutsch-hochdeutsche Diglossie annehmen, wenngleich Dialektkompetenzen lediglich bei einer stark schrumpfenden Minderheit vorhanden seien. ${ }^{11}$ Außerdem betont Schmidt eine weitere Besonderheit des norddeutschen Sprachraums. Anders als beispielsweise im Ruhrgebiet, das eine ähnliche sprachliche Entwicklungsstufe erreicht habe, sei im Norden „das Niederdeutsche als historisch eigenständige Sprache aber fest im Sprecherbewusstsein verankert

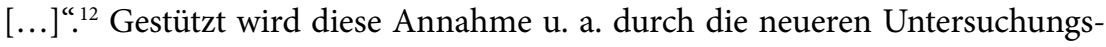
ergebnisse von Arendt, der zufolge die von ihr befragten Probanden „sich zumeist zustimmend zu der auch allgemein weit verbreiteten These, dass Niederdeutsch eine eigenständige Sprache sei und kein Dialekt", äußern. ${ }^{13}$

Es liegt daher nahe, Schmidts Zuordnung des Mecklenburgisch-Vorpommerschen zur vierten und letzten Stufe der Entdiglossierung einmal auf empirischer Grundlage zu hinterfragen. Die nachgewiesenen Restbereiche der Diglossie sowie die Verankerung des Niederdeutschen im Sprecherbewusstsein als eigenständige Sprache deuten darauf hin, dass der Endpunkt der Entwicklung, die standardsprachliche Monoglossie, hier noch nicht endgültig erreicht ist. Meine vorliegende Studie zielt darauf, das Modell von Schmidt auf seine Aktualität und Anwendbarkeit auf die gegenwärtige Sprachsituation in Mecklenburg-Vorpommern hin zu überprüfen. Dabei soll in erster Linie der Sprachgebrauchswandel des Niederdeutschen im Vordergrund stehen.

11 Vgl. Schmidt, 1998, S. 172. Schmidt bezieht sich hier auf Menke 1998 und Stellmacher 1997.

12 Schmidt, 1998.

13 Arendt 2010, S. 197-198. Diesen weit verbreiteten „Sprachtopos“ (Arendt 2010, S. 197-205) weist bereits Herrmann-Winter 1994, S. 460 nach, vgl. Arendt 2010, S. 198, Fußnote 302. 


\subsection{Zum sprachbiografischen Untersuchungsansatz}

Die mecklenburgisch-vorpommersche Mundart ist seit langem Untersuchungsgegenstand der Dialektologie und dementsprechend relativ gut erforscht. ${ }^{14}$ Dabei dominierten allerdings meist dialektografische und dialektgeografische Ansätze, die auch für die vergleichsweise wenigen Untersuchungen zur gegenwärtigen Dialektentwicklung in der Region bestimmend sind. ${ }^{15}$ Seltener werden soziolinguistische Methoden und Erklärungsmodelle herangezogen, um das Niederdeutsche der Gegenwart zu beschreiben und zu analysieren. Dabei sind unter anderem Fragen nach der Sprachkompetenz, den bevorzugten Gesprächspartnern, -themen und -situationen, den sozialen Merkmalen der Dialektsprecher sowie den Phasen und Motiven des Spracherwerbs von besonderer Bedeutung, um den Sprachwandel des Niederdeutschen zu erfassen. Derartige Forschungsansätze blieben bislang für den ostniederdeutschen Sprachraum, zu dem auch Mecklenburg-Vorpommern gehört, meist außerhalb der Betrachtung. ${ }^{16}$ Gleiches gilt für Studien zur Spracheinstellung und zur sozialsymbolischen Funktion des mecklenburgisch-vorpommerschen Dialektes, obgleich mit den aktuellen Untersuchungen von Arendt und Scharioth bereits wesentliche Forschungsdesiderate aufgearbeitet werden konnten. ${ }^{17}$

Abgesehen von der Untersuchung Scharioths zu Spracheinstellungen und Identitätskonstruktionen von Frauen in Schleswig-Holstein und MecklenburgVorpommern und der Untersuchung von Ehlers zur sprachlichen Akkulturation zugewanderter Vertriebener in Mecklenburg ${ }^{18}$ liegen sprachbiografische Untersuchungen, „bei denen die subjektive Sichtweise linguistischer Laien auf ihren sprachlichen Lebenslauf im Mittelpunkt des Forschungsinteresses steht ", ${ }^{19}$ mit Bezug auf das Mecklenburgische gegenwärtig noch nicht vor. Als Sprachbiografie oder auch Sprecherbiografie kann hierbei mit Bieberstedt „eine spezielle Form von Biographie bezeichnet werden, die den sprachlichen Lebenslauf eines Individuums, $d$. h. seine essentiellen sprachprägenden, sprachverändernden und sprachbezogenen Aktivitäten, Erlebnisse und Statusübergänge, verarbeitet und

14 Zum Forschungsstand vgl. auch den Beitrag von Rosenberg sowie die wissenschaftliche Bibliografie in diesem Band.

15 Vgl. etwa die Studien von Herrmann-Winter 2013; Hansen 2009; Köhncke 2010.

16 Vgl. Föllner 2004, S. 99-100.

17 Arendt 2010; Scharioth 2015.

18 Vgl. Ehlers 2011; Ehlers 2013; Ehlers 2017.

19 Bieberstedt 2017, S. 47.

Birte Arendt, Andreas Bieberstedt and Klaas-Hinrich Ehlers - 978-3-631-71893-3 
ihnen retrospektiv einen kohärenten Sinnzusammenhang zuschreibt ${ }^{\text {“ }}{ }^{20}$ Scharioth geht mit Bezug auf Macha von der Annahme aus, „dass der spezifische sprachliche Lebensweg mit seinen Implikationen für Einstellungen und Verhaltensdispositionen die entscheidende Prägung für aktuelles Sprachhandeln im Feld Dialekt/ Standardsprache darstellt “. ${ }^{21}$ Dieser individuenzentrierte Ansatz liegt auch der vorliegenden Fallstudie zugrunde, in der ausgewählte mecklenburgische Dialektsprecher aus der Gemeinde Satow zu ihrem sprachlichen Werdegang und Verhalten sowie zu ihren Spracheinstellungen interviewt wurden. Gleichzeitig wird angenommen, dass die individuellen Sprachbiografien exemplarischen Charakter besitzen und in gewisser Weise die allgemeine Sprachentwicklung in der Region widerspiegeln. So weist etwa Fix darauf hin, dass es berechtigt sei, „individuelle Erfahrung und Erinnerung als Quelle von Sprachwandel heranzuziehen“, und betont den ,sozialen Bezugsrahmen', innerhalb dessen sich der individuelle Erinnerungsprozess vollziehe: ${ }^{22}$ „Wenn man Erinnerungen erhebt, ist man also auf den Einzelnen verwiesen, kann sich aber zugleich darauf verlassen, eine sozial

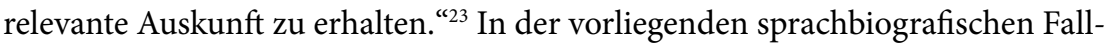
studie wurde der Sprachwandel primär auf familiärer Ebene (Mikroebene) analysiert. Dieser sollte Rückschlüsse bezüglich der Wandlungsprozesse auf der Meso- und Makroebene ermöglichen.

\subsection{Erhebungsort, Vorgehensweise und Korpus}

Die Erhebung der sprachbiografischen Interviews wurde in der Gemeinde Satow durchgeführt. Diese liegt im Norden des mecklenburgischen Dialektgebiets und besteht heute aus rund 5600 Einwohnern. Die Hansestädte Rostock und Wismar sind jeweils $25 \mathrm{~km}$ entfernt und die kleineren Städte Bad Doberan und Bützow etwa $20 \mathrm{~km} .{ }^{24}$

Vor dem Zweiten Weltkrieg umfasste der Ortskern Satows etwa 1000 Einwohner und fungierte mit landwirtschaftlichen Betrieben, Kleinhandel und Handwerk als eine Art wirtschaftliches Zentrum der näheren agrarischen Umgebung. ${ }^{25}$ Noch heute ist die Gemeinde, welche nun mehrere eingemeindete kleine Ortsteile

20 Bieberstedt 2017, S. 60. Zum Konzept der Sprachbiografie vgl. insb. Tophinke 2002.

21 Scharioth 2015, S. 32 unter Verweis auf Macha 1991, S. 217.

22 Fix 1995, S. 34.

23 Fix 1995, S. 34-35. Zur Verallgemeinerbarkeit des sprachbiografischen Einzelfalles vgl. auch Bieberstedt 2015; Bieberstedt 2017; Ehlers 2017.

24 Vgl. Gemeinde Satow. URL: http://www.gemeinde-satow.de/ [Zugriff am 09.09.2013].

25 Vgl. Seils 2012, S. 211.

Birte Arendt, Andreas Bieberstedt and Klaas-Hinrich Ehlers - 978-3-631-71893-3 
umfasst, stark landwirtschaftlich geprägt. Im mecklenburgischen Sprachgebiet ist die Gemeinde Satow eine prototypische Kommunikationsgemeinschaft im ländlich-kleinstädtischen Milieu, dennoch ist sie besonders durch ihre Nähe zu Rostock auch von Urbanisierungsprozessen beeinflusst. Viele Einwohner arbeiten in der Stadt, erledigen Einkäufe oder nutzen auf dem Land fehlende Freizeitangebote. Seit dem massiven Aufbau der Schwerindustrie im nahegelegenen Rostock in den 1950er Jahren und dem damit einhergehenden Ausbau einer weitreichenden Verkehrsinfrastruktur ist Satow in den wirtschaftlichen Verflechtungsraum der Großstadt Rostock eingebunden. Ein Großteil der Bevölkerung pendelt heute über die unmittelbar am Ort vorbeiführende Autobahn A 20 regelmäßig bzw. häufig zu Arbeit, Ausbildung und Einkauf in die Großstadt. Wie die meisten Ortschaften in Mecklenburg-Vorpommern war auch Satow nach dem Kriegsende von einer außerordentlich umfangreichen Zuwanderung von Flüchtlingen und Vertriebenen aus den östlichen deutschen Sprachgebieten betroffen, deren Anteil an der Wohnbevölkerung in Mecklenburg-Vorpommern nach dem Krieg zwischen $40 \%$ und $54 \%$ lag. ${ }^{26}$ Ein Spezifikum des Ortes Satow ist dabei, dass hier eine kompakte, etwa 300 Personen umfassende Gruppe von karpatendeutschen Vertriebenen aus dem Ort Oberstuben (Horná Štubňa) aus dem sogenannten Hauerland, einer ehemaligen deutschen Sprachinsel in der Mittelslowakei, angesiedelt worden ist. ${ }^{27}$

Um Aussagen über den Sprachwandel in der Gemeinde und über Zusammenhänge zur Sprecherbiografie treffen zu können, wurden narrative Interviews mit neun ortsansässigen Dialektsprechern durchgeführt. Da die Befragung Teil meines umfassenderen Dissertationsprojektes ist, bei dem es neben der Erhebung von Daten zu Spracheinstellung und Sprachgebrauch auch darum ging, objektsprachliche Daten zur strukturellen Entwicklung des Niederdeutschen zu erfassen, war eine aktive Sprachkompetenz in der mecklenburgischen Mundart das erste Kriterium bei der Wahl der Probanden. Wie stark diese Dialektkompetenz ausgeprägt ist, war bei der Auswahl der Probanden zunächst nicht ausschlaggebend. Zweitens sollten die Probanden über eine gewisse Ortsfestigkeit verfügen, damit diachrone Aussagen über die örtlichen Wandlungsprozesse getroffen werden können. Die Befragten sollten im Erhebungsort (Gemeinde Satow, inklusive der Ortsteile) wohnen. Aber sie sollten auch in der Gemeinde aufgewachsen und sozialisiert worden sein.

26 Vgl. Beer 2011, S. 106.

27 Vgl. Seils 2012, 206-217; Ehlers 2016. 
Zur diachronen Untersuchung des lokalen Sprachwandels wurden Probanden aus drei verschiedenen Generationen befragt. Die Fallstudie untersucht damit Sprachwandel als apparent-time change. Die Abgrenzung der untersuchten Alterskohorten als „Generationen“ trägt der Tatsache Rechnung, dass die lebensgeschichtlichen Erfahrungshorizonte der Angehörigen dieser Altersgruppen angesichts der politischen und gesellschaftlichen Entwicklung, mit denen sie konfrontiert waren, jeweils große Übereinstimmungen aufweisen. ${ }^{28}$ Durch die Gegenüberstellung der Erfahrungsberichte und Einstellungsäußerungen von Angehörigen verschiedener Generationen sollte es ermöglicht werden, auch Aussagen über den Zusammenhang von sprachlichem und gesellschaftlichem Wandel zu treffen.

Die älteste Generation (Generation 1), mit der Interviews durchgeführt wurden, kann man als Kriegs- und Nachkriegsgeneration bezeichnen. $\mathrm{Zu}$ dieser Generation zählen die Probanden, die bis 1950 geboren worden sind. Die Probanden dieser Generation erlebten ihre Kindheit in der Zeit während des Zweiten Weltkriegs und/oder in der Notzeit der ersten Nachkriegsjahre und wurden in dieser Zeit (auch sprachlich) sozialisiert. Ihr Berufsleben fällt in die Phase des fortschreitenden gesellschaftlichen und wirtschaftlichen Aufbaus der DDR, die vielfach gute Bedingungen für einen beruflichen und sozialen Aufstieg bot. Diese Altersgruppe deckt sich weitestgehend mit der „funktionierenden Generation“ im Generationenmodell der DDR-Geschichte von Ahbe und Gries. ${ }^{29}$

Zur zweiten Generation, die hier untersucht wird, zählen die Probanden, die zwischen 1950 und 1975 geboren wurden. Diese Generation erfuhr ihren Spracherwerb und ihre kindliche und schulische Sozialisation in der gesellschaftlich und wirtschaftlich konsolidierten Deutschen Demokratischen Republik, in der die jüngeren Angehörigen dieser Gruppe auch bereits das Berufsleben aufnahmen. Die Familien der Angehörigen dieser Generation profitierten von der sozialen Sicherheit und dem relativen wirtschaftlichen Wohlstand in der DDR, wurden aber durch die Erfahrung des Mauerbaus und der Niederschlagung des Prager Frühlings 1968 mit der Alternativlosigkeit ihrer Lebenssituation konfrontiert. In ihrer beruflichen Karriere erlebten diese Personen nach der politischen Wende 1989 häufig einschneidende Veränderungen. In dem feiner ausdifferenzierten Generationenmodell von Ahbe/Gries umspannt die hier zusammengefasste Altersgruppe etwa die „integrierte Generation“ und die „entgrenzte Generation“. ${ }^{30}$

28 Zum Begriff der Generation vgl. Ruge 1995, S. 5-9. Der Begriff Generation wird hier somit nicht biologisch, sondern soziologisch-historisch aufgefasst.

29 Vgl. Ahbe/Gries 2006, S. 518-531.

30 Vgl. Ahbe/Gries 2006, S. 531-556.

Birte Arendt, Andreas Bieberstedt and Klaas-Hinrich Ehlers - 978-3-631-71893-3 
In die dritte hier untersuchte Generation fallen die Probanden, die nach 1975 geboren und sozialisiert worden sind. Ihre Kindheit verlebten diese Personen noch in der Endphase der DDR, die Ausbildung bzw. ihr Berufsleben begannen die Angehörigen dieser Generation nach 1989 dann aber in den stark veränderten sozialen und gesellschaftlichen Verhältnissen der Bundesrepublik. Von ihren Eltern übernahmen diese Personen häufig im Gefolge der weltweiten Protestbewegungen der späten 1960er Jahre, welche auch in die DDR vordrangen und im Kontext des Prager Frühlings wahrgenommen wurden, ${ }^{31}$ eine distanzierte bis kritische Haltung gegenüber der DDR. Sie erlebten außerdem als Kinder häufig die Karriereeinbrüche ihrer Eltern nach 1989 und deren z. T. gravierende Folgen für die Familien. Mit Ahbe/Giese kann diese Generation als die Generation der „Wendekinder" beschrieben werden. ${ }^{32}$

Zusammenfassend lässt sich die Einteilung meiner Probanden in folgender Tabelle darstellen:

\begin{tabular}{|l|l|l|l|}
\hline Generation & Probanden & Geburtsjahr & \multicolumn{1}{|c|}{ Sozialer und biografischer Kontext } \\
\hline Eins & 4 & bis 1950 & $\begin{array}{l}\text { Kindheit in Kriegs- und Nachkriegszeit, Beginn } \\
\text { des Berufsleben in Aufbauphase der DDR }\end{array}$ \\
\hline Zwei & 3 & $1951-1975$ & $\begin{array}{l}\text { Aufwachsen in konsolidierter Gesellschaft und } \\
\text { Wirtschaft der DDR, Erfahrung des Mauerbaus } \\
1961 \text { und Niederschlagung des Prager Frühlings } \\
1968,1989 \text { als biografischer Einschnitt in } \\
\text { Berufsleben (der Eltern) }\end{array}$ \\
\hline Drei & 2 & ab 1975 & $\begin{array}{l}\text { Kindheit/Schulzeit in Endphase der DDR, } \\
\text { Aufnahme Berufsleben nach politischer Wende } \\
\text { 1989, Erfahrung der Distanzierung der Eltern } \\
\text { gegenüber DDR und 1989 als Einschnitt in den } \\
\text { Familiengeschichten }\end{array}$ \\
\hline
\end{tabular}

Es ist anzunehmen, dass auch andere Einflussfaktoren wie Geschlecht, Beruf, Einkommen oder die jeweilige Zugehörigkeit zu sozialen Schichten auf den persönlichen Spracherwerb und -gebrauch einwirken können. Diese wurden aber bei der Auswahl der Probanden nicht berücksichtigt, da der Sprachwandel hier vor allem in seiner zeitlichen Entwicklung untersucht werden sollte.

Pro Generation sollten ursprünglich vier Probanden in einem narrativen Interview von ihrer Sprecherbiografie berichten. Während der Vorbereitung der

31 Vgl. Gehrke 2008.

32 Vgl. Ahbe/Gries 2006, S. 556-569.

Birte Arendt, Andreas Bieberstedt and Klaas-Hinrich Ehlers - 978-3-631-71893-3 
Untersuchung stellte sich jedoch heraus, dass es mit jünger werdenden Generationen zunehmend schwerer wurde, kompetente Dialektsprecher im Ort zu finden. Daher begrenzt sich das Datenmaterial für die zweite Generation auf drei und für die dritte Generation auf nur zwei Interviews.

Inhaltliche Schwerpunkte der Interviews waren Spracherwerb, Sprachgebrauch und Einstellungen zur Sprache. Die Datenerhebung erfolgte also durch die Durchführung eines (sprach)biografischen Interviews. Es wurde ein Leitfaden genutzt, der auf verschiedene Bereiche der Sprachbiografie eingeht. Gefragt wurde nach dem Spracherwerb in der frühen Kindheit, dem Sprachgebrauch während der Schulzeit und des Arbeitslebens, dem gegenwärtigen Sprachgebrauch im privaten und öffentlichen Raum, dem persönlichen und örtlichen Sprachwandel sowie nach den Spracheinstellungen. Außerdem wurden die Probanden gebeten, die hochdeutschen und plattdeutschen Sprachkompetenzen für sich und ihre Familienmitglieder einzuschätzen. Zunächst wurde den Gewährspersonen im Sinne des narrativen Interviews die Möglichkeit gegeben, ihre Biografie und Erinnerungen an die Sprachverhältnisse am Ort frei zu schildern. Daher wurden Fragen des Leitfadens nur dann herangezogen, wenn die Gewährsperson nicht schon selbstständig auf die gewünschten Aspekte eingegangen war. Ehepartner wurden in einem gemeinsamen Interview zur Sprecherbiografie befragt, denn ihre Sprecherbiografien entwickeln sich spätestens seit der Hochzeit gemeinsam und beeinflussen sich gegenseitig. Außerdem ergänzen sich die Partner im gemeinsamen Gespräch durch Denkanstöße, Fragen oder Beispiele gegenseitig. ${ }^{33}$ Die Interviews wurden literarisch transkribiert und orthografisch standardisiert. Für

33 Vgl. Fix/Barth 2000, S. 11. Im Anschluss an das Interview wurden die Probanden gebeten, einige Sprachproben abzugeben. Zunächst sollten einige Testsätze, eine Auswahl aus den sogenannten „Wenker-Sätzen“, in den Ortsdialekt übersetzt werden. Diese Übersetzungstests bieten eine einfache Möglichkeit, durch Merkmalsanalysen die Dialektkompetenz der Gewährspersonen zu bestimmen. Für eine standardsprachliche Gegenprobe lasen die Gewährspersonen anschließend die Äsop-Fabel „Nordwind und Sonne" auf Hochdeutsch vor, um auch für die standardnahen Sprechlagen Befunde zu strukturellen Veränderungen über die Generationsfolge zu gewinnen. Die Erhebung von Sprachproben schloss mit einem freien Monolog in der Mundart zu einem möglichst emotionalen Thema, um eine Probe von spontanen dialektalen Äußerungen zu erhalten. Dazu wurde den Probanden zur Anregung, je nach Alter, ein Foto vom zerbombten Rostock im Jahr 1942 oder vom Berliner Mauerfall gezeigt. Eine Ausnahme gab es hier bei der Gewährsperson 4. Da diese Gewährsperson beide Ereignisse nicht miterlebte, wurde sie gebeten, von ihrem letzten Urlaub zu berichten. Die Auswertung dieser objektsprachlichen Daten ist noch nicht abgeschlossen, sie werden in diesem Beitrag nicht berücksichtigt. 
die Interpretation wurden die Aussagen der Gewährspersonen inhaltsanalytisch untersucht und miteinander verglichen, um auf diese Weise Aufschluss über individuelle, individuell übergreifende bzw. generationenspezifische Erwerbs-, Gebrauchs- und Einstellungsmuster und sprachbezogene Konzeptualisierungen zu erlangen.

Die Gewährspersonen werden nachfolgend in Kürze vorgestellt:

Vier Gewährspersonen aus der Generation 1

Gewährsperson 1, männlich, wurde 1934 geboren. Aufgewachsen ist die Gewährsperson in der Nähe von Kröpelin. Kröpelin gehört zwar nicht mehr zur Gemeinde Satow, liegt aber nur ca. zehn Kilometer von Satow entfernt, sodass stets enge Verbindungen zwischen den Gemeinden bestanden. Außerdem wurde GP1 in die Untersuchung aufgenommen, da sie seit der Hochzeit 1957 in der Gemeinde Satow lebt und dort gearbeitet hat. GP1war zunächst als Traktorist und Brigadier tätig, wechselte am Ende der 1950er Jahre jedoch zunächst in die Buchhaltung der LPG und später in die Verwaltung. Anfang der 1990er Jahr ging GP1 in Rente.

Gewährsperson 2 ist die Ehefrau von GP1. Die Gewährsperson wurde 1936 in der Gemeinde Satow geboren, im Nachbarhaus ihres jetzigen Wohnhauses. Sie besuchte die Schule im Nachbardorf und arbeitete anschließend in einem landwirtschaftlichen Betrieb und später bei der LPG des Dorfes. In den 1980er Jahren war sie als Reinigungskraft in einem Studentenwohnheim in Rostock tätig, bis sie eine Stelle in der Strohaufbereitung des VEGs Hohen Luckow bekam, wo sie bis zur Rente 1990 arbeitete. Das Ehepaar GP1 und GP2 hat drei Kinder, welche allesamt heute im gleichen Ort oder im näheren Umkreis leben. Die Eltern beider Gewährspersonen stammen ebenfalls aus der näheren Umgebung Satows, seit 1934 ist die Familie im jetzigen Ortsteil Satows ansässig.

GP5 wurde 1930 in Rostock geboren, die Familie väterlicherseits stammt aus der Hansestadt, die Mutter aus Westpreußen. Nach dem Bombenangriff auf Rostock 1942 zog die Familie in die Gemeinde Satow, wo die Gewährsperson noch heute lebt. Dort besuchte sie auch die Schule und war dort beruflich in verschiedenen Bereichen tätig. Nach dem Schulabschluss arbeitete sie als Dienstmädchen beim örtlichen Pastor. Später arbeitete sie im Hort der Satower Schule und übernahm schließlich die Leitung der örtlichen Bibliothek. Seit der Wiedervereinigung ist die Gewährsperson in Rente. Der verstorbene Ehemann stammte aus dem deutschsprachigen „Hauerland“ in der Slowakei. Die einzige Tochter der Gewährsperson lebt in Rostock. Von 2001-2007 wohnte GP5 in Stuttgart bei ihrem damaligen Lebensgefährten. Die Gewährsperson ist Mitglied der örtlichen Plattdeutsch-Gruppe. 
GP9 wurde 1930 in der Gemeinde Satow geboren. Die Familie väterlicherseits stammt aus Rostock, die der Mutter aus der Gemeinde Satow. Die Gewährsperson besuchte zunächst die Grundschule und dann die Mittelschule in Rostock, die sie aufgrund des Krieges jedoch nicht abschließen konnte. Zunächst war sie dann als Hausmädchen tätig, bevor sie eine Stelle als Telefonistin bei der Post begann. Bevor sie in Rente ging, war sie bei einer Versicherung angestellt. 1950 heiratete die Gewährsperson einen Karpatendeutschen aus der Slowakei, in dem Jahr wurde auch das erste Kind, eine Tochter, geboren. Der Sohn kam 1966 zur Welt. Die Gewährsperson lebt noch immer in ihrem Elternhaus, in welchem nun auch die Familie der Enkeltochter lebt. Der Sohn wohnt im Haus nebenan, die Tochter ist nach Rostock gezogen.

Drei Gewährspersonen aus der Generation 2

Gewährsperson 3 wurde 1954 geboren und wuchs in der Gemeinde Satow auf, wo sie noch heute lebt. Die Gewährsperson besuchte die Schule des Dorfes und ging später auf die Erweiterte Oberschule in Bad Doberan, dann auf die Pädagogische Hochschule Bad Doberan, wo sie eine Ausbildung zur Lehrerin machte. 36 Jahre lang war sie als Lehrkraft tätig, davon 24 Jahre als Schulleiterin. 2013 ging die Gewährsperson in den Ruhestand, jedoch veranstaltet sie weiterhin PlattdeutschAngebote an ihrer ehemaligen Schule. Der Vater der Gewährsperson stammt ebenfalls aus der Gemeinde Satow, die Mutter kam als eine deutschsprachige Vertriebene aus der Slowakei nach Satow. Beide Kinder der Gewährsperson leben ebenfalls in der Gemeinde Satow.

Die zweite Gewährsperson dieser Generation, GP6, wurde 1956 geboren. Ihre Familie mütterlicherseits ist seit ca. 1880 im Wohnort in der Gemeinde Satow ansässig, die Familie väterlicherseits seit 1930. Und auch die zwei Kinder der Gewährsperson wohnen noch dort. 1977 heiratete die Gewährsperson, der Ehepartner stammt aus Sachsen. Die Gewährsperson besuchte die Schule in Satow, machte eine Ausbildung in der Rechtspflege und ist momentan als Versicherungsfachkraft tätig.

Gewährsperson 8 wurde 1951 geboren und befindet sich damit an der genauen Grenze zwischen den Generationen 1 und 2. Auch die Mutter der Gewährsperson stammt aus der Gemeinde. Der Vater stammte aus Ostpreußen. Die Gewährsperson besuchte erst die Satower Schule und ging anschließend auf die Erweiterte Oberschule in Bad Doberan. Heute ist die Gewährsperson in einem Kindergarten beschäftigt. Auch der Ehepartner der Gewährsperson stammt aus der Umgebung, einer Nachbargemeinde Satows. Sowohl die Geschwister als auch der einzige Sohn der Gewährsperson wohnen noch immer in der Gemeinde Satow. 
Zwei Gewährspersonen aus der Generation 3

Gewährsperson 4 wurde 1991 geboren. Die Gewährsperson wuchs in der Gemeinde Satow auf und ging dort zur Grundschule. Anschließend besuchte sie das Gymnasium in Bad Doberan. Momentan studiert die Gewährsperson an der Universität Rostock. Durch das Studium bedingt wechselte GP4 auch den Wohnort und zog in die Universitätsstadt. Dadurch, dass jedoch ihre Familie weiterhin in Satow lebt, sie dort noch viele Freunde hat und Mitglied im Satower Karnevalsverein ist, sind die Bindungen an Satow nach wie vor sehr stark, sie hält sich regelmäßig am Ort auf. Die Familie der Gewährsperson ist seit mehreren Generationen, mindestens seit 1910, in Satow ansässig.

Gewährsperson 7 ist 30 Jahre alt, sie wurde 1983 geboren. Sie verbrachte ihr gesamtes Leben in der Gemeinde Satow, besuchte die Satower Grund- und Realschule und machte in einem örtlichen Betrieb eine Ausbildung im Groß- und Außenhandel, wo sie noch immer tätig ist. Die Gewährsperson ist außerdem Mitglied des örtlichen Posaunenchors. Die Familie von GP7 ist väterlicherseits seit über 100 Jahren im Ort ansässig. GP7 wohnt noch immer mit ihrer Großmutter und ihrem Vater in demselben Haus, das 1916 von der Familie gebaut wurde. Die Mutter der Gewährsperson stammt jedoch aus Thüringen, der Bruder lebt mittlerweile in Wilhelmshaven.

\section{Sprachgebrauchswandel in der Gemeinde Satow: Untersuchungsergebnisse}

Die durchgeführten Interviews zeigen deutlich, dass es im Sprachgebrauch in der Gemeinde Satow einen starken Wandel gegeben hat. Die Interviewaussagen legen nahe, dass die Wandlungsprozesse alle gesellschaftlichen Ebenen betreffen. Sowohl im familiären Kontext, auf der Mikroebene, als auch auf der übergeordneten Makro- und Mesoebene lassen sich sprachrelevante Entwicklungstendenzen aufzeigen. Es wird deutlich, dass sich die Verwendung des Dialekts zunehmend auf die private Kommunikation und auf bestimmte Gesprächspartner bzw. -situationen beschränkt. Die Ursachen und Auswirkungen auf allen gesellschaftlichen Ebenen scheinen sich diesbezüglich wechselseitig zu beeinflussen.

\subsection{Individueller Sprachgebrauchswandel im Generationenvergleich}

\subsubsection{Generation 1 (geb. bis 1950)}

Die Probanden der ersten Generation berichten, dass sie den Dialekt noch regelmäßig verwenden. Das Ehepaar GP1 und GP2 spricht nach eigenen Aussagen untereinander ausschließlich Plattdeutsch. „Wir beide sprechen nur Platt. Da gibt's 
gar nichts anderes“, äußert sich GP2. Die anderen beiden Gewährspersonen dieser Generation (GP 5 und GP9) haben jeweils einen zugewanderten Vertriebenen geheiratet und dementsprechend in der Familie nur wenig Plattdeutsch gesprochen. Diese beiden Probanden berichten jedoch, dass ihre Ehepartner im Laufe der Zeit eine passive Dialektkompetenz entwickelten und deshalb von niederdeutschen Kommunikationen nicht gänzlich ausgeschlossen waren. GP9 berichtet:

„Der [Ehemann, M. B.] hat sich sehr gequält, um die plattdeutsche Sprache zu erlernen. Nämlich hier in der Schmiede wurde nur Plattdeutsch gesprochen. Mein Mann hat dann auch hier mitgearbeitet. Und 1950 haben wir geheiratet. [...] Mein Mann verstand jedes Wort, aber Plattdütsch reden hat er nie gelernt."

Auffällig ist, dass selbst GP1 und GP2, die bis heute Niederdeutsch miteinander sprechen, ihre eigenen Kinder im Hochdeutschen erzogen haben und bis in die Gegenwart mit ihren Kindern und Enkelkindern ausschließlich Hochdeutsch sprechen. Lediglich der älteste Sohn des Ehepaares versteht noch das Plattdeutsche, nur mit ihm wird gelegentlich im Dialekt gesprochen. Bei der sprachlichen Erziehung ihrer Kinder haben sich die Gewährspersonen dem Vernehmen nach aber nicht bewusst für das Hochdeutsche oder gegen das Plattdeutsche entschieden. Die Frage, warum sie mit ihren Kindern Hoch- und nicht Plattdeutsch sprachen, hatten sich die Gewährspersonen nach eigener Aussage vor der Interviewsituation nie gestellt. GP2 berichtet über die Kommunikation mit ihrer Tochter:

„Ja, gleich vom Kind an wurde immer Hochdeutsch gesprochen. Das ist nachher schon zur Gewohnheit geworden. Das hat sich so ergeben. Ich weiß es auch nicht warum. Ich weiß es nicht."

Wahrscheinlich haben allgemein verbreitete negative Einstellungen gegenüber dem Niederdeutschen den Abbruch der innerfamiliären Weitergabe des Dialekts an die Kinder der Generation 1 befördert. GP5 erinnert sich: „Plattdeutsch war ja damals nicht fein. Sogar die Bauern hier haben mit ihren Kindern Hochdeutsch gesprochen. Das Plattdeutsche war ja eine gewöhnliche Sprache.“

Eventuell widerspiegelt das Sprachverhalten gegenüber den Kindern auch einfach die außerfamiliären Sprachverhältnisse der Zeit. Die drei Kinder von GP1 und GP2 sind zwischen 1965 und 1971 geboren, eine Zeit, in der das Plattdeutsche bereits in vielen Domänen des Alltags, beispielsweise im öffentlichen Leben oder in der Arbeitswelt stark rückläufig war. GP2 berichtet beispielsweise: „Ich hab ja denn in Rostock gearbeitet und bei der Uni war ich denn hin und hab da sauber gemacht und hier auf der LPG hab ich ja auch gearbeitet. Da war denn nachher auch schon... Denn hat man da auch schon Hochdeutsch gesprochen, weil da auch viele waren, die gar kein Platt verstanden." 
Im Allgemeinen lässt sich für die Generation 1 feststellen, dass der Personenkreis, mit dem Plattdeutsch gesprochen wird, aktuell fast ausschließlich gleichaltrige Personen umfasst. GP1 und GP2 sprechen mit dem Bruder von GP2 ebenfalls Plattdeutsch, aber auch der ist nur wenige Jahre jünger. Ansonsten wird nur mit einigen gleichaltrigen Nachbarn und anderen Einwohnern aus dem Dorf Platt geredet, die man gut und lange kennt und von denen man weiß, dass sie ebenfalls den Dialekt beherrschen. GP1 erklärt, dass sie an der Färbung des Hochdeutschen erkennen könne, ob jemand Plattdeutsch beherrscht oder nicht. Nur in diesen Fällen spricht die Gewährsperson auch fremde Leute im Dialekt an. GP1 erklärt:

\begin{abstract}
„Also, ich krieg das mit. Wenn eine Person Hochdeutsch spricht, dann hab ich schon zu manchen gesagt, mit mir kannst du Platt sprechen. Man hört das raus, dass die auch Platt sprechen. Die haben 'ne andere... wie sagt man das... Die sprechen Hochdeutsch, aber das klingt irgendwie anders, als wenn das Mecklenburger da drin hängt. Zum Beispiel M., die spricht Hoch, aber die ist Mecklenburgerin. Die stammt aus K., und das hörst du auch, wenn sie Hochdeutsch spricht. Das klingt anders.“
\end{abstract}

GP5 und GP9 sind beide Mitglieder der örtlichen Plattdeutschgruppe, die plattdeutsche Gedichte und Sketche einübt und aufführt. Die Gruppe wurde nach der Wende gegründet. Auch in diesem Kreis wird mehrheitlich der Dialekt verwendet, allerdings sind alle Mitglieder der Gruppe ebenfalls über 70 Jahre alt. Das Niederdeutsche fungiert hier also als Medium für kulturelle und organisierte gesellschaftliche Unternehmungen. Die Pflege des Niederdeutschen ist dabei für die Mitglieder der "Plattschnakers“ eine wichtige Motivation für ihr Engagement in der Plattdeutschgruppe. Ein weiteres Gruppenmitglied erklärt:

„Aus der Öffentlichkeit ist es ganz verschwunden, bis auf solche Gruppen, wie unsere Plattschnakers, die das also noch pflegen und die versuchen, die Plattdeutsche Sprache zu erhalten. Wir suchen ganz dringend jüngere Leute, junge Leute, die mit uns gemeinsam pflegen. Aber da haben wir bis jetzt wenig Erfolg gehabt. " ${ }^{34}$

Eine Konversation auf Plattdeutsch mit jüngeren Menschen kommt in der ältesten Generation so gut wie gar nicht vor. Eine Ausnahme stellt dabei GP9 dar. Die Gewährsperson lebt mit der Familie der Enkeltochter unter einem Dach. Mittlerweile hat auch die Enkeltochter begonnen Plattdeutsch zu lernen, um die Tradition des Dialekts in der Familie zu erhalten. GP9:

34 Diese Gewährsperson ist männlich und wurde 1939 in Hinterpommern geboren. Sie wurde im Rahmen der Datenerhebung interviewt. Da diese Gewährsperson erst seit 1978 vor Ort wohnhaft ist, wurde sie bei den weiteren Analysen nicht berücksichtigt. Die Gewährsperson war von 1994-2003 ehrenamtlicher Bürgermeister von Satow und ist Mitglied der örtlichen Plattdeutschgruppe. 
„Miene Enkeldochter, die hie im Hus wahnt, [...] un die versäucht nu Plattdeutsch zu schnacken, versäucht. Un ich würd seggen, die kann dat eigentlich all ganz gaud. Sie kann dat eigentlich. Aber mit ihrer Kinder, A. is nu zweite Klass, die antworten mi up Hochdeutsch. Un ich spreck Plattdeutsch mit ehr in der Hoffnung, sie liern dat.“

Durch diese bewusste Entscheidung wird in dem Vier-Generationen-Haushalt auch mit den Urenkeln der Gewährsperson wieder vermehrt Plattdeutsch gesprochen. Wie das Zitat zeigt, befindet sich die Familie gegenwärtig noch in einer Phase der Wiedereinführung des plattdeutschen Sprachgebrauchs, die maßgeblich von der (Ur)Großmutter aus der Generation 1 initiiert wird.

\subsubsection{Generation 2 (geb. 1951-1975)}

In der Generation 2 ist der Gebrauch des Plattdeutschen den Erzählungen der drei Gewährspersonen nach stärker eingeschränkt als in der Generation 1. GP8 berichtet zum Beispiel, dass sie selbst kaum Plattdeutsch spricht. Obwohl der Ehepartner und auch der Sohn Plattdeutsch sprechen könnten, wird in der Familie nur Hochdeutsch kommuniziert. Gelegentlich nutzt die Gewährsperson den Dialekt in der Kommunikation mit der kleinen Enkeltochter, jedoch nur zum Schimpfen, weil das Platt weniger hart klinge als auf Hochdeutsch, erklärt GP8. Auch viele andere Gewährspersonen teilen die Auffassung, dass das Plattdeutsche weniger hart bzw. lustiger klingt. GP2 erklärt beispielsweise: „Aber manchmal kannst du auf Platt besser sagen wie auf Hochdeutsch, gibt's auch. Ja, es gibt auch lustige Wörter. Aber man kann auf Platt manche gar nicht so beleidigen als wenn man auf Hochdeutsch spricht. ${ }^{{ }^{3} 3}$

Die einzige Person, mit der sich GP8 noch häufiger auf Platt verständigt, ist der Bruder. Auch im Ort findet der Dialekt nach Aussagen der Angehörigen dieser Generation nicht mehr viel Verwendung, lediglich wenn man ältere Menschen trifft, von denen die Gewährsperson weiß, dass sie Platt sprechen, werden einige Worte auf Platt gewechselt. Seit kurzer Zeit verwendet die Gewährsperson das Plattdeutsche vermehrt während der Arbeit im Kindergarten, um die Kinder an den Dialekt heranzuführen. „Wir machen Plattdeutsch hier im Kindergarten. Die Kinder haben Freude dran und lernen schnell, wenn man das so spielerisch macht", sagt GP8.

35 Die Annahme, dass Schimpfen auf Niederdeutsch weniger verletzend wirke als auf Hochdeutsch, ist ein auch weit über Mecklenburg-Vorpommern hinaus weitverbreiteter Topos in Einstellungsäußerungen gegenüber dem Dialekt, vgl. Arendt 2010, S. 197 zum „Humortopos“; zu nahezu identischen Einstellungsäußerungen bei Hamburger Probanden Jürgens 2015, S. 305-309 sowie aktuell Jürgens/Schröder 2016. 
Auch GP3 bemüht sich um den Erhalt des Plattdeutschen und gibt Kurse in der Satower Schule. Sie erzählt: „Als ich eben älter wurde und gemerkt habe in der Schule...Halt, Stopp! Das ist vielleicht zehn Jahre her. Jetzt bin ich 36 Jahre im Dienst. Sagen wir mal vor zehn Jahren, da hab ich gedacht: Meine Güte, das verschwindet, wenn wir nichts machen." GP3 schildert ihren Plattdeutsch-Unterricht folgendermaßen:

„So, und man muss sie neugierig machen und sie motivieren. Und das versuchen wir nun eben, indem wir... na, in R. ist doch dieser Denkmalhof, wie haben die Leute früher gelebt. Dass man da eben den Anknüpfungspunkt findet. Und über Lieder, schöne Gedichte, man sucht jetzt. Und wir haben auch überlegt, ich hab da auch Material, richtig für Stundenaufbau und so, aber man muss sie erst mal ein bisschen motivieren und da fängt man mit Liedern am besten an und guckt, wie sie früher gelebt haben, und erzählt dies und das und jenes. Und versucht, dass sie sich das auch ein bisschen erschließen."

Niederdeutschkompetente Angehörige der Generation 2, die in Bildungsinstitutionen arbeiten, ergreifen in den Jahren zwischen 1989 und etwa 2000 die Initiative und beginnen sich innerhalb ihres Arbeitsumfeldes für den Spracherhalt einzusetzen. Dabei steht aber weniger die Alltagskommunikation im Vordergrund, sondern Gedichte und Lieder. Schüler und Kindergartenkinder müssen dabei an das Plattdeutsche bereits wie an eine Fremdsprache herangeführt werden.

Ganz ähnliche Sprachverhältnisse beschreiben auch die Gewährspersonen GP3 und GP6. Das Plattdeutsche wird auch von ihnen allenfalls gegenüber älteren Nachbarn, Bekannten und Familienmitgliedern verwendet. Mit Gleichaltrigen wird der Dialekt nur dann verwendet, wenn man von der Dialektkompetenz des Gesprächspartners weiß. Gegenüber jüngeren Personen wird der Dialekt kaum im Alltag verwendet, sondern wenn überhaupt, dann auch bei diesen beiden Gewährspersonen in der bewussten Absicht, sie an den Dialekt heranzuführen. „Und wenn sie [die Großmutter von GP6, M.B.] mit uns geredet hat: ,Mak de Dör tau!', denn... wenn ich das zu meinen Enkeltöchtern sage, die sind elf und acht. Denn ,Was hast du gesagt Oma?' Ich sag: ,Ihr sollt das nebenbei lernen“', erzählt GP6.

\subsubsection{Generation 3 (geb. nach 1975)}

Am stärksten begrenzt ist der Dialektgebrauch in der jüngsten Generation. Beide Gewährspersonen dieser Altersgruppe haben jeweils nur noch eine Bezugsperson, mit der die Kommunikation häufig auf Plattdeutsch stattfindet. GP7 nutzt das Plattdeutsche darüber hinaus noch vereinzelt, z. B. im Gespräch mit einem Arbeitskollegen und einem ehemaligen Mitschüler. Erinnert werden einzelne Kommunikationssituationen, so beim Bäcker. GP7 erzählt: 
„Was ich schon mal gemacht habe beim Bäcker, da stand ein älterer Mann, so Renter war er schon, und quatschte mich an ,Oh, de Diern kümmt!' Und da hat er wohl nicht mit gerechnet, dass ich auf Plattdeutsch antworten könnte oder dass ich ihn überhaupt verstehe. Und dann habe ich ihm aber auch auf Platt geantwortet und dann war er erst mal überrascht. Und die Bäckersfrau stand auch hinterm Tresen und machte große Augen. Und dann habe ich ihr auf Platt meine Bestellung aufgegeben."

Derartige Situationen werden aber ausdrücklich als Ausnahmesituationen dargestellt, die keine langen, umfangreichen Konversationen beinhalten. Außerdem ergäben sich solche Ausnahmen nur selten.

Auch innerhalb der eigenen Familien verwenden die Gewährspersonen der Generation 3 das Plattdeutsche nur noch selten. GP4 erzählt:

„Also, früher war ich ja jeden Tag bei meiner Oma. Sie hat mich ja zur Schule gebracht und, wenn Mutti und Papa arbeiten mussten, mich wieder abgeholt. Ich war jeden Nachmittag da und sie wohnt ja auch direkt neben mir und dadurch hab ich jeden Tag Platt gesprochen. Aber als ich nachher aufs Gymnasium gegangen bin mit der siebten Klasse, und ich selbstständiger wurde, meine Schulzeiten anders wurden und ich dann mehr zuhause war, ist das alles abgeschwächt.“

GP4 erklärt sogar, dass sie außer im Gespräch mit der eigenen Großmutter innerhalb wie außerhalb der Familie ausschließlich Hochdeutsch spricht. „Also wir untereinander reden alle Hochdeutsch und nur mit meiner Oma reden wir Plattdeutsch. [...] Also, es herrschte immer das Hochdeutsche vor. Und wie gesagt, wenn meine Oma da nicht so dieser treibende Kern wäre, dann wäre das auch in unserer Familie nicht“, sagt sie. Und sie vermutet, dass sie nach deren Tod wahrscheinlich gar kein Plattdeutsch mehr sprechen wird.

\subsection{Lokaler Sprachgebrauchswandel im Kontext historischen Wandels}

\subsubsection{Phase I: Niederdeutsch als dominantes lokales Kommunikationsmittel vor 1945}

Die Gewährspersonen der ältesten Generation berichten einheitlich, dass vor 1945 in der Gemeinde fast ausschließlich Plattdeutsch gesprochen wurde, der Dialekt war in allen Lebensbereichen die dominante Sprachform. Eine hochdeutsche Sprachdomäne stellte in dieser Zeit lediglich die örtliche Schule dar. GP9 erinnert sich, dass sie während ihrer Kindheit nur Plattdeutsch gesprochen habe, in der Familie, mit Freunden, mit den Lehrlingen des elterlichen Handwerksbetriebes oder beim Einkaufen. Ihrer Erinnerung nach haben auch alle anderen Dorfbewohner untereinander ausschließlich Plattdeutsch miteinander gesprochen. „Hier waren ja die ganzen Bauern und so, die haben unter sich [Platt gesprochen, 
M. B.]", so beschreibt GP5, die als Jugendliche aus der Stadt nach Satow zog, als „objektive“ Beobachterin den frühen Sprachgebrauch in Satow. Für sie erklärt sich dieser Sprachgebrauch im Unterschied zur Großstadt aus dem ländlichen Charakter Satows zur damaligen Zeit. Arendt spricht in diesem Zusammenhang von einem „Ländlichkeitstopos“, den sie „als Verstehensressource mit beeindruckender Erklärungskraft“ beschreibt, „der die eigenen Erfahrungen ebenso prägt wie er auch die Prognosen zum Sprachwandel determiniert “. ${ }^{36}$ Die Aussagen der beiden ersten Probanden werden auch durch die Gewährspersonen GP1 und GP2 bestätigt. Beide können sich nicht erinnern, dass sie vor dem Schuleintritt überhaupt Kontakt zum Hochdeutschen hatten: „Wir haben ja... Das Einzigste, was wir gelernt haben, war Hochdeutsch nachher in der Schule. Wir haben ja auch alle... wir haben ja hier Platt gesprochen. Wir sind ja mit Platt aufgewachsen“, sagt GP2. Auch GP1 erinnert sich:

„Ich bin 1941 zur Schule gekommen und da ging das ja mit Hochdeutsch los und da musste der Lehrer erst mal Platt mit uns sprechen und sagen, das ist das und das ist das. Wir hatten so einen ganz alten Herrn als Lehrer. Da habe ich erst Hochdeutsch gelernt.

Das hat sich dann so ergeben, zuhause Platt und [in der Schule Hochdeutsch, M. B.].“

Vor 1945 war für die damals jungen Angehörigen der Generation 1 die Schule dem Vernehmen nach die einzige Domäne ihres sprachlichen Umfeldes, in der Hochdeutsch gesprochen wurde. GP1 beschreibt hier gewissermaßen eine klassische Diglossie-Situation.

\subsubsection{Phase II: Rückgang des Niederdeutschgebrauchs nach 1945}

Einen massiven Umbruch im Sprachgebrauch in der Gemeinde Satow sehen die Gewährsleute nach dem Zweiten Weltkrieg. GP2 berichtet beispielsweise folgendermaßen: „Also verändert hat sich das nachher nach '45, wie dann die Umsiedler gekommen sind. Und die konnten sowieso kein Platt. Und da mussten wir ja mit denen auch Hochdeutsch sprechen. "Unter anderem durch eine hohe Anzahl nicht-mecklenburgischer Einwohner in der Gemeinde sei das Plattdeutsche zunehmend aus der Kommunikation in öffentlichen Bereichen, auch aus der Arbeitswelt verschwunden. Die Gewährspersonen GP1 und GP2 arbeiteten beide in der Landwirtschaftlichen Produktionsgenossenschaft (LPG). Selbst in einer typisch plattdeutschen Domäne, der Landwirtschaft, ging die Verwendung des Dialekts demnach zurück. Die Gewährspersonen erklären dies damit, dass viele sogenannte „Umsiedler“ in der LPG beschäftigt waren. Plattdeutsch sei nur

36 Arendt 2010, S. 156. Vgl. dazu auch Jürgens/Schröder 2016, S. 346-349 und 374-376. 
noch mit einzelnen Kollegen gesprochen worden, von denen man gewusst habe, dass sie Mecklenburger sind. Über die Kommunikation mit Arbeitskollegen der LPG sagt GP1: „Wenn man weiß oder wusste, das ist ein Mecklenburger, der kann das, dann haben wir dann Platt gesprochen. "Die Ehefrau GP2 schildert die Sprachverhältnisse in den heterogen zusammengesetzten Belegschaften am Arbeitsplatz wie folgt:

„Mit einigen schon ja... und auch wie ich nach Rostock gefahren bin, da wurde auch Hochdeutsch gesprochen und nachher, wie ich in H. [bei der LPG, M. B.] gearbeitet hab, ja... da war ja dann Herr S. als Schichtleiter, da haben wir Platt mit gesprochen. Aber die anderen haben alle Hochdeutsch gesprochen. Und Herr J., der hat ja auch Platt gesprochen. Und Frau S., mit der sprechen wir auch Platt."

Aber auch auf den privaten, familiären Sprachgebrauch habe sich die Zuwanderung von Ortsfremden ausgewirkt. Die Hälfte der Gewährspersonen aus der ersten und zweiten Generation berichtet, dass entweder ein Elternteil von ihnen „Umsiedler" war und/oder dass sie selbst einen Vertriebenen bzw. einen NichtMecklenburger geheiratet haben. Dadurch sei auch in der Familie zunehmend Hochdeutsch gesprochen worden, weil entweder in der elterlichen oder in der eigenen Ehe ein Partner das Mecklenburgische nicht beherrscht habe. Dies habe sich besonders auf die sprachliche Biografie der Kinder ausgewirkt, da diese entsprechend meist auf Hochdeutsch erzogen worden seien. GP9 berichtet zum Beispiel, dass der karpatendeutsche Ehepartner zwar gelernt habe, Plattdeutsch zu verstehen, es aber nicht sprechen gelernt habe. Die Kinder dieses Ehepaares können den Dialekt zwar sprechen, tun dies jedoch nur sehr selten. Sie erzählt: „F. [der Sohn, M. B.] kann auch Plattdütsch schnaken. Und C. [die ältere Tochter, M. B.] noch viel besser. Die schnacken auch mal Plattdütsch. Mit mir reden sie Plattdütsch.“

Dass die massive Zuwanderung von Ortsfremden in die lokalen Kommunikationsgemeinschaften von der alteingesessenen Bevölkerung regelmäßig als Erklärung für den Rückzug des Niederdeutschen aus den öffentlichen Domänen angeführt wird, berichtet auch Ehlers aus seiner umfangreichen Befragung von Zeitzeugen im Großraum Rostock. Der Verweis auf die Immigranten bietet nach Ehlers einen vordergründig sehr plausiblen Argumentationstopos, mit dem die komplexen Rahmenbedingungen des Sprachgebrauchswandels auf eine einfache Ursache reduziert werden und die Beteiligten sich von ihrem eigenen Anteil an der Entwicklung entlasten können. ${ }^{37}$ Erfahrungen, die dieser verbreiteten Erklärung zuwiderlaufen, werden dagegen von befragten Zeitzeugen kaum relevant

37 Ehlers 2013, S. 114.

Birte Arendt, Andreas Bieberstedt and Klaas-Hinrich Ehlers - 978-3-631-71893-3 
gemacht. So berichten meine Gewährspersonen aus Satow durchaus auch von einigen ihnen bekannten Zuwanderern, die den örtlichen Dialekt erworben und auch aktiv verwendet hätten. ${ }^{38}$ Gelegentlich stellen Gewährsleute klar, dass die Migrationsbewegungen der Kriegs- und Nachkriegsjahre eben nicht der alleinige Grund für den Rückgang des Dialekts sein können. So beschreibt es auch GP9. Sie erinnert sich, dass zwar mit dem Zuzug der Ortsfremden vermehrt Hochdeutsch gesprochen wurde, es aber trotzdem keinen schlagartigen Abriss in der Verwendung des Mecklenburgischen gegeben habe. Sie erinnert sich: „Dann kamen ja Umsiedler. Die kamen aus Ostpreußen. Später waren es welche aus Sudetenland und aus Schlesien und so weiter. Und die konnten ja kein Plattdeutsch. Und mit den Menschen mussten wir ja dann Hochdeutsch reden. "Ihrer Erinnerung nach war der Rückgang des Dialekts ein langwieriger Prozess, der sich über viele Jahre hinweg gezogen habe. Das lässt sich durch die bereits zitierten Aussagen von GP2 stützen, die aussagte, dass auch andere Faktoren zum Rückgang des Plattdeutschen führten. Sie nannte zum Beispiel das Pendeln zur Arbeit in Rostock. Und auch die Gründung der LPG trug wie bereits erwähnt dazu bei, da dort Menschen aus unterschiedlichen Regionen und Dialektgebieten in großen Belegschaften zusammenarbeiteten.

\subsubsection{Phase III: negative Einstellungen gegenüber dem Dialektgebrauch seit den 1960er Jahren}

Die Gewährspersonen beschreiben die den Nachkriegsjahren folgenden Jahrzehnte als eine Zeit, in der die Verwendung des Plattdeutschen bewusst vermieden wurde.

„Und nun, 'ne Zeit lang, fand ich persönlich, da haben die Leute sich geziert, auf Plattdeutsch zu schnacken. Das wier in den 60er/70er Jahren. Die haben sich geziert in dem Sinne, wer Pladdüütsch schnaken det, dei künn das Hochdeutsche nich, die künn das nich richtig. Un die ha Angst, dat sei mir un mich verwesseln un ut det Grund heben sei Hochdeutsch reden.",

erinnert sich GP9. GP6 erinnert sich an ein Gespräch mit einem Freund aus der Stadt. Sie fragte ihn, warum er kein Platt sprechen kann. GP6 erzählt:

„Wenn wi platt hebben spraken, denn het dat so heiten, ach dat is ja nur $\mathrm{n}$ dumm Buernjung. Dat is ja nicht fin. Un wenns nich fin is, denn bruchst dat ok nich liern. Un dat is so trurich. Un dorvun könn di ganzen jung Lüüd tu min Tied, die künn dat all verstan, aber spreken is nich. Un dat wier hier nich wirklich anners."

38 Ähnliche Fälle dokumentiert Ehlers 2011 und 2013. 
Eine ähnliche Aussage machte auch GP5, sie beschreibt eine Zeit, in der das Plattdeutsche "nicht modern war.“ Die Gewährspersonen ordnen diese Phase ungefähr in den 1960er und 1970er Jahren ein. Das Niederdeutsche war in dieser Zeit nach der Erinnerung der Gewährspersonen als ,bäurisch' und ,unfein stigmatisiert. Gegen die hochdeutsche Prestige-Norm zu verstoßen war, wie GP9 ausdrücklich erzählt, mit „Angst“ besetzt.

Hier bestätigen meine Gewährsleute aus Satow die Ergebnisse ähnlicher Befragungen von Bieberstedt im ländlich geprägten Hamburger Stadtrandviertel Kirchwerder, in denen die Befragten ebenfalls die entscheidende Umbruchphase im Gebrauch des Niederdeutschen in die 1960er Jahre verlegen. Diese Umbruchphase wird von den Hamburger Probanden ebenfalls als Periode des Sprachbewertungswandels und der Stigmatisierung des Dialektgebrauchs gekennzeichnet. ${ }^{39}$

\subsubsection{Phase IV: weiterer kommunikativer Dialektabbau neben bewusster Dialektpflege seit 1989}

GP5 stellte jedoch auch fest, dass sich nach der Wende der Sprachgebrauch des Plattdeutschen wieder verstärkt habe, als sich sowohl in Satow als auch in der Umgebung zahlreiche Plattdeutsch-Gruppen gründeten, die sich die Dialektpflege zur Aufgabe machten. Der politische Umbruch hatte nach Ansicht von GP5 einen entscheidenden Einfluss auf die Gründung der Plattdeutsch-Gruppen und damit die verstärkte Pflege des Dialekts. Die Freiheit der Vereinsgründung und der ungehinderten kulturellen Betätigung werden offensichtlich als wesentliche neue politische Rahmenbedingungen für eine institutionelle Dialektpflege angesehen. GP5:

„Das wurde dann ja besser noch. Schon die Gruppen, die sich dann so... Dann kam Doberan, hatt schon ne Gruppe, Warnemünde hatte schon ne Gruppe, Kühlungsborn hatte ne Gruppe und alles diese kleinen Orte, Satow denn. Und so haben sie sich denn so zusammen, so. Und haben dann, wie die Chöre, die entstanden ja nachher auch, und so kam Plattdeutsch auch. Ich finde, man hatte...Wir wollen mal ganz ehrlich sagen. Man konnte sagen, was man mal wollte. Bei dem andern... war ja nur immer...du durftest ja nicht viel sagen. Ich mein Plattdeutsch ja, aber du durftest ja nicht alles so bringen. Heute bist du frei und offen und kannst das einfach mal so bringen. [...] Wir sind doch jetzt frei, wir können doch jetzt sagen und tun was wir wollen."

39 Bieberstedt 2015, S. 232-233. Vgl. ebenso Bieberstedt 2016. Zum stereotypen Kategorisierung des Plattdeutschen als, ländlich' und ,Unterschichtssprache`vgl. Arendt 2010, S. 156, 192-195 und für den Raum Hamburg Jürgens 2015, S. 324-344. 
Das Engagement für den Dialekterhalt führte jedoch nicht zu einer stärkeren Verwendung des Plattdeutschen außerhalb dieser Gruppen. In der Alltagskommunikation nahm und nimmt der Dialektgebrauch im Ort nach wie vor ab. Der Rückzug des Plattdeutschen sowohl aus der öffentlichen als auch aus der privaten Kommunikation hat sich bis heute von Generation zu Generation verstärkt. Für die Phase seit der politischen Wende 1989 lässt sich feststellen, dass sich jenseits bewusster Dialektpflege der alltägliche Gebrauch des Dialekts inzwischen auf bestimmte Gesprächssituation und sogar auf bestimmte einzelne Gesprächspartner beschränkt. Diese sind meist gleichaltrige Nachbarn oder ehemalige Arbeitskollegen, in jedem Fall handelt es sich um Gesprächspartner, von deren Niederdeutschkompetenz man weiß. Den gegenwärtigen Sprachgebrauch beschreibt GP2 folgendermaßen:

„Überall [wird] nur Hochdeutsch gesprochen. [...] Man mag ja auch heute gar nicht mehr einfach Platt sprechen. Man müsste ... an und für sich müsste man ja eigentlich nur Platt sprechen, auch wenn man einkauft. Aber das macht man ja eigentlich gar nicht. [...] Man traut sich auch überhaupt gar nicht, die Leute mit Plattdeutsch anzusprechen, weil man genau weiß, die sprechen sowieso kein Platt."

Der Personenkreis der Gesprächspartner, mit denen die Gewährspersonen Plattdeutsch sprechen, wird von Generation zu Generation kleiner.

\section{Fazit}

Die sprachbiografischen Erzählungen der neun Gewährspersonen aus Satow belegen, dass die eingangs beschriebene Entwicklung der Entdiglossierung nach dem Modell von Schmidt in Mecklenburg noch nicht vollständig ihre vierte und letzte Stufe erreicht hat. Es gibt am Untersuchungsort bis heute eine „verborgene hochdeutsche-niederdeutsche Zweisprachigkeit“. ${ }^{40}$ Bei diesem Befund ist allerdings in Rechnung zu stellen, dass es sich um einen Untersuchungsort im Übergang zum ländlichen Raum handelt und dass es auch in diesem Ort bereits recht schwierig war, noch dialektkompetente Gewährspersonen in den jüngeren Generationen zu finden. Die hochdeutsch-niederdeutsche Diglossie nimmt also selbst in diesem ländlichen Kommunikationsraum nur einen marginalen Stellenwert ein. In den meisten Familien am Ort ist auch heute schon mit monoglossischen Sprachverhältnissen zu rechnen, in denen Sprachvariation nur noch innerhalb der standardnahen regiolektalen Sprachlagen, aber nicht mehr unter aktiver Einbeziehung auch des Niederdeutschen vollzogen wird.

40 Stellmacher 1990, S. 12. 
Die Gewährpersonen stellen in ihren Interviews selbst einen Zusammenhang zwischen dem Sprachwandel auf der Mikroebene (Sprachgebrauch in der eigenen Familie) und der Mesoebene (Sprachgebrach am Ort) mit allgemeinen gesellschaftlichen Makroentwicklungen her. Hier dominiert sehr deutlich der Verweis auf die Immigrationsbewegungen nach dem Krieg (Immigration von Flüchtlingen und Vertriebenen und spätere Arbeitsbinnenmigration innerhalb der DDR). Auf gesellschaftliche Makrobedingungen des eigenen und des örtlichen Sprachgebrauchswandels wird aber auch verwiesen, wenn ein Zusammenhang mit den Arbeitsverhältnissen in den seit 1952 gegründeten LPGs, mit den allgemein verbreiteten negativen Einstellungen gegenüber dem Dialektgebrauch (vor allem 1960er Jahre), mit der Arbeitsaufnahme als Berufspendler in der nahen Großstadt (mit der Industrialisierung Rostocks seit den 1950er Jahren zunehmend) hergestellt wird. Als einschneidende Zäsur in der persönlichen und örtlichen Sprachentwicklung ist offenbar auch die politische Wende 1989 erfahren worden, nach der niederdeutschkompetente Personen zum Teil zu einem aktiven Engagement für den Dialekterhalt übergingen.

Die Interviews dokumentieren dabei eindrucksvoll, wie schnell und durchgreifend sich der kommunikative Dialektabbau in Mecklenburg in der zweiten Hälfte des 20. Jahrhundert im familiären Mikroumfeld und in der Kommunikationsgemeinschaft des Dorfes vollzogen hat. Vor 1945 war der Dialekt demnach die gängige Alltagssprache in der Gemeinde. Plattdeutsch wurde von allen in allen Lebenslagen gesprochen. Mit dem starken Zuzug der nichtmecklenburgischen Einwohner nach dem Krieg verschwand nach Darstellung der Gewährspersonen die mecklenburgische Mundart aber zunehmend aus der Öffentlichkeit. Allerdings muss die in der älteren Forschung getroffene Feststellung, die Migrationsbewegungen der Kriegs- und Nachkriegsjahre seien ein entscheidender Auslöser für das Schwinden der Mundart in Mecklenburg gewesen, ${ }^{41}$ als zu pauschal zurückgewiesen werden. Die Aussagen der Gewährspersonen aus der Gemeinde Satow bestätigen nämlich auch die neueren Untersuchungsergebnisse von Ehlers, ${ }^{42}$ denen zufolge viele Umsiedler die mecklenburgische Mundart annahmen und verwendeten. Jedoch scheinen diese demografischen Prozesse für eine Bewusstseinsänderung bei den Alteingesessenen gesorgt zu haben. Sie setzten die Dialektkompetenz bei ihren Gesprächspartnern nun nicht mehr voraus und nutzten in der Öffentlichkeit oder im Gespräch mit weniger gut bekannten Personen das Hochdeutsche. Dadurch verschob sich der Sprachgebrauch der mecklenburgischen Mundart in den privaten Bereich.

41 Z. B. Gernentz, 1980, S. 134-135.

42 Vgl. Ehlers 2013.

Birte Arendt, Andreas Bieberstedt and Klaas-Hinrich Ehlers - 978-3-631-71893-3 
Die Aussagen der Gewährspersonen machen aber auch deutlich, dass auch weitere gesellschaftliche Faktoren den Sprachwandel vorantrieben. Beispielsweise wuchs die Mobilität der Arbeitnehmer und es gab ein verstärktes Pendeln zum Arbeitsplatz. GP2 verdeutlichte den damals vorherrschenden Topos, in der Stadt, Rostock, müsse man Hochdeutsch reden. Aber auch die Gründung der Landwirtschaftlichen Produktionsgenossenschaften, die die landwirtschaftlichen Familienbetriebe und traditionellen Gutswirtschaften ablösten, trug erheblich zum Rückgang des niederdeutschen Sprachgebrauchs am Arbeitsplatz bei. Dort arbeiteten viele Menschen aus unterschiedlichen Regionen und Dialektgebieten zusammen. Das Hochdeutsche übernahm die Funktion einer Mittlersprache am Arbeitsplatz.

So zeigt der Vergleich der Aussagen von Gewährsleuten verschiedener Generationen, dass der Gebrauch des Mecklenburgischen seit der Nachkriegszeit permanent rückläufig ist. Schon die Generation 1 hat den Dialekt meist nicht mehr an ihre Kinder weitergegeben. Die 1960er und 1970er Jahre werden von den Probanden als besonders dialektfeindlich dargestellt. Gegenwärtig wird der Dialekt nur noch in Situationen genutzt, in denen sich die Gesprächspartner gut kennen. Denn die Dialektkompetenz kann nicht mehr vorausgesetzt werden, sodass Plattdeutsch nur noch gesprochen wird, wenn sicher ist, dass der Gesprächspartner ebenfalls dialektkompetent ist. Die lokale Alltagskommunikation findet nur noch selten auf Plattdeutsch statt, da es in der Gemeinde, besonders unter den jüngeren Einwohnern, nur noch wenige Dialektsprecher gibt.

Damit in Zusammenhang lässt sich eine veränderte funktionale Differenzierung hinsichtlich des Gebrauchs des Plattdeutschen und des Hochdeutschen feststellen. Die Wahl zwischen dem Hochdeutschen und dem Niederdeutschen ist nicht mehr an bestimmte Kommunikationsräume, wie etwa den Unterschied zwischen Stadt und Land oder zwischen dem öffentlichen (z. B. vor 1945 die Schule, später der Arbeitsplatz) und dem privaten Bereich (Familie, Freunde) gebunden. Auch in den ehemaligen Domänen des Niederdeutschen dominiert inzwischen das Hochdeutsche. Vielmehr wird - innerhalb der Nahkommunikation - der Varietätengebrauch heute vorrangig vom Alter des Gesprächspartners bestimmt. Während älteren Einwohnern gegenüber noch oftmals Platt gesprochen wird, wird jüngeren Personen gegenüber Hochdeutsch gesprochen, selbst wenn diese über (wenn auch geringe oder nur passive) Dialektkompetenzen verfügen.

Die Gewährspersonen können genau aufzählen, mit wem sie Plattdeutsch sprechen. Von Generation zu Generation nimmt die Anzahl der dialektkompetenten Gesprächspartner ab. Vor allem in der Generation 2 und 3 handelt es sich dabei zum Großteil um ältere Verwandte und Bekannte aus der Generation 1. 
Aus diesem Grund ist mit hoher Wahrscheinlichkeit anzunehmen, dass der Gebrauch des Plattdeutschen ca. in den nächsten zehn Jahren nochmals einen gewaltigen Rückschritt machen wird, wenn die erste Generation nicht mehr existiert. Sämtliche Probanden sind aus diesem Grund überzeugt, dass das Plattdeutsche in der Gemeinde bald aussterben wird. GP2 sagt: „Ja, die älteren Leute sind alle ausgestorben und die jetzt so wohnen, die sprechen alle Hochdeutsch. Und da spricht auch keiner von Platt. Ja, so ist das so, wenn die Älteren aussterben, dann ist Schluss [mit dem Niederdeutschen, M. B.]." Insofern bestätigen die Aussagen der linguistischen Laien also grundsätzlich das theoretische Modell von Schmidt, wonach sich auch im Sprachraum Satow eine hochdeutsche Monoglossie in naher Zukunft durchsetzen wird.

Es ist andererseits aber auch festzustellen, dass etwa seit der politischen Wende 1989 für viele Dialektsprecher in der Gemeinde Satow der Erhalt des Niederdeutschen ein wichtiges Ziel geworden ist. Nach Aussagen der Probanden bemühen sich viele Mitglieder der restlichen niederdeutschen Sprachgemeinschaft um den Erhalt des mecklenburgisch-vorpommerschen Dialekts. Sie achten deshalb auf eine verstärkte Verwendung des Dialekts und bemühen sich, ihre Mundart an jüngere Generationen in ihren Familien weiterzugeben. Außerdem wird der Dialekt zunehmend als Kulturgut bei gesellschaftlichen Veranstaltungen gepflegt, zum Beispiel in den Kindergarten- und Schulunterricht oder bei Feiern und Festen, für die die örtliche Plattdeutschgruppe gern gebucht wird. Diese Art des Sprachgebrauchs wird neben einem bewussten, zielgerichteten Sprachgebrauch im Alltag genutzt, um Dialekt an jüngere Generationen weiterzugeben. „Nun muss man ihnen das natürlich auch ein bisschen nahebringen“, schlussfolgert GP3 über den Sinn des Plattdeutschkurses an der Satower Schule.

Während das Niederdeutsche nach den Erzählungen der Gewährspersonen in seiner Funktion als Kommunikationsmittel heute stark eingeschränkt ist, erfüllt es zunehmend eine kulturelle und identitätsstiftende Funktion. ${ }^{43}$ Die mecklenburgische Mundart gilt als Symbol für die Region und die Dialektkompetenz als Zeugnis der regionalen Verbundenheit. Ob aber die Bemühungen der Gewährspersonen und sprachpolitische Maßnahmen zum Erhalt der Mundart erfolgreich sein werden, ob jüngere Generationen zunehmend die Identitätsfunktion des Plattdeutschen für sich erkennen und ob dadurch der Dialekt doch überleben kann, muss in den nächsten Jahren erneut untersucht werden.

43 Einen ähnlichen Statuswechsel des Niederdeutschen von einer „Kommunikationssprache“ zu einer „Identifikationssprache“ stellt Jürgens 2015, S. 374-392 für Hamburg fest. 


\section{Literatur}

Ahbe, Thomas/Gries, Rainer: Gesellschaftsgeschichte als Generationengeschichte. Theoretische und methodologische Überlegungen am Beispiel der DDR. In: Schüle, Annegret/Ahbe, Thomas/Gries, Rainer (Hrsg.): Die DDR aus generationengeschichtlicher Perspektive. Eine Inventur. Leipzig 2006, S. 475-571.

Arendt, Birte: Niederdeutschdiskurse. Spracheinstellungen im Kontext von Laien, Printmedien und Politik. Berlin 2010.

Beer, Mathias: Flucht und Vertreibung der Deutschen. Voraussetzungen, Verlauf, Folgen. München 2011.

Bieberstedt, Andreas: „In meinem Elternhaus wurde nur Plattdeutsch gesprochen." Sprachbiographische Konzeptionen Hamburger Dialektsprecher zum frühen Spracherwerb. In: Langhanke, Robert (Hrsg.): Sprache, Literatur, Raum. Festgabe für Willy Diercks. Bielefeld 2015, S. 205-237.

Bieberstedt, Andreas: „Das hieß dann, die können kein richtiges Deutsch in der Schule." Autobiographische Äußerungen Hamburger Dialektsprecher zu ihrer schulischen Sprachsozialisation. In: Bieberstedt, Andreas/Ruge, Jürgen/ Schröder, Ingrid (Hrsg.): Hamburgisch. Struktur, Gebrauch, Wahrnehmung der Regionalsprache im urbanen Raum (Sprache in der Gesellschaft; 34). Frankfurt a. M. [u. a.] 2016, S. 251-306.

Bieberstedt, Andreas: Lebenslauf und Sprachbiographie. Versuch einer sprachbiographischen Modellbildung aus dialektologischer Perspektive. In: Jürgens, Carolin/Schröder, Ingrid (Hrsg.): Sprachliche Variation in autobiographischen Interviews. Theoretische und methodische Zugänge. Frankfurt a. M. [u. a.] 2017, S. 47-80. (i. Dr.)

Dahl, Eva-Sophie: Interferenz und Alternanz - zwei Typen der Sprachschichtenmischung im Norden der Deutschen Demokratischen Republik. In: Ising, Gerhard (Hrsg.): Aktuelle Probleme der sprachlichen Kommunikation. Berlin 1974, S. 339-388.

Ehlers, Klaas-Hinrich: Schlesische und sudetendeutsche Plattschnacker. Eine Fallstudie zur sprachlichen Integration der Vertriebenen in MecklenburgVorpommern. In: Bohemia. Zeitschrift für Geschichte und Kultur der böhmischen Länder 51, H. 2 (2011), S. 345-357.

Ehlers, Klaas-Hinrich: Führte die Immigration der Heimatvertriebenen nach 1945 zu Dialektverlust und Nivellierung regionalsprachlicher Differenzen? Beobachtungen aus einer Untersuchungsregion in Mecklenburg. In: Niederdeutsches Jahrbuch 136 (2013), S. 97-116.

Ehlers, Klaas-Hinrich: „Uns’re Leut“ - Akkulturation und Abgrenzung einer karpatendeutschen Vertriebenengruppe in Mecklenburg. In: Zückert, Martin/ Schvarc, Michal/Meier, Jörg (Hrsg.): Migration - Zentrum und Peripherie - 
kulturelle Vielfalt. Neue Zugänge zur Geschichte der Deutschen in der Slowakei (Reihe Digi-Ost 2016). Leipzig 2016, S. 161-197.

Ehlers, Klaas-Hinrich: Von der Sprachbiografie zur Sprachgebrauchsgeschichte: Die Rekonstruktion des Varietätengebrauchs auf den Rostocker Werften. In: Jürgens, Carolin/Schröder, Ingrid (Hrsg.): Sprachliche Variation in autobiographischen Interviews. Theoretische und methodische Zugänge. Frankfurt a. M. [u. a.] 2017, S. 143-165.

Elmentaler, Michael/Rosenberg, Peter (Hrsg.): Norddeutscher Sprachatlas (NOSA). Bd. 1: Regiolektale Sprachlagen (Reihe: Deutsche Dialektgeographie, Sprachvariation in Norddeutschland; 113.1). Hildesheim/Zürich/New York 2015.

Fix, Ulla: Das Generationengedächtnis und der Sprachwandel. Sprachbiographisches Erinnern als Methode zum Erfassen von Sprachgebrauchswandel. In: Lerchner, Gotthard (Hrsg.): Chronologische, areale und situative Varietäten des Deutschen in der Sprachhistoriographie. Festschrift für Rudolf Große. Frankfurt a. M. [u. a.] 1995, S. 31-38.

Fix, Ulla/Barth, Dagmar: Sprachbiographien. Sprache und Sprachgebrauch vor und nach der Wende von 1989 im Erinnern und Erleben von Zeitzeugen aus der DDR. Inhalte und Analysen narrativ-diskursiver Interviews (Leipziger Arbeiten zur Sprach- und Kommunikationsgeschichte; 7). Unter Mitarbeit von Franziska Beyer, Frankfurt a. M. [u. a.] 2000.

Föllner, Ursula: Zum Gebrauch des Niederdeutschen in der Gegenwart - soziolinguistische und pragmatische Aspekte. In: Stellmacher, Dieter (Hrsg.): Niederdeutsche Sprache und Literatur der Gegenwart, Hildesheim [u. a.] 2004, S. 99-148.

Gehrke, Bernd: Die 68er Proteste in der DDR. 2008. [Online-Ressource: http://www.bpb.de/apuz/31327/die-68er-proteste-in-der-ddr?p=all (Stand 28.02.2017)]

Gemeinde Satow. [Online-Ressource: http://www.gemeinde-satow.de/ (Stand 09.09.2013)]

Gernentz, Hans Joachim: Wann und wie wird heute niederdeutsch gesprochen? In: Theil, Hans-Joachim (Red.): Niederdeutsch heute. Materialien einer Arbeitstagung des Freundeskreises Niederdeutsche Sprache und Literatur im Kulturbund der DDR, Kreisleitung Rostock 16.-17. November 1974. Rostock 1975, S. $12-19$.

Gernentz, Hans Joachim: Niederdeutsch - gestern und heute. Beiträge zur Sprachsituation in den Nordbezirken der Deutschen Demokratischen Republik in Gegenwart und Geschichte, Rostock 1980. 
Hansen, Martin: Zum Wandel des Niederdeutschen auf der Insel Rügen zwischen dem 19. und 21. Jahrhundert - Ein diachronischer Vergleich anhand ausgewählter Sprachmerkmale. Masterarbeit Greifswald 2009.

Herrmann-Winter, Renate: Auswirkungen der sozialistischen Produktionsweise in der Landwirtschaft auf die sprachliche Kommunikation in den Nordbezirken der Deutschen Demokratischen Republik. In: Ising, Gerhard (Hrsg.): Aktuelle Probleme der sprachlichen Kommunikation. Soziolinguistische Studien zur sprachlichen Situation in der Deutschen Demokratischen Republik. Berlin 1974, S. 135-190.

Herrmann-Winter, Renate: „Der Dialekt erlaubt keine eigene Sprache, aber eine eigene Stimme ... "Überlegungen zur Bewertung des Niederdeutschen. In: Mattheier, Klaus J./Wiesinger, Peter (Hrsg.): Dialektologie des Deutschen. Forschungsstand und Entwicklungstendenzen. Tübingen 1994, S. 457-464.

Herrmann-Winter, Renate: Sprachatlas für Rügen und die vorpommersche Küste. Kartographie Martin Hansen. Rostock 2013.

Jürgens, Carolin: Niederdeutsch im Wandel. Sprachgebrauchswandel und Sprachwahrnehmung in Hamburg (Deutsche Dialektgeographie; 119). Hildesheim/ Zürich/New York 2015.

Jürgens, Carolin/Schröder, Ingrid: Sprachstereotype und ihre Realisierungen im Gespräch am Beispiel des Niederdeutschen. In: Bieberstedt, Andreas/Ruge, Jürgen/Schröder, Ingrid (Hrsg.): Hamburgisch. Struktur, Gebrauch, Wahrnehmung der Regionalsprache im urbanen Raum (Sprache in der Gesellschaft; 34). Frankfurt a. M. [u. a.] 2016, S. 345-385.

Kehrein, Roland: Regionalsprachliche Spektren im Raum. Zur linguistischen Struktur der Vertikale (ZDL Beihefte; 152). Stuttgart 2012.

Köhncke, André: Mecklenburgisch heute - Bestandsaufnahme eines niederdeutschen Dialekts. Rostock 2010. [Online-Ressource: urn:nbn:de:gbv:28diss2011-0137-3 (Stand: 24.11.2016)]

Lenz, Alexandra: Hyperdialektalismen und Hyperkorrektionen - Indizien für Varietätengrenzen. In: Lenz, Alexandra/Mattheier, Klaus J. (Hrsg.): Varietäten - Theorie und Empirie. Frankfurt a. M. [u. a.] 2005, S. 75-95.

Macha, Jürgen: Der flexible Sprecher. Untersuchungen zu Sprache und Sprachbewusstsein rheinischer Handwerksmeister. Köln/Weimar/Wien 1991.

Menke, Hubertus: Niederdeutsch - Eigenständige Sprache oder Varietät einer Sprache? In: Schmitsdorf, Eva/Hartl, Nina/Meurer, Barbara (Hrsg.): Lingua Germanica. Studien zur deutschen Philologie. Jochen Splett zum 60. Geburtstag. Münster/New York 1998, S. 171-184.

Möhn, Dieter: Missingsch. In: Munske, Horst Haider (Hrsg.): Deutsch im Kontakt mit germanischen Sprachen. Tübingen 2004, S. 119-140. 
Möller, Frerk: Plattdeutsch im 21. Jahrhundert. Bestandsaufnahme und Perspektiven. Mit einem Aufsatz von Michael Windzio (Schriften des Instituts für Niederdeutsche Sprache; 34). Leer 2008.

Möller, Frerk: Niederdeutsch im 21. Jahrhundert. Bestandsaufnahme und Perspektiven. In: Niederdeutsches Jahrbuch 133 (2010), S. 141-163.

Ruge, Jürgen: Der landwirtschaftliche Wortschatz in der Wilstermarsch. Generationenspezifische Untersuchungen zu seiner Entwicklung. Hamburg 1995.

Scharioth, Claudia: Regionales Sprechen und Identität: Eine Studie zum Sprachgebrauch, zu Spracheinstellungen und Identitätskonstruktionen von Frauen in Schleswig-Holstein und Mecklenburg-Vorpommern (Deutsche Dialektgeographie; 120). Hildesheim/Zürich/New York 2015.

Schmidt, Jürgen Erich: Moderne Dialektologie und regionale Sprachgeschichte. In: Zeitschrift für deutsche Philologie 117, Sonderheft (1998), S. 163-179.

Schmidt, Jürgen Erich/Herrgen, Joachim: Sprachdynamik. Eine Einführung in die moderne Regionalsprachenforschung (Grundlagen der Germanistik; 49). Berlin 2011.

Seils, Mirjam: Die fremde Hälfte. Aufnahme und Integration der Flüchtlinge und Vertriebenen in Mecklenburg nach 1945. Schwerin 2012.

Stellmacher, Dieter: Niederdeutsche Sprache. Eine Einführung. Bern [u. a.] 1990.

Stellmacher, Dieter: Sprachsituation in Norddeutschland. In: Stickel, Gerhard (Hrsg.): Varietäten des Deutschen. Regional- und Umgangssprachen (Institut für deutsche Sprache 1996). Berlin/New York 1997, S. 88-108.

Tophinke, Doris: Lebensgeschichte und Sprache. Zum Konzept der Sprachbiografie aus linguistischer Sicht. In: Adamzik, Kirsten/Roos, Eva (Hrsg.): Biografie linguistiche - Biographies langagieres - Biografias linguisticas - Sprachbiografien (Bulletin suisse de linguistique appliquee; 76). Neuchâtel 2002, S. 1-14. 


\section{Förderung und Vermittlung des Niederdeutschen in Bildungsinstitutionen}


Birte Arendt, Andreas Bieberstedt and Klaas-Hinrich Ehlers - 978-3-631-71893-3

Downloaded from PubFactory at 01/11/2019 10:11:06AM

via free access 
Julia Mittelstädt

\title{
Das Projekt „Niederdeutsch in der Kita“ - Bedingungen, Formen und Perspektiven der Niederdeutschvermittlung in Kindergärten Mecklenburg-Vorpommerns
}

\begin{abstract}
Due to the decreasing number of speakers with Low German as a first language, the institutional teaching of Low German has become more and more important. The article presents results of a pilot study from the year 2010 that examined conditions, forms and methods of teaching Low German as a second language to preschool children. Participants of this study were 20 preschools in different regions of Mecklenburg-Western Pomerania. In order to survey the outcome of the pilot study ex post the participating preschool teachers were asked to fill in a comprehensive questionnaire about their experiences with the project. The article discusses the basic results of that questionnaire survey.
\end{abstract}

\section{Einleitung}

Der nachfolgende Beitrag thematisiert die Vermittlung des Niederdeutschen in vorschulischen Einrichtungen Mecklenburg-Vorpommerns. Grundlage der Ausführungen ist eine Reihe von Fragebögen, die im Rahmen einer Pilotstudie aus den Jahren 2010-2011 zum Niederdeutsch-Erwerb in ausgewählten Kindergärten Mecklenburg-Vorpommerns erstellt wurden. Die Fragebögen wurden von den beteiligten Kindergärtnerinnen im Anschluss an das Projekt ausgefüllt und geben Auskunft über die jeweiligen sprachlichen Voraussetzungen, Ziele, Methoden und Ergebnisse des Projektes in den einzelnen Kindergärten. Ihre Auswertung liefert aufschlussreiche Ergebnisse zu den Konditionen, Möglichkeiten und Problemen vorschulischer Niederdeutsch-Vermittlung in Kindertageseinrichtungen und zeigt damit Wege zur Förderung des frühkindlichen niederdeutschen Dialekterwerbs auf.

\section{Niederdeutsch und frühkindlicher Zweitspracherwerb}

Der Atlas oft the World's Languages in Danger der UNESCO führt insgesamt 13 Varietäten des Deutschen bzw. in Deutschland gesprochene Sprachen auf, die mehr oder weniger stark gefährdet sind. Fünf der deutschen Regional- bzw. Minderheitensprachen werden heute durch die Charta der Regional- oder Min- 
derheitensprachen geschützt. ${ }^{1}$ Auch Niederdeutsch - klassifiziert in der Charta als Regionalsprache - gehört zu diesen Sprachen. Laut UNESCO ist der Status des Niederdeutschen „vulnerable“, was so viel wie verwundbar, gefährdet bedeutet und noch der geringste Grad der Gefährdung ist. Viele Menschen identifizieren sich weiterhin mit der niederdeutschen Sprache, in einigen Gegenden ist sie aber nur noch rudimentär vertreten. Besonders deutlich wird das im Vergleich von Stadt und Land. In den Städten wird kaum Niederdeutsch gesprochen, auf dem Land noch verhältnismäßig häufiger, wenngleich ebenfalls stark rückläufig. ${ }^{2}$ Der Dialektgebrauch ist weitgehend auf die älteste Sprechergeneration beschränkt, zudem wird das Niederdeutsche nur noch in wenigen Fällen innerhalb der Familie an die nachfolgenden Generationen weitergegeben. Als Erstsprache wird nahezu ausnahmslos das Hochdeutsche erworben, aber auch als Zweitsprache wird das Niederdeutsche gegenwärtig kaum noch vermittelt. Diese und weitere Faktoren führen dazu, dass die Zahl der Sprecher mit einer niederdeutschen Dialektkompetenz permanent abnimmt. Dem drohenden Verlust des Niederdeutschen muss folglich verstärkt durch eine gezielte institutionelle Sprachförderung begegnet werden.

Eine erfolgversprechende Möglichkeit, diese Sprache zu erhalten und wieder mehr zu etablieren, bildet eine möglichst frühe Heranführung von Kindern an das Niederdeutsche im Bereich der vorschulischen Erziehung. Dies ist inzwischen auch von der Landespolitik erkannt worden. Bezüglich Teil III der Sprachencharta hat sich Mecklenburg-Vorpommern verpflichtet, „falls die staatlichen Stellen keine unmittelbare Zuständigkeit im Bereich der vorschulischen Erziehung haben,

1 Die politische Definition von „Regionalsprache“ laut EU-Charta unterscheidet sich von der linguistischen Begriffsbestimmung. Im Sinne der EU-Charta bezeichnet der Begriff Minderheiten- oder Regionalsprache „Sprachen, i die herkömmlicherweise in einem bestimmten Gebiet eines Staates von Angehörigen dieses Staates gebraucht werden, die eine Gruppe bilden, deren Zahl kleiner ist als die der übrigen Bevölkerung des Staates, und ii die sich von der (den) Amtssprache(n) dieses Staates unterscheiden; er umfaßt [sic!] weder Dialekte der Amtssprache(n) des Staates noch die Sprachen von Zuwanderern." Europarat: Europäische Charta der Regional- oder Minderheitensprachen 1992, Teil I, Artikel 1. Linguistisch gesehen ist unter Regionalsprache dagegen mit Lenz 2008, S. 2 „der regional markierte sprechsprachliche Gesamtbereich unterhalb der normierten und kodifizierten Standardsprache“ zu verstehen Der linguistische Terminus umfasst also ein regional begrenztes Spektrum unterschiedlicher Varietäten und Sprechlagen des Deutschen und damit gerade eben Dialekte und Regiolekte.

2 Vgl. Möller 2008, S. 15-18. Diese statistische Erhebung zur Kenntnis und Verwendung des Plattdeutschen im 21. Jahrhundert zeigt u. a. das Phänomen des Stadt-LandGefälles in der Dialektkompetenz auf. 
die Anwendung der unter den Ziffern i bis iii vorgesehenen Maßnahmen zu begünstigen und/oder dazu zu ermutigen “3. Die Ziffern i bis iii fordern,

„die vorschulische Erziehung in den betreffenden Regional- oder Minderheitensprachen anzubieten (i) oder einen erheblichen Teil der vorschulischen Erziehung in den betreffenden Regional- oder Minderheitensprachen anzubieten (ii) oder eine der unter Ziffern i und ii vorgesehenen Maßnahmen zumindest auf diejenigen Schüler anzuwenden, deren Familien dies verlangen, wenn die Zahl der Schüler als genügend groß angesehen wird. “4

In diesem Zusammenhang wurde im Jahre 2010 ein Modellprojekt „Niederdeutsch im Kindergarten" gestartet, welches vom Ministerium für Bildung, Wissenschaft und Kultur des Landes Mecklenburg-Vorpommern gefördert wurde. Ziel des Projektes war die Überprüfung von Möglichkeiten, Formen und Methoden des frühkindlichen Erwerbs des Niederdeutschen als Zweitsprache in vorschulischen Bildungseinrichtungen. Damit sollte die konzeptionelle Basis für eine intendierte umfassende Niederdeutsch-Vermittlung im vorschulischen Bereich gelegt werden. Träger dieses Projektes war die „Stiftung Mecklenburg“5, die sich schon seit längerem für die Pflege der niederdeutschen Sprache und Kultur einsetzt. ${ }^{6}$ Insgesamt nahmen zwanzig Kindergärten an dem Projekt teil, die in ganz Mecklenburg-Vorpommern verteilt lagen. So beteiligten sich Einrichtungen aus den Städten Schwerin, Rostock und Greifswald, aber auch Einrichtungen aus kleineren Gemeinden, wie Banzkow, Lüssow, Plate oder Rambin auf Rügen. ${ }^{7}$ Es wurden ausschließlich Kindergärten ausgewählt, in denen bereits vorher Niederdeutsch angeboten wurde oder zumindest ein Erzieher oder eine Erzieherin Dialektkenntnisse aufweisen konnte. Das Projekt wurde in der Pilotphase 2010-2011 von Fachwissenschaftlern der Universitäten Rostock und Greifswald begleitet. ${ }^{8}$

Dass ein Zweitspracherwerb, in dem Fall der des Niederdeutschen, im frühen Kindesalter viele Vorteile hat, ist vielen Menschen oft nicht bewusst. Häufig bestehen Bedenken, ob man schon Kindern eine solche Aufgabe abverlangen

3 Vgl. Institut für niederdeutsche Sprache 2008, S. 12 (Sprachencharta, Teil III, Artikel 8, Abschnitt 1.a.iv).

4 Vgl. Institut für niederdeutsche Sprache 2008, S. 12 (Sprachencharta, Teil III, Artikel 8, Abschnitt 1.a.i-iii).

5 Die Stiftung Mecklenburg wurde im Jahr 1973 von Mitgliedern der Landsmannschaft Mecklenburg in Ratzeburg gegründet, hat mittlerweile ihren Sitz in Schwerin und widmet sich der Erforschung der Geschichte Mecklenburgs. Auf ihrer Internetseite www.stiftung-mecklenburg.de findet man weitere Informationen.

6 Vgl. Stiftung Mecklenburg: Plattdütsch in'n Kinnergorden. [Online-Ressource]

7 Vgl. Stiftung Mecklenburg: Plattdütsch in'n Kinnergorden. [Online-Ressource]

8 Vgl. Stiftung Mecklenburg: Plattdütsch in'n Kinnergorden. [Online-Ressource] Birte Arendt, Andreas Bieberstedt and Klaas-Hinrich Ehlers - 978-3-631-71893-3 
kann und dass ein früher Zweitspracherwerb den Erwerb der Erstsprache zu beeinträchtigen vermag. Innerhalb der Spracherwerbsforschung besteht heute allerdings weitgehender Konsens, dass ein Kind in einem Alter von etwa vier Jahren die grundlegenden Merkmale der Muttersprache gelernt hat und diese auch weitgehend fehlerfrei gebrauchen kann. ${ }^{9}$ Kinder haben zudem besonders günstige kognitive Voraussetzungen, mehrere Sprachen zu erlernen. Denn in der Zeit, in der sich Kinder im Kindergarten befinden, also bis etwa zum siebenten Lebensjahr, spricht man von der „sensiblen Phase“ des Spracherwerbs. Während dieses Entwicklungsabschnittes sind sie besonders aufnahmefähig für sprachliche Strukturen. Das Gehirn hat eine erhöhte Flexibilität und ist sehr gut in der Lage, Neues zu erfassen und zu verarbeiten..$^{10}$ Dadurch fällt es Kindern leichter, Sprachen zu lernen, als Erwachsenen und sie beherrschen diese schneller und besser. Bei einem frühen Zweitspracherwerb verstehen sich die Kinder später sehr gut darauf, diese Sprache anzuwenden. Für Erwachsene ist es hingegen bedeutend mühsamer, eine weitere Sprache zu lernen und erfolgreich damit umzugehen. ${ }^{11}$

Des Weiteren ist das menschliche Gehirn im Allgemeinen für Mehrsprachigkeit geschaffen. Mehr als $70 \%$ der Weltbevölkerung benutzen täglich mehr als eine Sprache. So ist Mehrsprachigkeit global betrachtet schon mehr die Regel als eine Ausnahme. ${ }^{12}$

Der Zeitraum zwischen dem sukzessiven Erlernen der Erstsprache und Zweitsprache ist bei Kindern noch gering. So können sie beim Zweitspracherwerb auf Strategien und Erfahrungen beim Lernen ihrer Muttersprache zurückgreifen. Damit haben sie bessere Voraussetzungen, die zweite Sprache zu lernen. Der Zweitspracherwerb wiederum verbessert die Kompetenzen in der Erstsprache, da die angeeigneten Spracherwerbsmechanismen und -strategien auf beide Sprachen angewendet werden können.

Darüber hinaus lernt man nicht nur, sich mit der Sprache auszudrücken, das heißt, mit anderen zu kommunizieren, sondern erwirbt auch das dazugehörige kulturelle und soziale Hintergrundwissen. Die mit dem Zweitspracherwerb verbundenen erweiterten Kommunikationsmöglichkeiten erhöhen zugleich auch die sozialen Kompetenzen der Kinder. ${ }^{13}$ Nicht zuletzt ist das Sprachbewusstsein dieser

9 Vgl. Adler 2011, S. 11.

10 Vgl. Arendt 2012, S. 6-7.

11 Vgl. Adler 2011, S. 104.

12 Vgl. Timm o. J., S. 3.

13 Vgl. Adler 2011, S. 104.

Birte Arendt, Andreas Bieberstedt and Klaas-Hinrich Ehlers - 978-3-631-71893-3 
Kinder stärker ausgeprägt. Sie verfügen über ein spezielles Sprachempfinden und eine höhere kommunikative Flexibilität. ${ }^{14}$

Die Vorteile eines frühen Zweitspracherwerbs liegen damit deutlich auf der Hand. Der Erwerb einer weiteren Sprache im Kindesalter ist in Hinsicht auf den potenziell erreichbaren Kompetenzgrad dem Zweitspracherwerb eines Erwachsenen deutlich überlegen. Für eine Sprache wie das Niederdeutsche, die im Regelfall nicht mehr als Erstsprache im Familienkreis erworben wird, bietet sich eine solche frühe Zweitsprachvermittlung im Kontext vorschulischer Bildungseinrichtungen folglich in besonderer Weise an.

\section{Das Pilotprojekt „Niederdeutsch in der Kita“}

\subsection{Zielsetzung und Durchführungsphase}

Im Folgenden stelle ich das Projekt „Niederdeutsch in der Kita“ genauer vor und werde dabei zunächst das Ziel, den Projektbeginn und die Durchführung in Augenschein nehmen. Das Hauptaugenmerk liegt in der anschließenden Auswertung eines Fragebogens ${ }^{15}$, der von den Erzieherinnen der Kitas im Rahmen des Projektes ausgefüllt wurde.

Ziel des Projektes war im engeren Sinne die Überprüfung von Formen und Methoden der Niederdeutschvermittlung in den ausgewählten Kindergärten. Die Erfahrungen des Pilotprojektes sollten als Fernziel die Basis für eine feste Etablierung des Niederdeutschen an Kindergärten in Mecklenburg-Vorpommern schaffen. Man wollte erreichen, dass die Sprache nach dem Prinzip der Immersion in den normalen Tagesablauf, beispielsweise beim Morgenkreis oder am Essenstisch, einfließt. Es wurde ein frühes Erlernen der Sprache bis hin zum selbstständigen Gebrauch angestrebt. Außerdem sollte den Kindern so der Einstieg in einen angestrebten Niederdeutschunterricht in der Schule erleichtert werden. ${ }^{16}$

Zum Start des Projektes wurde allen Kindergärten von der „Stiftung Mecklenburg" je eine Materialbox übergeben. Diese enthielt niederdeutsche Bücher, Lieder, Gedichte und Spiele. Außerdem wurden den Erzieherinnen Spracherwerbskurse angeboten, um ihre eigenen Kenntnisse zu erweitern und auszubilden. In mehreren Workshops wurden unter Anleitung der damaligen Landesbeauftragten für Niederdeutsch in Mecklenburg-Vorpommern, Susanne Bliemel,

14 Vgl. Haid 2012, S. 4.

15 Genauere Informationen zum Aufbau des Fragebogens im Kapitel 3.2 „Datengrundlage und analytischer Zugang“.

16 Vgl. dazu insbesondere den Beitrag von Fink in diesem Band. 
Erfahrungen untereinander ausgetauscht und Materialien entwickelt und ausprobiert, die als Ergebnis in der Broschüre „lürlürlütt“ (,klitzeklitzeklein') veröffentlicht wurden. ${ }^{17}$ Diese beinhaltete didaktische Hinweise für die Vermittlung des Niederdeutschen sowie Lieder, Gedichte und Geschichten auf Niederdeutsch, die an den Kindergärten benutzt werden konnten. ${ }^{18}$ Zusätzlich dazu besuchte die Landesbeauftragte jede Einrichtung persönlich und gestaltete dort eine Unterrichtseinheit und gab Anregungen für neue Vermittlungsformen und -methoden.

\subsection{Datengrundlage und analytischer Zugang}

Um genau zu erfahren, wie in jedem Kindergarten der „Unterricht“ des Niederdeutschen vonstattenging und welche Resonanz die Erzieherinnen erfuhren, wurde von Wissenschaftlern der Universitäten Greifswald und Rostock ein Fragebogen entwickelt, der im Jahr 2011 an die Kindergärten verschickt wurde. ${ }^{19}$ Der Fragebogen diente unter anderem dazu, genauer herauszufinden, wie die Niederdeutschvermittlung in den einzelnen Kindertagesstätten integriert wurde, welche sprachlichen Voraussetzungen in den jeweiligen Einrichtungen herrschten, welche Erfahrungen die beteiligten Erzieherinnen sammeln konnten, welche Formen des Erlernens zum größten Erfolg führten und ab welchem Alter folglich der Erwerb einer Zweitsprache Niederdeutsch angebracht ist.

Vierzehn der insgesamt zwanzig teilnehmenden Kindergärten beantworteten den Fragebogen. Dieser war unterteilt in folgende Kategorien: Grunddaten, Struktur des Kindergartens, sprachliche Voraussetzungen und Erfahrungen, Rahmen und Zielsetzungen, Durchführung, Erfahrungen, Perspektive und weitere Anmerkungen. Gefragt wurde unter der Kategorie ,sprachliche Voraussetzungen und Erfahrungen' nach bereits vorhandenen Kompetenzen im Niederdeutschen sowohl bei den Erzieherinnen und den Kindern als auch den auswärtigen Personen, sofern solche an der Niederdeutschvermittlung beteiligt waren. Weiterhin ging es bei den Fragen zu ,Rahmen und Zielsetzungen' darum, seit wann Niederdeutsch in der Kita angeboten wurde und welche Personen an der Vermittlung beteiligt waren. Beim Fragenkomplex zur ,Durchführung' wurde nach dem Vermittlungsprinzip gefragt sowie nach der Form der Vermittlung, der Regelmäßigkeit und dem zur Verfügung stehendem Lehrmaterial. Unter der Kategorie ,Erfahrungen

17 Vgl. Stiftung Mecklenburg 2012.

18 Vgl. Brodkorb 2012, S. 5.

19 Von wissenschaftlicher Seite waren die Vertreter der niederdeutschen Philologie an den Universitäten Rostock und Greifswald, Prof. Andreas Bieberstedt und Dr. Birte Arendt, in das Pilotprojekt eingebunden. 
wurde untersucht, welche Vermittlungsformen nach Ansicht der Erzieherinnen sehr gut und welche weniger gut geeignet waren und welche Probleme auftraten. Auch ob bereits Lerneffekte an den Kindern beobachtet wurden und welche Resonanz diese Vermittlung erfuhr, sei es von den Kindern selbst, den Eltern, Kollegen oder weiteren Personen, wurde hier erfragt. Der Punkt ,Perspektive' beinhaltete Fragen nach Wünschen und Forderungen an das Projekt, seine Verantwortlichen und an die Politik. Die Erzieherinnen berichteten von ihren Erfahrungen beim Umgang mit dem Plattdeutschen, der Etablierung in den Alltag und den Entwicklungen der Kinder.

\subsection{Ergebnisse}

Im Folgenden werden eine Auswahl der Fragen und ihre Auswertung vorgestellt. Diese Auswahl erfolgte nach dem Kriterium, einen guten Überblick über die Art und Weise der Niederdeutschvermittlung an den Kitas in Mecklenburg-Vorpommern zu geben und es nachvollziehbar zu machen, ob und welchen Fortschritt und Erfolg diese Vermittlung erzielte. So bleiben beispielsweise die Punkte ,Grunddaten' und ,Struktur des Kindergartens' in der Auswertung unberücksichtigt, da sie im hier besprochenen Zusammenhang lediglich von untergeordneter Bedeutung sind. Generell erfolgt die Auswertung aber strukturiert in der oben genannten Reihenfolge und baut so systematisch aufeinander auf.

Es wird jeweils zuerst die Frage genannt und dementsprechend erfolgt die Auswertung der gegebenen Antworten. Stehen mehrere Antwortmöglichkeiten zur Auswahl, helfen Balkendiagramme, einen besseren Überblick zu geben und die Auswertung bildlich nachvollziehbarer zu gestalten. So wird bei den betreffenden Fragen immer vorweg das Diagramm gezeigt und anschließend ausgewertet und interpretiert. Die Auswertung erfolgt ausschließlich in anonymisierter Form. Die Angaben in den Diagrammen erfolgen in absoluten Zahlen.

Um einen besseren Lesefluss zu gewährleisten, werde ich im Folgenden immer die weibliche Form der Anrede benutzen, da es vor allem Frauen sind, die in den Kindergärten beschäftigt sind. Es ist zu beachten, dass es sich bei den Aussagen der Befragten ausschließlich um subjektive Selbsteinschätzungen handelt.

\subsubsection{Sprachliche Voraussetzungen und Erfahrungen}

In diesem Abschnitt wird von den sprachlichen Voraussetzungen und Erfahrungen von Erzieherinnen, auswärtig beteiligten Personen und Kindern berichtet. Diese Fragestellung ist insofern wichtig, als sie die Grundlage verdeutlicht, inwieweit überhaupt Kenntnisse im Bereich des Niederdeutschen vorhanden waren, 
auf denen die Vermittlung aufgebaut wurde. Neben dem Aspekt, wie viele Erzieherinnen Plattdeutschkenntnisse besaßen, wurde auch nach Kompetenzen im Verstehen, Sprechen, Lesen und Schreiben gefragt.

\section{a) Erzieherinnen}

Frage: Wie viele Erzieherinnen in Ihrem Kindergarten besitzen eine plattdeutsche Sprachkompetenz?

Es gibt nur einen von 14 befragten Kindergärten, in dem keine der 13 dort arbeitenden Erzieherinnen eine Niederdeutschkompetenz besitzt. In fünf Kindergärten hat jeweils eine und in sechs Kindergärten haben jeweils zwei Erzieherinnen eine niederdeutsche Kompetenz. Es gibt zwei Kindergärten, in denen alle Erzieherinnen die notwendigen sprachlichen Voraussetzungen haben. In Prozent ausgedrückt sind etwa $27 \%$ aller Erzieherinnen der 14 Kindergärten in der Lage, die niederdeutsche Sprache anzuwenden. Einige von ihnen nahmen an den niederdeutschen Spracherwerbskursen teil, die im Rahmen des Projektes angeboten wurden, um sprachliche Kompetenzen zu erlernen und bereits bestehende Kenntnisse zu festigen und zu vertiefen.

Frage: Wie würden Sie Ihre Plattdeutschkompetenz in den folgenden Bereichen selbst einschätzen?

Abbildung 1: Plattdeutschkompetenzen der Erzieherinnen

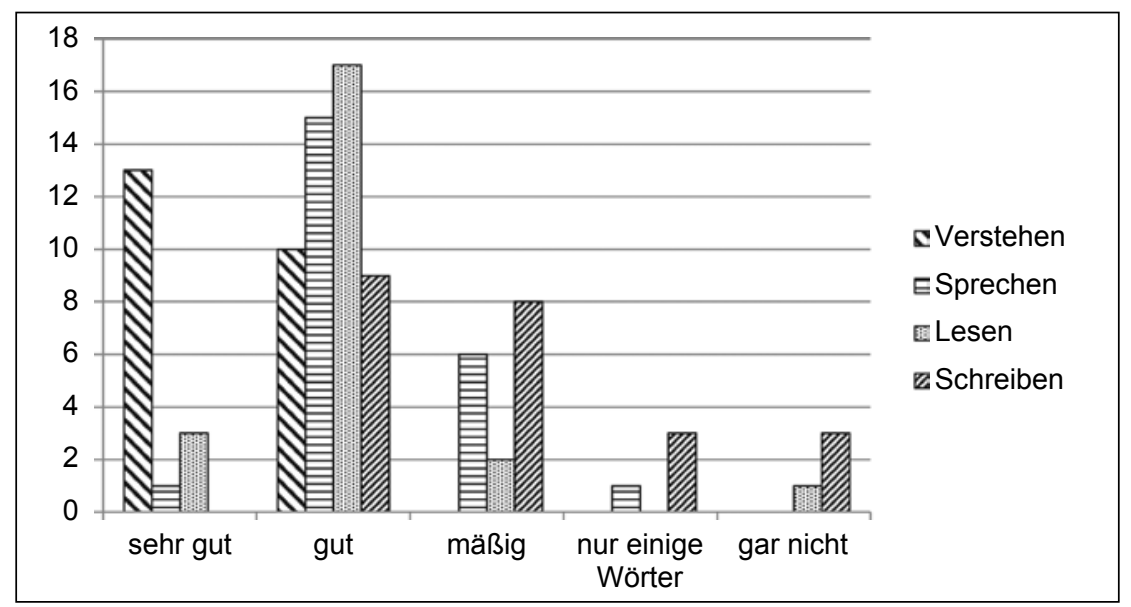


Diese Frage konnten pro Kindergarten mehrere Erzieherinnen beantworten. So haben insgesamt 23 Erzieherinnen die Selbsteinschätzungsfrage beantwortet. Im Diagramm (Abb. 1) ist deutlich zu erkennen, dass die Verstehenskompetenz am besten ausgeprägt ist. 13 Erzieherinnen verstehen Niederdeutsch „sehr gut“, zehn "gut“. Die Antwortmöglichkeiten „mäßig“, „nur einige Wörter“ und „gar nicht“ wurden überhaupt nicht angekreuzt. Das bedeutet, dass alle 23 Erzieherinnen Niederdeutsch „gut“ oder „sehr gut" verstehen. Die Kompetenzen im Sprechen und Lesen sind annähernd ähnlich verteilt. So schätzen sich die meisten Erzieherinnen als "gut" in diesen Fähigkeiten ein. Es gibt nur eine Person, die "gar nicht“ Niederdeutsch lesen kann und ebenfalls nur eine, die „nur einige Wörter“ Platt sprechen kann. Das Schreiben des Niederdeutschen schneidet am schlechtesten ab. Hier sieht sich keiner im Bereich „sehr gut“. 17 Erzieherinnen schätzen sich als „gut“ oder „mäßig“ ein. Aber auch jeweils drei meinen, dass sie „nur einige Wörter" bzw. „gar nicht“ Niederdeutsch schreiben können. Die Mehrheit aller Antworten konzentriert sich auf die Aussage „gut“. Das ist ein Wert, mit dem man den Kindern durchaus die Sprache näherbringen kann. Jedoch gibt es zu denken, dass lediglich eine Erzieherin ihre aktive Sprachkompetenz bei „sehr gut“ sieht. Hierin drückt sich die bereits oben angesprochene insgesamt geringe Dialektkompetenz in Mecklenburg-Vorpommern aus. Zudem ist es fraglich, ob bei solch einem Wert den Kindern fehlerfrei Niederdeutsch beigebracht werden kann.

\section{b) Auswärtige Personen}

Nicht in allen Kindergärten unterrichten die Erzieherinnen selber die Kinder. In neun von den 14 Kindergärten findet die Vermittlung durch auswärtige Personen statt. Zumeist sind es Rentner und Rentnerinnen, die an der Spracherziehung beteiligt sind. In zwei Fällen wird explizit angegeben, dass die Großeltern von jeweils einem Kind in die Kita kommen, um den Kindern das Niederdeutsche beizubringen. Auch Studenten und ehemalige Lehrer wirken an der Vermittlung mit, sowie weitere auswärtige Personen, die ehrenamtlich den Kindern Niederdeutsch beibringen. Die Niederdeutschkompetenz der auswärtigen Personen wurde in diesen Fällen von den Erzieherinnen bewertet. 
Frage: Für den Fall, dass auswärtige Personen in das Projekt einbezogen sind, wie würden Sie deren Plattdeutschkompetenz in den folgenden Bereichen einschätzen? Abbildung 2: Plattdeutschkompetenzen auswärtig einbezogener Personen

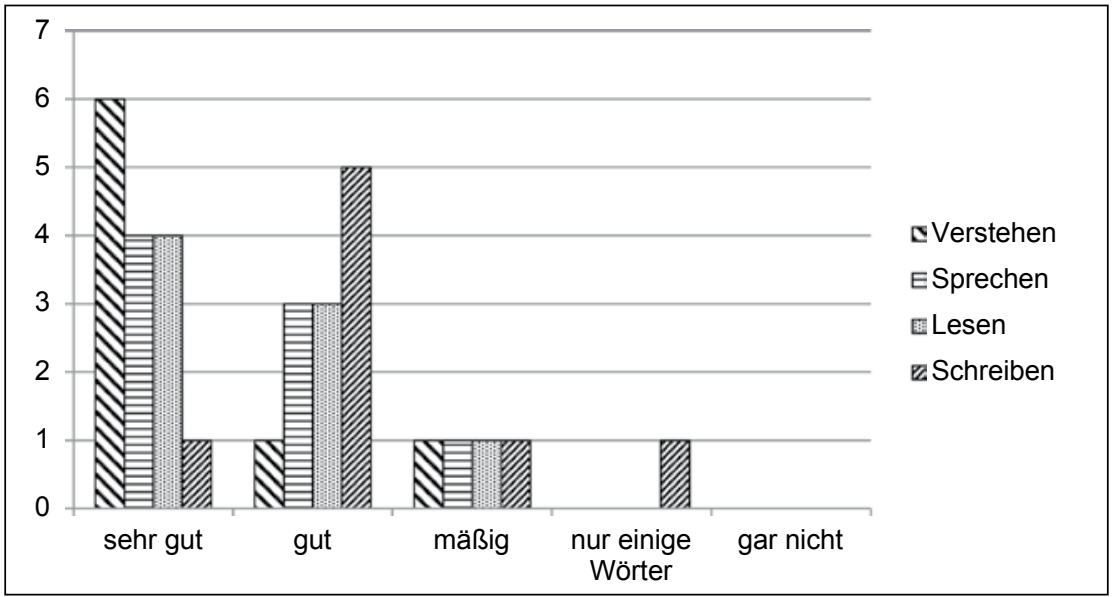

Dieses Diagramm (Abb. 2) veranschaulicht die Einschätzung der Erzieherinnen für die auswärtig einbezogenen Personen. Die Frage wurde insgesamt achtmal beantwortet. Die Kompetenzen der auswärtigen Personen werden als „sehr gut“ oder "gut" beschrieben. Die Verstehenskompetenz ist auch hier, wie zuvor bei den Erzieherinnen selbst, am höchsten ausgeprägt. So wird sie von ihnen in sechs von acht Fällen als „sehr gut“ eingestuft. Einmal wird das Verstehen als „mäßig“ eingeordnet. Es folgen das Sprechen und Lesen mit jeweils gleich vielen Punkten. Die Kompetenzen verteilen sich, mit sieben von acht Nennungen, vor allem bei "sehr gut" und "gut“. Das Schreiben ist auch hier der schlechteste Faktor, konzentriert sich aber bei der Antwort „gut" mit fünf Nennungen. Nur einmal wurde angegeben, dass die auswärtige Person „nur einige Wörter“ schreiben kann. Die Antwortmöglichkeit „gar nicht“ fällt komplett weg.

Im direkten Vergleich zur Selbsteinschätzung der Erzieherinnen fällt auf, dass die Fremdbewertungen für die auswärtigen Personen deutlich besser ausfallen. Wo sich die Selbstbewertungen der Kompetenzen bei den Erzieherinnen auf den Bereich "gut" konzentrieren und im Bereich „sehr gut“ lediglich das Verstehen noch einen hohen Wert erreicht, ist die Fremdbewertung der auswärtigen Personen mit „sehr gut“ sehr viel positiver. Möglicherweise liegt das an der unterschiedlichen Art der Bewertung, d. h. dass sich die Erzieherinnen selbst kritischer einschätzen und die Fremdbewertung positiver vornehmen. Gerade bei älteren 
auswärtigen Personen kann aber wohl auch tatsächlich von einer vergleichsweise hohen Kompetenz im Sprechen ausgegangen werden. Nach der repräsentativen Umfrage des Instituts für niederdeutsche Sprachforschung ist die aktive Niederdeutschkompetenz bei Personen über 50 Jahren durchschnittlich am höchsten. Die Einbindung der ältesten Sprechergeneration in den Sprachunterricht für die jüngste Generation erscheint also grundsätzlich sehr sinnvoll. ${ }^{20}$

\section{c) Kinder}

Frage: Welche sprachlichen Voraussetzungen besitzen die Kinder in Ihrem Kindergarten?

Bei einem Großteil der Kinder spricht man im Elternhaus kein Niederdeutsch. Es wird konkret nur von drei Fällen berichtet, bei denen der Vater, und von einem Fall, in dem die Mutter mit seinen/ihren Kindern manchmal Niederdeutsch sprechen. Die Erzieherinnen berichten aber, dass die Eltern durch Aushänge im Kindergartengebäude mitlernen und für Auftritte mit ihren Kindern Lieder und Texte üben. Es kommt durchaus öfter vor, dass die Großeltern oder andere ältere Verwandte, wie Urgroßeltern, Großtanten und Großonkel, noch Niederdeutsch sprechen und auch manchmal mit den Kindern versuchen, etwas zu reden. Zum Teil haben die Kinder auch über ältere Geschwister, die selber im Kindergarten oder in der Schule Niederdeutsch vermittelt bekommen haben, bereits einiges aufschnappen können. So gibt es durchaus Kinder, die schon ein paar Erfahrungen von zu Hause mitbringen, aber der Großteil hat keinerlei Voraussetzungen und Vorkenntnisse zum Erwerb der plattdeutschen Sprache. Daher muss Niederdeutsch heute im Kindergarten mehr oder weniger wie eine Fremdsprache vermittelt werden.

\subsubsection{Rahmenbedingungen der Niederdeutschvermittlung im Kindergarten}

Frage: Seit wann bieten Sie Niederdeutsch in Ihrer Einrichtung an?

In allen 14 Kindergärten, die sich an der Befragung beteiligt haben, wurde bereits vor Beginn des Projektes Niederdeutsch, wenngleich bisweilen nur ansatzweise, angeboten. Besonders hervorzuheben sind zwei Kindergärten, in denen schon seit etwa 20 Jahren Niederdeutsch fest im Programm integriert ist. Die anderen Einrichtungen begannen ab den 2000er Jahren, verstärkt im Zeitraum 2008-2010, mit der Niederdeutschvermittlung. 
Frage: Welche Erzieherinnen sind an dem Projekt beteiligt?

In den meisten Kindergärten sind ein bis zwei Erzieherinnen an dem Projekt beteiligt. Diese weisen auch bereits Niederdeutschkenntnisse auf. In einem Fall wird der Unterricht ausschließlich von auswärtigen Personen durchgeführt. In einem Kindergarten beteiligen sich alle, insgesamt drei Erzieherinnen, an dem Projekt und dem Unterrichten der Kinder.

Frage: Welche und wie viele Kinder beteiligen sich an dem Projekt?

Abbildung 3: Beteiligung der Kinder in verschiedenen Kindertagesstätten am Niederdeutschunterricht

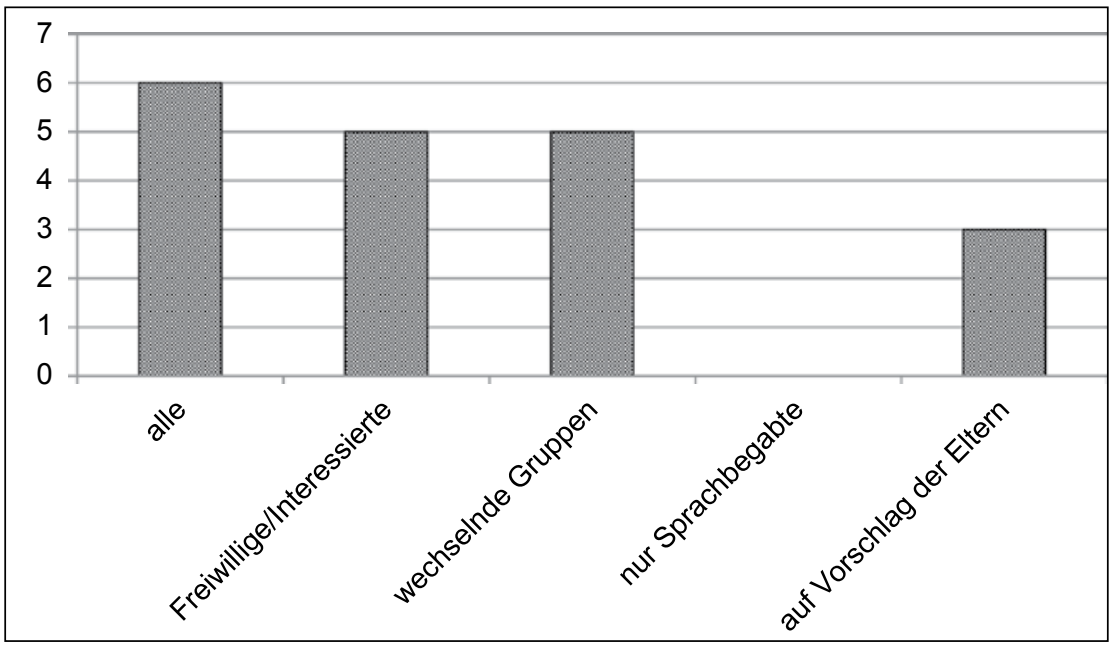

Bei der Beantwortung dieser Frage waren Mehrfachnennungen möglich. Es gibt keine Einrichtung, die nur mit besonders sprachbegabten Kindern arbeitet. In sechs Fällen beteiligen sich alle Kinder am Projekt, wobei noch zwischen den einzelnen Altersstufen unterschieden werden muss. So meinen einige Kindergärten mit „alle“ Kinder ab dem Kindergartenalter (nicht Krippe) und einige Kinder ab Vorschulalter. Es wird auch auf das Interesse der Kinder geachtet, nach dem Motto: „Wer keinen Spaß an der Sache hat, lernt auch nichts.“ In fünf Fällen erfolgt der Unterricht in wechselnden Gruppen und bei drei Kindergärten haben die Eltern das entscheidende Stimmrecht, ob die Gruppe Niederdeutsch lernt oder nicht. 


\subsubsection{Motive und Ziele der Erzieherinnen}

Frage: Was ist Ihre Motivation für die Vermittlung des Plattdeutschen an Kinder?

Für viele Erzieherinnen ist ein bedeutendes Motiv für die Vermittlung des Niederdeutschen, die Sprache nicht ins Vergessen geraten zu lassen. Hierzu schreibt eine Erzieherin aus der Kita in S.: „Die Kinder sollen lernen, dass die Sprache früher schon hier im Norden gesprochen wurde. Aber mit den Jahren nicht mehr so oft. Sie soll nicht vergessen werden." Niederdeutsch wird als Ausdruck der regionalen Kultur angesehen. So wünscht sich eine Erzieherin „Tradition und Sprachpflege für unsere Region“. Man möchte „einer ,aussterbenden' Sprache entgegenwirken“, Niederdeutsch erhalten und pflegen. Zu diesem Punkt schreibt eine Erzieherin: „Die plattdeutsche Sprache zu erhalten und zu pflegen ist für mich oberstes Gebot, Verpflichtung und Motivation. "Eine Erzieherin meint auch dazu, dass die Sprache lange ohne Wertschätzung und Beachtung im Schul- und Vorschulbereich geblieben sei, das aber geändert werden sollte, denn Platt sei etwas Besonderes in der Region. Man möchte ,einen Beitrag zur Sprachförderung für die Jüngsten" schaffen. Einige Erzieherinnen geben auch an, selbst mit der niederdeutschen Sprache aufgewachsen zu sein. Dazu meint eine Erzieherin „Ich liebe selber die plattdeutsche Sprache und möchte, dass sie erhalten bleibt." Sie möchten ihr Wissen weitergeben zum Erhalt der Sprache und haben große Freude an der Sprachvermittlung. Es wird auch angegeben, dass Sprachförderung und eine zweisprachige Erziehung der Kinder wichtig seien.

Nach Einschätzung der Erzieherinnen besteht ein großes Interesse von Seiten der Eltern und Großeltern an der Niederdeutschvermittlung. Die Kinder zeigen Freude und Spaß am Lernen. Sechs von den 14 Kitas haben ihrer Einrichtung einen plattdeutschen Namen gegeben, was auch als Botschaft für alle Außenstehenden verstanden wird, dass hier die Sprache gefördert wird. Außerdem möchte man sich mit diesem Angebot von anderen Kindergärten abheben, um auch für die Eltern interessant zu bleiben. Eine Rentnerin, die den Kindern in B. Niederdeutsch näherbringt, schreibt: „Ich bin plattdeutsch aufgewachsen und mecklenburge mit Leib und Seele. Plattdeutsch sprechen und Lesen gehört zu meinem Leben, da ich pädagogisch vorgebildet bin, möchte ich dieses gerne anderen weitergeben."

Zusammenfassend lässt sich sagen, dass Niederdeutsch von den Befragten durchweg als etwas Positives gesehen wird. Die Sprache gehört nach Meinung vieler Erzieherinnen zur Region und ist für viele noch wichtig, so sollen auch die Kinder an die Sprache herangeführt werden und sie kennenlernen. Ähnlich 
positive Einstellungen zum Niederdeutschen ermittelte für ganz Norddeutschland auch die Umfrage des Instituts für niederdeutsche Sprache. Dort gaben 91 \% der Befragten an, die Sprache sei für sie „heimatlich“, dicht gefolgt von den Bewertungen „humorvoll“ (85\%), „gemütlich“ (79\%) und „typisch norddeutsch“ (75\%). Die am wenigsten gegebenen Antworten waren „nicht zeitgemäß“ (30\%), "grob“ (27 \%) und „nur für alte Menschen“ $(21 \%) .{ }^{21}$ Auch die befragten Kindergartenerzieherinnen aus Mecklenburg-Vorpommern teilen diese positiven Einstellungen zum Niederdeutschen, die für sie eine starke Motivation darstellen, die Sprache zu erhalten. Damit in Zusammenhang bewegt der befürchtete Sprachentod des Niederdeutschen viele Erzieherinnen, sich in der frühkindlichen Vermittlung dieser Sprache zu engagieren.

Frage: Was wollen Sie erreichen, das heißt welche Ziele verfolgen Sie mit Ihrem Niederdeutschangebot?

Eine Erzieherin „möchte erreichen, dass die Kinder ungezwungen mit dem Plattdeutschen bekannt gemacht werden". Ähnlich meint auch eine Erzieherin aus R.: „Die Kinder sollen zum selbstverständlichen Gebrauch der plattdeutschen Sprache geführt werden. Wir wollen Plattdeutsch als Umgangssprache fördern und dabei bei den Kleinsten in unserer Kita beginnen. "Erzieherinnen und Kinder haben Freude an der regionalen Sprache und Interesse, diese zu verstehen und zu erlernen. Den Kindern sollen der Ursprung und die Entwicklung der niederdeutschen Sprache nahegebracht werden. Man möchte die „Sprache und somit auch die Kultur und Tradition wiederbeleben und weitergeben“, schildert eine Erzieherin aus G. In diesem Zusammenhang meint auch eine Erzieherin aus C., dass die „Stärkung von Heimatverbundenheit und Identitätsentwicklung“ wichtig sei. Den Kindern sollen die Grundlagen der Sprache vermittelt werden, damit sie in den Schulen mit ihrem Wissen weiterarbeiten können, soweit dort auch Niederdeutsch angeboten wird. Man möchte Sprachverständnis entwickeln und den Horizont der Kinder erweitern. Es seien vor allem Neugierde, Spaß und Freude wichtig, die beim spielerischen Umgang mit der Sprache geweckt würden und unverzichtbar seien. Auch wird sich gewünscht, dass „Plattdeutsch weitergeführt wird und nicht vergessen und ausstirbt“, wie eine Erzieherin aus R. äußert. In vielen Kindergärten treten die Kinder dann in der Öffentlichkeit auf und zeigen stolz ihre gelernten Programme.

Demnach ist es den Erzieherinnen und den Personen, die zusätzlich am Projekt beteiligt sind, wichtig, die Sprache zu erhalten, da sie zur Heimat gehört und ein

21 Vgl. Möller 2008, S. 24.

Birte Arendt, Andreas Bieberstedt and Klaas-Hinrich Ehlers - 978-3-631-71893-3 
Kulturgut ist, das es zu bewahren gilt. Um das zu erreichen, sollen die Kinder mit Freude an diese Sprache herangehen und eine positive Verbundenheit zum Niederdeutschen entwickeln. Allgemein stehen auch die Förderung des Verständnisses von Sprache und eine persönliche Weiterentwicklung durch den Umgang mit ihr im Fokus.

\subsubsection{Durchführung}

Frage: Welches Prinzip verfolgen Sie bei der Vermittlung des Plattdeutschen? Abbildung 4: Prinzipien der Vermittlung des Niederdeutschen in den Kindertagesstätten

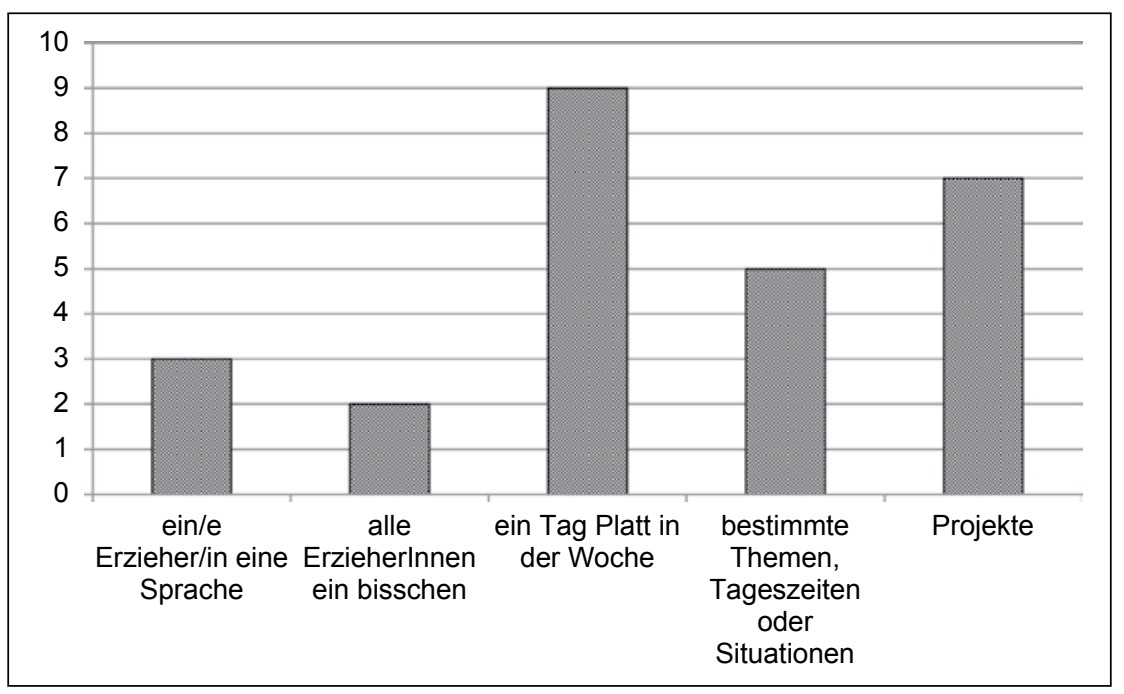

Bei dieser Frage waren Mehrfachantworten möglich, um alle Vermittlungsformen zu erfassen. Mit neun Nennungen ist das Prinzip „ein Tag Platt in der Woche“ am meisten vertreten. In fünf Einrichtungen wird das Prinzip verfolgt, dass nach „bestimmten Themen, Tageszeiten oder Situationen" unterrichtet wird. Drei Einrichtungen vermitteln nach dem Prinzip „eine Erzieherin eine Sprache“ und in zwei Kitas unterrichten „alle Erzieherinnen ein bisschen“. Es werden auch oft Projekte gemacht, wie beispielsweise für den Oma- und Opatag, den Muttitag, Wohngebiets- oder Jahreszeitenfeste. In einem Kindergarten wurde noch als weiterer Punkt angegeben, im Morgenkreis biblische Erzählungen durchzunehmen. Auch andere Kindergärten nutzen den Morgenkreis für erste niederdeutsche Sprüche oder Lieder. Zu den Mahlzeiten werden niederdeutsche Tischsprüche aufgesagt. In einer Kita erfolgt der Unterricht eher spontan und situationsabhängig, je nach- 
dem wie viel Zeit zur Verfügung steht und wie viel Erzieherinnen überhaupt an dem Tag anwesend sind. Eine Einrichtung hat einmal in der Woche einen kompletten plattdeutschen Tag, von der Begrüßung bis zur Verabschiedung. Auch an den anderen Tagen werden noch zusätzlich niederdeutsche Lieder und Sprüche gefestigt. In den meisten Kindergärten wird einmal wöchentlich, immer zu einem feststehenden Termin, Niederdeutsch gelernt. Wie schon erwähnt, nutzen einige Einrichtungen auch die anderen Tage zum Lernen von Tischsprüchen oder festigen das Gelernte.

Als das beste Verfahren, eine Sprache zu lernen, wird heute meist die sogenannte „Immersion“ angesehen, bei der die Kinder quasi in eine fremdsprachliche Umgebung ,eintauchen“ und die neue Sprache in alltäglichen Kontexten erfahren und erlernen. Dabei verfolgt man das Prinzip „eine Person - eine Sprache“22. Das bedeutet, die Lehrperson spricht konsequent in der neuen Sprache und übersetzt auch nicht, was sie gesagt hat, sondern veranschaulicht das Gesprochene. Dies geschieht durch Mimik und Gestik, dem Zeigen von Bildern und Symbolen, mit Hilfe der Verwendung von Handpuppen und auch durch das Vorspielen von Handlungen. Die zu lernende Sprache ist dann sowohl Unterrichts- als auch Umgangssprache. Die Kinder erschließen sich die Bedeutung von Worten und die Sprache selbstständig, indem sie Situationen und deren Zusammenhänge erkennen und begreifen. ${ }^{23}$ Der Erfolg, den man mit bilingualem Spracherwerb erfahren kann, wurde im ELIAS-Projekt bewiesen. Zehn bilinguale Kitas nahmen daran über zwei Jahre teil. Es stellte sich heraus, dass die Kinder deutliche Fortschritte beim Verständnis der Fremdsprache (Englisch) machten und auch bessere Lernerfolge erzielten, je intensiver sie mit der Fremdsprache in Kontakt kamen. ${ }^{24}$ Von den 14 Kindertagesstätten in Mecklenburg-Vorpommern, die an der Befragung teilnahmen, vermitteln nur drei das Niederdeutsche nach dem besonders erfolgsversprechenden Prinzip „eine Erzieherin eine Sprach““. Aber auch das Prinzip „,ein Tag Platt in der Woche" bietet den Kindern die Möglichkeit, das Niederdeutsche als Umgangssprache zu erleben und aus dem kommunikativen Zusammenhang heraus zu erwerben. Insgesamt könnte die Effizienz der Niederdeutschvermittlung in den Kindertagesstätten aber noch weiter erhöht werden, indem vermehrt die Vermittlungsprinzipien eingesetzt werden, die die größten Lernerfolge versprechen.

22 Vgl. FMKS e. V. 2016, S. 10-11 und Biedowicz in diesem Band.

23 FMKS e. V. 2016, S. 10-11.

24 FMKS e. V. 2016, S. 35.

Birte Arendt, Andreas Bieberstedt and Klaas-Hinrich Ehlers - 978-3-631-71893-3 
Frage: In welchen Formen taucht Niederdeutsch in Ihrer Einrichtung auf?

In allen Kindergärten wird mit Liedern, Gedichten, Reimen, Tischsprüchen, Sprichwörtern, Tänzen und Spielen gearbeitet. Auch Märchen werden behandelt. Wie oben schon genannt, führen viele Einrichtungen Projekte durch und erarbeiten Programme für Feiern und öffentliche Auftritte. In einigen Einrichtungen sind Aushänge und Wandzeitungen zu einzelnen Themen immer präsent. So sehen beispielsweise die Eltern, welche aktuellen Lieder und Gedichte gelernt werden, und können so aktiv in die Sprachvermittlung eingebunden werden. Es gibt Schilder im Garderobenbereich, auf denen Kleidungsstücke auf Niederdeutsch ausgehängt sind. Auch an Türen sind manchmal Schilder, auf denen auf Platt steht, welcher Raum sich dahinter befindet. Einige Kindergärten nehmen an Plattdeutsch-Wettbewerben teil. Die häufig erfolgreichen Ergebnisse hängen dann in Form von Urkunden an den Wänden.

In sechs von den 14 Kitas ist der Name der Einrichtung plattdeutsch. Die Namen lauten: „Lütt Sparling“, „Uns Kinnerhus“, „De lütten Landlüüd“, „Lütt Matten“, „Uns Lütten“ und „Lütte Meckelbörger“. Sie veranschaulichen so schon Außenstehenden, dass die Sprachvermittlung zum Programm des Kindergartens gehört. In drei Kitas wurde geschrieben, dass die Erzieherinnen im Unterricht mit einer Handpuppe arbeiten, die dann mit den Kindern Plattdeutsch spricht und lernt. Wenn ein Kind Geburtstag hat, wird ihm bisweilen ein Geburtstagslied in Plattdeutsch gesungen.

Frage: Welche Lehrmittel stehen Ihnen zur Verfügung oder haben Sie selbst entwickelt?

Alle am Projekt beteiligten Kindergärten haben von der Stiftung Mecklenburg eine Materialbox erhalten. Die Einrichtungen arbeiten mit Büchern, wie plattdeutsch-hochdeutschen Wörterbüchern, Liederbüchern und Bilderbüchern. Außerdem nutzen sie Spiele, Puzzles, Bildkarten, Broschüren, Fotos und zum Teil auch CDs und DVDs. Zur Recherche wird auch das Internet genutzt. Selbst entwickelte Lehrmittel sind umgeschriebene Tischsprüche, selbst übersetzte Lieder und Gedichte. Eine Kita hat ein Theaterstück selbst entwickelt, andere lassen sich neue Spiele einfallen. Eine Einrichtung hat zwei eigens für sie angefertigte Lieder. Die Erzieherinnen beschränken sich bei der Sprachvermittlung also keineswegs nur auf vorgegebene Lehrmittel, sondern zeigen eine beachtliche Kreativität, kindgerechte Lehrmaterialien zum Niederdeutschen auch selbst zu entwickeln. 
Im Unterricht werden die Kinder dann oft gefragt, was sie machen möchten. Welches Lied soll gesungen werden? Welches Gedicht soll aufgesagt werden? Welches Spiel soll gespielt werden? Die Kleinen können so den Unterricht selbst mitgestalten.

\subsubsection{Erfahrungen}

Frage: Mit welchen Vermittlungsformen haben Sie sehr gute Erfahrungen gemacht?

Die häufigste Antwort auf diese Frage war: Bilder. Es sei wichtig, dass alles, was gemacht wird, anschaulich dargestellt wird. So haben die Kinder eine genauere Vorstellung von dem, was sie machen, sagen und hören. Daher seien farbige, bunt gestaltete Bilderbücher und Bildkarten unverzichtbar. Geschichten, Gedichte und Reime, die mit Bildern unterstützt werden, prägen sich leichter bei den Kindern ein als bloßer Text. Bedeutend ist hierbei auch die Länge des Geschriebenen. Geschichten und Gedichte dürften nicht zu lang sein, da die Konzentrationsfähigkeit der Kindergartenkinder noch nicht so entwickelt sei wie später bei Schulkindern oder Erwachsenen. Dazu meint eine Erzieherin aus S.: „Besonders kommen Bilderbücher, die sehr farbig und bunt sind, an. Die Bilderbücher dürfen nicht zu lang sein. Auch eignen sich Fingerspiele recht gut, wo die Kinder selber gut mitmachen können."

Auch gut geeignet für eine anschauliche Vermittlung sind die Begleitung mit einem Instrument bei einzuübenden Liedern und die schon erwähnten Handpuppen. Das kommt bei Kindern nach Aussage der Erziehrinnen immer gut an. Die Atmosphäre sollte aufgelockert sein und nicht in Form von Frontalunterricht ablaufen. „Die besten Erfahrungen haben wir auf spielerische Weise, also hauptsächlich im Freispiel gemacht. Im täglichen Wiederholen von Liedern, Tänzen und Ähnlichem erfolgte die größte Sprachvermittlung. "So berichtet eine Erzieherin aus R. Weiterhin schreiben auch viele Erzieherinnen: Das Lernen erfolge am besten in spielerischer Form und durch tägliche Wiederholungen. Es helfe auch, die Sprache besser zu lernen, wenn man Geschichten oder Märchen vorliest, die den Kindern bereits bekannt seien. Auch meinen sie, es sollten feste Strukturen im Ablauf vorhanden sein, damit sich eine Regelmäßigkeit und Routine einstelle, aber keine Langeweile aufkomme. Einige Erzieherinnen berichten auch, dass die Möglichkeit, an Wettbewerben teilzunehmen, die Kinder motiviere. Insgesamt gesehen müssen die Methoden und Vermittlungsformen nach ihrer Meinung dem Alter der Kinder entsprechen und so auch angewandt werden. 
Frage: Welche Vermittlungsformen sind Ihrer Meinung nach weniger gut geeignet? Die Erzieherinnen berichten, dass die Kinder bei Hörgeschichten und längerem Vorlesen Probleme haben, dem genauen Wortlaut zu folgen. Eine Vermittlung ohne Anschauungsmaterial für die Kinder ist demnach nicht geeignet. So schreibt eine Erzieherin aus S., dass "plattdeutsche Märchen ohne Bilder" nicht geeignet seien: „Es muss immer anschaulich vermittelt werden und in spielerischer Form.“ Des Weiteren ist es schwierig, in zu großen Gruppen und verschiedenen Altersklassen gleichzeitig zu unterrichten. Besser sei es, dann Gruppen zu teilen und die Altersspanne nicht zu groß werden zu lassen. Sonst komme es zur Überforderung der Jüngsten und Unterforderung der Älteren. Wenn die Erzieherin nicht mit Spaß und Freude dabei sei, übertrage sich diese Stimmung schnell auf die Kinder. Ein reines Lernen von Vokabeln und einzelnen Wörtern sei ebenfalls unangebracht, und so sei Frontalunterricht auch viel zu steif für die Vermittlung. Dazu meint eine Erzieherin aus S.: „Einzelne Wörter geraten zu schnell in Vergessenheit, sie vertiefen sich besser in Liedern, Fingerspielen oder beim Vorlesen“. Beim Lernen von zu langen Theaterstücken fehle den Kindern am Ende die Konzentration weiterzumachen.

\section{Frage: Was bereitet Ihnen noch Probleme?}

Einige Erzieherinnen nennen den Zeitfaktor als Problem. Es nehme viel Zeit in Anspruch, Materialien aufzubereiten, Lehrmittel von Hochdeutsch ins Plattdeutsche zu übertragen. Es fehle auch manchmal die Zeit, um Lieder, Gedichte etc. regelmäßig zu wiederholen. Auch fällt es einigen Erzieherinnen schwer, über einen längeren Zeitraum Niederdeutsch zu sprechen, da es kaum Anlässe gibt, um ihre eigene Plattdeutschkompetenz zu festigen. Es gibt auch immer wieder Unsicherheiten bei einigen Wörtern, zu deren Beseitigung dann Wörterbücher zu Rate gezogen werden müssen, was wiederum Zeit in Anspruch nimmt.

Des Weiteren gebe es Probleme bei der intensiven Wortschatzvermittlung und bei Grammatikübungen, da das Vorsprechen allein nicht ausreiche und das Schreiben von Plattdeutsch noch zu schwer sei. So sei auch anfangs das Vorlesen von kaum vertrauten Geschichten mühsam. Eine Rentnerin schreibt, sie habe manchmal Probleme, Wörter, die situationsabhängig mehrere Bedeutungen im Plattdeutschen haben, auf kindliche Art zu vermitteln. Es fehlt auch teilweise an genügend Material für die Kinder, wie einige Erzieherinnen meinen. So berichtet eine Erzieherin aus einer Schweriner Kita: „Es gibt einfach zu wenig Bilderbücher auf Plattdeutsch, und auch eine CD mit den bekannten plattdeutschen Volksliedern habe ich bis jetzt vergeblich gesucht“. Ein weiteres Problem stellt die 
Möglichkeit des Übens von Texten zu Hause bei den Kindern dar. Denn dadurch, dass die Eltern selbst wenig oder kein Plattdeutsch sprechen können, ist es für sie schwer, mit ihren Kindern plattdeutsche Texte zu üben.

Frage: Mit welchen Kindern erscheint Ihnen die Arbeit am effektivsten?

Am effektivsten erscheint den Erzieherinnen die Arbeit mit Kindern, die Spaß und Freude am Plattdeutschen haben und gerne singen. Mit Kindern ab einem Alter von 3 bis 4 Jahren kann man nach Aussagen der Befragten schon einiges machen, mit Vorschulkindern ist der Unterricht noch wirkungsvoller. In einigen Kindergärten wird auch schon mit Krippenkindern etwas geübt. Hier haben die Erzieherinnen mit ersten Fingerspielen gute Erfahrungen. Generell gesehen kann man so in jedem Alter Fortschritte erreichen, als Hauptsache wird angesehen, dass der Unterricht dem Alter und Interessen der Kinder angepasst ist.

Frage: Welche Entwicklungen bzw. Veränderungen haben Sie evtl. an den Kindern bemerkt, welche Lerneffekte lassen sich bereits beobachten?

Die Erzieherinnen schreiben, dass die Kinder selbst versuchen, im Tagesablauf Niederdeutsch mit ihnen und untereinander zu sprechen. Sie greifen einzelne Redewendungen auf und integrieren diese in ihren Spielen. Die Kinder sind mit Spaß bei der Sache, haben Freude, Plattdeutsch zu sprechen und äußern selbst Wünsche nach Liedern und Gedichten. Sie freuen sich immer schon auf die nächste Unterrichtsstunde. Das Sprachverständnis ist deutlich gestiegen, ihr Selbstbewusstsein hat zugenommen und sie haben schnellere Reaktionen in der Wahrnehmung. Dazu meint eine Erzieherin aus M.: „Die Kinder sind allgemein selbstbewusster im Auftreten; zeigen schnellere Reaktionen in der Wahrnehmung; können sehr gut transferieren“. Des Weiteren ist ihre Aufnahme-, Konzentrationsund Merkfähigkeit gestiegen. Die Kinder singen mit Freude ihren Eltern vor und sind in der Lage, kleine Programme auf Plattdeutsch zu gestalten und „sind begeistert dabei“, wie eine Erzieherin aus R. berichtet. Sie sind stolz auf das, was sie können, berichten den Eltern von ihren Fortschritten und „genießen den Effekt, dass Eltern staunen“, wie eine Erzieherin aus S. schreibt. Dass auch die Eltern am Projekt „Niederdeutsch am Kindergarten“ zunehmend beteiligt werden, berichtet eine Erzieherin aus S.: „Sie haben ihre Eltern ins Boot gezogen. Die möchten oft Texte haben, damit sie auch mitmachen können." Darüber hinaus berichtet die Erzieherin: „Die Kinder sprechen gerne die Sprache, fordern sie auch, dass etwas damit gemacht wird, so z. B. Lieder gesungen werden oder Rollenspiele." Eine Erzieherin schreibt: „Die Kinder berichten nach der Stunde ihren Erzieherinnen stolz vom Erlernten. Sie freuen sich auf die nächste Stunde. Ihr Selbstbewusstsein wird gestärkt. Sie saugen Neues auf wie ein Schwamm.“ 


\section{Frage: Welche Resonanz haben Sie erlebt?}

Es wird vor allem berichtet, dass die Eltern und Großeltern begeistert sind, dass in der Einrichtung Plattdeutsch angeboten wird. Sie zeigen großes Interesse und arbeiten gut mit der Kita zusammen. Sie sind gespannt auf die Auftritte und sehen diese sehr gerne. Dazu schreibt eine Erzieherin aus S.: „Bei den Eltern ist die Resonanz sehr gut, sie möchten, dass es damit weitergeht. Auch die Kinder fragten gleich, wenn sie ,Großer Igel ${ }^{25}$ sind, ob es dann weitergeht“. Auch sonst kommen die Auftritte in den Ortschaften sehr gut an. Über einige Kindergärten wird in den Medien berichtet. Die Erzieherinnen erhalten immer wieder die Bestätigung weiterzumachen, denn die Sprache wird sehr gerne gehört. Die Kinder möchten auch in der Schule weiter Plattdeutsch lernen. Es besteht eine sehr große Nachfrage und es wird berichtet, dass weitere Einrichtungen darüber nachdenken, auch Plattdeutsch in ihr Angebot mit aufzunehmen. Eine Erzieherin schreibt: „Die Nachfrage ist sehr groß. Viele haben noch Verbindung/Erinnerungen, die mit dem Plattdeutschen zu tun haben. Weitere Einrichtungen wollen nachziehen“. Es wird jedoch auch berichtet, dass einzelne Personen dem Plattdeutschangebot eher reserviert gegenüberstehen. Dazu schreibt eine Erzieherin aus S.: „Einige Personen sind erstaunt und halten Plattdeutschunterricht nicht für nötig“. Insgesamt aber überwiegen die positive Resonanz und die Begeisterung für die plattdeutsche Sprache.

\subsubsection{Perspektive}

Frage: Was erhoffen Sie sich von diesem Projekt? Welche Forderungen/Wünsche haben Sie an uns?

Die Erzieherinnen erhoffen sich von dem Projekt, dass die niederdeutsche Sprache und das niederdeutsche Kulturgut erhalten bleiben. Niederdeutsch wird als die Sprache der Region, der Heimat verstanden, und diese solle stärker gefördert werden. So wünscht sich eine Erzieherin aus S. den „Erhalt der plattdeutschen Sprache“ und die „Förderung der Sensibilität für Kultur und Sprache der Heimat“. Außerdem sei es wichtig, ,dass Niederdeutsch im alltäglichen Sprachgebrauch wieder mehr an Bedeutung gewinnt", wie eine Erzieherin aus R. berichtet. Die Kinder sollen eine bleibende Erinnerung an das Plattdeutsche haben und es eventuell im späteren Leben, beispielsweise in der Schule, weiterführen und ihre Fähigkeiten in der Familie weiterverbreiten. Sie hoffen, dass die Sprache nicht verloren geht und ein fester Bestandteil der kindlichen Bildung in Mecklenburg-Vorpommern wird.

25 Bezeichnung einer Kindergartengruppe. 
Eine Erzieherin aus S. meint: „Mein Wunsch wäre, dass Plattdeutsch ein wichtiger Baustein im Entwicklungskonzept aller Kindergarteneinrichtungen wird und sich dann in der Schule fortsetzt, um es weiter zu festigen und zu erhalten“. Es wird auch mehr Pflege und Akzeptanz der Sprache in der Öffentlichkeit gefordert. Einige Erzieherinnen wünschen sich auch den Austausch von neuen Ideen und Erfahrungen untereinander und regelmäßige Kontakte zur Stiftung Mecklenburg. Hier meint eine Erzieherin aus S.: „Ich würde mich freuen, wenn es weiterhin möglich wäre, Zusammenkünfte mit den Plattdeutschlehrenden zu veranstalten“. Eine weitere Erzieherin schreibt dazu, sie wünsche sich ,gegenseitige Besuche und Reflexionen“. So könnten Anregungen und Impulse weitergegeben, Wissen erweitert und Kontakte geknüpft werden und eventuell Angebote an den Kitas vielfältiger ausfallen. In diesem Zusammenhang wünschen sich die Erzieherinnen auch, dass Weiterbildungen angeboten werden, die näher in der Umgebung liegen. Es gebe bis jetzt zu wenige Möglichkeiten für die Lehrenden, ihre Kompetenzen und eigene Sprachfähigkeit weiter auszubilden. Die wenigen Fortbildungsmöglichkeiten seien außerdem meist zu weit entfernt gelegen. Des Weiteren wünscht man sich, dass die Medien regelmäßig mit einbezogen werden, um mehr Aufmerksamkeit und Anerkennung in der Öffentlichkeit zu erhalten. Auch möchten einige Einrichtungen immer wieder neue Materialien erhalten, so dass eine größere Vielfalt des Angebots ermöglicht wird. Dazu schreibt eine Erzieherin aus S.: „Es würde uns helfen, regelmäßiger Material in die Hände zu bekommen. "Dafür schlägt sie ein monatliches oder jährliches Heft vor mit Themen wie Jahreszeitenbeschreibungen, Berufe und Feste und Feiern. Außerdem erhoffen sich die Erzieherinnen, noch weitere Einrichtungen zu begeistern und das Angebot des Erlernens der Sprache in der Schule fortzusetzen, um Plattdeutsch weiter zu festigen und zu erhalten.

Frage: Was sollte Ihrer Meinung nach von der Politik, der Verwaltung oder von anderen Stellen unternommen werden, um Sie zu unterstützen?

Die Wünsche an die Politik, Verwaltung und andere öffentliche Bereiche ähneln denen an das Projekt selbst. Auch hier erhofft man sich mehr Materialien und eine finanzielle Unterstützung der Einrichtungen. Man wünscht sich mehr qualifizierte Ausbilder. Lehrerinnen und Erzieherinnen „müssen in der Ausbildung diese Sprache vermittelt bekommen bzw. die Möglichkeit haben, sich damit auseinanderzusetzen“, fordert eine Erzieherin aus R. Das Erlernen der Sprache sollte zum festen Bestandteil der vorschulischen Erziehung werden und in den Schulen weitergeführt werden. Es wird sich auch mehr Öffentlichkeitsarbeit, mit besonderem Augenmerk auf die Presse, gewünscht, „damit uns nicht so viel Steine zwischen die Füße geworfen werden und es als nichtig herabgestuft wird“, so eine Erzieherin aus einer Kita in Schwerin. Abschließend erhoffen sich einige Lehrende 
mehr Anerkennung für ihre Tätigkeit als Plattdeutschunterrichtende und keine Abwertung des Projektes.

\subsubsection{Abschließende Anmerkungen}

Am Schluss des Fragebogens bekamen die Befragten noch einmal die Möglichkeit, abschließende Kommentare zum Projekt „Niederdeutsch im Kindergarten“ abzugeben.

Die Erzieherinnen haben berichtet, dass das Projekt „Niederdeutsch im Kindergarten" sehr gut angekommen ist. So haben sie viele Materialien kennengelernt und neue Erfahrungen sammeln können. Einige wünschen sich auch eine weitere Fortführung des Projektes, da Plattdeutsch unbedingt weiter gefördert werden soll und auch schon die Kleinsten mit einbezogen werden können. Man hätte sich nur zum Teil gewünscht, dass das Projekt schon früher hätte beginnen können, um noch mehr Kinder zu erreichen, um frühere Erfolge zu erzielen. Die Kitas bedanken sich für das Projekt und die Personen, die es auf die Beine gestellt haben.

\section{Entwicklungen nach dem Ende des Projektes}

Auch nach dem Auslaufen des Projekts „Niederdeutsch in der Kita“ wurde der Kontakt mit den beteiligten Einrichtungen aufrechterhalten und Informationen zum weiteren Verlauf der Entwicklung gesammelt. Diese sollen hier abschließend zusammengefasst werden. An den meisten Kindergärten hat sich demnach kaum etwas verändert, seitdem das Projekt zu Ende ist. Sie bieten noch immer Plattdeutsch an, arbeiten mit den Kindern in Gruppen und nutzen zahlreiche Materialien. Es wird meist einmal wöchentlich ein Kurs angeboten und an den anderen Tagen lässt man Plattdeutsch in den Tagesablauf, wie beim Morgenkreis oder bei den Mahlzeiten, einfließen. Zum Teil wird der Unterricht nur auf Nachfrage der Eltern angeboten. Wenn also die Mehrheit der Eltern einer Gruppe nicht wünscht, dass Niederdeutsch unterrichtet wird, fällt dieser aus. $\mathrm{Zu}$ Beginn des neuen Schuljahres wird dann wieder nachgefragt, auch um mögliche Neuzugänge bei der Entscheidung über die Niederdeutschvermittlung zu berücksichtigen.

Jedoch wünschen sich einige Einrichtungen immer noch mehr Unterstützung von Seiten der Politik und des Landes Mecklenburg-Vorpommern. Sie fühlen sich allein gelassen und verstehen das ihrer Meinung nach geringe Interesse an der Plattdeutscharbeit nicht. Die Erzieherinnen würden sich über regelmäßiges Feedback freuen, damit zu sehen ist, dass ihre Arbeit und Mühe auch geschätzt wird. Auch besteht noch oftmals der Wunsch nach mehr kindgerechten Materialien. Es fehlen Kinderbücher mit plattdeutschen Liedern, Märchen und Gedichten. 
Die Möglichkeit, den Unterricht vielseitig zu gestalten, ist damit eingeschränkt. Manche Kindergärten entwickeln weiterhin eigene Materialien und übersetzen sich eigenständig Geschichten, Lieder und Gedichte ins Plattdeutsche. Das ist eine sehr intensive Arbeit und nimmt viel Zeit in Anspruch, die einigen Einrichtungen fehlt. In einer Einrichtung kann das Angebot zukünftig nicht mehr durchgeführt werden, da die Erzieherin, die Niederdeutsch unterrichtet hat, die Einrichtung verlassen hat und es keine qualifizierten Nachfolger gibt.

Es gibt aber auch positive Entwicklungen. In einer Einrichtung wurde ein Plattdeutschstammtisch gegründet, der aus elf Erwachsenen der Region besteht und der in regelmäßigem Kontakt mit der Plattdeutschgruppe des Kindergartens steht. Diese Einrichtung wurde auch erstmalig für den Förderpreis „Kunst und Kulturarbeit mit Kindern und Jugendlichen“ nominiert, worauf alle sehr stolz sind.

\section{Schlussbetrachtungen}

Betrachtet man die Durchführung und Bewertung des Projekts „Niederdeutsch im Kindergarten“ nun abschließend, so war das Projekt insgesamt ein großer Erfolg auf Landesebene. Plattdeutsch wird bei den Kindern gut auf- und angenommen und begeistert umgesetzt.

Trotz der positiven Gesamtbilanz hat die Befragung der Kindergärten doch auch eine ganze Reihe von Problemen aufgedeckt, die die Vermittlung des Niederdeutschen an den Kindergärten in Mecklenburg-Vorpommern zurzeit noch erschweren. So muss die Tatsache, dass nur 27 \% der Erzieherinnen an den untersuchten Kindertagesstätten über ausreichende Sprachkompetenzen verfügt, $\mathrm{zu}$ denken geben. Dass insbesondere die Fähigkeit zum aktiven Niederdeutschsprechen nur eingeschränkt verbreitet ist, macht die Umsetzung des Niederdeutschunterrichts zu einem gewichtigen Personalproblem. Eine höhere Anzahl von Erzieherinnen, die des Plattdeutschen mächtig sind, ist unbedingt anzustreben. Daher ist es wichtig, den Wünschen der Erzieherinnen nachzugehen und mehr Sprachkurse und Weiterbildungen in der Nähe der jeweiligen Einrichtungen anzubieten. Die größtenteils noch mangelnde Schreibkompetenz könnte ebenfalls auf diese Weise verbessert werden. Zwar ist es für den Bereich der Kindergärten unproblematisch, wenn das Schreiben der niederdeutschen Sprache nicht geübt wird. Betrachtet man allerdings die nachfolgenden schulischen Bildungseinrichtungen, wiegt die fehlende Schreibkompetenz seitens der Erzieher schon bedeutend schwerer, da die Vermittlung von Lese- und Schreibkompetenzen eine wichtige Grundlage unter anderem für den Grammatikerwerb darstellt.

Auffallend ist die große Bereitschaft der auswärtigen Personen, die zum Teil ehrenamtlich die Kinder unterrichten. Auswärtige Personen leisten einen Großteil 
der Niederdeutschvermittlung in den Kindertagesstätten. Deren Kompetenzen im Niederdeutschen sind durchaus passabel mit nur wenigen Defiziten. Größtenteils helfen Rentnerinnen und Rentner, die die Sprache zwar oft in ihrer Kindheit und Jugend noch mit ihren Eltern gesprochen haben, im Laufe der Jahre aber auch nicht in jeder Situation mit dem Plattdeutschen konfrontiert werden und so etwas aus der Übung gekommen sein können. Auch hier ist also zumindest teilweise mit einer eingeschränkten Sprachkompetenz zu rechnen. Außerdem ist zu bedenken, dass die meisten auswärtigen Personen keine ausgebildeten Fachkräfte für Sprachunterricht sind. So mögen sie zwar vielfach bessere Sprachkompetenzen haben als die Erzieherinnen, könnten aber in didaktischer Hinsicht mit der Sprachvermittlung an Kleinkinder überfordert sein.

Das Unterrichtsprinzip der Immersion, das allgemein als ideale Vorgehensweise aufgefasst wird, wird an den Einrichtungen in Mecklenburg-Vorpommern leider nur selten verwirklicht. Nur in drei Kindergärten verfolgt man die Methode „ein/e Erzieher/in eine Sprache“. Oft ist dieses Prinzip einfach zu anspruchsvoll, um ihm gerecht zu werden. Es fehlt vor allem an Personal mit den nötigen sprachlichen Voraussetzungen. Die in den meisten Einrichtungen umgesetzten Vermittlungsmethoden können zwar erfolgreich positive Einstellungen zum Niederdeutschen vermitteln und auch erste Stufen des Sprachelernens mit Spaß verbinden, im Hinblick auf einen gefestigten Spracherwerb bleibt ihr langfristiger Erfolg jedoch fraglich.

Ein Problem besteht auch in der unzureichenden Fülle didaktischen Materials, mit welchem das Erlernen der plattdeutschen Sprache unterstützt und erleichtert werden kann. So wäre es hilfreich von Landesseite her, mehr Bilderbücher und spielerisches Material - auch bei den Kindern bereits gut bewährte Materialien $\mathrm{zu}$ entwickeln und für jeden verfügbar zu machen.

Ebenfalls für einen dauerhaften Erfolg ist es wichtig, den Spracherwerb in der Schule fortzuführen. Im Kindergarten wird der Grundstein gelegt, erste Kenntnisse werdem gewonnen. Diese müssen in der Schule vertieft werden, um ein gutes Sprachverständnis zu erhalten. Wird dies nicht gewährleistet, gehen die Erfolge, die die Kinder im Kindergarten erreicht haben, wieder verloren.

\section{Literatur}

Adler, Yvonne: Kinder lernen Sprache(n). Alltagsorientierte Sprachförderung in der Kindertagesstätte. Stuttgart 2011.

Arendt, Birte: Was spricht für einen frühen Zweitspracherwerb? In: Stiftung Mecklenburg (Hrsg.): Lürlürlütt. Handreichung zum Erlernen der niederdeutschen Sprache in der frühkindlichen Bildung. Rostock 2012, S. 6-8. 
Brodkorb, Mathias: Vorwort. In: Stiftung Mecklenburg (Hrsg.): Lürlürlütt. Handreichung zum Erlernen der niederdeutschen Sprache in der frühkindlichen Bildung. Rostock 2012, S. 5.

FMKS e. V. = Verein für frühe Mehrsprachigkeit an Kindertageseinrichtungen und Schulen e. V. (Hrsg.): Ich kann viele Sprachen lernen. Sprachen lernen im Sprachbad „Immersion“ in zwei- und mehrsprachigen Krippen, Kitas und Schulen. Kiel 2016.

Haid, Andrea: Kindlicher Spracherwerb. Mein Kind lernt mehr als eine Sprache. 2. Aufl. Wien 2012.

Institut für niederdeutsche Sprache (Hrsg.): Zehn Jahre Europäische Charta der Regional- oder Minderheitensprachen. Zwischenbericht zur Sprachpolitik für das Niederdeutsche (Schriften des Instituts für niederdeutsche Sprache: Dokumentation; 37). Leer 2008.

Möller, Frerk: Plattdeutsch im 21. Jahrhundert. Bestandsaufnahme und Perspektiven. Mit einem Aufsatz von Michael Windzio (Schriften des Instituts für Niederdeutsche Sprache; 34). Leer 2008.

Stiftung Mecklenburg (Hrsg.): Lürlürlütt. Handreichung zum Erlernen der niederdeutschen Sprache in der frühkindlichen Bildung, Rostock 2012.

Timm, Herbert: „Plattdüütsch för de Lütten“ Warum? Vorteile der mehrsprachigen Erziehung in Familie, Kindergarten und Grundschule. Salzhausen o. J.

\section{Online-Ressourcen:}

Europarat: Europäische Charta der Regional- oder Minderheitensprachen. Straßburg 5.11.1992. [Online-Ressource: http://www.coe.int/de/web/conventions/ full-list/-/conventions/rms/090000168007c089 (Stand: 3.12.2016)]

Stiftung Mecklenburg: Plattdütsch in'n Kinnergorden. [Online-Ressource: http:// www.stiftung-mecklenburg.de/index.php?option=com_content\&view=article \&id=71\%3Aplattduetsch-inn-kinnergorden-artikel\&catid=38\%3Averanstaltu ngen\&Itemid $=53$ (Stand: 16.11.2016)]

Lenz, Alexandra: Vom Dialekt zur regionalen Umgangssprache - Zur Vielfalt regionaler Sprechweisen. In: Munske, Horst Haider (Hrsg.): Sterben die Dialekte aus? Vorträge am Interdisziplinären Zentrum für Dialektforschung an der Friedrich-Alexander-Universität Erlangen-Nürnberg, 22.10.-10.12.2007. 2008. [Online-Ressource: http://www.dialektforschung.phil.uni-erlangen.de/ sterbendialekte (Stand: 29.10.2016)] 


\title{
Johanna Biedowicz
}

\section{Frühkindlicher Niederdeutscherwerb. Eine exemplarische Studie zu einem Greifswalder Kindergarten}

\begin{abstract}
Low German, defined as a regional language by the European Charter for Regional or Minority Languages, is strongly suspected to become a dead language. Signs of this development are a decreasing number of dialect speakers, the retreat of the Low German dialect from public communication, and the total domination of High German standard language in terms of a first language acquired in early childhood. Yet, in order to save a language from extinction, it is not enough to remember it. - It needs to be spoken.

Since children do not acquire Low German at home any longer, forms of dialect education in institutional contexts have to be implemented. Preschool language education might play a mayor role in this respect. The article presents results of a study with a single kindergarten in the Western Pomeranian city of Greifswald, which participated in a language project on teaching Low German to preschool children at an age from 4 to 6 . The methodological basis of the dialect education was the didactic concept of immersion. The article discusses language acquisition and multilingualism in preschool contexts as well as the acquisition of the Low German dialect as a secondary language by means of immersion.
\end{abstract}

\section{Einleitung}

Weltweit werden insgesamt circa sechstausend verschiedene Sprachen gesprochen. Die Mehrheit der Weltbevölkerung ist zwei- oder sogar mehrsprachig, Einsprachigkeit somit eher eine Ausnahmeerscheinung. ${ }^{1}$ Die aktuelle Spracherwerbs- und Mehrsprachigkeitsforschung betont die kognitiven, aber auch sozialen und kulturellen Vorteile und Gewinne von (individueller) Mehrsprachigkeit. Es wird darauf verwiesen, „daß sich die Mehrsprachigkeit begünstigend auf die Fähigkeiten in beiden Sprachen (additiv) “2 auswirkt. ${ }^{3}$ Auch der norddeutsche

1 Vgl. Riehl 2006, S. 3-4.

2 Tracy/Gawlitzek-Maiwald 2000, S. 499.

3 Gegenwärtig hat sich die Mehrsprachigkeitsforschung als ein zentraler und vielseitig ausdifferenzierter Forschungsbereich innerhalb der Linguistik etabliert. Für einen detaillierten Überblick über aktuelle und ältere Studien siehe z.B. Müller/Kupisch/ Schmitz/Cantone 2007, S. 52 oder de Houwer 2009, S. 337. 
Sprachraum ist von einer historisch gewachsenen und weiterhin existenten Mehrsprachigkeit mit einer Koexistenz von Niederdeutsch und Hochdeutsch geprägt. Gleichzeitig ist für die Gegenwart ein fortschreitender Abbau dieser spezifischen Form dialektaler Mehrsprachigkeit zu konstatieren, der durch die wachsende Dominanz des Hochdeutschen in sämtlichen Kommunikationsbereichen sowie durch einen Rückzug des Niederdeutschen aus der öffentlichen Kommunikation und einen rapiden Rückgang dialektkompetenter, -sprechender und -vermittelnder Personen gekennzeichnet ist. Die Regionalsprache Niederdeutsch droht damit - trotz ihrer wachsenden Akzeptanz als regionale Kultursprache - zu einer aussterbenden Sprache zu werden. ${ }^{4}$

Es soll im Folgenden nicht um die Existenzberechtigung des Niederdeutschen und deren Begründung gehen, sondern vielmehr um deren potenzielle zukünftige Sprecher. Im Mittelpunkt des Beitrages steht die gesteuerte Vermittlung des Niederdeutschen im Kindergarten als Zweitsprache als ein möglicher und vielversprechender Weg, mehrsprachige Kompetenzen bei Kindern auf- und auszubauen und gleichzeitig den Erhalt des Niederdeutschen als eine lebendige, d. h. tatsächlich gesprochene Regionalsprache zu fördern.

Bundesweit gibt es derzeit eine Vielzahl an Förderprogrammen für Regionaloder Minderheitensprachen, die auch auf den Bereich der kindlichen Spracherziehung zielen. ${ }^{5}$ Eine besondere Rolle nehmen in diesem Zusammenhang Kindergärten ein, die als eine Art „Sprachfenster" fungieren und in denen mehrsprachige Kompetenzen von Kindern häufig nach dem Prinzip der Immersion entwickelt werden. ${ }^{6}$

In Mecklenburg-Vorpommern haben sich - gefördert durch die Landespolitik - in den letzten Jahren eine ganze Reihe von Kindertagesstätten der Vermittlung des Niederdeutschen verschrieben. Auch ein Kindergarten ${ }^{7}$ in Greifswald hat sich für diese Form der Sprachförderung entschieden. Die in diesem Kindergarten praktizierte Sprachpädagogik ermöglicht es den Kindern, dem Niederdeutschen in ihrem natürlichen Alltag zu begegnen, beim Essen, Anziehen und

4 Der Begriff Regionalsprache wird hier als politischer Terminus im Sinne der EUCharta für Minderheiten- oder Regionalsprachen verstanden. Zu den Minderheitensprechern innerhalb Deutschlands gehören die dänisch sprechenden Südschleswiger, die Sorben, die Friesen sowie die Sinti und Roma, wobei Niederdeutsch eine Sonderstellung als Regionalsprache einnimmt.

5 Vgl. Bundesrat für Niederdeutsch 2009.

6 Vgl. u. a. Schulz 2009.

7 Auch wenn es formal Unterschiede zwischen den Bezeichnungen Kindergarten und Kindertagesstätte gibt, werden sie hier synonym verwendet. 
Erzählen, aber auch durch das Einüben von niederdeutschen Gedichten, Theaterstücken und Liedern, und auf diese Weise ihr Sprachgefühl und Sprachverstehen zu schulen.

Der vorliegende Artikel ist als exemplarische Fallstudie angelegt. Er entfaltet zunächst die theoretischen Grundlagen zum Zweitspracherwerb im Kindergarten und zur sprachdidaktischen Methode der Immersion (Kap. 2), stellt danach ein einzelnes Lehr-Lern-Projekt zum Niederdeutscherwerb an diesem Greifswalder Kindergarten vor, das in den Jahren 2011 und 2012 in Zusammenarbeit mit der Universität Greifswald durchgeführt wurde (Kap. 3), und präsentiert anschließend im analytischen Teil anhand von transkribierten Gesprächsausschnitten aus verschiedenen Sprachlernsituationen in diesem Kindergarten Einsichten in die durch das Projekt erzielten Sprachlernerfolge (Kap. 4). Im Fokus stehen die (spontanen) Kinderäußerungen während der Projektarbeit, die durch Videoaufnahmen dokumentiert wurden und in denen sich die Sprachlernprozesse und -erfolge dieser Kinder manifestieren. Für die Auswertung dieser Aufnahmen bedient sich die Untersuchung u. a. gesprächsanalytischer Verfahren. Abschließend werden aus den theoretischen und empirischen Befunden praktische Handlungsempfehlungen für die Niederdeutschvermittlung in vorschulischen Einrichtungen abgeleitet (Kap. 5).

\section{Niederdeutsch als Zweitsprache im Kindergarten}

\subsection{Niederdeutsch als Zweitsprache}

Das Niederdeutsche wird gegenwärtig nicht mehr als Erst- oder Muttersprache in einem ungeregelten Lernprozess erworben, sondern nahezu ausschließlich als eine Zweitsprache erlernt. So vermerkt etwa Bieberstedt in seiner Untersuchung zum Spracherwerb Hamburger Dialektsprecher:

Der grundlegende Sprachwandel, den der norddeutsche Raum in den vergangenen Jahrzehnten durchlaufen hat, betrifft nicht allein das sprachliche Spektrum zwischen nd. Basisdialekten und hd. regionaler Standardsprache sowie die Kenntnis und den Gebrauch regionaler Varietäten und die Einstellungen ihnen gegenüber, sondern ebenso die Formen und Konditionen dialektalen Spracherwerbs. ,Traditionelle` Erwerbsmodi, die bis in die 2. Hälfte des 20. Jh. dominierten und bei denen der Dialekt als Erstsprache im Elternhaus, das Hd. dagegen simultan als zweite Erstsprache oder erst sukzessiv als Zweitsprache in der Schule vermittelt wurde, sind durch neuere Formen des Erwerbs abgelöst, bei denen die hd. Standardsprache den wesentlichen Orientierungspunkt für die primäre Sprachsozialisation bildet. Der Erwerb des Hd. als Erstsprache stellt in der Gegenwart den absoluten Regelfall dar, zugleich eignen sich Dialektsprecher das Nd. 
häufig nicht mehr simultan als zweite Erstsprache an, sondern sukzessiv in neuen Erwerbszusammenhängen. ${ }^{8}$

Als Zweitspracherwerb wird hierbei allgemein jeder Spracherwerb bezeichnet, der über den (muttersprachlichen) Erstspracherwerb hinausgeht. Lernt ein Kind von Geburt an zwei Sprachen, nennt man diesen Prozess simultanen (gleichzeitigen) Spracherwerb oder auch doppelten Erstspracherwerb. ${ }^{9}$ Der konsekutive (nachfolgende) Spracherwerb beinhaltet alle zeitlich nachgelagerten Erwerbsprozesse. Einige Autoren ziehen die Grenze zwischen dem simultanen und sukzessiven Erwerb zweier Sprachen mit dem Beginn des dritten bis fünften Lebensjahres. ${ }^{10}$ Diese zeitliche Grenze wird von vielen Autoren allerdings als arbiträr, da fließend betrachtet. In den meisten Fällen werden die Bezeichnungen sukzessiver (,allmählich, nach und nach') und konsekutiver Spracherwerb synonym gebraucht.

Sprechen wir über einen Zweitspracherwerb im Kindergarten, wie im vorliegenden Fall, handelt es sich also um einen konsekutiven Sprachlernprozess, bei dem die Sprachpädagogik auf eine bereits vorhandene erstsprachliche (hochdeutsche) Kompetenz aufbauen kann.

\subsection{Sprachförderung im Kindergarten}

Im aktuellen Gesetz zur „Frühkindlichen Bildung“ des Ministeriums für Bildung und Wissenschaft des Landes Mecklenburg-Vorpommern von 2014 werden mehrere Bildungsziele hervorgehoben. ${ }^{11}$ Zentralen Stellenwert nimmt als pädagogische Maxime das lebenslange Lernen ein. Ebenso zentral ist die Sprachförderung, die bereits auf der frühkindlichen Entwicklungsstufe ansetzt. Ein zweisprachiger Kindergarten vermittelt neben der Förderung der Erstsprache und der Zielsprache auch weitere (kommunikative, soziale, kulturelle) Bildungskompetenzen. Häufig werden diese Bereiche im Kindergarten didaktisch nicht voneinander getrennt. Die Vermittlung einer weiteren Sprache stellt damit eine Sonderform der Kindergartenpädagogik dar.

Die (frühe) Zweisprachigkeit in einer Kita kann im Grunde durch zwei verschiedene Modelle praktiziert werden. Zum einen durch den Angebotsansatz, zum anderen durch die Immersion. Beide Methoden haben ihre Vor- und Nachteile. ${ }^{12}$

8 Bieberstedt 2015, S. 206.

9 Vgl. Tracy/Gawlitzek-Maiwald 2000, S. 499-503.

10 Vgl. Tracy 2008, S. 106.

11 Ministerium für Bildung, Wissenschaft und Kultur des Landes Mecklenburg-Vorpommern 2014.

12 Vgl. Nauwerck 2005, S. 84.

Birte Arendt, Andreas Bieberstedt and Klaas-Hinrich Ehlers - 978-3-631-71893-3 
So gehört der Angebotsansatz, bei dem sprachliche Lehrangebote gezielt und zeitlich begrenzt in den Kindergartenalltag eingebracht werden, zur Form der systematischen Sprachförderung, während die Immersion zu den integrativen und ganzheitlichen Verfahren ${ }^{13}$ zählt. Die Verfahren schließen sich jedoch nicht explizit aus. Allerdings wird der immersiven Methode - zumindest in einzelnen Bereichen wie dem hier diskutierten - eine gewichtigere Rolle bei der Fremdsprachenvermittlung zugeschrieben, da sie längerfristig orientiert arbeitet.

Das wohl wichtigste Element in einem Kindergartenalltag, der sich der Vermittlung einer weiteren Sprache angenommen hat, ist, neben dem Sprechen, das Hören. ${ }^{14}$ Das Gehör ist Basis grundlegender Spracherwerbsmechanismen im Kindergarten. Im Elementarbereich frühkindlichen Spracherwerbs ist eine wiederholte Informationsaufnahme durch abermaliges (und eigenständiges) Lesen natürlich noch nicht möglich. Demnach ist die kontinuierliche Repetition der Sprache und des Gesagten von immenser Wichtigkeit in der alltäglichen Kita-Arbeit. Sprache, ganz allgemein, wird durch unterschiedliche Methoden erworben, so stehen neben dem Nachahmen und dem Wiederholen auch das Variieren ${ }^{15}$ im Blickfeld des (kindlichen) Lernprozesses. Zusätzlich unterstützen Bewegung und Spiel die Aneignung der Fremdsprache.

\subsection{Zweitspracherwerb im Kindergarten durch Immersion}

Sprachlernangebote in Kindergärten, aber auch Grundschulen, werden überwiegend nach dem Prinzip der Immersion realisiert, das aktuell als aussichtsreiche und adressatengerechte Methode gilt. ${ }^{16}$ Das Prinzip des „Eintauchens“ (lateinisch immersus; englisch (to) immerse) beschreibt einen Prozess des Sprachlernens, bei dem die zu erlernende Sprache als Umgangs- und Situationssprache eingesetzt und somit alltäglich gemacht wird. Die Immersion basiert konzeptionell auf der Interlanguage-Hypothese. ${ }^{17}$ Das Eintauchen kann innerhalb einer Institution, wie

13 Einen ausführlichen und detaillierten Einblick in beide Ansätze bietet Nauwerck 2005 .

14 Vgl. Häussermann/Piepho 1996, S. 19.

15 Bildungskonzeption für 0- bis 10-jährige Kinder in Mecklenburg-Vorpommern. Förderung von Kindern unter 3 Jahren, o. J.

16 Vgl. Kersten/Fischer/Burmeister/Lommel 2009.

17 Die Interlanguage-Hypothese geht davon aus, dass beim Fremdspracherwerb eine Lernersprache (interlanguage) gebildet wird, die Züge der Ausgangs- und der Zielsprache aufweist und als autonomes und zugleich variables System angesehen werden kann, vgl. Klein 2000. „Grundsätzlich ist unter Spracherwerb der Aufbau einer interlanguage zu verstehen, also eines mentalen Konstrukts von Regeln, auf das Lerner zurückgreifen, um sich zu verständigen. Die interlanguage unterliegt einer kontinuierlichen Ver- 
einer Schule oder eines Kindergartens, stattfinden, aber auch außerhalb, in der Familie und der Umgebung, und hat als Sprachaneignungsmethode eine lange Tradition. Die Methode, so modern sie in den fachwissenschaftlichen Betrachtungen der westeuropäischen Welt auch erscheinen mag (seit den 1980ern), stellt keine Neuentwicklung im herkömmlichen Sinn dar. Die immersive Sprachaneignung ist eher der natürlichste Weg, eine fremde Sprache zu erwerben. ${ }^{18}$ Denn die Immersion arbeitet nach dem Prinzip eine Person = eine Sprache. So erlernen Kinder ihre Erstsprache prinzipiell immersiv. Aus diesem Grund erscheint das Immersionsverfahren kindgerechter ${ }^{19}$ als jede andere Vermittlungsart. Immersion bietet ferner großen Raum für Motivation und kommt ganz ohne Zwang oder Leistungsdruck aus. Es lässt sich festhalten, dass die Immersion eine Methode zur (Fremd-)Sprachenvermittlung ist, bei der die zu erlernende Sprache konsequent in den natürlichen Alltag und die Umgebung einfließt. Das Kind taucht nicht nur in eine Sprache ein, sondern in eine Welt, in der alles in dieser anderen Sprache geschieht und bewältigt wird.

\section{Das Projekt „Studierende lehren Plattdeutsch in der Kita“}

\subsection{Ausgangsbedingungen}

Die für die vorliegende Studie ausgewählte Greifswalder Kindertagesstätte ist eine von insgesamt 20 Kitas im Land Mecklenburg-Vorpommern, die an dem Projekt „Niederdeutsch in der frühkindlichen Bildung“ von 2010 bis 2012 teilnahmen. ${ }^{20}$ Im beobachteten Kindergarten bildet die niederdeutsche Sprache einen wichtigen Baustein des Alltags und ist - immer montags - im gesamten Gebäude hör- und für Besucher an Wandzeitungen auch lesbar. Darüber hinaus wird das Aufsagen von Gedichten als Abfragemethode eingesetzt, ebenso wie das Singen plattdeutscher Lieder. Zudem nehmen die Kinder der Kita regelmäßig erfolgreich an Plattdeutsch-Wettbewerben teil.

In den Jahren 2011-2012 entstand unter dem Namen „Studierende lehren Plattdeutsch in der Kita“ innerhalb des Trägerprojektes unter der Leitung von

änderung, die im Idealfall zu einer mentalen Grammatik führt, die auch ein Muttersprachler besitzt.“Weskamp 2001, S. 26.

18 Vgl. Wode 1995 und Le Pape-Racine 2007.

19 Die Vorteile der Immersionsmethode wie Effektivität, Kosteneinsparungen und kognitive Leistungssteigerung sind offensichtlich. Dennoch wird das Konzept durchaus auch kritisch reflektiert, vgl. Reinhold 2004, S. 28; Le Pape-Racine 2007, S. 27-29; Wode 2008, S. 102; Rachel 2010, S. 40-42.

$20 \mathrm{Zu}$ diesem Projekt vgl. auch den Beitrag von Mittelstädt in diesem Band. 
Dr. Birte Arendt eine Kooperation zwischen der Universität Greifswald und der Greifswalder Kindertagesstätte, in der Niederdeutschstudierende verschiedene Projekte der Niederdeutschvermittlung in dem Kindergarten realisierten. Die direkte Zusammenarbeit zwischen der Kita und der Universität stellte eine klare win-win-Situation für beide Institutionen dar. Die Erzieherinnen bekamen Unterstützung und neuen Input von außen, die Studierenden konnten praktische Erfahrungen im didaktischen Umgang mit dem Niederdeutschen gewinnen. Die Universität konnte ferner die Fortschritte im Gebrauch der Sprachvermittlung des Niederdeutschen dokumentieren und somit die Wichtigkeit des Projekts auch wissenschaftlich hervorheben und exemplarisch untermauern. Darüber hinaus lernten die Kinder auch junge Menschen kennen, die des Niederdeutschen mächtig sind, und erlebten die Sprache somit nicht nur als erzieherinnengebundene Sprache.

Bisher sind aus den theoretischen Überlegungen insgesamt die vier Projekte ${ }^{21}$ hervorgegangen: (1) „Klopp an! - eine interaktive Bilderbuchlektüre“, (2) „Fief lütte Tüffeln - eine Theaterinszenierung“, (3) „De Dierte - ein sachkundlichkünstlerischer Tierparkbesuch“ und (4) „De vertöwerten Geschenke - ein kollaborativ-musikalisches Weihnachtsspektakel“. Kern der Projekte (2) und (4) war das Theaterspiel. Diese Fokussierung ergab sich aus folgenden Überlegungen: Ein Theaterstück und die Inszenierung eines Stückes sind für die kindliche Entwicklung enorm wichtig. In Form des darstellenden Spiels und von Rollenspielen bekommen alle Kinder die gleiche Chance zu spielen. Theaterspielen fördert zudem das Selbstbewusstsein der Kinder und ihre Fantasie. Zudem erweist sich Theaterspielen als ganz und gar handlungsorientiert und handlungsanregend. ${ }^{22}$ Das Theaterspielen kann auch dazu dienen, sprachliche Barrieren zu überwinden. So kann und sollte das Theaterprojekt „Fief lütte Tüffeln“ den Kindern Spaß an der plattdeutschen Sprache vermitteln und ihnen ihre Scheu vor dieser nehmen. Zudem lässt sich Sprachvermittlung innerhalb von Kindergärten sehr gut über ein Theaterstück immersorisch umsetzen.

\subsection{Projektziele}

Die Ziele des Projektes lassen sich wie folgt konturieren: An erster Stelle stand die Vermittlung der niederdeutschen Sprache. Weitere Ziele waren der ganzheitliche

21 Bildmaterial und Grundinformationen zu den einzelnen Projekten finden sich auch auf der Homepage von Dr. Birte Arendt [Online-Ressource: https://germanistik.unigreifswald.de/arendt/ (Stand: 12.12.2016)] sowie in Arendt/Gąszczyk 2015.

22 Vgl. Andresen 2002. 
Umgang mit der Sprache als Medium des Alltagsgeschehens sowie das Lernen mit Kopf, Herz und Hand: Die entwickelten Projekte sollten die Kinder auf mehreren Ebenen berühren und verschiedene Kompetenzen fördern. Vom Medium Buch, über Musik und Bewegungsspiele bis hin zu Naturerlebnissen und kulinarischen Erfahrungen. Die verschiedenen Projekte haben aus verschiedenen multisensorischen Erfahrungsbereichen geschöpft, um möglichst ansprechende, ganzheitliche und nachhaltige Impressionen zu hinterlassen.

Durch die kontinuierliche und alltagsgebundene Nutzung des Niederdeutschen, lernten die Kinder ihr Sprachempfinden zu schärfen. Die Kinder sollten Niederdeutsch nicht nur als institutionelle, an den Kindergarten gebundene, sondern als reale Sprache erleben. Aus diesen Gründen bereiteten die beteiligten Studierenden unter Anleitung von Frau Dr. Arendt nicht nur kontinuierlich und kleinschrittig die Theaterstücke und andere Projekte vor, sondern vermittelten diese den Kindern ebenso kleinschrittig und kindgerecht über mehrere Wochen und Monate hinweg. Doch nicht die bloße Sprachvermittlung war wichtig, sondern auch der methodische Zugang. So mussten sich nicht nur die Projektteilnehmer, sondern auch die Projekte an die sprachlichen Bedingungen der Kinder anpassen. Die Maximen der Vorbereitungen lagen also auf der Kontinuität und der Progression der Sprachvermittlung. ${ }^{23}$

\subsection{Datenbasis}

Die empirische Basis der Untersuchung bilden Daten aus dem Projekt „Studierende lehren Plattdeutsch in der Kita“. Die Studie arbeitet mit Audio- und Videoaufnahmen von überwiegend einsprachig aufwachsenden Kindern im Alter von vier bis sechs Jahren der Greifswalder Kindertagesstätte. Die Anzahl der teilnehmenden Kinder belief sich in der Regel zwischen 8-15 Personen, je nach Projekt. Die Projektteilnehmer sowie die Erzieherinnen und Kinder trafen sich einmal wöchentlich, immer vormittags, in den Räumlichkeiten der Kita. Diese Räume gehören zur alltäglichen Umgebung der Kinder. Mit der Wahl des Aufnahmeortes verbinden sich jedoch nicht nur Vorteile. So ist der Kindergarten immer noch eine institutionelle Umgebung und die Kinder würden sich vermutlich zu Hause, in ihrer Privatheit oder im Freien anders verhalten als in einem geschlossenen Gruppenraum.

23 Vgl. Knapp/Kucharz/Gasteiger-Klicpera 2010, S. 131.

Birte Arendt, Andreas Bieberstedt and Klaas-Hinrich Ehlers - 978-3-631-71893-3 
Im Fokus der nachfolgenden Darstellung stehen Daten des Theaterprojekts „Fieflütte Tüffeln“. ${ }^{24}$ Über einen Zeitraum von vier Wochen waren die Studierenden je einmal pro Woche für ca. 1-1 1/2 Stunden im Kindergarten, um mit einer Gruppe von ca. 12 Kindern ein Theaterstück zu erarbeiten. Es liegen knapp drei Stunden Videomaterial zu dem Teilprojekt „Fief lütte Tüffeln“ vor. ${ }^{25}$

Es liegt zunächst die Vermutung nahe, dass bei dem Einstudieren eines Theaterstückes nur gesteuerte Gespräche im Vordergrund stehen. Natürlich sollen die Kinder den Rollen entsprechende Textpassagen auswendig können. Jedoch macht das imitierte Sprechen keineswegs die gesamte Arbeit einer Theaterinszenierung aus. So wurde den Kindern zunächst das Theaterstück auf mehreren Ebenen vorgestellt, um es möglichst anschaulich und ganzheitlich darzustellen: visuell mittels Zeichnungen, haptisch durch reale Kartoffeln (Tüffeln), akustisch durch das Imitieren von bestimmten Tieren oder Gegenständen und durch Bewegungseinheiten. Die Aufnahmen fanden in einem habitualisierten Kontext statt, der in einer Theaterinszenierung als Abschluss der Projekteinheit gipfelt. Von relevanten Passagen der Videoaufnahmen wurden nach den GAT-Konventionen Basistranskripte erstellt. ${ }^{26}$

$\mathrm{Da}$ es sich bei den vorgestellten Gesprächstranskripten lediglich um Ausschnitte und damit dezidiert um Momentaufnahmen handelt, können durch die Analyse keine longitudinalen sprachlichen Entwicklungen oder allgemeine Regeln des Zweitspracherwerbs aufgedeckt werden. ${ }^{27}$ Aus den Beobachtungen können aber Zusammenhänge erschlossen werden, die ihrerseits valide sind und sich durchaus auf weitere Handlungsebenen beim Niederdeutscherwerb in der Kita übertragen lassen.

\section{Analysen und Ergebnisse}

Die folgenden Ausführungen basieren auf drei Transkriptausschnitten, die aus drei unterschiedlichen Aufnahmen stammen und die im Abstand von jeweils

24 Eine verkürzte Form des Theaterstückes findet sich unter dem Namen der Verfasserin dieses Beitrages in dem Buch „Lürlürlütt“, das 2012 unter der Redaktion von Susanne Bliemel von der Stiftung Mecklenburg herausgegeben wurde, Stiftung Mecklenburg 2012, S. 69.

25 Zum Beobachterparadoxon und den daraus resultierenden Problemen verweise ich auf Klann-Delius 1996, S. 24-26.

26 Bei den Transkripten wird sich nach den GAT-Konventionen von Selting [u. a.] 1998, S. 91-122 gerichtet, vgl. auch den Anhang dieses Beitrags.

27 Zum Für und Wider der Dauer und Häufigkeit und anderer Charakteristika einer longitudinalen und somit aussagekräftigen Studie siehe Klann-Delius 1996, S. 17-20. 
einer Woche entstanden sind (T1: 30.9.2011; T2: 07.10.2011; T3: 14.10.2011). Sie illustrieren verschiedene Formen des Spracherwerbs, die vom rein rezeptiven Sprachverstehen (T1 in 4.1) über die Imitation (T2 in 4.2) bis hin zum eigenständigen Sprechen reichen (T3 in 4.3). Damit stellen diese Formen zugleich Abbilder einer klaren Lernprogression dar.

\subsection{Sprachverstehen in der Einstiegsphase - Gesprächstranskript T1}

Das erste Gesprächstranskript (T1) ${ }^{28}$ geht auf eine Aufnahme aus der ersten Projektsitzung am 30.09.2011 zurück, die eine Gesamtdauer von mehr als 30 Minuten aufweist, und zeigt den Beginn einer Szene, in der die vier Universitätsstudierenden (U 1-4) den anwesenden sieben Kindern (K 1-7; vier Jungen, drei Mädchen, alle im Alter von 4-6 Jahren) im Gespräch begegnen. Die Umgebung ist den Kindern bekannt, und auch die Gesprächspartner sind keine Fremden für die Kinder. Allerdings ist das Thema des Gespräches von Anfang an festgelegt. Das gesamte Gespräch zirkuliert um das Thema „Tüffeln“. Die Gruppenerzieherin war ebenfalls anwesend.

Transkriptausschnitt T1: 30.09.2011, Einstiegsphase

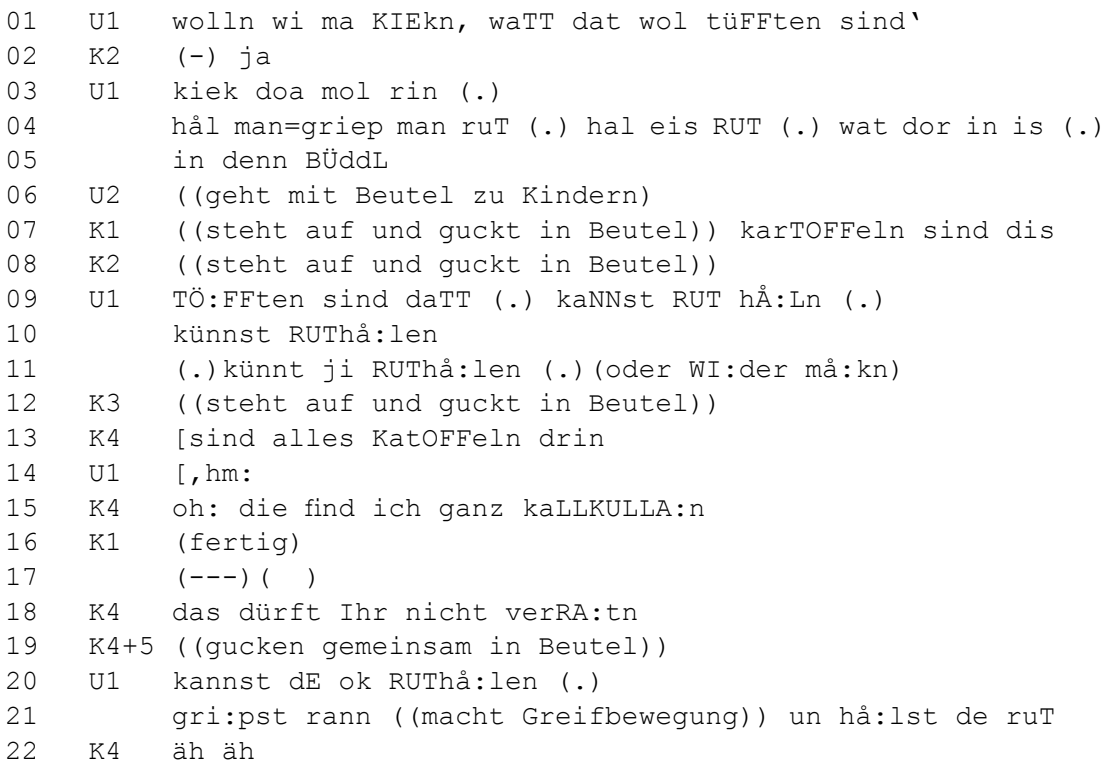

28 Die Konventionen zur Transkription sind im Anhang zusammengefasst. 


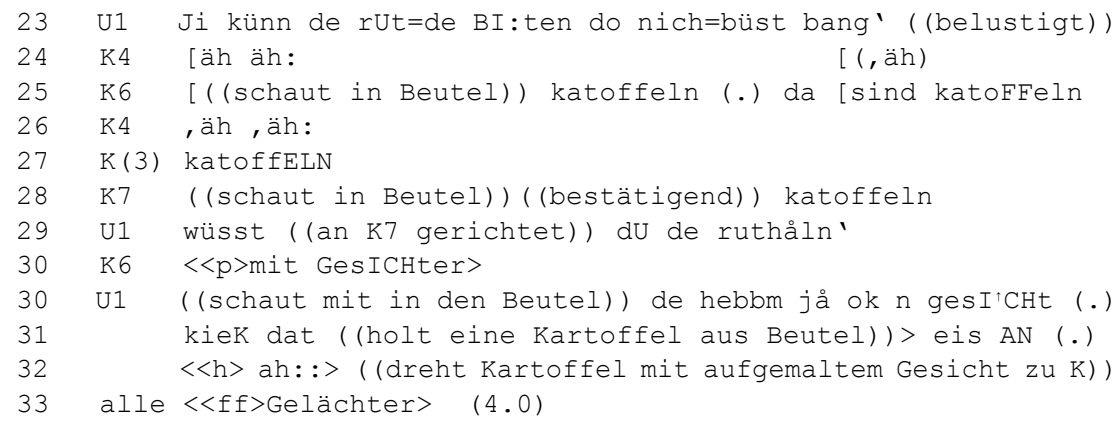

Das Transkript beginnt mit einer als Frage formulierten Einleitung von U1 (Z. 01). Daran anschließend folgt, bevor die Reaktion lautlich realisiert wird, eine Pause. Durch die Zustimmung eines Kindes (Z. 02) beginnt das Gespräch. Augenscheinlich ist, dass die Rollen der Interakteure klar verteilt sind. Der Sprechanteil von U1 ist wesentlich höher als der der Kinder. Jedoch darf die Rolle des Hörers innerhalb eines Gespräches nicht als rein passiver Part verstanden werden. Die reaktiven nonverbalen Handlungen der Kinder in den Zeilen 06, 07, 08, 13, 22, 26 und 28 lassen die Vermutung zu, dass die Kinder das Gesagte und somit die Anweisungen von $U 1$ verstehen. Die Transkription zeigt deutlich in den benannten Zeilen, dass ein sprachliches Verstehen der realisierten Aufforderungen vorhanden ist. Zunächst äußern sich die Kinder überhaupt nicht, während ihr Redeanteil zum Ende des Transkriptes hin steigt.

Beinahe jeder Gesprächszug (Turn) von U1 beinhaltet die Aufforderung, in den Beutel zu greifen. Zudem zeigt $U 1$ in Z. 04 und 05 einige schnelle Turn-Abfolgen. Diese kann man als Verbesserungstaktik ansehen oder als Versuch, den Kindern die Aufforderung sprachlich so verständlich wie möglich zu machen. Ferner ist U1 bemüht, den Fokus von dem einzelnen Kind auch auf die anderen als Einheit zu übertragen (Z. 11-12; 23). Hierbei sucht $U 1$ immer wieder zuerst den Augenkontakt zum jeweilig handelnden Kind, wiederholt als Folgereaktion auf die nicht geleistete Reaktion des Kindes die Aufforderung und richtet sie zugleich an alle.

Das $K 4$ bejaht (Z. 02) die einleitende Frage und erweist sich ebenso wie die anderen Kinder zunächst als handlungsgehemmt, indem sie zwar in den Beutel schauen, aber nicht hineingreifen. Als K1 sagt, was sich im Beutel befindet (Z. 07), greift $U 1$ dies sprachlich auf, um es von der Ausgangssprache der Kinder, dem Hochdeutschen, in die Zielsprache Niederdeutsch zu konvertieren. Das Kind mit dem höchsten Redeanteil innerhalb dieser Sequenz ist $K 4$. Diese Redeanteile setzen sich aus verschiedenen Elementen zusammen: So folgt der Feststellung (Z. 13) eine sprachlich interessante Aussage, die hier als kindlicher Phantasie- 
Ausdruck (Z. 15) festgehalten wird: Möglicherweise ist der Begriff „kalkulla“ eine Zusammensetzung aus verschiedenen Begriffen, wie den Begriffen Kartoffeln und kugelig oder kugelrund. Zeile 17 stellt eine Unruhephase dar, die zwar von kurzer Dauer war, aber eine Transkription unmöglich machte. Daran anschließend folgt ein Appell von K4 (Z. 18), gerichtet an die anderen Kinder aus der Gruppe. Im weiteren Verlauf äußert $K 4$ mehrfach verneinende Rezeptionssignale (Z. 24, 26), die als Reaktion auf die wiederholte Aufforderung U1 zu verstehen sind.

U1 versucht wiederholt, die Kinder verbal zu instruieren und durch gestische Kommunikation zu motivieren (Z. 01, 21). Es gibt auch sich überlappende Gesprächsbeiträge. In Z. 15 ist die Partikel $h m$ als bestätigendes Element zur Aussage von $K 4 \mathrm{zu}$ verstehen. Die rasche Turnabfolge von $U 1$ in Z. 23 ist als Reaktion auf die verneinenden Signale von $K 4 \mathrm{zu}$ verstehen. Wobei $U 1$ hier eine Frage angliedert, die eher rhetorische Natur ist und mit einem Lachen am Ende der Formulierung schließt. Ebenso ist diese Abfolge als Kooperation zwischen U1 und $K 4$ zu verstehen, da $U 1$ unmittelbar auf die Äußerung K4s reagiert. Am Ende dieses Transkriptes holt U1 eine Kartoffel aus dem Beutel heraus. Dies erweist sich als Überraschungsmoment, das gleichzeitig als Motivationsträger fungiert, da gemeinsames Lachen verbindet.

Die Aussagen der Kinder sind meist sehr kurz, in Form von Dreiwortäußerungen, mit Ausnahme von K4. Die Äußerungen der Studenten sind ebenfalls knapp gehalten, mit vielen Kurzsätzen und einigen Fragesätzen. Auf den ersten Blick wirkt dieses Transkript beinahe monologisch, doch wie die genauere Betrachtung zeigt, sind hier ebenso sprachliches Wissen und Verständnis aufseiten der Kinder gegeben. Dies zeigt zum einen die Bejahung zu Beginn des Transkriptes als auch die Aufforderung, nicht alles zu verraten (Z. 18) und die wiederholte Feststellung der Kinder, dass Kartoffeln in dem Beutel seien (Z. 07, 13, 25, 28).

\subsection{Imitationen in der Rollenverteilung - Gesprächstranskript T2}

Das zweite Gesprächstranskript T2 ${ }^{29}$ stammt aus der Probenphase vom 07.10.2011. Es zeigt einen transkribierten Ausschnitt aus einer 16 Minuten und 52 Sekunden

29 Neben den beiden Erzieherinnen E1 und E2 sind insgesamt sechs von sieben Universitätsmitgliedern (U1-U7) und zudem mehrere Kinder anwesend. Von den insgesamt anwesenden 19 Kindern wurden die aus dem Transkript T1 bereits bekannten sieben Kinder in ihrer Nomenklatur belassen (K1-7) und weitere aktive Kinder fortlaufend mit K8-19 nummeriert, soweit die eindeutige Zuordnung möglich war. An einigen Stellen ist die Transkription nur schwer, manchmal nicht möglich gewesen, da es zeitweise zu einer immensen Lautstärke kam, die eine spezifizierte Transkription nicht zuließ. 
langen Gesamtszene. Dieser Ausschnitt zeigt den Einstieg in das Theaterstück. Nachdem die Studierenden das Theaterstück zuvor vorgespielt hatten, werden nun die Rollen unter den Kindern verteilt.

In dieser Szene beginnt $U 1$ mit einer als Aussage formulierten Frage (Z. 01), um daran anschließend eine dezidierte Frage zu formulieren. Da das Zählen auf Niederdeutsch bereits bekannt ist, beginnt $U 1$ langsam mit dem Zählen und nimmt unterstützend die in die Höhe gestreckte Hand zum Abzählen dazu. Die folgenden Turns sind die Äußerungen der Kinder, die laut und langsam die Zahlen bis fünf auf Niederdeutsch nachsprechen. Die Kinder produzieren in Form der direkten Imitation hier spielerisch niederdeutsche Äußerungen. Am Ende der Einheit greift U1 die Zahl, die das Ergebnis der gestellten Frage darstellt, wieder auf.

Transkriptausschnitt T2: 07.10.2011, Rollenverteilung

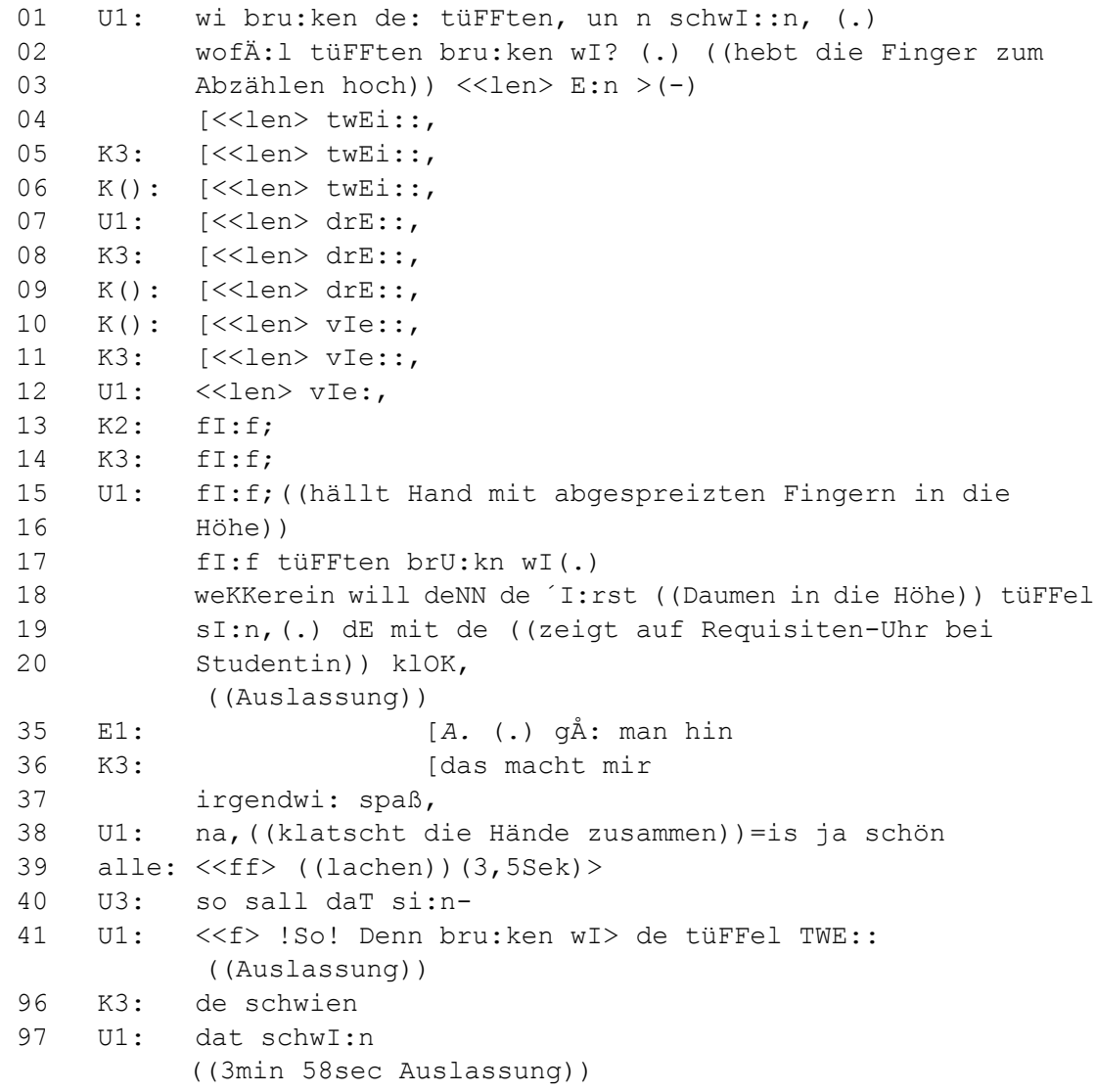




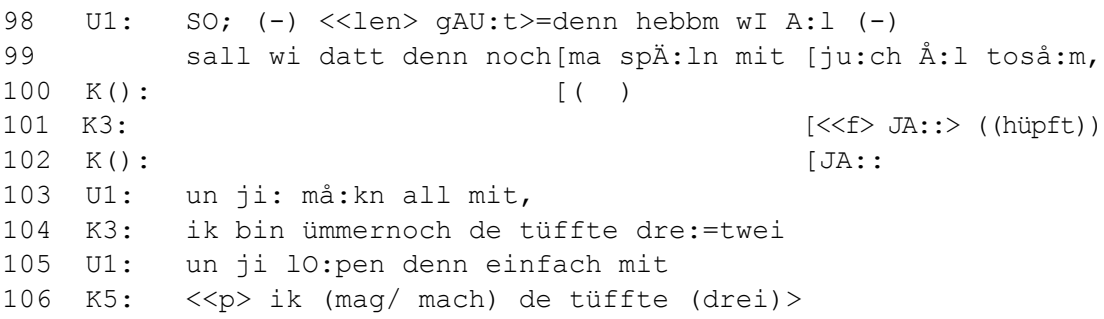

Im Wesentlichen ist das gesamte Transkript ein Aktion-Reaktion-Szenario. Diese Szene lebt allerdings nicht allein von den sprachlichen Äußerungen, sondern vermehrt von den Gesten (Z. 02-03 15-16, 19-20 u. a.) und prosodischen Kommunikationsanteilen. Bei den Kindern sticht wiederholt $K 3^{30}$ hervor. Dieses Kind hat nicht nur Spaß am Projekt (Z. 36-37, 101), es äußert sich auch, abgesehen von dem Zählen, als einziges Kind auf Niederdeutsch (Z. 96, 104). Seine Äußerungen gehen auch über die reine Imitation hinaus. Auf den ersten Blick scheinen diese Äußerungen nicht von großer Bedeutung zu sein, doch stellen sie eine Tatsache eindeutig dar: K3 verbindet die Anwesenheit der Studierenden mit Niederdeutsch. Das Kind versucht scheinbar, den Ansprüchen der Erwachsenen gerecht zu werden, indem es in der "Niederdeutsch-Zeit mit den Studierenden“ auch Niederdeutsch spricht. Das sprachliche Empfinden des Kindes ist soweit fortgeschritten, dass es seine eigenen sprachlichen Defizite selbstständig korrigiert (Z. 104), auch in der Zielsprache. Ähnliches lässt sich beim Kind K5 beobachten: Es bildet ganz frei, ebenso wie K3, einen vollständigen Satz auf Niederdeutsch (Z. 106).

\subsection{Kindliche Sprachproduktion in der Probenphase - Gesprächstranskript T3}

Bei diesem Transkript T3 handelt es sich um einen knappen Ausschnitt von insgesamt 60 Sekunden, der einen Vorbereitungsmoment zur Probe des Theaterstückes „Fief lütte Tüffeln“ darstellt.

Dieses Transkript ist in zwei Abschnitte geteilt. Im ersten Bereich (Z. 01-11) ist zu Beginn eine verbale Einheit zweier Studierender (Z. 01-02) zu sehen. In Z. 04 nimmt ein Kind das letzte Wort von $U 2$ auf, um einen thematisch passenden Gesprächszug anzuschließen, es kommt zu einer Überlappung mit K2. Das Kind

30 Aus dem Informationsgespräch vom 28.05.2013 mit der Leiterin der Einrichtung und der Erzieherin ging hervor, dass $K 3$ von Hause aus in einer stark plattdeutsch geprägten Sozialisation aufwächst, die durch die Eltern und Großeltern unterstützt und gefördert wird. 
K2 (fiel bereits im T2 positiv auf) äußert sich niederdeutsch (Z. 06), tut dies jedoch mit einer vorangestellten Ankündigung (Z. 05), den Text bereits gelernt zu haben. Auch hier treffen wir wieder auf eine Form des Gedächtnistrainings, dem initiierten und imitierten Lernen, in diesem Fall das Auswendiglernen. U2 zeigt sich erstaunt und erfreut über die Äußerung von $K 3$ und lobt das Kind. Das einwortige Lob von U3 wird am beendeten Turn von U2 aufgegriffen. U2 spricht das Kind zudem mit Namen an, um die Nähe zwischen den Lernenden und Lehrenden aufzubauen und Sicherheit zu geben.

Transkriptausschnitt T3: 14.10.2011, Probephase

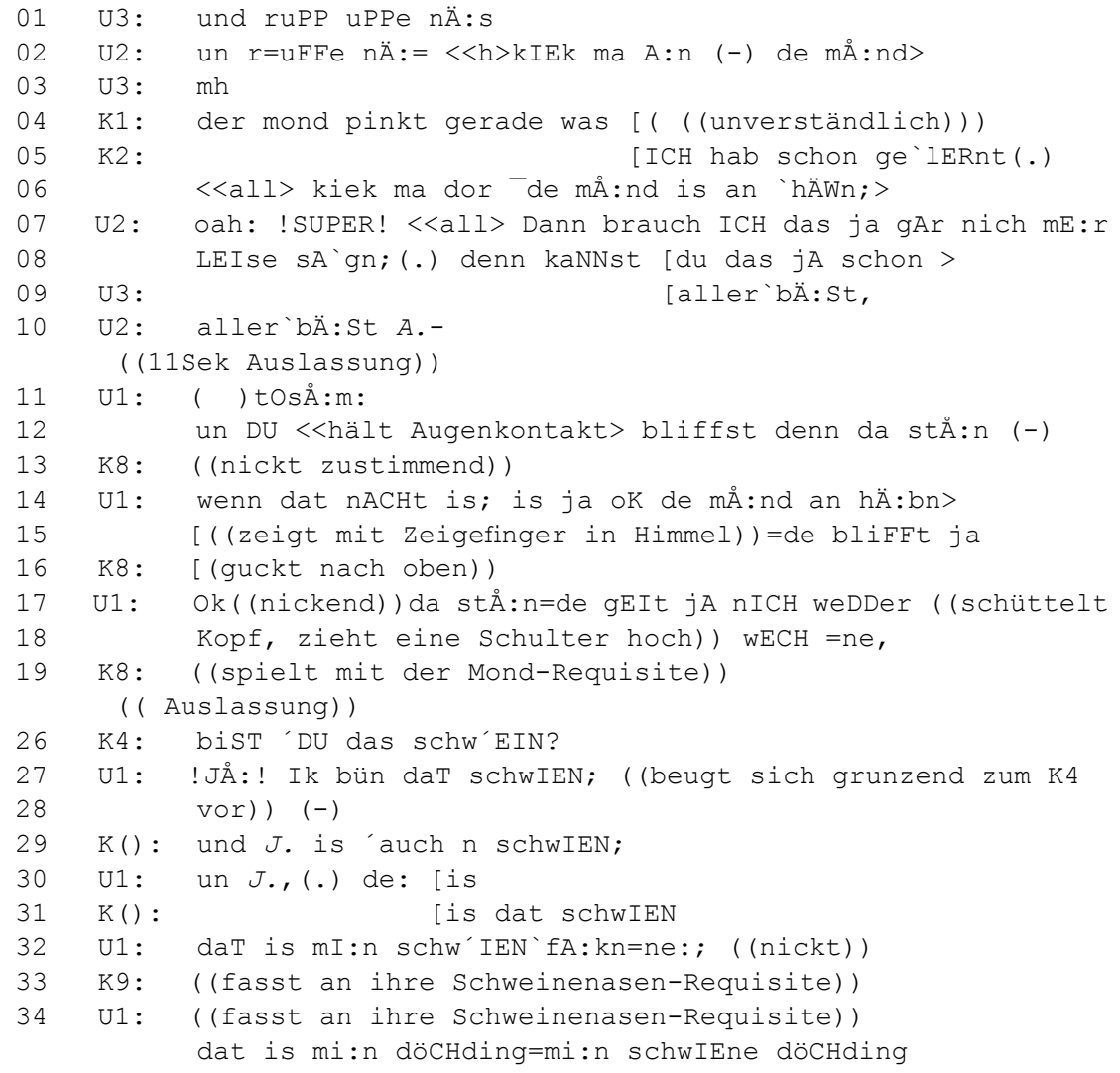

Der zweite Teil des Transkriptes (Z.11-35) zeigt U1 im Gespräch mit K8. Auch hier hat $U 1$ einen hohen Wortanteil und versucht, das Gesagte durch gezielte Mimik und Gestik zu unterstreichen oder hervorzuheben. $U 1$ versucht durch ein- 
sprachige (niederdeutsche) Erklärungsversuche, $K 8$ zu verdeutlichen, was seine Rolle zu tun hat, de Månd, und wie er sich auf der Bühne bewegen muss.

Daran anschließend beginnt eine Abfolge, in dem sich $K 4$ (sprachlich bereits im T1 hervorgetreten) wieder einbringt. $K 4$ stellt $U 1$ eine Frage nach dem Kostüm (Z. 26). U1 greift das Gesagte auf, bejaht die Frage und wandelt die Antwort in die Zielsprache (Z. 27) um. Dies ist der Anlass, für ein anderes Kind, auf dasjenige Kind zu verweisen, das im Theaterstück ebenfalls den Part des Schweins übernimmt (Z. 29). Und auch hier ist es zunächst ein Wort auf Niederdeutsch Schwien und in Z. 31 eine vollständige syntaktische Einheit is dat schwien.

\subsection{Auswertung und Zusammenfassung der Befund}

Aus den Beobachtungen der Gesprächstranskripte geht hervor, dass die Kinder des beobachteten Kindergartens (zunehmend) in der Lage sind, Niederdeutsch zu verstehen. Dies zeigt sich in allen drei Transkripten. Aufforderungen wie in T1, Z. 03 werden umgesetzt, gemeinsames Memorieren der Zahlen bis fief wie in T2, Z. 03-14 oder aber imitiertes Sprechen in Form von gelernten Textpassagen wie in T3, Z. 06 und auch freie Sprechakte in der Zielsprache Niederdeutsch sind zu finden (T2, Z. 96, 104, 106; T3, Z. 26).

Die Projektgruppe begegnet den Kindern in erster Linie sprachlich. Die Studierenden waren bemüht, die Kinder auch durch verschiedene Hilfsmittel zu motivieren, wie Plakate, Requisiten verschiedenster Art, Bewegungseinheiten und Verkleidungen sowie Accessoires. Die Kinder werden auf unterschiedlichen Wegen an die Sprache herangeführt. Der Zweitspracherwerb ist ein über Jahre andauernder Prozess. Nichtsdestoweniger ist bekannt, dass Kinder bereits früh an Sprachen herangeführt werden können und zudem einen passiven Wortschatz und ein implizites Wissen in Bezug auf die Zweitsprache erwerben können. Dies äußert sich durch das Verstehen und Reagieren auf Aufforderungen. Das Reagieren auf Aufforderungen oder Fragen wird hier als Verstehen der Zweitsprache aufgenommen. Dieses Phänomen konnte bei einigen Kindern beobachtet werden. So kommen die sieben Kinder im T1 der Aufforderung „kiek mol in“(Z. 03) nach, artikulieren aber zunächst wenig bis gar nichts und zeigen somit nur außersprachliche Reaktionen. Doch im weiteren Verlauf des Transkriptes zeigt sich, dass sie auch verbal, wenngleich noch nicht in der Zielsprache, auf Aufforderungen und Fragen reagieren.

Es ist ferner bekannt, dass Kommunikationsversuche in fremden Sprachen mit dem Äußern von Ein- und Zweiwortäußerungen oder kurzen Wortketten beginnen. Diese werden meist aus einstudierten Liedern oder Gedichten oder Reimen (auditiv) herausgefiltert und als abrufbare sprachliche Einheiten im mentalen Le- 
xikon gespeichert. ${ }^{31}$ Das Einüben einiger Basiselemente lässt sich über das gezielte Einprägen gut vermitteln. Dieser Methode bedienten sich auch die Studierenden beim Erarbeiten des Theaterstückes. Die Kinder mussten ihre Textpassagen auswendig lernen. Nicht nur das Lernen von Theatertexten, auch das Singen von Liedern und Aufsagen von Gedichten dient der gezielten Sprachförderung. In diesen Texten „werden oft typische sprachliche Muster präsentiert ${ }^{\text {“32 }}$, die sich ein Kind durch wiederholtes Aufsagen oder Singen besser aneignen kann. Ein Kind konnte den Text bereits sehr früh abrufen (T3, Z. 05-06). Dieser Prozess wird als imitatives Sprechen der L2 verstanden. Die ersten zweisprachigen Äußerungen lassen sich als Resultate auf vorfabrizierte, auswendig gelernte Einheiten und sprachliche Formeln, zurückführen. Das imitative Sprechen dient primär dem Einprägen und Einüben bestimmter Sprachformeln. Es ist ein wesentliches Merkmal des Angebotsansatzes. Das auswendig gelernte Material manifestiert sich dann als explizites Wissen zunächst in simplen Sätzen mit Komponenten aus der Erstund Zweitsprache.

Dieses Spracherwerbsphänomen ließ sich auch bei den Kindern aus der Kita beobachten. Sie zeigten sich in der Lage, ihren auswendig gelernten und somit sprachlich-statischen Input, auf andere sprachliche Ereignisse zu transformieren: das freie Sprechen. So lösten sich einige Zahlen aus dem statischen Zustand des bloßen Abzählens und wurden in anderen Kontexten wiederverwendet (T2, Z. 104, 106). Diese Form der Äußerung ist nicht mehr nur imitiertes Sprechen. Es kann als reaktives Sprechen ${ }^{33}$ verstanden werden. Reaktives Sprechen meint hier das ungelenkte Sprechen als Reaktion auf eine vorangegangene Sprechhandlung. Dies ist ein grundlegender verbaler Schritt in die Zweisprachigkeit durch die eigenständige Anwendung bereits erlernter Sprachelemente. Der erste Schritt wurde durch ein erlerntes Ordnungssystem vorbereitet. Es handelt sich hierbei um reproduzierte und nicht bewusst analysierte Sprachformeln.

In einem zweiten Schritt müssen die Kinder befähigt und bestärkt werden, ihre sprachlichen Ordnungssysteme und die entstandenen Sprachroutinen weiter zu entwickeln und neu zu verknüpfen und durch ein zunächst als Chaos erscheinendes Sprachfeld zu gehen, um letztlich zu zwei eigenständigen Sprachen zu gelangen. Dieses sprachliche Chaos ist durch Fehler wie Interferenzen, Code-

31 Vgl. Koll-Stobbe 1994, S. 61.

32 Knapp/Kucharz/Gasteiger-Klicpera 2010, S. 13.

33 Das reaktive Sprechen ist keine wissenschaftliche Größe, wird hier aber als bewusste Reaktion auf sprachliche Äußerungen verstanden. Diese Form der Sprechhandlung soll den aktiven Part der Kinder in den Fokus rücken. Sie reaktivieren ihr bereits vorhandenes Wissen und verbalisieren es. 
Switchings und Übergeneralisierungen geprägt. Diese Fehlerphänomene sind aber unerlässlich und notwendig in der Sprachentwicklung. Auf dem Weg zu einer weiteren Sprache sind Lob und Anerkennung, auch in der Chaosphase, von größter Bedeutung. Der größte gemeinsamer Nenner unter den individuellen Spracherwerbsprozessen lässt sich wie folgt formulieren: Es ist ein kreativer und offener Konstruktionsprozess. ${ }^{34}$ Jedes Kind entwickelt sich sprachlich weiter und wird dabei über Spiel- und Handlungssituationen, in denen die Sprache, das Sprechen und die Aktion nicht nur im Fokus stehen, sondern eine Einheit bilden, gefördert.

Einige abschließende Betrachtungen sollen auch die sprachlichen Äußerungen der Projektteilnehmer berücksichtigen. So ließ sich im T1 schon zu Beginn der Aufzeichnung feststellen, dass U1 viele inhaltliche Wiederholungen durch Synonyme ausdrückt, die als Aussagesätze formuliert waren. Auch waren kaum komplexe grammatische Äußerungen anzutreffen, sondern eher einfache und knappe Sätze. Häufig wurden auch Fragen formuliert oder durch die Partikel ne suggeriert. Diese vereinfachten sprachlichen Handlungsabläufe dienen zunächst der einfachen Verständigung. Sie wirken teilweise auch förderlich auf den Spracherwerb ein. ${ }^{35}$ Erwähnung sollten auch die hochfrequentierten para- und nonverbalen Kommunikationselemente finden, die weitestgehend als Stützfunktion zu den sprachlichen Einheiten verwendet wurden.

\section{Fazit und Handlungsempfehlungen}

In diesem Abschnitt sollen die folgenden drei Punkte detaillierter beleuchtet und bewertet werden: 1) die Greifswalder Kita und ihr Umgang mit der Sprachvermittlung, 2) die Rolle der Niederdeutschbeauftragten in Mecklenburg-Vorpommern und 3) die Rolle der weiterführenden Institutionen, allen voran der Grundschulen.

1) Dass die untersuchte Kindertagesstätte sich der Vermittlung der niederdeutschen Sprache verschrieben hat, ist zunächst anerkennenswert. Allerdings ist dieser Kindergarten keine dezidiert ausgewiesene zweisprachige Einrichtung. Doch könnten die Bemühungen in diese Richtung gehen. Zusätzlich muss an dieser Stelle darauf aufmerksam gemacht werden, dass die Vermittlung einer weiteren Sprache sich besonders dann als effektiv und gehaltvoll sowie langfristig erfolgreich erweisen kann, wenn sie bereits im Elementarbereich einen hohen Stellenwert einnimmt. Dazu gehört zum einen der konstante Umgang mit der

34 Vgl. Günther/Günther 2004, S. 129.

35 Vgl. Knapp/Kucharz/Gasteiger-Klicpera 2010, S. 13.

Birte Arendt, Andreas Bieberstedt and Klaas-Hinrich Ehlers - 978-3-631-71893-3 
Zielsprache innerhalb eines umfassenden zeitlichen Rahmens. Dass in der Greifswalder Kita nur der Montag Plattdeutsch-Tag ist, reicht vermutlich nicht aus, um den Kindern mittel- oder gar langfristig die Möglichkeit zum Erwerb des Niederdeutschen als weitere Sprache zu gewährleisten.

Allerdings wird durch den scheinbar unbewusst praktizierten Angebotsansatz sanft an die "neue und fremde Sprache“ herangeführt. Immerhin scheint der minimale Wochenumfang des Sprachangebots auszureichen, um an den Klang des Niederdeutschen heranzuführen, ebenso das Interesse und die Neugier an der Zielsprache hervorzurufen und somit positive Spracheinstellungen und Spracherwerbsvoraussetzungen zu bewirken. Auch wenn dem Angebotsansatz als Sprachvermittlungsmethode weniger Erfolgschancen eingeräumt werden, ${ }^{36}$ ist er in einem eigentlich einsprachig dominierten Kindergarten durchaus zu empfehlen, da er nachweislich die Kinder an eine Zielsprache heranführen kann. ${ }^{37}$ Kinder erwerben im Angebotsansatz durch wiederholte Lieder und Reime gezielte Sprachroutinen in Form von imitativem Sprechen. Dieses imitative Sprechen befähigt die Kinder zur Herausbildung eines Basisvokabulars zu ausgesuchten Themen und Wortfeldern und sensibilisiert sie zudem für den Klang und die grundlegenden strukturellen Gegebenheiten anderer Sprachen. Dieses Basiswissen kann den Kindern einfache Sprechhandlungen in der Zielsprache ermöglichen (Begrüßung, Verabschiedung, Danken, Phrasen). Sie können durchaus, das haben auch Untersuchungen von Nauwerck gezeigt, durch den Angebotsansatz die Zielsprache auf einfachem Sprachniveau erlernen. Jedoch unterscheidet sich das imitierte Sprechen in seiner Qualität deutlich vom reaktiven Sprechen als ungelenkter Handlungsaktion.

Deshalb soll an dieser Stelle noch einmal das Prinzip der Immersion hervorgehoben werden: Das immersive Verfahren baut keinerlei Leistungsdruck oder Zwang zur Sprache auf. Eine Person = eine Sprache, das ist die immersive Maxime, die zur kontinuierlichen und längerfristigen Sprachvermittlung völlig ausreicht. ${ }^{38}$ Vielleicht können die Erzieherinnen, unabhängig vom Institut für

36 Vgl. Nauwerck 2005, S. 88.

37 Vgl. Nauwerck 2005, S. 88-89 und 96.

38 Weder durch intensive Beobachtungen noch durch gezielte Fragen an die Erzieherinnen konnte eindeutig geklärt werden, ob in diesem Kindergarten der sukzessive Zweitspracherwerb mittels minimaler Immersion oder aber durch den Angebotsansatz vermittelt wird. Ich gehe davon aus, dass die Kita eine Mischform der Methoden praktiziert, die sich, wie ich bereits feststellte, nicht zwingend ausschließen müssen. 
Qualitätsentwicklung Mecklenburg-Vorpommern (IQMV) ${ }^{39}$ niederdeutsche Patenschaften eingehen: Möglicherweise erklären sich (Groß-)Eltern zu einer PlattPatenschaft bereit und können so einen Beitrag zur Plattdeutsch-Vermittlung leisten. Das wäre eine Erfahrung, die langfristig betrachtet bei allen Beteiligten Nutzen stiften könnte. Die Erzieherinnen bekämen (sprachliche) Unterstützung, die Kinder bekämen neue Eindrücke und lernten Niederdeutsch nicht nur als institutionell-gebundene Sprache kennen und die Paten könnten ihr Wissen an die Kinder weitergeben. Natürlich ist dies mit einem nicht unerheblichen zeitlichen und organisatorischen Aufwand verbunden, dennoch sollte es als Vorschlag zur weiteren Förderung und Unterstützung in Betracht gezogen werden. Doch der Rückhalt aus den Familien der betreuten Kinder allein reicht nicht aus. Die Kitas und ihre Fachkräfte brauchen auch die Unterstützung aus der direkten Umgebung und Subventionen von den landespolitischen und ministerialen Institutionen.

2) $\mathrm{Zu}$ Beginn des Jahres 2013 wurde die Stelle der Niederdeutschbeauftragten für Mecklenburg-Vorpommern neu besetzt. Bezüglich des Plattdeutschen ist die Niederdeutschbeauftragte die nächste Ansprechpartnerin für die Kitas. Es gab bis zum jetzigen Zeitpunkt bereits mehrere Tagungen und Treffen, um einen Austausch an Erfahrungen zu organisieren und Anregungen zu sammeln und zu strukturieren. Im Zuge des Projekts des IQMV „Frühkindliche Bildung im Kindergarten und der Grundschule", wäre es ratsam, und auch von den Erzieherinnen der Greifswalder Kita ausdrücklich gewünscht, zeitnah wieder ein solches Treffen, besser noch eine Art Verband zu initiieren. Es fehlt, das haben auch die Erzieherinnen bestätigt, nicht nur am gegenseitigen Austausch, um von der Arbeit und den Ideen anderer lernen und profitieren zu können; ebenso fehlt insbesondere eine Vernetzung zwischen den Pädagogen. ${ }^{40}$ Ferner sollten in regelmäßigen Abständen Fort- und Sprachbildungsseminare für Pädagogen angeboten werden, damit eine sprachlich qualitativ hochwertige Vermittlung gewährleistet werden kann. Die 20 Kitas in Mecklenburg-Vorpommern, die an dem Projekt

39 Das Institut für Qualitätsentwicklung ist für die Ausbildung der Lehrkräfte in Mecklenburg-Vorpommern, deren Fort- und Weiterbildung sowie für die Weiterentwicklung des Unterrichts an den Bildungseinrichtungen das Landes zuständig, vgl. [Online-Ressource: http://www.regierung-mv.de/Landesregierung/bm/ Ministerium/Beh\%C3\%B6rden-\&-Institutionen/Institut-f\%C3\%BCr-Qualit\% C3\%A4tsentwicklung-M\%E2\%80\%93V/ (Stand: 12.12.2016)].

40 Vgl. die gleichlautenden Forderungen von Kita-Erzieherinnen, die im Beitrag von Mittelstädt in diesem Band angeführt werden. Vernetzung und Austauschmöglichkeiten scheinen somit ein generelles Desiderat in der vorschulischen Niederdeutschvermittlung in Mecklenburg-Vorpommern darzustellen. 
teilnehmen, stehen nur bedingt in Kontakt mit anderen Plattdeutsch fördernden Kitas des Landes. Hier sollten mehr Fördermaßnahmen und Unterstützung ins Leben gerufen werden. Vielleicht wäre es sogar möglich, in gewissen Abständen Treffen zu organisieren, an dem ausgewählte Plattdeutsch-Kitas norddeutschlandweit teilnehmen könnten. So wäre es möglich, ein Grenzen überschreitendes „Plattdeutsch-in-der-Kita-Netzwerk“ entstehen zu lassen. In Zeiten der Social Media wäre sogar an eine digitale Materialsammlung zu bestimmten Themen vorstellbar.

3) Doch viel wichtiger als eine mögliche community-Präsenz im Internet ist die Zusammenarbeit mit den Primarschulen. Denn nicht zuletzt ist die Kontinuität beim Spracherwerb wichtig. Daraus leitet sich die Empfehlung ab, dass der Zweitspracherwerb in der Grundschule nahtlos weitergeführt wird. Nur so lassen sich kontinuierliche Sprachbiografien aufbauen. Um dies gewährleisten zu können, reicht es allerdings nicht aus, Niederdeutsch als Wahlfach am Nachmittag anzubieten. Niederdeutsch muss aus der Rolle eines marginalen Unterrichtsfaches bzw. -stoffes befreit werden. Zu diesem Zweck ist die Einbindung des Niederdeutschen in die obligatorischen Fächer eine Grundvoraussetzung. Die Vermittlung der Sprache mittels Immersion kann und sollte gleichzeitig durchaus auch in der Grundschule weiter vorangetrieben werden. Es ist, wie bereits mehrfach hervorgehoben, der kindgerechte Sprachaneignungsweg.

Durch die strukturelle Nähe des Niederdeutschen einerseits zum Hochdeutschen, andererseits zu den skandinavischen Sprachen, dem Niederländischen und dem Englischen können Kinder doppelt vom Niederdeutschen profitieren. Das Niederdeutsche vermag in der Schule quasi als „Gelenk“ zwischen dem Hochdeutschen und anderen Sprachen und als eine Art erste Fremdsprache fungieren. In dieser Funktion als Gelenksprache kann das Niederdeutsche als Brücke zwischen zwei Sprachen dienen.

Abschließend möchte ich noch auf zwei Dinge aufmerksam machen. Zum einen ist eine frühe Mehrsprachigkeit kein Patentrezept für die sprachliche Entwicklung von Kindern. Sie kann aber durchaus die naturgegebenen und individuellen sprachlichen Fähigkeiten des Kindes optimal nutzen und weiterentwickeln. Was den Erwerb einer Regionalsprache wie des Niederdeutschen so interessant macht, ist die Tatsache, dass die kognitiven Vorteile, die die frühe Zwei- oder Mehrsprachigkeit bringt, erhalten bleiben. Selbst wenn das Kind später den Kontakt zu dieser Sprache verlieren sollte, wird der Regionalspracherwerb nicht unwesentlich zu einem potenziell daran anschließenden erleichterten Erwerb weiterer Sprachen beigetragen haben. Zudem können die Regionalsprachen Deutschlands durch die frühe Mehrsprachigkeit lebendig gehalten werden. Mit der „Charta der Regional- 
oder Minderheitensprachen" begann, zumindest in politischer Hinsicht, eine neue Ära für das Niederdeutsche. ${ }^{41} \mathrm{Ob}$ die Forderung nach vermehrtem Einsatz des Niederdeutschen in den Bildungsinstitutionen dafür sorgen kann, dass die Kinder und (zukünftigen) Jugendlichen den Sprachschwund aufhalten können, wird sich in den nächsten Jahren zeigen.

„Es geht im vielsprachigen Europa nicht darum, Englisch zu lernen. Es geht darum, das Sprachenlernen zu lernen." ${ }^{42}$ Diesem Ausspruch ist zuzustimmen, jedoch würde eine Ergänzung um eine soziale Dimension den Satz zusätzlich abrunden. Wie die Beispiele der vorliegenden Arbeit gezeigt haben, geht es nicht nur um das bloße Sprachenlernen, sondern, und da kann das Einüben von Theaterstücken als typisches Element für die Niederdeutsch-Vermittlung ansetzen, um die Verknüpfung von Generationen, um das Miteinander, um das Gemeinsam-inAktion-Treten: vom imitativem Sprechen hin zum freien Sprechen.

\section{Literatur}

Andresen, Helga: Interaktion, Sprache und Spiel. Zur Funktion des Rollenspiels für die Sprachentwicklung im Vorschulalter. Tübingen 2002.

Arendt, Birte/Gąszczyk, Alicja: Second language immersion programmes at kindergarten - as illustrated by Polish and Low German. In: Minsterium für Bildung und Kultur des Saarlandes (Hrsg.): Multilingualism creates opportunities. Saarbrücken 2015, S. 54-68.

Bieberstedt, Andreas: „In meinem Elternhaus wurde nur Platt gesprochen.“ Sprachbiographische Konzeptionen Hamburger Dialektsprecher zum frühen Spracherwerb. In: Langhanke, Robert (Hrsg.): Sprache, Literatur, Raum. Festgabe für Willy Diercks. Bielefeld 2015, S. 205-237.

Bildungskonzeption für 0- bis 10-jährige Kinder in Mecklenburg-Vorpommern. Förderung von Kindern unter 3 Jahren. [Online-Ressource: http://www. bildung-mv.de/export/sites/bildungsserver/downloads/Bildungskonzeptionfuer-0-bis-10-jaehrige-Kinder-in-Mecklenburg-Vorpommern.pdf (Stand: 01.12.2016)]

Bundesrat für Niederdeutsch (Hrsg): Zehn Jahre Europäische Charta der Regional- oder Minderheitensprachen. Zwischenbericht zur Sprachpolitik für das Niederdeutsche. Leer 2008.

41 Vgl. Bundesrat für Niederdeutsch 2008.

42 Nath 2009, S. 4. Ich habe an dieser Stelle die Hervorhebung Naths (im Original Majuskelschreibweise) bewusst übernommen, weil die Aussage ein eindeutiger Appell an die Zuhörer ihres Vortrags war und nicht nur als ein Hinweis zu verstehen ist. 
Bundesrat für Niederdeutsch (Hrsg.): Plattdeutsch, die Region und die Welt. Wege in eine moderne Mehrsprachigkeit. Leer 2009.

Günther, Britta/ Günther, Herbert: Erstsprache und Zweitsprache. Einführung aus pädagogischer Sicht. Weinheim/Basel 2004.

Häussermann, Ulrich/Piepho, Hans-Eberhard: Aufgabenhandbuch Deutsch als Fremdsprache. Abriß einer Aufgaben- und Übungstypologie. München 1996.

Houwer, Annik de: Bilingual First Language Acquisition. Bristol 2009.

Institut für Niederdeutsche Sprache. 2016. [Online-Ressource: http://www.insbremen.de/de/sprache/umfrage.html (Stand: 1.12.2016)]

Kersten, Kristin/Fischer, Uta/Burmeister, Petra/Lommel, Annette: Immersion in der Grundschule. Ein Leitfaden. 2009. [Online-Ressource: http://www.fmksonline.de/shop.html (Stand: 12.12.2016)]

Klann-Delius, Gisela: Einige praktische Probleme von Langzeituntersuchungen und ihre methodologischen Implikationen. In: Ehlich, Konrad (Hrsg.): Kindliche Sprachentwicklung. Konzepte und Empirie. Opladen 1996, S. 17-29.

Klein, Wolfgang: Prozesse des Zweitspracherwerbs. In: Grimm, Hannelore (Hrsg.): Sprachentwicklung. Enzyklopädie der Psychologie. 3. Bd. Göttingen [u. a.] 2000, S. 537-570.

Knapp, Werner/Kucharz, Diemut/Gasteiger-Klicpera, Barbara: Sprache fördern im Kindergarten. Umsetzung wissenschaftlicher Erkenntnisse in die Praxis. Weinheim/Basel 2010.

Koll-Stobbe, Amei: Verstehen von Bedeutungen: Situative Wortbildungen und mentales Lexikon. In: Börner, Wolfgang/Vogel, Klaus (Hrsg.): Kognitive Linguistik und Fremdsprachenerwerb. Das mentale Lexikon. Tübingen 1994, S. 51-68.

Le Pape-Racine, Christine: Erfolgreich über Immersion. In: Euro-Schulen. Gemeinnützige Gesellschaft für berufliche Bildung und Beschäftigung Sachsen $\mathrm{mbH}$ (Hrsg.): Fachtagung „Erfolgreich über Immersion zur Mehrsprachigkeit in der Europaregion Neisse-Nisa-Nysa“ 28./29.09.2007. Görlitz 2007, S. 17-35. [Online-Ressource: http://www.pontes-pontes.eu/fileadmin/userfiles/files_de/ Dokumentation_der_Fachtagung_2007_pdf.pdf (Stand: 12.12.2016)]

Ministerium für Bildung, Wissenschaft und Kultur des Landes MecklenburgVorpommern: Gesetz zur Förderung von Kindern in Kindertageseinrichtungen und in Kindertagespflege (Kindertagesförderungsgesetz - KiföG $\mathrm{M}-\mathrm{V}$ ): 01.04.2004, letzte berücksichtigte Änderung: $\$ \$ 18$ und 24 geändert durch Artikel 3 des Gesetzes* vom 3. November 2014 (GVOBl. M-V S. 594). 2014. [Online-Ressource: http://www.landesrecht-mv.de/jportal/portal/ page/bsmvprod.psmlshowdoccase $=1 \&$ doc.id=jlr-KTEinrGMVrahmen\&doc. part $=X \&$ doc.origin $=b s \& s t=\operatorname{lr}($ Stand: 01.12 .2016$)]$ 
Müller, Natascha/Kupisch, Tanja/Schmitz, Katrin/Cantone, Katja (Hrsg.): Einführung in die Mehrsprachigkeitsforschung. 2. Aufl. Tübingen 2007.

Nath, Cornelia: Frühe Mehrsprachigkeit im Niederdeutschen. 2009. [OnlineRessource: http://www.landtag.ltsh.de/export/sites/landtagsh/parlament/ niederdeutsch/data/Vortrag_Cornelia-Nath.pdf (Stand: 12.12.2016)].

Nauwerck, Patricia: Zweisprachigkeit im Kindergarten: Konzepte und Bedingungen für das Gelingen. Freiburg im Breisgau 2005.

Rachel, Christine: Das Modell der Immersion in der sorbischsprachigen Lausitz. Erzählwerkstatt als Chance der integrativen Sprachförderung. 2010. [OnlineRessource: http://witaj-sprachzentrum.de/files/rachelic_bachelorowe_dzelo. pdf (Stand: 12.12.2016)]

Reinhold, Yvette: Zweitspracherwerb im Vorschulalter. Darstellung des Kooperationsprojektes Grundschule-Kindertagesstätte Großrückerswalde. 2004. [Online-Ressource: http://www.kita-bildungsserver.de/downloads/downloadstarten/? did=140 (Stand: 12.12.2016)]

Riehl, Claudia-Maria: Mehrsprachigkeit ist der Normalfall. In: Migration. Informationsdienst der Landesarbeitsgemeinschaft der kommunalen Migrantenvertretung NRW, Nr. 25 (2006). [Online-Ressource: http://docplayer. org/22710124-Infodienst-der-landesarbeitsgemeinschaft-der-kommunalenmigrantenvertretungen-nrw-mehrsprachigkeit-ein-reichtum-fuer-alle.html (Stand: 12.12.2016)]

Schulz, Jana: Die Rolle der Sprecher bei der Weitergabe ihrer Sprache. In: Bundesrat für Niederdeutsch (Hrsg.): Plattdeutsch, die Region und die Welt. Wege in eine moderne Mehrsprachigkeit. Leer 2009, S. 48-54.

Selting, Margret/Auer, Peter/Barden, Birgit/Bergmann, Jörg/Couper-Kuhlen, Elizabeth/Günthner, Susanne/Meier, Christoph/Quasthoff, Uta/Schlobinski, Peter/Uhmann, Susanne: Gesprächsanalytisches Transkriptionssystem (GAT). In: LB - Linguistische Berichte 173 (1998), S. 91-122. [Online-Ressource: http://www.uni-potsdam.de/u/slavistik/vc/rlmprcht/textling/comment/gat. pdf (Stand: 12.12.2016)]

Stiftung Mecklenburg: Lürlürlütt. Handreichung zum Erlernen der niederdeutschen Sprache in der frühkindlichen Bildung. Redaktion Susanne Bliemel. Rostock 2012.

Tracy, Rosemarie/Gawlitzek-Maiwald, Ira: Bilingualismus in der frühen Kindheit. In: Grimm, Hannelore (Hrsg.) Sprachentwicklung. Göttingen 2000, S. 495-529.

Tracy, Rosemarie: Wie Kinder Sprachen lernen: Und wie wir sie dabei unterstützen können. 2. Aufl. Tübingen 2008.

Weskamp, Ralf: Fachdidaktik: Grundlagen Konzepte. Anglistik-Amerikanistik. Berlin 2001. 
Wode, Henning: Immersion und ihr Bedingungsgefüge. In: Ministerium für Bildung, Familie, Frauen und Kultur des Saarlandes (Hrsg.): Mehrsprachiges Aufwachsen in der frühen Kindheit. Fakten, Voraussetzungen, Möglichkeiten für einen gelenkten Spracherwerb. Weimar 2008.

Wode, Henning: Lernen in der Fremdsprache: Grundzüge von Immersion und bilingualem Unterricht. Ismaning 1995.

\section{Anhang}

Zusammenfassung grundlegender GAT-Konventionen der Transkripte (nach Selting u. a. 1998):

Sequenzielle Struktur/Verlaufsstruktur

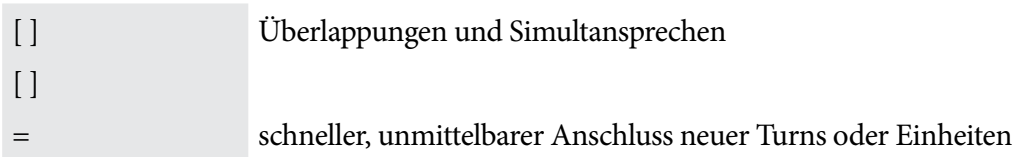

Pausen

(.) Mikropause

(-), (--), (--) kurze, mittlere, längere Pausen von ca. 0.25-0.75 Sek.; bis ca. 1 Sek. geschätzte Pause, bei mehr als ca. 1 Sek. Dauer gemessene Pause (Angabe mit zwei Stellen hinter dem Punkt)

Sonstige segmentale Konventionen

$\begin{array}{ll}\text { und=äh } & \text { Verschleifungen innerhalb von Einheiten } \\ : \text { : :: ::: } & \text { Dehnung, Längung, je nach Dauer } \\ \text { äh, öh, etc. } & \text { Verzögerungssignale, sog. ,gefüllte Pausen“ }\end{array}$

Rezeptionssignale

\begin{tabular}{l|l}
$\mathrm{hm}, \mathrm{ja}$, nein, nee & einsilbige Signale \\
$\mathrm{hm}=\mathrm{hm}, \mathrm{ja}=\mathrm{a}$, & zweisilbige Signale \\
nei=ein, nee=e & \\
'hm'hm & mit Glottalverschlüssen, meistens verneinend
\end{tabular}

Akzentuierung

$\begin{array}{ll}\text { akZENT } & \text { Primär- bzw. Hauptakzent } \\ \text { ak!ZENT! } & \text { extra starker Akzent }\end{array}$


(solche) vermuteter Wortlaut

al(s)o vermuteter Laut oder Silbe

(solche/welche) mögliche Alternativen

((..)) Auslassung im Transkript 
Anne Fink

\title{
Die Umsetzung der Verwaltungsvorschrift "Niederdeutsch in der Schule“ - eine exemplarische Studie zum Niederdeutschangebot in den Schulen Greifswalds
}

\begin{abstract}
In 1999 the federal state Mecklenburg-Vorpommern obliged to implement Low German into the school's range of courses by signing on the European Charter for Regional or Minority languages. Since 2004 the administrative regulation "Low German at School" is effective in Mecklenburg-Vorpommern, which prescribes Low German as continuous teaching principle for all schools. Since even many years after the definition of these language-political requirements Low German education at schools is not compulsorily established in this federal state, this paper presents data from an exemplary study in Greifswald. By means of questionnaire surveys, narrative interviews with teachers and work shadowing, the author reviews the concrete implementation of the public requirements for the Low German school lessons.

Her case analysis comes to the conclusion that neither the extent of the school's Low German education opportunity nor its realization in school lessons fully complies with the public requirements.
\end{abstract}

\section{Einführung}

Seit dem 01.01.1999 ist in der Bundesrepublik Deutschland die Europäische Charta der Regional- oder Minderheitensprachen (nachfolgend Sprachencharta) in Kraft. ${ }^{1}$ Neben den Minderheitensprachen Friesisch, Sorbisch, Dänisch und Romanes ist seither auch das Niederdeutsche als schützenswerte Regionalsprache anerkannt. Mit der Ratifizierung der Sprachencharta haben sich die Bundesländer Brandenburg, Nordrhein-Westfalen, Sachsen-Anhalt, Freie Hansestadt Bremen, Freie und Hansestadt Hamburg, Mecklenburg-Vorpommern, Niedersachsen und Schleswig-Holstein zu einem aktiven und entschlossenen Sprachenschutz des Niederdeutschen bekannt. In der Folge entwickelte man spezielle rechtlich verbindliche Maßnahmen für die einzelnen unterzeichnenden Bundesländer. So wurde in Mecklenburg-Vorpommern der Niederdeutschunterricht im Schulge-

1 Vgl. Sprachencharta 1999.

Birte Arendt, Andreas Bieberstedt and Klaas-Hinrich Ehlers - 978-3-631-71893-3 
setz des Landes verankert und die Verwaltungsvorschrift „Niederdeutsch in der Schule“ vom 9. März 2004 des Ministeriums für Bildung, Wissenschaft und Kultur Mecklenburg-Vorpommern (nachfolgend Verwaltungsvorschrift) erlassen. ${ }^{2}$ Mit diesen und anderen Maßnahmen greift die Politik in den Sprachgebrauch der Bevölkerung positiv reglementierend ein und wird zu einem wichtigen sprachenpolitischen Akteur.

Allerdings ist auch 2016, 17 Jahre nach Inkrafttreten der Sprachencharta und der darauf aufbauenden sprachpolitischen Fördermaßnahmen, ein zunehmender Gebrauch des Niederdeutschen im norddeutschen Sprachgebiet nicht erkennbar. Das Gegenteil ist der Fall: Einer repräsentativen Umfrage des Instituts für niederdeutsche Sprache im Jahre 2007 zufolge ist die Zahl der kompetenten Niederdeutschsprecher_innen seit der letzten repräsentativen Umfrage aus dem Jahr 1984 um mehr als die Hälfte zurückgegangen. ${ }^{3}$ Dies kann nur bedeuten, dass kaum noch Spracherwerb stattfindet. Dennoch zeigt die Umfrage des Instituts für niederdeutsche Sprache, dass das Niederdeutsche weiterhin eine vitale Sprache ist. ${ }^{4}$ Besetzt mit positiven Konnotationen wie „heimatlich“, „humorvoll“ und „gemütlich“ erfährt das Niederdeutsche in der Bevölkerung eine neue Form der Wertschätzung. ${ }^{5}$ Zudem befürworten 73 \% der Befragten im niederdeutschen Sprachgebiet, dass das Niederdeutsche stärker gefördert werden solle, 81 \% sprechen sich ausdrücklich für mehr Lehrangebote in den Schulen aus. ${ }^{6}$

Der folgende Beitrag nimmt das Missverhältnis von offiziellen sprachenpolitischen Fördermaßnahmen und den offensichtlich ausbleibenden bzw. geringen Wirkungen für den Spracherhalt des Niederdeutschen zum Ausgangspunkt einer empirischen Untersuchung. Dabei soll der Bereich des Bildungswesens im Zentrum stehen, denn hier ist ein direktes Eingreifen der Politik in den Sprachenschutz gewährleistet, da Einrichtungen wie Schulen, Hochschulen und Uni-

2 Verwaltungsvorschrift 2004. Exemplarisch für die Sprachenpolitik des Niederdeutschen wird an dieser Stelle nur auf die Sprachencharta sowie die Verwaltungsvorschrift eingegangen. Genauere Betrachtungen sprachenpolitischer Grundlagen wie z. B. der "Schweriner Thesen“ und der „Rahmenpläne“ sowie des Begriffsverständnisses von "Sprachenpolitik“ in Piontkowski [Fink] 2012 und Arendt 2010, S. 242-269. Es ist darauf hinzuweisen, dass das Niederdeutsche schon lange vor der Sprachencharta einen Baustein an den Schulen in Mecklenburg-Vorpommern darstellte, vgl. dazu Osnowski 1998.

3 Vgl. Möller 2008, S. 12.

4 Vgl. Möller 2008, S. 83.

5 Vgl. Möller 2008, S. 24.

6 Vgl. Möller 2008, S. 55.

Birte Arendt, Andreas Bieberstedt and Klaas-Hinrich Ehlers - 978-3-631-71893-3 
versitäten staatliche Institutionen sind. Am Fallbeispiel der Stadt Greifswald soll untersucht werden, in welchem Umfang und in welchen Realisierungsformen die amtlichen Vorschriften für das Niederdeutschangebot an den Schulen tatsächlich umgesetzt werden. Nach einer knappen Skizze der amtlichen Vorgaben für das Niederdeutschangebot an den Schulen Mecklenburg-Vorpommerns (Abschnitt 2) und einer Darlegung meiner Untersuchungsmethoden (Abschnitt 3.1) wird das schulische Angebot in der Stadt Greifswald zunächst in einem umfassenden Überblick betrachtet (Abschnitt 3.2). Es schließt sich die Analyse der konkreten Realisierung der Niederdeutschvermittlung im Schulalltag einer beispielhaft ausgewählten Schule an (Abschnitt 3.3). Die Erhebung meiner quantitativen und qualitativen Datengrundlage erfolgte im Jahr 2011, meine Fallstudie kann also eine detaillierte Momentaufnahme der schulischen Umsetzung amtlicher Fördermaßnahmen zwölf Jahre nach der Unterzeichnung der Sprachencharta geben.

\section{Sprachenpolitik des Niederdeutschen}

Mit der Unterzeichnung der entsprechenden Artikel der Sprachencharta zum Bildungsbereich hat sich das Land Mecklenburg-Vorpommern selbst verpflichtet, „unbeschadet des Unterrichts der Amtssprache(n) des Staates“ sowohl „innerhalb des Grundschulunterrichts“ als auch „innerhalb des Unterrichts im Sekundarbereich den Unterricht der betreffenden Regional- oder Minderheitensprache als integrierenden Teil des Lehrplans vorzusehen“"7 Weitere konkretisierende Vorgaben macht die Sprachencharta allerdings nicht. Die Art der Umsetzung der unterzeichneten Artikel zur Förderung der Regionalsprache Niederdeutsch obliegt dem Bundesland Mecklenburg-Vorpommern. Aus den recht allgemeinen Formulierungen des Maßnahmenkatalogs mussten daher weitere verbindliche Vorgaben zur Realisierung im Schulunterricht des Landes folgen.

Insbesondere ist auslegungs- und regelungsbedürftig, wie eine schulische Berücksichtigung des Niederdeutschen „als integrierender Teil des Lehrplans“ realisiert werden soll. Der 2011 erschienene Handkommentar zur Sprachencharta bietet eine eindeutige Erläuterung der Wortgruppe „integrierender Teil des Lehrplans". Langenfeld betont in ihrem Kommentar, dass das Niederdeutsche ein „integriertes Angebot ${ }^{\text {“8 }}$ des Lehrplans sein müsse. Mit der Unterzeichnung der zitierten Artikel der Sprachencharta hat sich das Land Mecklenburg-Vorpommern

7 Sprachencharta 1999, Teil III, Art. 8 Abs. 1 b (iii), c (iii). Vgl. Mittelstädt in diesem Band.

8 Langenfeld 2011, S. 206.

Birte Arendt, Andreas Bieberstedt and Klaas-Hinrich Ehlers - 978-3-631-71893-3 
demnach zu einer flächendeckenden Einführung des Niederdeutschen verpflichtet. Was dies speziell bedeutet, gibt das folgende Zitat aus dem Handkommentar wieder:

„Die geschützte Sprache ist in diesem Fall nicht Unterrichtsmedium, sondern Unterrichtsfach. Nicht ausreichend ist insoweit ein freiwilliges zusätzliches Angebot (etwa im Rahmen einer Arbeitsgemeinschaft), sondern es muss sich um ein in das reguläre Curriculum integriertes Angebot als Zweitsprache handeln, das freilich nicht als Pflichtfach ausgestaltet sein muss, wohl aber als reguläres Schulfach versetzungsrelevant ist."

Es ist demnach nicht ausreichend, das Niederdeutschangebot als einen integrierten Teil innerhalb eines einzelnen Unterrichtsfaches, also etwa im Deutschunterricht, zu realisieren. Es muss auch erklärtes Ziel der Schulen sein, bei der Vermittlung des Niederdeutschen ein sehr gutes Sprachniveau zu erreichen. Die Konsequenz daraus kann nach Langenfeld nur bedeuten, das Niederdeutsche als ein Fach mit mehreren Wochenstunden zu etablieren. ${ }^{10}$

Nach der Ratifizierung der Sprachencharta bedurfte es weiterer Maßnahmen und verbindlicher Regelungen für den Schutz und die Förderung des Niederdeutschen. Ein wichtiger Schritt war hier der Erlass der Verwaltungsvorschrift des Ministeriums für Bildung, Wissenschaft und Kultur Mecklenburg-Vorpommern „Niederdeutsch in der Schule“ vom 9. März 2004. ${ }^{11}$ Die Verwaltungsvorschrift ist in sieben Punkte gegliedert, von denen hier einige Punkte angesprochen werden sollen. Der erste Punkt gibt Ziele und Aufgaben für die Vermittlung des Niederdeutschen in den allgemein bildenden Schulen vor. Hier wird das Niederdeutsche als „wesentlicher Teil des Kulturgutes“12 des Landes verankert und der Rückgang der Sprecherzahlen bedauert. Das Konzept der regionalen Identität in Verbindung mit der Warnung vor dem Verlust eines Kulturguts bildet demnach eine Grundlage für den Sprachenschutz. ${ }^{13}$

Zudem wird in der Verwaltungsvorschrift die besondere Rolle der Schule als vermittelnde Instanz betont. So heißt es im ersten Absatz der Verwaltungsvor-

9 Langenfeld 2011, S. 206.

10 Vgl. Langenfeld 2011, S. 207.

11 Verwaltungsvorschrift 2004. Diese Verwaltungsvorschrift stützt sich auf $\$ 2$ Abs. 3 des Schulgesetzes für Mecklenburg-Vorpommern, hier in der Fassung von 2003. Der genannte Paragraph ist bei der neuen Fassung von 2006 unverändert geblieben, vgl. Schulgesetz 2006.

12 Verwaltungsvorschrift 2004, o. S., 1. Abs.

13 Zur Problematik dieses Kulturgut-Konzepts innerhalb der Sprachenpolitik vgl. Arendt 2012. 
schrift: „Daraus erwächst eine hohe Verantwortung für die Pflege des Niederdeutschen in der Schule. "14 Aus dieser Verantwortung ergibt sich als Aufgabe der Schulen:

„Verbindliche Aufgabe der Schule (unabhängig von der Schulart und der Jahrgangsstufe) ist es, Kenntnisse über niederdeutsche Literatur, Kunst und Kultur zu vermitteln. Daneben soll die Schule zum Gebrauch der niederdeutschen Sprache ermuntern und insbesondere die Fähigkeit fördern, Niederdeutsch zu sprechen. [...] Niederdeutsch kann und soll nicht als eigenes Fach mit einem Stundenanteil ausgewiesen werden, muss aber an allen Schulen ein durchgängiges Unterrichtsprinzip sein." ${ }^{\text {"15 }}$

Durch die Benennung verbindlicher Aufgaben und der Zielstellung, zum Gebrauch der Sprache anzuregen, verankert Mecklenburg-Vorpommern die Förderung des Niederdeutschen im schulischen Bereich. Allerdings „kann und soll“16 das Niederdeutsche kein eigenes Unterrichtsfach bilden, vielmehr wird hier das Konzept eines „durchgängigen Unterrichtsprinzips ${ }^{\text {“17 }}$ bevorzugt.

Der zweite Punkt der Verwaltungsvorschrift konkretisiert dann, was unter dem durchgängigen Unterrichtsprinzip verstanden werden soll. In beispielhafter Aufzählung wird beschrieben, wie das Niederdeutsche in den Unterricht eingebracht werden kann. Demnach soll im Unterricht u. a. die „Bedeutsamkeit und Spezifik“ des Niederdeutschen mit einem Bezug zum Hochdeutschen herausgestellt werden, die „Bindung an die Region“ mit der Identitätsbildung und Persönlichkeitsentwicklung verknüpft werden sowie das Sprechen und Verstehen gefördert und verbessert werden. Aber auch „Methoden und Verfahren des Spracherwerbs“ sollen im Unterricht vermittelt werden. ${ }^{18}$ Zur Realisierung dieser Empfehlungen können alle Unterrichtsfächer beitragen. Im Wahlpflichtbereich sowie im Projektunterricht kann dies noch intensiviert werden.

Schwerpunkte hierbei werden durch die Rahmenpläne gesetzt. Im Grundschulbereich soll das Niederdeutsche im Sachkunde-, Deutsch- und Musikunterricht behandelt werden. In der Orientierungsstufe soll dies vor allem im Deutsch- und im Musikunterricht erfolgen. In den Regionalen Schulen und Gesamtschulen soll das Niederdeutsche im Fachunterricht sowie im Wahlpflicht- oder Neigungsunterricht eingebracht werden. An gymnasialen Schulen soll das Niederdeutsche

14 Verwaltungsvorschrift 2004, o. S., 1. Abs.

15 Verwaltungsvorschrift 2004, o. S., 2. und 3. Abs.

16 Verwaltungsvorschrift 2004, o. S., 3. Abs.

17 Verwaltungsvorschrift 2004, o. S., 3. Abs.; nachfolgend wird diese Bezeichnung so übernommen und nicht weiter als Zitat kenntlich gemacht.

18 Vgl. Verwaltungsvorschrift 2004, o. S., Punkt 2.

Birte Arendt, Andreas Bieberstedt and Klaas-Hinrich Ehlers - 978-3-631-71893-3 
in den Fachunterricht sowie in den Wahlbereich integriert werden. Erkennbar wird hier, dass das Niederdeutsche nach der Verwaltungsvorschrift ab der siebten Klassenstufe vermehrt im Wahlpflicht- bzw. Neigungsunterricht, also im außerfachlichen Bereich, Berücksichtigung finden soll.

Die Verwaltungsvorschrift beinhaltet auch Beispiele, wie das Niederdeutsche mittels des "fächerübergreifenden Unterrichtsprinzips“ in den Unterricht eingebunden werden kann. Im Deutschunterricht können demnach beispielsweise Texte aus der Region und Literatur niederdeutscher Autoren dienlich sein. Aber auch die Orthographie und die niederdeutsche Sprachgeschichte können im Deutschunterricht angesprochen werden. Im Musikunterricht bietet sich das niederdeutsche Liedgut an. Der Kunstunterricht gibt die Möglichkeit, niederdeutsche Inschriften und die Landeskunst zu behandeln. Selbst der Biologieunterricht könne dem fächerübergreifenden Unterrichtsprinzip folgen, indem die regionale Flora und Fauna mit den niederdeutschen Bezeichnungen gelehrt wird.

Ein weiterer Abschnitt der Vorschrift gibt Anregungen für den Wahlpflichtunterricht bzw. Neigungsunterricht. Diese schulischen Angebotsformen sollen genutzt werden, um beispielsweise niederdeutsche Stücke oder Hörspiele aufzuarbeiten sowie die Schulveranstaltungen mit einem niederdeutschen Beitrag zu beleben. In der Verwaltungsvorschrift wird den Schulen des Landes MecklenburgVorpommern ausdrücklich empfohlen, an außerschulischen Veranstaltungen wie den Plattdeutschwettbewerben oder Lesewettbewerben teilzunehmen, die im Rahmen des Unterrichts vorbereitet werden. ${ }^{19}$

Der dritte bis fünfte Punkt der Verwaltungsvorschrift regelt die Nominierung der Niederdeutschberater_innen. So soll am Landesinstitut für Schule und Ausbildung, an den staatlichen Schulämtern sowie an den Schulen jeweils ein(e) Niederdeutschberater_in tätig werden. Die Aufgabe dieser Beratung ist vornehmlich eine koordinierende und unterstützende Tätigkeit.

17 Jahre nach Inkrafttreten der Sprachencharta ist die Förderung des Niederdeutschen noch nicht durchgängig realisiert worden. Innerhalb des Sprachencharta-Prozesses ist das turnusmäßige Berichtsverfahren eine wesentliche Säule, denn es erlegt den unterzeichnenden Bundesländern auf, in regelmäßigen Abständen die jeweils erreichten Ergebnisse kritisch zu reflektieren und dem Bundesinnenministerium darüber Rechenschaft abzulegen. Am 15. Juni 2010 ist der vierte Staatenbericht gemäß Art. 15 Abs. 1 vom Bundesministerium des Inneren herausgegeben worden. Der Bericht des Sachverständigenausschusses zu diesem ist am 25. Mai 2011 erschienen. Ein Ergebnis dieses Berichtsverfahrens

19 Vgl. Verwaltungsvorschrift 2004, o. S., Punkt. 2. 
war, dass für Mecklenburg-Vorpommern die gezeichneten Verpflichtungen für den Grundschul- und den Sekundarbereich als nicht erfüllt angesehen wurden. Grund für diese Beurteilung ist, dass es nach wie vor keine Strukturierung für einen systematischen Unterricht in Niederdeutsch gab. ${ }^{20}$ Es wurde dem Land Mecklenburg-Vorpommern deshalb empfohlen, das Niederdeutsche „systematisch als [festen; Anm. A. F.] Bestandteil des Lehrplans an Grund- und Sekundarschulen“ zu unterrichten. ${ }^{21}$

Meine Fallstudie zum Niederdeutschunterricht in der Stadt Greifswald kann über diese allgemeine Empfehlung hinaus in einer Momentaufnahme aus dem Jahr 2011 aufzeigen, welche konkreten Defizite im schulischen Niederdeutschangebot in Mecklenburg-Vorpommern auch 12 Jahre nach der Unterzeichnung der Sprachencharta bestanden, die größtenteils bis heute anzutreffen sind, und damit Überlegungen unterstützen, wie die Förderung des Niederdeutschen im schulischen Bereich zu verbessern wäre. Da zum Zeitpunkt der Beitragserstellung (2016) keine andere verbindliche rechtliche Grundlage der Niederdeutschvermittlung im Land Mecklenburg-Vorpommern vorliegt, sind die erhobenen Daten von 2011 bis heute grundsätzlich als aktuell zu betrachten. Noch immer ist die Verwaltungsvorschrift von 2004 die schulartenübergreifende verbindliche Handlungsbasis.

Gleichwohl gibt es neuere Entwicklungen, die auf eine zunehmende Institutionalisierung des Niederdeutschen an den Schulen Mecklenburg-Vorpommerns hindeuten. So wird in einem Modellprojekt ab 2016 an sechs Gymnasien bzw. Gesamtschulen Niederdeutsch als Unterrichtsfach mit dem Status einer zweiten Fremdsprache eingeführt, wodurch auch ein Abitur auf Niederdeutsch möglich sein soll. ${ }^{22}$ Darüber hinaus nimmt Niederdeutsch einen zentralen Stellenwert im Landesprogramm „Meine Heimat - mein modernes Mecklenburg-Vorpommern“ ein, ${ }^{23}$ das für den Zeitraum 2016 bis 2020 verabschiedet wurde. Dort heißt es im geplanten Maßnahmenkatalog: ,verbindliche Verankerung der Niederdeutschen Sprachförderung und Heimatpflege in der Bildungskonzeption für 0-10-Jährige“, „Ermöglichung der Einrichtung des Faches Niederdeutsch als Zweite Fremdsprache an weiterführenden Schulen ab Klasse 7“ und „Einbindung von regionaler - auch niederdeutscher - Literatur in den Unterricht“ ${ }^{24}$ Das heißt, dass Niederdeutsch sowohl als eigenständiges Fach in der Sekundarstufe II eingerichtet

20 Vgl. Vierter Bericht des Sachverständigenausschusses 2011, S. 80.

21 Vierter Bericht des Sachverständigenausschusses 2011, S. 80.

22 Vgl. Abitur op Platt 2016.

23 Landesprogramm 2016.

24 Landesprogramm 2016, S. 7.

Birte Arendt, Andreas Bieberstedt and Klaas-Hinrich Ehlers - 978-3-631-71893-3 
wird als auch ab dem Kindergartenalter institutionell zu fördern ist. Inwieweit diese Absichtserklärungen wirklich greifen werden, bleibt abzuwarten. Grundsätzlich stellt diese Niederdeutschfokussierung im Landesprogramm „Meine Heimat - Mein modernes Mecklenburg-Vorpommern" eine politische Aufwertung der niederdeutschen Sprache und ein artikuliertes Bekenntnis zur schulischen Vermittlung dar.

\section{Niederdeutsch in den Schulen Mecklenburg-Vorpommerns - eine exemplarische Studie zu Greifswald}

\subsection{Methodik und Vorgehensweise}

Um den Stand des Niederdeutschunterrichts in den Greifswalder Schulen untersuchen zu können, wurde eine Methodenkombination aus quantitativen und qualitativen Verfahrensweisen gewählt. ${ }^{25}$ Durch die gegenseitige Ergänzung der beiden Methoden konnte ein umfassendes Bild der Stellung des Niederdeutschen an den Greifswalder Schulen erlangt werden. Am Anfang stand eine quantitative Datenerhebung: Dafür wurden die Greifswalder Schulen telefonisch und per EMail kontaktiert mit der Bitte, von Lehrer_innen einen Fragebogen zur Vermittlung des Niederdeutschen an der jeweiligen Schule ausfüllen zu lassen. Dieser Fragebogen enthielt offene und geschlossene Fragen, die das Ziel hatten, einen Überblick zu gewinnen, welche Schulen das Niederdeutsche in welcher Angebotsform unterrichten bzw. in den Schulalltag integrieren. Die Fragebogenmethode wurde gewählt, um eine hohe Vergleichbarkeit der Antworten bei der Auswertung zu gewährleisten. Des Weiteren konnte an vier Schulen hospitiert werden und die Beobachtungen wurden protokollarisch festgehalten. Die Hospitation wird in diesem Zusammenhang verstanden als „eine eher passive Teilnahme an Veranstaltungen einer pädagogischen Einrichtung ${ }^{16}$, die das Ziel verfolgt, einen Einblick in den schulischen Alltag zu erhalten. Die ausgewerteten Daten konnten zusätzlich mit weiteren quantitativen Daten aus einem internen Bericht des Staatlichen Schulamtes Greifswald aus dem Jahr 2003 verglichen werden.

Auf dem Wege der quantitativen Erhebung sind die Strukturen und Bedingungen des Niederdeutschunterrichtes allerdings nur unzureichend zu ermitteln, die tatsächliche Behandlung und der Stellenwert des Niederdeutschen im Schulunterricht kann auf diesem Wege nicht ausreichend geklärt werden. Deshalb wurden zusätzlich zur Fragebogenerhebung narrative Interviews mit sieben Lehrkräften

25 Vgl. dazu Kelle/Erzberger 2010, S. 305-306.

26 Schaub/Zenke 2007, S. 292.

Birte Arendt, Andreas Bieberstedt and Klaas-Hinrich Ehlers - 978-3-631-71893-3 
verschiedener Schulen geführt, um auch die Vorteile qualitativer Datengewinnung zu nutzen. So hat die „qualitative Forschung [...] den Anspruch, Lebenswelten ,von innen heraus' aus der Sicht der handelnden Menschen zu beschreiben“ ${ }^{27}$ Die qualitative Vorgehensweise ist also stark am Alltag der Betroffenen (in diesem Falle dem der Lehrer_innen) orientiert und kann damit ein besseres Verständnis ihrer sozialen Wirklichkeit (hier des Schulalltags) schaffen. Aus diesem Grunde vermag das narrative Interview zu rekonstruieren, was es aus der Sichtweise der Lehrer_innen bedeutet, das Niederdeutsche in die Schule zu bringen.

Die Befragten wurden mittels eines ersten narrativen Impulses zum Erzählen angeregt ${ }^{28}$ und legten im Verlauf der narrativen Interviews ihre persönlichen Erfahrungen mit dem Niederdeutschunterricht an ihrer Schule dar. In den Interviews gaben die befragten Lehrer_innen nicht nur detaillierte Informationen zum Lehrangebot der Schulen, sondern sie brachten auch ihre subjektiven Einstellungen zum Niederdeutschen und dessen Vermittlung im Schulalltag auf vielfältige Weise zum Ausdruck. Einstellungen werden im Kontext dieses Beitrags verstanden als "gesellschaftlich gewachsene und in der Sozialisation erworbene Bewertungsstrukturen [...], die sich in verschiedenen Formen ausprägen und handlungsleitende Funktion haben“. ${ }^{29}$ Sie sind mentale Ereignisse der Bewertung hinsichtlich eines Objekts (hier Niederdeutsch und Niederdeutschunterricht), welches nach bestimmten Kriterien klassifiziert und geprüft wird. Einstellungen entstehen in sozialen Interaktionen, durch Erfahrungen und Erlebtes. Ebenso sind Einstellungen wesentlich für die Her- und Darstellung der eigenen Identität. ${ }^{30}$

\subsection{Niederdeutsch an den Schulen in Greifswald - ein Überblick}

Nach offiziellen Angaben der Stadtverwaltung gab es 2011 insgesamt 15 bildende Schulen in Greifswald. ${ }^{31}$ Zehn dieser Schulen waren auf kommunaler Ebene verankert: fünf Grundschulen, zwei Regionale Schulen, eine integrierte Gesamtschule und zwei Gymnasien. Darüber hinaus gab es fünf Schulen in freien Trägerschaften in Greifswald.

27 Flick/von Kardorff/Steinke 2010, S. 14.

28 Vgl. Hopf 2010, S. 355.

29 Arendt 2010, S. 8.

30 Vgl. Arendt 2010, S. 8 sowie weiterführend zur „narrativen Identität“ Lucius-Hoene/ Deppermann 2004, S. 167.

31 Vgl. Angaben der Internetpräsenz der Universitäts- und Hansestadt Greifswald www. greifswald.de (Stand Dezember 2011). 
Obwohl die Schulen in freier Trägerschaft nach $₫ 117$ zur "Schulgestaltung“ des Schulgesetzes Mecklenburg-Vorpommern nicht an die Vorschriften der öffentlichen Verwaltung gebunden sind, wurden sie bei der Erhebung mit berücksichtigt. ${ }^{32}$ Zwar betrifft die Verwaltungsvorschrift „Niederdeutsch in der Schule" die Schulen in freien Trägerschaften nicht, da sie die Lehrinhalte und die Organisation des Unterrichts weitgehend eigenständig gestalten dürfen. Die Schulen freier Träger werden aber dennoch in die Untersuchung einbezogen, da grundsätzlich die Möglichkeit besteht, dass sie das Niederdeutsche aus eigenen Erwägungen in den Schulalltag integrieren. Nicht berücksichtigt wurden hingegen eine Allgemeine Förderschule, ein Abendgymnasium, eine Berufliche Schule, eine Volkshochschule sowie eine Musikschule, da diese nicht in den Bereich der primären Schullaufbahn gehören. Insgesamt bildet also die Befragung von zehn kommunalen und fünf freien Schulen die Grundlage für den folgenden quantitativen Überblick.

Insgesamt lässt sich feststellen, dass das Niederdeutsche 2011 an sechs Greifswalder Schulen im Unterricht berücksichtigt wurde. Fünf Schulen gaben dagegen nach telefonischer Auskunft und den Antworten in ausgefüllten Fragebögen an, das Niederdeutsche nicht einzubeziehen. Als Begründungen wurde zum Teil genannt, dass wegen der Fülle des Lehrstoffes die Zeit für die Behandlung des Niederdeutschen fehle. In anderen Fällen wurde auf das Desinteresse der Schüler, aber auch auf das Fehlen sprachkompetenter Fachkräfte verwiesen. Vier Schulen der Stadt Greifswald wollten sich bezüglich des Niederdeutschangebots an ihrer Schule nicht äußern, daher können im Folgenden nur die Angaben von elf Schulen ausgewertet werden.

Insgesamt boten demnach $40 \%$ der 15 Schulen in Greifswald das Niederdeutsche in irgendeiner Form an. Der durchaus bemerkenswerte Anteil von Schulen mit Niederdeutschangeboten erhöht sich noch, wenn man allein die zehn kommunalen Schulen der Stadt betrachtet. Hier lag der Anteil der Schulen mit einem Niederdeutschangebot sogar bei $60 \%$ (= sechs Schulen). Eine der kommunalen Schulen machte „keine Angaben“ zum Lehrangebot im Niederdeutschen (10\%), drei dieser Schulen boten ihrer Auskunft zufolge keinen Niederdeutschunterricht an (30\%). Es lässt sich also feststellen, dass ein überwiegender Teil der kommunalen Schulen das Niederdeutsche berücksichtigte.

Alle sechs Schulen mit Niederdeutschangebot hatten die Universitäts- und Hansestadt Greifswald als öffentlichen Träger. An zwei dieser Schulen wurde das Niederdeutsche - hauptsächlich im Fach Deutsch - integrativ unterrichtet. Vier kommunale Schulen boten das Niederdeutsche in einer anderen Form an, 
z. B. als Angebot der Vollen Halbtagsschule (nachfolgend VHTS-Angebot), als so genannte „Mittagsfreizeit“, als Ganztagsschulkurs (nachfolgend GTS-Kurs) oder als Angebot im Hort.

Von den fünf Greifswalder Schulen in freier Trägerschaft boten zwei das Niederdeutsche nicht an, drei dieser Schulen wollten keine Angaben über ihr Lehrangebot machen. Die nachfolgende Abbildung verdeutlicht noch einmal die Verteilung der Integration des Niederdeutschen nach Schulart und Form.

\section{Abb. 1: Verteilung des Niederdeutschangebots nach Schulart und Form ${ }^{33}$}
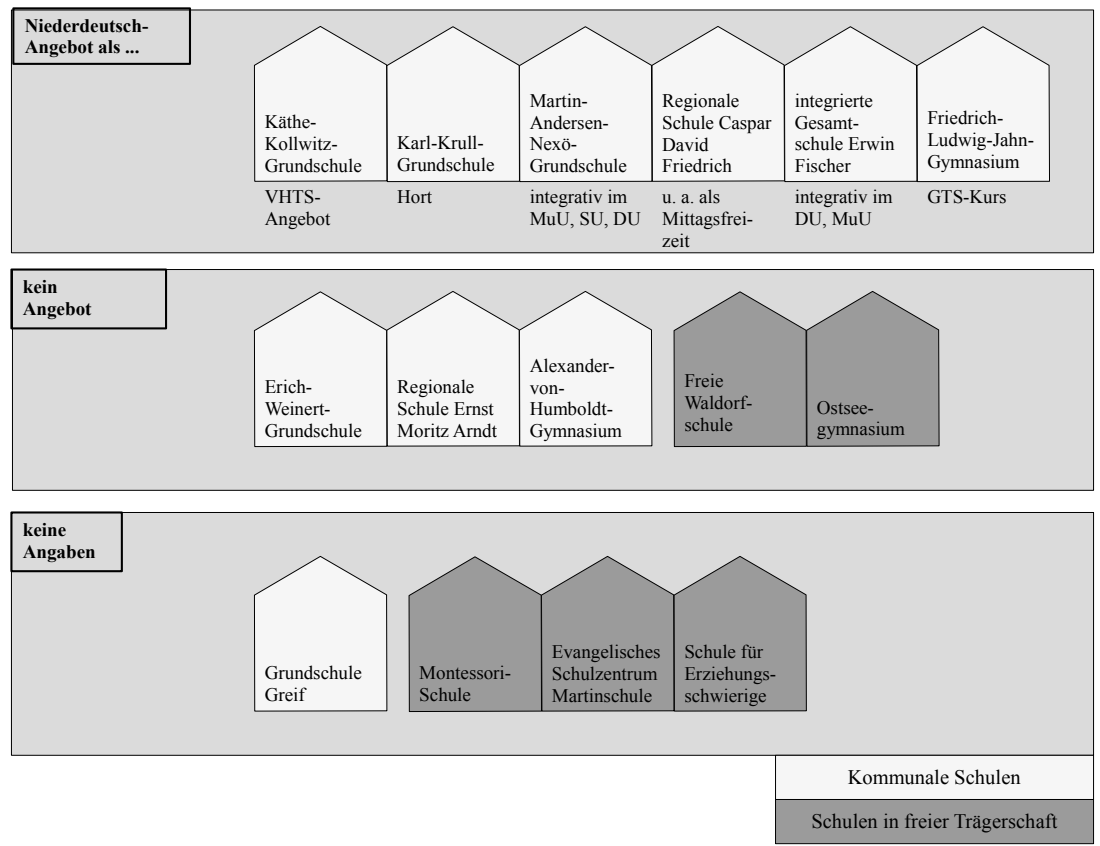

Laut einer internen Befragung des Staatlichen Schulamtes Greifswald aus dem Jahre 2003 unterrichteten damals neun von insgesamt 16 (56,25 \%) Schulen in der Stadt Greifswald das Niederdeutsche. ${ }^{34}$ Ein Abgleich mit den oben dargestellten Zahlenverhältnissen aus dem Jahr 2011 (6 von 15 Schulen, 40 \%) scheint auf den ersten

33 Dabei werden hier und nachfolgend der Musikunterricht mit MuU, der Sachunterricht mit SU und der Deutschunterricht mit DU abgekürzt.

34 Vgl. Alexander 2008, S. 6. 
Blick nahezulegen, dass die Anzahl der schulischen Niederdeutschangebote innerhalb von acht Jahren um ca. 16 Prozentpunkte gesunken ist, und dies trotz intensiver sprachenpolitischer Bemühungen. Der interne Bericht des Staatlichen Schulamtes Greifswald „Niederdeutsch“ zu den Daten von 2003 ist aber nur sehr bedingt mit den Ergebnissen meiner Befragung zu vergleichen. Zum einen hat sich die Schullandschaft der Universitäts- und Hansestadt Greifswald im Zeitraum zwischen 2003 und 2011 durch Schulschließungen und Zusammenlegungen verändert. Dies erklärt die abweichende Anzahl der Schulen. Zum anderen wurden bei den beiden Erhebungen unterschiedliche Schulen befragt. 2003 wurden die Schulen in freien Trägerschaften nicht berücksichtigt, dagegen aber Schulen wie z. B. das Abendgymnasium und die Allgemeine Förderschule. Diese wurden bei der Erhebung 2011 nicht berücksichtigt, da sie nicht der primären Schullaufbahn angehören.

Aus diesem Grunde werden im Folgenden nur die Schulen gegenübergestellt und in ihrem Niederdeutschangebot miteinander verglichen, die sowohl 2003 als auch 2011 befragt wurden. Dies ergibt für einen weiteren Vergleich insgesamt zehn Schulen. Es zeigt sich, dass lediglich bei einer Schule das Niederdeutschangebot entfallen ist. Hingegen konnten gleich zwei Schulen das Niederdeutsche neu einführen und drei Schulen konnten ihr Angebot sogar erweitern. Neben diesen Veränderungen gab es in einem Fall eine Verlagerung des Niederdeutschangebots vom Unterricht in den Hortbereich. Die folgende Tabelle verdeutlicht diese Veränderungen. ${ }^{35}$

Tabelle 1: Vergleich des Niederdeutschangebotes an zehn Greifswalder Schulen in den Jahren 2003 und 2011

\begin{tabular}{|l|l|l|l|}
\hline \multicolumn{1}{|c|}{ Schule } & \multicolumn{1}{c|}{2003} & \multicolumn{1}{c|}{2011} & \multicolumn{1}{c|}{ Veränderung } \\
\hline $\begin{array}{l}\text { Erich-Weinert- } \\
\text { Grundschule }\end{array}$ & $\begin{array}{l}\text { integrativ im SU, } \\
\text { MuU }\end{array}$ & kein Angebot & entfallen \\
\hline $\begin{array}{l}\text { Martin-Andersen- } \\
\text { Nexö-Grundschule }\end{array}$ & integrativ im SU & $\begin{array}{l}\text { integrativ im MuU, } \\
\text { SU, DU }\end{array}$ & $\begin{array}{l}\text { Niederdeutsch- } \\
\text { angebot erweitert }\end{array}$ \\
\hline $\begin{array}{l}\text { integrierte Gesamt- } \\
\text { schule Erwin Fischer }\end{array}$ & integrativ im DU & $\begin{array}{l}\text { integrativ im DU, } \\
\text { MuU }\end{array}$ & $\begin{array}{l}\text { Niederdeutsch- } \\
\text { angebot erweitert }\end{array}$ \\
\hline
\end{tabular}

35 Die Angaben aus dem Jahre 2003 wurden aus den Fragebögen „Niederdeutsch an den

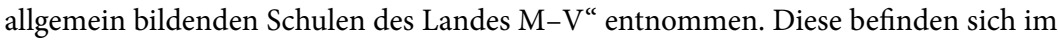
Anhang des internen Berichts des Staatlichen Schulamtes Greifswald, vgl. Alexander 2008. Auch hier wird Musikunterricht mit MuU, Sachunterricht mit SU und Deutschunterricht mit DU abgekürzt. GTS: Ganztagsschule, VHTS-Volle Halbtagsschule. 


\begin{tabular}{|l|l|l|l|}
\hline \multicolumn{1}{|c|}{ Schule } & \multicolumn{1}{c|}{ 2003 } & \multicolumn{1}{c|}{ 2011 } & \multicolumn{1}{c|}{ Veränderung } \\
\hline $\begin{array}{l}\text { Regionale Schule Ernst } \\
\text { Moritz Arndt }\end{array}$ & kein Angebot & kein Angebot & $\begin{array}{l}\text { keine Verände- } \\
\text { rung }\end{array}$ \\
\hline $\begin{array}{l}\text { Karl-Krull- } \\
\text { Grundschule }\end{array}$ & integrativ im DU, SU & Hort & Verlagerung \\
\hline Grundschule Greif & integrativ im SU, DU & keine Angaben & -- \\
\hline $\begin{array}{l}\text { Alexander-von- } \\
\text { Humboldt-Gymnasium }\end{array}$ & kein Angebot & kein Angebot & $\begin{array}{l}\text { keine Verände- } \\
\text { rung }\end{array}$ \\
\hline $\begin{array}{l}\text { Käthe-Kollwitz- } \\
\text { Grundschule }\end{array}$ & kein Angebot & VHTS-Angebot & eingeführt \\
\hline $\begin{array}{l}\text { Regionale Schule } \\
\text { Caspar David Friedrich }\end{array}$ & integrativ im DU, & $\begin{array}{l}\text { WuUhlpflicht, Mittags- } \\
\text { freizeit, integrativ } \\
\text { im DU }\end{array}$ & $\begin{array}{l}\text { Niederdeutsch- } \\
\text { angebot erweitert }\end{array}$ \\
\hline $\begin{array}{l}\text { Friedrich-Ludwig- } \\
\text { Jahn-Gymnasium }\end{array}$ & kein Angebot & GTS-Kurs & eingeführt \\
\hline
\end{tabular}

Die Befragung zeigt, dass das Niederdeutsche auch an den Schulen mit Niederdeutschangebot keineswegs in jedem Unterrichtsfach Berücksichtigung fand. Überwiegend wurde das Niederdeutsche in das Fach Deutsch, seltener in den Musikunterricht und für den Grundschulbereich gelegentlich auch in den Sachunterricht integriert. In den regulären Schulfächern wurde das Niederdeutsche also, wenn überhaupt, nur partiell unterrichtet. Von einem durchgängigen Unterrichtsprinzip kann somit leider nicht gesprochen werden. An den Greifswalder Schulen, wo eine Hospitation stattfinden konnte, gab es neben dem Unterricht vier weitere Formen (VHTS-Angebot, Hortangebot, Mittagsfreizeit und GTSKurs) der Vermittlung des Niederdeutschen. Diese zusätzlichen Angebote wurden in der Regel im Ganztagsschulbereich als eine Art Wahlkurs bzw. Neigungsunterricht außerhalb des regulären Unterrichts realisiert.

Fasst man die detaillierten Angaben dieser zehn Schulen unter dem allgemeinen Aspekt zusammen, an wie vielen Schulen Niederdeutsch angeboten wurde, so wird ersichtlich, dass die Zahl der Niederdeutschangebote hier konstant geblieben ist. Diese Verteilung ist in der folgenden Tabelle $2 \mathrm{zu}$ sehen: 
Tabelle 2: Niederdeutschangebot an zehn Greifswalder Schulen 2003 und 2011

\begin{tabular}{|l|c|c|}
\hline Anzahl der Schulen & $\mathbf{2 0 0 3}$ & $\mathbf{2 0 1 1}$ \\
\hline mit Niederdeutschangebot & 6 & 6 \\
\hline ohne Angebot & 4 & 3 \\
\hline keine Angaben & 0 & 1 \\
\hline
\end{tabular}

Dennoch ist die konstant niedrige Zahl der Niederdeutschangebote in den Greifswalder Schulen zu bedauern, zumal seit dem Erlass der Verwaltungsvorschrift „Niederdeutsch in der Schule“ im Jahr 2004 eigentlich allen Schulen in öffentlichen Trägerschaften die Aufgabe gestellt worden ist, das Niederdeutsche als ein durchgängiges Unterrichtsprinzip zu etablieren. Außerdem fand der Niederdeutschunterricht schon im Schulgesetz von 2003 Verankerung, das stärkere Bemühungen seitens der Schulen erforderte. Gleichwohl bleibt positiv festzuhalten, dass drei der verglichenen Greifswalder Schulen ihr Angebot noch erweitern konnten und zwei weitere Schulen das Niederdeutsche etabliert haben.

\subsection{Niederdeutschunterricht aus der Binnensicht der Lehrkräfte einer Schule}

Im Folgenden soll der Fokus auf die konkrete Umsetzung des Niederdeutschangebots im Schulalltag einer beispielhaft herausgegriffenen Schule gelegt werden. Ein komplexes Bild dieser Umsetzung aus der Binnensicht der Akteure lässt sich über narrative Interviews mit unmittelbar beteiligten Lehrkräften gewinnen, deren geschilderte persönliche Erfahrungen und Bewertungen einander in Teilen ergänzen, in Teilen aber auch voneinander abweichen. Die ausgewählte Schule wurde 2011 von ca. 310 Schülern besucht. Zum Kollegium gehörten 34 Lehrer_ innen. Mit zwei Lehrerinnen dieser Schule (Frau C und Frau H) sind Einzelinterviews geführt worden, nachdem in einer Niederdeutsch-Stunde hospitiert werden konnte.

Das Interview mit Frau H wurde direkt in der Schule geführt und konnte somit am Wirkungs- und Handlungsort der Gewährsperson mit unmittelbarem Bezug zu ihrem schulischen Alltag stattfinden. Das Interview mit der Gewährsperson C konnte hingegen aus organisatorischen Gründen nur zu Hause erfolgen. ${ }^{36}$

36 Die Transkription erfolgte in Anlehnung an die Methode der Basistranskription nach GAT; vgl. Selting [u. a.] 1998. 
Tabelle 3: Übersicht der Gewährspersonen

\begin{tabular}{|c|c|c|l|l|}
\hline GP & Geschlecht & Dienstjahre & Fächerkombination & \multicolumn{1}{|c|}{$\begin{array}{c}\text { Ausbildung } \\
\text { Niederdeutsch }\end{array}$} \\
\hline H & w & 30 & $\begin{array}{l}\text { Deutsch, } \\
\text { Sport } \\
\text { (Sonderpädagogik) }\end{array}$ & $\begin{array}{l}\text { in der Lehrerausbildung } \\
\text { enthalten gewesen }\end{array}$ \\
\hline C & w & 35 & $\begin{array}{l}\text { Deutsch, } \\
\text { Geschichte }\end{array}$ & $\begin{array}{l}\text { keine, Zertifikatskurs nicht } \\
\text { abgeschlossen }\end{array}$ \\
\hline
\end{tabular}

Das vorherige Hospitieren im Unterricht der Gewährspersonen erwies sich als ein wichtiger und vertrauensbildender Schritt für die Vorbereitung der Interviews, da in kurzen Gesprächen nach dem Unterricht bzw. nach den Kursangeboten bereits ein gegenseitiges Kennenlernen möglich war. Zu Beginn des Interviews wurden den beiden Gewährspersonen die gleiche Frage, „Wie finden Sie das Plattdeutsche?", als narrativer Impuls gestellt. Mittels dieser „Eisbrecherfrage“ sollte den Gewährspersonen die Scheu vor dem Aufnahmegerät genommen werden. Außerdem sicherte die gleiche Ausgangsfrage der Interviews deren Vergleichbarkeit.

In der untersuchten Schule gab es vielfältige Niederdeutschangebote. Zum einen wurde Niederdeutsch in den Deutschunterricht integriert und mindestens zweimal pro Schuljahr in Unterrichtseinheiten behandelt, die sich über mehrere Stunden erstreckten. Dies wurde von der Lehrerin $\mathrm{H}$ in den Klassen fünf bis acht realisiert. Zum anderen gab es einmal für die siebente und für die zehnte Klasse jeweils einen Wahlpflichtkurs, der nach Klassenstufen getrennt unterrichtet wurde. Aus der siebenten Klassenstufe nahmen dieses Angebot 2011 drei Schüler_innen wahr, in der zehnten Klassenstufe wählten fünf Schüler_innen den Wahlpflichtkurs. Aus organisatorischen Gründen wurden diese beiden Wahlpflichtkurse an einem Wochentag in die so genannte „Mittagsfreizeit“ von 12:15 bis 13:05 gelegt, wobei der erste Teil dieser Zeitspanne für die siebente Klassenstufe und der zweite Teil der „Mittagsfreizeit“ für die Schüler_innen der zehnte Klassenstufe vorbehalten war. Im Wahlpflichtunterricht stand das produktive Arbeiten mit niederdeutschen Texten im Vordergrund. Eine Benotung erfolgte in diesem Kurs nicht.

Weiterhin wurde das Niederdeutsche an der untersuchten Schule als ein zusätzliches Angebot der betreuten Pausengestaltung ebenfalls in der Mittagsfreizeit angeboten. Bei dieser Angebotsform stand die aktive Beschäftigung der Schüler_innen im Vordergrund. So wurden beispielsweise niederdeutsche Lieder gehört, Puzzles mit niederdeutschen Wörtern zusammengefügt oder einzelne 
niederdeutsche Wörter beim Malen vermittelt. Die Schüler_innen konnten ihre Mittagsfreizeit individuell gestalten und Angebote beispielsweise aus den Bereichen wie Sport oder Basteln wählen. Jedes Pausenangebot wurde einmal in der Woche realisiert. Die niederdeutsche Mittagspausenbeschäftigung wurde zur Zeit der Befragung von fünf Schüler_innen aus der fünften Klassenstufe und von vier Schüler_innen der sechsten Klassenstufe immer an einem Wochentag wahrgenommen. Auch hier wurde nach Klassenstufen getrennt. Insgesamt nahmen das Niederdeutschangebot in den Wahlpflichtkursen und in der Mittagsfreizeit 17 Schüler_innen verschiedener Klassenstufen wahr. Die Niederdeutsch-Kurse außerhalb des Deutschunterrichts erreichten damit nur eine sehr begrenzte Zahl von Schüler_innen (etwa 5,5\% der Schülerschaft, $\mathrm{n}=310$ ).

In den Interviews der beiden Gewährspersonen kamen nicht nur Einzelheiten zur konkreten Umsetzung des Niederdeutschangebotes an ihrer Schule zur Sprache, sondern es wurden auch ihre persönlichen Einstellungen zum Niederdeutschen und ihre Motive für ihr Engagement bei der Vermittlung des Niederdeutschen thematisiert. Beide Lehrerinnen äußerten sehr positive Einstellungen zum Niederdeutschen, das für beide in einer engen Beziehung zur Familie und zur Region steht.

So betont Frau H, nachdem sie den ersten Erzählimpuls bekommen hat, dass sie das Niederdeutsche seit ihrer Kindheit aus ihrer Familie kenne und es „schon immer" als positiv empfunden habe. Diese Einstellung wird in der folgenden Sequenz wiedergegeben. ${ }^{37}$

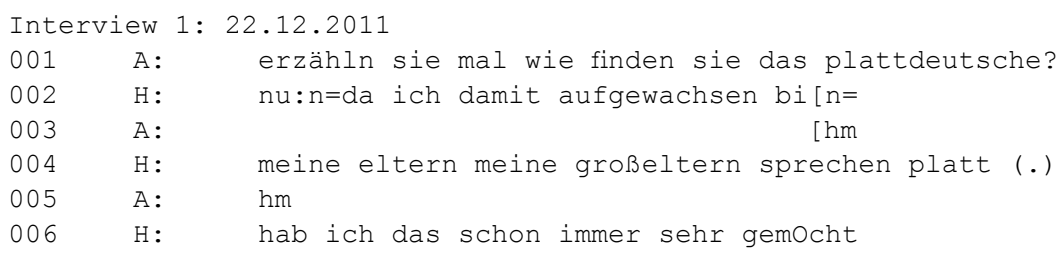

Aber nicht nur die Familie hat bei Frau H einen Einfluss auf die Einstellungsbildung. Der Klang und die Wirkung der niederdeutschen Sprache sind für sie ebenfalls entscheidende Aspekte, wie der folgende Ausschnitt wiedergibt.

37 Als Lesehilfe für die Transkripte seien hier nur die wichtigsten verwendeten Zeichenkonventionen angeführt: Simultansprechen der Gesprächspartner wird in eckige Klammern gefasst, einfache runde Klammern markieren Pausen, Großbuchstaben Akzentsetzungen im Wort. Dehnungen von Vokalen und Konsonanten werden mit Doppelpunkt bezeichnet. Näheres siehe Basistranskription nach GAT; vgl. Selting [u. a.] 1998, vgl. auch den Anhang zum Beitrag von Biedowicz in diesem Band. 
Interview 1: 22.12.2011

$013 \mathrm{H}$ : nA ich mag die sprache sehr weil sie viel weicher is als das

014 normal [e deu]tsch

015 A: $\quad[\mathrm{hm}=\mathrm{hm}]$

$016 \mathrm{H}$ : man kann vieles äh=äh sANfter ausdrücken ( $\mathrm{sa} \mathrm{s}$ ) ob das nun

017 ein lob oder ein tadel ist-

$018 \mathrm{~A}: \mathrm{hm}$

$019 \mathrm{H}$ : und ich finde leute die so äh äh platt sprechen auch immer

020 sehr (.) gemütlich die haben immer irgendwie etwas (.) wie

021 soll ich das sagen auch freundliches an sich die sprache an

022 sich is freundicher als das normale deutsch.

Frau H empfindet das Niederdeutsche als eine weiche Sprache (vgl. Z. 013) im Gegensatz zum „normalen“ Deutsch. Diese phonetische und pragmatische Charakterisierung der Sprache überträgt die Gewährsperson auch auf die Sprecher_ innen des Niederdeutschen (vgl. Z. 019 bis 022). So haben diese für sie ebenfalls "gemütliche“ (Z. 020) und „freundliche“ (vgl. Z. 021) Charakterzüge.

Eine weitere Spracheinstellung wird hier durch die zweimalige Abgrenzung des Niederdeutschen vom „normalen Deutsch“ (vgl. Z. 014 und 022) deutlich. Das Niederdeutsche ist für Frau H demnach nicht die „normale“ Sprache, nicht das „Standarddeutsche“, sondern das „Unnormale“. Durch diese Qualifizierung ist bei Frau $\mathrm{H}$ jedoch keine Abwertung des Niederdeutschen ausgedrückt. Es erfolgt hier im Gegenteil implizit eine negative Bewertung des Hochdeutschen. Dies ist nach Ansicht der Interviewpartnerin nicht so „weich“ und „freundlich“ wie das Niederdeutsche. Frau H gibt mit diesen kontrastierenden Bewertungen zu erkennen, dass sie bewusst zwischen Hochdeutsch als der Standardsprache in Deutschland und Niederdeutsch als der Regionalsprache unterscheidet, die vor allem für die Kommunikation emotionaler Inhalte im Bereich zwischen einander nahe stehenden Personen geeignet scheint. ${ }^{38}$

Im weiteren Verlauf des Gespräches verdeutlicht Frau H, dass sie sich der gegenwärtigen Situation des Niederdeutschen und der rückläufigen Sprecherzahlen bewusst ist. Das Bestreben, dem Verlust der Sprache entgegenzuwirken, ist für sie die zentrale Motivation, das Niederdeutsche immer wieder in den Deutschunterricht einzubringen: „Und man möchte natürlich ähm nicht dass diese Sprache/ verloren geht“" (Interview 1, Z. 030-031). In diesem Zusammenhang verdeutlicht Frau H ihren Schüler_innen, wenn sie Unterrichtseinheiten zum Niederdeutschen gestaltet, dass sie selbst ein persönliches Interesse an der Sprache hat. Es ist ihr ein wichtiges Anliegen, dass den Schüler_innen das Niederdeutsche im Gedächtnis

38 Zum Stereotyp des ,weichen und gemütlichen ' Niederdeutsch vgl. auch Jürgens 2015, S. 297-314. 
bleiben soll. Das „Nicht-Vergessen“ zeichnet sich bei Frau H als eine Handlungsleitlinie ihres Deutschunterrichts ab. Dies wird durch die nachfolgende Sequenz noch einmal hervorgehoben.

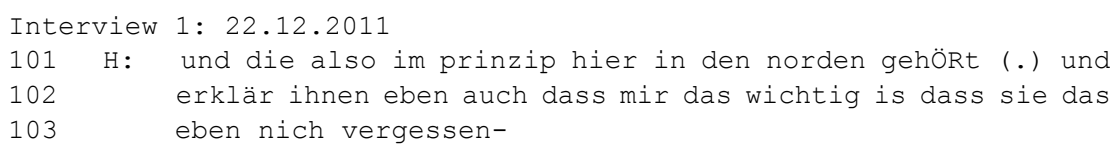

Das Niederdeutsche gehört für Frau H in den Norden und deshalb ist ihr es auch wichtig, dass ihre Schüler_innen diesen Zusammenhang zwischen der Sprache und der Region verstehen und im besten Falle selbst verinnerlichen.

Die Einstellung zum Niederdeutschen und zu dessen schulischer Vermittlung ähnelt bei der zweiten Interviewpartnerin von der exemplarisch untersuchten Schule sehr stark den Aussagen von Frau H. Auch für Frau C ist das Niederdeutsche eng mit ihrer Familie, der Stadt und der Region verbunden. In ihrem Interview entwirft sie das Bild einer regelrechten Greifswalder ,Familiendynastie (Z. 0428), in die sie sich einreiht.

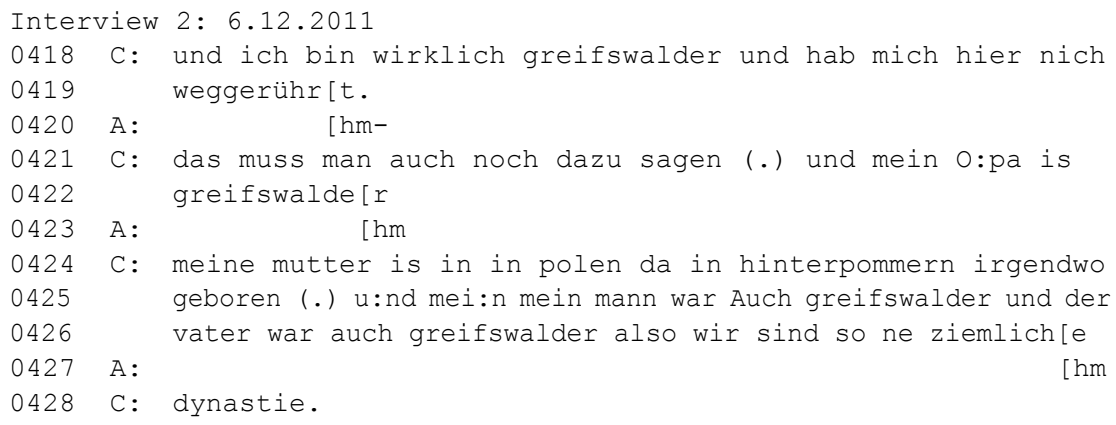

$[\mathrm{hm}$

Indem Frau C aufzählt, dass ihr Großvater, ihr Vater, ihr Ehemann und ihr Schwiegervater Greifswalder sind, verdeutlicht sie, wie sehr ihre Familie mit der Stadt und allgemein mit der Region verbunden ist. Der große Nachdruck, mit dem sie hier ihre „wirklich greifswalder“ Identität (Z. 0418-0419) konstruiert, wird auch dadurch deutlich, dass die mütterliche Familienlinie dabei offenbar ausgeblendet bleibt. Die feste Verbundenheit mit der Stadt wird auch durch Betonung ihrer großen Ortstreue („,hier nicht weggerührt“, Z. 0418-0419) unterstrichen.

Auch Frau C hat einen familiären Bezug zum Niederdeutschen vor allem über den Großvater (vgl. „Äh:m mein großvater hat/plattdeutsch gesproche[n;“ Interview 2, Z. 0006-0007). Des Weiteren erinnert sie sich, dass in ihrer Umgebung das Niederdeutsche früher häufig gesprochen wurde (vgl. ,ich hab das auch noch so in der dörflichen (.) abgeschiedenheit/ kennengelernt dass sich alle platt unterhalten 
[so-“, Interview 2, Z. 0009-0010). Die Nennung der „dörflichen abgeschiedenheit“ (Z. 0009) zeigt, dass Frau C sich nicht nur mit der Stadt Greifswald im Speziellen verbunden fühlt, sondern auch mit der weiteren Region. Dies äußert sie auch konkret in der folgenden Aussage: „also ich finde das wIchtig Eben dass wir hier aus $=\mathrm{m}$ no:rden/dass das bei uns ist" (Interview 2, Z. 0124-0125).

Auch für Frau $\mathrm{C}$ macht das Niederdeutsche einen Teil der regionalen Identität aus. Dies motiviert auch ihr Handeln in der Schule. Ganz ähnlich wie Frau H hat auch Frau C den zurückgehenden Gebrauch des Niederdeutschen aus eigener Anschauung im Familienumfeld erfahren und sie wertet diese Entwicklung als einen „Verlust“. Ihr schulisches Engagement für das Niederdeutsche motiviert sie als persönliche Bemühung, den drohenden Sprachverlust abzuwenden. Diese Einstellung gibt Frau C mit folgender Aussage wieder: ,ich find das is n grOßer vERlust und dem wollt ich einfach/entgegenwirken." (Interview 2, Z. 0155-0156).

Beide befragten Lehrerinnen thematisieren auch ihre eigene eingeschränkte Niederdeutschkompetenz und reflektieren über ihre sprachlichen Voraussetzungen für den Niederdeutschunterricht. Frau H schätzt ihr Verstehen als sehr gut ein, räumt aber Schwierigkeiten mit dem aktiven Sprechen ein: „Äh ich verste:h es auch sehr gut (.) A:ber sprechen (.)/wEniger (.) geht=so;" (Interview 7, Z. 008-009). Um das Niederdeutsche dennoch in den Unterricht einbeziehen zu können, bereitet sie niederdeutsche Texte besonders intensiv vor, um ihre eingeschränkte aktive Sprechkompetenz zu kompensieren: „Also äh äh::m=kann man sich als lehrer ja auch bestimmte/texte vorbereiten dann fällt das sprechen ja auch nich/schwer-" (Interview 7, Z. 033-035). Ihre geringere Kompetenz verunsichert Frau $\mathrm{H}$ im Unterricht allerdings nicht, sondern sie geht damit gegenüber den Schülern offen um und sieht darin sogar eine Möglichkeit, sich mit den Schüler_innen auf eine Ebene zu stellen und ihnen Hemmungen vor dem eigenen Lesen und Sprechen zu nehmen.

Interview 1: 22.12.2011

$037 \mathrm{H}$ :

$((\ldots))$ zum

038 anfang natürlich (.) muss man ihnen die hemmschwelle nehmen

039 und ihnen auch erklären dass man selber das nich so gut kann

Allerdings hat Frau $\mathrm{H}$ feststellen können, dass andere Kolleginnen und Kollegen mit einer geringen Sprechkompetenz nicht genauso problemlos umgehen können wie sie („es gibt sehr viel kollegen im die die platt verstE:hn-/aber sie hamm alle eine hemmschwelle das zu spreche[n." Interview 7, Z. 229-231). Sie selbst erachtet ihre Sprechkompetenz als ausreichend, um mit entsprechender Vorbereitung das Niederdeutsche in den Deutschunterricht einzubringen. 
Auch Frau C schränkt den Grad ihrer Verstehens- und Sprechkompetenz ein. So äußert sie sich zu Beginn des Interviews mit Blick auf den Niederdeutschgebrauch ihres Großvaters, dass sie das Niederdeutsche „eigentlich“ auch verstehe (,u:nd ich hab dann eben gedacht eigentlich kann ich:s/verste:hen (.) “Interview 2, Z. 0012-0013). Im privaten Umfeld sucht Frau C nach Möglichkeiten, ihre Kompetenzen aufzubessern, indem sie sich regelmäßig mit Freunden und Bekannten trifft und mit ihnen auf Niederdeutsch kommuniziert. Sie räumt ein, dass sie bei diesen niederdeutschen Gesprächen noch Fehler mache, aber hofft auch, dass sich ihr Sprechen dabei allmählich immer weiter verbessert („und auf die art und weise denn das sprechen eben $n$ bisschen/ mehr lernen." Interview 2, Z. 0724-0725). Frau $\mathrm{C}$ hat einen hohen Anspruch an sich selbst und betrachtet ihre Sprechkompetenz im Niederdeutschen stets kritisch. Da sie gleichzeitig eine große regionale Verbundenheit und hohe Wertschätzung für das Niederdeutsche empfindet, überträgt sie ihre kritische Sicht ebenso auf die Realisierung des Niederdeutschen an ihrer Schule.

Beide befragte Lehrerinnen bewerten den Stellenwert des Niederdeutschen an ihrer Schule im Jahr 2011 recht unterschiedlich. Für Frau H ist das Niederdeutsche ein ganz normaler Bestandteil des Deutschunterrichts und wird von ihr mindestens zweimal pro Schuljahr über mehrere Stunden unterrichtet. Hierbei ist die didaktische Realisierung überwiegend auf Lesen und Textarbeit beschränkt. Das Niederdeutsche selbst wird nicht als Unterrichtsmedium verwendet und folglich kaum aktiv genutzt. Nur in den unteren Klassenstufen arbeitet Frau $\mathrm{H}$ auch mit Darstellendem Spiel im Niederdeutschen. Wenn sie feststellt, dass ihr Niederdeutschangebot bei den Schüler_innen auf größeres Interesse stößt, dehnt sie diese Unterrichtseinheiten weiter aus. Dabei orientiert sich Frau H auch am Rahmenplan Deutsch, der niederdeutsche Themenstellungen enthält.

Nach Abstimmung des Rahmenplans mit anderen Deutschlehrer_innen dieser Schule, geht Frau $\mathrm{H}$ davon aus, dass jede Fachlehrkraft für Deutsch das Niederdeutsche im Unterricht behandelt. Dies wird in der folgenden Aussage deutlich.

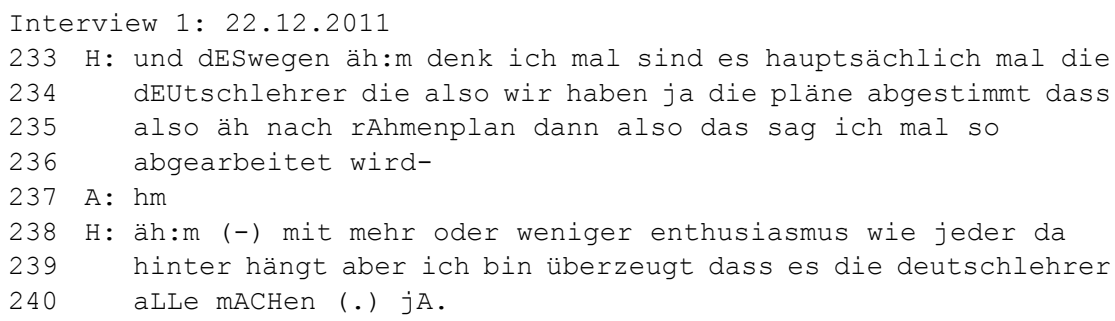


Je nach eigener Sprachkompetenz und Einstellung wird das Niederdeutsche demnach von allen Kolleg_innen mehr oder weniger intensiv in den Deutschunterricht integriert. Unter diesen Voraussetzungen kann nach Frau $\mathrm{H}$ das Niederdeutsche bereits als ein "ganz normaler" Bestandteil des Deutschunterrichts angesehen werden.

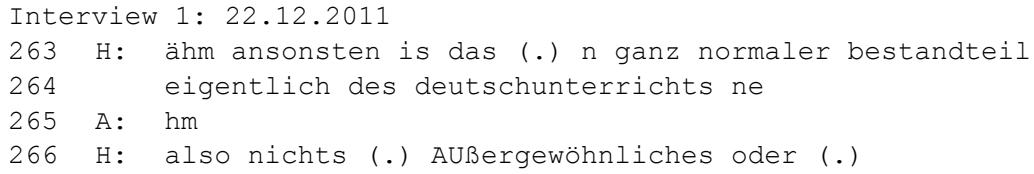

Nach Ansicht von Frau H ist das Niederdeutsche mit Hilfe der Verankerung in den Rahmenplänen also bereits recht gut in die Schule integriert und es hat sich bereits eine Routine für den Niederdeutschunterricht entwickelt.

Weniger positiv bewertet Frau C zum Zeitpunkt der Befragung den Stellenwert des Niederdeutschen an dieser Schule. Ihrer Erfahrung nach nimmt das Niederdeutsche einen immer geringeren Platz in der Schule ein. So erzählt Frau C im Zusammenhang mit der gegebenen Situation:

Interview 2: 6.12.2011

$0197 \mathrm{C}$ :

[na JA es is immer wE] Iter

0198

rE:duziert wOrden das is äh das wAHLpflichtfach ä::h ist (.)

0199

$\ddot{A}: \mathrm{H}$ ja ist immer mehr reduziert worden (.) es gibt kEIne noten

0200 me:hr im wAHLpflichtfac $[h$,

Da das Niederdeutsche kein selbstständiges Unterrichtsfach darstelle, werde es an dieser Schule u. a. als Wahlpflichtfach angeboten. Der Stellenwert eines solchen Faches ist nach Frau $\mathrm{C}$ weiter herabgestuft worden, seit es keine Leistungsbewertungen für die Schüler_innen mehr gibt. Dies bedeute zugleich, dass das Wahlpflichtfach nicht mehr relevant für eine Versetzung und für den Schulabschluss ist. Es werden nur die eingebrachten Stunden berücksichtigt. Der Stellenwert des Niederdeutschen in der Schule hat sich nach Frau $\mathrm{C}$ auch durch die Einbindung in die Mittagsfreizeit verringert. Der schulischen Regelung entsprechend soll die Ausgestaltung der Mittagsfreizeit keinen Unterrichtscharakter haben. Hier steht die Beschäftigung der Schüler_innen im Vordergrund und Wissensvermittlung hat in der Mittagspause nur eine untergeordnete Bedeutung. Dem entsprechend beschränkt sich Frau $\mathrm{C}$ auf die spielerische Vermittlung einzelner niederdeutscher Wörter, z. B. beim Basteln und Malen. Frau C berichtet auch, dass von den Schüler_innen ebenfalls kein Wissenstransfer in der Mittagsfreizeit gewünscht ist: „Sie mÖ:gen/(.) NICHT in der mittagsfreizeit auch noch ne sprache lerne[n.“ (Z. 0229-0230). Den Stellenwert des Niederdeutschen an ihrer Schule beurteilt Frau C zusammenfassend wie folgt: 


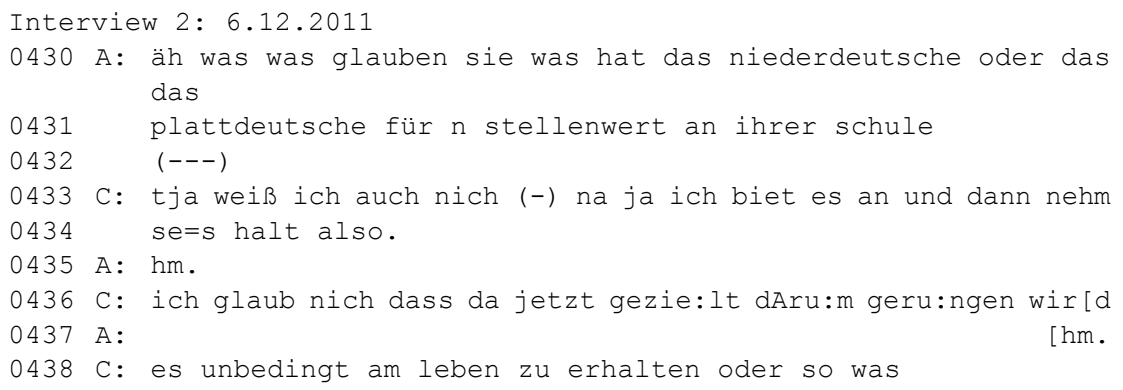

Nach einer kurzen Bedenkzeit konstatiert Frau $C$ hier, dass das Niederdeutsche zwar akzeptiert werde (vgl. Z. 0433-0434), aber ein echtes schulisches Engagement fehle, um die Regionalsprache „am leben zu erhalten“ (Z. 0434). Hier wird einer Unzufriedenheit mit der schulischen Situation Ausdruck verliehen, in der auch eine gewisse Resignation mitschwingt. Die unterschiedlichen Bewertungen des Niederdeutschangebotes der Schule bei den beiden Interviewpartnerinnen hängen offensichtlich auch mit ihren unterschiedlichen Erfahrungskontexten im Deutschunterricht einerseits und im Bereich des außerunterrichtlichen Zusatzangebotes in Wahlpflichtkursen und in der Mittagsfreizeit andererseits zusammen.

\section{Zusammenfassung und Ausblick}

Die bisher ergriffenen Maßnahmen des Landes Mecklenburg-Vorpommern sind vom Sachverständigenausschuss, der die Umsetzungen der Sprachencharta turnusmäßig zu überprüfen hat, grundsätzlich lobend hervorgehoben worden. ${ }^{39}$ Dennoch sind die von Mecklenburg-Vorpommern übernommenen Verpflichtungen der Sprachencharta bis 2011 demnach nur zum Teil erfüllt worden. Der Ausschuss hielt die Behörden weiterhin dazu an, ihre Maßnahmen fortzuführen und das Niederdeutsche „systematisch als [festen; Anm. A. F.] Bestandteil des Lehrplans an Grund- und Sekundarschulen ${ }^{\text {" }}{ }^{40}$ zu unterrichten. Diese Empfehlung resultiert u. a. aus einem entstandenen Widerspruch zwischen den Verpflichtungen der Sprachencharta und den Bestimmungen der Verwaltungsvorschrift. Mit den gezeichneten Maßnahmen der Sprachencharta, die den schulischen Bereich betreffen, hat Mecklenburg-Vorpommern sich zu einer flächendeckenden Einführung des Niederdeutschen als reguläres Unterrichtsfach verpflichtet. ${ }^{41}$ Dagegen

39 Vgl. Vierter Bericht des Sachverständigenausschusses 2011, S. 79.

40 Vierter Bericht des Sachverständigenausschusses 2011, S. 79.

41 Vgl. Langenfeld 2011, S. 200. 
stellt die Verwaltungsvorschrift klar heraus, dass das Niederdeutsche nicht als eigenständiges Fach mit ausgewiesenem Stundenanteil unterrichtet werden soll. Stattdessen hat sich Mecklenburg-Vorpommern für die Methode eines „durchgängigen Unterrichtsprinzips“ entschieden. Hierbei kann jedes reguläre Unterrichtsfach sowie Wahlkurse bzw. Neigungsunterricht einbezogen werden.

Die Umsetzung der Verpflichtungen der Sprachencharta und der Bestimmungen der Verwaltungsvorschrift wurde in diesem Beitrag am Beispiel der Universitäts- und Hansestadt Greifswald mit Hilfe einer quantitativen und qualitativen Befragung untersucht. Demnach wurde das Niederdeutsche im Jahr 2011 in Greifswald an sechs kommunalen Schulen, d.h. an 60 \% aller Schulen in öffentlicher Trägerschaft, eingebracht. Auffällig ist, dass dabei auch drei Grundschulen das Niederdeutsche etabliert hatten. Dass auch die Schulen in freien Trägerschaften das Niederdeutsche im Schulalltag berücksichtigen, konnte nicht bestätigt werden. Der Vergleich mit dem internen Bericht „Niederdeutsch“ des Staatlichen Schulamtes Greifswald hat gezeigt, dass seit 2003 die Anzahl der Schulen mit einem Niederdeutschangebot konstant geblieben ist. Vor dem Hintergrund der Verwaltungsvorschrift „Niederdeutsch“ kann der Anteil kommunaler Schulen mit Niederdeutschangebot in Greifswald allerdings nicht zufrieden stellen, zumal an diesen Schulen das Niederdeutsche keineswegs wie gefordert als durchgängiges Unterrichtsprinzip realisiert wurde. Niederdeutsch wurde vielmehr nur über einzelne Unterrichtseinheiten vor allem in den Deutschunterricht integriert, seltener in den Musikunterricht und in den Sachunterricht der Grundschule. Darüber hinaus boten Greifswalder Schulen das Niederdeutsche allerdings auch in den schulischen Zusatzangeboten des Horts, der sogenannten „Mittagsfreizeit“, der Vollen Halbtagsschule und der Ganztagsschule an.

Auch in der hier exemplarisch näher betrachteten Greifswalder Schule wurde das Niederdeutsche in vielfältigen Angebotsformen vermittelt. Es wurde hier einerseits in jeder Klassenstufe jeweils über zwei Unterrichtseinheiten im regulären Deutschunterricht behandelt, andererseits gab es Niederdeutschkurse im Wahlpflichtbereich und in der betreuten Mittagsfreizeit. Die Niederdeutschangebote außerhalb des regulären Deutschunterrichts erreichten an der Schule aber nur wenige Schüler_innen (etwa 5,5 \% der Schülerschaft). Von den unmittelbar beteiligten Lehrkräften wurden diese verschiedenen Angebote und ihr Stellenwert im Unterrichtsprofil der Schule unterschiedlich bewertet. Im Deutschunterricht sei die Integration des Niederdeutschen nach Einschätzung von der Lehrerin $\mathrm{H}$ inzwischen "normal“ und zu einer Routine geworden, die durch den Rahmenplan gut abgesichert sei und - so ihre Vermutung - auch von Kolleg_innen regelmäßig „mit mehr oder weniger Enthusiasmus“ umgesetzt werde. Die im 
Wahlpflichtbereich und in der Mittagsfreizeit tätige Frau C beurteilt 2011 den Stellenwert des Niederdeutschen an derselben Schule dagegen skeptischer. Die Relevanz des Niederdeutschunterrichts sei durch die Aufhebung der Benotung der entsprechenden Wahlpflichtkurse deutlich gesunken. In den Angeboten der Mittagsfreizeit sei ergiebige Sprachvermittlung weder vorgesehen noch von den Schüler_innen gewollt. Ein ernsthaftes Engagement für das Niederdeutsche vermisste sie an ihrer Schule.

Die Befragung hat deutlich gemacht, wie sehr die Umsetzung des Niederdeutschunterrichtes an den Schulen von dem persönlichen Engagement und der Sprachkompetenz der einzelnen Lehrkräfte abhängt. Die mangelnde Sprachkompetenz der Lehrkräfte wurde wiederholt als Begründung von Greifswalder Schulen dafür angeführt, dass sie kein Niederdeutschangebot bereitstellen könnten. Auch für die hier exemplarisch untersuchte Schule wird von einer Gewährsperson angegeben, dass die mangelnde Sprachkompetenz für ihre Kolleg_innen eine „Hemmschwelle“ für die Behandlung des Niederdeutschen darstelle. Beide Interviewpartnerinnen, die an der Schule Niederdeutsch unterrichten, räumen ein, dass sie ihre eigene Sprachkompetenz eingeschränkt sehen, schildern aber Strategien, wie sie diesen Mangel für den Bedarf des Niederdeutschunterrichts zu kompensieren suchen. Festzuhalten ist, dass das Niederdeutschangebot auch aus der Perspektive der Lehrenden Fremdsprachenunterricht ist.

Das Engagement für die Vermittlung des Niederdeutschen scheint sich vor allem aus der persönlichen Motivation einzelner Lehrkräfte zu speisen. So verweisen beide Interviewpartnerinnen auf ihre enge familiäre Bindung an das Niederdeutsche und sehen es als Teil der regionalen Identität. Vor der eigenen Erinnerung an den familiären Sprachgebrauch insbesondere der Großelterngeneration wird der gegenwärtige Entwicklungsstand des Niederdeutschen als drohender „Verlust“ der Sprache konzeptualisiert. Die starke Motivation für die schulische Vermittlung des Niederdeutschen ist bei beiden Probandinnen demgemäß der Versuch, die Sprache vor dem „Vergessen“ zu bewahren und sie „am Leben zu halten“.

Diese Motivation der engagierten Lehrenden deckt sich weitgehend mit den sprachpolitischen Intentionen der Sprachencharta und der Verwaltungsvorschrift „Niederdeutsch in der Schule“ des Landes Mecklenburg-Vorpommern. Es scheint allerdings sehr fraglich, ob das gegenwärtige Niederdeutschangebot nach Umfang und Art seiner Realisierung geeignet ist, das Ziel des Spracherhalts erfolgreich umzusetzen. Entgegen den sprachpolitischen Absichtserklärungen und der Verwaltungsvorschrift wird Niederdeutsch an den kommunalen Schulen Greifswalds bis heute weder als eigenständiges Unterrichtsfach noch konsequent als 
durchgängiges Unterrichtsprinzip angeboten. Es wird an einer Reihe von Schulen öffentlicher Trägerschaft sogar gar nicht unterrichtet. An den Schulen mit Niederdeutschangebot wird Niederdeutsch nur in begrenzten Unterrichtseinheiten in wenigen Schulfächern, in erster Linie im Deutschunterricht, behandelt. In regulären Fächern wird das Niederdeutsche also, wenn überhaupt, nur partiell unterrichtet. Im Übrigen werden die Niederdeutschangebote in den Randbereich des Schulalltags in Kursen platziert, die für die Schüler_innen wenig Relevanz haben. Die Schülerzahlen in diesen außerfachlichen Niederdeutschangeboten dürften dementsprechend nicht nur an der beispielhaft untersuchten Schule gering ausfallen. Zudem kann in diesen Angeboten noch nicht einmal eine schuljährliche Realisierung gesichert werden, da ein Kurs häufig erst bei einer Teilnehmerzahl von drei Schüler_innen geöffnet wird. Der institutionell festgelegte Stellenwert des Niederdeutschen in der Schule entscheidet daher wesentlich über eine kontinuierliche Weiterführung. Ein langfristig erfolgreicher Spracherwerb könnte aber nur im Rahmen eines kontinuierlichen Angebots gewährleistet werden, in welchem die Bildungskette nicht abbricht.

Fraglich ist auch, ob die Art der Realisierung des Niederdeutschangebots dem Ziel der Spracherhalts gerecht werden kann. Bedingt schon durch die allgemein eher geringe Sprechkompetenz unter den heutigen Lehrkräften beläuft sich der Niederdeutschunterricht offenbar in der Hauptsache auf Textarbeit und Leseübungen. In Bereichen wie der Mittagsfreizeit ist der Anspruch der Sprachvermittlung noch wesentlich geringer. Das aktive Sprechen der Sprache wird demgemäß kaum gefördert und keinesfalls wird das Niederdeutsche als Unterrichtssprache eingesetzt. Angesichts ähnlicher Beobachtungen stellte Arendt 2009 die These auf, dass die niederdeutsche Sprachenpolitik sich sogar kontraproduktiv auf den Sprachgebrauch auswirke, da mehr über das Niederdeutsche als auf Niederdeutsch gesprochen werde. ${ }^{42}$

Es ist unbedingt anzuerkennen, dass das dargestellte Niederdeutschangebot an den Greifswalder Schulen geeignet ist, metasprachliches Wissen über das Niederdeutsche zu vermitteln und bei den Schüler_innen Interesse für die Regionalsprache zu wecken. Ein engagiertes Niederdeutschangebot kann gewiss dazu beitragen, positive Einstellungen gegenüber dem einstmals schlecht angesehenen Niederdeutschen zu verbreiten und zu festigen. Die Spracheinstellungsforschung zeigt allerdings auch, dass die positive Einstellung gegenüber einer Sprache keineswegs zwangsläufig auch deren aktiven Gebrauch befördert. ${ }^{43}$ Nur

42 Vgl. Arendt 2009, S. 40 und Arendt 2012, S. 113-115.

43 Vgl. Auer/ Hinskens 1996, S. 21-22, vgl. Arendt 2012, S. 102.

Birte Arendt, Andreas Bieberstedt and Klaas-Hinrich Ehlers - 978-3-631-71893-3 
der aktive Gebrauch einer Sprache ist aber wesentlich für deren langfristigen Erhalt. Unterrichtsangebote, die sich hauptsächlich in einem „Sprechen über das Niederdeutsche" erschöpfen, scheinen wenig geeignet, den aktiven Spracherwerb zu unterstützen und bergen überdies die Gefahr, eine Konservierung des Sprachstands zu begünstigen. Wandlungen und Anpassungen an kommunikative Bedürfnisse sind jedoch unabdingbare Voraussetzungen für das Überleben einer Sprache. Der institutionelle Sprachenschutz kann in der gegenwärtigen Realisierung im übertragenen Sinne zum Denkmalschutz degradieren.

Die aktuell geplanten und noch in Bearbeitung befindlichen sprachenpolitischen Maßnahmen zeigen die Bemühungen des Landes Mecklenburg-Vorpommern, die vorhandenen Defizite durch Umstrukturierung und Erneuerung der Verwaltungsvorschrift und mittels eines künftigen Landessprachplans zu reduzieren. Dies zeigt zudem, dass das Land sich weiterhin in einem Entwicklungsprozess des Sprachenschutzes befindet. Es ist meines Erachtens unabdingbar, die Motivation der Schüler_innen zu einem aktiven Gebrauch des Niederdeutschen weiter zu steigern. Dies kann z. B. geschehen, indem das Niederdeutsche als ein gleichwertiges und damit versetzungsrelevantes Unterrichtsfach in den Lehrplan eingebunden wird und gleichrangig neben anderen Fremdsprachen wählbar ist, wie es das in Kap. 2 angesprochene Modellprojekt von 2016 vorsieht. Durch die Gleichstellung in Bezug auf andere moderne Fremdsprachen erfährt das Niederdeutsche eine erkennbare Aufwertung.

\section{Literaturverzeichnis}

Abitur op Platt. Niederdeutsch-Gymnasien und -Gesamtschulen stehen fest. [Online-Ressource: http://www.bildung-mv.de/aktuell/2016/abitur-up-plattniederdeutsch-gymnasien-und-gesamtschulen-stehen-festpage/index.html (Stand: 12.12.2016)]

Alexander, Dittmar: Niederdeutsch. [interner Bericht des Staatlichen Schulamtes Greifswald]. Unveröffentlichtes Manuskript. o. O. 2008.

Arendt, Birte: Krankgepflegt? Was Sprachenpolitik bewirken kann. In: Aptum. Zeitschrift für Sprachkritik und Sprachkultur 3, H.1 (2009), S. 38-60.

Arendt, Birte: Niederdeutschdiskurse. Spracheinstellungen im Kontext von Laien, Printmedien und Politik. Berlin 2010.

Arendt, Birte: Niederdeutschdiskurse. Spracheinstellungen im Kontext von Laien, Printmedien und Politik. In: Niederdeutsches Jahrbuch 135 (2012), S. 101-117.

Auer, Peter/Hinskens, Frans: The convergence and divergence of dialects in Europe. New and not so new developments in an old area. In: Sociolinguistica 10 (1996), S. 1-30. 
Flick, Uwe/Kardorff, Ernst von/Steinke, Ines: Was ist qualitative Forschung? Einleitung und Überblick. In: Flick, Uwe/Kardorff, Ernst von/Steinke, Ines (Hrsg.): Qualitative Forschung. Ein Handbuch. Reinbek bei Hamburg 2010, S. 13-29.

Hopf, Christel: Qualitative Interviews - ein Überblick. In: Flick, Uwe/Kardorff, Ernst von/Steinke, Ines (Hrsg.): Qualitative Forschung. Ein Handbuch. Reinbek bei Hamburg 2010, S. 349-360.

Jürgens, Carolin: Niederdeutsch im Wandel. Sprachgebrauchswandel und Sprachwahrnehmung in Hamburg. Hildesheim/Zürich/New York 2015.

Kelle, Udo/Erzberger, Christian: Qualitative und quantitative Methoden: kein Gegensatz. In: Flick, Uwe/Kardorff, Ernst von/Steinke, Ines (Hrsg.): Qualitative Forschung. Ein Handbuch. Reinbek bei Hamburg 2010, S. 299-309.

Landesprogramm: , Meine Heimat - Mein modernes Mecklenburg-Vorpommern. ‘ Heimathafen - Heimatkunde - Heimatsprache - Heimatbildung - Heimatkultur. 2016. [Online-Ressource: http://www.regierung-mv.de/serviceassistent/_ php/download.php?datei_id=1573584 (Stand: 12.10.2016)]

Langenfeld, Christine: Artikel 8. In: Boysen, Sigrid/Engbers, Jutta/Hilpold, Peter/ Körfgen, Marco/Langenfeld, Christine/Rein, Detlev/Richter, Dagmar/Rier, Klaus: Europäische Regional- oder Minderheitensprachen. Handkommentar. Zürich/St. Gallen 2011, S. 183-221.

Lucius-Hoene, Gabriele/Deppermann, Arnulf: Narrative Identität und Positionierung. In: Gesprächsforschung 5 (2004), S. 166-183. [Online-Ressource: http://www.gespraechsforschung-online.de/heft2004/heft2004.html (Stand: 12.10.2016)]

Möller, Frerk: Plattdeutsch im 21. Jahrhundert. Bestandsaufnahme und Perspektiven. Mit einem Aufsatz von Michael Windzio. Leer 2008.

Osnowski, Stefan: Niederdeutsch in den Deutschlehrplänen der DDR. In: Herrmann-Winter, Renate (Hrsg.): Heimatsprache zwischen ideologischer Einbindung und Ausgrenzung. Frankfurt a. M. 1998, S. 271-279.

Piontkowski [Fink], Anne: Sprachenpolitik im Bereich der Bildung am Beispiel der Regionalsprache Niederdeutsch in Mecklenburg-Vorpommern. Unveröff. Staatsexamensarbeit Greifswald 2012.

Schaub, Horst/Zenke, Karl (Hrsg.): Wörterbuch Pädagogik. München 2007.

Schulgesetz für das Land Mecklenburg-Vorpommern. Vom 13. Februar 2006, geändert durch Gesetz vom 16.2.2009. [Online-Ressource: http://www.kulturellebildung-mv.de/wp-content/uploads/2011/12/Schulgesetz_MV_2009.pdf (Stand: 12.12.2016)]

Selting, Margret/Auer, Peter/Barden, Birgit/Bergmann, Jörg/Couper-Kuhlen, Elizabeth/Günthner, Susanne/Meier, Christoph/Quasthoff, Uta/Schlobinski, Peter/Uhmann, Susanne: Gesprächsanalytisches Transkriptionssystem (GAT). 
[Online-Ressource: http://uni-potsdam.de/u/slavistik/vc/rlmprcht/textling/ comment/gat.pdf (Stand: 12/2011)].

Sprachencharta 1999: Europäische Charta der Regional- oder Minderheitensprachen. Nichtamtliche deutsche Übersetzung. [Online-Ressource: http:// conventions.coe.int/Treaty/ger/Treaties/Html/148.htm (Stand: 12.12.2016]

Verwaltungsvorschrift: Niederdeutsch in der Schule. Verwaltungsvorschrift des Ministeriums für Bildung, Wissenschaft und Kultur (vom 09. März 2004). [Online-Ressource: http://service.mvnet.de/_php/download.php?datei_id=48 (Stand: 12.12.2016)].

Vierter Bericht des Sachverständigenausschusses über die Anwendung der Charta in Deutschland 2011. [Online-Ressource: http://www.coe.int/t/dg4/ education/minlang/Report/EvaluationReports/GermanyECRML4_de.pdf (Stand: 12/2011)]. 
Birte Arendt

\title{
Niederdeutsch als Studienfach - Formen, Entwicklungen und studentische Motivationen am Beispiel der Universität Greifswald
}

\begin{abstract}
While there is a wide range of studies focussing on Low German from a structural or sociolinguistic point of view, considerably less studies discuss Low German as a subject of study at university. Due to several changes in this field and the general importance of universities for teacher training, research on the different forms and motivation to study Low German is still an urgent desideratum.

Little is known about the reasons for choosing Low German as a course of study. Therefore it is the paper's objective to shed light on both the changing forms in which Low German can be taken up as a course of study as well as the motivation of the students who actually use these opportunities. This is demontrated by the analysis of a case example, the university of Greifswald in Mecklenburg-Western Pomerania.

Drawing on an online survey among students of Low German who were asked to explain their reasons of choosing this course of study, two main research questions are addressed: (1) Why did students choose Low German? (2) Which individual background was influencing their decision? The results indicate a type of student whose choice of study is affected by individual preferences, social relationships and considerations on usefulness. The results will be discussed in relation to sociological concepts of generations, particularly of the currently discussed "generation y".
\end{abstract}

\section{Einleitung: Niederdeutsch in Bildungsinstitutionen}

Der nachfolgende Beitrag fokussiert das Niederdeutschstudium an der Universität in Greifswald in seinen Formen, seiner Entwicklung und aus der Perspektive studentischer Motivationen. Er beleuchtet mit einer Bildungsinstitution einen unter sozio-pragmatischer Perspektive zentralen Einflussfaktor der niederdeutschen Sprachgeschichte. ${ }^{1}$

1 Der Zusammenhang zwischen Sprache(n) und Institutionen wird evident, wenn wir erstens die Aufrechterhaltung und Etablierung von Institutionen auf der Basis sprachlicher Handlungen ansiedeln, zweitens Institutionen mit Kiesendahl 2011 und Ehlich/Rehbein 1994 als Formen geregelten sozialen Handelns mit eingeschränkten individuellen (auch verbalen) Handlungsmöglichkeiten zur Prozessierung je spezifischer Bündel gesellschaftlicher Zwecke verstehen und drittens davon ausgehen, dass institutionelle Kommunikation sich aus den rollenbezogenen typisierten sprachlichen 
Im Folgenden wird das Niederdeutschstudium sowohl aus der Perspektive der institutionellen Agenten als auch aus der Perspektive der studentischen Klienten beschrieben. Aus der Agentenperspektive wird das Niederdeutschstudium mit seinen Studienangeboten dargestellt (Kap. 2). Gleichwohl ist die Wahl eines Studienfaches wie des Niederdeutschen immer auch die Entscheidung von Individuen. Insofern gehört zur Beschreibung institutioneller Zusammenhänge auch die Rekonstruktion der Klientensicht. Deshalb sollen in diesem Beitrag Daten vorgestellt werden, die die Frage beantworten, warum sich welche Studierenden für ein Niederdeutschstudium entschieden haben (Kap. 3). Das Ziel besteht in einer möglichen Typisierung aktuell Studierender auf der Basis ihrer Studienmotive. Abschließend werden die Befunde zusammengefasst und mit sozialwissenschaftlichen Theorien zur Generationsspezifik interpretiert (Kap. 4).

\subsection{Niederdeutsch an Universitäten}

Sowohl die Rolle des Niederdeutschen in den Bildungsinstitutionen selbst als auch der Stand der wissenschaftlichen Beschreibung der institutionellen Niederdeutschvermittlung ist durchaus heterogen. Während hinsichtlich der Erfassung der schulischen Angebote eine relativ breite Daten- und Forschungslage zu verzeichnen ist, bildet der universitäre Bereich ein deutliches Forschungsdesiderat. ${ }^{2}$

Bieberstedt beschreibt die universitäre Situation des Niederdeutschen als ambivalent: Einerseits sei der Fortbestand des Niederdeutschen als akademische Fachdisziplin ebenso positiv zu bewerten wie die Etablierung in der Lehre in verschiedenen, vor allem Integrations- und Schwerpunktmodellen. ${ }^{3}$ So bieten derartige Schwerpunktsetzungen im Niederdeutschen u. a. die Universitäten Greifswald und Flensburg in den Lehramtsstudiengängen und die Universität Oldenburg in den Bachelor- und Master-Studiengängen an sowie die Universität Rostock in Form eigenständiger Module. ${ }^{4}$ Andererseits unterliegt das Universitätsfach

Handlungsmustern der Agenten und Klienten zusammensetzt. Institutionen können z. B. regeln, in welcher Sprache bzw. Varietät in Form welcher Vertextungsmuster über welchen Sachverhalt sprachlich gehandelt wird.

2 Vgl. als Ausnahmen Gernentz 1998; Möhn 2005; Arendt 2009a; Bieberstedt 2009.

3 Vgl. Bieberstedt 2009, S. 34.

4 Vgl.z. B.zur Darstellung des Lernbereichs an der Universität Flensburg [Online-Ressource: https://www.uni-flensburg.de/portal-studium-und-lehre/studiengaenge/master/ lehramt-an-grundschulen/lernbereiche-wahlpflicht/niederdeutsch/\#unfold-c9733 (Stand: 12.12.2016)] und des Zertifikats Niederdeutsch an der Universität Oldenburg [Online-Ressource: https://www.uni-oldenburg.de/germanistik/niederdeutsch/ niederdeutsch/niederdeutsch-oldenburg/ (Stand: 12.12.2016)]. 
sowohl auf der Ebene der Professuren als auch auf der des wissenschaftlichen Mittelbaus finanziellen Kürzungen. Festzustellen sei auch, dass im Fach Niederdeutsch ein deutlicher Schwerpunkt auf sprachwissenschaftlichen Themen liege. Das ist insofern problematisch, als dass zukünftig insbesondere auch didaktische Themen der Niederdeutschvermittlung zu bearbeiten sind, da die Schule die maßgebliche Instanz der künftigen Niederdeutschvermittlung bildet (vgl. Kap. 1.2). ${ }^{5}$

Wenngleich die Universität im Zentrum des Beitrags steht, sollen im Kapitel 1.2 auch ausgewählte Etappen der Beziehung zwischen der Institution Schule und Niederdeutsch beschrieben werden, da sie als historisch relevante Basis auch für universitäre Angebote zu betrachten sind. Erstens richten sich viele universitäre Niederdeutschangebote an Lehramtsstudierende. ${ }^{6}$ Zweitens spielt für deren Akzeptanz die institutionelle Einbindung von Niederdeutsch an den Schulen eine entscheidende Rolle. Und wenn Niederdeutsch als Unterrichtsfach an den Schulen etabliert wird, so muss die Universität drittens nicht nur Studienangebote für angehende NiederdeutschlehrerInnen vorhalten, sondern auch die Korpusplanung aktiv begleiten und didaktische Konzepte zur Sprachvermittlung erarbeiten.

\subsection{Niederdeutsch an den Schulen}

Die schulische Einbindung in Form eines eigenständigen Unterrichtsfaches Niederdeutsch ist bzw. wird aktuell in den Bundesländern Hamburg, SchleswigHolstein und Mecklenburg-Vorpommern realisiert. ${ }^{7}$ Die Schule wird - aktuellen Umfragen zufolge - als entscheidende Instanz der Sprachvermittlung und damit auch des Spracherhalts angesehen: So sprachen sich in der Erhebung des Instituts für niederdeutsche Sprache (INS) von 200773 \% der Befragten für eine stärkere Plattdeutschförderung aus und befürworteten mit $81 \%$ - und damit den meisten Stimmen - insbesondere „mehr Lehrangebote in den Schulen“. 8 Arendt weist in einer Studierendenbefragung nach, dass sich 68 \% von 69 befragten Germanistikstudierenden für die schulische Vermittlung des Niederdeutschen aussprechen

5 Vgl. Bieberstedt 2009, S. 34-35; Arendt 2009a, S. 47.

6 Vgl. Bieberstedt 2009. Einen guten Einstieg in die Recherche der aktuellen Niederdeutschangebote an den norddeutschen Hochschulen bietet auch eine entsprechende Seite des Instituts für niederdeutsche Sprache INS in Bremen, vgl. Institut für niederdeutsche Sprache 2016.

7 Vgl. Ehlers/Goltz/Henschen 2013.

8 Möller 2008, S. 55. 
und die Sprache eine hohe Wertschätzung genießt. ${ }^{9}$ Die Sprache soll - laut den Befragten - vermittelt werden, wenngleich sich die Germanistikstudierenden selbst aufgrund ihrer geringen Sprechkompetenz (nur $14 \%$ sprechen sehr gut und gut niederdeutsch) dazu nicht in der Lage sehen. Die Schule nimmt insofern eine zentrale Rolle als zukünftiger Ort des institutionell gelenkten Spracherwerbs ein und die Universität muss u. a. mit der Ausbildung von NiederdeutschlehrerInnen diesem Umstand Rechnung tragen.

Möhn zeichnete in den 1980er Jahren noch das Bild einer „mehrhundertjährigen Problemgeschichte“, einer Geschichte der fortschreitenden Verdrängung des Niederdeutschen aus der Schule mit der Zielvarietät „Hochdeutsch“ ab dem 17. Jahrhundert. ${ }^{10} \mathrm{Ab}$ den 1980er Jahren zeigt sich im Zusammenhang mit der sogenannten "Mundartwelle“ in der damaligen BRD zugleich eine zunehmende Einbindung des Niederdeutschen an den Schulen. Allerdings erfolgte die schulische Integration der Regionalsprache eher als durchgängiges Unterrichtsprinzip, nach welchem Niederdeutsch in verschiedenen Fächern, maßgeblich aber dem Deutschunterricht, thematisiert werden sollte. ${ }^{11}$ Rösler weist darauf hin, dass das Niederdeutsche in der DDR zwar nie Unterrichtsfach gewesen sei, aber auf der Basis individueller Angebote von einzelnen engagierten LehrerInnen - meist außerhalb des regulären Unterrichts - auch Teil der schulischen Bildungsinstitution war. ${ }^{12}$ Unisono urteilen Hennings [u. a.], dass Niederdeutsch in der schulischen Praxis „in hohem Maße ehrenamtlich betrieben“ wird. ${ }^{13}$

Für das 21. Jahrhundert entwerfen Ehlers/Goltz/Henschen ein ganz neues Bild: Niederdeutsch wird nicht mehr aus der Schule verdrängt, sondern es erfährt aktuell eine zunehmende Integration in die Schule, partiell sogar als eigenes Schulfach. ${ }^{14}$ Als wichtige Ursachen dafür sind sowohl die gesunkenen Sprecherzahlen als auch die Anerkennung des Niederdeutschen als Regionalsprache im Sinne der

9 Vgl. Arendt 2006, S. 254. Inwiefern die aktuelle Umfrage des INS aus dem Jahr 2016 diese Befunde bestätigt oder revidiert, werden die Ergebnisse zeigen. Bisher liegen lediglich Daten zur aktiven und passiven Kompetenz vor, vgl. [Online-Ressource: http://www.ins-bremen.de/de/sprache/umfrage-2016/erste-ergebnisse.html (Stand: 12.12.2016)].

10 Möhn 1983, S. 631.

11 Vgl. Feldmann 1997.

12 Vgl. Rösler 1998. Osnowski 1998 zeigt in seiner Analyse von Deutschlehrplänen aus der DDR einerseits, dass das Niederdeutsche dort kaum erwähnt wird, dass aber andererseits ab den 1946er Jahren kontinuierlich auf Mundart und Umgangssprachen verwiesen wurde, was sich insbesondere ab den 1980er Jahren steigert.

13 Vgl. Hennings [u. a.] 2005, S. 7.

14 Ehlers/Goltz/Henschen 2013; vgl. den Beitrag von Fink in diesem Band. 
„Europäischen Charta für Regional- oder Minderheitensprachen“ zu nennen. Hier entfaltet insbesondere der Artikel 8 der Charta seine Wirkung, der die Implementierung der Sprache im Bildungssektor regelt. ${ }^{15}$

\section{Niederdeutsch an der Universität Greifswald: Vom Beifach und zurück}

Im Folgenden soll die neuere Entwicklung des Niederdeutschstudiums exemplarisch an der Universität Greifswald untersucht werden und dabei ein Schwerpunkt auf die Lehramtsausbildung gelegt werden.

\subsection{Studienmodelle im Verlauf}

An der Universität Greifswald war es seit 1993 ununterbrochen möglich, Niederdeutsch als Studienfach zu belegen. Allerdings wechselten die Formen des Studiums mehrfach: erstens aufgrund von Veränderungen der aktuellen Hochschulentwicklung, zweitens in Reaktion auf geänderte Arbeitsmarktbedingungen z. B. für angehende LehrerInnen und drittens aufgrund aktueller Entwicklungen der niederdeutschen Sprache selbst. Wie die Abbildung 1 verdeutlicht, ${ }^{16}$ wurden an der Universität dafür die folgenden Studiengänge konzipiert, erprobt und geschlossen: Von 1993 bis 2007 und ab 2012 gab bzw. gibt es ein Beifach-Studium Niederdeutsch, wobei das Beifach ab 2012 - als Ergebnis der Bologna-Reform modularisiert zu studieren ist. Von 2009 bis 2012 wurde mit dem Schwerpunkt Niederdeutsch ein integratives Modell im Lehramtsstudium Deutsch angeboten, das im Vergleich zu vorherigen und anderen Niederdeutschstudienformen hohe Einschreibzahlen aufwies. ${ }^{17}$

Im Rahmen des Magisterstudiums wurde ab dem Wintersemester 1997/98 Niederdeutsch als Magister-Nebenfach angeboten, das einen Gesamtumfang von 36 Semesterwochenstunden aufwies. Dieses Studienangebot wurde allerdings nur von insgesamt vier Studierenden genutzt. Noch weniger Einschreibungen hatte der Bachelor-Teilstudiengang Niederdeutsch mit nur zwei Studierenden und der Master Niederdeutsch, der ab dem Wintersemester 2007/08 angeboten wurde, mit nur einem Studenten. ${ }^{18}$

15 Vgl. Ehlers/Goltz/Henschen 2013; Möller 2008 und Institut für niederdeutsche Sprache 2008.

16 Die Abbildung findet sich in einer älteren Fassung in Arendt 2009a.

17 Die genannten Studienangebote im Lehramt Deutsch werden im Kap. 2.2 genauer besprochen.

$18 \mathrm{Zu}$ den Studieninhalten vgl. Arendt 2009a. 
Die heterogene Einschreibungslage fordert Erklärungen und verdeutlicht zugleich die eingangs erwähnte Notwendigkeit, die Motivation der Studierenden systematisch zu erfassen und zu analysieren. Insbesondere das Kapitel 3 fragt deshalb danach, was die Studierenden motiviert hat, sich in den Schwerpunkt einzuschreiben und diesen partiell auch abzuschließen. ${ }^{19}$ Lag es an der integrativen Studienform, an einem neuen Bewusstsein für die niederdeutsche Sprache, an sprachpolitischen Änderungen im Zuge der Charta? Um diese Fragen fundiert zu beantworten, wurde unter den Studierenden des Schwerpunktes Niederdeutsch eine Umfrage durchgeführt.

Abbildung 1: Entwicklung der Niederdeutsch-Studienmodelle an der Universität Greifswald

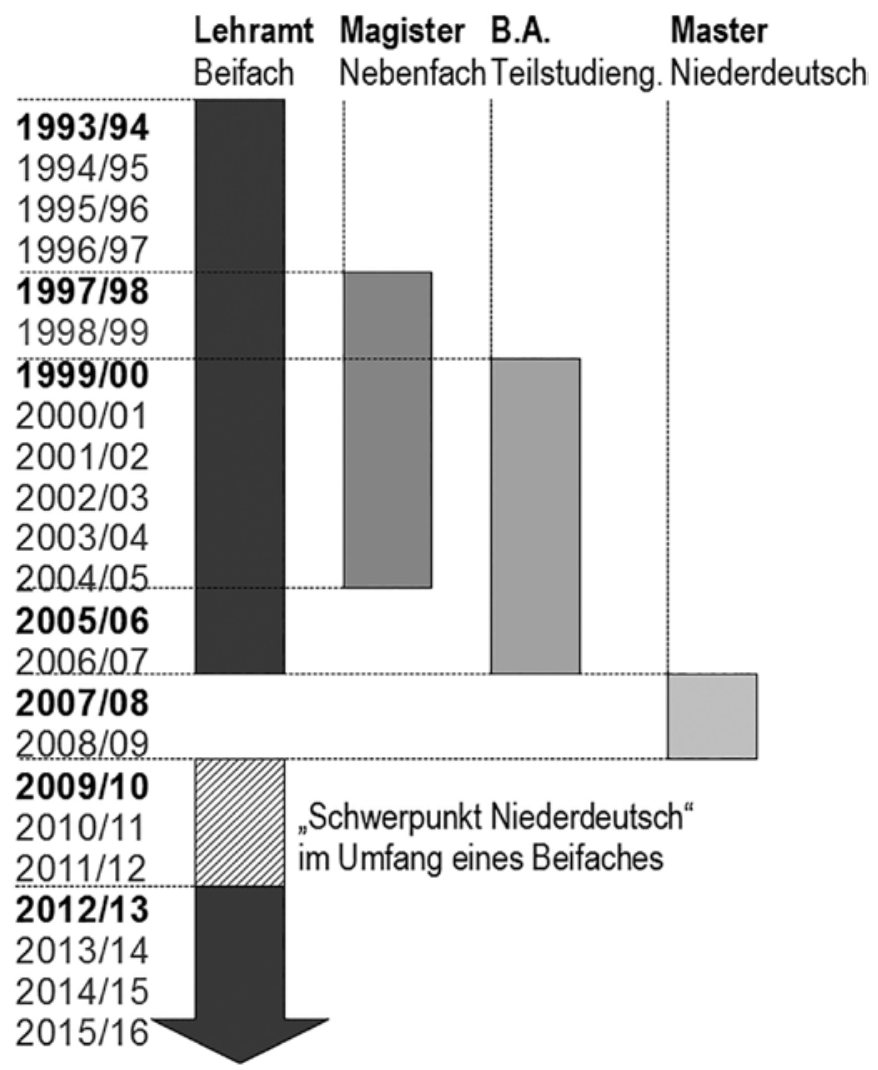

19 Insgesamt wurden bis 12/2016 zehn Zeugnisse ausgestellt, weitere sind in Vorbereitung. 


\subsection{Niederdeutsch im Lehramtsbereich}

\subsubsection{Schwerpunkt Niederdeutsch}

Der Schwerpunkt Niederdeutsch konnte von Studierenden des Lehramts Deutsch integrativ und studienbegleitend belegt werden. Integrativ bedeutet hierbei, dass Studienleistungen in Form von Pro- und Hauptseminararbeiten aus dem Lehramt Deutsch und dem Schwerpunkt Niederdeutsch partiell wechselseitig anerkannt wurden. Gleichwohl war das Anforderungsspektrum der fünf Module, bestehend aus (1) Spracherwerb, (2) Mittelniederdeutsche Sprache und Literatur, (3) Niederdeutsche Literatur, (4) Sprachwissenschaftliche Grundlagen des Niederdeutschen und (5) Niederdeutsch in der Schule, sehr umfangreich. Insbesondere das Modul 5 erforderte von den Studierenden neben sprachlichen auch didaktische Fähigkeiten, da in schulpraktischen Übungen an Greifswalder Schulen Niederdeutsch zu unterrichten war. Das Zertifikat über die Absolvierung des Schwerpunktes wurde von der philosophischen Fakultät ausgestellt und vom Lehrerprüfungsamt als reguläres Beifach anerkannt.

Dieses integrative Modell wurde sehr gut von den Studierenden angenommen. So waren pro Semester im Schnitt vier bis maximal zehn (Sommersemester 2010) Einschreibungen zu verzeichnen, so dass sich insgesamt 54 Studierende im Laufe von fünf Jahren, in denen der Schwerpunkt bisher angeboten wurde, angemeldet hatten. ${ }^{20}$ Damit haben allein in einem Semester mehr Studierende dieses Niederdeutschangebot gewählt als im Gesamtzeitraum der vorherigen Studienangebote zum Magister- und Bachelor-Nebenfach oder zum Masterstudium.

\subsubsection{Modularisiertes Beifach}

Seit dem Wintersemester 2012/13 ist es möglich, das Beifach Niederdeutsch im Lehramtsstudiengang zu studieren. Das Studium soll dazu befähigen, Niederdeutschunterricht an Schulen zu erteilen. Auch dieses Modell ist insofern integrativ angelegt, als dass die Studierenden des Lehramtes Deutsch von den für ein Beifach obligatorischen 30 Leistungspunkten nur 15 Leistungspunkte erreichen müssen, da die Basismodule zugleich Bestandteile des regulären Deutschstudiums sind. Insgesamt sind somit lediglich die drei Module (1) Niederdeutsche Sprachgeschichte, (2) Regionale Varietäten und (3) Praxis Niederdeutsch zusätzlich zu

20 Wenngleich ab Wintersemester 2012/13 das Beifach Niederdeutsch im Rahmen des modularisierten Lehramtsstudiums angeboten wurde, war es für die Studierenden des nicht-modularisierten Lehramts weiterhin möglich, den Schwerpunkt zu wählen. Im Wintersemester 2014/15 erfolgte die vorerst letzte Einschreibung. 
studierenden. ${ }^{21}$ Im Vergleich zum Schwerpunkt Niederdeutsch ist das Anforderungsspektrum für die Studierenden geringer. Konstitutive Bestandteile sind aber auch hier der Spracherwerb und die schulpraktischen Übungen.

Der Studiengang wurde bisher von den Studierenden gut angenommen, wenngleich bisher keine Spitzeneinschreibungen wie beim Schwerpunkt Niederdeutsch zu verzeichnen sind. Aktuell sind acht Studierende in das Beifach Niederdeutsch eingeschrieben, davon fünf für das Lehramt Gymnasium und drei für das Lehramt Regionalschule. Zum Wintersemester 2016/17 erfolgten sieben Neueinschreibungen, was eine deutliche Steigerung bedeutet. ${ }^{22}$

\section{Studentische Motivationen zum Niederdeutschstudium}

Um die in Kapitel 1 gestellte Frage nach der Motivation der Studierenden zu beantworten und die Akzeptanz nicht allein quantitativ aus der Zahl der Einschreibungen bzw. von Studienabbrüchen zu ermitteln, wurde im Zeitraum MaiJuli 2015 eine Online-Umfrage unter Niederdeutsch-Studierenden der Universität Greifswald durchgeführt. ${ }^{23}$ Das Ziel bestand in der Rekonstruktion der Motivation und der individuellen (Klienten-)Perspektive der Studierenden des Schwerpunkt Niederdeutsch (vgl. Kap. 2.2.1). Im Zentrum stand die Beantwortung der folgenden motivationalen und sprachbiographischen Fragen: Warum studieren die Befragten Niederdeutsch? Welche persönlichen Voraussetzungen bringen sie mit? Welche Erfahrungen haben sie mit dem Studium gemacht?

Indem die Befragten gebeten wurden, ihr Verhalten (Studienfachwahl) rückblickend aus subjektiven Motiven herzuleiten, wurde eine konativ orientierte Einstellungskomponente ${ }^{24}$ erfragt. Insofern handelt es sich um eine „retrospektive Studie ${ }^{\text {“25 }}$, bei der ein vergangenes Verhalten verbal plausibilisiert wird. Inwiefern mit den Äußerungen tatsächlich die für die Person zentralen verhaltenssteuernden Aspekte erfasst wurden, lässt sich nicht sicher sagen. Es liegen vielmehr Daten

21 Vgl. PSO Beifach Niederdeutsch 2013.

22 Angaben des Studierendensekretariats der Universität Greifswald von 08/2016 sowie $11 / 2016$.

23 Ich danke den Studierenden Kristin Boos, Kristina Deibele und Annika Pinn, dass sie ihre Umfrage auch an meine Forschungsziele angepasst und mir die Daten zur Analyse zur Verfügung gestellt haben.

24 Zum Drei-Komponentenmodell von Einstellungen in der Sozialpsychologie vgl. einführend Bierhoff 2006, S. 327-329. Zu methodologischen Aspekten der Erhebung von attitudinalen Daten maßgeblich in Gesprächen vgl. Arendt 2014. Zu Einstellungsäußerungen zum Niederdeutschen unter Jugendlichen vgl. Arendt 2006; Arendt 2012.

25 Flick 2009, S. 255.

Birte Arendt, Andreas Bieberstedt and Klaas-Hinrich Ehlers - 978-3-631-71893-3 
vor, in denen die Befragten in den Antworten eine für sie und den Adressaten sinnvolle Ordnung der Vergangenheit entworfen haben. Trotz der Limitation geben die Daten erstens Einblicke in mögliche Plausibilisierungen für die Aufnahme eines Niederdeutschstudiums und erlauben zweitens eine motivational orientierte Typisierung aktueller Niederdeutschstudierender auf der Basis ihrer Motivationen. $^{26}$

\subsection{Daten und Methode: Induktive Typisierung anhand einer Online-Befragung}

Zur Umsetzung der Befragung wurde über das Internetportal SurveyMonkey eine Online-Umfrage entworfen, da es als für alle Beteiligten praktikabelste Lösung erschien und die Rücklaufquoten bei Online-Befragungen vergleichsweise hoch sind. Der aus nur sechs Fragen aufgebaute kurze Fragebogen erfasste im ersten Teil persönliche Daten (1. Geschlecht, 2. Alter, 3./4. Beginn des Studiums und des Niederdeutschstudiums und 5. Herkunftsraum). Der zweite Teil des Fragebogens fokussierte das Untersuchungsziel in einer offenen, sechsten Frage mit folgendem Erzählimpuls: „Warum hast Du angefangen, an der Ernst-Moritz-Arndt Universität Niederdeutsch zu studieren? Vertell uns Deine Geschichte - es gibt kein richtig oder falsch." Wir haben uns für diese Art des offenen Fragens entschieden, da es uns - wie in der qualitativen Sozialforschung zentral - um die Rekonstruktion individueller Relevanzsysteme ging, die methodisch adäquat mit Narrationen erhoben werden können. ${ }^{27}$ Die offene Frage erlaubt sowohl eine argumentative Themenentfaltung, initiiert durch die Etablierung von Begründungspflicht durch

26 Eine ähnliche, wenn auch inhaltlich anders ausgerichtete Typisierung nimmt Möller 1996 anhand der GETAS-Umfrage vor. Seine typisierende Bündelung von erhobenen Daten erscheint zielführend und hat die vorliegende Untersuchung - hinsichtlich dieses Aspektes - inspiriert.

27 Vgl. Mayring 2009, S. 469. Die Konzentration auf einen Erzählimpuls in Form einer offenen Frage ergab sich aus dem typischen Vorgehen, wie es auch in qualitativen Interviews gewählt wird. Hopf 2009, S. 350 betont, dass qualitative Interviews ein adäquates Erhebungsinstrument zur Rekonstruktion individueller Relevanzsysteme darstellt, wenn sie schreibt: „Durch die Möglichkeit, Situationsdeutungen oder Handlungsmotive in offener Form zu erfragen, Alltagstheorien und Selbstinterpretationen differenziert und offen zu erheben, und durch die Möglichkeit der diskursiven Verständigung über Interpretationen sind mit offenen und teilstandardisierten Interviews wichtige Chancen einer empirischen Umsetzung handlungstheoretischer Konzeptionen in Soziologie und Psychologie gegeben." Wenngleich die Möglichkeit einer diskursiven Verständigung im Online-Fragebogen fehlt, kann meines Erachtens die offene Frage die geschilderten Wissensbestände erheben. 
das Interrogativadverb warum, als auch eine narrative Beantwortung, initiiert durch die direktive Sprechhandlung mit dem verbum dicendi vertell im Imperativ aktiv.

Der Link zur Umfrage wurde an alle zum Zeitpunkt der Erhebung eingeschriebenen 47 Studierenden geschickt. Als zusätzliche Motivation zur Bearbeitung des Bogens wurden unter allen Antwortenden zwei Bücher verlost. Die Reduktion auf den spezifischen Personenkreis ergab sich folgerichtig aus der Untersuchungsfrage, stellt aber gleichwohl eine wichtige Einschränkung des Geltungsbereichs der Ergebnisse dar.

Die Datenbasis besteht aus insgesamt 26 beantworteten Fragebögen. Die Rücklaufquote liegt bei 55 \%: 18 der 26 Studierenden haben auf die offene Frage geantwortet und dabei überwiegend argumentative Vertextungsmuster gewählt. Im Durchschnitt wurde diese mit 82 Wörtern in jeweils durchschnittlich fünf Sätzen beantwortet. Insgesamt umfasst das Kernkorpus, d. h. die Antworten auf den Erzählimpuls der sechsten Frage, 1468 Wörter.

Kern der Analyse ist die qualitativ-quantitative Auswertung der Antworten zur Frage nach der Motivation für das Studium. Die genannten Aspekte wurden induktiv zu Kategorien gebündelt und typisiert und mit der Analysesoftware MAXQDA ausgewertet. Darüber hinaus erwies sich die Analyse niederdeutschsprachiger Phrasen innerhalb der Antworten als relevant.

\subsection{Ergebnisse}

Im Folgenden werden die Fragen dergestalt gebündelt ausgewertet, dass die Antworten auf die Fragen 1-5 zu den Primärdaten der Befragten im Subkapitel 3.2.1 zusammengefasst werden und die Antworten auf die offene Frage in Kap. 3.2.2. Diese Ordnung ergibt sich sowohl aus der unterschiedlichen Gewichtung der Fragen in Bezug auf die Untersuchungsfrage des Artikels nach der Motivation als auch bezüglich der Auswertbarkeit.

\subsubsection{Wer studiert Niederdeutsch?}

Die Angaben zu den Primärdaten geben Aufschluss darüber, woher die Studierenden stammen, die Niederdeutsch studieren, und welchen Alters und Geschlechts sie sind. Von den 26 Antwortenden waren 21, d. h. $80 \%$, weiblich und 5, d. h. $19 \%$, männlich. Diese Verteilung weicht nur minimal vom Geschlechterverhältnis der damaligen Schwerpunkt-Studierenden mit 15 \% männlichen Studierenden 
(7 von 47) ab. ${ }^{28}$ Insofern entspricht die Verteilung hinsichtlich des Geschlechts der Gesamtheit, die sie abbilden soll. Das heißt aber zugleich, dass die männlichen Studierenden sich mit $71 \%$ an der Umfrage beteiligt haben und die weiblichen mit nur $52 \%$. Davon waren mit 53 \% (14) die meisten Studierenden im Alter von 26-30 Jahren, $38 \%$ (10) zwischen 20 und 25 Jahren und $8 \%$ (2) älter als 30 Jahre. 25 von 26 haben ihr Niederdeutschstudium nicht mit dem Beginn ihres Deutschstudiums begonnen. Das entspricht den Empfehlungen des Musterstudienplanes. ${ }^{29}$

Von den befragten Studierenden stammen mit $42 \%$ (11) die meisten aus Mecklenburg-Vorpommern, was einem üblichen Trend der Lehramtsstudierenden entspricht. Sechs Studierende kommen aus den anderen nördlichen Bundesländern: aus Schleswig-Holstein (4), Hamburg (1) und Niedersachsen (1). Die übrigen Studierenden kommen wie folgt aus südlicher gelegenen neuen Bundesländern: Vier Studierende stammen aus Brandenburg, drei aus Berlin und zwei aus Sachsen-Anhalt.

Abbildung 2: Herkunft der Studierenden des Schwerpunkts Niederdeutsch (2015)

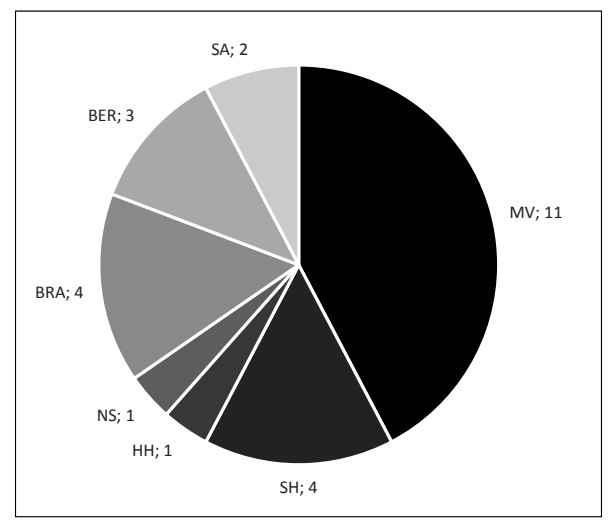

28 Diese geringe Menge an männlichen Studierenden entspricht den normalen Verhältnissen des Germanistikstudiums an der Universität Greifswald. Im Wintersemester waren $27 \%$ der Studierenden, die Deutsch als erstes Fach belegt haben, männlich, vgl. [Online-Ressource: https://www.uni-greifswald.de/fileadmin/uni-greifswald/1_ Universitaet/1.1_Information/1.1.6_Zahlen_und_Fakten/Studierende/WS_15_ Bericht.pdf (Stand: 12.12.2016)].

29 Vgl. SO Deutsch LA Gym 2009. 
Abbildung 2 visualisiert die Zahlen und verdeutlicht auch auf dieser Ebene die Dominanz (17 von 26) der Herkunft aus den nördlichen Bundesländern. Dieses - nicht überraschende - Merkmal ist somit charakteristisch für die Niederdeutschstudierenden. Die räumlich verankerte Varietät wird dominant von einer auch räumlich charakterisierten Gruppe studiert, deren inhaltliches Merkmal der Norden Deutschlands ist. Wenn wir auf der Grundlage der oben schon genannten INS-Umfrage von 2007 davon ausgehen, dass „den Norddeutschen“ und damit auch den Studierenden die Sprache Niederdeutsch auch vor dem Studium bekannt war, ${ }^{30}$ so scheint sich die Kenntnis der Sprache - nicht zwingend im Sinne einer aktiven Kompetenz - positiv auf die Aufnahme eines Niederdeutschstudiums auszuwirken.

\subsubsection{Warum wird Niederdeutsch studiert?}

Die Antworten auf die sechste - offene - Frage nach der Motivation ${ }^{31}$ für die Aufnahme des Niederdeutschstudiums wirken insofern relativ homogen, als dass keine $18^{32}$ individuell unterschiedlichen Gründe genannt werden. Vielmehr kristallisieren sich die folgenden sieben Motivationsbereiche - geordnet in absteigender Reihenfolge nach der Anzahl der Nennungen - heraus: ${ }^{33}$

1. individuelle Präferenzen $(n=12)$

2. soziale Beziehungen $(n=11)$

3. Nützlichkeit des Studiums $(\mathrm{n}=8)$

4. Spracherhalt $(\mathrm{n}=8)$

5. Studienform $(\mathrm{n}=7)$

6. Heimat $(n=6)$

7. eigener Spracherwerb $(n=5)$

30 Vgl. Möller 2008, S. 25.

31 Vgl. zu Fragen der Motivation zum Niederdeutschlernen an Volkshochschulen den Aufsatz von Reuter in diesem Band. Ein Vergleich der Daten zeigt viele Gemeinsamkeiten.

32 Innerhalb der 26 beantworteten Fragebögen wurde nur 18mal auch die offene Frage beantwortet (vgl. Kap. 3.1).

33 Es wurden keine Doppelkodierungen vorgenommen. Das heißt pro Antwort einer Gewährsperson wurde jeweils nur eine Kodierung für eine der genannten Motivationen (z. B. ,individuelle Präferenzen') vergeben, auch wenn die betreffenden Aussagen ausführlicher entwickelt wurden. Gleichwohl konnten dieselben Aussagen zusätzlich auch mit weiteren Motivationskategorien (z. B. ,soziale Beziehungen') kodiert werden, wenn derartige Gesichtspunkte in der Aussage der Gewährsperson ebenfalls berührt wurden. 
Abbildung 3 visualisiert die genannten Motivationsbereiche in ihren quantitativen Verhältnissen. Es lässt sich feststellen, dass mit dem hohen Anteil der individuellen Präferenzen (66 \%) und den sozialen Beziehungen (61 \%) dominant intrasubjektive Gründe als motivational wirksam benannt wurden.

\section{Abbildung 3: Verteilung der Gründe für das Niederdeutschstudium (absolute Zahlen)}

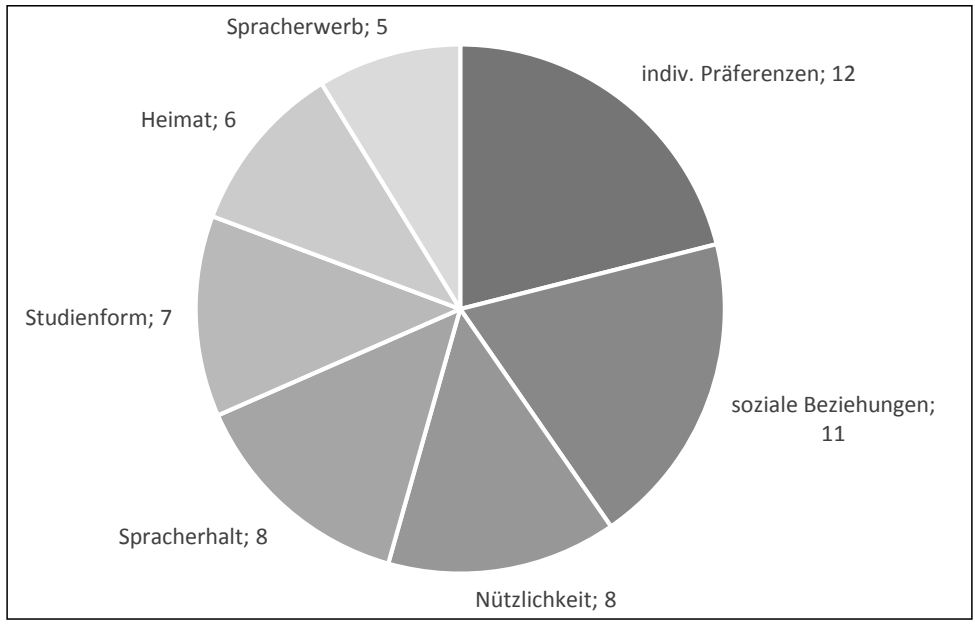

Am häufigsten wurden somit von den Studierenden Gründe wie Interesse (2MVw+ 26; 25BRAw+20), Spaß (4NSw+26; 19MVw+20), Begeisterung (9BRAw+20) und Sympathie $(12 \mathrm{BRAw}+26)$ genannt $(\mathrm{n}=12)$, die als individuelle Präferenzen klassifiziert wurden. ${ }^{34}$

Im Folgenden sollen ausgewählte Beispiele die konkreten Vertextungen dieser individuellen Studienmotive illustrieren:

1. Beispiel: $2 \mathrm{MV}+26$ : „Ich studiere Niederdeutsch aus Interesse an der Sprache. ${ }^{{ }^{35}}$

2. Beispiel: $4 \mathrm{NSw}+26$ : „Ich habe aus Spaß mit dem Sprachkurs begonnen.“

34 In Klammern werden die Umfragebogensiglen genannt, die sich aus der Umfragenummer, der Abkürzung für das Bundesland, dem Geschlecht und der Altersgruppe zusammensetzen. So handelt es sich bei $2 \mathrm{MVw}+26$ um eine Studentin aus MecklenburgVorpommern im Alter zwischen 26 und 30 Jahren.

35 Die Antworten werden dem Wortlaut der schriftlichen Fragebogenantworten entsprechend übernommen. Korrekturen oder anderweitige Kennzeichnungen von Standardabweichungen werden nicht vorgenommen, da dies erstens nicht im Fokus des Beitrages steht und zweitens ein Verstehen trotz eventueller Fehler stets möglich ist. 
3. Beispiel: $19 \mathrm{MVw}+20$ : „Weil ich selbst Nddt. in der Schule hatte und es mir sehr viel Spaß gemacht hat."

4. Beispiel: $26 \mathrm{MVw}+20$ : „Ich habe Plattdeutsch schon immer gemocht.“

Die Studierenden konstruieren ihre Studienfachwahl somit als Ergebnis individueller, intrinsischer Motivationen. Sie positionieren sich damit gleichsam als selbstbestimmte Personen, die sich in ihrer Studienwahl von individuellen partiell unterhaltenden (Spaß) und emotiven (Sympathie) Gründen leiten lassen. Voraussetzung für derartige Entscheidungen ist ein basales Wissen um die niederdeutsche Sprache sowie um die Möglichkeit des Studiums. Die sich hier zeigende sprachenaffine Einstellung überrascht nicht, da es sich durchweg um Germanistikstudierende handelt, die diese Einstellungskomponenten insofern schon bei der Wahl ihres Hauptfaches offenbarten.

Interessant war in diesem Zusammenhang die Äußerung einer Studentin aus Brandenburg, die die Beschäftigung mit Niederdeutsch quasi als Rehabilitation ihrer eigenen Vergangenheit erlebte:

5. Beispiel: 15BRAw+26: „Da ich ursprünglich aus Hamburg komme, habe ich eine Verbindung zum Plattdeutschen gehabt (war keine aktive Sprecherin - auch sonst niemand in der Familie, aber es gab doch einige plattdeutsche Wörter in meinem Wortschatz, von denen mir erst später bewusst wurde, dass sie plattdeutsch und kein familieninternes Kauderwelsch sind, weil man mich in Berlin teilweise nicht verstand). ${ }^{36}$

Am zweithäufigsten wurden von den befragten Studierenden soziale Beziehungen dergestalt relevant gesetzt, dass auf Verwandte und Bekannte verwiesen wurde, die niederdeutsch sprechen bzw. zum Studium angeregt haben $(\mathrm{n}=11)$. Innerhalb dieser Kategorie dominiert die Familie mit fünf Erwähnungen, gefolgt von den Großeltern mit drei Nennungen und den Bekannten mit zwei Nennungen. So artikuliert 21MVm+30 familiäre Gründe und 13MVw+26 äußert: „Ein Teil meiner Familie spricht schon ihr ganzes Leben lang Niederdeutsch." Als weitere Einflussgrößen sozialer Art wurden Freunde $(8 \mathrm{SAm}+26)$ und Kommilitonen $(9 B R A w+20)$ genannt.

36 An diesem Zitat wird zugleich die Problematik der Einordnung der Äußerungen offensichtlich. Aufgrund der dargestellten starken persönlichen Involviertheit wurde die Äußerung als ,individuelles Interesse“ kodiert. Es wäre unter Umständen auch eine Einordnung als Heimatbezug möglich. Gleichwohl liegt keine explizite Wertschätzung der Heimat vor, was für Äußerungen dieser Kategorie als typisch anzusehen war. 
6. Beispiel: 6SHw+26: „Mein Interesse am Plattdeutschen ist durch meine Großeltern gewachsen. Beide sprechen es, allerdings nicht permanent. Vor allem, wenn es um witzige Anekdoten geht, die sie sich mit ihren Verwandten und Bekannten erzählen, aber auch, wenn sie sich ,kappeln :-) [...] Ich erinnere mich noch genau wie stolz ich war, als ich zu meinem Opa sagte: ,Ich glaube „hüt Nacht“ bedeutet „heute Nacht!' und er es mit dem Satz ,Du büst ja ne richtig plietsche Diern!' bejaht hat. Das Angebot der Uni-Greifswald hat mich an diese Momente erinnern lassen. Das war der Anstoß für meine Anmeldung."

Aus der langen Äußerung spricht nicht nur eine sehr intensive Beziehung zu den Großeltern, sondern sie zeigt zugleich, dass die Niederdeutschkompetenz auch als familiär geschätzter Wert etabliert wurde. Insofern ist die Aufnahme des Studiums auch als Effekt familiär tradierter Wertvorstellungen zu begreifen. Darüber wird implizit das Verstehen-Können der Anekdoten - zumindest aus kindlicher Sicht als motivational interpretiert, da es bewusst erinnert wurde.

7. Beispiel: $16 \mathrm{MVw}+26$ : „Der wichtigste Grund ist aber, dass meine liebe Uromi, die heute leider nicht mehr unter uns weilt, damals ausschließlich Platt mit mir gesprochen hat. Und ich ärgere mich bis heute, dass ich damals nie versucht habe, ihr auf Platt zu antworten oder sie darauf bestanden hat, ich habe immer Hochdeutsch gesprochen. Ich habe damals alles verstanden, konnte aber nichts sagen. Das will ich heute nachholen. Platt erinnert mich einfach an meine Omi und das ist schön. :)“

Die Äußerungen im Beispiel 7 etablieren eine sehr intensive Beziehung der Studentin zu ihrer Urgroßmutter, die zugleich die Basis der Wahl für das Niederdeutschstudium war. Mit der Äußerung „der wichtigste Grund“ erfolgt eine explizit markierte Priorisierung dieses Grundes. In der bemerkenswerten Äußerung „Das will ich heute nachholen“ zeigt sich meines Erachtens der Wunsch, die Geschichte „zurückdrehen“ zu können und der Person durch den Erwerb der eigenen Kompetenz nahe zu sein. Diese Motivation offenbart insofern auch konservative und - mehr noch - konservierende Züge.

Wenngleich im folgenden Zitat nicht dominant familiäre Bindungen geschildert werden, so stellen sich doch soziale Beziehungen sehr spezifischer Art als motivational relevant dar:

8. Beispiel: 12BRAw+26: „Mein Interesse daran entwickelte ich hauptsächlich durch Fahrten zum Trakehner Hengstmarkt in Neumünster, da meine Familie dort immer bei einem älteren Ehepaar unter kam. Dieses herz- 
liche, sympathische Ehepaar sprach ausschließlich platt, es war ihnen egal, ob man sie verstand oder nicht. Ich verstand zum Glück sehr viel, meine Eltern und mein Bruder auch.“

Die Studentin schildert Erlebnisse aus der Kindheit und Jugend als ausschlaggebend für ihre Studienfachwahl. Auch im obigen Zitat sind es Personen und die Beziehung zu ihnen, die prägend gewirkt haben. Hier ist es ein Ehepaar, das maßgeblich durch sein Alter und sein positives soziales Verhalten (herzlich, sympathisch) charakterisiert wird. Die Schilderung rahmt das Ehepaar gleichsam implizit als Vorbild.

Die Studierenden verorten sich mit diesen Nennungen in einem sozialen Umfeld, das stark familiär geprägt ist. Die Familie wird somit als prägend für Studienentscheidungen und insbesondere für die Entscheidung zum Niederdeutschstudium angesehen. Damit kann diese Studierendenkohorte zugleich durch eine hohe Relevanz sozialer Bindungen charakterisiert werden, auf die sie ihre Entscheidungen gründet.

Die enge Beziehung zwischen Familie und Niederdeutsch ist indes nicht neu. Vielmehr weist die INS-Umfrage von 2007 die Familie bei den damals Befragten als primären Ort des Spracherwerbs aus. ${ }^{37}$ Für die aktuell Studierenden wurde diese Rolle primär durch die Großelterngeneration übernommen, wie auch wiederholt in - bisher nicht systematisch erhobenen - Gesprächen zur Studienberatung vor der Aufnahme des Niederdeutschstudiums geschildert wurde. Es scheint so, als ob aktuell die dritte Generation vermehrt Niederdeutsch studiert eine Generation also, die Niederdeutsch maßgeblich mit ihren Großeltern in Verbindung bringt und es von diesen auch erlernt hat.

Darüber hinaus verwies Diercks bereits in den 1990er Jahren auf die familiäre Interpretation von Niederdeutsch bei den Jugendlichen mit nicht-handwerklicher Ausbildung. Die Konstruktion eines dominant familiär konnotierten Niederdeutschen zeigt sich bei den Haupt- und RealschülerInnen mit handwerklichen Berufen nicht, weil ihnen das Niederdeutsche vor allem als Betriebssprache vertraut war. ${ }^{38}$ Insofern kann die gymnasiale und akademische Bildung der Befragten an der Universität Greifswald durchaus steuernden Einfluss auf diese familiäre Interpretation haben.

Am dritthäufigsten wurde die Nützlichkeit des Studiums als Motivation für die Studienfachwahl betont $(\mathrm{n}=8)$. Durch explizite Hinweise auf den „Bonus auf dem Arbeitsmarkt" (15BRAw+26), bessere "Chancen auf einen Referendariatsplatz"

37 Vgl. Möller 2008, S. 45.

38 Vgl. Diercks 1994, S. 142.

Birte Arendt, Andreas Bieberstedt and Klaas-Hinrich Ehlers - 978-3-631-71893-3 
$(16 \mathrm{MVw}+20)$ oder auf den „allgemeinen Nutzen von Zusatzqualifikationen“ $(26 \mathrm{MVw}+26)$ zeigen sich die Studierenden als zielorientiert und investitionsbereit. Konkret wurden derartige Gründe wie folgt versprachlicht:

9. Beispiel: $4 \mathrm{NSw}+26:$ „Da ich gerne in Schleswig-Holstein oder Hamburg unterrichten möchte, und in diesen beiden Bundesländern platt deutsch unterrichtet und erwünscht ist, habe ich aus meinem Interesse einen Vorteil für meine spätere Bewerbung gemacht.“

Die Studentin aus Niedersachsen kennzeichnet im Beispiel 9 eine inhaltlich klar konturierte Berufsfokussierung, die den Ort bzw. die Region der Arbeit einschließt. Um diese erwünschte regionale Bindung zu etablieren, werden auch zusätzliche Bildungsangebote wahrgenommen. Bemerkenswert ist jedoch der Hinweis, dass an erster Stelle das eigene Interesse stand, das dann positiv umgedeutet wurde: „habe ich aus meinem Interesse einen Vorteil [...] gemacht“. Insofern nimmt die Studentin hier eine Hierarchisierung der Gründe zugunsten der individuellen Präferenzen vor.

10. Beispiel: $18 \mathrm{HHw}+26$ : „Bezogen auf den schulischen Kontext erhoffe ich mir aber auch gute Einstellungschancen durch das abgeschlossene Beifach-Studium.“

Nachdem 18HHw+26 Heimatverbundenheit und Spracherhalt als Gründe für ihre Studienfachwahl genannt hat, geht sie auf den Nutzen des Studiums zur Verbesserung ihrer Einstellungschancen ein. Zugleich präsupponiert dieses Ansinnen eine als prekär eingeschätzte Lage auf dem Arbeitsmarkt, die zur Investition in die Bildung nötigt. Die Studierenden erkennen das Niederdeutschstudium als Baustein zur Verbesserung ihrer Chancen an und rechnen sich zugleich ohne Zusatzqualifikationen nur geringe Chancen aus. Für ihre Karriere sind die Studierenden somit bereit, in Bildung auch zusätzlicher Art zu investieren.

Auf einen Nutzen als Brückensprache des Niederdeutschen sowie als potenzielles Verständigungsmittel hat nur eine Studentin hingewiesen. ${ }^{39}$

11. Beispiel: „Außerdem hilft platt auch, um sich in anderen Ländern zu verständigen, z. B: Belgien, und meine Englischkenntnisse haben sich verbessert, seitdem ich Niederdeutsch studiere.“

Die Äußerung charakterisiert den Nutzen der Sprache im Erlernen anderer Sprachen wie dem Englischen. Der Verweis auf eben diese Sprache lässt meines

39 Vgl. zum Niederdeutschen als Brückensprache den Beitrag von Biedowicz in diesem Band. 
Erachtens auf sprachhistorische Kenntnisse zur ingwäonischen Sprachfamilie aus dem Studium bzw. auf Erfahrung sprachstruktureller Parallelen schließen.

Ebenfalls an dritter Stelle wurde in den Antworten auf einen notwendigen Spracherhalt rekurriert $(\mathrm{n}=8)$. Mit Verweisen auf den notwendigen Erhalt der Sprache (9BRAw+20: „damit die Sprache erhalten bleibt“) sowie die Bewahrung vor dem Vergessen (19MVw+20: „dass es nicht in Vergessenheit gerät“) wird zugleich ein Bedrohungsszenario etabliert, das das Niederdeutsche als schutzbedürftig darstellt und einen nahenden Untergang projiziert.

Etwas pragmatischer, aber auf gleicher Basis argumentieren Studierende, die die notwendige Weitergabe der Sprache wie folgt relevant setzen.

12. Beispiel: 12BRAw+26: „Wenn ich Lehrerin bin und meine Schule mitspielt, würde ich sehr gerne Plattdeutsch anbieten, damit auch noch mehr Kinder/ Jugendliche die Sprachkompetenz erwerben und Freude daran haben. Sollte ich nicht an einer Schule bleiben, sondern an eine Uni gehen, um zu lehren, würde ich dort Plattdeutsch anbieten, wenn es möglich ist. Ich hoffe sehr, dass Niederdeutsch weiterhin studiert werden kann und dass es auch an Schulen angeboten wird.“

Wenngleich im Beispiel 12 kein expliziter Hinweis auf den Spracherhalt gegeben wird, so ist der als notwendig erachtete Kompetenzerwerb bei den Kindern wohl als Indikator für eine derartige motivationale Basis anzusehen.

13. Beispiel: $14 \mathrm{MVw}+20$ : „Meiner Meinung nach, gehört die plattdeutsche Sprache in diese Region, wie der Königsstuhl zu Rügen. Es ist mir wichtig, dass Plattdeutsch bewahrt und an die nächste Generation weitergegeben wird.“

Neben der Naturalisierung des regionalen Bezugs der Sprache durch die Analogie mit dem Königsstuhl fällt die - für sprachpolitische Kontexte typische - Weitergabemetapher auf. Niederdeutsch wird damit gleichsam materialisiert - als Container - und evaluativ als wertvoller Schatz konzeptualisiert. Wir erkennen darin eine bereits für sprachpatriotische Diskurse des 19. Jahrhunderts typische Hypostasierung der Sprache. Die Äußernden selbst inszenieren sich gleichsam als verantwortungsbewusst insbesondere in Bezug auf gesellschaftliche Aufgaben.

In diesem Kontext werden auch Hochwertwörter, wie Tradition $(16 \mathrm{MVw}+20)$, Kultur $(16 \mathrm{MVw}+20)$ und kulturelle Wurzeln $(13 \mathrm{MVw}+26)$, von den Befragten angeführt, um die Notwendigkeit des Erhalts zu begründen. Es zeigt sich in diesen Argumentationen eine insbesondere für sprachpolitische Texte typische Hierarchie von Gründetypen. ${ }^{40}$

40 Vgl. Arendt 2009b.

Birte Arendt, Andreas Bieberstedt and Klaas-Hinrich Ehlers - 978-3-631-71893-3 
Damit inszenieren sich die Studierenden als gesellschaftlich engagiert und verantwortungsbewusst Handelnde. Zugleich zeigen sie ein sozial-pragmatisch orientiertes Verständnis von Sprachwandel, bei dem die SprecherInnen eine entscheidende Rolle spielen. Dass es sich dabei um ein im Studium erworbenes Wissen handelt, das positiv und handlungswirksam in das eigene Verhalten integriert wurde, ist zu vermuten. Derartige Äußerungen können als selbstwertdienlich dergestalt angesehen werden, dass sich die Studierenden damit zugleich als relevante Einflussgröße des Sprachwandels konturieren.

Die besondere Form des Studiums und insofern etwas Greifswald-Spezifisches wurde am fünfthäufigsten erwähnt $(\mathrm{n}=7)$. Dabei wurde sowohl auf das integrative Modell $(9 \mathrm{BRAw}+20)$ als auch die besonders motivierende und kenntnisreiche Arbeit der Lehrpersonen $(17 \mathrm{MVw}+26)$ verwiesen. Ein Student aus SachsenAnhalt verwies auf die Differenz zum Germanistikstudium, das ihm „tau drög worn is" $(8 \mathrm{SAm}+26){ }^{41}$

14. Beispiel: $24 \mathrm{MVw}+26$ : „Mich hat ebenso das Format als Schwerpunkt Niederdeutsch sowie die gute und freundliche Betreuung der Lehrenden gereizt."

Auch in der Äußerung im Beispiel 14 wird die Qualität der zwischenmenschlichen Beziehung als ein ausschlaggebender Faktor genannt. Dieser Aspekt korreliert inhaltlich mit dem am zweithäufigsten genannten Faktor der sozialen Beziehungen.

Darüber hinaus finden sich auch eher allgemeinere Verweise auf Differenzen zwischen Studienformen, deren Bezug zur Frage erst durch interpretatorische Inferenzleistungen gezogen werden kann, wie im Beispiel 15:

15. Beispiel: 10SHw+26: „Ich habe erst an der Uni Osnabrück begonnen, war aber mit dem Bachelor-System nicht sonderlich zufrieden. Aus diesem Grund habe ich mich für das alte Studiensystem entschieden und bin so nach Greifswald gekommen."

Wenn man die Äußerung als thematisch responsive Antwort auf die Frage werten will, so scheint der Studentin das Studium des alten Systems die Möglichkeit gegeben zu haben, den Schwerpunkt Niederdeutsch belegen zu können.

Der Bezug zur Heimat und die Relevanz dieses Konzepts für die eigene Lebensplanung wurde in sechs Äußerungen thematisiert und war damit die sechsthäufigste Antwort $(n=6)$. Gleichwohl zeigen sich eine starke Verbundenheit mit der

41 Auf die niederdeutschen Passagen in den Äußerungen wird im Abschluss des Kapitels eingegangen.

Birte Arendt, Andreas Bieberstedt and Klaas-Hinrich Ehlers - 978-3-631-71893-3 
Region und der Wunsch, in dieser zu arbeiten, auch in Äußerungen ohne explizit verbalisierten Heimat- bzw. Regionalbezug, wie etwa im Beispiel 9. Wenngleich also die Anzahl der Nennungen vergleichsweise gering ist, ist die Verbundenheit mit der Region aus dem genannten Grund als relativ hoch einzuschätzen.

16. Beispiel: $21 \mathrm{MVm}+30$ : „Aus familiären Gründen und aus Sehnsucht zu meiner Heimat."

Ein Student aus Mecklenburg-Vorpommern entwirft in Beispiel 16 die Heimat als romantischen Sehnsuchtsort und durch die Konstruktion „Sehnsucht nach“ zugleich als etwas (noch) nicht Erreichtes. Das aufgenommene Niederdeutschstudium erlaubt ihm, dieser Sehnsucht nach Heimat nachzugehen. Darüber erfolgt eine auch in anderen Antworten anklingende Engführung der Konzepte Niederdeutsch - Heimat - Region. Diese drei Komponenten bilden somit für die Studierenden nicht nur eine sinnvolle Relation, sondern auch ein Angebot zur persönlichen Verortung. Heimat erscheint erstens positiv konnotiert. Der Dialekt und hier das Niederdeutsche werden zweitens als Mittel einer aktiven sprachlichen Verbeheimatung angesehen und angenommen.

17. Beispiel: $18 \mathrm{HHw}+26$ : „Ich habe das Niederdeutsch-Studium aufgenommen, weil ich eine starke Verbundenheit zu Norddeutschland verspüre und Plattdeutsch hierzulande einen kulturellen Schatz darstellt.“

Die Studentin im Beispiel 17 postuliert an erster Stelle der Antwort eine emotive Beziehung („starke Verbundenheit“) zu einer Region, deren konstitutives Merkmal der Norden Deutschland ist. Das Konzept „Norddeutschland“ scheint derart tradiert und zum gefestigten Wissensbestand zu gehören, dass keinerlei weitere Erklärungen notwendig sind. Ob sie sich dabei auf ein bestimmtes Bundesland, die Küstenregion oder das niederdeutsche Sprachgebiet insgesamt bezieht, bleibt offen. ${ }^{42}$

Wenngleich die Motivation, Niederdeutsch zu lernen, d. h. der Spracherwerb, bereits partiell in den anderen Kategorien anklang, wie etwa im Beispiel 8, so wurde dieser Wunsch doch auch explizit als Grundlage der Studienaufnahme angegeben $(\mathrm{n}=5)$.

18. Beispiel: $2 \mathrm{MVw}+26:$ „Ich wollte meine Fähigkeiten im Niederdeutschen ausbauen."

42 Vermutlich ist letzteres der Fall, da in der Fragebogenkonzeption ein klarer Bezug zur niederdeutschen Sprache gegeben ist. 
19. Beispiel: $14 \mathrm{MVw}+20$ : „Plattdeutsch zu verstehen finde ich schon ziemlich toll, es aber auch selbst sprechen zu können, hat mich sehr motiviert."

20. Beispiel: $13 \mathrm{MVw}+26$ : „auch wenn ich vielleicht nie wie sie [die Familie, B.A.] sprechen werde, möchte ich doch fähig sein es zu verstehen und bestmöglich zu verwenden. Besser ein verändertes nicht ganz ursprüngliches Plattdeutsch als gar keins."

Die Äußerungen in den Beispielen 18 bis 20 benennen explizit den Wunsch nach aktiver Sprachkompetenz als einen Motivationsfaktor zur Aufnahme des Studiums. In Beispiel 20 schwingt im letzten Satz zugleich die Befürchtung mit, dass die im Studium erworbene Kompetenz im Gegensatz zum familiär erworbenen „ursprünglichen Platt“ verändert und insofern potenziell defizitär sein könnte. Diese Haltung zeigte sich auch bei anderen befragten Jugendlichen, wobei hier wie dort zugleich eine normentolerante und gebrauchsorientierte Auffassung von Niederdeutschkompetenz dominiert.

In ihren Aussagen auf dem Fragebogen haben die Studierenden nicht nur metasprachlich auf die niederdeutsche Sprache Bezug genommen, sondern sie teilweise auch objektsprachlich eingesetzt. In fünf von 18 Antworten wurden niederdeutsche Lexeme bzw. Passagen in einem typischen emblematischen Gebrauch realisiert. Vom Gesamtumfang der 1468 Wörter wurden 20 als niederdeutsch identifiziert. Das entspricht 1,3\%. Wenngleich kein expliziter Appell zum Verfassen der Antworten auf Niederdeutsch gegeben war, hätte die sprachliche Form des Erzählimpulses „Vertell uns Deine Geschichte!“ eine entsprechende Vertextung ermöglicht. Die niederdeutschen Versatzstücke können insofern auch als Triggereffekt des Impulses aufgefasst werden. Anders also als in Online-Foren, wo Niederdeutsch durchaus auch zunehmend verwendet wird, ${ }^{43}$ zeigt sich in diesen Antworten die typische vor allem aus den Printmedien bekannte Puzzletaktik. ${ }^{44}$ Funktional dienen sie als:

- Nachweis der Sprachkompetenz:

21. Beispiel: $8 \mathrm{SAm}+26$ : „nachdem ich mit dem Gedanken spielte, nicht mehr Germanistik zu studieren, weil es mir tau drög worn is"

22. Beispiel: $2 M V w+26:$ „wenn ich Menschen platt snacken höre, erweckt es ein Heimatgefühl in mir"

- der Inszenierung von Authentizität (Stilisierungen), im Beispiel 6: kabbeln und die Äußerung des Großvaters „Du bist ja ne richtig plietsche Diern!“

43 Reershemius 2015.

44 Vgl. Arendt 2010b. 
- dem symbolisch doppelt kodierten Ausdruck einer positiven Werthaltung:

23. Beispiel: $18 \mathrm{HHw}+26$ : „Außerdem ist das Plattdeutsche einfach eine smucke Spraak :)“

Das Beispiel 23 erfasse ich insofern als doppelt kodierten Ausdruck, da sowohl explizit durch eine Prädikativkonstruktion mit positiv konnotiertem Adjektiv eine Wertung erfolgt als auch durch den symbolischen Mehrwert, der durch die niederdeutschsprachige Vertextung erzeugt wird. Die niederdeutsche Phrase fungiert gleichsam als formaler Beleg für das inhaltlich Gesagte.

\section{Zusammenfassung und Ausblick auf eine „Generation“ von Niederdeutschstudierenden}

Seit 1993 ist es an der Universität Greifswald ununterbrochen möglich, Niederdeutsch als eigenes Fach zu studieren, was von den Studierenden - spätestens seit 2009 mit dem integrativen Schwerpunkt und ab 2012 mit dem modularisierten Beifach - gut bis sehr gut angenommen wurde (vgl. Kap. 2). Diese Studienmodelle bieten mit dem Ziel einer schulischen Niederdeutschvermittlung nicht nur sprachwissenschaftliche Themen, sondern auch literaturwissenschaftliche sowie didaktische und beinhalten den Aufbau einer Sprachkompetenz im Niederdeutschen. Die überwiegend aus den nördlichen Bundesländern stammenden weiblichen Studierenden entscheiden sich aufgrund individueller - heimat- und interessenbezogener -, sozialer und pragmatischer Gründe für ein Niederdeutschstudium (vgl. Kap. 3).

Als Ergebnis der Befragung lässt sich festhalten, dass die Studierenden des Schwerpunkt Niederdeutsch überwiegend aus Norddeutschland stammen. Die Studienfachwahl rekonstruieren sie aus sieben verschiedenen Typen von Begründungen, bei denen individuelle Präferenzen und soziale sowie regionale Bindungen dominieren. Mit der Betonung der Nützlichkeit und dem Verweis auf die integrative Form des Studiums kommen utilitaristische Gründe zur Geltung. Bildung wird als Investitionsgut für die Zukunft konzeptualisiert. Der Wunsch nach Sprachkompetenz kann als spezifische Form von Selbstverwirklichung angesehen werden. Zugleich positionieren sich die Studierenden als verantwortungsbewusst und gesellschaftlich engagiert, wenn sie den Spracherhalt aus ihrem Handeln ableiten. Heimat stellt für viele von ihnen ein sinnstiftendes Konzept dar, das klar traditionell räumlich verankert ist.

Im Sinne einer möglichen Typisierung auf der Grundlage der Motivation für die Studienfachwahl zeichnen sich die Niederdeutschstudierenden durch enge soziale, insbesondere familiäre, und regionale Bindungen aus. Niederdeutsch 
stellt für einige von ihnen ein Mittel der aktiven Verbeheimatung dar. Sie treiben ihre Selbstverwirklichung durch utilitaristisch geprüfte Investitionen in Bildung sowie durch die Orientierung auf ihre individuellen Interessen voran. Mit einer grundsätzlich sprachaffinen Haltung übernehmen sie aktiv Verantwortung für den Erhalt des Niederdeutschen, indem sie ihr eigenes Handeln - zumindest in den expliziten Stellungnahmen - an diesem Ziel ausrichten.

Wenngleich die pragmatischen Gesichtspunkte der Studienfachwahl bereits vor der Untersuchung als Hypothesen postuliert wurden und studienfachwahltypisch sind, ${ }^{45}$ so scheinen die weiteren Gründe spezifischer und werfen Fragen wie die folgenden auf: Wie lassen sich die genannten Aspekte erklären? Gibt es Konzepte, die die verschiedenen Typen der Studienmotivationen zusammenfassend deuten können? Oder wurde mit den Daten eine spezifische und einmalige Kohorte von Niederdeutschstudierenden der Universität Greifswald beschrieben?

Innerhalb sozialwissenschaftlicher Forschungen hat sich zur Typisierung von Personengruppen annähernd gleichen Alters, wovon bei der untersuchten Gruppe ausgegangen werden kann, das Konzept der Generation etabliert. Im Folgenden soll deshalb der Versuch unternommen werden, aktuelle Generationskonzepte in ihrem Erklärungspotenzial für die untersuchten Niederdeutschstudierenden zu prüfen, um die entworfene Typisierung in übergeordnete Zusammenhänge einzubetten.

Innerhalb des rekonstruierten studentischen Wertesystems erfolgt eine Konzentration auf soziale Nahbezüge. Einflusspersonen als indirekt wirkende opinion leaders im Sinne Lazarsfeld sind Großeltern und Bekannte und damit sozial nahestehende Personen. ${ }^{46}$ Durch den wiederholten Bezug auf die Großeltern konstruieren die Studierenden sich selbst als Angehörige einer „dritten Generation“. Dieser Befund aus den Daten gleicht Erfahrungen aus Gesprächen, die ich mit Niederdeutschstudierenden beim Antritt ihres Studiums führte, jedoch nicht systematisch dokumentiert habe. Das Niederdeutsche als Sprache der Großelterngeneration stellt sich als sprachbiographisch prägende Erfahrung der meisten Studierenden dar. Eine spezifische dritte Generation wird auch innerhalb der sozialwissenschaftlichen Migrationsforschung beschrieben. ${ }^{47}$ Laut Studien, die maßgeblich von Hansen inspiriert wurden, sei diese Generation durch eine konservative Perspektive auf das Ursprungsland und eine Orientierung an Traditio-

45 Vgl. Bieberstedt 2009, S. 35.

46 Vgl. Jäckel 2007.

47 Vgl. z. B. Bülbül-Emanet 2015.

Birte Arendt, Andreas Bieberstedt and Klaas-Hinrich Ehlers - 978-3-631-71893-3 
nen gekennzeichnet. ${ }^{48}$ Wenngleich kein „Ursprungsland“ im eigentlichen Sinne aus den Antworten ableitbar ist, so offenbart sich im Verweis auf Traditionen, einen nötigen Spracherhalt sowie die „Heimat" doch zugleich eine konservative Grundhaltung, die eine - partiell romantische - Erinnerungskultur etabliert und wertschätzt (vgl. Beispiel 7, 13 und 16). ${ }^{49}$

Der explizite Heimat- und Regionalbezug der Studierenden kann auch als Ergebnis der Glokalisierung im Sinne Robertsons gedeutet werden, ${ }^{50}$ bei der Identitätsangebote dominant auf lokaler Ebene genutzt werden und ein regional definierter Raum als sinngebendes Bezugssystem einer persönlichen Verortung, einer Heimat, fungiert.

Darüber hinaus bieten soziologisch-pädagogische Ansätze zur Konstruktion einer „Generation Y“, der zwischen 1980-1999 Geborenen, explanative Parallelen. Nach Hurrelmann/Albrecht, die diese Generation einschlägig beschrieben haben, zeichnet sich diese erstens durch eine besondere Wertschätzung von Bildung aus, in die investiert wird, um sich möglichst viele Optionen für den Arbeitsmarkt und damit zugleich einen Platz in der Gesellschaft offen zu halten (vgl. 3. Grund: Nützlichkeit des Studiums). Zweitens kennzeichne diese Generation, die auch als "Egotaktiker" bezeichnet werden, ${ }^{51}$ ein dominant interessengeleitetes Abwägen, das auf das Wohlbefinden der eigenen Person gerichtet ist (vgl. 1. Grund: individuelle Präferenzen, 7. Grund: eigener Spracherwerb, 5. Grund: Studienform). Drittens wird die Familie als wichtige Ressource für Lebensqualität angesehen (vgl. 2. Grund: soziale Bindungen), wenngleich mit tradierten Familienmodellen gebrochen wird.

Alle Generationskonzepte sind letztlich immer holzschnittartige Abstraktionen individueller Wirklichkeiten. Darin liegt ihre Erklärungskraft, aber zugleich auch -schwäche. Die übergreifenden Generationskonzepte der Sozialwissenschaften deuten aber darauf hin, dass wir es weniger mit einer spezifischen Kohorte von Niederdeutschstudierenden zu tun haben, sondern sich aktuelle generations-

48 Hansen 1938; vgl. Aumüller 2010, S. 8. Zugleich problematisieren aktuelle Studien den „Generationen-Begriff“ als zu starr, um die heterogene Wirklichkeit der jetzigen Generation von MigrantInnen erfassen zu können, vgl. u. a. Aumüller 2010.

49 Gleichwohl weisen aktuelle Studien zur dritten Generation darauf hin, dass es in gewissem Gegensatz zu Hansens Befunden vielmehr zu Pluralität und zu einer „Ausdifferenzierung der Lebensstile“ (Aumüller 2010, S. 9) komme.

50 Vgl. Robertsons 1998; Arendt 2010a. In eine ähnliche Richtung der Provinzialisierung als bedingter Reflex auf die Globalisierung weist auch Zenklusens 2006 Konzept des „Hyperprovinzialismus“.

51 Hurrelmann 2002, S. 33; vgl. Hurrelmann/Albrecht 2014. 
typische Bedürfnisse durch ein Niederdeutschstudium befriedigen lassen. Oder anders gesagt: Das Studium des Niederdeutschen ist mitnichten eine Angelegenheit für ältere soziophobe ,Nerds'. Vielmehr zeichnen sich die aktuell Studierenden, wie sie in der vorliegenden Untersuchung exemplarisch für die Universität Greifswald konturiert wurden, durch das Bewusstsein und die Wertschätzung sozialer Beziehungen, ein ausgeprägtes Interesse an individueller Selbstverwirklichung und ein utilitaristisch geprägtes Engagement für Bildung aus. Wenn man den Befunden von Hurrelmann/Albrecht zur Charakterisierung einer aktuellen

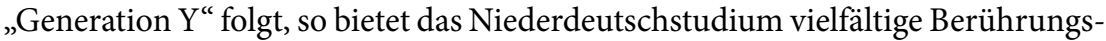
punkte in motivationaler Hinsicht. Es bleibt abzuwarten, welche Beziehung auch zukünftige Generationen zum Niederdeutschen und einem möglichen Studium aufbauen.

Was bedeutet das für zukünftige Studienangebote? Unter Berücksichtigung der Dominanz individuell bedeutsamer Gründe wäre als motivationaler Anreiz für künftige Studierende die Betonung des Spaßfaktors und der Exklusivität des Studiums von möglichem Gewinn. Es könnte darüber hinaus als Angebot für die individuelle Selbstverwirklichung konturiert werden. Wenn die Ressource der sozialen Beziehung auch für die aktive Motivation von zukünftigen Studierenden genutzt werden sollte, dann ist dafür zu sorgen, dass es NiederdeutschsprecherInnen mit Vorbildcharakter gibt, die für die Studierenden sozial bedeutsam sind. Das können Familienangehörige und Freunde, aber im besten Fall auch LehrerInnen und DozentInnen sein.

Für die nächste Generation - die heutigen SchülerInnen - sollte die Beschäftigung mit Niederdeutsch vor allem Spaß machen, wie eine kleine Umfrage im Rahmen der Kinder- und Jugenduniversität 2016 erbrachte..$^{52}$ Niederdeutsch wollen sie - ebenso wie die Studierenden - aus sozialen Gründen und insbesondere wegen der Großeltern lernen. So antwortete eine zehnjährige Schülerin aus Grimmen auf die Frage, wie sie einen möglichen Plattdeutschunterricht finden würde: „Finde ich gut, weil ich gerne meiner Oma eine Freude machen würde und damit ich versteh was sie erzählen.“

Gleichzeitig werden auch von den SchülerInnen individuelle und pragmatische Gründe genannt, die Erwachsene bei ihrer Motivationsplanung vielleicht unberücksichtigt gelassen hätten. So antwortet eine elfährige Schülerin auf dieselbe Frage: „Find ich gut, weil es interessant ist zu wissen wie die Vorfahren vielleicht gesprochen haben und wenn andere kein Plattdeutsch können kann

52 Im Zusammenhang des Vortrages „Kann, mutt, löppt - Niederdeutsch im 21. Jahrhundert“ im Juni 2016 wurden fünf 10-11jährige Mädchen befragt. 
man immer Geheimnisse auf Plattdeutsch sagen zu seiner Freundin oder so." Plattdeutsch als Geheimsprache bietet vielleicht einen Ansatz, das Interesse unter den SchülerInnen zu wecken. Es zeigt in seiner Referenz auf ein kinderkulturelles Wertesystem zugleich, dass die Motivation von individuellen Relevanzsystemen geprägt ist, deren genaue Kenntnis erst ein Maßschneidern auf Bedürfnisse ermöglicht. Der vorliegende Artikel hat einen ersten Beitrag zur Rekonstruktion derartiger Motivationsbestände für Niederdeutschstudierende geliefert.

\section{Literatur}

Arendt, Birte: Qualitative Interviews als interaktive ko-konstruktive Prozesse. Kontextsensitivität in mikroanalytischer Perspektive. In: Studler, Rebekka/ Counz, Christina (Hrsg.): Sprechen über Sprache. Perspektiven und neue Methoden der Spracheinstellungsforschung (Stauffenburg Linguistik; 81). Tübingen 2014, S. 7-30.

Arendt, Birte: „deswegen hab ich mich auch nich getr!AU!t zu sprechen“. Spracheinstellungsmuster und Sprachgebrauch Jugendlicher bezüglich des Niederdeutschen im sozialen Netzwerk »Plattdüütschkring «. In: Muttersprache. Vierteljahresschrift der Gesellschaft für deutsche Sprache 2012, S. 1-25.

Arendt, Birte: Plattdeutsche Glokalisierung. Identitätsinszenierung auf subnationaler Ebene. In: Schiewe, Jürgen/Lipczuk, Ryszard/ Nerlicki; Krzysztof /Westphal, Werner (Hrsg.): Kommunikation für Europa II. Sprache und Identität. Frankfurt a. M. [u. a.] 2010a, S. 67-76.

Arendt, Birte: Welches Bild wird in der Regionalpresse vom Niederdeutschen gezeichnet? In: Bundesraat för Nedderdüütsch (Hrsg.): Zwischen Kulturauftrag und Unterhaltungsprogramm. Plattdeutsch in den Medien. Leer 2010b, S. 57-62.

Arendt, Birte: Entwicklung des Niederdeutschstudiums an der Ernst-MoritzArndt-Universität Greifswald. In: Bundesraat för Nedderdüütsch (Hrsg.): Plattdeutsch, die Region und die Welt. Wege in eine moderne Mehrsprachigkeit. Leer 2009a, S. 39-47.

Arendt, Birte: Krankgepflegt? Was Sprachpolitik bewirken kann. In: Aptum. Zeitschrift für Sprachkritik und Sprachkultur 1, 2009b, S. 38-60.

Arendt, Birte: „Dialekt rulZ... klingt phatt ... - Hochdeutsch ruled, scheiß auf den Rest!“ Niederdeutsch im Diskurs unter Jugendlichen. In: Dürscheid, Christa/ Spitzmüller, Jürgen (Hrsg.): Perspektiven der Jugendsprachforschung. Frankfurt a. M. [u. a.] 2006, S. 241-259. 
Aumüller, Jutta: Wie viele Generationen dauert Integration? Wie Begriffe unser Bild von Gesellschaft prägen. In: Heinrich-Böll-Stiftung (Hrsg.): Bis in die dritte Generation? Lebensrealitäten junger MigrantInnen. Berlin 2010, S. 7-11.

Bieberstedt, Andreas: Niederdeutsch an den Hochschulen. Gegenwärtiger Stand und Perspektiven. In: Bundesraat för Nedderdüütsch (Hrsg.): Plattdeutsch, die Region und die Welt. Leer 2009, S. 34-38.

Bierhoff, Hans Werner: Sozialpsychologie. Ein Lehrbuch. Stuttgart 2006.

Bülbül-Emanet, Sevnur: Lebensweltgestaltung junger Frauen mit türkischem Migrationshintergrund in der dritten Generation. Wiesbaden 2015.

Diercks, Willy: Niederdeutsch in der Stadt Schleswig. Zu Attitüden und zu Sprachverwendung (ZDL Beihefte; 86). Stuttgart 1994.

Ehlers, Christiane/ Goltz, Reinhardt/Henschen, Walter: Auf dem Stundenplan: Plattdeutsch. Leer 2013.

Ehlich, Konrad/Rehbein, Jochen: Institutionsanalyse. Prolegomena zur Untersuchung von Kommunikation in Institutionen. In: Brünner, Gisela/Gräfen, Gabriele (Hrsg.): Texte und Diskurse. Methoden und Forschungsergebnisse der Funktionalen Pragmatik. Opladen 1994, S. 287-327.

Feldmann, Christa: Plattdüütsch. Diskussionsstand und Grundlagen zur Arbeit in den allgemeinbildenden Schulen Schleswig-Holsteins. Kiel 1997.

Flick, Uwe: Design und Prozess qualitativer Forschung. In: Flick, Uwe/Kardorff, Ernst von/Steinke, Ines (Hrsg.): Qualitative Forschung. Reinbek bei Hamburg 2009, S. 252-265.

Gernentz, Hans Joachim: Niederdeutsch an der Universität Rostock 1946 bis 1983. In: Herrmann-Winter, Renate (Hrsg.): Heimatsprache zwischen Ausgrenzung und ideologischer Einbindung. Niederdeutsch in der DDR. Frankfurt a. M. [u. a.] 1998, S. 101-108.

Hansen, Marcus Lee: The Problem of the Third Generation Immigrant. In: Sollors, Werner (Hrsg.): Theories of Ethnicity: A Classical Reader. New York 1938, S. 202-215.

Hennings, Jürgen/Goltz, Reinhard/Gerdes, Dirk: Schule und Plattdeutsch gehören zusammen. In: De Spieker. Heimatbund für niederdeutsche Kultur e. V. (Hrsg.): Plattdeutsch in der Schule. Symposion an der Carl von Ossietzky Universität Oldenburg am 3. September 2004. Oldenburg 2005, S. 7-8.

Hopf, Renate: Qualitative Interviews - ein Überblick. In: Flick, Uwe/Kardorff, Ernst von/Steinke, Ines (Hrsg.): Qualitative Forschung. Reinbek bei Hamburg 2009, S. 349-360.

Hurrelmann, Klaus/Albrecht, Erik: Die heimlichen Revolutionäre. Wie die Generation Y unsere Welt verändert. Weinheim/Basel 2014. 
Hurrelmann, Klaus: Jugend 2002. 14. Shell-Jugendstudie. Frankfurt a. M. 2002.

Institut für Niederdeutsche Sprache (Hrsg.): Zwischenbericht zur Sprachpolitik für das Niederdeutsche. Leer 2008.

Institut für Niederdeutsche Sprache: Platt an Hochschulen. 2016. [Online-Ressource: http://www.ins-bremen.de/de/sprache/bildung/platt-an-hochschulen. html (Stand: 12.12.2016)]

Jäckel, Michael: Medienwirkung. Ein Studienbuch zur Einführung. 4., überarb. und erw. Aufl. Wiesbaden 2007.

Kiesendahl, Jana: Status und Kommunikation. Ein Vergleich von Sprachhandlungen in universitären E-Mails und Sprechstundengesprächen. Berlin 2011.

Mayring, Philipp: Qualitative Inhaltsanalyse. In: Flick, Uwe/Kardorff, Ernst von/ Steinke, Ines (Hrsg.): Qualitative Forschung. Reinbek bei Hamburg 2009, S. $468-475$.

Möhn, Dieter: Niederdeutsch in der Schule. In: Cordes, Gerhard/Möhn, Dieter (Hrsg.): Handbuch zur niederdeutschen Sprach- und Literaturwissenschaft. Berlin 1983, S. 631-659.

Möhn, Dieter: Ausbildungsangebote im Wandel. Niederdeutsch an der Universität. In: Heimatbund für niederdeutsche Kultur e. V. (Hrsg.): Plattdeutsch in der Schule. Oldenburg 2005, S. 34-46.

Möller, Frerk: Der typisierte Plattsprecher. Bremen 1996.

Möller, Frerk: Plattdeutsch im 21. Jahrhundert. Bestandsaufnahme und Perspektiven. Mit einem Aufsatz von Michael Windzio (Schriften des Instituts für Niederdeutsche Sprache; 34). Leer 2008.

Osnowski, Stefan: Niederdeutsch in den Deutsch-Lehrplänen der DDR. In: Herrmann-Winter, Renate (Hrsg.): Heimatsprache zwischen Ausgrenzung und ideologischer Einbindung. Niederdeutsch in der DDR. Frankfurt a. M. [u. a.] 1998, S. 271-279.

PSO Beifach Niederdeutsch 2013: Prüfungs- und Studienordnung für das Beifach Niederdeutsch im Lehramtsstudiengang an der Philosophischen Fakultät der Ernst-Moritz-Arndt-Universität Greifswald vom 24. September 2013. Greifswald 2013.

Reershemius, Gertrud: Kleinere, regionale und Minderheitensprachen im Web 2.0 am Beispiel des Niederdeutschen. In: Fredsted, Elin/Langhanke, Robert/Westergaard, Astrid (Hrsg.): Modernisierung in kleinen und regionalen Sprachen (Kleine und regionale Sprachen: KURS; 1). Hildesheim 2015, S. 53-84.

Robertson, Roland: Glokalisierung: Homogenität und Heterogenität in Raum und Zeit, In: Beck, Ulrich (Hrsg.): Perspektiven der Weltgesellschaft. Frankfurt a. M. 1998, S. 192-220. 
Rösler, Irmtraud: Niederdeutsch und Schule. In: Herrmann-Winter, Renate (Hrsg.): Heimatsprache zwischen Ausgrenzung und ideologischer Einbindung. Niederdeutsch in der DDR. Frankfurt a. M. [u. a.] 1998, S. 257-269.

SO Deutsch LA Gym 2009: Studienordnung für den Teilstudiengang Deutsch (Lehramt an Gymnasien) an der Ernst-Moritz-Arndt-Universität Greifswald vom 17. August 2009. Greifswald 2009.

Zenklusen, Stefan: Im Archipel Coolag - soziognostische Denk-Zettel aus postfordistischer Jetztzeit. Berlin 2006. 
Birte Arendt, Andreas Bieberstedt and Klaas-Hinrich Ehlers - 978-3-631-71893-3

Downloaded from PubFactory at 01/11/2019 10:11:06AM

via free access 


\title{
Niederdeutschunterricht an der Volkshochschule - Kaffeeklatschersatz oder Spracherwerbskonzept mit Zukunft?
}

\begin{abstract}
Im times where Low German is not only no longer being passed on to future generations, but also progressively losing its standing as a means of everyday communication, a notable and ever-growing importance is now being placed on the explicit acquisition of this language. Although the focus of these language policy measures largely concerns pre-school and school education, Low German is also already a common teaching medium for courses in adult education, above all in adult education centres. The extent at which this framework represents a learning approach to preserve the Low German language, or whether it is simply a branch of the Low German culture sector to afford already competent speakers of the language a means to a pastime, will be determined in this article by way of an analysis of adult education centres in the central and northern regions of Vorpommern.

On the one hand, it becomes apparent that these classes are directly comparable with other foreign language courses in terms of length, structure and methodology. On the other hand, these courses do distinguish themselves on account of the course participants' rather unusual type of strong intrinsic motivation and extensive prior knowledge. Moreover, the conclusions of this research will only pertain to specific Low German regions, or courses to be precise, due to the significant heterogeneity in course layouts and participant structures. Nevertheless, the chances of Low German playing any role in the future of adult education in Mecklenburg-Vorpommern appear to be slim owing to the current lack of skilled teachers as well as motivated learners.
\end{abstract}

\section{Einleitung}

In Zeiten, in denen das Niederdeutsche nicht mehr selbstverständlich als Mutteroder Zweitsprache an die Folgegeneration weitergegeben wird und zudem seine Rolle als Alltagskommunikationsmittel mehr und mehr verliert, kommt dem gesteuerten Zweitspracherwerb eine gestiegene Bedeutung zu, um dem drastischen Absinken der Sprecherzahlen entgegenzuwirken. Das sprachpolitische Desiderat eines umfassenden Niederdeutschlernkonzepts findet sich sehr anschaulich formuliert in den „Schweriner Thesen zur Bildungspolitik“ des Bundesraats för Nedderdüütsch aus dem Jahr 2007. Hier heißt es: 
„2. Um die plattdeutsche Sprache dauerhaft zu sichern, bedarf es eines Gesamtkonzeptes, das sich über alle Altersgruppen erstreckt und die Bereiche Kindergarten, Schule, Hochschule sowie Erwachsenenbildung umfasst. [...] 12. Die Bundesländer sind aufgefordert, für das Niederdeutsche einen Rahmenplan des lebenslangen Lernens zu entwickeln. “1

Der Fokus aller sprachpolitischen Bemühungen im Falle des Niederdeutschen liegt derzeit klar auf den Bereichen Kindergarten und Schule, und dies obwohl der Erwachsenenbildung in der Literatur zur Bewahrung kleiner Sprachen theoretisch durchaus Bedeutung beigemessen wird, weil die interessierten Erwachsenen zu Multiplikatoren der zu pflegenden Sprache werden können, indem sie etwa die Regionalsprache in Kindergärten und Schulen unterrichten, Kulturarbeit in vielfältiger Weise leisten oder wenigstens mit ihren Enkelkindern sprechen. ${ }^{2}$ Lediglich die Bundesländer Bremen und Hamburg haben sich durch die Unterzeichnung der Europäischen Charta der Regional- oder Minderheitensprachen ${ }^{3}$ überhaupt verpflichtet, Niederdeutschkurse in der Erwachsenenbildung anzubieten. Niedersachsen, Schleswig-Holstein und Brandenburg ermutigen lediglich dazu, weil sie keinen direkten administrativen Zugriff auf die Volkshochschulen haben und die anderen norddeutschen Bundesländer haben diesen Artikel nicht übernommen. ${ }^{4}$

Dennoch wurde und wird das Niederdeutsche, wenn auch in regional quantitativ wie qualitativ stark unterschiedlichem Maße an vielen norddeutschen Volkshochschulen unterrichtet. Für den westdeutschen Raum stellt Appenzeller einen „schwachen Höhepunkt“ des Niederdeutschangebots an Volkshochschulen (VHS) bereits Anfang der 1980er Jahre fest, auf den allerdings ein starker Rückgang in den 1990ern erfolgte. ${ }^{5}$ Mecklenburg-Vorpommern (bzw. die damaligen Kreise Rostock, Neubrandenburg und Schwerin) war zu DDR-Zeiten von einer eher restriktiven Niederdeutsch-Politik geprägt und erlebte einen - in seinen Ausmaßen zweifellos überschaubaren - „Hype“ der Niederdeutschkurse, wie ihn die VHS-Dozentin der Kreisvolkshochschule Nordvorpommern beschreibt, nach der Wende bzw. sogar erst im Nachklang der Aufnahme des Niederdeutschen in die Europäische Charta der Regional- oder Minderheitensprachen. ${ }^{6}$

1 Bundesraat för Nedderdüütsch 2007, S. 1. Hervorhebung im Original.

2 Vgl. Schebesta 2005.

3 Bundesraat för Nedderdüütsch 2016, Artikel 8: Bildung.

4 Vgl. Möller 2004, S. 297.

5 Appenzeller 2004, S. 26.

6 Aus dem Interview der Dozentin der Kreisvolkshochschule (KVHS) Nordvorpommern vom Juli 2014. Die Interviewpartnerin wird im folgenden Text mit der Sigle MV-2 zitiert. 
Dass Niederdeutsch in der Erwachsenenbildung zu jeder Zeit sowohl wissenschaftlich als auch alltagspraktisch ein ziemlich randständiger Bereich geblieben ist, wird insgesamt niemanden verwundern. Während die Notwendigkeit des Niederdeutschunterrichts in Kindergarten und Schule mit Blick auf die Zukunft der Regionalsprache sofort einleuchtet bzw. sich leicht argumentativ begründen lässt, weckt das Konzept des regionalsprachlichen Volkshochschulkurses in der Regel einige Zweifel, die sich auf ihre Sinnhaftigkeit beziehen. Wer frequentiert überhaupt VHS-Kurse, um eine „kleine Sprache“ zu lernen oder einen Dialekt, als der das Niederdeutsche im Alltagswissen der Laien heute vielfach noch gilt? Was sind die Motive der Niederdeutschlehrkräfte und wie effektiv kann ein Kurs überhaupt sein, der für zwei Stunden einmal in der Woche von häufig nicht ausgebildeten Dozierenden ohne festen Rahmenplan gehalten wird? Von fachwissenschaftlicher Seite lässt sich darüber hinaus auch in Frage stellen, ob diese Kurse überhaupt ausschließlich dem Spracherwerb gelten sollen und damit als Mittel zur Bewahrung des Niederdeutschen gedacht sind. So hält Goossens etwa fest:

„Seit einigen Jahren werden von Volkshochschulen und Heimatvereinen Plattdeutschkurse veranstaltet, die den Interessierten Gelegenheit geben, den Dialekt zu erlernen und die Sprechfertigkeit zu üben. Dieser Unterricht der Mundart als Fremdsprache ist aber m. E. nicht so sehr als konservierender Faktor zu deuten, sondern vielmehr als ein Aspekt der Erscheinung, Dialekt als Kulturfaktor' " ${ }^{7}$

Tatsächlich hat sich das Niederdeutsche von der alltagssprachlichen Nähesprache, als die es Jahrhunderte lang funktioniert hatte, zunehmend zu einer Freizeit- und Kultursprache mit hohem identifikatorischen Wert entwickelt. In der für eine kleine Sprache außergewöhnlich ausdifferenzierten niederdeutschen Kultursze$n e^{8}$ ist das Niederdeutsche vom reinen Kommunikationsmittel zum „Kulturprodukt" und als solches zum Objekt der Freizeitbeschäftigung geworden, ob nun der Heimatverein zur Diskussion der Werke von Regionalschriftstellern einlädt, der Norddeutsche Rundfunk die beste niederdeutsche Band ehrt oder zahlreiche Laienbühnen niederdeutsche Stücke inszenieren. ${ }^{9}$ In diesem Sinne könnten auch die VHS-Niederdeutschkurse bloße Ausläufer dieses Kulturbetriebes sein und

7 Goossens 1993, S. 401.

8 Zur niederdeutschen Kulturszene Wirrer 1990; Appenzeller 2004; Möller 2004.

9 Die Dozentin aus Nordvorpommern, die selbst als Schauspielerin aktiv ist, berichtet in diesem Zusammenhang auch von Jugendlichen, die leidenschaftlich niederdeutsches Theater spielen, aber hierfür alle Stücke mühselig auswendig lernen müssen, da sie selbst keine Alltagskompetenz in der Sprache besitzen. 
als solche ihren Teilnehmenden schlicht eine Plattform liefern, um sich in ihrer Freizeit in organisierter Form mit der Regionalsprache zu befassen. In diesem Falle wäre der Nutzen für Spracherwerb und Spracherhalt wohl tatsächlich als eher gering einzustufen, denn dann wären die VHS-Kurse eher Stammtisch- oder Kaffeeklatschersatz für Niederdeutschsprechende, die kein natürliches Kommunikationsumfeld mehr haben.

Inwieweit das Format Volkshochschulkurs im Sinne der Regionalsprachencharta das Lehr- und Lernkonzept der Sprachvermittlung und damit des Sprachenschutzes umzusetzen geeignet ist oder lediglich Gelegenheit zur Auseinandersetzung mit norddeutscher Regionalkultur bietet, soll in diesem Beitrag erörtert werden. Hierzu wird in Form einer Fallstudie von Kursen aus Mittel- und Nordvorpommern die gegenwärtigen Unterrichtspraxis an Volkskochschulen im Bundesland Mecklenburg-Vorpommern untersucht und mit den Volkshochschulen im übrigen niederdeutschen Sprachraum abgeglichen.

\section{Die Untersuchung}

Schwerpunktmäßig in den Jahren 2010 und 2011 und im Rahmen von Nacherhebungen bis ins Jahr 2014 habe ich eine teils quantitativ, teils qualitativ angelegte Erhebung an Volkshochschulen im gesamten niederdeutschsprachigen Raum durchgeführt. Auf die ursprünglich auch geplanten teilnehmende Beobachtungen im Niederdeutschunterricht der Volkshochschulen habe ich verzichten müssen. ${ }^{10}$ In meine Untersuchung habe ich ausschließlich solche Kursangebote einbezogen, die im VHS-Kursprogramm einen expliziten Sprachvermittlungscharakter erkennen ließen. Die weitaus zahlreicheren „Klönschnacks“ und „plattdeutschen Lesenachmittage“ wurden bewusst ausgeklammert. Inwieweit mir dies allerdings tatsächlich gelungen ist, muss ganz im Sinne der oben skizzierten Fragestellung durch die Auswertung der Daten geklärt werden.

Mittels eines überwiegend quantitativ auszuwertenden Fragebogens wurden zunächst sowohl Niederdeutschlehrende als auch -lernende unter anderem nach ihrer Niederdeutschkompetenz, ihrem sprachlichen Hintergrund und ihren Motivationen befragt. Die Dozierenden sollten darüber hinaus Angaben über

10 Die geplante teilnehmende Beobachtung wurde von einem Großteil der VHSDozierenden nicht gestattet. Darüber hinaus legten die wenigen getätigten Kursbesuche den Verdacht nahe, dass einige Dozierende den Unterricht sehr auf meinen Besuch ausgerichtet hatten und sich so kein authentisches Bild vom normalen Ablauf der Kurse gewinnen ließ. 
Kurszielsetzungen, Unterrichtsmethoden und eingesetzte Materialien machen. Aufbauend auf dieser ersten quantitativen Annäherung sollten jeweils anderthalbstündige Experteninterviews mit ausgewählten Dozierenden tiefere Einblicke in die Unterrichtskonzeption gewähren.

Meine Stichprobe umfasst insgesamt 242 Lernenden- und 28 Lehrendenfragebögen sowie 15 Experteninterviews von insgesamt 27 Volkshochschulen aus den Bundesländern Niedersachsen, Schleswig-Holstein, Hamburg, Bremen, Brandenburg, Mecklenburg-Vorpommern und Nordrhein-Westfalen, wobei die Karte (vgl. Abb. 1) die Unterschiede in der regionalen Verteilung erkennen lässt. Auch durch aufwendige Nacherhebungen ist es mir nicht gelungen eine ausgeglichenere Streuung der Erhebungsstandorte zu erreichen. Während es beispielsweise in Nordrhein-Westfalen und um Hamburg herum eine erstaunliche Ballung von Niederdeutschangeboten gibt, ließen sich in Mecklenburg-Vorpommern auch nach mehrmaliger Nachfrage nicht mehr als vier Volkshochschulen des Bundeslandes finden, an denen in den zurückliegenden Jahren Niederdeutsch angeboten wurde und zum Teil auch heute noch wird. Als Gründe für die Abwesenheit von Niederdeutsch im Kursprogramm wurden von den VHS-Leitungen sowohl die mangelnde Nachfrage seitens der Teilnehmenden als auch ein fehlendes Angebot an geeigneten Lehrkräften genannt. ${ }^{11} \mathrm{Da}$ mir für Mecklenburg-Vorpommern letztlich nur 11 Lernenden- und 2 Lehrendenfragebögen sowie die dazugehörigen Interviews vorliegen, kann ich hier lediglich eine Art Fallanalyse der Volkshochschulkurse in der Region Uecker-Randow (im Folgenden MV-1) und Nordvorpommern (MV-2) vor dem Hintergrund der Gesamtstichprobe vornehmen. Da sich bei der quantitativen Auswertung rein rechnerisch keine grundlegenden Abweichungen der mecklenburg-vorpommerschen Teilstichprobe vom Gesamtdatensatz aller 27 VHS ergeben haben und sich einige bundeslandspezifische Besonderheiten darüber hinaus sehr eindrucksvoll durch das qualitative Interviewmaterial illustrieren lassen, scheint es mir dennoch möglich, die Fragestellung mit aller gebotenen Vorsicht auch gezielt für Mecklenburg-Vorpommern zu bearbeiten.

11 Herrmann-Winter konnte in den Jahre um 1980 in den Nordbezirken der DDR noch an sechs Standorten Niederdeutschkurse an Volkshochschulen untersuchen, spricht aber davon, dass die Teilnehmerzahlen damals schon „rückläufig“ gewesen seien, Herrmann-Winter 1985, S. 297. 
Abbildung 1: Das Erhebungsgebiet (2010-2014). Stichproben in den namentlich aufgeführten Volkshochschulen

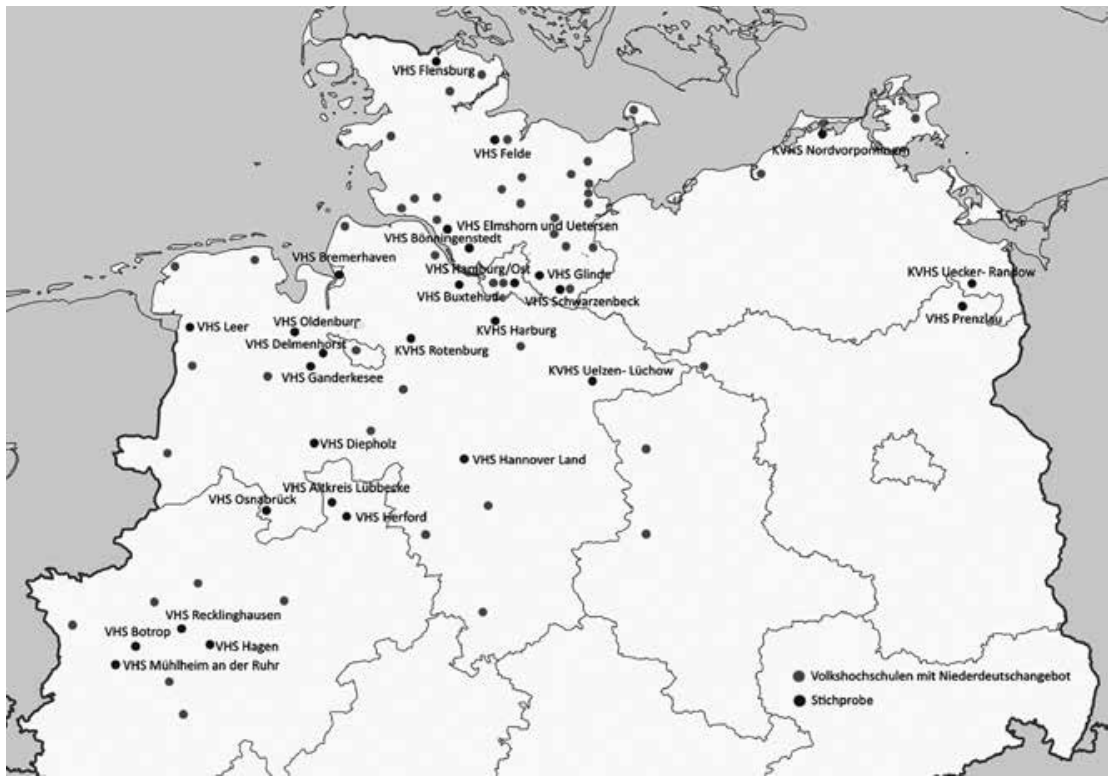

\section{Auswertung: VHS-Niederdeutschkurse als Freizeitvertreib oder Sprachkurs?}

Um die aufgeworfene Frage nach dem Charakter der VHS-Niederdeutschkurse adäquat beantworten zu können, sollen im Folgenden fünf Aspekte aus dem Datenmaterial heraus schlaglichtartig beleuchtet werden, die in ihrer Zusammenschau eine gute Indizienlage versprechen: 1. Was lässt sich über das Format Niederdeutsch-VHS-Kurs im Vergleich zu anderen Sprachkursen allgemein festhalten und 2. durch welche Unterrichtsmethoden zeichnet es sich aus? 3. Wer sind die Teilnehmenden dieser Kurse? Handelt es sich um die typischen Dialektsprechenden, also um schlechter gebildete sogenannte „NORMs“, ${ }^{12}$ die bereits über Niederdeutschkompetenz verfügen? 4. Welche Kompetenzen können und sollen in diesen Kursen überhaupt vermittelt werden? 5. Welche Motive bewegen Lehrende wie Lernende dazu, ihre Freizeit mit Niederdeutschunterricht zu füllen?

12 Im Rückgriff auf Chambers/Trudgill 1980, S. 33 werden in der Dialektologie die typischen Dialektsprecher als „non-mobile older rural males“ (NORMs) charakterisiert. 
Kurzum: Steht die Weitergabe der Sprache im Zentrum der Kurszielsetzungen oder ist es tatsächlich so, wie die Dozentin aus Nordvorpommern resigniert als Resümee festhält:

„Und die kommen auch nicht, weil sie Plattdeutsch lernen wollen. Die können es meistens. Die kommen einfach, weil sie sonst keinen haben, der es noch mit ihnen spricht. Das geht nicht ums Lernen! Also schon lange nicht mehr! Das war vielleicht so vor zwanzig Jahren noch so, ja, die da kamen, die wollten es noch lernen. Aber die jetzt kommen, sind die, die einfach sagen, wir haben sonst keine Gelegenheit mehr. So muss man das, glaube ich, auf den Punkt bringen. ${ }^{{ }^{13}}$

\subsection{Zum Format Niederdeutschkurs}

Zunächst lässt sich feststellen, dass das Format VHS-Niederdeutschkurs den äußeren Rahmenbedingungen und Veranstaltungshinweisen gemäß im Wesentlichen anderen fremdsprachlichen Angeboten an den Volkshochschulen entspricht. Es handelt sich zumeist um wöchentlich stattfindende, anderthalbstündige Kurse, die die Vermittlung oder Auffrischung von Basiskompetenzen versprechen. Eine für Fremdsprachen übliche Differenzierung nach Leistungsniveaus in Anfänger- und Fortgeschrittenenkurse findet hingegen in aller Regel nicht statt. Üblicherweise werden neue Kursteilnehmende einfach in den laufenden Kursbetrieb integriert. Diese mangelnde Ausdifferenzierung der Lernendenniveaus als Indiz für den eingangs unterstellten ausschließlichen Freizeitcharakter des Kursformats zu werten, liegt nahe. Durch die ständigen Neuzugänge von Lernenden dürfte eine konsequente Kompetenzsteigerung im fortgeschrittenen Bereich behindert werden und andererseits könnten die vorhandenen Fähigkeiten der fortgeschrittenen Kursteilnehmenden ihrerseits den Lernfortschritt der Anfängerinnen und Anfänger aufhalten bzw. zu deren Entmutigung beitragen. Letztendlich ist der Hauptgrund für den gemeinsamen Unterricht von Lernenden unterschiedlicher Kompetenzniveaus schlichtweg in der mangelnden Nachfrage nach Niederdeutschkursen zu sehen, denn die meisten Volkshochschulen legen eine Mindestteilnehmendenzahl von acht bis zehn Personen fest, die im Falle von Niederdeutschkursen häufig nicht ohne Weiteres erreicht wird.

Dass eine Mehrheit von 59 \% der Gesamtstichprobe der Lernenden $(\mathrm{n}=242)$ nicht zum ersten Mal einen Niederdeutschkurs besucht, kann - in Ermangelung der Aufstiegsmöglichkeiten in einen Fortgeschrittenenkurs - also zunächst wenig verwundern. Allerdings gibt immerhin ein Fünftel aller Befragten an, bereits mehr als zehn Kurse besucht zu haben, was meines Erachtens weniger für kon-

13 MV-2, KVHS Nordvorpommern, Juli 2014.

Birte Arendt, Andreas Bieberstedt and Klaas-Hinrich Ehlers - 978-3-631-71893-3 
sequent fortgeführte Sprachvermittlung als vielmehr für eine gewisse „Stammtischqualität" der Kurse spricht. Während die fehlende Kompetenzdifferenzierung von keinem der befragten Dozierenden als große Schwierigkeit eingestuft wird, besteht durchaus Kritik am Umfang des Lehrangebots. So die Dozentin der KVHS Uecker-Randow: „Und ich kann nicht in vierzig Stunden Plattdeutsch lernen. Das geht mit keiner Sache. Auch mit einer Fremdsprache ist das doch auch so. "14

Auch in den Augen der meisten anderen Niederdeutsch-Dozierenden kann und soll der VHS-Unterricht lediglich einen Einstieg in Sprachlernen und -gebrauch darstellen. Allerdings lassen sich hier durchaus regionale Unterschiede feststellen, die den Sprachverhältnissen in den unterschiedlichen Niederdeutschgebieten geschuldet sind. In den mundartschwächeren Gebieten fehlt es außerhalb des Kurses oft schlicht an Möglichkeiten, das Erlernte einzusetzen und zu vertiefen. Für Neueinsteigende kann der VHS-Kurs in diesen Gegenden also nur einen Ort der Sprachbegegnung darstellen, an dem sich diejenigen treffen, die sonst keine Gesprächspartner mehr finden.

\subsection{Unterrichtsmethoden}

Die große Mehrzahl der Dozierenden (11 von 15) geben im Experteninterview an, dass Niederdeutschunterricht an der Volkshochschule grundsätzlich wie Fremdsprachenunterricht aufgebaut sei. Dass sich diese Aussagen dabei nicht nur auf das bloße Kursformat beziehen, sondern durchaus auch die Unterrichtsmethodik meinen, lässt sich aus den ausführlichen Beschreibungen des Kursaufbaus erschließen. Wie im Fremdsprachenunterricht kommt eine sehr große Bandbreite an Unterrichtsmethoden zum Einsatz, wobei die verschiedenen Lehrkräfte natürlich individuelle Präferenzen haben und je nach Konzeption des Kurses auch unterschiedliche Schwerpunkte setzen müssen. Ein Literaturkurs, wie er beispielsweise in Elmshorn angeboten wird, erfordert einfach mehr Textarbeit als ein Konversationskurs. ${ }^{15}$

Generell lässt sich sagen, dass ganz im Sinne der Tatsache, dass das Niederdeutsche Jahrhunderte lang eine überwiegend gesprochene Alltagssprache war, in den VHS-Kursen der Domäne der Mündlichkeit die weitaus größte Aufmerksamkeit geschenkt wird. Im Schnitt wird also der größte Teil der Unterrichtszeit für die

14 MV-1, KVHS Uecker-Randow, Juli 2010.

15 Da eine ausführliche Darstellung der unterschiedlichen Kursformate an dieser Stelle zu weit führen würde, sei auf Arbatzat 1990, S. 198 verwiesen. Er beschreibt ausführlich und sehr treffend verschiedene Kurskonzeptionen, die sich auch in meiner Untersuchung wiederfinden. Vgl. Holm 1981. 
Vermittlung sprechsprachlicher Kompetenz verwendet, während das Schreiben nur eine marginale Rolle spielt - größtenteils zur Fixierung des Erlernten. Dementsprechend kommen verstärkt auflockernde Unterrichtselemente wie Rollenspiele, das Lesen mit verteilten Rollen oder Singen von Liedern zum Einsatz, die bevorzugten Textsorten sind Kurzgeschichten, Schwänke, Witze, Rätsel und Reime, die zumeist zur Konversationsanregung genutzt werden. Die Dozentin aus Nordvorpommern fasst ihre Vorgehensweise folgendermaßen zusammen:

\begin{abstract}
„Man nimmt sich die Szenen des täglichen Lebens, man lässt den Tag Revue passieren und weiß, was mach ich morgens? Ich geh arbeiten, ich esse Mittag. Was esse ich? Dafür muss ich einkaufen. Man hat ja schon einen vorgegebenen Wortschatz aus dem Hochdeutschen. Bitteschön, den zu übersetzen sollte ja nicht die Schwierigkeit sein! Man bündelt das thematisch zusammen. Man fängt, wie wenn man jemandem das Sprechen beibringt, mit dem kleinen, einfachen Satz an, wie bei jeder Fremdsprache und buchselt das hoch. Und ich glaube, da macht Plattdeutsch zu keiner anderen Sprache auch nur irgendwie einen Unterschied. ${ }^{" 16}$
\end{abstract}

So naheliegend, wie diese Unterrichtsmethoden tatsächlich auch in Anfängerkursen anderen Sprachunterrichts zu vermuten sind, so ist ein weiterer elementarer Bestandteil von Fremdsprachenunterricht unter den Niederdeutschdozierenden umstritten: Nur neun von fünfzehn Befragten geben im Interview an, überhaupt Grammatik zu unterrichten. Häufig ist die Entscheidung gegen den Grammatikunterricht wie im Fall der Dozentin aus Pasewalk schlicht zielgruppengebunden, da sie ihn beispielsweise angesichts von zu unterrichtenden Erzieherinnengruppen in Kombination mit der geringen Unterrichtszeit als unzweckmäßig empfindet: „Und wenn sie von vorn anfangen wollen und richtig exakt wie Grammatik, Rechtschreibung... komme ich nicht vorwärts und schaffe ich nicht das, was ich bei Erziehern will, dass sie den Kindern mit Liedern, mit Gedichten das Plattdeutsche näher bringen. Das passiert am meisten über die Lieder und die kleinen Gedichte, ne. ${ }^{\text {"17 }}$ Den potenziellen Multiplikatoren des Niederdeutschen in den Kindergärten wird bei der Vertiefung ihrer Sprachkompetenz an der VHS die Grammatik der Regionalsprache also nicht eigens vermittelt.

Sehr häufig wird von den befragten Niederdeutschdozierenden befürchtet, der Grammatikunterricht könnte den Grundgedanken des Niederdeutschunterrichts korrumpieren, ihm nämlich den Spaß rauben: „Ja! Plattdeutsch muss Spaß machen, das ist das allerallererste! Alles andere findet sich!“18

16 MV-2, KVHS Nordvorpommern, Juli 2014.

17 MV-1, KVHS Uecker-Randow, Juli 2010.

18 MV-2, KVHS Nordvorpommern, Juli 2014.

Birte Arendt, Andreas Bieberstedt and Klaas-Hinrich Ehlers - 978-3-631-71893-3 
In diesem Sinne betonen auch beide Dozentinnen aus MecklenburgVorpommern, sie würden Niederdeutsch zwar wie eine Fremdsprache unterrichten, gestalten den Unterricht aber eher wie ein Freizeitprogramm, indem sie beispielsweise viel Theater spielen, selbst Gedichte rezitieren oder Lieder einüben lassen. Besondere Bedeutung scheint diese Art der Kulturvermittlung in Mecklenburg-Vorpommern insofern zu haben, als beide die Relevanz des Niederdeutschen im kulturellen Nahbereich unterstreichen: Auch sie selbst benötigen das Niederdeutsche vor allen Dingen, um auf lokalen und regionalen Festen aufzutreten oder einfach nur Familienfeiern aufzulockern. Insgesamt kann der Fokus auf spaßerhaltende Unterrichtselemente wohl allein deshalb kaum verwundern, weil es sich beim Kursbesuch eben um eine vollkommen freiwillig ausgeübte Freizeitbeschäftigung handelt.

\subsection{Die Kursteilnehmenden: Alter, Geschlecht und Bildungsgrad}

Auch wenn das für die Fragestellung hier weniger zielführend ist, lässt sich auf die Frage, ob es sich bei den Kursteilnehmenden um ,typische Dialektsprecher, also um schlechter gebildete NORMs (s. o.) handelt, die lediglich ihrem Freizeitvergnügen nachgehen, zunächst Folgendes feststellen: Zumindest die $\mathrm{Zu}-$ schreibung männlich trifft hier bei einer Mehrheit nicht zu, denn 58,5\% der befragten Lernenden der Gesamtstichprobe bzw. sogar 72,2\% der mecklenburgvorpommerschen Teilstichprobe sind weiblich. Auch das Attribut non mobile dürfte auf die VHS-Zielgruppe insofern weniger zutreffen, als ein Großteil der Volkshochschulen Kreisvolkshochschulen mit einem großen Einzugsgebiet sind, die eine gewisse Mobilität und damit auch einen Austausch jenseits der Ortsebene notwendig machen. Bestätigen lässt sich hingegen die Vermutung, dass es sich bei den Kursteilnehmenden größtenteils um ältere Personen handelt. Zwar reicht die Spannweite für die Gesamtstichprobe von 12 bis 89 Jahren, aber der Mittelwert liegt dennoch bei 60,1 Jahren. 46,3\% der Gesamtstichprobe sind damit älter als 66 Jahre, nur 5,2 \% jünger als 30 Jahre (vgl. Tabelle 1). In der mecklenburgischvorpommerschen Stichprobe ist dieser Altersschnitt zugunsten der zweitältesten Altersgruppe verschoben. Hier sind immerhin 6 von 11 Befragten zwischen 46 und 65, aber nur eine einzige Person ist jünger. ${ }^{19}$

19 Gegenüber einer Befragung der Teilnehmenden von Niederdeutschkursen in Volkshochschulen in Wismar, Rostock, Greifswald, Schwerin, Waren und Neubrandenburg aus den Jahren 1979-1981 ist das Durchschnittsalter heutiger Kursteilnehmer in Mecklenburg-Vorpommern deutlich gestiegen. Es betrug um 1980 noch etwa 45 Jahre, auch damals überwog aber schon der Anteil weiblicher Teilnehmerinnen deutlich, vgl. Herrmann-Winter 1985, S. 301. 
Tabelle 1: Altersgruppen der Kursteilnehmenden

\begin{tabular}{|l|c|c|}
\hline $\begin{array}{l}\text { Alter in } \\
\text { Gruppen }\end{array}$ & Gesamtstichprobe in Prozent & Mecklenburg-Vorpommern in Prozent \\
\hline unter 30 & 5,2 & 9,1 \\
\hline 30 bis 45 & 11,7 & 0,0 \\
\hline 45 bis 65 & 36,8 & 54,5 \\
\hline $66+$ & 46,3 & 36,4 \\
\hline
\end{tabular}

Zwar kann es generell auch jenseits der Unterstellung größerer Dialektkompetenz und -verwendung wenig verwundern, dass es die älteren Leute sind, die sich vorrangig für das Format Niederdeutschkurs interessieren, da Menschen mittleren Alters angesichts von Erwerbstätigkeit und Kindererziehung auch deutlich weniger freie Zeit zur Verfügung steht, die sie mit VHS-Angeboten füllen könnten. Dennoch ist dieses Ergebnis sehr eindeutig und würde für Englisch oder Spanisch oder jede andere Sprache, mit der instrumenteller Nutzen verbunden wird, nicht so ausfallen.

Was die Zuschreibung schlechter Bildung angeht, die eventuell der Frequentierung eines Sprachlernangebots entgegenstehen würde, lässt sich konstatieren, dass sich auch diese Hypothese durchaus in der Tendenz bestätigen lässt. Allerdings scheint der Zusammenhang aber nicht so stark zu sein wie erwartet. Knapp zwei Drittel der Befragten in Mecklenburg-Vorpommern und gute zwei Drittel der Gesamtstichprobe verfügen nur über einen mittleren oder niedrigeren Schulabschluss. Die typischen Teilnehmenden an VHS-Kursen zum Niederdeutschen sind also keine NORMs, sondern größtenteils mobile, ältere Frauen. Und sie haben womöglich, wie Frauen dieser Altersgruppe typischerweise, eher keine höheren Schulabschlüsse machen können.

\subsection{Niederdeutsch-Kompetenz}

Verlässt man sich bezüglich der Kompetenzzuschreibung der Teilnehmenden auf die Aussagen der beiden Dozierenden aus Vorpommern, ergibt sich ein höchst uneinheitliches Bild. Während MV-2 konstatiert, ihr Kurs würde von den Teilnehmenden nicht besucht „um zu lernen, weil die es schon können“ ${ }^{20}$, sind laut MV-1 „im Höchstfalle zwei dabei, (...) die das schon mitbringen. Der Rest muss von vorn anfangen. ${ }^{\text {"21 }}$ Diese große Diskrepanz kann durchaus der im dialektschwachen Mittelpommern und dialektstärkeren Vorpommern differierenden generellen Niederdeutschkompetenz geschuldet sein, verdient aber eine nähere

20 MV-2, KVHS Nordvorpommern, Juli 2014.

21 MV-1, KVHS Uecker-Randow, Juli 2010. 
Betrachtung. 17,9 \% der Gesamtstichprobe der Lernenden gaben an, vor Beginn des Niederdeutschkurses bereits alles zu verstehen und im Gespräch alles sagen zu können, was sie wollten. ${ }^{22}$ Das legt nahe, dass dem Kursbesuch bei diesen Kursteilnehmenden tatsächlich eine Motivationslage jenseits des Wunsches nach Spracherwerb zugrunde liegt. Gar keine Niederdeutschkompetenz attestierte sich hingegen eine verschwindend kleine Gruppe von 1,8 \% der Befragten. Die überwiegende Mehrheit verfügte nach eigener Aussage vor Kursbeginn schon über eine passive Sprachkompetenz (vgl. Abb. 2).

Abbildung 2: Vorhandene Sprachkompetenz der befragten Kursteilnehmenden vor Kursbeginn in Prozent $(n=242)$
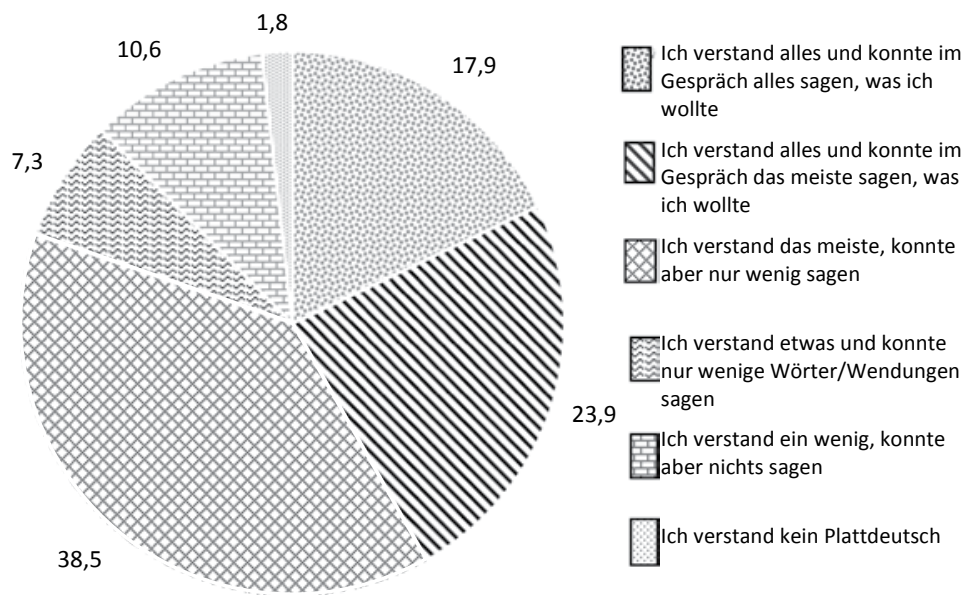

Dementsprechend positiv fällt zum Befragungszeitpunkt auch die Einschätzung der verschiedenen Teilbereiche ihrer derzeitigen Sprachkompetenz aus. 92,4\% aller Befragten sind der Meinung, auf Niederdeutsch alles oder das meiste verstehen, $85,7 \%$ alles oder das meiste lesen zu können. Immerhin 57 \% geben darüber hinaus an, über entsprechend gute aktive mündliche Kompetenz zu verfügen. Lediglich der Wert für die zugeschriebene Schreibkompetenz fällt niedriger aus. Nur 35,4 \% können ihrer Aussage zufolge alles oder das meiste auf Niederdeutsch schreiben, was sie möchten.

22 Um die unterschiedlichen Ausprägungen aktiver und passiver Kompetenz ansatzweise erfassen zu können, waren auf die Frage in meinem Fragebogen „Verfügten Sie vor Beginn des Kurses über Plattdeutschkenntnisse“ die in Abbildung 2 angeführten Antwortmöglichkeiten vorgesehen. 
Abbildung 3: Aktuelle Sprachkompetenz der befragten Teilnehmenden an Niederdeutsch-Kursen der VHS

Gesamtstichprobe

Sprechen

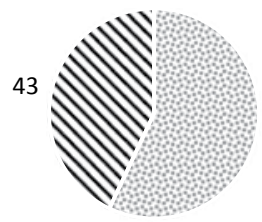

57

Verstehen

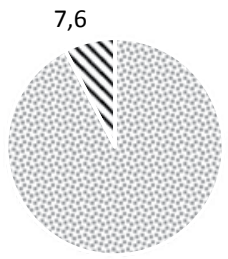

92,4
Mecklenburg-Vorpommern

Sprechen

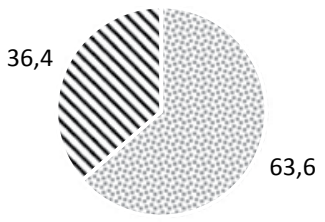

Verstehen

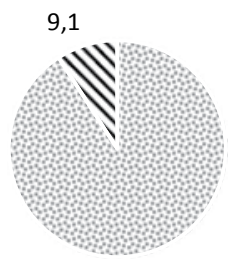

90,9

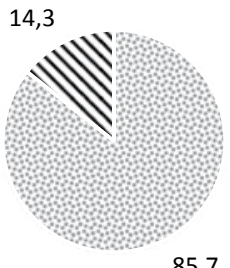

85,7

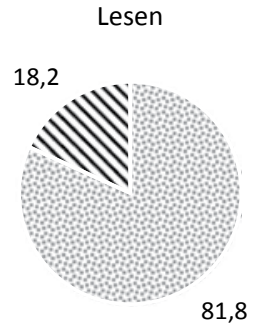

Schreiben

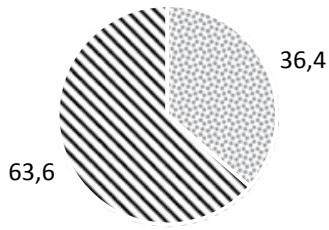

alles/das meiste

etwas/nichts $\mathbf{S}$

Birte Arendt, Andreas Bieberstedt and Klaas-Hinrich Ehlers - 978-3-631-71893-3 
Letztendlich scheinen die Ergebnisse hier im Wesentlichen den Kompetenzverteilungen der großen Sprachstandserhebungen zu entsprechen, in denen sich auch der starke passive plattdeutsche Hintergrund sowie der Fokus auf die gesprochene Sprache manifestierten. ${ }^{23}$ Dass in meiner Befragung der Wert für die vorhandene Schreibkompetenz deutlich höher angesiedelt ist, ${ }^{24}$ dürfte der Unterrichtspraxis geschuldet sein, die auch den Teilnehmenden in mehr oder weniger starkem Umfang eine schriftliche Fixierung des Erlernten abverlangt und somit ihre Schreibkompetenz automatisch fördert.

Möchte man schließlich die Frage beantworten, ob die Teilnehmenden der Niederdeutschkurse bereits so kompetent sind, dass der Kursbesuch weniger dem Erlernen der Sprache als vielmehr dem "Schnacken“ gilt, lässt sich hier vorläufig feststellen, dass der Anteil bereits kompetenter Sprechender verglichen mit anderen VHS-Sprachkursen unvergleichlich hoch sein dürfte, es andererseits aber doch eine große Zahl von Teilnehmenden gibt, die tatsächlich vor Beginn des Kurses nur ein wenig oder gar kein Niederdeutsch konnten und bei denen man ein vorrangiges Interesse am Erwerb von Sprachkompetenz voraussetzen kann. ${ }^{25}$

\subsection{Motivation}

Dass sich die Motivationslage, die für den Besuch eines Sprachkurses ausschlaggebend ist, im Falle des Niederdeutschen deutlich anders darstellt als bei anderen Sprachen, liegt unmittelbar auf der Hand, wenn man die Entwicklungsgeschichte und jetzige Stellung des Niederdeutschen in der Gesellschaft betrachtet. Anders als etwa beim Englischen, Spanischen oder im Falle der hier vertretenen UeckerRandow-Region vielleicht auch beim Polnischen ist der rein praktische Nutzen des Niederdeutschen ob seiner geringen funktionalen und arealen Reichweite deutlich eingeschränkt. So überwiegen in den kategorisierten Freitextantworten ${ }^{26}$

23 Stellmacher 1987 zur GETAS-Umfrage und Möller 2008 zur repräsentativen Sprachstandserhebung des Instituts für Niederdeutsch (INS).

24 Während sich in meiner Befragung ganze 35,4 \% der Gesamtstichprobe und 36,4 \% der mecklenburgisch-vorpommerschen Teilstichprobe zugestehen, alles oder das meiste schreiben zu können, geben z. B. in der INS-Studie nur $2 \%$ der Befragten gute und niemand sehr gute Schreibkompetenz an.

25 Ähnlich große Unterschiede in der Niederdeutschkompetenz, die die Teilnehmenden in die VHS-Kurse mitbrachten, stellte schon Herrmann-Winter 1985, S. 302 fest.

26 Die Kategorisierung der teilweise sehr ausführlichen Freitextantworten erfolgte größtenteils induktiv, d. h. aus dem Material selbst. Auf eine erste deduktive Herangehensweise lassen sich die Kategorien berufliche Gründe, soziales Umfeld und Spracherhalt 
der Teilnehmenden-Fragebögen auch klar intrinsische Motive. ${ }^{27}$ Extrinsische Motive für die Kursteilnahme werden nur von wenigen Befragten genannt: 5,7 \% der Befragten nennen etwa berufliche Gründe, $13 \%$ sprechen von kommunikativer Notwendigkeit bzw. von angestrebter Verbesserung im sozialen Umfeld und 3,9 \% gaben an, sich als Zugezogene mithilfe des Niederdeutschkurses auch sprachlich in die neue (Heimat-)Region integrieren zu wollen.

Wie Abbildung 4 ferner zeigt, fallen dagegen identifikatorische Gründe insgesamt und hier vor allem der Bezug zum eigenen niederdeutschen Hintergrund verhältnismäßig stark ins Gewicht. Einen direkten Hinweis auf die Bedeutung des Kurses bzw. des Niederdeutschen als Freizeitvertreib haben hingegen zunächst nur 10,9\% der Teilnehmenden gegeben. Nimmt man allerdings die 29,6 \% der etwas unspezifischeren Kategorie Spaß an der Sprache hinzu, in der alle Antworten vereint sind, die in irgendeiner Weise Sympathiebekundungen gegenüber der Sprache selbst oder dem Niederdeutschlernen beinhalten, lässt sich durchaus der große Wert des Niederdeutschen als Objekt der Freizeitbeschäftigung ableiten. Dass zumindest ein Teil der Befragten beim Beantworten der Frage offenbar den potenziellen Lerneffekt des Kurses im Hinterkopf hatte, spiegeln die 20,9\% der Antworten wider, in denen sich der Wunsch nach dem Ausbau bereits vorhandener Kompetenz erkennen lässt.

Auch wenn die Prozentwerte ob der geringen Fallzahl mit Vorsicht zu genießen sind, kann man mit Blick auf die mecklenburgisch-vorpommersche Teilstichprobe einerseits einen Fokus auf Hobby/Freizeitgestaltung und andererseits einen auf Spracherhalt erkennen (vgl. Abb. 4). Beides stützt die Vermutung, dass das Niederdeutsche in Mecklenburg-Vorpommern mittlerweile eine besondere Rolle als Objekt der Freizeitgestaltung und weniger als Alltagskommunikationsmittel spielt. Familientraditionen, die das Niederdeutsche als Kommunikationsmittel des Nahbereichs stützen könnten, sind in Mecklenburg-Vorpommern offenbar abgerissen. Auch das stärkste extrinsische Motiv des Spracherwerbs zur besseren

zurückführen, da mit Antworten in diesen Bereichen zu rechnen war. Die hier zu vernachlässigende Kategorie sonstige Gründe beinhaltet ansonsten nicht zuzuordnende Antworten, z. B. durch einen Freund zum Kurs mitgenommen worden zu sein, den Kursleiter zu mögen oder jetzt Zeit für den Kursbesuch zu haben.

27 Unter intrinsischer Motivation versteht man, etwas um seiner selbst willen zu tun, z. B. weil es eine besondere Herausforderung darstellt oder schlicht, weil es einfach Spaß macht, während bei extrinsischer Motivation das Ziel im Vordergrund steht, für bestimmte Leistungen, wie etwa im Beruf, Sanktionen aller Art zu vermeiden, vgl. dazu auch Myers 2004, S. 330-331. 
beruflichen Kommunikation spielt offenbar keine Rolle. ${ }^{28}$ Ebenfalls schwer scheint auch das Argument des Heimischwerdens im Vergleich zum Durchschnitt der Gesamtstichprobe zu wiegen. Das könnte darauf hindeuten, dass dem Niederdeutschen in Mecklenburg-Vorpommern zumindest von außen eine große identifikatorische Bedeutung zugeschrieben wird. Allerdings sollte der Befund hier nicht überinterpretiert werden, denn diese Angaben beruhen in absoluten Zahlen nur auf den Aussagen von drei von elf Personen.

Abbildung 4: Motivation für den Kursbesuch in Prozent (Kategorisierte FreitextMehrfachantworten)

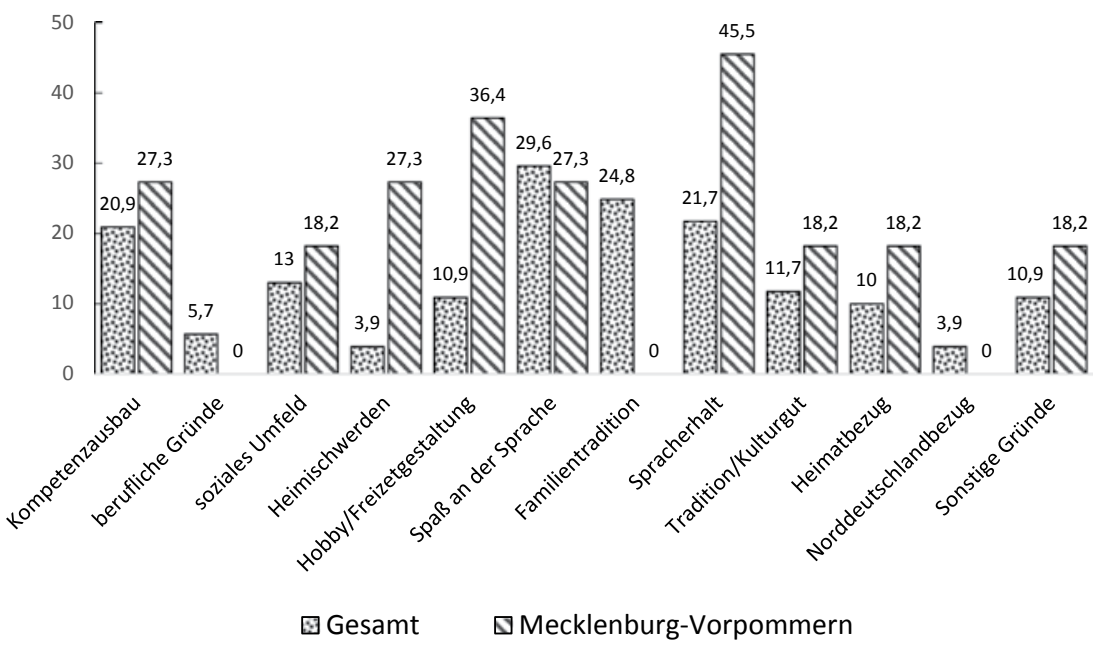

Die Dozierenden geben im Experteninterview weitaus häufiger sprachpflegerische Motive an als die Niederdeutsch-Lernenden. Es gibt zwar niemanden, der davon überzeugt ist, mit VHS-Kursen den Sprachverlust aufhalten zu können, es findet sich aber doch - mit Abstufungen - größtenteils die Überzeugung, dass der Unterricht an der VHS diesen Sprachabbau verlangsamen könne, indem er beispielsweise durch die Sprachbegegnung das Bewusstsein für das Niederdeutsche positiv beeinflusst, Anreiz zum Weiterlernen gibt und die Beschäftigung mit niederdeutschen Kulturgütern wie literarischen Erzeugnissen fördert.

28 In den Jahren um 1980 hatte die Motivation „Plattdeutsch bei der Arbeit ,gut gebrauchen “ zu können, noch eine gewisse, wenn auch damals schon untergeordnete Rolle für den Besuch von VHS-Kursen gespielt, Herrmann-Winter 1985, S. 306. 
Gerade die beiden Dozierenden aus Mecklenburg-Vorpommern messen dem VHS-Unterricht allerdings lediglich eine Erinnerungsfunktion bei: „Platt blifft in uns, bleibt in uns. So habe ich es einfach genannt. Wenn ich jetzt sage: Platt mütt läben oder överlävt oder all solche Sachen, dann müssen wir alle ein bisschen mehr dafür tun. Müssten sie alle ein bisschen aufwachen. ${ }^{\text {"29 }}$ Und die Dozentin aus Nordvorpommern führt auf die Frage nach ihren Motiven und Zielsetzungen aus, dass ein VHS-Kurs „, wie ein Museum“ sei und fügt hinzu:

„Vor zehn Jahren habe ich noch gesagt, Traditionen hochhalten heißt nicht Asche bewahren, sondern die Flamme weitertragen. Jetzt sage ich, bei Plattdeutsch sind wir bei Ascheverwahren angekommen. Alles, was wir an Hype mal hatten und alles, was mal gut funktioniert hat, ist so nach und nach tot und nackt und bloßgelegt. ${ }^{{ }^{30}}$

Die pessimistischen Einschätzungen der beiden Dozierenden aus Vorpommern entsprechen den Fragebogenantworten der Lernenden aus dieser Region, die darauf hindeuten, dass das Niederdeutsche für die Befragten hier - im Unterschied $\mathrm{zu}$ anderen norddeutschen Regionen (vgl. Abb. 4) - seine Rolle als Kommunikationsmittel in den Familien oder am Arbeitsplatz heute ganz eingebüßt hat.

\section{Fazit: Zwischen Hobby und Sprachbewahrung}

Die Frage, ob es sich bei dem Niederdeutschangebot an Volkshochschulen um Freizeitvertreib bzw. um Kulturveranstaltungen oder aber um seriöse Sprachlernangebote handelt, lässt sich hier wohl nicht eindeutig - oder nur eindeutig mit sowohl als auch beantworten. Einmal ganz davon abgesehen, dass die im Kurs erworbenen Kenntnisse im Fach Niederdeutsch nicht abgeprüft bzw. bewertet und nur selten mit einem Zertifikat honoriert werden, ist das Niederdeutschangebot mit Blick auf Umfang und Aufbau der Kurse sowie die angewandte Methodik durchaus mit anderem Fremdsprachenunterricht an der Volkshochschule vergleichbar. Dass aber Niederdeutschkurse ebenso eindeutig in die Kategorie Freizeitvertreib/Kulturveranstaltungen fallen, legt vor allem die Motivationslage der Teilnehmenden nahe. Mit dem Erlernen der Sprache wird weniger instrumenteller Nutzen, als vielmehr ein hohes Maß an Spaß und identifikatorischem Potential mit der Heimat verbunden. Der Anteil an bereits kompetenten Kursteilnehmenden ist verhältnismäßig hoch und kann wohl als deutlichstes Indiz für ein mangelndes Interesse an Sprachvermittlung geltend gemacht werden. Andererseits kann man der ebenfalls recht großen Gruppe derer, die angeben,

29 MV-1, KVHS Uecker-Randow, Juli 2010.

30 MV-2, KVHS Nordvorpommern, Juli 2014.

Birte Arendt, Andreas Bieberstedt and Klaas-Hinrich Ehlers - 978-3-631-71893-3 
über nur wenige passive Niederdeutschkenntnisse zu verfügen, den Sprachlernwillen wohl kaum absprechen. In der Gesamtschau aller Daten lässt sich hier also vor allem festhalten, dass sich Niederdeutschkurse durch eine große Heterogenität an zugrundeliegenden Interessenlagen und Kompetenzniveaus der Teilnehmenden auszeichnen..$^{31}$ Die hier im Titel bewusst plakativ gestellte OderFrage lässt sich dementsprechend auch nur für einzelne Gruppen innerhalb der Niederdeutschkurse beantworten, denn neben denen, die das Niederdeutsche fast muttersprachlich beherrschen und lediglich ein ansprechendes kommunikatives Umfeld suchen, das sie woanders nicht mehr finden, sitzen diejenigen, die es ob nun zwecks Anwendung in einem Pflegeberuf oder zur Integration in eine bestehende Dorfgemeinschaft von Beginn an neu erlernen und zu angenehmen Freizeitplaudereien noch gar nicht in der Lage sind; bei ersteren überwiegt eindeutig der Freizeit- und Kulturcharakter des Kurses, bei letzteren das Sprachlernangebot.

Was aber lässt sich abschließend aus der Untersuchung der gegenwärtigen Situation über die Zukunft des Formats Niederdeutschkurs an den Volkshochschulen ableiten? Auch diese sprachpolitisch durchaus interessante Frage wird sich am Ende nur für einzelne Niederdeutschregionen bzw. einzelne Kursangebote adäquat beantworten lassen. Grundsätzlich können seriös geplante Kurse - und das scheinen die derzeitigen ja durchaus zu sein - als Mittel der Sprachbewahrung gesehen werden, denn wie eingangs bereits erwähnt können sie Multiplikatorinnen und Multiplikatoren der Sprachpflege hervorbringen, die sich ihrerseits für den Erhalt des Niederdeutschen einsetzen. Allerdings zeigt die Analyse des Ist-Zustandes für Regionen wie Mecklenburg-Vorpommern recht deutlich, dass das Potential eher gering zu sein scheint, denn wo weder Kursteilnehmende noch qualifizierte Lehrende vorhanden sind, müssen auch gut durchdachte Kurskonzepte wie in Nordvorpommern zwangsläufig ins Leere laufen.

\section{Literatur}

Appenzeller, Gerrit: Das kulturelle Umfeld des Niederdeutschen und die EUCharta. Ergebnisse einer Untersuchung an der INS-Presse. In: Lehmberg, Maik (Hrsg.): Sprache, Sprechen, Sprichwörter. Festschrift für Dieter Stellmacher zum 65. Geburtstag (ZDL Beiheft; 126). Wiesbaden 2004, S. 25-35.

31 Arbatzat 1990, S. 196-197. Eine ähnlich große Heterogenität sowohl der sprachlichen Voraussetzungen als auch der damit verbundenen Teilnahmemotivationen hatte schon Herrmann-Winter 1985 bei ihrer Untersuchung der damaligen VHS-Kurse in den Nordbezirken der DDR festgestellt. Vgl. auch den persönlichen Erfahrungsbericht aus den 1970er Jahren von Gernentz 1998. 
Arbatzat, Hartmut: Niederdeutsche Sprachkurse. In: Speckmann, Rolf (Hrsg.): Niederdeutsch morgen. Perspektiven in Europa. Beiträge zum Kongress des Instituts für Niederdeutsche Sprache, Lüneburg 19.-21.10.1990. Leer 1990, S. 195-201.

Bundesraat för Nedderdüütsch: Schweriner Thesen zur Bildungspolitik. Beschlossen vom Bundesraat för Nedderdüütsch am 4. Oktober 2007. [OnlineRessource: http://bundesraat-nd.de/index.php?option=com_content\&view=a rticle\&id $=47 \% 3$ Aschweriner-thesen $\&$ catid $=37 \% 3$ Apositionenbildungkat $\&$ Ite mid=59\&lang=de (Stand 11.12.2016)]

Bundesraat för Nedderdüütsch: Die Sprachencharta und ihre Umsetzung. 2016 [Online-Ressource: http://www.bundesraat-nd.de/index.php?option=com_co ntent\&view $=$ article\&id=86\&Itemid=66\&lang $=$ de $($ Stand 11.12.2016) $]$

Chambers, John/Trudgill, Peter: Dialectology. Cambridge [u. a.] 1980.

Gernentz, Marianne: Meine Erfahrungen mit Niederdeutsch-Kursen an der Volkshochschule Rostock in den siebziger Jahren. In: Herrmann-Winter, Renate (Hrsg.): Heimatsprache zwischen Ausgrenzung und ideologischer Einbindung. Niederdeutsch in der DDR. Frankfurt a. M. [u. a.] 1998, S. 399-404.

Goossens, Jan: „Dialektverfall“ und „Mundartenrenaissance“ in Westniederdeutschland und im Osten der Niederlande. In: Stickel, Gerhard (Hrsg.): Varietäten des Deutschen. Regional- und Umgangssprache. Berlin/New York 1997, S. 399-404.

Herrmann-Winter, Renate: Urteile über Niederdeutsch in den Nordbezirken der DDR. In: Zeitschrift für Phonetik, Sprachwissenschaft und Kommunikationsforschung 38, H. 3 (1985), S. 297-308.

Holm, Volker: Niederdeutsch an Volkshochschulen. Arbeitsheft für Teilnehmer an Volkshochschul-Kursen. Kiel 1981.

Möller, Frerk: Niederdeutsch: das sozio-kulturelle Umfeld. In: Stellmacher, Dieter (Hrsg.): Niederdeutsche Sprache und Literatur der Gegenwart. Hildesheim/ Zürich/New York 2004, S. 281-358.

Möller, Frerk: Plattdeutsch im 21. Jahrhundert. Bestandsaufnahme und Perspektiven. Mit einem Aufsatz von Michael Windzio. Leer 2008.

Myers, David G.: Psychology. 7. Aufl. New York 2004.

Stellmacher, Dieter: Wer spricht Platt? Zur Lage des Niederdeutschen heute. Eine kurzgefaßte Bestandsaufnahme. Leer 1987.

Wirrer, Jan: Die niederdeutsche Kulturszene als Gegenstand der empirischen Literaturwissenschaft. In: Niederdeutsches Jahrbuch 113 (1990), S. 44-69. 
Birte Arendt, Andreas Bieberstedt and Klaas-Hinrich Ehlers - 978-3-631-71893-3

Downloaded from PubFactory at 01/11/2019 10:11:06AM

via free access 


\section{Regionalsprache in Mecklenburg-Vorpommern seit dem 18. Jahrhundert. Eine Bibliographie der sprachwissenschaftlichen Forschungsliteratur. Begründet von Ingrid Schröder, fortgesetzt von Andreas Bieberstedt und Klaas-Hinrich Ehlers. Bearbeitet von Corina Altmann und Enrico Lippmann.}

Die nachfolgend abgedruckte Bibliographie liefert eine Zusammenschau der sprachwissenschaftlichen Forschungsliteratur zur Regionalsprache in Mecklenburg-Vorpommern seit 1700, d. h. seit der nachmittelniederdeutschen Zeit. Verzeichnet sind Überblicksdarstellungen, Einzelstudien und wissenschaftliche Beiträge, die sich unter verschiedensten Perspektiven mit der Entwicklung und dem aktuellen Stand der neuniederdeutschen Dialektvarianten und der hochdeutschen regionalen Umgangssprache auf dem Gebiet des heutigen Bundeslandes Mecklenburg-Vorpommern (und zum Teil darüber hinaus) auseinandersetzen. ${ }^{1}$ Dies umfasst Darstellungen etwa zu Sprachstruktur und Sprachareal ebenso wie zur Soziolinguistik, Pragmatik und Perzeptionslinguistik sowie zur Vermittlung, Förderung und Pflege der Regionalsprache. Nicht erfasst sind explizit literaturwissenschaftliche und ethnographische Darstellungen, wobei in verschiedenen Fällen keine trennscharfe Unterscheidung möglich war (etwa bei Arbeiten zum Sprachgebrauch Fritz Reuters). Das Bemühen ging dahin, die vorhandene Forschungsliteratur erstmalig systematisch und möglichst vollständig zu erfassen.

Die Bibliographie ist thematisch in drei Teile gegliedert. Der erste Teil liefert einen Überblick über Bibliographien zur Region und Regionalsprache in

1 Nicht erfasst sind Kongressberichte, Rezensionen, reine Textsammlungen u. ä. Wissenschaftliche Abschlussarbeiten (Staatsexamens-, Magister- und Masterarbeiten) wurden nur in Ausnahmefällen bei entsprechender wissenschaftlicher Qualität und Relevanz berücksichtigt. Ebenfalls nicht in die Bibliographie aufgenommen wurden wissenschaftliche Darstellungen, die sich allgemein auf den deutschen Dialektraum bzw. auf allgemeine Fragen der Regionalsprachenforschung beziehen, ohne die Sprachverhältnisse in Mecklenburg-Vorpommern explizit zu berücksichtigen. 
Mecklenburg-Vorpommern. Die nachfolgenden zwei Teile erfassen die eigentliche Forschungsliteratur. Teil 2 listet Darstellungen zum niederdeutschen Dialekt in Mecklenburg-Vorpommern auf, Teil 3 erfasst Darstellungen zum Regiolekt und Regionalakzent in Mecklenburg-Vorpommern. Innerhalb dieser beiden Teile wurden jeweils thematische Unterkategorien gebildet. Thematisch übergreifende Darstellungen wurden hierbei im Zweifelsfall mehreren Kategorien zugeordnet und erscheinen daher mehrfach. Dem gegenwärtigen Forschungsstand entsprechend ist der dritte Teil zum Regiolekt vorläufig weniger ausdifferenziert als der zweite Teil der Bibliographie, der die weitaus umfangreichere Forschungsliteratur zum Niederdeutschen dokumentiert.

Die Bibliographie basiert in ihrem Grundbestand auf den Recherchen von Ingrid Schröder (Universität Hamburg, ehemals Universität Greifswald), von der die bis zum Jahre 2003 erschienene Forschungsliteratur aufgearbeitet wurde. Die Ergänzung, Erweiterung und Überarbeitung der Bibliographie wird von Andreas Bieberstedt und Klaas-Hinrich Ehlers verantwortet. Bibliographien der Forschung zur Literatur und Volkskunde mit Bezug auf Mecklenburg-Vorpommern sind für die Folgebände dieser Publikationsreihe geplant. Perspektivisch werden diese Bibliographien durch eine Gesamtbibliographie zur Sprache, Literatur und Volkskunde in Mecklenburg-Vorpommern abgelöst, die in Zukunft als fortlaufend aktualisierte Online-Ressource von den Internetseiten der Universität Rostock abgerufen werden kann.

1 Bibliographien

2 (Neu)Niederdeutsch

2.1 Überblicksdarstellungen zum (Neu)Niederdeutschen allgemein

2.2 Überblicksdarstellungen zum Mecklenburgisch-Vorpommerschen

2.2.1 Sprachareal im Überblick

2.2.2 Sprachgeschichte im Überblick

2.3 Grammatik und Dialektographie

2.3.1 Grammatiken

2.3.2 Phonologie/Phonetik

2.3.3 Graphie

2.3.4 Morphologie

2.3.5 Syntax

2.4 Lexikologie, Lexikographie und Wörterbücher

2.5 Namenkunde

2.6 Fach- und Sondersprachenforschung

2.7 Soziolinguistik, Pragmatik und Perzeptionslinguistik

2.8 Sprachhistoriographie

Birte Arendt, Andreas Bieberstedt and Klaas-Hinrich Ehlers - 978-3-631-71893-3 
2.9 Niederdeutsch in den Medien (Rundfunk, Fernsehen, Printmedien, Internet)

2.10 Niederdeutsch in kulturellen Institutionen (Kirche, Bühne usw.)

2.11 Vermittlung, Förderung und Pflege des Niederdeutschen

3 Regiolekt in Mecklenburg-Vorpommern

3.1 Überblicksdarstellungen zu den Regiolekten in Norddeutschland

3.2 Überblicksdarstellungen zum Regiolekt in Mecklenburg-Vorpommern

3.3 Grammatik und Regiolektographie

3.4 Soziolinguistik, Pragmatik und Perzeptionslinguistik

\section{Bibliographien ${ }^{2}$}

Baarck, Gerhard (Bearb.): Mecklenburgische Bibliographie. Regionalbibliographie für die Bezirke Rostock, Schwerin und Neubrandenburg (MecklenburgVorpommern). Hrsg. von der Mecklenburgischen Landesbibliothek Schwerin. Schwerin 1966-1992.

Gattner, Monika: Mundart in Mecklenburg (Regionalbibliographien aus der Willi-Bredel-Bibliothek Rostock). Rostock 1984.

Grewolls, Grete (Bearb.): Mecklenburg-Vorpommersche Bibliographie. Hrsg. von der Mecklenburgischen Landesbibliothek Schwerin. Schwerin 1993-1995.

Hahn, Walther von: Fachsprachen im Niederdeutschen. Eine bibliographische Sammlung. Berlin 1979.

Hartig, Joachim/Goltz, Reinhard/Schröder, Ingrid [u. a.]: Niederdeutsche Bibliographie. In: Korrespondenzblatt des Vereins für niederdeutsche Sprachforschung $77 \mathrm{ff}$. (1970 ff.).

Wiesinger, Peter/Raffin, Elisabeth: Bibliographie zur Grammatik der deutschen Dialekte. Laut-, Formen-, Wortbildungs- und Satzlehre 1800 bis 1980 (Europäische Hochschulschriften, Reihe 1, Deutsche Sprache und Literatur; 509). Frankfurt a. M. [u. a.] 1982.

Wiesinger, Peter: Bibliographie zur Grammatik der deutschen Dialekte. Laut-, Formen-, Wortbildungs- und Satzlehre. 1981 bis 1985 und Nachträge aus früheren Jahren. Frankfurt a. M. [u. a.] 1987.

2 Erfasst sind sowohl Bibliographien speziell zu Mecklenburg-Vorpommern als auch solche generell zum Niederdeutschen. 


\section{2 (Neu) Niederdeutsch}

\section{1 Überblicksdarstellungen zum (Neu)Niederdeutschen allgemein}

Deutscher Sprachatlas. Auf Grund des von Georg Wenker begründeten Sprachatlas des Deutschen Reichs in vereinfachter Form begonnen von Ferdinand Wrede, fortgesetzt von Walther Mitzka und Bernhard Martin. 2 Bde. Marburg/ Lahn 1926-1956.

Foerste, William: Die Geschichte der niederdeutschen Mundarten. In: Stammler, Wolfgang (Hrsg.): Deutsche Philologie im Aufriß. 1. Bd., 2., überarb. Aufl. Berlin 1957, Sp. 1729-1898.

Foerste, William: Einheit und Vielfalt der niederdeutschen Mundarten (Schriften zur Heimatkunde und Heimatpflege; 4). Münster 1960.

Frings, Theodor: Aufbau und Gliederung des Niederdeutschen. In: Beiträge zur Geschichte der deutschen Sprache und Literatur 91 (1969), S. 106-129.

Goossens, Jan (Hrsg.): Niederdeutsch. Sprache und Literatur. 1. Bd. Neumünster 1983.

Hildebrandt, Reiner: Typologie der arealen lexikalischen Gliederung deutscher Dialekte aufgrund des Deutschen Wortatlasses. In: Besch, Werner [u. a.] (Hrsg.): Dialektologie. Ein Handbuch zur deutschen und allgemeinen Dialektologieforschung (Handbücher zur Sprach- und Kommunikationswissenschaft; 1.2). 2. Halbbd. Berlin/New York 1983, Sp. 1331-1366.

Jellinghaus, Hermann: Zur Einteilung der niederdeutschen Mundarten - ein Versuch. Kiel 1884.

König, Werner/Elspaß, Stephan/Möller, Robert: dtv-Atlas zur deutschen Sprache. Tafeln und Texte. Graphiken von Hans-Joachim Paul. 18., durchges. und korr. Aufl. München 2015.

Mitzka, Walther/Schmitt, Ludwig Erich/Hildebrandt, Reiner (Hrsg.): Deutscher Wortatlas (Deutscher Sprachatlas: Reihe Wortatlas). 22 Bde. Gießen 1951-1980.

Möller, Frerk: Niederdeutsch: das soziokulturelle Umfeld. In: Stellmacher, Dieter (Hrsg.): Niederdeutsche Sprache und Literatur der Gegenwart. Hildesheim/ Zürich/New York 2004, S. 281-358.

Niebaum, Hermann: Niederdeutsch in Geschichte und Gegenwart. In: Lindow, Wolfgang/Schuppenhauer, Claus (Hrsg.): Niederdeutsch - 5 Vorträge zur Einführung (Schriften des Instituts für Niederdeutsche Sprache. Reihe: Dokumentation; 12). Leer 1986, S. 7-42.

Niebaum, Hermann: Zur Lexik und Lexikographie des Niederdeutschen. In: Stellmacher, Dieter (Hrsg.): Niederdeutsche Sprache und Literatur der Gegenwart. Hildesheim/Zürich/New York 2004, S. 149-189. 
Niekerken, Walther: Von den Grenzen der niederdeutschen Sprache. In: Hoffmann, Gustav/Jürgensen, Gustav (Hrsg.): Hart, warr nich mööd. Festschrift für Christian Boeck. Zum 85. Geburtstag am 10. März 1960. HamburgWellingsbüttel 1960, S. 214-223.

Panzer, Baldur/Thümmel, Wolf: Die Einteilung der niederdeutschen Mundarten aufgrund der strukturellen Entwicklung des Vokalismus (Linguistische Reihe; 7). München 1971.

Peßler, Wilhelm: Plattdeutscher Wortatlas von Norddeutschland nach eigenen Forschungen und mit eigenen Aufnahmen. Hannover 1928.

Peters, Robert: Zur Sprachgeschichte des niederdeutschen Raumes. In: Zeitschrift für deutsche Philologie 117 (1998), S. 108-127.

Riemann, Erhard: Niederdeutsche Sprachlandschaften. In: Mehlem, Richard/Seedorf, Wilhelm (Hrsg.): Niederdeutsch. Ein Handbuch zur Pflege der Heimatsprache. Hannover 1957, S. 20-28.

Sanders, Willy: Sachsensprache, Hansesprache, Plattdeutsch. Göttingen 1982.

Schirmunski, Viktor M.: Deutsche Mundartkunde. Vergleichende Laut- und Formenlehre der deutschen Mundarten. Aus dem Russischen übers. und wissenschaftlich bearb. von Wolfgang Fleischer (Deutsche Akademie der Wissenschaften zu Berlin. Veröffentlichungen des Instituts für deutsche Sprache und Literatur; 25). Berlin 1962. Neuaufl., hrsg. und kommentiert von Larissa Naiditsch. Unter Mitarb. von Peter Wiesinger. Frankfurt a. M. 2010.

Schmidt, Jürgen Erich/Herrgen, Joachim/Kehrein Roland (Hrsg.): Regionalsprache.de (REDE). Forschungsplattform zu den modernen Regionalsprachen des Deutschen. Bearbeitet von Dennis Bock, Brigitte Ganswindt, Heiko Girnth, Simon Kasper, Roland Kehrein, Alfred Lameli, Slawomir Messner, Christoph Purschke, Anna Wolańska. Marburg: Forschungszentrum Deutscher Sprachatlas. $2008 \mathrm{ff}$. [Online-Ressource]

Schröder, Ingrid: Niederdeutsch in der Gegenwart: Sprachgebiet - Grammatisches Binnendifferenzierung. In: Stellmacher, Dieter (Hrsg.): Niederdeutsche Sprache und Literatur der Gegenwart. Hildesheim/Zürich/New York 2004, S. 35-97.

Schuppenhauer, Claus (Bearb.): Niederdeutsch heute. Kenntnisse - Erfahrungen - Meinungen. Leer 1976.

Schuppenhauer, Claus/Werlen, Iwar: Stand und Tendenzen in der Domänenverteilung zwischen Dialekt und deutscher Standardsprache. In: Besch, Werner [u. a.] (Hrsg.): Dialektologie. Ein Handbuch zur deutschen und allgemeinen Dialektologieforschung (Handbücher der Sprach- und Kommunikationswissenschaft; 1.2). 2. Halbbd. Berlin/New York 1983, Sp. 1411-1427.

Stellmacher, Dieter: Niederdeutsche Sprache. Eine Einführung (Germanistische Lehrbuchsammlung; 26). 2., überarb. Aufl. Berlin 2000. 
Teuchert, Hermann: Die sprachliche Beziehung zwischen dem niederdeutschen Osten und Westen. In: Korrespondenzblatt des Vereins für niederdeutsche Sprachforschung 55 (1942), S. 96-98.

Wiesinger, Peter: Die Einteilung der deutschen Dialekte. In: Besch, Werner [u. a.] (Hrsg.): Dialektologie. Ein Handbuch zur deutschen und allgemeinen Dialektologieforschung (Handbücher der Sprach- und Kommunikationswissenschaft; 1.2). 2. Halbbd. Berlin/New York 1983, Sp. 807-899.

\section{2 Überblicksdarstellungen zum Mecklenburgisch-Vorpommerschen}

\subsubsection{Sprachareal im Überblick}

Brose, Gerhard: Zur Dialektgeographie der pommersch-neumärkischen Grenzzone. In: Zeitschrift für Mundartforschung 23 (1955), S. 22-37.

Brose, Gerhard: Ein Streifzug durch Pommerns Mundarten. In: Carolinum 33 (1967), S. 78-87; 34 (1968), S. 88-95; 34 (1968), S. 75-77; 34 (1968), S. 64-68; 35 (1969), S. 100-104.

Bruhn, Max: Sprachliche Eigenarten im vorpommersch-mecklenburgischen Platt. In: Pommern. Kunst, Geschichte, Volkstum 5, H. 3 (1967), S. 10-11; 5, H. 4 (1967), S. 12-13; 6, H. 1 (1968), S. 33-34.

Dahl, Eva-Sophie: Interferenz und Alternanz - zwei Typen der Sprachschichtenmischung im Norden der Deutschen Demokratischen Republik. In: Ising, Gerhard (Hrsg.): Aktuelle Probleme der sprachlichen Kommunikation. Berlin 1974, S. 339-388.

Dietz, Johann Christian Friedrich: Über die mecklenburgisch-plattdeutsche Mundart in Bemerkungen zu Richey's Dialectologia Hamburgensis. In: Niederdeutsches Jahrbuch 20 (1894), S. 123-131.

Dost, Wolfgang: Untersuchungen zu den sprachlichen Existenzformen Mundart und Umgangssprache im Raum Wittstock unter Einschluß eines nördlichen Vorlandes. Diss. Rostock 1976.

Gernentz, Hans Joachim: Niederdeutsch - gestern und heute. Beiträge zur Sprachsituation in den Nordbezirken der Deutschen Demokratischen Republik in Geschichte und Gegenwart (Hinstorff Bökerie; 11). Rostock 1980.

Gernentz, Hans Joachim: Mecklenburg und seine Sprache. In: De Kennung 18, H. 2 (1995), S. 85-97.

Gundlach, Jürgen: Einführung in die mecklenburgischen Sprachverhältnisse. In: Korrespondenzblatt des Vereins für niederdeutsche Sprachforschung 65 (1958), S. 22. 
Gundlach, Jürgen: Volkssprache. In: Bentzien, Ulrich/Neumann, Siegfried (Hrsg.): Mecklenburgische Volkskunde. Rostock 1988, S. 423-438.

Gundlach, Jürgen: Niederdeutsche Sprache und Literatur in den drei Nordbezirken der DDR. In: Bericht der 42. Bevensen-Tagung vom 15.-17. September 1989 in Bad Bevensen. Bad Bevensen 1989, S. 92-96.

Herrmann-Winter, Renate: Niederdeutsch in Pommern. In: Pommern. Geschichte. Kultur. Wissenschaft. Hrsg. von der E.-M.-Arndt-Universität Greifswald. Greifswald 1991, S. 325-330.

Herrmann-Winter, Renate: Plattdeutsch in Vorpommern. In: Vorpommern Geschichte, Sprache, Volkskultur. Hrsg. vom Volkskulturinstitut Mecklenburg und Vorpommern im Kulturbund e. V. Rostock. Rostock/Stralsund 1991, S. $18-24$.

Herrmann-Winter, Renate: Mecklenburgisch-vorpommersches Niederdeutsch. In: Konturen. Magazin für Sprache, Literatur und Landschaft 2 (1993), S. 35-37.

Herrmann-Winter, Renate: Sprache und Sprechen in Pommern. In: Niederdeutsches Jahrbuch 118 (1995), S. 165-187.

Holsten, Robert: Sprachgrenzen im pommerschen Plattdeutsch. Pyritz 1913.

Holsten, Robert: Der Kampf der niedersächsischen und niederfränkischen Mundart in Pommern. In: Zeitschrift für Mundartforschung 18 (1942), S. 122-134.

Jacobs, Hugo: Dialektgeographie Südmecklenburgs zwischen Lübz und Hagenow. In: Teuthonista 2 (1925/26), S. 46-55 und 107-133; 3 (1926/27), S. 119-152 und 241-262.

Kruse, Erich: Dialektgeographie von Südmecklenburg und den angrenzenden Elbmarschen Brandenburgs und Hannovers. Diss. Rostock 1923.

Mischke, Kurt: Die niederdeutsche Sprache in Pommern. Aus der in Vorbereitung befindlichen „Dialektgeographie Pommerns und der Grenzmark“. In: Witt, Werner: Wirtschafts- und verkehrsgeographischer Atlas von Pommern. Hrsg. vom Geographischen Institut der Ernst-Moritz-Arndt-Universität Greifswald. Stettin 1934.

Mitzka, Walther: Zur Erforschung der ostniederdeutschen Mundarten. In: Schmitt, Ludwig Erich (Hrsg.): Germanische Dialektologie II. Festschrift für Walther Mitzka zum 80. Geburtstag (Zeitschrift für Mundartforschung. Beihefte, N. F.; 6). Wiesbaden 1968, S. 604-609.

Neumann, Siegfried: Volkskunde und Niederdeutsch in MecklenburgVorpommern. Ein niederdeutscher Mundart-Sprachraum in der volkskundlichen Forschung. Rostock 1997.

Prowatke, Christa: Plattdeutsch. Eine Einführung in die Sprache der Heimat (Landeskundliche Arbeitsblätter für Schüler und Lehrer). Schwerin 1993. 
Rösler, Irmtraud: Landesgrenze $=$ Sprachgrenze? Das Niederdeutsche im Dömitzer Raum im Vergleich zu seinen Nachbarn. In: Museum Festung Dömitz (Hrsg.): Der Festungskurier. Beiträge zur Mecklenburgischen Landes- und Regionalgeschichte vom Tag der Landesgeschichte im November 2004 in Dömitz. 5. Bd. Rostock 2005, S. 29-41.

Schönfeld, Helmut: East Low German. In: Russ, Charles V. J. (Hrsg.): The dialects of modern German. A linguistic survey. Stanford 1989, S. 91-135.

Seelmann, Wilhelm: Die Rostocker Mundart. In: Niederdeutsches Jahrbuch 53 (1917), S. 3-5.

Stellmacher, Dieter: Ostniederdeutsch. In: Althaus, Hans-Peter/Henne, Helmut/ Wiegand, Herbert Ernst (Hrsg.): Lexikon der Germanistischen Linguistik. 3. Bd., 2., vollständig neu bearb. und erw. Aufl. Tübingen 1980, S. 464-468.

Teuchert, Hermann: Grammophonaufnahmen mecklenburgischer Mundart. In: Mecklenburg. Zeitschrift des Heimatbundes Mecklenburg (Landesverein des Bundes Heimatschutz) 21 (1926), S. 24-28.

Teuchert, Hermann: Deutsche Mundarten. Mecklenburgisch (Lautbibliothek. Phonetische Platten und Umschriften; 21). Berlin 1927.

Teuchert, Hermann: Der mecklenburgische Sprachraum. In: Vierter Jahresbericht für das Jahr 1928. Hrsg. von der Mecklenburgischen Landes-UniversitätsGesellschaft. Rostock 1928, S. 15-30.

Teuchert, Hermann/Schmitt, Alfred: Deutsche Mundarten. Mecklenburgisch II und Pommersch (Lautbibliothek. Phonetische Platten und Umschriften; 35). Berlin 1933.

Teuchert, Hermann: Sprachliche Gliederung Mecklenburgs. In: Korrespondenzblatt des Vereins für niederdeutsche Sprachforschung 46 (1933), S. 26-29.

Teuchert, Hermann: Die mecklenburgische Sprachlandschaft. In: Schulz, Ernst (Hrsg.): Mecklenburg. Ein deutsches Land im Wandel der Zeit. Rostock 1938, S. $157-166$.

\subsubsection{Sprachgeschichte im Überblick}

Eichler, Ernst: Slawen und Deutsche in ihren Sprechbeziehungen östlich der Elbe und Saale. In: Stellmacher, Dieter (Hrsg.): Sprachkontakte. Niederländisch, Deutsch und Slawisch östlich von Elbe und Saale (Wittenberger Beiträge zur deutschen Sprache und Kultur; 3). Frankfurt a. M. [u. a.] 2004, S. 55-71.

Gernentz, Hans Joachim: Niederdeutsch - gestern und heute. Beiträge zur Sprachsituation in den Nordbezirken der Deutschen Demokratischen Republik in Geschichte und Gegenwart (Hinstorff Bökerie; 11). Rostock 1980.

Goossens, Jan: Die ostelbischen „Siedlungsmundarten“ aus niederdeutscher und niederländischer Perspektive. In: Stellmacher, Dieter (Hrsg.): Sprachkontakte. 
Niederländisch, Deutsch und Slawisch östlich von Elbe und Saale (Wittenberger Beiträge zur deutschen Sprache und Kultur; 3). Frankfurt a. M. [u. a.] 2004, S. 25-54.

Gundlach, Jürgen: Volkssprache. In: Bentzien, Ulrich/Neumann, Siegfried (Hrsg.): Mecklenburgische Volkskunde. Rostock 1988, S. 423-438.

Lerchner, Gotthard: Niederländisch, Niederdeutsch und Slawisch in siedlungsgeschichtlichen Kontaktbeziehungen: Möglichkeiten und Grenzen ihrer kulturmorphologischen Beschreibung. In: Stellmacher, Dieter (Hrsg.): Sprachkontakte. Niederländisch, Deutsch und Slawisch östlich von Elbe und Saale (Wittenberger Beiträge zur deutschen Sprache und Kultur; 3). Frankfurt a. M. [u. a.] 2004, S. 13-24.

Müns, Wolfgang: Niederdeutsch in Mecklenburg-Vorpommern von 1853 bis zur Gegenwart - eine Betrachtung im Überblick. In: Bericht der 48. BevensenTagung vom 15.-17. September 1995 in Bad Bevensen. Bad Bevensen 1995, S. 33-54.

Rösler, Irmtraud: Sprachenwechsel in Norddeutschland (am Beispiel Mecklenburg). In: Wiesinger, Peter/Derkits, Hans (Hrsg.): Akten des X. Internationalen Germanistenkongresses Wien 2000 „Zeitenwende - Die Germanistik auf dem Weg vom 20. ins 21. Jahrhundert" (Jahrbuch für Internationale Germanistik. Reihe A: Kongressberichte; 55). 3. Bd. Bern [u. a.] 2002, S. 73-78.

Rösler, Irmtraud: Aspekte einer Sprachgeschichte des Ostniederdeutschen. In: Besch, Werner [u. a.] (Hrsg.): Sprachgeschichte. Ein Handbuch zur Geschichte der deutschen Sprache und ihrer Erforschung (Handbücher zur Sprach- und Kommunikationswissenschaft; 2.4). 3. Teilbd., 2., vollständig neu bearb. und erw. Aufl. Berlin/New York 2003, Sp. 2698-2713.

Rösler, Irmtraud: Sprachwechsel in Mecklenburg. In: Niederdeutsches Jahrbuch 129 (2006), S. 139-156.

Teuchert, Hermann: Entwurf einer mecklenburgischen Sprachgeschichte. In: Wissenschaftliche Zeitschrift der Universität Rostock. Gesellschafts- und sprachwissenschaftliche Reihe 7, H. 1 (1957/58), S. 197-202.

Weltzien, Otto: John Brinckmans Leben und Werke nebst einer Abhandlung über die Geschichte der niederdeutschen Sprache. In: Weltzien, Otto (Hrsg.): John Brinckmans sämtliche Werke in fünf Bänden mit Einleitungen und Anmerkungen. Leipzig 1903, S. III-XXX. 


\subsection{Grammatik und Dialektographie}

\subsubsection{Grammatiken}

Gilow, Carl Christian: Leitfaden zur plattdeutschen Sprache, mit besonderer Berücksichtigung der südwestlich-vorpommerschen Mundart. Anclam 1868.

Grimme, Hubert: Plattdeutsche Mundarten. Berlin/Leipzig 1910.

Mussäus, Johann Jacob Nathanael: Versuch einer plattdeutschen Sprachlehre, mit besonderer Berücksichtigung der mecklenburgischen Mundart. Neu-Strelitz/ Neu-Brandenburg 1829.

Nerger, Karl: Grammatik des meklenburgischen Dialektes älterer und neuerer Zeit. Laut- und Flexionslehre. Leipzig 1869.

Ritter, Johann G. C.: Grammatik der mecklenburgisch-plattdeutschen Mundart. Rostock/Schwerin 1832.

Wiggers, Julius: Grammatik der plattdeutschen Sprache in Grundlage der Mecklenburgisch-Vorpommerschen Mundart. Leipzig 1857. Nachdruck in: Kikut. Plattdütsch gistern un hüt 21 (1998), S. 23-42 (Teil 1); 22 (1999) (Teil 2).

\subsubsection{Phonologie/Phonetik}

Beckmann, Paul: Der Lautstand der Rostocker Mundart auf historischer Grundlage. In: Teuthonista 4 (1927/28), S. 125-130.

Beckmann, Paul: Monophthonge und Zwielaute in und um Rostock. In: Niederdeutsches Jahrbuch 83 (1960), S. 127-130.

Bellmann, Günter/Göschel, Joachim: Tonbandaufnahme ostdeutscher Mundarten 1962-1965. Gesamtkatalog (Deutsche Dialektgeographie; 73). Marburg 1970.

Bischoff, Karl: Zur Chronologie von lauenburgisch-mecklenburgisch fëld (,Feld'). In: Korrespondenzblatt des Vereins für niederdeutsche Sprachforschung 70 (1963), S. 62.

Boll, Franz: Zur Aussprache und Rechtschreibung des Plattdeutschen. In: Archiv für Landeskunde in den Großherzogthümern Mecklenburg und Revue der Landwirtschaft 5 (1855), S. 625-639.

Dost, Wolfgang: Untersuchungen zu den sprachlichen Existenzformen. Mundart und Umgangssprache im Norden der Mark Brandenburg (Raum Wittstock). In: Beiträge zur Erforschung der deutschen Sprache 10 (1991), S. 240-266.

Gundlach, Jürgen: Plattdeutsch in Mecklenburg heute. Bericht über die Tonbandaufnahmen der mecklenburgischen Mundart 1962/63. In: Rostocker Beiträge. Regionalgeschichtliches Jahrbuch der mecklenburgischen Seestädte 1 (1966), S. 173-194. 
Hansen, Martin: Zum Wandel des Niederdeutschen auf der Insel Rügen zwischen dem 19. und 21. Jahrhundert. Ein diachronischer Vergleich anhand ausgewählter Sprachmerkmale. Masterarbeit Greifswald 2009.

Herrmann-Winter, Renate: Sprachatlas für Rügen und die vorpommersche Küste. Kartographie Martin Hansen. Rostock 2013.

Höfer, Albert: Die neuniederdeutschen Lautverhältnisse, besonders Neuvorpommerns. In: Zeitschrift für die Wissenschaft der Sprache 3, H. 3 (1851), S. 375-396.

Holst, Clara: Zur Aussprache in Fritz Reuters Heimat. In: Niederdeutsches Jahrbuch 33 (1907), S. 143-158.

Kehrein, Roland: Regionalsprachliche Spektren im Raum. Zur linguistischen Struktur der Vertikale (ZDL Beihefte; 152). Stuttgart 2012.

Kerckhove, Michael van de: Intonationssystem einer Mundart. In: Zeitschrift für Phonetik und allgemeine Sprachwissenschaft 2 (1948), S. 52-65.

Klatt, Hans Heinrich: Zur Lautverbindung sk in Südwestmecklenburg. In: Korrespondenzblatt des Vereins für niederdeutsche Sprachforschung 69 (1962), S. 39-40.

Köhncke, André: Untersuchungen zur gegenwärtigen mecklenburgischen Mundart. In: Niederdeutsches Jahrbuch 129 (2006), S. 157-184.

Köhncke, André: Mecklenburgisch heute - Bestandsaufnahme eines niederdeutschen Dialekts. Rostock 2010. [Online-Ressource: urn:nbn:de:gbv:28diss2011-0137-3 (Stand: 24.11.2016)]

Kolz, Willy: Das Lautsystem der haupttonigen Silben des Westmecklenburgischen Dialekts. Schönberg in Mecklenburg 1914.

Lasch, Agathe: Zur Chronologie von -tk- > -t $(\mathrm{t})$ - in der mecklenburgischvorpommerschen Mundart. In: Zeitschrift für deutsche Mundarten (1912), S. 166-173. Nachdruck in: Peters, Robert/Sodmann, Timothy (Hrsg.): Lasch, Agathe - Ausgewählte Schriften zur niederdeutschen Philologie. Neumünster 1979, S. 463-470.

Latendorf, Friedrich: Zu Zeitschrift V, 449 ff. [Mecklenburgisches Material zu: Tierbezeichnungen; Unorganisches $\mathrm{n}$ vor vokalisch anlautenden Wörtern; das Verbum sich ralen; Bezeichnung der Ameise]. In: Die Deutschen Mundarten. Vierteljahrsschrift für Dichtung, Forschung und Kritik 6 (1859), S. 229-232.

Mitzka, Walther: eifrig mit $r$-Schwund im Pommerschen. In: Niederdeutsches Jahrbuch 95 (1972), S. 195-198.

Nerger, Karl: Über die tonlangen Vocale des Niederdeutschen. In: Germania 11 (1866), S. 452-457. 
Pedd, Andreas: Besonderheiten des Mecklenburger Platt. Eine Untersuchung ausgewählter Phoneme. Staatsexamensarbeit Rostock 2003.

Pfaff, Hermann: Die Vocale des mittelpommerschen Dialects. Leipzig 1898.

Prowatke, Christa: Zum gegenwärtigen Lautstand der niederdeutschen Mundart in der DDR. Untersuchungen von Wortmaterial aus den drei Nordbezirken unter Berücksichtigung phonologischer Erkenntnisse. Diss. Rostock 1973.

Prowatke, Christa: Untersuchungen von niederdeutschem Wortmaterial aus den drei Nordbezirken der DDR unter Berücksichtigung phonologischer Erkenntnisse. In: Wissenschaftliche Zeitschrift der Universität Rostock. Gesellschaftsund sprachwissenschaftliche Reihe 24 (1975), S. 395-401.

Prowatke, Christa: Zur Distribution der vokalischen und konsonantischen Phoneme in der niederdeutschen Mundart. In: Wissenschaftliche Zeitschrift der Universität Rostock. Gesellschafts- und sprachwissenschaftliche Reihe 27 (1978), S. 71-76.

Rehm, Friedrich: Der plattdeutsche A-Klang, wie in Slätel. In: Mecklenburg. Zeitschrift des Heimatbundes Mecklenburg (Landesverein des Bundes Heimatschutz) 25 (1930), S. 53-55.

Schmidt, Gerhard: Der Vokalismus der Mundart von Barth. Greifswald 1912.

Seelmann, Wilh[elm]: Mundartliches aus Mecklenburg. In: Korrespondenzblatt des Vereins für niederdeutsche Sprachforschung 43, H. 3 (1930), S. 46-48.

Specht, Fritz/Teuchert, Hermann: Mecklenburgisch '-len > '-dḷ. In: Teuthonista $2(1925 / 26)$, S. 77-78.

Teuchert, Hermann: Der Lautstand im Südteil des Landes Stargard. In: Teuthonista 10 (1934), S. 2-34.

Warnkross, Julius: Die Lautlehre des Wolgaster Platt. Greifswald 1912.

Watanabe, Kakuji: Die Lautlehre der mecklenburgischen Mundart in den Werken Fritz Reuters. In: Tezukayama-Daigaku-kiyo. Bulletin of Tezukayama University $2 / 1$ (1965), S. 125-165.

Winter, Renate: Kollatsch-Tollatsch. Ein Beitrag zum Phonem /k/ und seinen phonetischen Varianten in den pommerschen Mundarten. In: Lingua Posnaniensis 11 (1966), S. 121-130.

\subsubsection{Graphie}

Boll, Franz: Zur Aussprache und Rechtschreibung des Plattdeutschen. In: Archiv für Landeskunde in den Großherzogthümern Mecklenburg und Revüe der Landwirthschaft 5 (1855), S. 625-639. 
Haeger, Fritz: Plattdeutsche Einheitsrechtschreibung und Mecklenburger Mundart. In: Mecklenburg. Zeitschrift des Heimatbundes Mecklenburg (Landesverein des Bundes Heimatschutz) 31 (1936), S. 53-58.

Lisch, Georg Friedrich: Soll man Meklenburg oder Mecklenburg schreiben. In: Freimütiges Abendblatt 14 (1832), Nr. 718, Sp. 793-796. Nachdruck in: Stier und Greif. Blätter zur Kultur- und Landesgeschichte in MecklenburgVorpommern. Sonderheft: 1000 Jahre Mecklenburg (1995), S. 34-36.

Lisch, Georg Friedrich: Meklenburg und meklenburgisch. In: Jahrbücher des Vereins für meklenburgische Geschichte und Altertumskunde 1 (1836), S. 174. Nachdruck in: Stier und Greif. Blätter zur Kultur- und Landesgeschichte in Mecklenburg-Vorpommern. Sonderheft: 1000 Jahre Mecklenburg (1995), S. 37.

Prowatke, Christa: Empfehlungen zur Schreibweise der niederdeutschen Mundart. In: Prowatke, Christa (Hrsg.): Up Platt is ook hüt noch wat. Niederdeutsche Prosa und Lyrik der Gegenwart (Hinstorff-Bökerie; 9). Rostock 1980, S. $162-168$.

Prowatke, Christa: Zur Schreibung des Niederdeutschen bei Fritz Reuter. In: Kikut. Plattdütsch gistern un hüt 10 (1985), S. 76-81.

Rösler, Irmtraud: Zur Verschriftung des Mecklenburger Platt. In: Nerius, Dieter (Hrsg.): Aktuelle Probleme der gegenwärtigen Linguistik: Schriftlinguistik Lexikologie - Textlinguistik (Rostocker Beiträge zur Sprachwissenschaft; 10). Rostock 2000, S. 115-134.

Rakow, Peter-Joachim: Zu den folgenden Aufsätzen von Lisch, Wex und Techen. Soll man Mecklenburg oder Meklenburg schreiben? In: Stier und Greif. Blätter zur Kultur- und Landesgeschichte in Mecklenburg-Vorpommern. Sonderheft: 1000 Jahre Mecklenburg (1995), S. 33.

Taylor, Dennis Q.: Fritz Reuter as a Phonorthographer. A Linguistic Commentary on His Phonology-Orthography Based on Rhymed Words in Four Unrevised Poems. In: Christiansen, Heinz C. (Hrsg.): Fritz-Reuter-Gedenkschrift (Amsterdamer Publikationen zur Sprache und Literatur; 18). Amsterdam 1975, S. 75-84.

Techen, Friedrich: Der Name Meklenburg und seine Schreibung. In: Mecklenburgische Monatshefte 1 (1925), S. 100-101. Nachdruck in: Stier und Greif. Blätter zur Kultur- und Landesgeschichte in Mecklenburg-Vorpommern. Sonderheft: 1000 Jahre Mecklenburg (1995), S. 44-47.

Teuchert, Hermann: Zur plattdeutschen Rechtschreibung. In: Zeitschrift für deutsche Mundarten 3 (1914), S. 228-237.

Wex, Carl: Wie ist Mecklenburg deutsch zu schreiben und wie lateinisch zu benennen? Schwerin 1856. Nachdruck in: Stier und Greif. Blätter zur Kultur- 
und Landesgeschichte in Mecklenburg-Vorpommern. Sonderheft: 1000 Jahre Mecklenburg (1995), S. 38-43.

\subsubsection{Morphologie}

Beckmann, Paul: Reste der alten Diminutivendung -ke, -ken im Mecklenburgischen. In: Niederdeutsches Jahrbuch 85 (1962), S. 126-128.

Dahl, Eva-Sophie: Zu Fragen des Geschlechtswechsels und der Doppelgeschlechtigkeit im neueren mecklenburgischen Niederdeutschen. In: Beiträge zur Geschichte der deutschen Sprache und Literatur 83 (1961), S. 195-204.

Dahl, Eva-Sophie: Substantivische Komposita in der mecklenburgischen Mundart und in der Hochsprache. Ein Vergleich nach Bildungstypen und deren Auftreten. In: Wissenschaftliche Zeitschrift der Universität Rostock. Gesellschaftsund sprachwissenschaftliche Reihe 27 (1978), S. 29-36.

Dussart, André: Analyse einiger Hypothesen über die Entstehung der umgelauteten Präterita im Mecklenburgischen. In: Korrespondenzblatt des Vereins für niederdeutsche Sprachforschung 75 (1968), S. 56-60.

Gillhoff, Johannes: Beiträge zur Lehre von der Inklination im Niederdeutschen. In: Gillhoff, Johannes: Das mecklenburgische Volksrätsel. Parchim 1892, S. IX-XVI.

Grimme, Hubert: Plattdeutsche Mundarten. Berlin/Leipzig 1910.

Hansen, Martin: Zum Wandel des Niederdeutschen auf der Insel Rügen zwischen dem 19. und 21. Jahrhundert. Ein diachronischer Vergleich anhand ausgewählter Sprachmerkmale. Masterarbeit Greifswald 2009.

Höfer, Albert: Das Verbum der neuniederdeutschen Mundart Neu-Vorpommerns. In: Zeitschrift für die Wissenschaft der Sprache 1 (1846), S. 379-392.

Kock, Ernst A.: Die niederdeutschen Relativpronomen (Lunds Universitets årsskrift. Afdeln. 1: Teologi, juridik och humanistiska ämnen; 39). Lund 1904.

Köhncke, André: Mecklenburgisch heute - Bestandsaufnahme eines niederdeutschen Dialekts. Rostock 2010. [Online-Ressource: urn:nbn:de:gbv:28diss2011-0137-3 (Stand: 24.11.2016)]

Koskensalo, Annikki: Die von deutschen Wortstämmen abgeleiteten -ierenVerben im Ostniederdeutschen. Ein Werkstattbericht. In: Lehmberg, Maik (Hrsg.): Sprache, Sprechen, Sprichwörter. Festschrift für Dieter Stellmacher zum 65. Geburtstag (ZDL Beihefte; 126). Stuttgart 2004, S. 79-89.

Krogmann, Willy: Mudding. Die Herkunft der mecklenburgisch-vorpommerschen Bildungen auf -ing (Mecklenburgische Forschungsgemeinschaft. Sitzungsberichte und Vorträge; 1). Hamburg 1953. 
Lasch, Agathe: Zur Deminutivbildung in der mecklenburgisch-vorpommerschen Mundart. In: Niederdeutsches Jahrbuch 38 (1912), S. 81-104.

Schmuck, Mirjam: Personennamen als Quelle der Grammatikalisierung. Der -ing-Diminutiv in Mecklenburg-Vorpommern. In: Beiträge zur Namenforschung 44, H. 1 (2009), S. 35-63.

Teuchert, Hermann: Mecklenburgisch hôner „Hühner“. In: Korrespondenzblatt des Vereins für niederdeutsche Sprachforschung 39 (1924), S. 23.

Teuchert, Hermann: In de Wind „in den Wind“. In: Niederdeutsches Jahrbuch 54 (1928), S. 115-121.

Teuchert, Hermann: Mudding „Mütterchen“. Die Herkunft des mecklenburgischvorpommerschen -ing. In: Zeitschrift für Mundartforschung 21 (1953), S. 83-101.

Wiese, Joachim: Mittelniederdeutsche Wortbildungsvarianten in ihrer Bedeutung für sprachliche Entwicklungen im Neuniederdeutschen. In: Wissenschaftliche Zeitschrift der Universität Rostock. Gesellschafts- und sprachwissenschaftliche Reihe 35 (1986), S. 113-115.

Winter, Renate: Suffixe der slawischen Lehnwörter im Pommerschen und ihr Einfluß auf die niederdeutsche Wortbildung. In: Niederdeutsches Jahrbuch 90 (1967), S. 106-121.

Wossidlo, Richard: Imperativische Bildungen im Niederdeutschen. In: XXI. Jahresbericht über das Städtische Gymnasium zu Waren. Waren 1890, S. 1-18.

Wossidlo, Richard: Die Präpositionen und präpositionalen Adverbien in der Mecklenburger Mundart. In: Niederdeutsches Jahrbuch 20 (1894), S. 40-56.

Wossidlo, Richard: Niederdeutsche Adjektive auf -ern. In: Korrespondenzblatt des Vereins für niederdeutsche Sprachforschung 32 (1911), S. 19.

\subsubsection{Syntax}

Bader, Angela/Bader, Ulf-Hermann: Syntaktische Strukturen in neuniederdeutschen narrativen Texten. In: Rösler, Irmtraud (Hrsg): „Ik lerde kunst dor lust." Ältere Sprache und Literatur in Forschung und Lehre. Festschrift zum 65. Geburtstag von Prof. Dr. phil. habil. Christa Baufeld (Rostocker Beiträge zur Sprachwissenschaft; 7). Rostock 1999, S. 9-27.

Dützmann, Heinz: Syntax von Nomen und Verb im Ostlüneburgischen auf Grund der Mundart von Kaarßen. In: Zeitschrift für Mundartforschung 15 (1939), S. 1-24.

Fleischer, Jürg: ... und habe es ihr gesagt. Zur dialektalen Abfolge pronominaler Objekte (eine Auswertung von Wenkersatz 9). In: Glaser, Elvira/Schmidt, 
Jürgen Erich/Frey, Natascha (Hrsg.): Dynamik des Dialekts - Wandel und Variation. (ZDL Beihefte; 144). Stuttgart 2011, S. 77-100.

Grimme, Hubert: Plattdeutsche Mundarten. Berlin/Leipzig 1910.

Hansen, Martin: Zum Wandel des Niederdeutschen auf der Insel Rügen zwischen dem 19. und 21. Jahrhundert. Ein diachronischer Vergleich anhand ausgewählter Sprachmerkmale. Masterarbeit Greifswald 2009.

Hoefer, Albert: Das Verbum der neuniederdeutschen Mundart NeuVorpommerns. In: Hoefer, Albert (Hrsg.): Zeitschrift für die Wissenschaft der Sprache. 1. Bd. Greifswald 1846, S. 379-392.

Lierow, Hartwig Georg Heinrich: Beiträge zur Syntax des Verbums in der mecklenburgischen Mundart. In: Achter Jahresbericht der städtischen Realschule mit Progymnasium zu Oschatz. Oschatz 1904, S. 3-22.

Mackel, Emil: Umschreibendes dôn. In: Korrespondenzblatt des Vereins für niederdeutsche Sprachforschung 41 (1927/28), S. 59.

Weber, Thilo: Neue Fragen an alte Daten. Niederdeutsche Syntaxgeographie auf der Grundlage von Zwirner- und DDR-Korpus. In: Langhanke, Robert [u. a.] (Hrsg.): Niederdeutsche Syntax. (Germanistische Linguistik; 220). Hildesheim/ Zürich/New York 2012, S. 157-179.

Weise, Oskar: Zu Reuters Syntax. In: Zeitschrift für deutsche Mundarten (1910), S. 291-307.

Wossidlo, Richard: Negative Verbindung zweier Ausdrücke im Mecklenburger Platt [vom Typ: de kihrt sik nich an gott un de welt]. In: Korrespondenzblatt des Vereins für niederdeutsche Sprachforschung 14 (1889/90), S. 18-22.

Wossidlo, Richard: Der Gebrauch des Infinitivs im Mecklenburger Platt. In: Korrespondenzblatt des Vereins für niederdeutsche Sprachforschung 17 (1893), S. 60-66.

\subsection{Lexikologie, Lexikographie und Wörterbücher}

Bentzien, Ulrich: Zur Wortgeographie von Kolter (,Pflugmesser') in Mecklenburg. In: Korrespondenzblatt des Vereins für niederdeutsche Sprachforschung 70 (1963), S. 11-12.

Bentzien, Ulrich: Wörter der modernen Technik in der mecklenburgischen Mundart. In: Niederdeutsches Jahrbuch 87 (1964), S. 87-106.

Bentzien, Ulrich: Wie nannte der Bauer vor 200 Jahren seine Tiere? In: Zwischen Maurine und Wallensteingraben. Heimatkalender für das nordwestliche Mecklenburg 1, Beiheft 1 (1966), S. 22-28.

Bielfeldt, Hans Holm: Slawische Wörter im Deutschen Vorpommerns. In: Zeitschrift für Slawistik 5 (1960), S. 207-211. 
Bielfeldt, Hans Holm: Die slawischen eigentlichen Reliktwörter in den deutschen Mundarten. In: Zeitschrift für Slawistik 8 (1963), S. 155-172.

Blume, Rudolf: Wortgeographie des Landes Stargard. In: Teuthonista 9 (1933), S. 193-207.

Cammin, Friedrich: Der mecklenburgische Haken. In: Niederdeutsches Jahrbuch 48 (1922), S. 36-38.

Dähnert, Johann Carl: Platt-Deutsches Wörter-Buch nach der alten und neuen Pommerschen und Rügischen Mundart. Stralsund 1781.

Dittmar, Lisa: Die gegenwärtige Sprachsituation auf dem Fischland und dem Darß. In: Wissenschaftliche Zeitschrift der Universität Rostock. Gesellschaftsund sprachwissenschaftliche Reihe 11 (1962), S. 313-318.

Frederiksen, Jan: Schwedische Sprachreste in Pommern. In: Niederdeutsche Mitteilungen 16/18 (1960/62), S. 91-107.

Friebertshäuser, Hans: Das Mecklenburgische und das BrandenburgischBerlinische Wörterbuch. In: Friebertshäuser, Hans (Hrsg.): Dialektlexikographie. Berichte über Stand und Methoden deutscher Dialektwörterbücher. Festgabe für Luise Berthold zum 85. Geburtstag am 27.1.1976. Wiesbaden 1976, S. 211-215.

Friebertshäuser, Hans: Die großlandschaftlichen Wörterbücher der deutschen Dialekte. Areale und lexikologische Beschreibung. In: Besch, Werner [u. a.] (Hrsg.): Dialektologie. Ein Handbuch zur deutschen und allgemeinen Dialektologieforschung (Handbücher der Sprach- und Kommunikationswissenschaft; 1.2). 2. Halbbd. Berlin/New York 1983, Sp. 1283-1294.

Fuchs, Günther/Lüdemann, Hans-Ulrich: Das Mecklenburgisch-Vorpommerische Schimpfwörterbuch: bannich deftige Wörter. Nidderau 1993.

Gillhoff, Johannes: Mecklenburgische Idiotismen. In: 16. Bericht über die städtischen Mittel- und Elementarschulen der Vorderstadt Parchim. Parchim 1889, S. $1-10$.

Gilow, Christian: De Minsch. Anclam 1869.

Gilow, Christian: De Diéré, as man to seggt un wat's seggen. 2 Bde. Anclam 1871-74.

Gilow, Christian: De Planten, as man to seggt un wat's seggen. Botanisches und Niederdeutsches Wörterbuch für Landwirthe, Ärzte, Apotheker, Theologen und Philologen. 7 Bde. Anclam 1872-1878.

Gosselck, Johannes: Die Anfänge der Wörterbucharbeiten in Mecklenburg vor 25 Jahren. In: Mecklenburgische Monatshefte 15 (1939), S. 60-63. 
Groth, Max: Das Kartenspiel „Schafkopf“ in den pommerschen Mundarten. In: Korrespondenzblatt des Vereins für niederdeutsche Sprachforschung 73 (1966), S. 30-31 und 34-40.

Gülzow, Erich: Fladrun. In: Niederdeutsches Jahrbuch 50 (1937), S. 43-44.

Gülzow, Erich: Schwedische Wörter in Westpommern. In: Korrespondenzblatt des Vereins für niederdeutsche Sprachforschung 53 (1940), S. 5-8 und 28; 54 (1941), S. 22-23; 58 (1951), S. 30-32.

Gundlach, Jürgen: Von Aant bis Zäg. Plattdeutsche Wörter und Wendungen. Leipzig 1982.

Gundlach, Jürgen/Rothe, Christian: Diatopik und Historizität im Dialektwörterbuch, dargestellt am Beispiel des Mecklenburgischen Wörterbuchs. In: Fahning, Wolfgang/Spangenberg, Karl/Wilhelmi, Bernd (Hrsg.): Dialektlexikographie. Berichte und Analysen zur Arbeit an Dialektwörterbüchern. Jena 1988, S. 65-85.

Gundlach, Jürgen: Das Mecklenburgische Wörterbuch von Richard Wossidlo und Hermann Teuchert. Seine Geschichte und seine Aussage. In: Niederdeutsches Jahrbuch 115 (1992), S. 145-158.

Gundlach, Jürgen: Das Mecklenburgische Wörterbuch. Vollendung eines großlandschaftlichen Dialektwörterbuchs. In: Stier und Greif. Blätter zur Kulturund Landesgeschichte in Mecklenburg-Vorpommern 5 (1995), S. 36-42.

Gundlach, Jürgen: Richard Wossidlos schwierige Position bei der Gestaltung des Mecklenburgischen Wörterbuches. In: Bunners, Christian/Bichel, Ulf/ Grote, Jürgen (Hrsg.): Fritz Reuter, Richard Wossidlo, Mecklenburgische Volksliteratur (Beiträge der Fritz Reuter Gesellschaft; 13). Rostock 2003, S. 71-83.

Hagenow, Käthe von: Etwas vom Mecklenburgischen Wörterbuch. In: Mecklenburg. Zeitschrift des Heimatbundes Mecklenburg (Landesverein des Bundes Heimatschutz) 34 (1939), S. 36-40.

Hansen, Martin: Zum Wandel des Niederdeutschen auf der Insel Rügen zwischen dem 19. und 21. Jahrhundert. Ein diachronischer Vergleich anhand ausgewählter Sprachmerkmale. Masterarbeit Greifswald 2009.

Herrmann-Winter, Renate: Johann Carl Dähnerts Platt=Deutsches Wörter=Buch in seiner und unserer Zeit. In: Niederdeutsche Mitteilungen 26 (1970), S. 73-98.

Herrmann-Winter, Renate: Wortgut aus niederdeutschen Pressetexten der DDR im Urteil von Mundartsprechern. In: Das Niederdeutsche in Geschichte und Gegenwart. Hrsg. vom Zentralinstitut für Sprachwissenschaft der Akademie der Wissenschaften der DDR (Linguistische Studien. Reihe A: Arbeitsberichte; 75.1). Berlin 1981, S. 96-104. 
Herrmann-Winter, Renate: Zu aktuellen Ansprüchen an die Lexikographie einer regionalen Sprachform. In: Bahner, Werner/Schildt, Joachim/Viehweger, Dieter (Hrsg.): Proceedings of the Fourteenth International Congress of Linguists. 2. Bd. Berlin 1987, S. 1503-1505.

Herrmann-Winter, Renate: Geschichte, Geschicke und Ansehen pommerscher Dialektwörterbücher. In: Stier und Greif. Blätter zur Kultur- und Landesgeschichte in Mecklenburg-Vorpommern 3 (1993), S. 27-31.

Herrmann-Winter, Renate: Das Pommersche Wörterbuch in Greifswald. In: Mitteldeutsches Jahrbuch für Kultur und Geschichte 1 (1994), S. 346-347.

Herrmann-Winter, Renate: Das Pommersche Wörterbuch. Vorläufer - Ansätze - Konzepte. In: Schmidt, Roderich (Hrsg.): Tausend Jahre pommersche Geschichte (Veröffentlichungen der Historischen Kommission für Pommern. Reihe 5: Forschungen zur pommerschen Geschichte; 31). Köln/Weimar/Wien 1999, S. 281-306.

Herrmann-Winter, Renate: Neues hochdeutsch-plattdeutsches Wörterbuch für den mecklenburgisch-vorpommerschen Sprachraum. Sinngleiche und sinnähnliche Wörter, Phrasen und Redensarten. 2. Aufl. Rostock 2003.

Herrmann-Winter, Renate: Plattdeutsch-hochdeutsches Wörterbuch für den mecklenburgisch-vorpommerschen Sprachraum. 6. Aufl. Rostock 2013.

Herrmann-Winter, Renate: Sprachatlas für Rügen und die vorpommersche Küste. Kartographie Martin Hansen. Rostock 2013.

Holsten, Robert: Wie heißt der Wacholder auf Rügen? In: Zeitschrift für Mundartforschung 15 (1939), S. 174-182.

Holsten, Robert: Holm in Pommern. In: Korrespondenzblatt des Vereins für niederdeutsche Sprachforschung 57 (1950), S. 2-6; 58 (1951), S. 4-5.

Ihrke, Walter: Doppelbenennungen zweiseitig verbundener Teile beim Spinnrad nach mecklenburgischen Belegen. In: Korrespondenzblatt des Vereins für niederdeutsche Sprachforschung 65 (1958), S. 33-38.

Kaestner, Walter: Mecklenburgisch snop „Flachsbündel“. In: Niederdeutsches Wort 16 (1976), S. 183-186.

Kaestner, Walter: Niederdeutsch-slavische Interferenzen. In: Cordes, Gerhard/ Möhn, Dieter (Hrsg.): Handbuch zur niederdeutschen Sprach- und Literaturwissenschaft. Berlin 1983, S. 678-729.

Kaiser, Karl: Bezeichnungen für den Marienkäfer in Pommern. In: Zeitschrift für Mundartforschung 12 (1936), S. 89-98.

Köhncke, André: Mecklenburgisch heute - Bestandsaufnahme eines niederdeutschen Dialekts. Rostock 2010. [Online-Ressource: urn:nbn:de:gbv:28diss2011-0137-3 (Stand: 24.11.2016)] 
Köhncke, André: Untersuchungen zur gegenwärtigen mecklenburgischen Mundart. In: Niederdeutsches Jahrbuch 129 (2006), S. 157-184.

Knoop, Otto: Die Namen des Regenwurmes in Pommern. In: Blätter für Pommersche Volkskunde. Monatsschrift für Sage und Märchen, Sitte und Brauch, Schwank und Streich, Lied, Rätsel und Sprachliches in Pommern. 4, Nr. 2 (1895), S. 29-31

Knoop, Otto: Die Namen der Ameise in Pommern. In: Blätter für Pommersche Volkskunde. Monatsschrift für Sage und Märchen, Sitte und Brauch, Schwank und Streich, Lied, Rätsel und Sprachliches in Pommern. 4, Nr. 7 (1896), S. 111.

Kosegarten, Johann Gottfried Ludwig: Wörterbuch der Niederdeutschen Sprache älterer und neuerer Zeit. 3 Bde. Greifswald 1856-1860.

Mecklenburgisches Wörterbuch. Hrsg. von der Sächsischen Akademie der Wissenschaften zu Leipzig. Aus den Sammlungen Richard Wossidlos und aus den Ergänzungen und nach der Anlage Hermann Teucherts. 7 Bde. Berlin 1942-1992. Unveränd., verkleinerter Nachdruck der Erstauflage von 1937-1992. Neumünster 1996. Nachtrag und Index bearb. unter d. Leitung von Christian Rothe. Neumünster 1998.

Mensing, Otto: Zur Geschichte der älteren niederdeutschen Wörterbücher. In: Volkskundliche Beiträge. Festschrift Richard Wossidlo, am 26. Januar 1939 zum Dank dargebracht von Freunden und Verehrern und dem Verlag. Neumünster 1939, S. 88-96.

Mentz, Richard: Französisches im mecklenburgischen Platt und in den Nachbardialekten. Beilage zum Jahresbericht des Realprogymnasiums zu Delitzsch. 2 Bde. Delitzsch 1897/98.

Mi [Georg Friedrich Sibeth]: Wörterbuch der mecklenburgisch-vorpommerschen Mundart. Leipzig 1876.

Mohr, Werner: Einiges aus der plattdeutschen Wortkunde. In: Mecklenburg. Zeitschrift des Heimatbundes Mecklenburg (Landesverein des Bundes Heimatschutz) 20 (1925), S. 78-93.

Mohr, Werner: Aus der plattdeutschen Wortkunde. In: Mecklenburg. Zeitschrift des Heimatbundes Mecklenburg (Landesverein des Bundes Heimatschutz) 24 (1929), S. 130-138; 25 (1930), S. 13-24.

Neumann, Siegfried: Kuffert und Lad: Zur Kontinuität volkstümlicher Terminologie. In: Korrespondenzblatt des Vereins für niederdeutsche Sprachforschung 71 (1964), S. 30-32 und S. 40.

Niebaum, Hermann: Zu Lexik und Lexikographie des Niederdeutschen. In: Stellmacher, Dieter (Hrsg.): Niederdeutsche Sprache und Literatur der Gegenwart. Hildesheim/Zürich/New York, S. 149-189. 
Pommersches Wörterbuch. Begründet von Wolfgang Stammler. Fortgesetzt von Hans-Friedrich Rosenfeld. Hrsg. von Renate Herrmann-Winter. Sächsische Akademie der Wissenschaften Leipzig. Kommission für die Mundartwörterbücher. Bd. 1 ff. Berlin 1997 ff.

Prowatke, Christa (Hrsg): Plattdeutsches Wörterverzeichnis. Rostock 1996.

Rehfeld, Irmgard: Fladrun. In: Korrespondenzblatt des Vereins für niederdeutsche Sprachforschung 66 (1959), S. 47-50.

Rosenfeld, Hans-Friedrich: $\mathrm{Zu}$ den pommerschen Haustierbezeichnungen. Aus der Werkstatt des pommerschen Wörterbuches. In: Niederdeutsche Mitteilungen 3 (1947), S. 55-81. Nachdruck in: Kuhn, Hugo/Rosenfeld, Hellmut/ Schubert, Hans-Jürgen (Hrsg.): Ausgewählte Schriften zur deutschen Literaturgeschichte, germanischen Sprach- und Kulturgeschichte und zur deutschen Wort-, Mundart- und Volkskunde nebst Bibliographie aller Publikationen des Autors 1923-1974. Festschrift zum 75. Geburtstag von Hans-Friedrich Rosenfeld 5. Dezember 1974 (Göppinger Arbeiten zur Germanistik; 125). 2. Bd. Göppingen 1974, S. 616-643.

Rosenfeld, Hans-Friedrich: Nd. Buddel, hd. Flasche ,Tölpel, Dummkopf', it. Fiasco ,Mißerfolg', franz. Bouteille ,Fehler, Schnitzer'. In: Neuphilologische Mitteilungen 53 (1952), S. 277-287; 54 (1953), S. 327-356. Nachdruck in: Kuhn, Hugo/ Rosenfeld, Hellmut/Schubert, Hans-Jürgen (Hrsg.): Ausgewählte Schriften zur deutschen Literaturgeschichte, germanischen Sprach- und Kulturgeschichte und zur deutschen Wort-, Mundart- und Volkskunde nebst Bibliographie aller Publikationen des Autors 1923-1974. Festschrift zum 75. Geburtstag von Hans-Friedrich Rosenfeld 5. Dezember 1974 (Göppinger Arbeiten zur Germanistik; 125). 2. Bd. Göppingen 1974, S. 735-774.

Rosenfeld, Hans-Friedrich: Spinnen und Weben im pommerschen Platt. Festschrift für Emil Öhmann. In: Annales Academiae Scientiarum Fennicae B 84 (1954), S. 463-511. Nachdruck in: Kuhn, Hugo/Rosenfeld, Hellmut/Schubert, Hans-Jürgen (Hrsg.): Ausgewählte Schriften zur deutschen Literaturgeschichte, germanischen Sprach- und Kulturgeschichte und zur deutschen Wort-, Mundart- und Volkskunde nebst Bibliographie aller Publikationen des Autors 1923-1974. Festschrift zum 75. Geburtstag von Hans-Friedrich Rosenfeld 5. Dezember 1974 (Göppinger Arbeiten zur Germanistik; 125). 2. Bd. Göppingen 1974, S. 489-537.

Rosenfeld, Hans-Friedrich: Ausdrucksfähigkeit und Bildkraft der niederdeutschen Sprache, dargelegt an der Bezeichnung des Bezechten (Sonderschrift des Stader Geschichts- und Heimatvereins; 7). Neumünster 1956.

Rosenfeld, Hans-Friedrich: Urtümliche Holzbearbeitung nach Wort, Sache und Brauchtum diesseits und jenseits der Ostsee. In: Deutsches Jahrbuch für Volkskunde 2 (1956), S. 147-178. 
Rosenfeld, Hans-Friedrich: West- und Ostfälisches in der Pommerschen Mundart. In: Westfälische Forschungen 9 (1956), S. 122-144. Nachdruck in: Kuhn, Hugo/ Rosenfeld, Hellmut/Schubert, Hans-Jürgen (Hrsg.): Ausgewählte Schriften zur deutschen Literaturgeschichte, germanischen Sprach- und Kulturgeschichte und zur deutschen Wort-, Mundart- und Volkskunde nebst Bibliographie aller Publikationen des Autors 1923-1974. Festschrift zum 75. Geburtstag von Hans-Friedrich Rosenfeld 5. Dezember 1974 (Göppinger Arbeiten zur Germanistik; 125). 2. Bd. Göppingen 1974, S. 574-597.

Rosenfeld, Hans-Friedrich: Niederdeutsche Zahlwortstudien. 1. Die Elferzählung. Ein niederdeutsches Zahlenproblem. Zugleich ein Beitrag zur Volkskunde der Zahlen; 2. Kardinalia; 3. Ordinalia; 4. Andere Zahlarten; 5. Die Zahlbegriffe; 6. Nachtrag zur Elferzählung. In: Niederdeutsches Jahrbuch 79 (1956), S. 115-140; 80 (1957), S. 69-92; 81 (1958), S. 59-103. Nachdruck in: Kuhn, Hugo/Rosenfeld, Hellmut/Schubert, Hans-Jürgen (Hrsg.): Ausgewählte Schriften zur deutschen Literaturgeschichte, germanischen Sprachund Kulturgeschichte und zur deutschen Wort-, Mundart- und Volkskunde nebst Bibliographie aller Publikationen des Autors 1923-1974. Festschrift zum 75. Geburtstag von Hans-Friedrich Rosenfeld 5. Dezember 1974 (Göppinger Arbeiten zur Germanistik; 124). 1. Bd. Göppingen 1974, S. 369-463.

Rosenfeld, Hans-Friedrich: Pommersche Haustierkosenamen. In: Niederdeutsches Jahrbuch 82 (1959), S. 237-253. Nachdruck in: Kuhn, Hugo/Rosenfeld, Hellmut/Schubert, Hans-Jürgen (Hrsg.): Ausgewählte Schriften zur deutschen Literaturgeschichte, germanischen Sprach- und Kulturgeschichte und zur deutschen Wort-, Mundart- und Volkskunde nebst Bibliographie aller Publikationen des Autors 1923-1974. Festschrift zum 75. Geburtstag von HansFriedrich Rosenfeld 5. Dezember 1974 (Göppinger Arbeiten zur Germanistik; 125). 2. Bd. Göppingen 1974, S. 599-615.

Rosenfeld, Hans-Friedrich: Kindesfoot. Ursprung und Verbreitung einer hansisch-niederdeutschen Brauchtumsbezeichnung. Zugleich ein Beitrag zur Benennung der Kindelfeiern (Commentationes humanarum litterarum; 36.1). Helsinki 1964.

Schönfeld, Helmut: Das Pommersche Wörterbuch. In: Berichte über dialektologische Forschungen in der Deutschen Demokratischen Republik. Hrsg. von der Deutschen Akademie der Wissenschaften Berlin, Institut für deutsche Sprache. Berlin 1965, S. 5-10.

Schröder, Edward: Papphahn. Ein mecklenburgischer Münzname. In: Niederdeutsches Jahrbuch 33 (1907), S. 119-121.

Teuchert, Hermann: Das Mecklenburgische Wörterbuch. In: Mecklenburg. Zeitschrift des Heimatbundes Mecklenburg (Landesverein des Bundes Heimatschutz) 21 (1926), S. 50-52, S. 86-88, S. 114-115. 
Teuchert, Hermann: Mecklenburgisch gaut „gut“. In: Karg-Gasterstädt, Elisabeth/ Erben, Johannes (Hrsg.): Fragen und Forschungen im Bereich und Umkreis der germanischen Philologie. Festgabe für Theodor Frings zum 70. Geburtstag am 23. Juli 1956. Berlin 1956, S. 251-260.

Teuchert, Hermann: Slawische Lehnwörter in ostdeutschen Mundarten. In: Zeitschrift für Mundartforschung 26 (1958), S. 13-31.

Teuchert, Hermann: Sprachschichten im Mecklenburgischen Wörterbuch. Zum 100. Geburtstage Richard Wossidlos am 26. Januar 1959. In: Wissenschaftliche Zeitschrift der Universität Rostock. Gesellschafts- und sprachwissenschaftliche Reihe 8 (1958/59), S. 1-9.

Teuchert, Hermann: Das Mecklenburgische Wörterbuch. In: Deutsches Jahrbuch für Volkskunde 5 (1959), S. 133-136.

Teuchert, Hermann: Das Mecklenburgische Wörterbuch. In: Berichte über dialektologische Forschungen in der Deutschen Demokratischen Republik. Hrsg. von der Deutschen Akademie der Wissenschaften Berlin, Institut für deutsche Sprache. Berlin 1965, S. 1-4.

Teuchert, Hermann: Mecklenburgisch Lü̈̈nge „Lichtschein“- pomoranisch usw. łuna. In: Zeitschrift für Slawistik 12 (1967), S. 505-506.

Törnquist, Nils: Vorpommerisch fladrun, „Bettwärmer“. In: Korrespondenzblatt des Vereins für niederdeutsche Sprachforschung 65 (1958), S. 42-44.

Vollmer, Matthias: Zur Dialektlexikographie in Pommern. Kosegartens Wörterbuch der Niederdeutschen Sprache älterer und neuerer Zeit. In: Niederdeutsches Jahrbuch 131 (2008), S. 113-132.

Vollmer, Matthias: Die ostpommerschen Dialekte. 2 Bde. Habilitationsschrift Greifswald 2012.

Winter, Renate: Zur Etymologie von nd. Spann,Eimer. In: Wissenschaftliche Zeitschrift der Universität Greifswald. Gesellschafts- und sprachwissenschaftliche Reihe 9 (1959/60), S. 379-381.

Winter, Renate: Das Pommersche Wörterbuch in Greifswald. Seine Geschichte und seine gegenwärtigen Aufgaben. In: Sprawozdania Poznanskiego Towarzystwa Przyjaciol Nauk 72, H. 2 (1964), S. 194-197.

Winter, Renate: Die Milch und ihre Verarbeitung im niederdeutschen Wortschatz der ehemaligen Provinz Pommern. Diss. Rostock 1963. Autoreferat in: Demos 5 (1964), Sp. 235-254.

Winter, Renate: Butterersatz und Kunstbutter in den pommerschen Mundarten. In: Korrespondenzblatt des Vereins für niederdeutsche Sprachforschung 72 (1965), S. 54-56.

Winter, Renate: Bildhafte Ausdrucksformen im Niederdeutschen (Pommerschen) aus dem Bereich der bäuerlichen Milchwirtschaft. In: Wissenschaftliche ZeitBirte Arendt, Andreas Bieberstedt and Klaas-Hinrich Ehlers - 978-3-631-71893-3 
schrift der Universität Rostock. Gesellschafts- und sprachwissenschaftliche Reihe 15 (1966), S. 685-696.

Winter, Renate: Zur bäuerlichen Butterbereitung im ehemaligen Pommern. Eine Sach- und Wortstudie. In: Deutsches Jahrbuch für Volkskunde 12 (1966), S. 312-333.

Winter, Renate: $\mathrm{Zu}$ einigen slawischen Fischnamen in Pommern. In: Krauss, Werner/Stieber, Zdzisław/Bělič, Jaromír [u. a.] (Hrsg.): Slawisch-deutsche Wechselbeziehungen in Sprache, Literatur und Kultur (Deutsche Akademie der Wissenschaften zu Berlin. Veröffentlichungen des Instituts für Slawistik; 44). Berlin 1969, S. 285-295.

Wossidlo, Richard: Synonyma aus Mecklenburg für schelten. In: Korrespondenzblatt des Vereins für niederdeutsche Sprachforschung 9 (1884), S. 74-75.

Wossidlo, Richard: Im Stich lassen. In: Korrespondenzblatt des Vereins für niederdeutsche Sprachforschung 10 (1885), S. 60.

Wossidlo, Richard: Einige beachtenswerthe Wortbedeutungen im Mecklenburger Platt. In: Festschrift zur Feier des 50jährigen Dienst-Jubiläums des Gymnasialdirektors Dr. Nölting. Hrsg. von dem Lehrerkollegium der Großen Stadtschule zu Wismar. Wismar 1886, S. 167-172.

Wossidlo, Richard: Dreihundert Ausdrücke für prügeln aus Mecklenburg. In: Korrespondenzblatt des Vereins für niederdeutsche Sprachforschung 12 (1887), S. 72-74.

Wossidlo, Richard: Gott und Teufel im Munde des Mecklenburgischen Volkes. In: Korrespondenzblatt des Vereins für niederdeutsche Sprachforschung 15 (1891), S. 18-32 und 44-48.

Wossidlo, Richard: Das Naturleben im Munde des Mecklenburger Volkes. In: Zeitschrift für Volkskunde 5 (1895), S. 302-325 und 424-448.

Wossidlo, Richard: Muscheblicks. In: Korrespondenzblatt des Vereins für niederdeutsche Sprachforschung 32 (1911), S. 47.

Wossidlo, Richard: Die volkstümlichen Pflanzennamen Mecklenburgs. In: Mecklenburg. Zeitschrift des Heimatbundes Mecklenburg (Landesverein des Bundes Heimatschutz) 11 (1916), S. 94-99.

Wossidlo, Richard: Peervolk; Hohalieren. In: Korrespondenzblatt des Vereins für niederdeutsche Sprachforschung 37 (1919), S. 12.

Wossidlo, Richard: Mollang. In: Korrespondenzblatt des Vereins für niederdeutsche Sprachforschung 37 (1919), S. 45-46.

Wossidlo, Richard: Fierk. In: Korrespondenzblatt des Vereins für niederdeutsche Sprachforschung 37 (1919), S. 26-27. 
Wossidlo, Richard: Einige Bemerkungen zum mecklenburgischen Wörterbuche. In: Uns plattdütsch Heimat. Nachrichtenblatt von den plattdütschen Landesverband Meckelborg 4 (1929), S. 4-6 und 17-20.

Wossidlo, Richard: Kummaf; henharden; Ulenspeigel; sinen Tappen krigen; Kurfürst; Mit Cölnischer Munte betalen. In: Korrespondenzblatt des Vereins für niederdeutsche Sprachforschung 32 (1911), S. 49-51.

Zuck, Paul: Loen in Mecklenburg. In: Korrespondenzblatt des Vereins für niederdeutsche Sprachforschung 64 (1957), S. 31-32.

\subsection{Namenkunde}

Anonym: Einiges zu den älteren Straßennamen Rostocks. In: Kommunalpolitische Schriftenreihe der Seestadt Rostock 1: Das Antlitz der Stadt (1936), S. 23-26.

Bader, Ulf-Hermann: Plattdeutsche Volkssprache und Eigennamen Vorpommerns in einigen von Gustav Ehrismann betreuten Dissertationen. In: Bräuer, Rolf (Hrsg.): Mediävistische Literaturgeschichtsschreibung. Gustav Ehrismann zum Gedächtnis. Göppingen 1992, S. 301-309.

Bahlow, Hans: Mecklenburgisches Namenbüchlein. Ein Führer durch Mecklenburgs Familiennamen. Rostock 1932.

Bahlow, Hans: Der Zug nach dem Osten im Spiegel der niederdeutschen Namenforschung, insbesondere in Mecklenburg. In: Teuthonista 9 (1933), S. 222-233.

Bahlow, Hans: Die mecklenburgische Namenlandschaft. In: Volkskundliche Beiträge. Festschrift Richard Wossidlo, am 26. Januar 1939 zum Dank dargebracht von Freunden und Verehrern und dem Verlag. Neumünster 1939, S. 45-51.

Bahlow, Hans: Niederdeutsches Namenbuch. Walluf bei Wiesbaden 1972.

Bahlow, Hans: Niederdeutsche Namenwelt. Erscheinungsbild und Wesensgehalt. Mit 500 Namen. Hamburg 1973.

Bahlow, Hans: Pommersche Familiennamen. Ihr Geschichts- und Heimatwert. Neustadt, Aisch 1982.

Beyersdorf, Oskar: Über die slawischen Städtenamen Pommerns. o. O. 1874.

Bilek, Julius/Schall, Hermann: Slavische Ortsnamen aus Mecklenburg. Berlin 1957.

Bosse, Heinrich: Die Forst-, Flur- und Gewässernamen der Ueckermünder Heide (Veröffentlichungen der historischen Kommission für Pommern. Reihe 5: Forschungen zur pommerschen Geschichte; 2). Köln/Graz 1962.

Curschmann, Fritz: Die deutschen Ortsnamen im nordostdeutschen Kolonialgebiet (Forschungen zur deutschen Landes- und Volkskunde; 19/2). Stuttgart 1910. 
Dahl, Eva-Sophie: Die alten Rostocker Straßennamen. In: Wissenschaftliche Zeitschrift der Universität Rostock. Gesellschafts- und sprachwissenschaftliche Reihe 4 (1954/55), S. 135-150.

Ebbinghaus, Karl: Hiddenseer Ökelnamen. In: Beiträge zur Volkskunde Vorpommerns. Hrsg. vom Mecklenburgischen Folklorezentrum der drei Nordbezirke in Zusammenarbeit mit der Akademie der Wissenschaften der DDR, Zentralinstitut für Geschichte (WB Kulturgeschichte/Volkskunde). Rostock 1989, S. 15-18.

Eichler, Ernst/Mühlner, Werner: Die Namen der Städte in MecklenburgVorpommern. Herkunft und Bedeutung. Unter Mitarbeit von Hans Walther. Rostock 2002.

Ewe, Herbert: Die Flurnamen von Rügen und ihre geographische Bedeutung für die Insel. 1.-3. Bd. Diss. Greifswald 1959.

Ewe, Herbert: Die Flurnamenbildungen auf Rügen seit der Romantik. In: Erdmann, Gustav/Eichstaedt, Alfons (Hrsg.): Worte und Werte. Bruno Markwardt zum 60. Geburtstag. Berlin 1961, S. 92-98.

Felgenhauer, Joachim: Flurnamen aus dem Landkreis Demmin. In: Archäologische Berichte aus Mecklenburg-Vorpommern 7 (2000), S. 344-349.

Francke, Norbert/Krieger, Bärbel: Die Familiennamen der Juden in Mecklenburg. Mehr als 2000 jüdische Familien aus 53 Orten der Herzogtümer MecklenburgSchwerin und Mecklenburg-Strelitz im 18. und 19. Jahrhundert (Schriften des Vereins für Jüdische Geschichte und Kultur in Mecklenburg und Vorpommern e. V.). Schwerin 2001.

Francke, Norbert: Familiennamen jüdischer Mecklenburger. In: Susemihl, Bohnsack und anderes von den Herbsttagungen 2000 und 2001 (Schriften des Vereins für mecklenburgische Familien- und Personengeschichte; 4). Rostock 2002, S. $14-25$.

Geinitz, Eugen: Die Namen der mecklenburgischen Sölle. In: Mecklenburg. Zeitschrift des Heimatbundes Mecklenburg (Landesverein des Bundes Heimatschutz) 10 (1915), S. 14-28; 11 (1916), S. 12-13. Ergänzung von Peek, Hermann in: Ebd. 12 (1917), S. 70.

Glamann, Wilhelm: Malchiner Familiennamen. In: Mecklenburg. Zeitschrift des Heimatbundes Mecklenburg (Landesverein des Bundes Heimatschutz) 28 (1933), S. 86-90 und S. 109-116.

Görschner, Richard: Neubrandenburger Straßennamen. In: Mecklenburg. Zeitschrift des Heimatbundes Mecklenburg (Landesverein des Bundes Heimatschutz) 16 (1921), S. 40-42. 
Gosselck, Johannes: Die Pinnower Flurnamen. In: Mecklenburg. Zeitschrift des Heimatbundes Mecklenburg (Landesverein des Bundes Heimatschutz) 35 (1940), S. 57-60.

Grempe, Günther: Die slawischen Ortsnamen des Kreises Greifswald. Greifswald 1957.

Greve, Dieter: Stadt Boizenburg, Dörfer des Amtes Boizenburg-Land. Erster Teil (Flurnamenatlas für das südliche Westmecklenburg; 1). Schwerin 2011.

Haas, Alfred: Vorslawische Reste in pommerschen Ortsnamen. Sonderdruck aus der Beilage „Ostpommersche Heimat" der Zeitung für Ostpommern. o. O. 1929.

Haefs, Hanswilhelm: Ortsnamen und Ortgeschichten auf Rügen mitsamt Hiddensee und Mönchgut. Anmerkungen zur Geschichte. München 2003.

Haeger, Fritz: Die deutschen Ortsnamen Mecklenburgs seit dem Beginn der Kolonisation (Wissenschaftliche Schriftenreihe des Heimatbundes Mecklenburg; 2). Wismar 1935.

Hänsel, Magdalene: Die rügenschen Fischerflurnamen (Einzelschriften der Gesellschaft für pommersche Geschichte und Altertumskunde; 1). Hrsg. von der Gesellschaft für pommersche Geschichte und Altertumskunde. Stettin 1938.

Herrmann-Winter, Renate: Substitutionsprozesse in Ortsnamen. Zur Aussprache der auf -itz, -vitz und -witz auslautenden slawischen Ortsnamen auf Rügen. Mit 7 Karten. In: Eichler, Ernst (Hrsg.): Onomastica Slavogermanica XI. Berlin 1976, S. 85-96.

Holsten, Robert: Die pommersche Flurnamenforschung, eine nationale Wissenschaft. In: Pommersche Heimatpflege 1, H. 4 (1930), S. 159-163.

Holsten, Robert: Die ältesten deutschen Flurnamen in Pommern. In: Baltische Studien 35 (1933), S. 1-42.

Holsten, Robert: Lau, Leu und Le in pommerschen Flurnamen. In: Teuthonista 10 (1934), S. 252-255.

Holsten, Robert: Stüwt, ein pommerscher Waldname. In: Zeitschrift für Mundartenforschung 13 (1937), S. 223-227.

Holsten, Robert: Anger in pommerschen Flurnamen, ein niederfränkisches Merkmal. In: Zeitschrift für Mundartforschung 15 (1939), S. 39-43.

Holsten, Robert: Tanz und Spiel in pommerschen Flurnamen. In: Volkskundliche Beiträge. Festschrift Richard Wossidlo, am 26. Januar 1939 zum Dank dargebracht von Freunden und Verehrern und dem Verlag. Neumünster 1939, S. 77-87.

Holsten, Robert: Von der Sprache Rügens. In: Monatsblätter der Gesellschaft für pommersche Geschichte und Altertumskunde 54 (1940), S. 21-26. 
Holsten, Robert: Die Pommersche Flurnamensammlung (Veröffentlichungen der historischen Kommission für Pommern. Reihe 5: Forschungen zur pommerschen Geschichte; 6). Köln/Graz 1963.

Jordan, Bernd: Historische Orts- und Flurnamen der Stadt Lassan. In: Heimathefte für Mecklenburg und Vorpommern 10, H. 2 (2000), S. 30-31.

Kaestner, Walter: Zum mecklenburgischen Ortsnamen Findenwirunshier. In: Zeitschrift für Slawistik 25 (1980), S. 555-557.

Keuthe, Burghard: Ist „Klein“ im Ortsnamen mit „wendisch“ identisch? In: Pütt. Schriftenreihe des Heimatbundes e. V. Parchim in Mecklenburg (2001), S. 43-49.

Kohls, Fritz: Die Orts- und Flurnamen des Kreises Grimmen (Vorpommern). Diss. Greifswald 1930.

Kühn, Ingrid: Neue Namen zwischen Mecklenburg-Vorpommern und dem Freistaat Sachsen. In: Namenkundliche Informationen. Beiheft 20 (1999), S. 203-214.

Kühnel, Paul: Die slawischen Ortsnamen in Meklenburg. In: Jahrbücher des Vereins für meklenburgische Geschichte und Alterthumskunde 46 (1881), S. 3-168.

Kühnel, Paul: Die slavischen Ortsnamen in Mecklenburg-Strelitz. Wissenschaftliche Beilage zum Programm des Gymnasiums zu Neubrandenburg. 2 Bde. Neubrandenburg 1881-83.

Laabs, Eberhard: Der Familienname Laabs und seine pommersche Geschichte. Herbolzheim 2003.

Langner, F.: Flurnamen aus Neustadt-Glewe. In: Mecklenburg. Zeitschrift für Mecklenburg und Vorpommern 37, H. 2 (1995), S. 5-6.

Legrand, Horst: Die Bützower Straßennamen im Wandel der Zeiten (Unsere regionale Heimatgeschichte; 9). Bützow 2002.

Lorenz, F.: Der Name der Warnen in Ortsnamen Mecklenburgs. In: Mecklenburg. Zeitschrift des Heimatbundes Mecklenburg (Landesverein des Bundes Heimatschutz) 31 (1936), S. 59-60.

Madaus, Christian: Dorfnamen in Mecklenburg. In: Mecklenburg. Zeitschrift für Mecklenburg und Vorpommern 41, H. 6 (1999), S. 2.

Mucke, Erich: Die Namen der Ortschaften des Kreises Anklam und ihre Bedeutung. Anklam 1907.

Neumann, Walter: Die Flurnamen des Amtes Grevesmühlen. Wismar/Rostock 1932. 
Neumann, Walter: Kiekindemark und seine Flurnamen. In: Mecklenburg. Zeitschrift des Heimatbundes Mecklenburg (Landesverein des Bundes Heimatschutz) 33 (1938), S. 85-88.

Neumann, Walter: Wiese und Weide. Ein Beitrag zur mecklenburgischen Flurnamenkunde und Flurnamengeographie. In: Volkskundliche Beiträge. Festschrift Richard Wossidlo, am 26. Januar 1939 zum Dank dargebracht von Freunden und Verehrern und dem Verlag. Neumünster 1939, S. 58-76.

Niemeyer, Manfred: Kreis Nordvorpommern. Quellen- und Literatursammlung zu den Ortsnamen (Greifswalder Beiträge zur Ortsnamenkunde; 7). Greifswald 2007.

Niemeyer, Manfred: Ostvorpommern I. Quellen- und Literatursammlung zu den Ortsnamen. Usedom (Greifswalder Beiträge zur Ortsnamenkunde; 1). Greifswald 2001.

Niemeyer, Manfred: Ostvorpommern II. Quellen- und Literatursammlung zu den Ortsnamen. Festland (Greifswalder Beiträge zur Ortsnamenkunde; 2). Greifswald 2001.

Niemeyer, Manfred: Rügen I. Quellen- und Literatursammlung zu den Ortsnamen. A - M (Greifswalder Beiträge zur Ortsnamenkunde; 3). Greifswald 2001.

Niemeyer, Manfred: Rügen II. Quellen- und Literatursammlung zu den Ortsnamen. N - Z (Greifswalder Beiträge zur Ortsnamenkunde; 4). Greifswald 2002.

Niemeyer, Manfred: Siedlungsnamen slawischer Herkunft auf Rügen. In: RugiaJournal (2002), S. 29-35.

Peek, Hermann: Mecklenburgische Orts- und Flurnamen, die mit Krug zusammengesetzt sind. In: Mecklenburg. Zeitschrift des Heimatbundes Mecklenburg (Landesverein des Bundes Heimatschutz) 13 (1918), S. 7-12; Ergänzung von Wossidlo, Richard in: Ebd., S. 12-15.

Pfitzner, Martin: Das alte und das neue Strelitz: Volkskunde, Sagen, Flurnamen. Reprint der Orig.-Ausg. Neustrelitz 1938 mit einer Schrifterläuterung und einem Nachwort von Jörg Sachse versehen. Neustrelitz 1996.

Rahn, Dietrich: Die Orts- und Flurnamen des Stadt- und Landkreises Greifswald. Ihre Entstehung und ihre Bedeutung für die Pommersche Heimatkunde. Diss. Greifswald 1923.

Rieck, Carl: Die Personennamen von Woldegk und Umgegend. In: Mecklenburg. Zeitschrift des Heimatbundes Mecklenburg (Landesverein des Bundes Heimatschutz) 13 (1918), S. 44-66.

Schall, Hermann: Probleme der Namensforschung in Mecklenburg. In: Jahrbuch für Volkskunde und Kulturgeschichte 5 (1959), S. 137-145. 
Schlifkowitz, Heinrich: Typische Ortsnamen zwischen Elbe und Weichsel. Ortsnamen auf -au, -ow, -witz, -itz, -schütz und -in. Karlsruhe 1992.

Schlimpert, Gerhard: Alte Gewässernamen in Brandenburg und Mecklenburg. In: Eichler, Ernst (Hrsg.): Probleme der älteren Namenschichten Leipziger Symposion, 21. bis 22. November 1989. (Beiträge zur Namenforschung. N. F.; 32) Heidelberg 1991, S. 43-52.

Schmaltz, K.: Was die Sternberger Flurnamen erzählen. In: Mecklenburg. Zeitschrift des Heimatbundes Mecklenburg (Landesverein des Bundes Heimatschutz) 4 (1909), S. 21-32.

Schmidt, Herbert: Die Orts- und Flurnamen von Mönchgut. Sammlung und Deutungsversuche. Anklam 1921.

Schmuck, Mirjam: Personennamen als Quelle der Grammatikalisierung. Der -ing-Diminutiv in Mecklenburg-Vorpommern. In: Beiträge zur Namenforschung 44, H. 1 (2009), S. 35-63.

Schommartz, Günther: Vom Nutzen der Flurnamen zur Deutung von Ortsnamen am Beispiel von Ahrenshoop auf dem Vordarß. In: Stier und Greif. Blätter zur Kultur- und Landesgeschichte in Mecklenburg-Vorpommern 15 (2005), S. 131-145.

Schröder, Walter Johannes: Die volkstümlichen Pflanzennamen Mecklenburgs. Grundzüge der Namenbildung. In: Volkskundliche Beiträge. Festschrift Richard Wossidlo, am 26. Januar 1939 zum Dank dargebracht von Freunden und Verehrern und dem Verlag. Neumünster 1939, S. 25-44.

Schulz, Erwin: Ortsnamen in Mecklenburg-Strelitz von 1170-1572 (Greifswalder Beiträge zur Ortnamenkunde; 6). Greifswald 2004.

Siering, Waldemar/Siering, Robert: Flurnamen aus Dörfern zwischen Krakow und Teterow. Neubrandenburg 1999.

Siering, Waldemar/Siering, Robert: Orte mit kuriosen Namen in MecklenburgVorpommern. Von Aalbude bis Zitterpenningshagen. Friedland 2012.

Simon, Irmgard: Düüker, Droos, Budde, Klaonenkasper, Belzebuck. Über niederdeutsche Teufelsnamen und ihre Verwendung. In: Niederdeutsches Wort 36 (1996), S. 107-133.

Sommerfeldt, Karl-Ernst: Straßennamen in Mecklenburg-Vorpommern nach der Wende. In: Deutschunterricht 45, H. 9 (1992), S. 408-412.

Sommerfeldt, Karl-Ernst: Beiträge zur regionalen Namenkunde. Namen in Mecklenburg-Vorpommern (Sprache - System und Tätigkeit; 48). Frankfurt a. M. [u. a.] 2004.

Sommerfeldt, Karl-Ernst: Zur Entwicklung der Namen einer Stadt. Dargestellt am Beispiel der Stadt Güstrow in Mecklenburg-Vorpommern. Frankfurt a. M. [u. a.] 2004. 
Spruth, Herbert: Deutungen der Orts- und Personennamen Musswiek - Mussewiek in Rügen und Westpommern. In: Korrespondenzblatt des Vereins für niederdeutsche Sprachforschung 62 (1952), S. 52-54.

Staak, Gerhard: Der Flurname „up dei Süße“ und das Beiwort „süß“ in mecklenburgischen Flurnamen. In: Mecklenburg. Zeitschrift des Heimatbundes Mecklenburg (Landesverein des Bundes Heimatschutz) 26 (1931), S. 5-8.

Steinmann, Ulrich: Die Flurnamen der Hagenower Feldmark. In: Mecklenburg. Zeitschrift des Heimatbundes Mecklenburg (Landesverein des Bundes Heimatschutz) 25 (1930), S. 89-95 und S. 110-115.

Strathmann, Peter: Die Vogelbezeichnungen im Niederdeutschen SchleswigHolsteins und Mecklenburgs. Eine kommentierende systematisch-onomasiologische Untersuchung (Name und Wort; 17). 3 Teile. Neumünster 2008.

Techen, Friedrich: Die Straßennamen Wismars. In: Jahrbücher des Vereins für meklenburgische Geschichte und Alterthumskunde 66 (1901), S. 65-114.

Techen, Friedrich: Die Flurnamen der Wismarschen Feldmark. In: Mecklenburg. Zeitschrift des Heimatbundes Mecklenburg (Landesverein des Bundes Heimatschutz) 19 (1924), S. 33-41.

Techen, Friedrich: Über die Straßennamen norddeutscher Städte. In: Nordelbingen. Beiträge zur Heimatforschung in Schleswig-Holstein, Hamburg und Lübeck 5 (1926), S. 526-553.

Trautmann, Reinhold: Die wendischen Ortsnamen Ostholsteins, Lübecks, Lauenburgs und Mecklenburgs (Quellen und Forschungen zur Geschichte SchleswigHolsteins; 21). Neumünster 1939.

Trautmann, Reinhold: Die elb- und ostseeslawischen Ortsnamen. 1. Teil Berlin 1948.

Trautmann, Reinhold: Die elb- und ostseeslawischen Ortsnamen. 2. Teil Berlin 1949.

Trautmann, Reinhold: Die slavischen Ortsnamen Mecklenburgs und Holsteins (Abhandlungen der Sächsischen Akademie der Wissenschaften zu Leipzig. Philologisch-historische Klasse; 45.3). Berlin 1950.

Willich, Cornelia: Zum Projekt „Mecklenburgisches Namenbuch“. In: Namenkundliche Informationen 67/68 (1995), S. 103-107.

Willich, Cornelia: Zur Widerspiegelung der mittelalterlichen Siedlungsentwicklung im Namenschatz des nordwestlichen Mecklenburg. In: Ernst, Peter (Hrsg.): Ortsnamen und Siedlungsgeschichte. Akten des Symposiums in Wien vom 28.-30. September 2000. Heidelberg 2002, S. 253-260.

Witkowski, Teodolius: Die Ortsnamen des Kreises Stralsund (Veröffentlichungen des Instituts für Slawistik der Deutschen Akademie der Wissenschaften zu Berlin; 36). Berlin 1965. 
Witkowski, Teodolius: Slawische Namenforschung im niederdeutschen Sprachgebiet. In: Niederdeutsches Jahrbuch 89 (1966), S. 103-121.

Witkowski, Teodolius: Die Ortsnamen des Kreises Greifswald (Berliner Beiträge zur Namenforschung). Weimar 1978.

Wossidlo, Richard: Der typische Gebrauch der Vornamen im mecklenburger Platt. In: Korrespondenzblatt des Vereins für niederdeutsche Sprachforschung 9 (1884), S. 81-88; 10 (1885), S. 41.

Wossidlo, Richard: Mecklenburgische Krug- und Gehöftsnamen. In: Korrespondenzblatt des Vereins für niederdeutsche Sprachforschung 9 (1884), S. 92-93.

Wossidlo, Richard: Zu dem Ortsnamen mitten in der Welt. In: Jahrbücher des Vereins für Mecklenburgische Geschichte und Altertumskunde 57 (1892). Quartalbericht April 1892, S. 15-16.

Zessin, Wolfgang: Reflexionen über den Ursprung zoologischer und botanischer Familiennamen aus Mecklenburg und Pommern. In: Sedina-Archiv 48, 1/2 (2002), S. 23-30.

Zühlsdorff, Werner: Flurnamenatlas des südlichen Südwest-Mecklenburg, 1.-3. Teil Berlin 1970-1972.

Zühlsdorff, Werner: Flurnamenatlas von Südwestmecklenburg: Raum Parchim, Neustadt-Glewe, Hagenow. Berlin 1988.

\subsection{Fach- und Sondersprachenforschung}

Bandlow, Ernst: Unsere Seemannssprache. In: Heimatkalender des Kreises Kolberg-Körlin. Körlin/Kolberg 1927, S. 52-53.

Eichhoff, Jürgen: Die Sprache des niederdeutschen Reepschlägerhandwerks (Niederdeutsche Studien; 16). Marburg (Lahn) 1965.

Gosselck, Johannes: Die Sprache der mecklenburgischen Fischer. In: Mecklenburg. Zeitschrift des Heimatbundes Mecklenburg (Landesverein des Bundes Heimatschutz) 26 (1931), S. 8-12.

Gosselck, Johannes: Fischerglaube in Sprache und Brauch. In: Mecklenburg. Zeitschrift des Heimatbundes Mecklenburg (Landesverein des Bundes Heimatschutz) 29 (1934), S. 7-15.

Rassow, Margarethe: Fischersprache und Brauchtum im Lande zwischen dem Darß und der unteren Oder (Veröffentlichungen des Instituts für Deutsche Sprache und Literatur der Deutsche Akademie der Wissenschaften zu Berlin; 12). Berlin 1958.

Rehfeld, Irmgard: Sprache und volkskundliche Geltung des Wasser- und Windmüllers im ehemaligen Pommern. 2 Bde. Diss. Greifswald 1960. 
Wossidlo, Richard: Wind und Wasser im Munde des mecklenburgischen Seemannes. In: Mecklenburgische Monatshefte 1 (1925), S. 352-354.

Wossidlo, Richard: Aus dem Leben des plattdeutschen Seemanns. In: Uns' plattdütsch Heimat 9 (1933), S. 86-94.

Wossidlo, Richard: Reise, Quartier, in Gottesnaam. Das Seemannsleben auf den alten Segelschiffen im Munde alter Fahrensleute. Im Auftrage des Kuratoriums der Wossidlo-Stiftung aus dem Nachlaß Richard Wossidlos hrsg. von Paul Beckmann. 2 Bde. Rostock 1940/1943.

\subsection{Soziolinguistik, Pragmatik und Perzeptionslinguistik}

Arendt, Birte: Vom Ärgernis zum Kulturgut - politische Einstellungen zum Niederdeutschen in Mecklenburg-Vorpommern von 1945 bis heute. In: Arendt, Birte/Lippmann, Enrico (Hrsg.): Die Konstanz des Wandels im Niederdeutschen. Politische und historische Aspekte einer Sprache. Hamburg 2005, S. 191-222.

Arendt, Birte: Wie Metasprachdiskurse Wirklichkeiten konstruieren. Eine Untersuchung von Spracheinstellungen zum Niederdeutschen. In: Lipczuk, Ryszard [u. a.] (Hrsg.): Diskurslinguistik - Systemlinguistik. Theorien, Texte, Fallstudien. Hamburg 2010, S. 273-287.

Arendt, Birte: Niederdeutschdiskurse. Spracheinstellungen im Kontext von Laien, Printmedien und Politik. Berlin 2010.

Arendt, Birte: Plattdeutsche Glokalisierung. Identitätsinszenierung auf subnationaler Ebene. In: Schiewe, Jürgen [u. a.] (Hrsg.): Kommunikation für Europa II. Sprache und Identität. Frankfurt a. M. [u. a.] 2010, S. 67-76.

Arendt, Birte: Laientheoretische Konzeptionen von Sprache und Dialekt am Beispiel des Niederdeutschen. Eine kontextsensitive Analyse von Spracheinstellungsäußerungen sowie ihre methodologische Fundierung. In: Niederdeutsches Wort 51 (2011), S. 135-162.

Arendt, Birte: Diskursprägung und ihre Konsequenzen. Kulturfundierte Sprachkonzepte im sprachpolitischen Diskurs am Beispiel der Regionalsprache Niederdeutsch. In: Kämper, Heidrun/Kilian, Jörg (Hrsg.): Wort - Begriff - Diskurs. Deutscher Wortschatz und europäische Semantik. Bremen 2012, S. 289-304.

Arendt, Birte: Niederdeutschdiskurse. Spracheinstellungen im Kontext von Laien, Printmedien und Politik. In: Niederdeutsches Jahrbuch 135 (2012), S. 101-117.

Arendt, Birte: Language Ideology of the European Union under a Critical Perspective - on the Example of the Regional Language Low German. In: Koll-Stobbe, Amei/Knospe, Sebastian (Hrsg.): Language Contact Around the Globe. Proceedings of the LCTG3 Conference. Frankfurt a. M. [u. a.] 2014, S. 299-318. 
Bader, Ulf-Hermann: Zum Verhältnis von Textstil und Stilrezeption - Ergebnisse von Informantentests an einem niederdeutschen Text. In: Greifswalder Germanistische Forschungen 12 (1990), S. 110-126.

Bader, Ulf-Hermann: Das Niederdeutsche in der Honecker-Ära - kulturelles Erbe und folkloristische Renaissance. In: Herrmann-Winter, Renate (Hrsg.): Heimatsprache zwischen Ausgrenzung und ideologischer Einbindung. Niederdeutsch in der DDR. Frankfurt a. M. [u. a.] 1998, S. 47-55.

Bichel, Ulf: „Wenn einer dauhn deiht, wat hei deiht.... Söken na den rechten Platz för plattdüütsche Spraak un Literatur in unse Daag. In: Bichel, Ulf/Griephan, Hans-Joachim/de Voss, Helmut (Hrsg.): Fritz Reuters Freiheitsidee. Dichter für Liberalismus und Demokratie. Vorträge zu den Internationalen Reuter-Tagen vom 23. bis 25. März 1990 in Lübeck-Travemünde (Beiträge der Fritz Reuter Gesellschaft; 2). Neubrandenburg 1992, S. 107-114.

Chudnitzki, Wladimir S.: Zur kommunikativen Funktion der sprachlichen Existenzformen in Mecklenburg. Soziolinguistische Untersuchungen in der Schiffswerft Rechlin und ihrer engeren Umgebung. In: Beiträge zur Erforschung der deutschen Sprache 10 (1991), S. 223-239.

Dittmar, Lisa: Die gegenwärtige Sprachsituation auf dem Fischland und dem Darß. In: Wissenschaftliche Zeitschrift der Universität Rostock. Gesellschaftsund sprachwissenschaftliche Reihe 11 (1962), S. 313-318.

Dost, Wolfgang: Zur Einwirkung der kommunikativen Bedingungen in der DDR auf die regionale Abgrenzung im Bereich der Umgangssprache und der Mundart, dargestellt an der Entwicklung im Raum Wittstock-Röbel. In: Das Niederdeutsche in Geschichte und Gegenwart. Hrsg. vom Zentralinstitut für Sprachwissenschaft der Akademie der Wissenschaften der DDR (Linguistische Studien. Reihe A: Arbeitsberichte; 75.2). Berlin 1981, S. 121-127.

Dröge, Kurt: Der ,mittelpommersche Keil'. Genese eines kulturwissenschaftlichen Stereotyps. In: Buchholz, Werner/Mangelsdorf, Günter (Hrsg.): Land am Meer. Pommern im Spiegel seiner Geschichte. Roderich Schmidt zum 70. Geburtstag. Köln/Weimar/Wien 1995, S. 759-785.

Ehlers, Klaas-Hinrich: Schlesische und sudetendeutsche Plattschnacker. Eine Fallstudie zur sprachlichen Integration der Vertriebenen in MecklenburgVorpommern. In: Bohemia. Zeitschrift für Geschichte und Kultur der böhmischen Länder 51, H. 2 (2011), S. 345-357.

Ehlers, Klaas-Hinrich: Forschungsprojekt: Kontaktlinguistische Untersuchungen zur sprachlichen Akkulturation Heimatvertriebener in Mecklenburg. In: brücken. Germanistisches Jahrbuch Tschechien-Slowakei NF 19, H. 1-2 (2012), S. 358-374.

Ehlers, Klaas-Hinrich: De Ierste Weltkrieg op Mäkelborger Platt. Heinrich Zilles Vadding-Hefte. In: Niederdeutsches Wort 55 (2015), S. 55-72. 
Ehlers, Klaas-Hinrich: „Uns’re Leut“ - Akkulturation und Abgrenzung einer karpatendeutschen Vertriebenengruppe in Mecklenburg. In: Zückert, Martin/ Schvarc, Michal/Meier, Jörg (Hrsg.): Migration - Zentrum und Peripherie kulturelle Vielfalt. Neue Zugänge zur Geschichte der Deutschen in der Slowakei. (Reihe Digi-Ost 2016) Leipzig 2016, S. 161-197.

Ehlers, Klaas-Hinrich: Von der Sprachbiografie zur Sprachgebrauchsgeschichte: Die Rekonstruktion des Varietätengebrauchs auf den Rostocker Werften. In: Jürgens, Carolin/Schröder, Ingrid (Hrsg.): Sprachliche Variation in autobiographischen Interviews. Theoretische und methodische Zugänge. Frankfurt a. M. [u. a.] 2017, S. 143-165.

Gernentz, Hans Joachim: Der Rückgang der Mundarten in Norddeutschland. In: Deutsch als Fremdsprache 2 (1965), S. 13-17.

Gernentz, Hans Joachim: Über den Auflösungsprozeß der Mundart im Norden der Deutschen Demokratischen Republik. In: Actes du Xe Congres International des Linguistes. 1. Bd. Bukarest 1969, S. 677-684.

Gernentz, Hans Joachim: Die kommunikative Funktion der niederdeutschen Mundart und hochdeutschen Umgangssprache im Norden der Deutschen Demokratischen Republik, unter besonderer Berücksichtigung der Interferenz und der Alternanz zwischen diesen beiden sprachlichen Existenzformen. In: Studia Germanica Gandensia 15 (1974), S. 209-244.

Gernentz, Hans Joachim: System und Verwendung der Existenzformen des Deutschen im Norden der Deutschen Demokratischen Republik. In: Wissenschaftliche Zeitschrift der Universität Rostock. Gesellschafts- und sprachwissenschaftliche Reihe 24 (1975), S. 385-393.

Gernentz, Hans Joachim: Wann und wie wird heute niederdeutsch gesprochen? In: Theil, Hans-Joachim (Red.): Niederdeutsch heute. Materialien einer Arbeitstagung des Freundeskreises Niederdeutsche Sprache und Literatur im Kulturbund der DDR, Kreisleitung Rostock 16.-17. November 1974. Rostock 1975, S. 12-19.

Gernentz, Hans Joachim: Zur Verbreitung und Bewertung der niederdeutschen Mundart in den drei Nordbezirken der DDR. In: Prowatke, Christa (Bearb.): Niederdeutsche Mundart und Literatur in der DDR. Hrsg. vom Mecklenburgischen Folklorezentrum für die drei Nordbezirke. Rostock 1985, S. 3-11.

Gernentz, Hans Joachim: Zur Entwicklung und Bewertung des Niederdeutschen in Mecklenburg und Vorpommern. In: Greifswalder Germanistische Forschungen 11 (1989), S. 48-58.

Herrmann-Winter, Renate: Auswirkungen der sozialistischen Produktionsweise in der Landwirtschaft auf die sprachliche Kommunikation in den Nordbezirken der DDR. Unter Mitarbeit von Christa Baufeld, Irmgard Rosenthal, Max Groth und Jürgen Gundlach. In: Ising, Gerhard (Hrsg.): Aktuelle Probleme der Birte Arendt, Andreas Bieberstedt and Klaas-Hinrich Ehlers - 978-3-631-71893-3 
sprachlichen Kommunikation. Soziologische Studien zur sprachlichen Situation in der DDR (Reihe Sprache und Gesellschaft; 2). Berlin 1974, S. 135-190.

Herrmann-Winter, Renate: Soziolinguistische Aspekte empirischer Erhebungen zur sprachlichen Varianz. In: Hartung, Wolfdietrich (Hrsg.): Normen in der sprachlichen Kommunikation (Reihe Sprache und Gesellschaft; 11). Berlin 1977, S. 209-246.

Herrmann-Winter, Renate: $\mathrm{Zu}$ einigen Problemen der Bewertung sprachlicher Varianten durch Sprecher. In: Gesellschaftliche Funktionen und Strukturen sprachlicher Kommunikation. Hrsg. vom Zentralinstitut für Sprachwissenschaft der Akademie der Wissenschaften der DDR (Linguistische Studien. Reihe A: Arbeitsberichte; 72.1). Berlin 1980, S. 140-149.

Herrmann-Winter, Renate: Niederdeutsch heute. In: Sprachpflege 30, H. 11 (1981), S. 165-167.

Herrmann-Winter, Renate: Urteile über Niederdeutsch in den Nordbezirken der DDR. In: Zeitschrift für Phonetik, Sprachwissenschaft und Kommunikationsforschung 38, H. 3 (1985), S. 297-308.

Herrmann-Winter, Renate: Niederdeutsch in der Metakommunikation. Befragungen zur kommunikativen Leistungsfähigkeit einer sprachlichen Varietät. In: Wissenschaftliche Zeitschrift der Universität Rostock. Gesellschafts- und sprachwissenschaftliche Reihe 35, H. 8 (1986), S. 108-112.

Herrmann-Winter, Renate: Zum Zusammenhang von Verwendungsweisen und Bewertungen des Niederdeutschen im Norden der DDR. In: Zeitschrift für Phonetik, Sprachwissenschaft und Kommunikationsforschung 40, H. 6 (1987), S. 822-829.

Herrmann-Winter, Renate: Frau Apotheker kaufte ihren Hut hochdeutsch. Prominente über platt. Rostock 1989.

Herrmann-Winter, Renate: Zu Fragen der Erforschung gesprochener Sprache im Norden der DDR. In: Berthold, Hans (Red.): Theoretische Positionen der Beschreibung gesprochener und geschriebener Sprache: Protokollband. Pädagogische Hochschule Leipzig 1989, S. 83-93.

Herrmann-Winter, Renate: Standardsprache und Mundarten in der Deutschen Demokratischen Republik. In: Stickel, Gerhard (Hrsg.): Deutsche Gegenwartssprache. Tendenzen und Perspektiven (Jahrbuch des Instituts für deutsche Sprache 1989). Berlin/New York 1990, S. 184-191.

Herrmann-Winter, Renate: „Der Dialekt erlaubt keine eigene Sprache, aber eine eigene Stimme...". Überlegungen zur Bewertung des Niederdeutschen. In: Mattheier, Klaus/Wiesinger, Peter (Hrsg.): Dialektologie des Deutschen. Forschungsstand und Entwicklungstendenzen. Tübingen 1994, S. 457-464. 
Herrmann, Winter: Notwehr ist erlaubt. Niederdeutsch im Urteil von Verehreren und Verächtern. Texte aus Mecklenburg und Pommern vom 16. bis zum 20. Jahrhundert. Rostock 1995.

Herrmann-Winter, Renate: Norddeutsche Volkssprache unter der Arbeiter-undBauern-Macht bis 1970. In: Herrmann-Winter, Renate (Hrsg.): Heimatsprache zwischen Ausgrenzung und ideologischer Einbindung. Niederdeutsch in der DDR. Frankfurt a. M. [u. a.] 1998, S. 15-47.

Latendorf, Friedrich: Niederdeutsch und Neudeutsch. Offener Brief an Edmund Höfer. Poesneck 1879.

Möller, Frerk: Plattdeutsch im 21. Jahrhundert. Bestandsaufnahme und Perspektiven. Mit einem Aufsatz von Michael Windzio (Schriften des Instituts für Niederdeutsche Sprache; 34). Leer 2008.

Möller, Frerk: Niederdeutsch im 21. Jahrhundert. Bestandsaufnahme und Perspektiven. In: Niederdeutsches Jahrbuch 133 (2010), S. 141-164.

Plewnia, Albrecht/Rothe, Astrid: Sprache - Einstellung - Regionalität. In: Eichinger, Ludwig [u. a.] (Hrsg.): Sprache und Einstellungen. Spracheinstellungen aus sprachwissenschaftlicher und sozialpsychologischer Sicht. Tübingen 2012, S. 9-118.

Prowatke, Christa: Erfahrungen im Umgang mit der niederdeutschen Mundart. In: Prowatke, Christa (Bearb.): Niederdeutsche Mundart und Literatur in der DDR. Hrsg. vom Mecklenburgischen Folklorezentrum für die drei Nordbezirke. Rostock 1985, S. 12-17.

Rösler, Irmtraud: „Dat Land steiht för de plattdüütsche Sprak in un bringt ehr Pläg” vöran“. Soziolinguistische Beobachtungen zur kommunikativen Geltung des Niederdeutschen in Mecklenburg-Vorpommern. In: Rösler, Irmtraud/ Sommerfeldt, Karl-Ernst (Hrsg.): Probleme der Sprache nach der Wende. Beiträge des Kolloquiums in Rostock am 16. November 1996 (Sprache - System und Tätigkeit; 23). 2., korr. Aufl. Frankfurt a. M. [u. a.] 1998, S. 33-44.

Rösler, Irmtraud: Dat Segg Ik Up Platt. Zur kommunikativen Geltung des Niederdeutschen in Mecklenburg-Vorpommern. In: Lehmberg, Maik (Hrsg.): Sprache, Sprechen, Sprichwörter. Festschrift für Dieter Stellmacher zum 65. Geburtstag (ZDL Beihefte; 126). Stuttgart 2004, S. 151-158.

Scharnhorst, Jürgen: Die Theorie der Sprachkultur und ihre Anwendung im Norden der DDR. In: Das Niederdeutsche in Geschichte und Gegenwart. Hrsg. vom Zentralinstitut für Sprachwissenschaft der Akademie der Wissenschaften der DDR (Linguistische Studien. Reihe A: Arbeitsberichte; 75.1). Berlin 1981, S. 30-48.

Schönfeld, Helmut: Gruppenspezifische Unterschiede bei der Verwendung und Bewertung des Niederdeutschen in der DDR. In: Das Niederdeutsche in Ge- 
schichte und Gegenwart. Hrsg. vom Zentralinstitut für Sprachwissenschaft der Akademie der Wissenschaften der DDR (Linguistische Studien. Reihe A: Arbeitsberichte; 75.2). Berlin 1981, S. 112-120.

Schütt, Peter: Zwischen Platt und Parteichinesisch. Auf den Spuren des Niederdeutschen in Mecklenburg. In: MUT 333 (1995), S. 78-87.

Stellmacher, Dieter: Heimatsprache zwischen Ausgrenzung und ideologischer Einbindung - einige Anmerkungen von außen. In: Herrmann-Winter, Renate (Hrsg.): Heimatsprache zwischen Ausgrenzung und ideologischer Einbindung. Niederdeutsch in der DDR. Frankfurt a. M. [u. a.] 1998, S. 57-65.

Völschow, Werner: Erinnerungen und Überlegungen zum Umgang mit dem Niederdeutschen zwischen 1970 und 1990. In: Herrmann-Winter, Renate (Hrsg.): Heimatsprache zwischen Ausgrenzung und ideologischer Einbindung. Niederdeutsch in der DDR. Frankfurt a. M. [u. a.] 1998, S. 35-360.

\subsection{Sprachhistoriographie}

Anonym: Das plattdeutsche Rostock. In: Kommunalpolitische Schriftenreihe der Seestadt Rostock: Das Antlitz der Stadt (1936), S. 34-36.

Beckmann, Paul: Die Weiterentwicklung der Mecklenburger Mundart nach dem Tode Fritz Reuters. In: Wissenschaftliche Zeitschrift der Universität Rostock. Gesellschafts- und sprachwissenschaftliche Reihe 4 (1954/55), S. 129-133.

Ehlers, Klaas-Hinrich: Führte die Immigration der Heimatvertriebenen nach $1945 \mathrm{zu}$ Dialektverlust und Nivellierung regionalsprachlicher Differenzen? Beobachtungen aus einer Untersuchungsregion in Mecklenburg. In: Niederdeutsches Jahrbuch 136 (2013), S. 97-116.

Ehlers, Klaas-Hinrich: Von der Sprachbiografie zur Sprachgebrauchsgeschichte: Die Rekonstruktion des Varietätengebrauchs auf den Rostocker Werften. In: Jürgens, Carolin / Schröder, Ingrid (Hrsg.): Sprachliche Variation in autobiographischen Interviews. Theoretische und methodische Zugänge. Frankfurt a. M. 2017, S. 143-165.

Gernentz, Hans Joachim: Die historischen Voraussetzungen für die kommunikative Funktion des Niederdeutschen in der DDR. In: Das Niederdeutsche in Geschichte und Gegenwart. Hrsg. vom Zentralinstitut für Sprachwissenschaft der Akademie der Wissenschaften der DDR (Linguistische Studien. Reihe A: Arbeitsberichte; 75.1). Berlin 1981, S. 19-29.

Gernentz, Hans Joachim: Zur Entwicklung und Bewertung des Niederdeutschen in Mecklenburg und Vorpommern. In: Greifswalder Germanistische Forschungen 11 (1989), S. 48-58. 
Hansen, Martin: Zum Wandel des Niederdeutschen auf der Insel Rügen zwischen dem 19. und 21. Jahrhundert. Ein diachronischer Vergleich anhand ausgewählter Sprachmerkmale. Masterarbeit Greifswald 2009.

Herrmann-Winter, Renate: $\mathrm{Zu}$ Prestige und Bewertung von Niederdeutsch im Wandel der Jahrhunderte. In: Buske, Norbert (Hrsg.): Niederdeutsche Bibeltradition. Entwicklung und Gebrauch des Niederdeutschen in der Kirche. Berlin/Altenburg 1990, S. 42-57.

Herrmann-Winter, Renate: Urteile über Niederdeutsch aus dem 18. und 19. Jahrhundert. In: Niederdeutsches Jahrbuch 115 (1992), S. 123-144.

Herrmann-Winter, Renate: Notwehr ist erlaubt. Niederdeutsch im Urteil von Verehrern und Verächtern. Texte aus Mecklenburg und Pommern vom 16. bis zum 20. Jahrhundert. Rostock 1995.

Herrmann-Winter, Renate: Mundartrenaissance in den sogenannten neuen Bundesländern. In: Stickel, Gerhard (Hrsg.): Varietäten des Deutschen. Regionalund Umgangssprachen. Berlin/New York 1997, S. 396-398.

Holsten, Robert: Zur Geschichte der vorpommerschen Mundart. In: Teuthonista $5(1928 / 29)$, S. 77-79.

Kohfeldt, Gustav: Plattdeutsche mecklenburgische Bauerngespräche aus der Zeit der Karl Leopold'schen Streitigkeiten (1719-1734). In: Niederdeutsches Jahrbuch 33 (1907), S. 159-164.

Kunze, Peter: Low German in Mecklenburg, before and after the „Wende“. Plattdütsch in Meckelnborg, vör un na de Wend. In: Burmeister, Robert (Hrsg.): Low German Conference in Schleswig-Holstein. Kiel 1998, S. 35-46.

Mackel, Emil: Über die Entstehung der Mundarten mit besonderer Berücksichtigung Mecklenburgs und der Priegnitz. o. O. 1906.

Müns, Wolfgang: Niederdeutsch in Mecklenburg-Vorpommern von 1853 bis zur Gegenwart - eine Betrachtung im Überblick. In: Bericht der 48. BevensenTagung vom 15.-17. September 1995 in Bad Bevensen. Bad Bevensen 1995, S. 33-54.

Prowatke, Christa (Bearb.): Niederdeutsche Mundart und Literatur in der DDR. Hrsg. vom Mecklenburgischen Folklorezentrum für die drei Nordbezirke. Rostock 1985.

Rösler, Irmtraud: Kum, wi suupen eens tosamen, Schnack mi doch wat nÿes vör. - Ein politisches Bauerngespräch aus dem Jahre 1718. In: Peters, Robert/ Pütz, Horst P. (Hrsg.): Vulpis Adolatio. Festschrift für Hubertus Menke zum 60. Geburtstag (Germanistische Bibliothek; 11). Heidelberg 2001, S. 625-637.

Rösler, Irmtraud: Aspekte einer Sprachgeschichte des Ostniederdeutschen. In: Besch, Werner [u. a.] (Hrsg.): Sprachgeschichte. Ein Handbuch zur Geschichte der deutschen Sprache und ihrer Erforschung (Handbücher zur Sprach- und 
Kommunikationswissenschaft; 2.3). 3. Teilbd. 2., vollständig neu bearb. und erw. Aufl. Berlin/New York 2003, Sp. 2698-2713.

Rösler, Irmtraud: Sprachwechsel in Mecklenburg. In: Niederdeutsches Jahrbuch 129 (2006), S. 139-156.

Schildt, Joachim: Soziolinguistische Probleme des Sprachwandels - dargestellt am Beispiel des Niederdeutschen. In: Das Niederdeutsche in Geschichte und Gegenwart. Hrsg. vom Zentralinstitut für Sprachwissenschaft der Akademie der Wissenschaften der DDR (Linguistische Studien. Reihe A: Arbeitsberichte; 75.1). Berlin 1981, S. 6-18.

Schönfeld, Helmut: Sprache und Sprachvariation in der Stadt. Zu sprachlichen Entwicklungen und zur Sprachvariation in Berlin und anderen Städten im Nordteil der DDR (Linguistische Studien. Reihe A: Arbeitsberichte; 197). Berlin 1989.

Teuchert, Hermann: Beiträge zur Geschichte der mecklenburgischen Mundart. In: Niederdeutsches Jahrbuch 82 (1959), S. 207-236.

Teuchert, Hermann: Entwurf einer mecklenburgischen Sprachgeschichte. In: Wissenschaftliche Zeitschrift der Universität Rostock. Gesellschafts- und sprachwissenschaftliche Reihe 7, H. 1 (1957/58), S. 197-202.

Weltzien, Otto: John Brinckmans Leben und Werke nebst einer Abhandlung über die Geschichte der niederdeutschen Sprache. In: Weltzien, Otto (Hrsg.): John Brinckmans sämtliche Werke in fünf Bänden mit Einleitungen und Anmerkungen. Leipzig 1903, S. III-XXX.

\subsection{Niederdeutsch in den Medien (Rundfunk, Fernsehen, Printmedien, Internet)}

Angrick, Dieter W.: Ein (Berufs-)Leben für den „Leuchtturm“. Die Sprache Fritz Reuters in der Wochenendbeilage der Norddeutschen Zeitung 1952-1991. In: Bichel, Ulf/Griephan, Hans-Joachim (Hrsg.): Zwischen Niedergang und Aufbruch. Plattdeutsche Dichtung von 1945 bis 1990. Vorträge zu den ReuterTagen vom 19. bis 21. März 1993 in Lübeck-Travemünde (Beiträge der Fritz Reuter Gesellschaft; 4). Neubrandenburg 1995, S. 65-76.

Angrick, Dieter W.: Plattdeutsch im Printmedium „Norddeutsche Zeitung“. In: Herrmann-Winter, Renate (Hrsg.): Heimatsprache zwischen Ausgrenzung und ideologischer Einbindung. Niederdeutsch in der DDR. Frankfurt a. M. [u. a.] 1998, S. 325-333.

Arendt, Birte: Welches Bild wird in der Regionalpresse vom Niederdeutschen gezeichnet? In: Bundesraat för Nedderdüütsch (Hrsg.): Zwischen Kulturauftrag und Unterhaltungsprogramm. Plattdeutsch in den Medien. Leer 2010, S. 57-62. 
Arendt, Birte: Niederdeutschdiskurse. Spracheinstellungen im Kontext von Laien, Printmedien und Politik. Berlin 2010.

Arendt, Birte: „deswegen hab ich mich auch nich getr!AU!t zu sprechen. "Spracheinstellungsmuster und Sprachgebrauch Jugendlicher bezüglich des Niederdeutschen im sozialen Netzwerk „Plattdüütschkring“. In: Muttersprache. Vierteljahresschrift der Gesellschaft für deutsche Sprache (2012), S. 1-25.

Arendt, Birte: Niederdeutschdiskurse. Spracheinstellungen im Kontext von Laien, Printmedien und Politik. In: Niederdeutsches Jahrbuch 135 (2012), S. 101-117.

Beckmann, Paul: Der Rundfunk und das Plattdeutsche. In: Mecklenburgische Monatshefte 7 (1931), S. 6-8.

Hensel, Hanjo: Niederdeutsch im DDR-Rundfunk. In: Herrmann-Winter, Renate (Hrsg.): Heimatsprache zwischen Ausgrenzung und ideologischer Einbindung. Niederdeutsch in der DDR. Frankfurt a. M. [u. a.] 1998, S. 335-346.

Herrmann-Winter, Renate: Wortgut aus niederdeutschen Pressetexten der DDR im Urteil von Mundartsprechern. In: Das Niederdeutsche in Geschichte und Gegenwart. Hrsg. vom Zentralinstitut für Sprachwissenschaft der Akademie der Wissenschaften der DDR (Linguistische Studien. Reihe A: Arbeitsberichte; 75.1). Berlin 1981, S. 96-104.

Herrmann-Winter, Renate: Niederdeutsche Presseüberschriften. In: Lösch, Wolfgang (Bearb.): Umgangssprache und Dialekte in der DDR. Jena 1986, S. 88-99.

Laudahn, Heinz: Plattdütsch im Funk. Über Möglichkeiten und Grenzen des Niederdeutschen im regionalen Rundfunkprogramm des Nordens. In: Kikut. Plattdütsch gistern un hüt 1 (1976), S. 3-4.

Schmitt, Christoph: Mecklenburg im Hörfunk der Weimarer Republik. Richard Wossidlo und die Nordische Rundfunk AG. In: Stier und Greif. Blätter zur Kultur- und Landesgeschichte in Mecklenburg-Vorpommern 8 (1998), S. 76-83.

Völschow, Werner: Plattdeutsch im Funk. In: Kikut. Plattdütsch gistern un hüt 6 (1981), S. 27-28.

Zänger, Horst: Radio in Mecklenburg-Vorpommern. In: Heimathefte für Mecklenburg-Vorpommern 4, H. 4 (1994), S. 5-9.

Zänger, Horst: Rundfunk in Mecklenburg. Ein chronologischer Streifzug. In: Stier und Greif. Blätter zur Kultur- und Landesgeschichte in MecklenburgVorpommern 4 (1994), S. 8-13. 
Zänger, Horst: Geschichten aus 50 Jahren Rundfunk. Chronik des Landesrundfunks Mecklenburg-Vorpommern. Schwerin 1995.

\subsection{Niederdeutsch in kulturellen Institutionen (Kirche, Bühne usw.)}

Ackermann, G[eorg]. C[hristian]. B[enedikt]: Ueber die Sprache des gemeinen Mannes in Meklenburg, mit Hinsicht auf Kanzelvortrag. In: Neue Monatsschrift von und fuer Mecklenburg 4. 1stes Stueck (1794), S. 25-31.

Bellmann, Johann Diedrich: Die Ehrwürdigen Väter. Niederdeutsch als Kirchensprache im 19. Jahrhundert. In: Quickborn 68 (1978), S. 76-84.

Bunners, Christian: Die heutige Verwendung des Niederdeutschen in Kirchengemeinden der DDR - Brauchtumspflege oder missionarische Elemente? In: Buske, Norbert (Hrsg.): Niederdeutsche Bibeltradition. Entwicklung und Gebrauch des Niederdeutschen in der Kirche. Berlin/Altenburg 1990, S. 139-153.

Dahl, Dietrich: Zur niederdeutschen Bühnenarbeit. In: Theil, Hans-Joachim (Red.): Niederdeutsch heute. Materialien einer Arbeitstagung des Freundeskreises Niederdeutsche Sprache und Literatur im Kulturbund der DDR, Kreisleitung Rostock 16.-17. November 1974. Rostock 1975, S. 53-55.

Holtz, Gottfried: Niederdeutsch als Kirchensprache. In: Wissenschaftliche Zeischrift der Universität Rostock, Gesellschafts- und sprachwissenschaftliche Reihe 4, H. 2 (1954/55), S. 151-165.

Homuth, Karl: Bemühungen um die niederdeutsche Bibel im 20. Jahrhundert. Diss. Rostock 1974.

Homuth, Karl: Plattdeutsch und Kirche in Mecklenburg. In: De Kennung 4 (1981), S. 39-45.

Köhn, Friedrich: Die Kirchensprache Niederdeutschlands. In: Köhn, Friedrich (Hrsg.): Sünndagsklocken. Stadt- und Dörp-Predigten. Wolgast 1922, S. 3-30.

Kröger, Heinrich: Plattdüütsch in de Kark in der DDR - aus der Sicht und Strategie eines Westdeutschen. In: Herrmann-Winter, Renate (Hrsg.): Heimatsprache zwischen Ausgrenzung und ideologischer Einbindung. Niederdeutsch in der DDR. Frankfurt a. M. [u. a.] 1998, S. 287-316.

Kröger, Heinrich: Plattdüütsch in de Kark in drei Jahrhunderten. 3 Bde. Hannover 1996-1998, Hermannsburg 2001.

Möller, Frerk: Niederdeutsch: das soziokulturelle Umfeld. In: Stellmacher, Dieter (Hrsg.): Niederdeutsche Sprache und Literatur der Gegenwart. Hildesheim/ Zürich/New York 2004, S. 281-358.

Passig, Willi: Karl Friedrich Steinhagen un Plattdütsch as Kirchensprak in Rostock. In: De Kennung 15 (1992), S. 32-35. 


\subsection{Vermittlung, Förderung und Pflege des Niederdeutschen}

Arendt, Birte: Niederdeutschpolitik in Mecklenburg-Vorpommern. Im Zeichen der Sprachencharta. In: Blanke, Detlev (Red.): Internationale Plansprachen Entwicklung und Vergleich. Beiträge der 14. Jahrestagung der Gesellschaft für Interlinguistik e. V., 5.-7. November 2004 in Berlin. Berlin 2005, S. 95-113.

Arendt, Birte: Entwicklung des Niederdeutschstudiums an der Ernst-MoritzArndt-Universität Greifswald. In: Bundesrat für Niederdeutsch (Hrsg.): Plattdeutsch, die Region und die Welt. Wege in eine moderne Mehrsprachigkeit. Leer 2009, S. 39-47.

Arendt, Birte: Niederdeutschdiskurse. Spracheinstellungen im Kontext von Laien, Printmedien und Politik. Berlin 2010.

Arendt, Birte: Niederdeutschdiskurse. Spracheinstellungen im Kontext von Laien, Printmedien und Politik. In: Niederdeutsches Jahrbuch 135 (2012), S. 101-117.

Arendt, Birte: Diskursprägung und ihre Konsequenzen. Kulturfundierte Sprachkonzepte im sprachpolitischen Diskurs am Beispiel der Regionalsprache Niederdeutsch. In: Kämper, Heidrun/Kilian, Jörg (Hrsg.): Wort - Begriff - Diskurs. Deutscher Wortschatz und europäische Semantik. Bremen 2012, S. 289-304.

Arendt, Birte: Was spricht für einen frühen Zweitspracherwerb? In: Stiftung Mecklenburg (Hrsg.): Lürlürlütt. Handreichungen zum Erlernen der niederdeutschen Sprache in der frühkindlichen Bildung. Rostock 2012, S. 6-8.

Arendt, Birte/Gąszczyk, Alicja: Second language immersion programmes at kindergarten - as illustrated by Polish and Low German. In: Minsterium für Bildung und Kultur des Saarlandes (Hrsg.): Multilingualism creates opportunities. Saarbrücken 2015, S. 54-68.

Bader, Ulf-Hermann: Mundartpflege und Kommunikationsfähigkeit im Niederdeutschen. $\mathrm{Zu}$ einigen Aspekten der niederdeutschen Mundartpflege. In: Determinanten und Faktoren der Kommunikationsfähigkeit. 2. Interdisziplinäres Symposium, Greifswald, November 1988. Ernst-Moritz-Arndt-Universität Greifswald, Institut für Deutsche Philologie (Wissenschaftliche Beiträge der E.-M.-Arndt-Universität Greifswald). Greifswald 1990, S. 154-164.

Bieberstedt, Andreas: Niederdeutsch in der universitären Lehre. Stand und Perspektiven. In: Korrespondenzblatt des Vereins für niederdeutsche Sprachforschung 116, H. 1 (2009), S. 11-16.

Bieberstedt, Andreas: Niederdeutsch an den Hochschulen. Gegenwärtiger Stand und Perspektiven. In: Bundesrat für Niederdeutsch (Hrsg.): Plattdeutsch, die Region und die Welt. Wege in eine moderne Mehrsprachigkeit - Positionen und Bilanzen (Schriften des Instituts für Niederdeutsche Sprache; 39). Leer 2010, S. 34-38. 
Bieberstedt, Andreas: Das Modellprojekt „Plattdeutsch im Kindergarten“ - Was berichten die Teilnehmer? In: Stiftung Mecklenburg (Hrsg.): Lürlürlütt. Handreichungen zum Erlernen der niederdeutschen Sprache in der frühkindlichen Bildung. Rostock 2012, S. 9-11.

Bentzien, Ulrich: Etappen der Mundartpflege in Mecklenburg. In: Jahrbuch für Volkskunde und Kulturgeschichte 23 (1980), S. 152-157.

Bentzien, Ulrich: Historische Etappen der Mundartpflege im Norden der DDR. In: Das Niederdeutsche in Geschichte und Gegenwart. Hrsg. vom Zentralinstitut für Sprachwissenschaft der Akademie der Wissenschaften der DDR (Linguistische Studien. Reihe A: Arbeitsberichte; 75.2). Berlin 1981, S. 147-154.

Bliemel, Susanne: Lehrerausbildung in Mecklenburg-Vorpommern. In: 61. Bevensen-Tagung. Berichtsheft (2009), S. 45-49.

Die Niederdeutsch-Beiräte in Mecklenburg-Vorpommern und SchleswigHostein. Aufgaben, Leistungen und Pläne. In: Schuppenhauer, Claus (Hrsg.): Plattdeutsch in Literatur und Gesellschaft. Eine Tagung zum 130. Todestag von John Brinckman, veranstaltet im Namen der Niederdeutsch-Beiräte aus Mecklenburg-Vorpommern und Schleswig-Holstein (Schriften des Instituts für niederdeutsche Sprache. Reihe: Dokumentation; 21). Leer 2001, S. 9-19.

Diedrich, Susanne: Zur Situation des Niederdeutschen in der Schule in Mecklenburg-Vorpommern. In: Schuppenhauer, Claus (Hrsg.): Plattdeutsch in Literatur und Gesellschaft. Eine Tagung zum 130. Todestag von John Brinckman, veranstaltet im Namen der Niederdeutsch-Beiräte aus MecklenburgVorpommern und Schleswig-Holstein (Schriften des Instituts für Niederdeutsche Sprache. Reihe: Dokumentation; 21). Leer 2001 S. 45-49.

Diedrich, Susanne: „Plattdüütsch? Wir haben doch ganz andere Probleme!“ In: Niederdeutschpflege und Ehrenamt. Aktuelle Vermittlungsstrategien für die Regionalsprache Niederdeutsch. Wissenschaftliche Konferenz des Volkskulturinstituts Mecklenburg und Vorpommern e. V., des Niedersächsischen Heimatbundes und des Landesheimatbundes Sachsen-Anhalt e. V. am 02./03. November 2001 in Salzwedel (Beiträge zur Regional- und Landeskultur Sachsen-Anhalts; 24). Halle 2002, S. 65-82.

Fanslau, Gunda: Einige Betrachtungen über die Pflege der niederdeutschen Sprache und Literatur am Musischen Gymnasium Neubrandenburg. In: Heimathefte für Mecklenburg und Vorpommern 6, H. 3 (1996), S. 43-44.

Gassen, Kurt: Die niederdeutsche Abteilung der Universitätsbibliothek Greifswald ( $\mathrm{Zu}$ ihrem 25jährigen Bestehen). In: Pommersche Heimatpflege 2, H. 2 (1931), S. 112-115.

Gassen, Kurt: Die niederdeutsche Bewegung der Gegenwart. Pflege plattdeutscher Sprache und Literatur durch Bibliotheken, Institute, Vereine und Bühnen des 
niederdeutschen Sprachgebietes (Aus den Schätzen der Universitätsbibliothek zu Greifswald; 8). Greifswald 1933.

Gernentz, Hans Joachim: Niederdeutsch an der Universität Rostock 1946 bis 1983.

In: Herrmann-Winter, Renate (Hrsg.): Heimatsprache zwischen Ausgrenzung und ideologischer Einbindung. Niederdeutsch in der DDR. Frankfurt a. M. [u. a.] 1998, S. 101-107.

Gernentz, Marianne: Meine Erfahrungen mit Niederdeutsch-Kursen an der Volkshochschule Rostock in den siebziger Jahren. In: Herrmann-Winter, Renate (Hrsg.): Heimatsprache zwischen Ausgrenzung und ideologischer Einbindung. Niederdeutsch in der DDR. Frankfurt a. M. [u. a.] 1998, S. 281-285.

Gundlach, Heinz: Die niederdeutsche Sprachpflege in der DDR. In: Buske, Norbert (Hrsg.): Niederdeutsche Bibeltradition. Entwicklung und Gebrauch des Niederdeutschen in der Kirche. Berlin/Altenburg 1990, S. 71-81.

Gundlach, Jürgen: Niederdeutsch an der Deutschen Akademie der Wissenschaften zu Berlin - Erfahrungen einer Außenstelle. In: Herrmann-Winter, Renate (Hrsg.): Heimatsprache zwischen Ausgrenzung und ideologischer Einbindung: Niederdeutsch in der DDR. Frankfurt a. M. [u. a.] 1998, S. 109-124.

Herrmann-Winter, Renate: Kurt Gassen und die niederdeutsche Sammlung der Universitätsbibliothek Greifswald. In: Baltische Studien 83 (1997), S. 87-94.

Herrmann-Winter, Renate: Zur Geschichte der Dialektgeographie in Pommern. In: Asmus, Ivo/Porada, Haik Thomas/Schleinert, Dirk (Hrsg.): Geographische und historische Beiträge zur Landeskunde Pommerns. Festschrift für Eginhard Wegner zum 80. Geburtstag (Sonderband der Greifswalder Geographischen Arbeiten - Wissenschaftliche Beiträge des Geographischen Instituts der Universität Greifswald). Schwerin 1998, S. 299-304.

Hückstädt, Arnold: Zur Pflege der niederdeutschen Literatur und der niederdeutschen Mundart im Norden der DDR. In: Prowatke, Christa (Bearb.): Niederdeutsche Mundart und Literatur in der DDR. Hrsg. vom Mecklenburgischen Folklorezentrum für die drei Nordbezirke. Rostock 1985, S. 41-53.

Kauffold, Peter: Zur Zukunft des Niederdeutschen in Vorschule, Schule und Hochschule. In: Schuppenhauer, Claus (Hrsg.): Plattdeutsch in Literatur und Gesellschaft. Eine Tagung zum 130. Todestag von John Brinckman, veranstaltet im Namen der Niederdeutsch-Beiräte aus Mecklenburg-Vorpommern und Schleswig-Holstein (Schriften des Instituts für Niederdeutsche Sprache. Reihe: Dokumentation; 21). Leer 2001, S. 27-29.

Keuchel, Brigitte: Die Einführung des Rahmenplans für Niederdeutsch an den Schulen und die Beschäftigung mit niederdeutscher Literatur. In: Heimathefte für Mecklenburg und Vorpommern 6, H. 3 (1996), S. 40-43. 
Keuchel, Brigitte: Konferenz zur Evaluation des Rahmenplans für Niederdeutsch. In: Heimathefte für Mecklenburg und Vorpommern 7, H. 3 (1997), S. 37-39. Keuchel, Brigitte: Umsetzung des Rahmenplans Niederdeutsch. In: Heimathefte für Mecklenburg und Vorpommern 7, H. 4 (1997), S. 42.

Keuchel, Brigitte: Erhebung zum Fachbereich Niederdeutsch an den Schulen in Mecklenburg-Vorpommern. In: Heimathefte für Mecklenburg und Vorpommern 8, H. 3 (1998), S. 46-49.

Kohfeldt, Gustav: Die Universität Rostock und das Niederdeutsche. In: Niederdeutsches Jahrbuch 44 (1918), S. 73-94.

Neumann, Siegfried: Zur Forschungstradition und -perspektive des Instituts für Volkskunde in Mecklenburg-Vorpommern (mit dem Wossidlo-Archiv). In: Schmitt, Christoph (Hrsg.): Informationen zur Volkskunde. Beiträge zu einem Kolloquium an der Universität Rostock am 11.4.1997 in Warnemünde (Kleine Schriften des Wossidlo-Archivs; 4). Rostock 1997 S. 41-51.

Osnowski, Stefan: Niederdeutsch in den Deutsch-Lehrplänen der DDR. In: Herrmann-Winter, Renate (Hrsg.): Heimatsprache zwischen Ausgrenzung und ideologischer Einbindung. Niederdeutsch in der DDR. Frankfurt a. M. [u. a.] 1998, S. 271-279.

Reifferscheid, Alexander: Über Pommerns Anteil an der niederdeutschen Sprachforschung. In: Niederdeutsches Jahrbuch 13 (1887), S. 33-42

Rösler, Irmtraud: Niederdeutsch und Schule. In: Herrmann-Winter, Renate (Hrsg.): Heimatsprache zwischen Ausgrenzung und ideologischer Einbindung. Niederdeutsch in der DDR. Frankfurt a. M. [u. a.] 1998, S. 257-269.

Schmidt, Marion: Zur Folklorepflege in den Nordbezirken der DDR. In: Jahrbuch für Volkskunde und Kulturgeschichte 23 (1980), S. 149-151.

Schönfeld, Helmut: Sprache - Heimatgeschichte. Mundart, Gruppensprachen, Umgangssprache, Namengut als Gegenstand von Forschung und Pflege (Arbeitsmaterial des ZFA Kulturgeschichte/Volkskunde). Berlin 1983.

Schröder, Ingrid: Niederdeutsch an den Hochschulen in MecklenburgVorpommern. In: Schuppenhauer, Claus (Hrsg.): Plattdeutsch in Literatur und Gesellschaft. Eine Tagung zum 130. Todestag von John Brinckman, veranstaltet im Namen der Niederdeutsch-Beiräte aus Mecklenburg-Vorpommern und Schleswig-Holstein (Schriften des Instituts für Niederdeutsche Sprache. Reihe: Dokumentation; 21). Leer 2001, S. 35-43.

Schröder, Ingrid: Die Zukunft des akademischen Faches Niederdeutsch. In: Stellmacher, Dieter (Hrsg.): Zur Wissenschaft vom Niederdeutschen. Beiträge zu einem Fachjubiläum und Dokumentation eines Kapitels germanischer Fachgeschichte an der Georg-August-Universität Göttingen. Neumünster 2005, S. 43-66. 
Spiewok, Wolfgang: Kulturpolitische Aspekte der Pflege des Niederdeutschen. In: Theil, Hans-Joachim (Red.): Niederdeutsch heute. Materialien einer Arbeitstagung des Freundeskreises Niederdeutsche Sprache und Literatur im Kulturbund der DDR, Kreisleitung Rostock 16.-17. November 1974. Rostock 1975, S. 3-11.

Strehlow, Gerda: Niederdeutschpflege im Mecklenburgischen Folklorezentrum. In: Herrmann-Winter, Renate (Hrsg.): Heimatsprache zwischen Ausgrenzung und ideologischer Einbindung. Niederdeutsch in der DDR. Frankfurt a. M. [u. a.] 1998, S. 347-354.

Strehlow, Gerda: Möglichkeiten der Zusammenarbeit zwischen plattdeutschen Vereinen, Schulen und Kitas. In: Niederdeutschpflege und Ehrenamt. Aktuelle Vermittlungsstrategien für die Regionalsprache Niederdeutsch. Wissenschaftliche Konferenz des Volkskulturinstituts Mecklenburg und Vorpommern e. V., des Niedersächsischen Heimatbundes und des Landesheimatbundes SachsenAnhalt e. V. am 02./03. November 2001 in Salzwedel (Beiträge zur Regionalund Landeskultur Sachsen-Anhalts; 24). Halle 2002, S. 83-85.

Teuchert, Hermann: Niederdeutsche Forschung an der Universität. In: Mecklenburgische Monatshefte 9 (1933), S. 281-283.

Töteberg, Michael/Theilig, Ulrike: „Up Platt is ook hüt noch wat“. Niederdeutsche Gegenwartsliteratur und Pflege des Plattdeutschen in der DDR. In: Michelsen, Friedrich W./Spiekermann, Gerd (Hrsg.): Dat en Spoor blifft. Ulf Bichel zum 60. Geburtstag. Göttingen 1985, S. 151-161.

Töteberg, Michael: Mundartpflege, plattdeutsche Volkskunst und niederdeutsche Gegenwartsliteratur in der DDR. In: Reichardt, Hartmut (Hrsg.): Konjunktur und Kommerz mit Plattdeutsch (Loccumer Protokolle 1986; 6). RehburgLoccum 1987, S. 29-35.

Wirrer, Jan: „Dat Negere rägelt dat Gesetz.“ Anmerkungen zur niederdeutschen Übersetzung der Verfassung von Mecklenburg-Vorpommern. In: Niederdeutsches Wort 43 (2003), S. 253-269.

\section{Regiolekt in Mecklenburg-Vorpommern}

\section{1 Überblicksdarstellungen zu den Regiolekten in Norddeutschland}

Berend, Nina: Regionale Gebrauchsstandards - Gibt es sie und wie kann man sie beschreiben? In: Eichinger, Ludwig/Kallmeyer, Werner (Hrsg.): Standardvariation. Wie viel Variation verträgt die deutsche Sprache. Berlin/New York, S. $143-170$.

Eichhoff, Jürgen: Wortatlas der deutschen Umgangssprachen. Bd. 1: Bern/München 1977; Bd. 2: Bern/München 1978; Bd. 3: München [u. a.] 1993. 
Elmentaler, Michael/Rosenberg, Peter: Norddeutscher Sprachatlas (NOSA). Bd. 1: Regiolektale Sprachlagen. Unter Mitarbeit von Andresen, Liv/Ehlers, KlaasHinrich/Eichhorn, Krisin/Langhanke, Robert/Reuter, Hannah/Scharioth, Claudia/ Wilcken, Viola. Kartografie: Ulrike Schwedler. (Deutsche Dialektgeographie; 113.1). Hildesheim/Zürich/New York 2015.

Elspaß, Stephan/Möller, Robert: Atlas der deutschen Alltagssprache (AdA). 2003 ff. [Online-Ressource: http://www.atlas-alltagssprache.de/ (Stand: 24.11.2016)]

Kleiner, Stefan: Atlas zur Aussprache des deutschen Gebrauchsstandards (AADG). Unter Mitarbeit von Ralf Knöbl. Mannheim 2011 ff. [Online-Ressource: http:// prowiki.ids-mannheim.de/bin/view/AADG/ (Stand: 24.11.2016)]

König, Werner/Elspass, Stephan/Möller, Robert: dtv-Atlas zur deutschen Sprache. Tafeln und Texte. Graphiken von Hans-Joachim Paul. 18., durchges. und korr. Aufl. München 2015.

Kretschmer, Paul: Wortgeographie der hochdeutschen Umgangssprache. Göttingen 1918.

Lauf, Raphaela: ,Regional markiert'. Großräumliche Umgangssprache(n) im niederdeutschen Raum. In: Niederdeutsches Jahrbuch 119 (1996), S. 193-218.

Martens, Hiltrud/Martens, Peter: Niederdeutsch-bedingte Abweichungen von der hochdeutschen Standardaussprache. In: Behme, Helena (Hrsg.): Angewandte Sprechwissenschaft. Interdisziplinäre Beiträge zur mündlichen Kommunikation. Stuttgart 1988, S. 123-136.

Mihm, Arend: Die Rolle der Umgangssprachen seit der Mitte des 20. Jahrhunderts. In: Besch, Werner [u. a.] (Hrsg.): Sprachgeschichte. Ein Handbuch zur Geschichte der deutschen Sprache und ihrer Erforschung (Handbücher zur Sprach- und Kommunikationswissenschaft; 2.2). 2. Teilb., 2., vollständig neu bearb. und erw. Aufl. Berlin/New York 2000, Sp. 2106-2137.

Möhn, Dieter: Missingsch. In: Munske, Horst Haider (Hrsg.): Deutsch im Kontakt mit germanischen Sprachen. Tübingen 2004. S. 119-140.

Möller, Robert: Wortgebrauchsräume in der nord- und mitteldeutschen Alltagssprache - eine Untersuchung zum Wortatlas der deutschen Umgangssprachen. In: Niederdeutsches Wort 42 (2001), S. 1-31.

Möller, Robert: Zur diatopischen Gliederung des alltagssprachlichen Wortgebrauchs. Eine dialektometrische Auswertung von Jürgen Eichhoff: Wortatlas der deutschen Umgangssprachen. In: Zeitschrift für Dialektologie und Linguistik 70/1 (2003), S. 259-297.

Protze, Helmut: Wortatlas der städtischen Umgangssprachen. Zur theoretischen Differenzierung der Sprache in Mecklenburg-Vorpommern, Brandenburg, 
Berlin, Sachsen-Anhalt, Sachsen und Thüringen. (Mitteldeutsche Forschungen; 114). Köln/Weimar/Wien 1997.

\section{2 Überblicksdarstellungen zum Regiolekt in Mecklenburg-Vorpommern}

Dahl, Eva-Sophie: Interferenz und Alternanz - zwei Typen der Sprachschichtenmischung im Norden der Deutschen Demokratischen Republik. In: Ising, Gerhard (Hrsg.): Aktuelle Probleme der sprachlichen Kommunikation. (Sprache und Gesellschaft; 2) Berlin 1974, S. 339-388.

Gernentz, Hans Joachim: System und Verwendung der Existenzformen des Deutschen im Norden der Deutschen Demokratischen Republik. In: Wissenschaftliche Zeitschrift der Universität Rostock. Gesellschafts- und sprachwissenschaftliche Reihe 24 (1975), S. 385-393.

Herrmann-Winter, Renate: Standardsprache und Mundarten in der Deutschen Demokratischen Republik. In: Stickel, Gerhard (Hrsg.): Deutsche Gegenwartssprache. Tendenzen und Perspektiven (Jahrbuch des Instituts für deutsche Sprache 1989). Berlin/New York 1990, S. 184-191.

Rosenberg, Peter: Mecklenburgisch-vorpommerscher Raum. In: Elmentaler, Michael/Rosenberg, Peter: Norddeutscher Sprachatlas (NOSA). Bd. 1: Regiolektale Sprachlagen. Hildesheim/Zürich/New York 2015, S. 47-55.

Schönfeld, Helmut: Sprache und Sprachvariation in der Stadt. Zur sprachlichen Entwicklung und Sprachvariation in Berlin und anderen Städten im Nordteil der DDR. (Linguistische Studien. Reihe A: Arbeitsberichte; 197). Berlin 1989.

\subsection{Grammatik und Regiolektographie}

Born, Karl: Die mecklenburgsche und pommersche Aussprache des Hochdeutschen mit ihren Maengeln. In: Freimuethiges Abendblatt 15, Nr. 763 (1833), Sp. 649-656.

Dahl, Eva-Sophie: Niederdeutsches in der hochdeutschen Rostocker Umgangssprache des 18. Jahrhunderts. In: Wissenschaftliche Zeitschrift der Universität Rostock. Gesellschafts- und sprachwissenschaftliche Reihe 7, H. 1 (1957/58), S. 68-79.

Dahl, Eva-Sophie: Interferenz und Alternanz - zwei Typen der Sprachschichtenmischung im Norden der Deutschen Demokratischen Republik. In: Ising, Gerhard (Hrsg.): Aktuelle Probleme der sprachlichen Kommunikation. (Sprache und Gesellschaft; 2) Berlin 1974, S. 339-388.

Dahl, Eva-Sophie: Substantivische Komposita in der mecklenburgischen Mundart und in der Hochsprache. Ein Vergleich nach Bildungstypen und deren Auf- 
treten. In: Wissenschaftliche Zeitschrift der Universität Rostock. Gesellschaftsund sprachwissenschaftliche Reihe 27 (1978), S. 29-36.

Dahl, Eva-Sophie: Niederdeutsche Verbalkomposita als Interferenzmerkmale im gesprochenen Hochdeutsch der Nordbezirke. Grundlagen und Grenzen ihres Auftretens. In: Zentralinstitut für Sprachwissenschaft der Akademie der Wissenschaften der DDR (Hrsg.): Das Niederdeutsche in Geschichte und Gegenwart. 1. Bd. Berlin 1981, S. 105-111.

Dost, Wolfgang: Untersuchungen zu den sprachlichen Existenzformen. Mundart und Umgangssprache im Norden der Mark Brandenburg (Raum Wittstock). In: Beiträge zur Erforschung der deutschen Sprache 10 (1991), S. 240-266.

Dost, Wolfgang: Zur Einwirkung der kommunikativen Bedingungen in der DDR auf die regionale Abgrenzung im Bereich der Umgangssprache und der Mundart, dargestellt an der Entwicklung im Raum Wittstock-Roebel. In: Das Niederdeutsche in Geschichte und Gegenwart. Hrsg. vom Zentralinstitut für Sprachwissenschaft der Akademie der Wissenschaften der DDR (Linguistische Studien. Reihe A: Arbeitsberichte; 75.1). Berlin 1981, S. 121-127.

Ehlers, Klaas-Hinrich: „Dürfen wir essing?“ Beobachtungen zur Übernahme des mecklenburgischen ing-Diminutivs in das regionale Hochdeutsch. In: Niederdeutsches Jahrbuch 134 (2011), S. 79-92.

Herrmann-Winter, Renate: Studien zur gesprochenen Sprache im Norden der DDR. Soziolinguistische Untersuchungen im Kreis Greifswald. Berlin 1979.

Jordan, Kurt: Umgangssprachliche Ausdrücke aus dem alten Pommern. In: Pommern. Kultur und Geschichte 2 (1991), S. 13-15, S. 29-30; 7 (1997), S. 30-31.

Jürgens, Carolin: Kontakt - Spaltung - Dopplung. Zur Variation der Pronominaladverbien in der norddeutschen Umgangssprache. In: Hettler, Yvonne/Jürgens, Carolin/Langhanke, Robert/Purschke, Christoph (Hrsg.): Variation, Wandel, Wissen. Studien zum Hochdeutschen und zum Niederdeutschen. (Sprache in der Gesellschaft; 32). Frankfurt a. M. [u. a.] 2013, S. 129-143.

Kehrein, Roland: Regionalsprachliche Spektren im Raum. Zur linguistischen Struktur der Vertikale (ZDL Beihefte; 152). Stuttgart 2012.

Skoczek, Robert: Der endogene Aussprachestandard in Norddeutschland und seine Auto- und Heterostereotype. Diss. Lublin 2007.

Skoczek, Robert: Zum phonetischen Nachlass des Niederdeutschen in der Aktualisierung der Standardsprache in Mecklenburg. In: Grucza, Franticzek (Hrsg.): Vielheit und Einheit der Germanistik weltweit. Bd. 17: Diachronische, diatopische und typologische Aspekte des Sprachwandels. Frankfurt a. M. [u. a.] 2013, S. 383-393.

Teuchert, Hermann: Missingsch. In: Beiträge zur Geschichte der deutschen Sprache und Literatur 82 (1961), S. 245-265. 


\subsection{Soziolinguistik, Pragmatik und Perzeptionslinguistik}

Bichel, Ulf: Typisches und Privates im Missingsch des Inspektor Bräsig. In: Bichel, Ulf/Minssen, Friedrich/de Voss, Helmut (Hrsg.): Vom Reichtum des Erzählens. Fritz Reuter 1810-1874. München/Wien 1985, S. 76-92.

Boehm, Carl: Der Broiler lebt: Die deutsche Sprache im Wandel zwischen DDR und BRD. Ergebnisse einer interdisziplinären Untersuchung im Bereich der mecklenburgischen Landeshauptstadt Schwerin im Sommer 1991. In: Zeitschrift für Germanistik, NF. 2, H. 2 (1992), S. 320-340.

Chudnitzki, Wladimir S.: Zur kommunikativen Funktion der sprachlichen Existenzformen in Mecklenburg. Soziolinguistische Untersuchungen in der Schiffswerft Rechlin und ihrer engeren Umgebung. In: Beiträge zur Erforschung der deutschen Sprache 10 (1991), S. 223-239.

Ehlers, Klaas-Hinrich: „Dürfen wir essing?“ Beobachtungen zur Übernahme des mecklenburgischen ing-Diminutivs in das regionale Hochdeutsch. In: Niederdeutsches Jahrbuch 134 (2011), S. 79-92.

Ehlers, Klaas-Hinrich: Führte die Immigration der Heimatvertriebenen nach 1945 zu Dialektverlust und Nivellierung regionalsprachlicher Differenzen? Beobachtungen aus einer Unterschungsregion in Mecklenburg. In: Niederdeutsches Jahrbuch 136 (2013), S. 97-116.

Ewald, Petra: Auffälligkeit um jeden Preis? Zur Berichterstattung über alltägliche Kriminalität in lokalen Tageszeitungen Mecklenburg-Vorpommerns. In: Rösler, Irmtraud/Sommerfeldt, Karl-Ernst (Hrsg.): Probleme der Sprache nach der Wende. Beiträge des Kolloquiums in Rostock am 16. November 1996. Frankfurt a. M. [u. a.] 1997, S. 81-91.

Gernentz, Hans Joachim: Die kommunikative Funktion der niederdeutschen Mundart und hochdeutschen Umgangssprache im Norden der Deutschen Demokratischen Republik, unter besonderer Berücksichtigung der Interferenz und der Alternanz zwischen diesen beiden sprachlichen Existenzformen. In: Studia Germanica Gandensia 15 (1974), S. 209-244.

Gundlach, Jürgen: Missingsch bei Reuter. Form und Funktion In: Kikut. Plattdütsch gistern un hüt 10 (1985), S. 68-75.

Herrmann-Winter, Renate: Auswirkungen der sozialistischen Produktionsweise in der Landwirtschaft auf die sprachliche Kommunikation in den Nordbezirken der Deutschen Demokratischen Republik. In: Ising, Gerhard (Hrsg.): Aktuelle Probleme der sprachlichen Kommunikation (Sprache und Gesellschaft; 2). Berlin 1974, S. 135-190. 
Herrmann-Winter, Renate: Studien zur gesprochenen Sprache im Norden der DDR. Soziolinguistische Untersuchungen im Kreis Greifswald. Berlin 1979.

Huesmann, Annette: Zwischen Dialekt und Standard. Empirische Untersuchung zur Soziolinguistik des Varietätenspektrums in Deutschland. Tübingen 1998.

Lohoff, Heinrich: Die systematische Bekämpfung ortsüblicher Fehler im Sprechen und im Schreiben. (Mit zwei Beispiel-Sammlungen) (Zur Volksschulpädagogik; 18). Langensalza 1910.

Scharioth, Claudia: Regionales Sprechen und Identität. Eine Studie zum Sprachgebrauch, zu Spracheinstellungen und zu Identitätskonstruktionen von Frauen in Schleswig-Holstein und Mecklenburg-Vorpommern (Deutsche Dialektgeographie; 120). Hildesheim/Zürich/New York 2015.

Schomaker, Angi: „Wenn Fischköppe snacken“ - eine Untersuchung zur laienlinguistischen Wahrnehmung der Mecklenburger Umgangssprache. Staatsexamensarbeit Rostock 2013. 